Nevada

Environmental

Restoration

Project

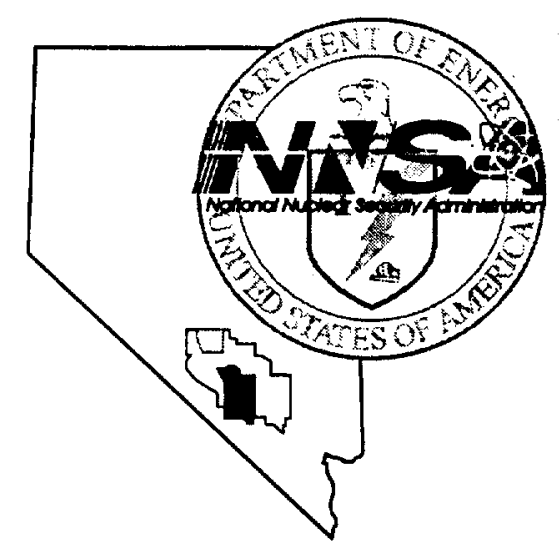

Streamlined Approach for

Environmental Restoration Plan for Corrective Action Unit 398: Area 25 Spill Sites, Nevada Test Site, Nevada

Controlled Copy No.:

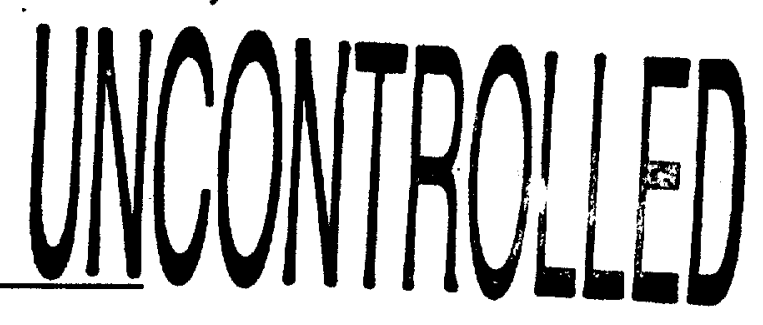

Revision: 0

November 2001

Environmental Restoration

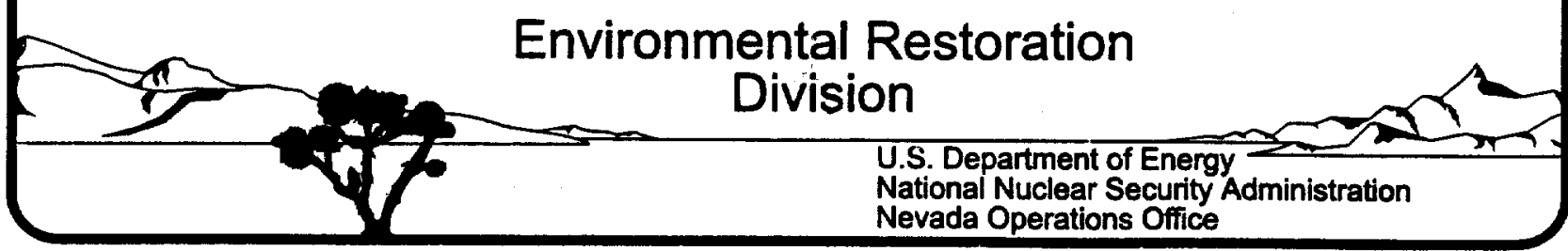




\section{DISCLAIMER STATEMENT}

Reference herein to any specific commercial product, process, or service by trade name, trademark, manufacturer, or otherwise, does not necessarily constitute or imply its endorsement, recommendation, or favoring by the U.S. Government or any agency thereof or its contractors or subcontractors.

\section{AVAILABILITY STATEMENT}

Available for sale to the public from-

U.S. Department of Commerce

National Technical Information Service

5285 Port Royal Road

Springfield, VA 22161-0002

Telephone: 800.553 .6847

Fax: 703.605.6900

E-mail: orders@ntis.fedworld.gov

Online ordering: http://www.ntis.gov/ordering.htm

Available electronically at http://www.doe.gov/bridge

Available for a processing fee to U.S. Department of Energy and its contractors, in paper, fromU.S. Department of Energy

Office of Scientific and Technical Information

P.O. Box 62

Oak Ridge, TN 37831-0062

Telephone: 865.576 .8401

Fax: 865.576.5728

E-mail: reports@adonis.osti.gov 


\section{Department of Energy \\ National Nuclear Security Administration \\ Nevada Operations Office \\ P.O. Box 98518 \\ Las Vegas, NV 89193-8518}

JAN 242002 DOE/NU/11718--657

Southern Nevada Public Reading Room

P.O. Box 98521 , M/S NLV 040

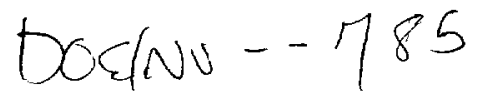

Las Vegas, NV 89193-8521

Northern Nevada Public Reading Room

$$
\text { Jogia for ODF } 1 / 29 / 62
$$

Nevada State Library and Archives

100 N. Stewart St.

Carson City, NV 89701-4285

SUBMITTAL OF THE STREAMLINED APPROACH FOR ENVIRONMENTAL

RESTORATION PLAN FOR CORRECTIVE ACTOIN UNIT 398: AREA 25 SPILL SITES, NEVADA TEST SITE, NEVADA, REVISION 0, NOVEMBER 2001

The Federal Facility Agreement and Consent Order (FFACO) requires that the enclosed document is available to the public through the FFACO Public Reading Rooms. The following copies have been provided for submittal into the FFACO Public Reading Room collection in accordance with the DOE/NV Distribution Process for FFACO Documents.

$$
\begin{array}{ll}
\text { Southern Nevada Public Reading Room: } & 1 \text { controlled copy } \\
& 1 \text { uncontrolled copy }
\end{array}
$$

Northern Nevada Public Reading Room: 1 uncontrolled copy

Please direct comments and questions to Sabine T. Curtis, of my staff, at (702) 295-0542.

ERD:010.STC

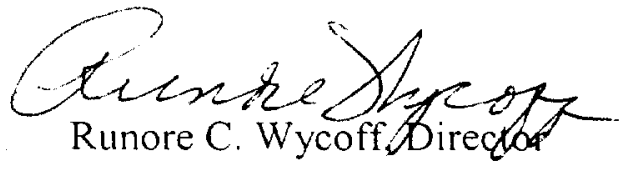

Environmental Restoration Division

Enclosure:

As stated

cc w/encl. (controlled):

R.B. Hudson, BN, Las Vegas, NV

FFACO Support Office, IT, Las Vegas, NV 
cc w/encl. (uncontrolled):

TIRC, NNSA/NV, Las Vegas, NV

BN, Document Production (electronic copy to OSTI)

cc w/o encl:

M. A. DeBurle, NDEP, Carson City, NV

Eric Shanholtz, DTRA, Mercury, NV

LTC P. M. Loomis, DTRA, M/S 645, Mercury, NV

DTRA Environmental, M/S 645, Mercury, NV

BN Technical Information Officer, M/S NLV048, Las Vegas, NV

G. M. Romano, IT, Las Vegas, NV

K. A. Hoar, ESHD, NNSA/NV, Las Vegas, NV

J. M. Ford, OPAI, NNSA/NV, Las Vegas, NV

P. L. Hall, EM, NNSA/NV, Las Vegas, NV

J. L. Appenzeller-Wing, ERD, NNSA/NV, Las Vegas, NV 


\title{
STREAMLINED APPROACH FOR ENVIRONMENTAL RESTORATION PLAN FOR CORRECTIVE ACTION UNIT 398: AREA 25 SPILL SITES, NEVADA TEST SITE, NEVADA
}

\author{
Prepared for: \\ U.S. Department of Energy \\ National Nuclear Security Administration \\ Nevada Operations Office \\ Under Contract No. DE-AC08-96-NV11718
}

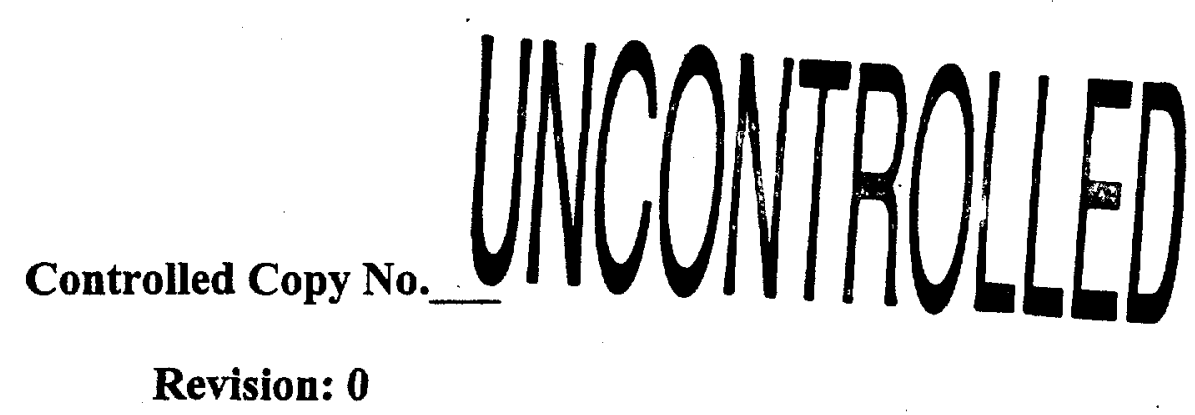

November 2001 


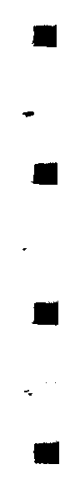

THIS PAGE INTENTIONALLY LEFT BLANK

$\mathbf{0}$

- 


\section{STREAMLINED APPROACH FOR ENVIRONMENTAL RESTORATION PLAN FOR CORRECTIVE ACTION UNIT 398: AREA 25 SPILL SITES, NEVADA TEST SITE, NEVADA}

Approved by: $\frac{\text { Sabine Cuntis }}{\text { fo Janet L. Appenzeller-Wing, Project Manager }}$

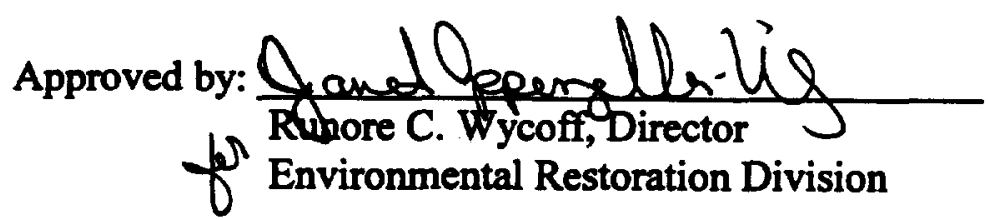

Date: $11 / 13 / 01$

Date: $11 / 13 / 01$ 


\section{THIS PAGE INTENTIONALLY LEFT BLANK}




\section{TABLE OF CONTENTS}

ACRONYMS AND ABBREVIATIONS $\ldots \ldots \ldots \ldots \ldots \ldots \ldots \ldots \ldots \ldots \ldots$ ix

EXECUTIVE SUMMARY $\ldots \ldots \ldots \ldots \ldots \ldots \ldots \ldots \ldots \ldots \ldots \ldots \ldots \ldots \ldots \ldots \ldots \ldots$

$1.0 \quad$ INTRODUCTION $\ldots \ldots \ldots \ldots \ldots \ldots \ldots \ldots \ldots \ldots \ldots \ldots \ldots \ldots \ldots \ldots \ldots$

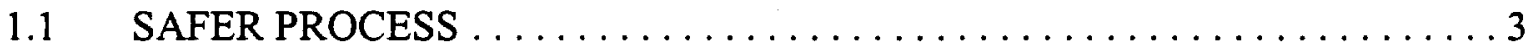

1.2 SUMMARY OF PROPOSED CORRECTIVE ACTION $\ldots \ldots \ldots \ldots \ldots \ldots$

$1.3 \quad$ HOLD/DECISION POINTS $\ldots \ldots \ldots \ldots \ldots \ldots \ldots \ldots \ldots \ldots \ldots \ldots \ldots$

1.4 SAFER WORK PLAN CONTENTS $\ldots \ldots \ldots \ldots \ldots \ldots \ldots \ldots \ldots \ldots \ldots$

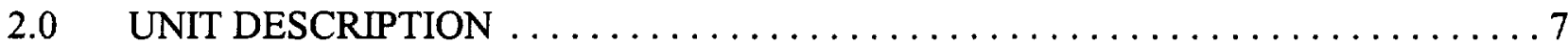

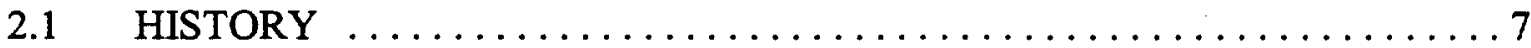

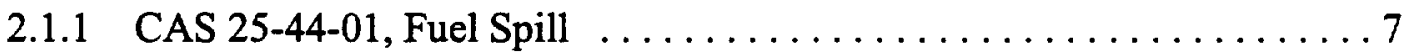

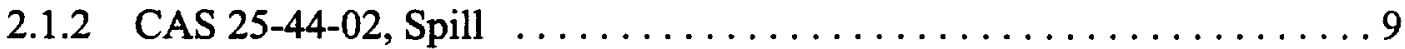

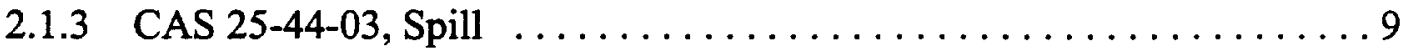

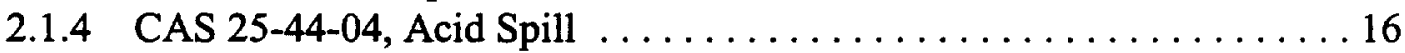

2.1.5 CAS 25-25-02, Oil Spills .................... 16

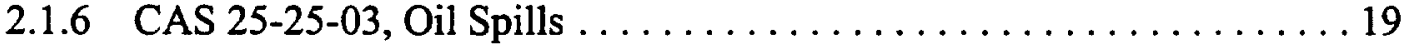

2.1 .7 CAS $25-25-04$, Oil Spills . . . . . . . . . . . . . . . . . 19

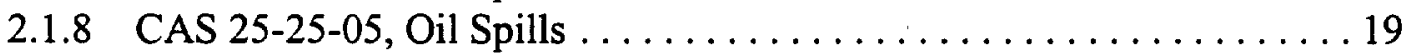

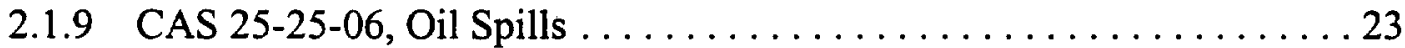

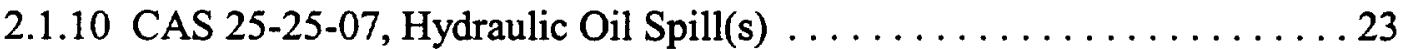

2.1.11 CAS 25-25-08, Hydraulic Oil Spill(s) ............... 25

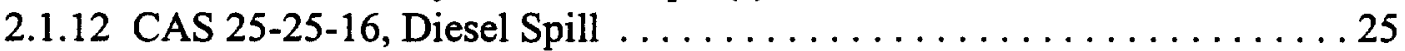

2.1.13 CAS 25-25-17, Subsurface Hydraulic Oil Spill . . . . . . . . . . . 25

2.2 SITE LOCATION AND DESCRIPTION $\ldots \ldots \ldots \ldots \ldots \ldots \ldots \ldots \ldots \ldots$

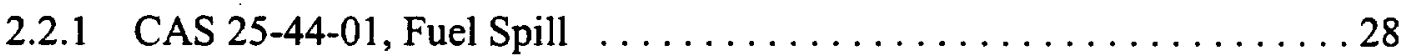

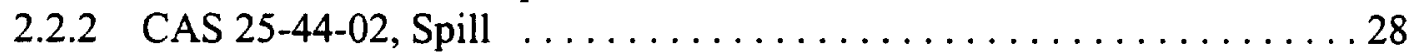

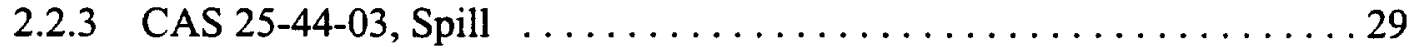

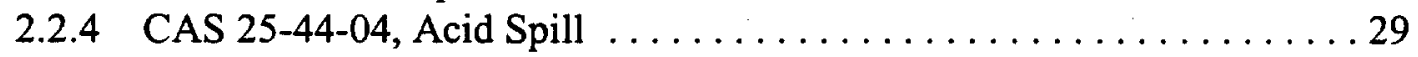

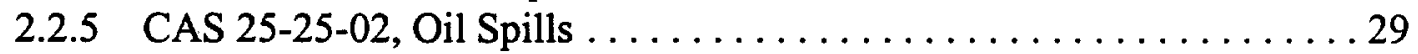

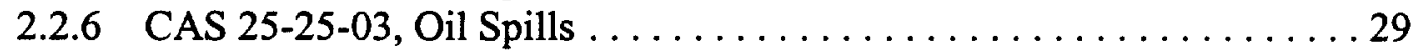

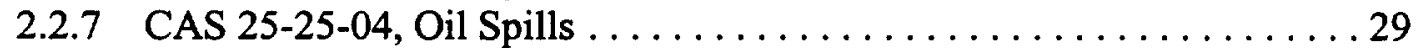

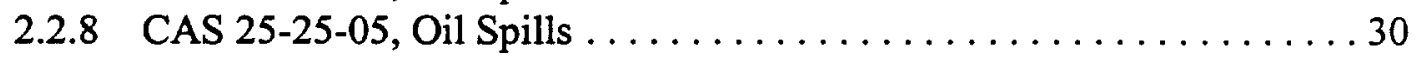

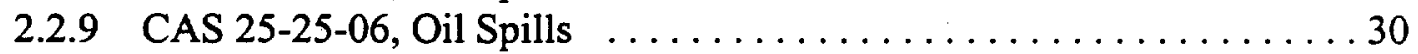

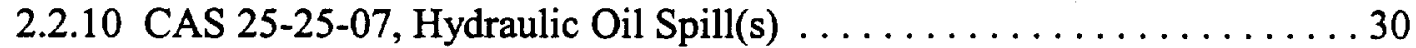

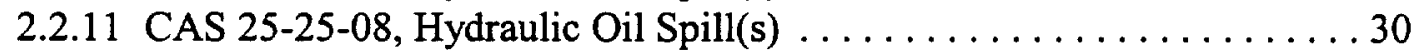

2.2.12 CAS 25-25-16, Diesel Spill .................... 30

2.2.13 CAS 25-25-17, Subsurface Hydraulic Oil Spill . . . . . . . . . . . 31

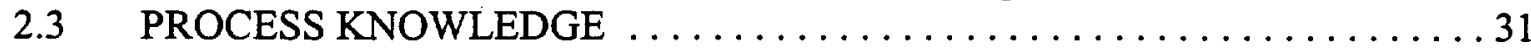

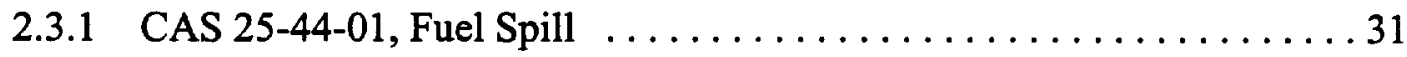

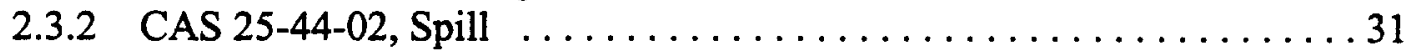

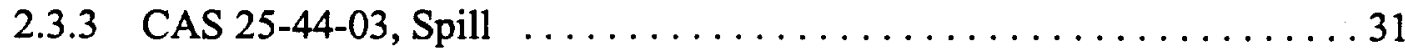

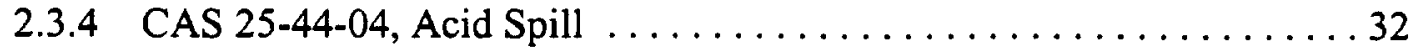




\section{TABLE OF CONTENTS (continued)}

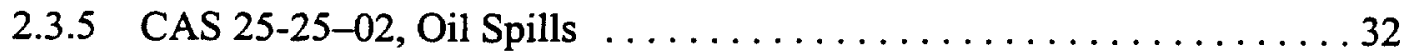

2.3.6 CAS 25-25-03, Oil Spills .......................... 32

2.3.7 CAS 25-25-04, Oil Spills .......................... 32

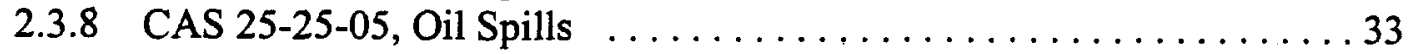

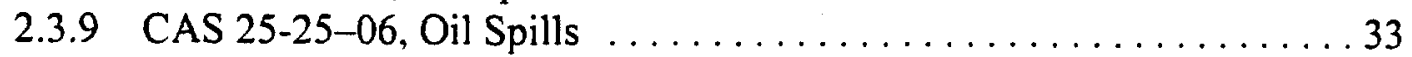

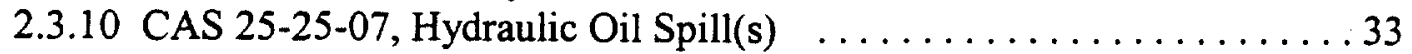

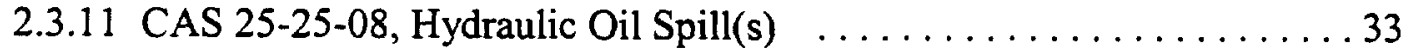

2.3.12 CAS 25-25-16, Diesel Spill ........................... 33

2.3.13 CAS 25-25-17, Subsurface Hydraulic Oil Spill .............. 34

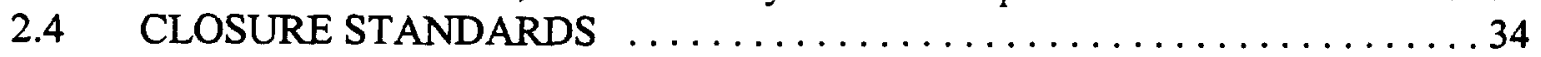

3.0 FIELD ACTIVITIES AND CLOSURE OBJECTIVES $\ldots \ldots \ldots \ldots \ldots \ldots \ldots \ldots \ldots$

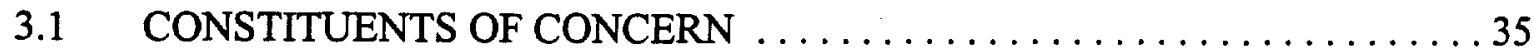

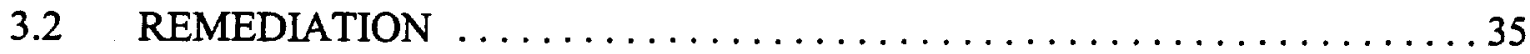

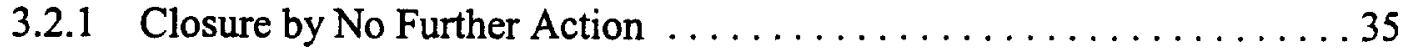

3.2.2 Clean Closure by Excavation and Disposal ................ 35

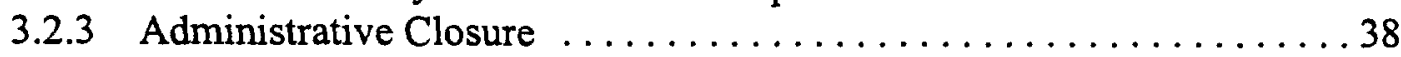

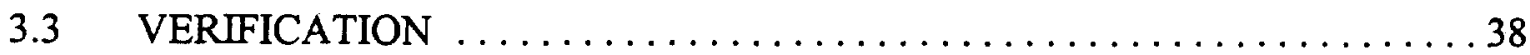

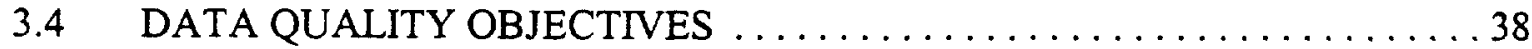

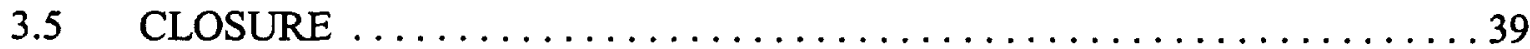

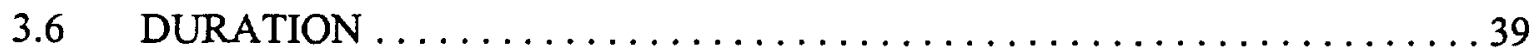

4.0 REPORTS AND RECORDS AVAILABILITY $\ldots \ldots \ldots \ldots \ldots \ldots \ldots \ldots \ldots$

$5.0 \quad$ INVESTIGATION/REMEDIATION WASTE MANAGEMENT $\ldots \ldots \ldots \ldots \ldots . \ldots 43$

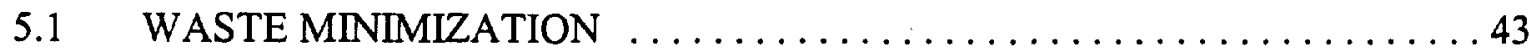

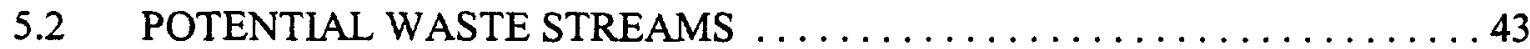

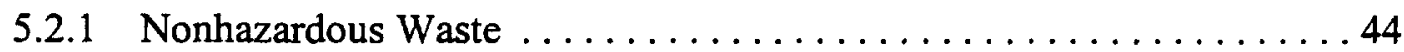

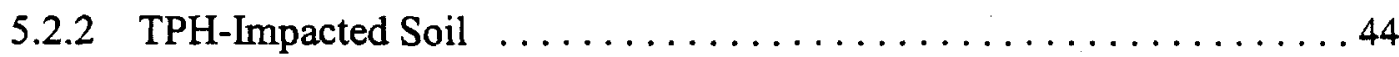

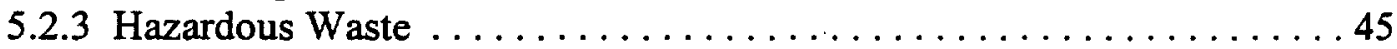

5.3 CONTAINER MANAGEMENT $\ldots \ldots \ldots \ldots \ldots \ldots \ldots \ldots \ldots \ldots \ldots \ldots$

6.0 QUALITY ASSURANCE/QUALITY CONTROL . . . . . . . . . . . . . . . . 47

6.1 PROPOSED FIELD SAMPLE COLLECTION ACTIVITIES $\ldots \ldots \ldots \ldots \ldots 47$

6.2 PROPOSED LABORATORY/ANALYTICAL DATA QUALITY INDICATORS 


\section{TABLE OF CONTENTS (continued)}

\section{APPENDICES}

APPENDIX A-1 CHARACTERIZATION SAMPLE ANALYTICAL RESULTS

APPENDLX A-2 DATA QUALITY OBJECTIVES

APPENDIX A-3 PROJECT ORGANIZATION

APPENDIX A-4 NDEP DOCUMENT REVIEW SHEET

DISTRIBUTION LIST

\section{FIGURES}

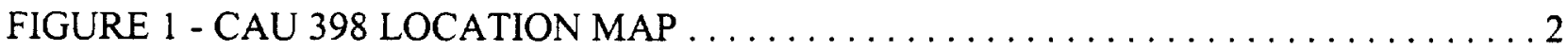

FIGURE 2 - CAS $25-44-01$ SPILL SITE LOCATION $\ldots \ldots \ldots \ldots \ldots \ldots \ldots \ldots \ldots$

FIGURE 3 - CAS $25-44-02$ SPILL SITE LOCATION . . . . . . . . . . . . . . 14

FIGURE 4 - CAS $25-44-03$ SPILL SITE LOCATION $\ldots \ldots \ldots \ldots \ldots \ldots \ldots \ldots \ldots$

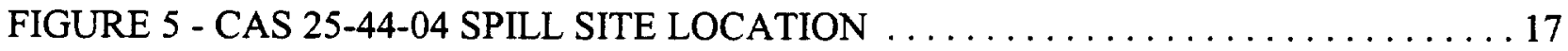

FIGURE 6 - CAS $25-25-02$ SPILL SITE LOCATION $\ldots \ldots \ldots \ldots \ldots \ldots \ldots \ldots \ldots$

FIGURE 7 - CAS $25-25-03$ SPILL SITE LOCATION . . . . . . . . . . . . . . 20

FIGURE 8 - CAS $25-25-04$ SPILL SITE LOCATION $\ldots \ldots \ldots \ldots \ldots \ldots \ldots \ldots \ldots$

FIGURE 9 - CAS $25-25-05$ SPILL SITE LOCATION . . . . . . . . . . . . . . . 22

FIGURE 10 - CAS $25-25-06$ SPILL SITE LOCATION . . . . . . . . . . . . . . . . . . . 24

FIGURE 11 - CAS $25-25-16$ SPILL SITE LOCATION $\ldots \ldots \ldots \ldots \ldots \ldots \ldots \ldots \ldots$

FIGURE 12 - CAS 25-25-17 SPILL SITE LOCATION . . . . . . . . . . . . . 27

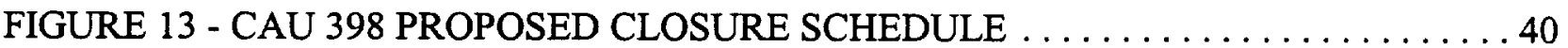




\section{TABLE OF CONTENTS (continued)}

\section{TABLES}

TABLE 1 - CAU SAMPLE ANALYTICAL RESULTS $\ldots \ldots \ldots \ldots \ldots \ldots \ldots \ldots$

TABLE 2 - CAU 398 CONSTITUENTS OF CONCERN $\ldots \ldots \ldots \ldots \ldots \ldots \ldots \ldots$

TABLE 3 - POTENTIAL WASTE STREAMS BY CAS AT CAU $398 \ldots \ldots \ldots \ldots \ldots$

TABLE 4 - MANAGEMENT OF WASTE TYPES TO BE PRODUCED

DURING CAU 398 CLOSURE . . . . . . . . . . . . . . . . . . . . . 44

TABLE 5 - LABORATORY/ANALYTICAL DATA QUALITY INDICATORS . . . . . . . 49

TABLE 6 - LABORATORY TPH PROCEDURE FOR CAU $398 \ldots \ldots \ldots \ldots \ldots \ldots \ldots$ 


\section{ACRONYMS AND ABBREVIATIONS}

BN

CAS

CAU

$\mathrm{cm}$

$\mathrm{COC}$

COPC

CR

CSM

DOE

DQI

DQO

E-MAD

EPA

ETS-1

FFACO

$\mathrm{ft}$

gal

in

IT

L

m

$\mathrm{m}^{3}$

$\mathrm{mg} / \mathrm{kg}$

$\mathrm{mg} / \mathrm{L}$

NAC

NDEM

NDEP

NNSA/NV
Bechtel Nevada

Corrective Action Site

Corrective Action Unit

centimeter(s)

constituent of concern

constituent of potential concern

Closure Report

conceptual site model

U.S. Department of Energy

data quality indicator

data quality objective

Engine-Maintenance, Assembly, and Disassembly

U.S. Environmental Protection Agency

Engine Test Stand-1

Federal Facility Agreement and Consent Order

foot/feet

gallon(s)

inch(s)

International Technology Corporation

liter(s)

meter(s)

cubic meter(s)

milligram(s) per kilogram

milligram(s) per liter

Nevada Administrative Code

Nevada Division of Emergency Management

Nevada Division of Environmental Protection

U.S. Department of Energy National Nuclear Security Administration Nevada Operations Office 


\section{ACRONYMS AND ABBREVIATIONS (continued)}

NTS

PA

$\mathrm{pCi} / \mathrm{g}$

PCB

PPE

ppm

PRG

QA

QC

RCRA

REECo

RPD

SAA

SAFER

SDG

SVOC

TCLP

TPH

TSCA

$\mu \mathrm{g} / \mathrm{kg}$

$\mu \mathrm{g} / \mathrm{L}$

VOC

$\mathrm{yd}^{3}$

$\% \mathrm{R}$
Nevada Test Site

preliminary assessment

picocuries per gram

polychlorinated biphenyls

personal protective equipment

parts per million

preliminary remediation goals

quality assurance

quality control

Resource Conservation and Recovery Act

Reynolds Electrical \& Engineering Company

relative percent difference

satellite accumulation area

Streamlined Approach for Environmental Restoration

sample delivery group

semivolatile organic compound

Toxicity Characteristic Leaching Procedure

total petroleum hydrocarbon

Toxic Substance Control Act

microgram(s) per kilogram

microgram(s) per liter

volatile organic compound

cubic yard(s)

percent recovery 


\section{EXECUTIVE SUMMARY}

This Streamlined Approach for Environmental Restoration Plan addresses the activities necessary to close Corrective Action Unit (CAU) 398: Area 25 Spill Sites. CAU 398, located in Area 25 of the Nevada Test Site, is currently listed in Appendix III of the Federal Facility Agreement and Consent Order (FFACO) (FFACO, 1996) and consists of the following 13 Corrective Action Sites (CASs):

- CAS 25-44-01, Fuel Spill. This site is described as a fuel spill on soil that covers a concrete pad. The origins and use of the spill material are unknown.

- CAS 25-44-02, Spill. This site consists of a spill to soil from leaking drums. The source of the drums is unknown. The drums have been removed from the site and disposed.

- CAS 25-44-03, Spill. This site is described as a spill from leaking drums onto a concrete pad and surrounding soil.

- CAS 25-44-04, Acid Spill. This site consists of spills from two tanks used for a water demineralization process. Tank T-2002 contained sodium hydroxide and Tank T-2003 contained sulfuric acid. The tanks have been removed from the site.

- CAS 25-25-02, Oil Spills. This site is described as an oil/fuel spill associated with leaking drums that were removed in 1992.

- CAS 25-25-03, Oil Spills. This site is described as a spill adjacent to a tipped-over drum. The source of the drum is unknown; the drum was removed in 1991.

- CAS 25-25-04, Oil Spills. This site consists of an area on the north side of the EngineMaintenance, Assembly, and Disassembly (E-MAD) facility, where used oils and cooling fluids from metal machining operations were poured directly onto the ground.

- CAS 25-25-05, Oil Spills. This site consists of oil/hydraulic fluid spills located where heavy equipment was once stored.

- CAS 25-25-06, Oil Spills. This site consists of diesel fuel stains beneath the location of two generators that have since been removed.

- CAS 25-25-07, Hydraulic Oil Spill(s). This site consists of a hydraulic oil spill that was released from a tunnel-boring machine left on site when $\mathrm{X}$-Tunnel was placed on inactive status.

- CAS 25-25-08, Hydraulic Oil Spill(s). This site consists of a hydraulic oil spill that was released from a tunnel-boring machine left on site when Y-Tunnel was placed on inactive status.

- CAS 25-25-16, Diesel Spill. The site consists of a diesel release from an above-groud storage tank used to store diesel fuel for a boiler located in Building 3320. This tank and much of the impacted soil were removed in 1998.

- CAS 25-25-17, Subsurface Hydraulic Oil Spill(s). This site consists of a hydraulic oil spill associated with the historical operations of an oil recovery system. The spill is located in a significant power/utility corridor feeding into the E-MAD facility. 
Based on the results of previous CAS soil/material sampling (see Section 2.1 and Appendix A-1), closure of the 13 CASs that comprise CAU 398 will be completed by the activities listed below.

Three CASs will be closed by taking no further action. At these CASs, sample results revealed no evidence of constituents of potential concern (COPCs) above action levels. Therefore, the following sites will be closed with no further action.

- $\quad$ CAS 25-44-01, Fuel Spill

- CAS 25-44-04, Acid Spill

- CAS 25-25-06, Oil Spills

Seven CASs will be clean-closed by removal of impacted soil. Sufficient information exists for the following CASs to be clean-closed by excavation and disposal of impacted soil.

- CAS 25-44-02, Spill

- CAS 25-44-03, Spill

- CAS 25-25-02, Oil Spills

- CAS 25-25-03, Oil Spills

- $\quad$ CAS 25-25-04, Oil Spills

- CAS 25-25-05, Oil Spills

- CAS 25-25-16, Diesel Spill

The three CASs listed below will be closed in place administratively. The only COPC at these sites is total petroleum hydrocarbons as diesel/oil. Given specific site conditions (e.g., utilities, limited space, bedrock, and limited lighting), a risk assessment of each of these sites, based on the "A through K" evaluation as presented in Nevada Administrative Code (NAC) 445A.227, will be performed (NAC, 2000). Use Restrictions will also be implemented for each of the following CASs.

- CAS 25-25-07, Hydraulic Oil Spill(s)

- $\quad$ CAS 25-25-08, Hydraulic Oil Spill(s)

- CAS 25-25-17, Subsurface Hydraulic Oil Spill 


\subsection{INTRODUCTION}

This Streamlined Approach for Environmental Restoration (SAFER) plan addresses the activities necessary to close Corrective Action Unit (CAU) 398: Area 25 Spill Sites. CAU 398, located in Area 25 of the Nevada Test Site, is currently listed in Appendix III of the Federal Facility Agreement and Consent Order (FFACO) (FFACO, 1996), and consists of the following 13 Corrective Action Sites (CASs) (Figure 1):

- CAS 25-44-01, a fuel spill on soil that covers a concrete pad. The origins and use of the spill material are unknown, but the spill is suspected to be railroad bedding material.

- CAS 25-44-02, a spill of liquid to the soil from leaking drums.

- CAS 25-44-03, a spill of oil from two leaking drums onto a concrete pad and surrounding soil.

- CAS 25-44-04, a spill from two tanks containing sulfuric acid and sodium hydroxide used for a water demineralization process.

- CAS 25-25-02, a fuel or oil spill from leaking drums that were removed in 1992.

- CAS 25-25-03, an oil spill adjacent to a tipped-over drum. The source of the drum is not listed, although it is noted that the drum was removed in 1991.

CAS 25-25-04, an area on the north side of the Engine-Maintenance, Assembly, and Disassembly (E-MAD) facility, where oils and cooling fluids from metal machining operations were poured directly onto the ground.

- CAS 25-25-05, an area of oil and/or hydraulic fluid spills beneath the heavy equipment once stored there.

- CAS 25-25-06, an area of diesel fuel staining beneath two generators that have since been removed.

- CAS 25-25-07, an area of hydraulic oil spills associated with a tunnel-boring machine abandoned inside $\mathrm{X}$-Tunnel.

- CAS 25-25-08, an area of hydraulic fluid spills associated with a tunnel-boring machine abandoned inside Y-Tunnel.

- CAS 25-25-16, a diesel fuel spill from an above-groud storage tank located near Building 3320 at Engine Test Stand-1 (ETS-1) that was removed in 1998.

- CAS 25-25-17, a hydraulic oil spill associated with the historical operations of a vacuum pump oil recovery system at the E-MAD facility. 


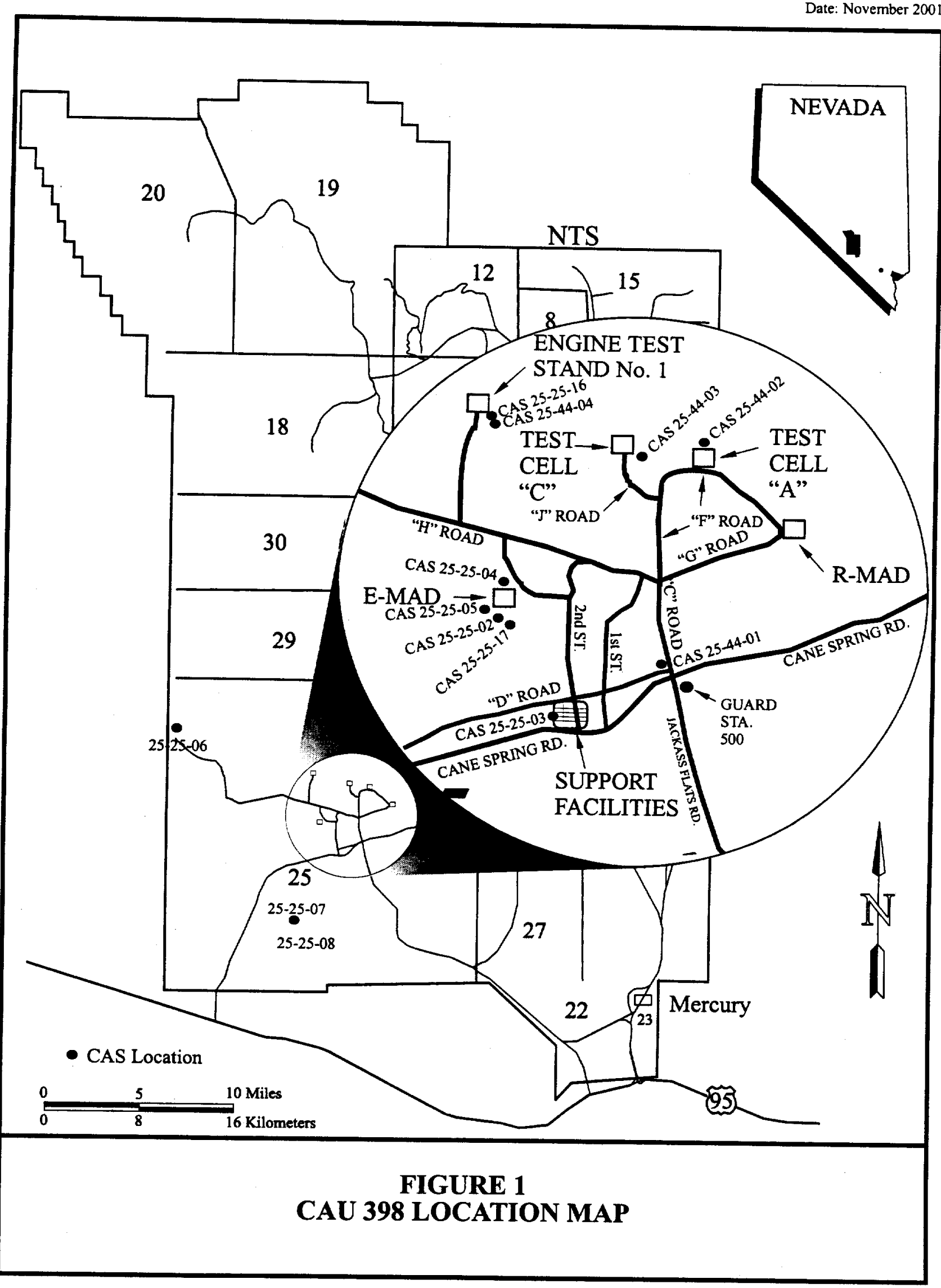




\subsection{SAFER PROCESS}

The SAFER process will be used at CAUs where the parties agree that enough information exists about the nature and extent of contamination to propose an appropriate corrective action prior to the completion of a Corrective Action Investigation.

The SAFER process combines elements of the data quality objectives (DQOs) process and the observational approach to help plan and conduct corrective actions. DQOs are used to identify a problem and define the type and quality of data needed to complete the investigation phase of the process. The purpose of the investigation phase in the SAFER process is to verify the adequacy of existing information to implement the corrective action. The observational approach provides a framework for managing uncertainty and planning decision making.

Use of the SAFER process allows technical decisions to be made based on incomplete but sufficient information and the experience of the decision maker. Any uncertainties are addressed by documented assumptions that are verified by sampling and analysis, data evaluation, and onsite observation as planned activities progress, and by contingency plans, as necessary.

Remediation and closure may proceed simultaneously with site characterization as sufficient data are gathered to confirm or disprove the assumptions made in selecting the closure method. If at any time during the site closure new information is developed that indicates that the closure method should be revised, the closure activities will be modified to implement the revised closure method.

Closure of CAU 398 will be accomplished primarily through clean closure of hydrocarbonimpacted soil by excavation and disposal, although three sites will be closed with administrative controls, implementing a Use Restriction with no further action, and three will be closed with no further action because they contain no constituents of concern (COCs) above regulatory levels.

\subsection{SUMMARY OF PROPOSED CORRECTIVE ACTION}

Based on the results of previous site soil/material sampling (see Section 2.1 and Appendix A-1), closure of the 13 CASs that comprise CAU 398 will be completed by the activities described in the following paragraphs.

Analytical results show no evidence of constituents of potential concern (COPCs) above action levels at the following three CASs; therefore, they will be closed by taking no further action.

- $\quad$ CAS 25-44-01, Fuel Spill

- $\quad$ CAS 25-44-04, Acid Spill

- $\quad$ CAS 25-25-06, Oil Spills

Analytical results show that the following seven CASs can be clean-closed by excavation and disposal of impacted soil; therefore, they will be clean-closed by remoyal of impacted soil: 
- CAS 25-44-02, Spill

- CAS 25-44-03, Spill

- $\quad$ CAS 25-25-02, Oil Spills

- CAS 25-25-03, Oil Spills

- CAS 25-25-04, Oil Spills

- CAS 25-25-05, Oil Spills

- CAS 25-25-16, Diesel Spill

At three CASs, specific site conditions (e.g., utilities, limited space, bedrock, and limited lighting) make clean closure difficult or potentially dangerous to site workers. In addition, the only COPC present at these CASs is total petroleum hydrocarbons (TPH). For these reasons, a risk assessment, based on the "A through $\mathrm{K}$ " evaluation as presented in Nevada Administrative Code (NAC) 445A.2272 (NAC, 2000), will be made and Use Restrictions will be implemented for each of these sites. The following CASs will be closed in place administratively:

- CAS 25-25-07, Hydraulic Oil Spill(s)

- CAS 25-25-08, Hydraulic Oil Spill(s)

- CAS 25-25-17, Subsurface Hydraulic Oil Spill

\subsection{HOLD/DECISION POINTS}

During closure activities, certain conditions affecting the project schedule and budget may require decisions to be made prior to continuing work. Work will be temporarily suspended if any of the following conditions occur:

- Out-of-scope work activities such as the detection of other COCs, a considerably larger release than originally planned for, and/or other technical factors requiring the preparation of a Record of Technical Change.

- The discovery of soil exceeding action limits where none was found during waste identification activities.

- Unsafe condition or work practice posing a threat to personnel, equipment, or the environment not originally documented in the Site-Specific Health and Safety Plan.

If any of these conditions occur, work will stop and the U.S. Department of Energy (DOE), National Nuclear Security Administration Nevada Operations Office (NNSANV) and/or the Nevada Division of Environmental Protection (NDEP) will be notified. Work will continue when a resolution has been agreed upon and a Record of Technical Change, if required, has been approved.

\subsection{SAFER WORK PLAN CONTENTS}

This SAFER Work Plan has been developed to support the proposed characterization and closure of CAU 398 according to the required format, which includes the following: 
- Introduction (Section 1.0)

- Unit Description (Section 2.0)

- $\quad$ Field Activities and Closure Objectives (Section 3.0)

- $\quad$ Reports and Records Availability (Section 4.0)

- Investigation/Remediation Waste Management (Section 5.0)

- $\quad$ Quality Assurance/Quality Control (Section 6.0)

- References (Section 7.0)

This plan was developed using guidance provided from the following documents:

- Nevada Environmental Restoration Project, Project Management Plan, Revision 0, U.S. Department of Energy, Nevada Operations Office, 1994.

- Federal Facility Agreement and Consent Order (FFACO). 1996. Agreed to by the Nevada Division of Environmental Protection, U.S. Department of Energy, and U.S. Department of Defense.

- Nevada Administrative Code, Section 445A.2272. July 2000.

- Occupational Safety and Health Administration. 2001. Title 29 Code of Federal Regulations 1910.120, "Hazardous Waste Operations and Emergency Response," Washington, D.C.

- U.S. Department of Energy, Nevada Operations Office. 1996. Industrial Sites Quality Assurance Project Plan, Nevada Test Site, Nevada, DOE/NV-372. Las Vegas, NV.

- U.S. Environmental Protection Agency. 1996a. Region IX Preliminary Remediation Goals (PRGs), San Francisco, CA.

- U.S. Environmental Protection Agency. 1996c. Title 40 Code of Federal Regulations 261.24, "Toxicity Characteristic," Washington, D.C. 
SAFER PLAN - CAU 398

Area 25 Spill Sites

Section: Introduction

Revision: 0

Date: November 10, 2001

\section{THIS PAGE INTENTIONALLY LEFT BLANK}




\subsection{UNIT DESCRIPTION}

CAU 398 is located in Area 25 of the NTS (Figure 1) and consists of the following 13 CASs:

- CAS 25-44-01, near the northwest corner of the intersection of Roads C and D on a concrete pad at what was known as the Fuel Storage Facility.

- CAS 25-44-02, on and near a concrete pad on the north side of Building 3117 at Test Cell A.

- CAS 25-44-03, on a concrete pad on the southeast side of Test Cell C (Building 4838).

- CAS 25-44-04, on a concrete pad on the east side of Building 3320 at the ETS-1 facility.

- CAS 25-25-02, adjacent to a small concrete loading ramp on the south side of the E-MAD facility (i.e., Building 3900 ).

- CAS 25-25-03, south of Building 4838 (i.e., the Gas Station), originally described as a spill adjacent to a tipped-over drum.

- CAS 25-25-04, on the north side of the E-MAD facility near a building that once stored flammable material.

- CAS 25-25-05, on the northeast side of the E-MAD facility where heavy equipment was once stored.

- CAS 25-25-06, at the Drill Hole Wash Monitoring Station, west of the Yucca Mountain facility.

- CAS 25-25-07, adjacent to a boring machine in an escape drift of X-Tunnel.

- CAS 25-25-08, adjacent to a boring machine at the end of the main drift of Y-Tunnel.

- $\quad$ CAS 25-25-16, east of the ETS-1 (Building 3320) entrance near Building 3320.

- CAS 25-25-17, on the south side of E-MAD facility (Building 3900.)

\subsection{HISTORY}

\subsubsection{CAS 25-44-01, Fuel Spill}

Historical information about CAS 25-44-01 (Figure 2) is limited. The origins and use of the spill material are unknown, although it is suspected to be railroad bedding material. There is no process knowledge of the area being used for fuel storage. The area surrounding the CAS is littered with construction debris, indicating that it may have been used as a dumping area for excess construction material. 


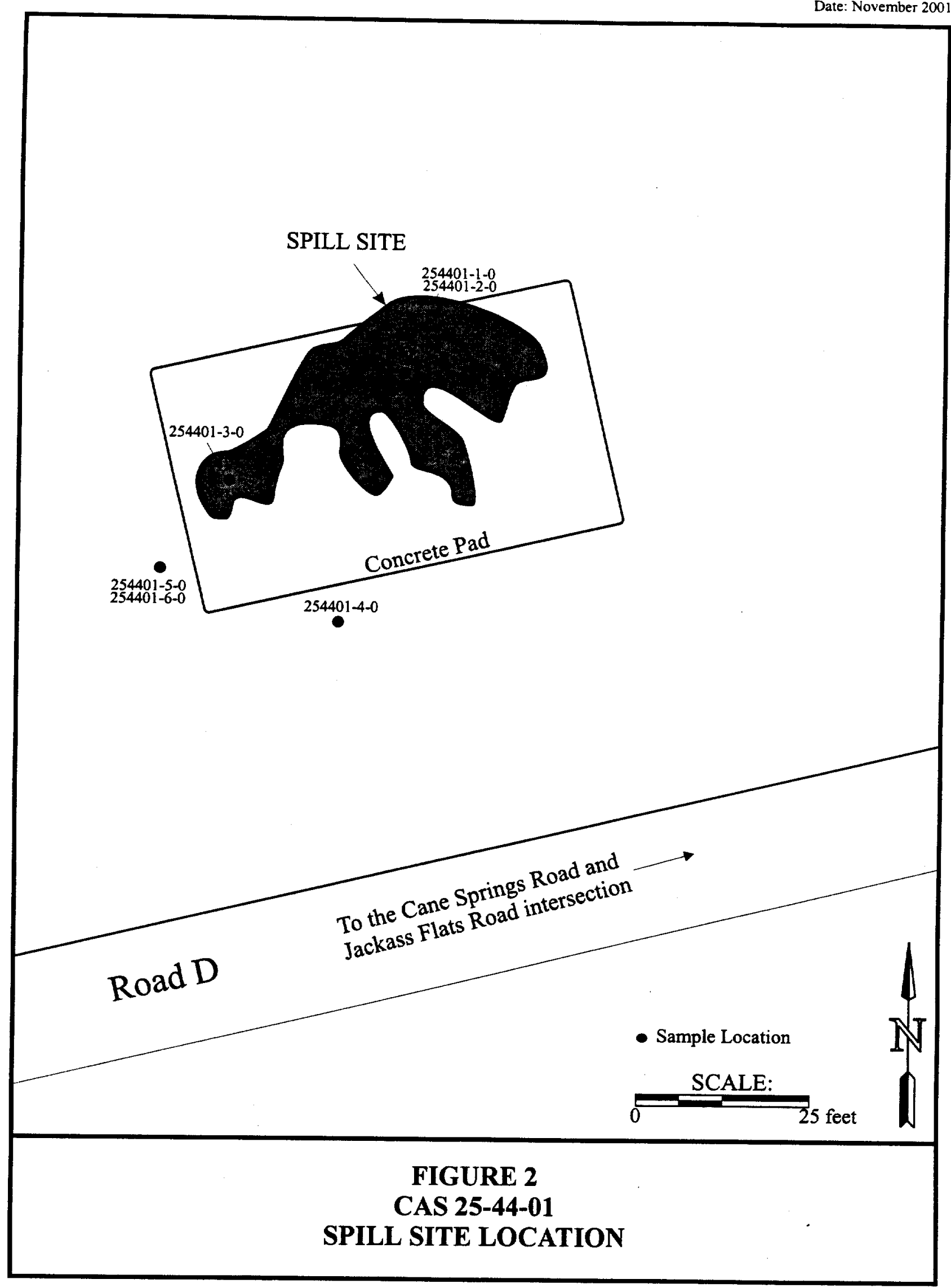


The site is listed as a fuel spill (FFACO, 1996). The International Technology Corporation (IT) Industrial Sites Preliminary Assessment (PA) group visited the site in 1996 and 1998 and determined it to be a fuel spill on soil that covers a concrete pad. The dimensions of the spill were measured to be 18 by 12 meters $(\mathrm{m})$ ( 60 by 40 feet [ft]) with the depth from 0 to 13 centimeters $(\mathrm{cm})(0$ to 5 inches [in]). Samples were collected from the rock and soil on the concrete pad. Sample results identified Resource Conservation and Recovery Act (RCRA) metals as the only COPC.

Bechtel Nevada (BN) collected six samples from the site on May 24, 2001. The spill material sampled was dense and metallic and was analyzed for Toxicity Characteristic Leaching Procedure (TCLP) metals (U.S. Environmental Protection Agency [EPA], 1996b) and gamma spectroscopy. Samples of the soil surrounding the down gradient sides of the pad were also collected and analyzed for full-scan TPH. Sample results show no COCs present above regulatory limits (EPA, 1996a; 1996c; NAC, 2000) (Table 1).

\subsubsection{CAS 25-44-02, Spill}

CAS 25-44-02 (Figure 3) consists of a spill to the soil caused by leaking drums. A literature search and site visit by IT in 1993 determined that the barrels had been removed but staining remained. The shipping manifest indicated that the waste contained various oils, lead, and chromium. A previous contractor collected samples from one of the drums and from soil in the spill area. The samples were analyzed for polychlorinated biphenyls (PCBs), TPH, and total chlorinated hydrocarbons. The only COC detected was TPH in the soil.

BN collected eight soil samples from around the pad and the suspected spill area on May 23, 2001. It appeared that the area had been disturbed (i.e., the impacted soil had been removed). The samples were analyzed for full-scan TPH, TCLP chromium and lead, and gamma spectroscopy. Sample results show that TPH is the only COC present above regulatory limits (EPA, 1996a; 1996c; NAC, 2000) (Table 1).

\subsubsection{CAS 25-44-03, Spill}

CAS 25-44-03 (Figure 4) was a temporary drum storage area where the contents of two drums labeled as containing PCBs leaked onto the pad and surrounding soil. On December 14, 1990, the drums were removed and shipped to the Area 6 PCB storage facility (IT, 2000). The drums were later shipped off site for disposal after being characterized as non-PCB. The source of the drums is unknown, but likely resulted from the draining of transformers or other electrical equipment. Available documentation indicates that the oil contained no PCBs.

The IT Industrial Sites PA group conducted a site assessment on December 2, 1999, and identified the staining on the pad and soil staining extending approximately $2 \mathrm{~m}(7 \mathrm{ft})$ east of the pad. BN collected five samples of the spilled material and surrounding soil on May 24, 2001. Samples were analyzed for full-scan TPH, PCBs, TCLP metals, and gamma spectroscopy. Sample results indicated the presence of TPH (i.e., diesel/oil range) above regulatory levels in the spill material (Table 1). 
TABLE 1 - CAU 398 SAMPLE ANALYTICAL RESULTS

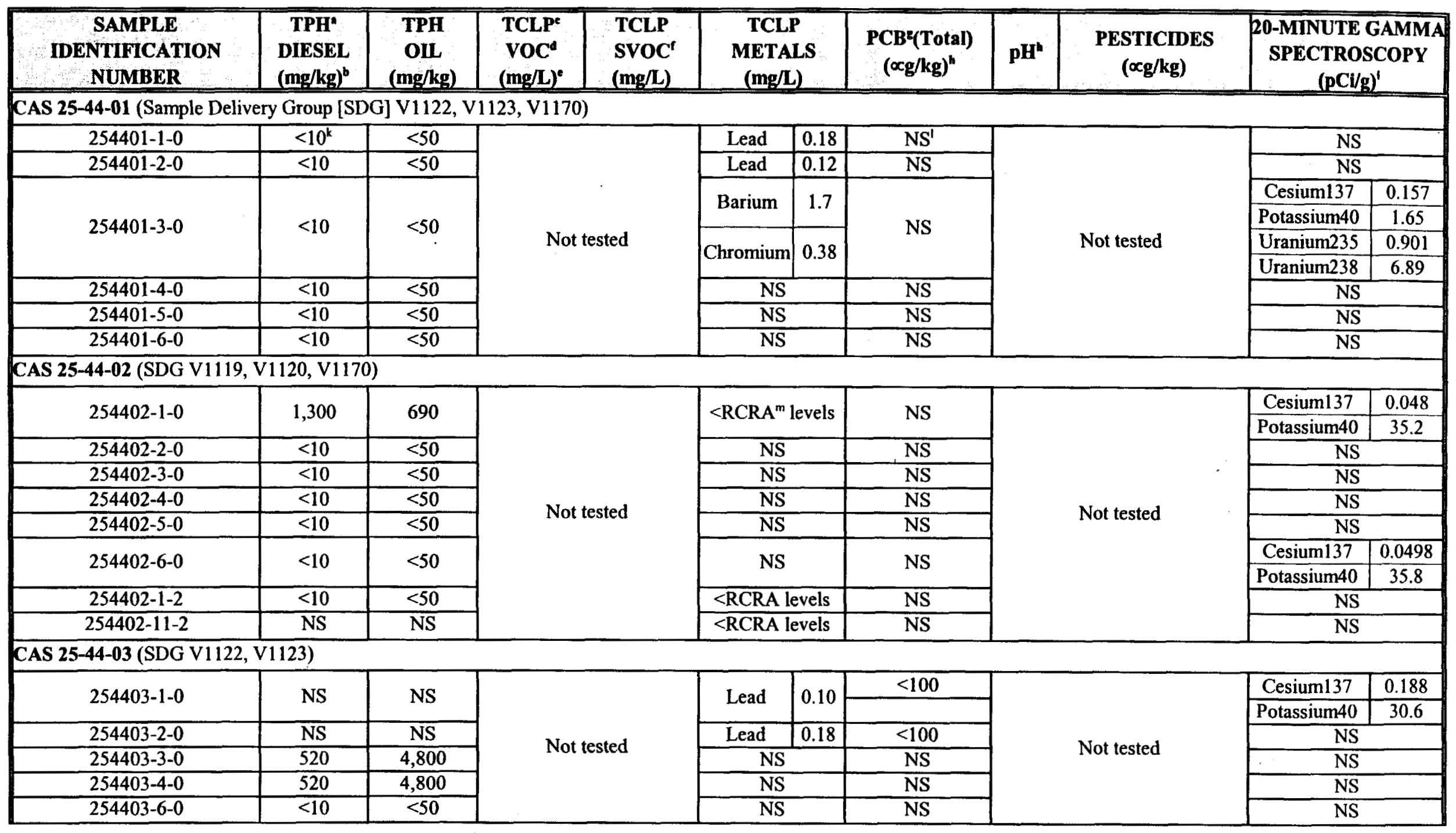


TABLE 1 - CAU 398 SAMPLE ANALYTICAL RESULTS (continued)

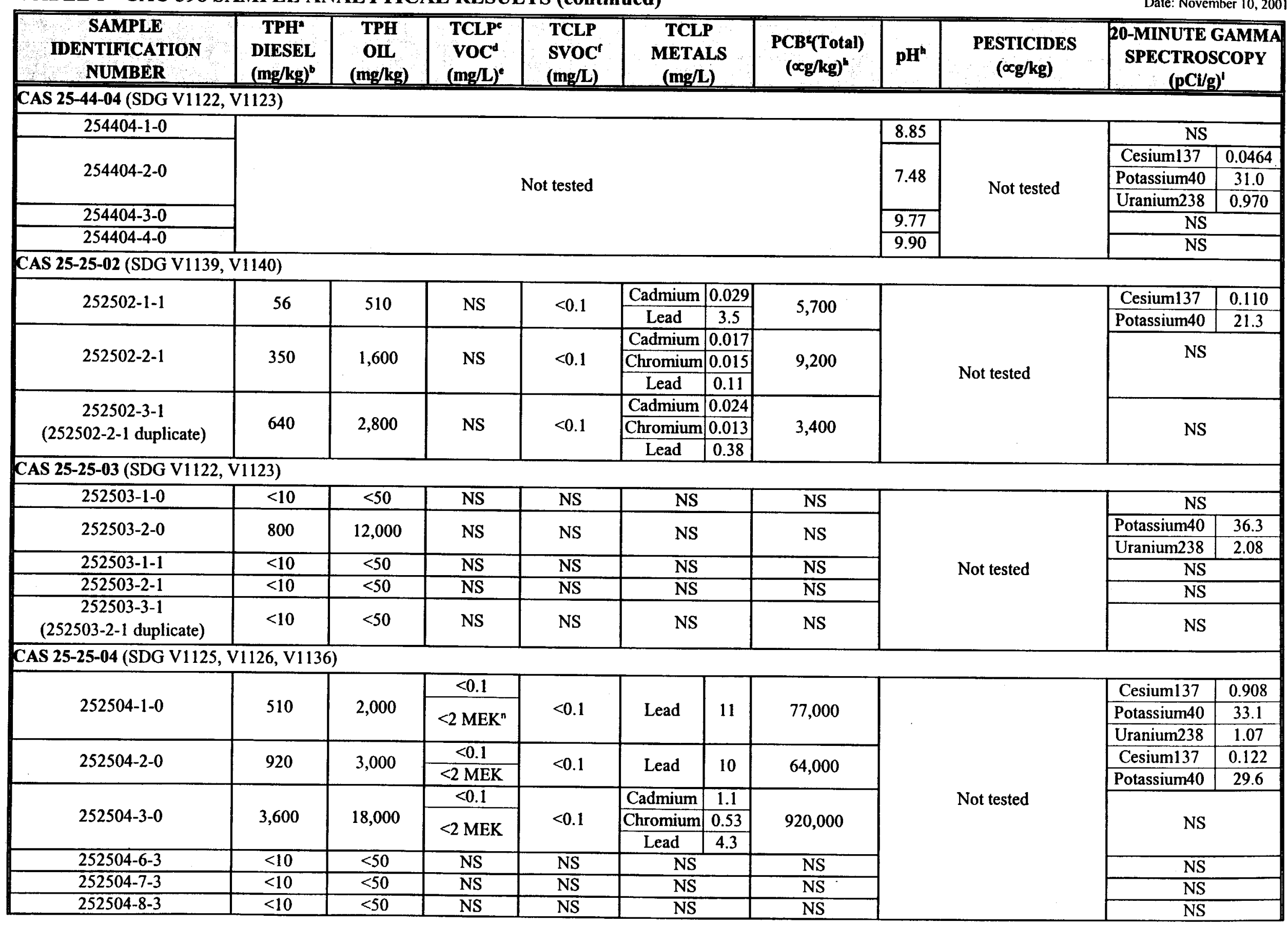


TABLE 1 - CAU 398 SAMPLE ANALYTICAL RESULTS (continued)

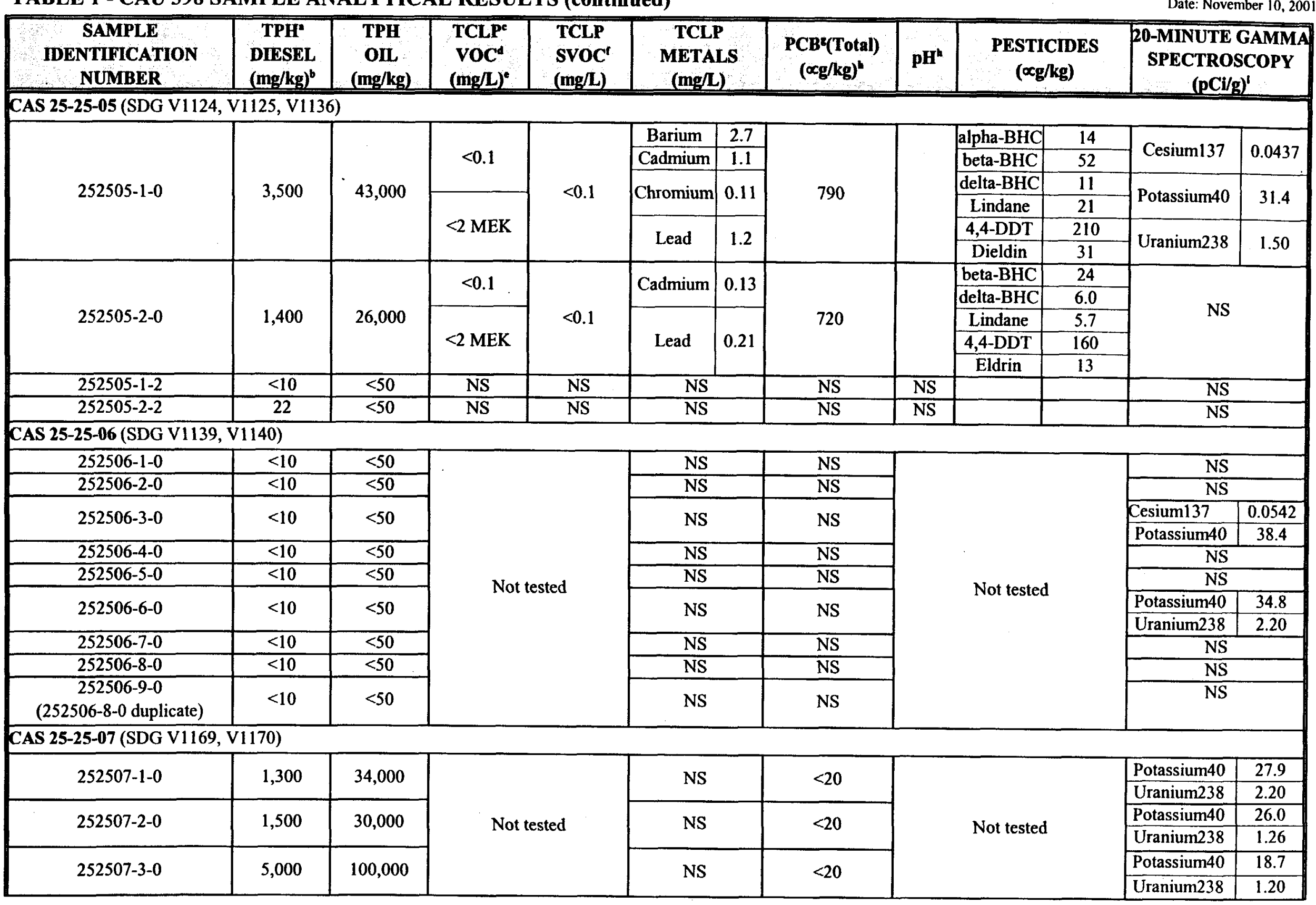


TABLE 1 - CAU 398 SAMPLE ANALYTICAL RESULTS (continued)

\begin{tabular}{|c|c|c|c|c|c|c|c|c|c|c|}
\hline $\begin{array}{c}\text { SAMPLE } \\
\text { IDENTIFICATION } \\
\text { NUMBER }\end{array}$ & $\begin{array}{r}\text { TPH } \\
\text { DIESEL } \\
(\mathbf{m g} / \mathbf{k g})^{\mathbf{b}} \\
\end{array}$ & $\begin{array}{c}\text { TPH } \\
\text { OLL } \\
\text { (mg/kg) }\end{array}$ & $\begin{array}{l}\text { TCLPe } \\
\text { VOCd } \\
(\mathrm{mg} / \mathrm{L})^{\mathrm{e}}\end{array}$ & $\begin{array}{l}\text { TCLP } \\
\text { SVOC' } \\
(\mathrm{mg} / \mathrm{L})\end{array}$ & $\begin{array}{l}\text { TCLP } \\
\text { METALS } \\
(\mathrm{mg} / \mathrm{L}) \\
\end{array}$ & $\begin{array}{l}\text { PCB } \text { (Total) } \\
(\propto g / \mathbf{k g})^{k}\end{array}$ & $\mathbf{p H}^{\mathbf{k}}$ & $\begin{array}{l}\text { PESTICIDES } \\
(\propto \mathrm{g} / \mathrm{kg})\end{array}$ & \multicolumn{2}{|c|}{$\begin{array}{c}\text { 20-MINUTE GAMMA } \\
\text { SPECTROSCOPY } \\
\text { (pCi/g) })^{1}\end{array}$} \\
\hline \multicolumn{11}{|c|}{ CAS 25-25-08 (SDG V1169, V1170) } \\
\hline \multirow{3}{*}{$252508-1-0$} & \multirow{3}{*}{9,500} & \multirow{3}{*}{110,000} & \multirow{4}{*}{\multicolumn{2}{|c|}{ Not tested }} & & \multirow{3}{*}{$<20$} & \multirow{4}{*}{\multicolumn{2}{|c|}{ Not tested }} & Potassium40 & 21.1 \\
\hline & & & & & NS & & & & Uranium235 & 0.182 \\
\hline & & & & & & & & & Uranium238 & 1.76 \\
\hline $252508-2-0$ & 10,000 & 130,000 & & & NS & $<20$ & & & Potassium40 & 21.2 \\
\hline \multicolumn{11}{|c|}{ CAS 25-25-16 (SDG V1124, V1125) } \\
\hline $252516-1-0$ & $<10$ & $<50$ & \multirow{10}{*}{\multicolumn{2}{|c|}{ Not tested }} & NS & NS & \multirow{10}{*}{\multicolumn{2}{|c|}{ Not tested }} & Potassium40 & 35.3 \\
\hline $252516-2-0$ & $<10$ & $<50$ & & & NS & NS & & & \multicolumn{2}{|l|}{$\begin{array}{r}\mathrm{NS} \\
\end{array}$} \\
\hline $252516-3-0$ & $<10$ & $<50$ & & & NS & NS & & & \multicolumn{2}{|l|}{ NS } \\
\hline $252516-4-0$ & $<10$ & $<50$ & & & NS & NS & & & \multicolumn{2}{|l|}{ NS } \\
\hline \multirow{2}{*}{$252516-5-2$} & \multirow{2}{*}{$<10$} & \multirow{2}{*}{$<50$} & & & \multirow{2}{*}{ NS } & \multirow{2}{*}{ NS } & & & Potassium40 & 35.4 \\
\hline & & & & & & & & & Uranium238 & 1.69 \\
\hline $252516-6-1$ & $<10$ & $<50$ & & & NS & NS & & & \multicolumn{2}{|l|}{ NS } \\
\hline $252516-7-1$ & $<10$ & $<50$ & & & NS & NS & & & \multicolumn{2}{|l|}{ NS } \\
\hline $252516-8-0$ & 220 & 1,100 & & & NS & NS & & & \multicolumn{2}{|l|}{ NS } \\
\hline $\begin{array}{c}252516-9-0 \\
\text { (252516-8-0 duplicate) }\end{array}$ & 140 & 570 & & & NS & NS & & & \multicolumn{2}{|l|}{ NS } \\
\hline \multicolumn{11}{|c|}{ CAS 25-25-17 (SDG V1139, V1140) } \\
\hline \multirow{2}{*}{$252517-1-1$} & \multirow{2}{*}{47} & \multirow{2}{*}{600} & \multirow{4}{*}{\multicolumn{2}{|c|}{ Not tested }} & \multirow{2}{*}{ NS } & \multirow{2}{*}{78} & \multirow{4}{*}{\multicolumn{2}{|c|}{ Not tested }} & Potassium40 & 29.4 \\
\hline & & & & & & & & & Uranium238 & 1.45 \\
\hline $252517-2-1$ & $<10$ & 530 & & & NS & 620 & & & \multicolumn{2}{|l|}{ NS } \\
\hline $\begin{array}{c}252517-3-1 \\
\text { (252517-2-1 duplicate) } \\
\end{array}$ & 25 & $<50$ & & & NS & 110 & & & \multicolumn{2}{|l|}{ NS } \\
\hline
\end{tabular}

atotal petroleum hydrocarbons

bmilligram(s) per kilogram

'Toxicity Characteristic Leaching Procedure (SW-846 extraction method 1311 [EPA, 1996])

¿volatile organic compounds

emilligram(s) per liter

semivolatile organic compounds

gpolychlorinated biphynels. hmicrogram(s) per kilogram

${ }^{i} \log 10$ of the concentration of hydrogen ions In solution

${ }^{\mathrm{j}}$ picocurie(s) per gram

$k_{<}<$indicates result was less than laboratory reporting limit (e.g., $<50 \mathrm{mg} / \mathrm{kg}$ indicates that TPH oil was not detected at the reporting limit of $50 \mathrm{mg} / \mathrm{kg}$ )

not sampled

methyl ethyl ketone

Note: The analytical data presented in Appendix A-1 are arrange by SDG in increasing order. 


\section{TEST CELL A}

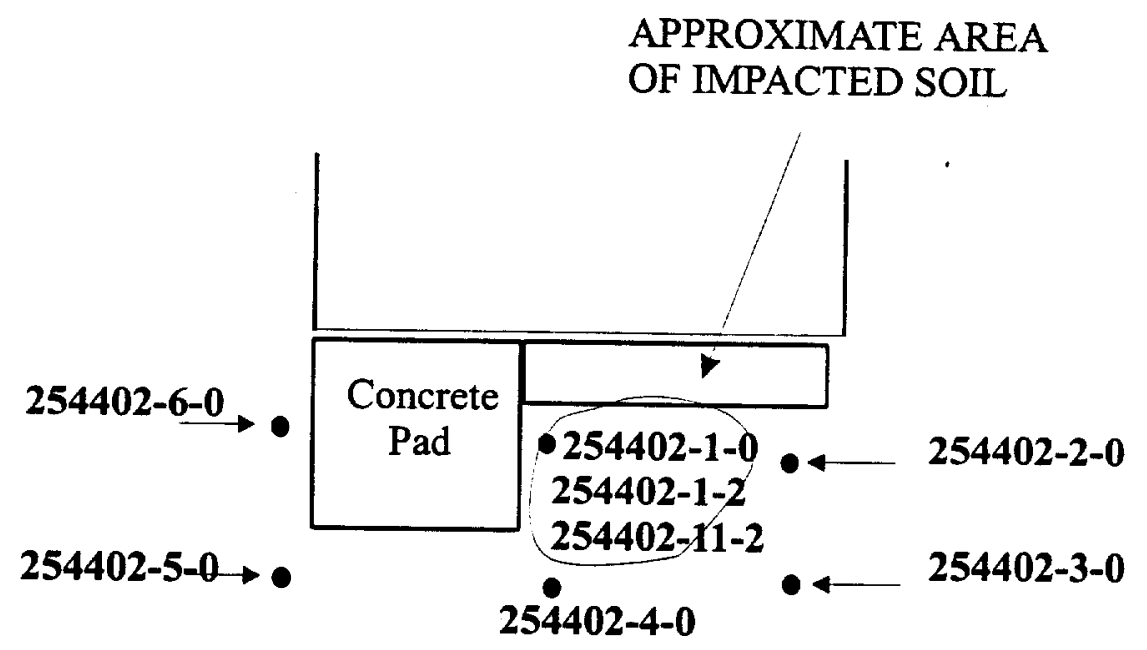

- Sample Location

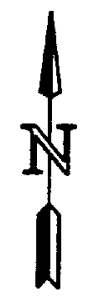

FIGURE 3

CAS 25-44-02

SPILL SITE LOCATION 


\section{TEST CELL C}

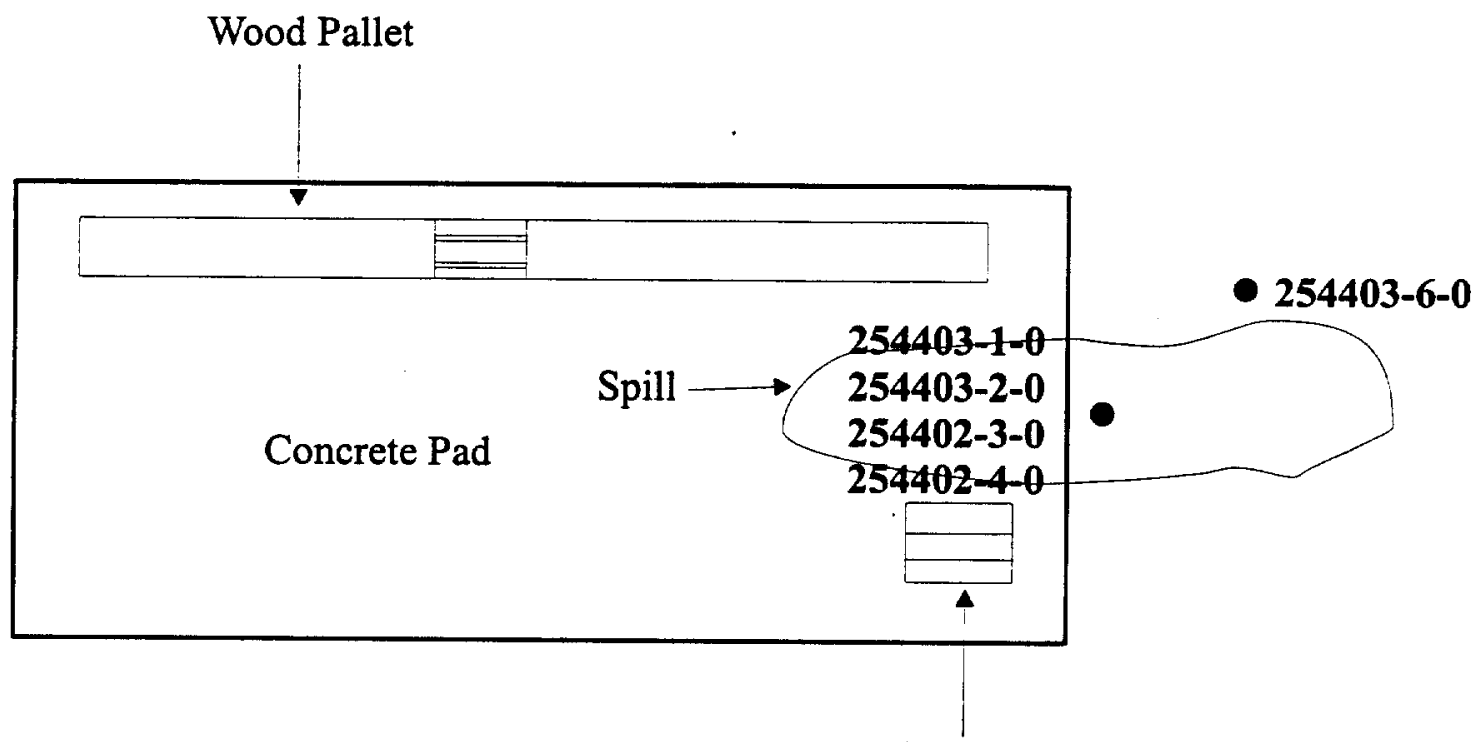

Wood Pallet

- Sample Location

SCALE:

010 feet

FIGURE 4

CAS 25-44-03

SPILL SITE LOCATION 


\subsubsection{CAS 25-44-04, Acid Spill}

CAS 25-44-04 (Figure 5) consists of spills from two tanks used for a water demineralization process (IT, 2000). Tank T-2002 contained sodium hydroxide and Tank T-2003 contained sulfuric acid (BN, 1998a; 1998b). Each tank had an 18,927-liter (L) (5,000-gallon [gal]) capacity. Engineering drawings indicate that the tanks were installed in the early $1960 \mathrm{~s}$. The tanks were removed from the site in 1998.

A site visit by IT on December 2,1999, determined the spill to be approximately 10 by $5 \mathrm{~m}$ ( 33 by $16 \mathrm{ft})$. The spill extended another $3 \mathrm{~m}(10 \mathrm{ft})$ east off the pad into a small gully. The spill was identified by the red-brown and yellow staining. The spill from the sulfuric acid tank was believed to have occurred over a period of time due to corrosion of the tank bottom. The spill was reported to the Nevada Division of Emergency Management (NDEM) as NDEM \#980819-3014. The sodium hydroxide spill (NDEM \#980811-3001) occurred on August 10, 1998, during tank removal activities. At that time, approximately 2.3 cubic meters $\left(\mathrm{m}^{3}\right)$ (3 cubic yards $\left.\left[\mathrm{yd}^{3}\right]\right)$ of impacted soil were removed and disposed of in the U10c Landfill.

BN collected four samples of the material on the pad and surrounding soil on May 24, 2001. These samples were analyzed for soil $\mathrm{pH}$ and gamma spectroscopy. The results indicated that the $\mathrm{pH}$ of the samples ranged from 7.48 to 9.90 , which is not RCRA-corrosive. Analytical results for the BN samples collected at this CAS are summarized in Table 1.

\subsubsection{CAS 25-25-02, Oil Spills}

Available documentation for CAS 25-25-02 (Figure 6) indicates that the spill was associated with leaking drums that were removed in 1992 (Reynolds Electrical \& Engineering Company [REECo], 1992). No documentation exists to indicate that the spill was removed. The source of the drums is not listed in the documentation, although since the loading ramp leads to the boiler room, the drums could have been waste oil or fuel from operations conducted there. The spill was described as 3 by $3 \mathrm{~m}$ ( 10 by $10 \mathrm{ft}$ ) and approximately $10 \mathrm{~cm}$ ( $4 \mathrm{in}$ ) below ground surface, and appeared to have been covered with soil.

BN collected two samples of the spill material and soil on May 31, 2001. One sample was collected from the original IT sample location. The other sample was collected from the southwest corner of the loading ramp from an obvious spill area of a dark grey to black tar-like material. Both samples were analyzed for full-scan TPH, PCBs, TCLP semivolatile organic compounds (SVOCs), TCLP metals, and gamma spectroscopy. Results indicate that the soil and spill material contained both TPH and PCBs above regulatory limits. No other chemical or radiological constituent was present above action levels. Analytical results for the $\mathrm{BN}$ samples collected at this CAS are summarized in Table 1. 


\section{ENGINE TEST STAND-1 \\ (ETS-1)}

Former Location of

Sulfuric Acid Tank

BUILDING

3320

- Sample Location

SCALE:

0

20 feet

Former Location of

Sodium Hydroxide Tank

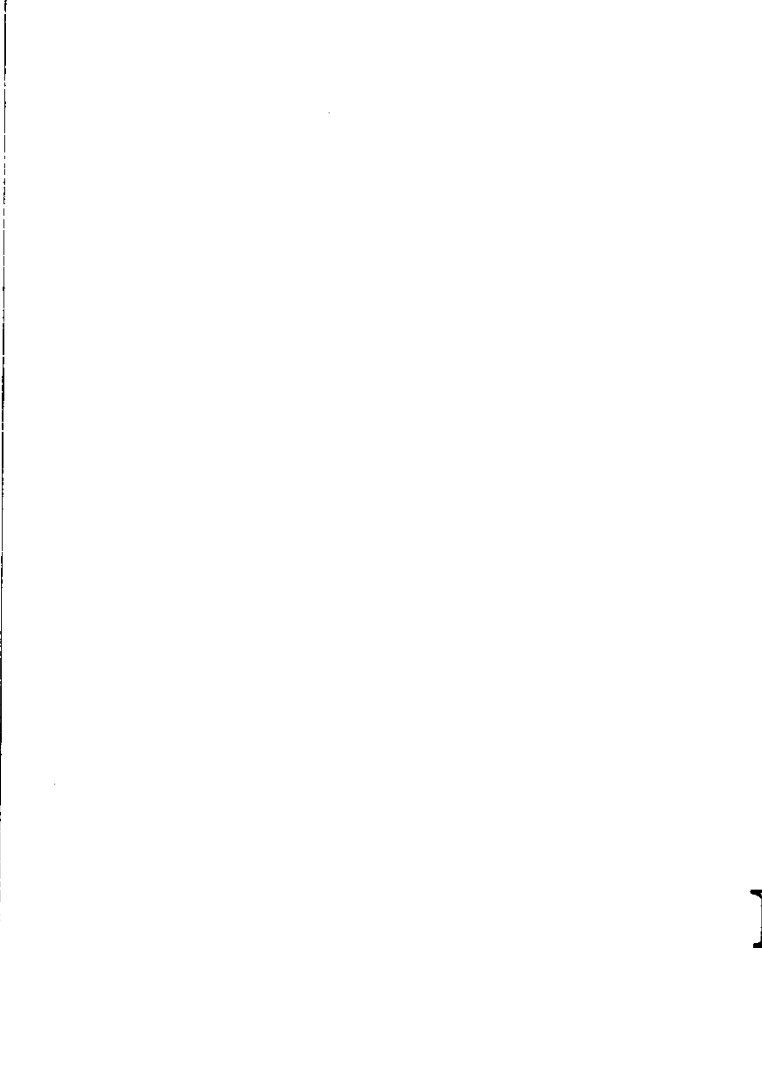

FIGURE 5

CAS 25-44-04

SPILL SITE LOCATION 


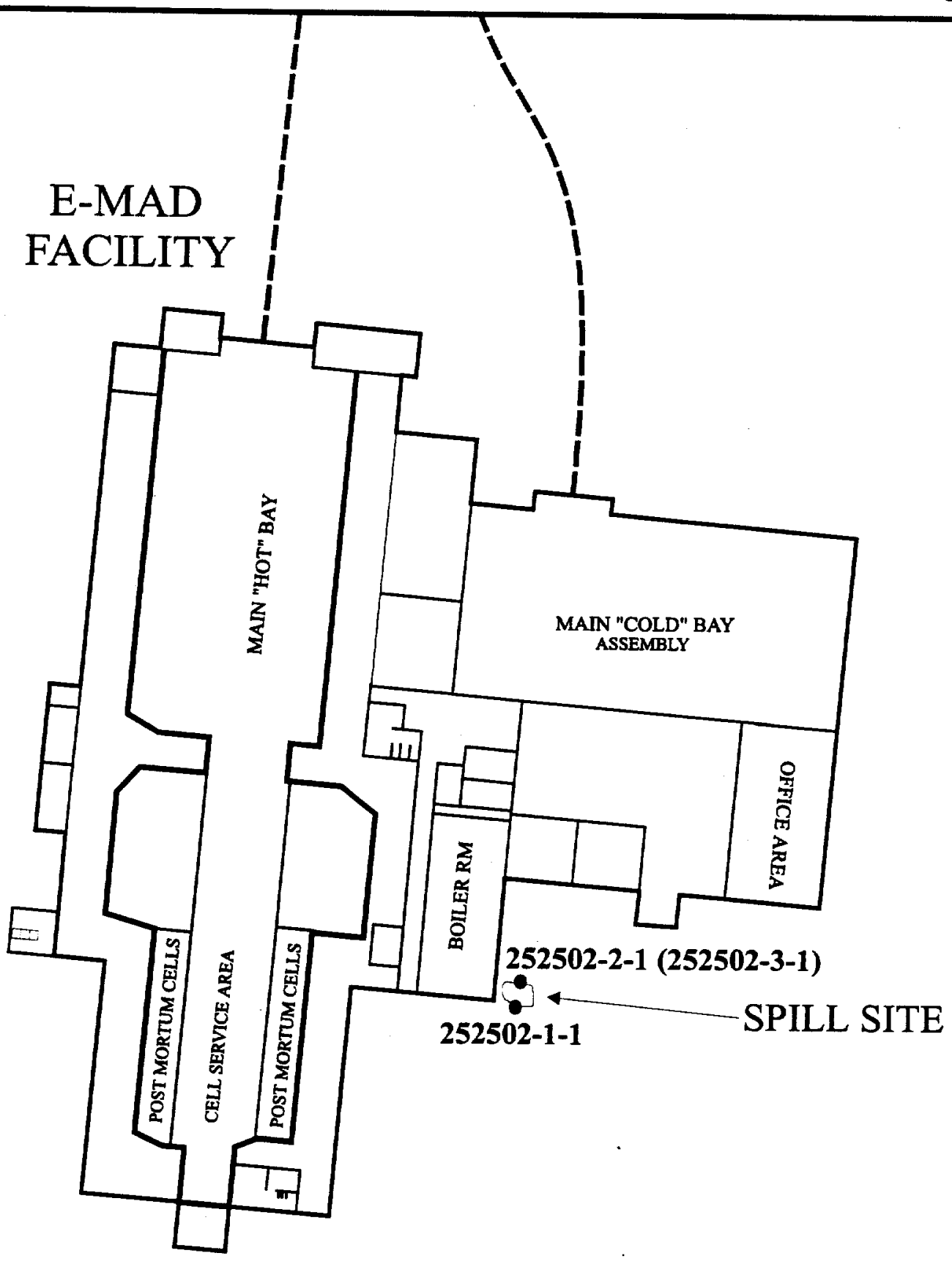

- Sample Location

SCALE:

FIGURE 6

CAS 25-25-02

SPILL SITE LOCATION 


\subsubsection{CAS 25-25-03, Oil Spills}

CAS 25-25-03 (Figure 7) was originally documented as a spill adjacent to a tipped-over drum. Documentation does not list the source of the drum, although it may have been vehicle maintenance operations at the gas station. The drum was documented as being removed in July 1991 (REECo, 1991b); in 1993 the spill was measured to be 2 by 1 by $1 \mathrm{~m}$ ( 6 by 3 by $3 \mathrm{ft}$ ) (REECo, 1993).

BN collected five samples from two locations on May 24, 2001. One location was at the site of

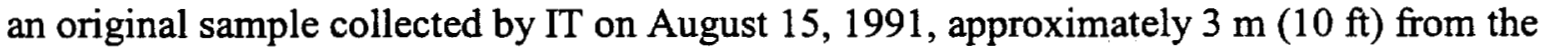
southwest corner of the paved parking area. The other location was approximately $15 \mathrm{~m}(50 \mathrm{ft})$ southwest of the first location near the IT CAS identification stake. Samples were collected from the surface and at a depth of $0.3 \mathrm{~m}(1 \mathrm{ft})$ from each location. Samples were analyzed for fullscan TPH and gamma spectroscopy. Sample results show that TPH is the only COC above regulatory limits present at this site (Table 1).

\subsubsection{CAS 25-25-04, Oil Spills}

CAS 25-25-04 (Figure 8) was documented as extensive oil spills outside a former storage building at E-MAD (REECo, 1991a). Unspecified flammable materials were stored in the Flammable Material Storage Facility, which was built in 1966 (Space Nuclear Propulsion Office, 1970). An estimated 133 to $151 \mathrm{~L}$ ( 35 to 40 gal) of waste liquids were discharged to the soil. Waste may have included cutting oils (i.e., sulphur-based) and coolants. The spill area is approximately 5 by $5 \mathrm{~m}(15$ by $15 \mathrm{ft})$.

Six samples of the area were collected by BN on May 26, 2001. Samples were collected from the original IT locations as well as others within the CAS from the surface and a depth of $1 \mathrm{~m}$ $(3 \mathrm{ft})$. Surface samples were analyzed for full-scan TPH, PCBs, TCLP VOCs, TCLP SVOCs, TCLP metals, and gamma spectroscopy. Subsurface samples were analyzed only for full-scan TPH. The results indicated the presence of PCBs, TPH, and lead. One sample was collected from a small area on the surface of what appeared to be machine shop waste. The analytical results from this area indicated PCBs and TPH (i.e., diesel/oil range) above regulatory limits. This area was approximately 30 by $30 \mathrm{~cm}$ ( 1 by $1 \mathrm{ft})$ and appeared to be only surficial. All analytical results for samples collected from the $1-\mathrm{m}(3-\mathrm{ft})$ depth were below regulatory limits. Therefore, the COCs above regulatory limits for this CAS include TPH, PCBs, and RCRA metals (Table 1).

\subsubsection{CAS 25-25-05, Oil Spills}

CAS 25-25-05 (Figure 9) consists of oil and/or hydraulic fluid spills associated with heavy equipment stored there. There are two larger oil spills (approximately 1.5 by $1.5 \mathrm{~m}$ ) and four smaller spills (approximately 0.3 by $1.5 \mathrm{~m}$ ) within the footprint of the heavy equipment. The spills are presumed to be from the same source, an equipment oil tank, but from different parts of the equipment. 


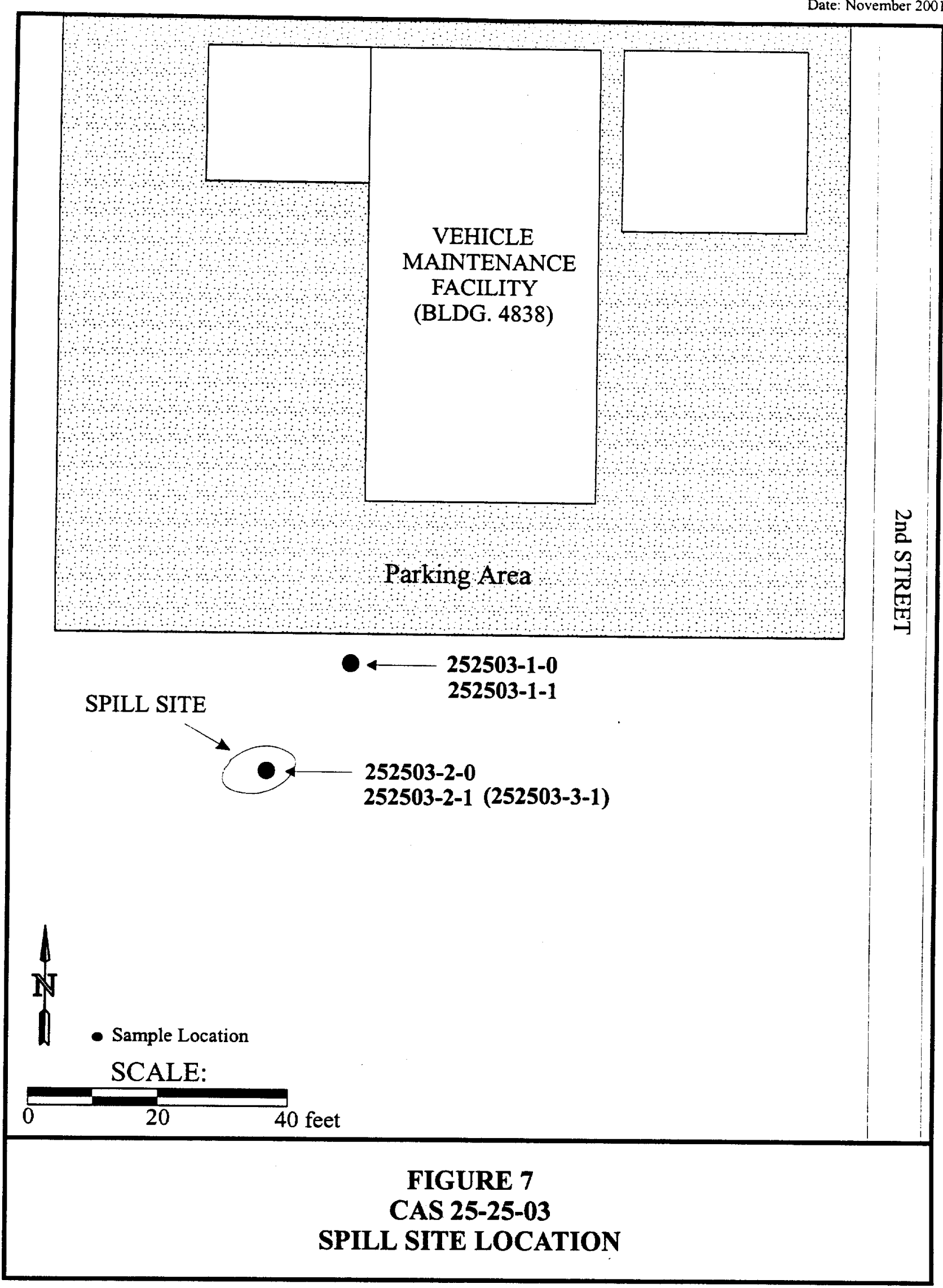


Date: November 2001

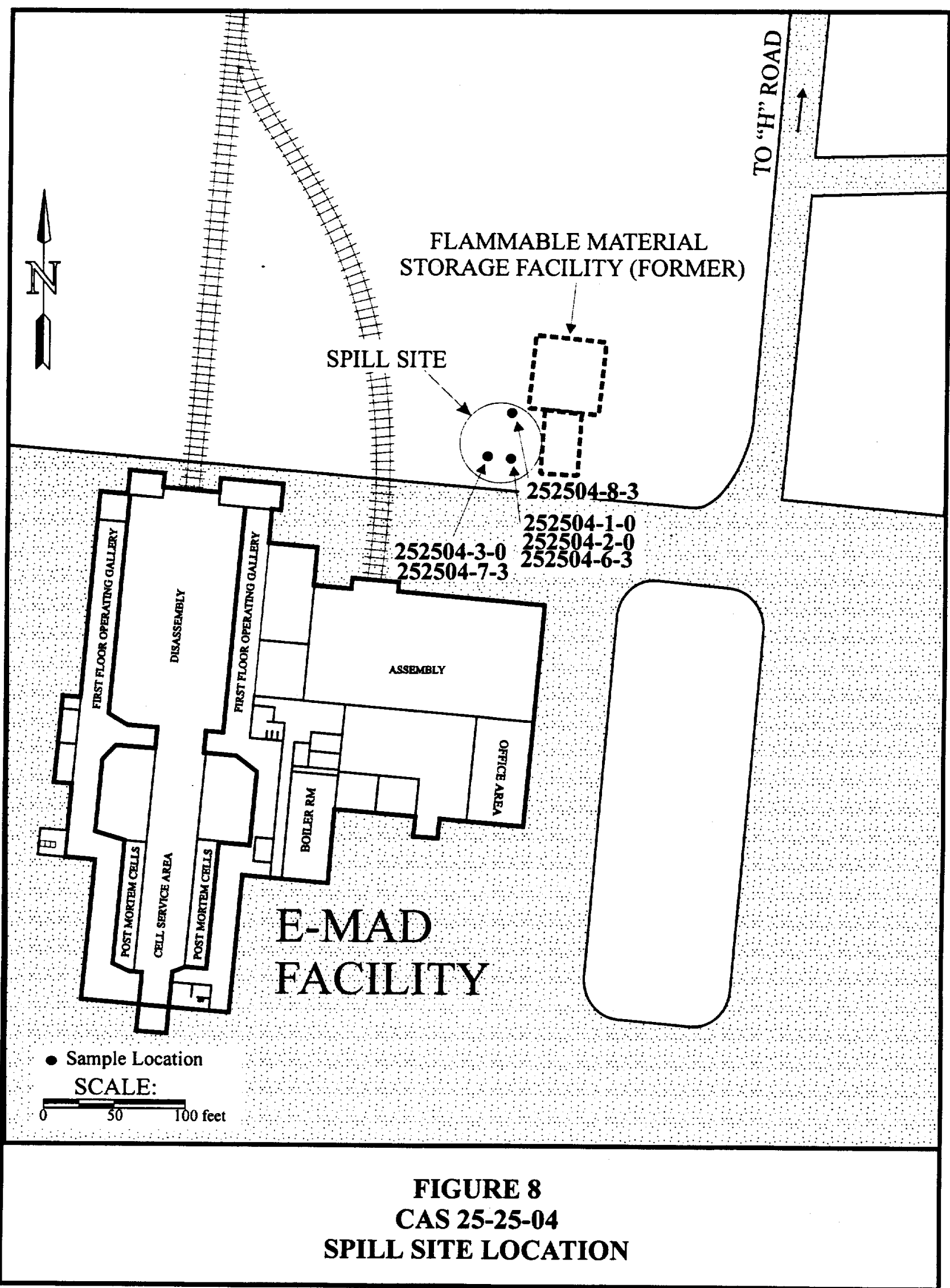




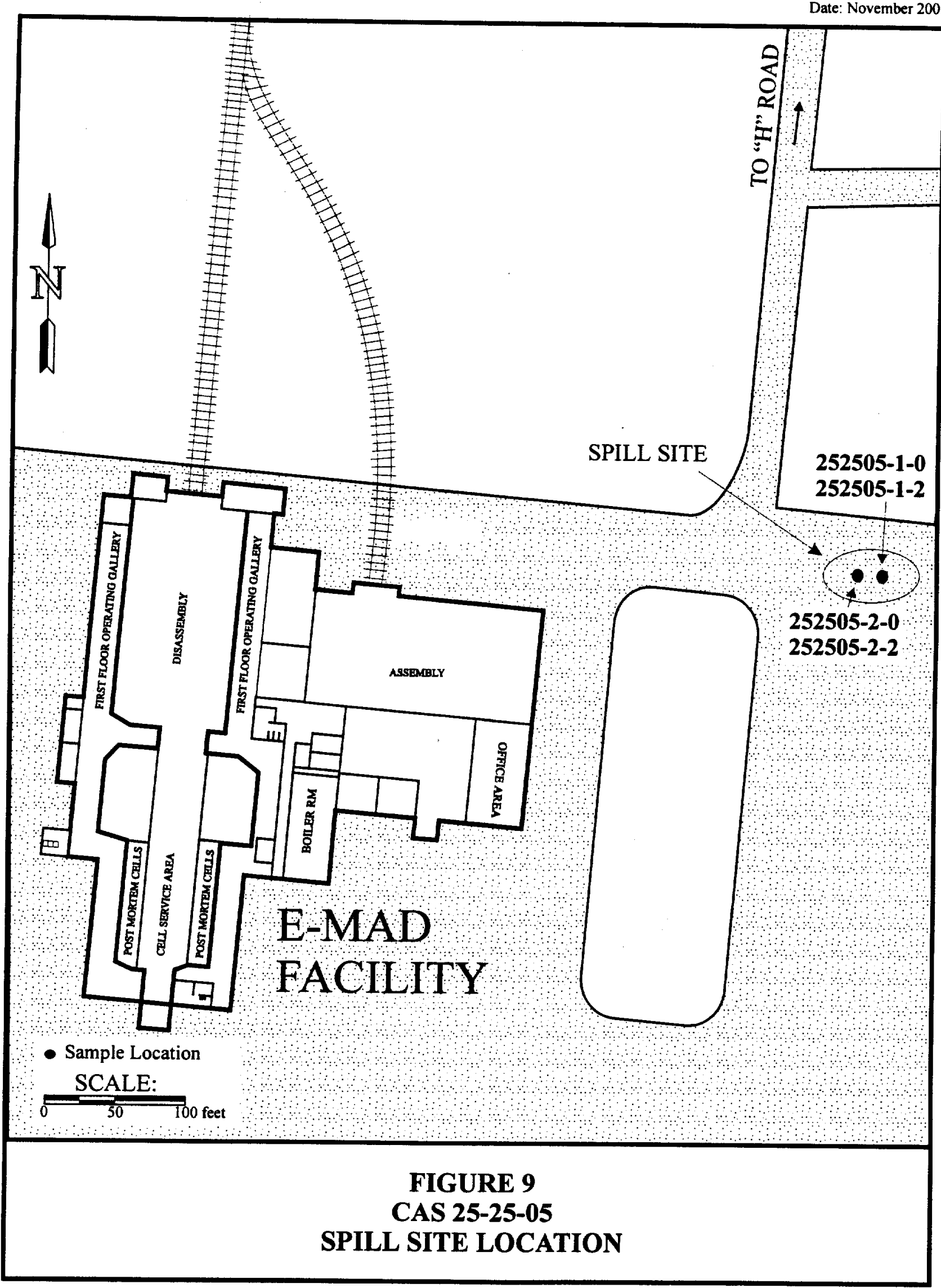


BN collected four samples from the spill site on May 25, 2001. Samples were collected from the surface and from a depth of $0.6 \mathrm{~m}(2 \mathrm{ft})$. Surface samples were analyzed for full-scan TPH, PCBs, TCLP VOCs, TCLP SVOCs, TCLP metals, pesticides, and gamma spectroscopy.

Samples collected from the subsurface were analyzed only for full-scan TPH. Surface samples showed high TPH concentrations in the diesel/oil range. Also, TCLP cadmium was detected in concentrations as high as 1.1 milligrams per liter $(\mathrm{mg} / \mathrm{L})$. Results of samples collected from the $0.6-\mathrm{m}$ (2-ft) depth were below TPH regulatory levels. Sample results indicate the presence of TPH and cadmium at concentrations above regulatory limits in surface samples (Table 1).

\subsubsection{CAS 25-25-06, Oil Spills}

CAS 25-25-06 (Figure 10) was documented as diesel fuel stains beneath two generators at the Area 25 Drill Hole Wash Monitoring Station (IT, 2000). The generators likely provided power for two trailers and monitoring equipment. A January 1996 site visit by the IT Industrial Sites PA group determined that the generators were gone and no soil staining was apparent. Two trailers remained. A later IT site visit on March 5, 1998, revealed that the larger of the two trailers was gone. However, dark-brown staining was evident on the soil where the larger trailer had been. The staining was approximately 9 by $2 \mathrm{~m}$ ( 28 by $8 \mathrm{ft})$. No samples were collected (IT, 2000).

A BN site visit on May 31, 2001, revealed no indication of the staining identified earlier. Based on descriptions and photographs, the location of the previously stained area was noted and nine samples were collected. Samples were analyzed for full-scan TPH and gamma spectroscopy. Sample results indicated that TPH and radiological constituents are not present in the soil above regulatory limits (Table 1).

\subsubsection{CAS 25-25-07, Hydraulic Oil Spill(s)}

CAS 25-25-07 is located adjacent to a boring machine in an escape drift of X-Tunnel, which was left in place when X-Tunnel was placed on inactive status in 1982. Over time the hydraulic lines leaked fluid to the surrounding soil. The lines may also have leaked due to damage from the removal of salvageable parts from the boring machine. It is estimated that approximately 1,514 L (400 gal) of hydraulic fluid may have been released (IT, 2000).

A sample of the hydraulic reservoir was collected by IT on January 31, 1997, and analyzed for PCBs. Results indicated that PCBs were below regulatory levels. The reservoir was later drained. BN collected three samples of the impacted soil on June 20,2001. The samples were analyzed for full-scan TPH, PCBs, and gamma spectroscopy. Due to the presence of fixed depleted uranium within the drift, a radiological control technician was present during sampling activities. No radiological constituents exceeding action levels were detected in the sampling areas. Sample results indicate the presence of TPH concentrations in the diesel/oil range above regulatory limits (Table 1). 


\section{DRILL HOLE WASH MONITORING STATION}
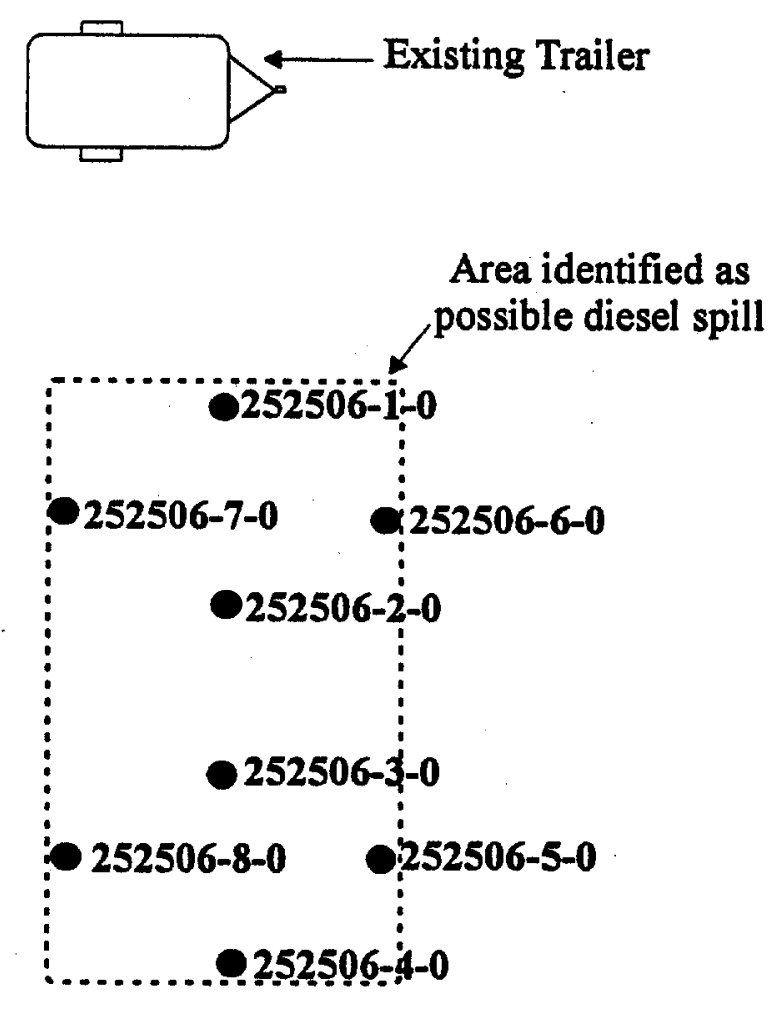

- Sample Location SCALE:

$=10$ feet

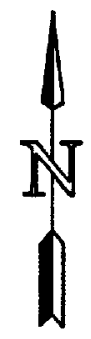

\section{FIGURE 10}

SPILL SITE LOCATION

CAS 25-25-06 


\subsubsection{CAS 25-25-08, Hydraulic Oil Spill(s)}

CAS 25-25-08 is adjacent to a boring machine at the end of the main drift of Y-Tunnel, which was left in place when Y-Tunnel was placed on inactive status in 1982 . Over time, the hydraulic lines leaked fluid to the surrounding soil. The lines may also have leaked due to damage from the removal of salvageable parts from the boring machine. It is estimated that approximately $1,514 \mathrm{~L}$ (400 gal) of hydraulic fluid may have been released. It is believed that the spill has existed since 1982 (IT, 2000).

BN collected two samples of the impacted soil on June 20,2001. The samples were analyzed for full-scan TPH, PCBs, and gamma spectroscopy. A radiological control technician was present during sampling activities as a precautionary measure. No radiological constituents exceeding action levels were detected in the sampling areas. Sample results indicated the presence of TPH concentrations in the diesel/oil range above regulatory levels (Table 1).

\subsubsection{CAS 25-25-16, Diesel Spill}

CAS 25-25-16 (Figure 11) consists of soil staining from a diesel release of an above-groud storage tank (Tank T-2001), a 79,000-L (21,000-gal) capacity steel tank used to store diesel fuel for a boiler located in Building 3320.

The tank was removed during the August 1998 closure of CAU 126. There was no evidence that the tank had leaked. However, the soil surrounding a valve connected to the drain pipe was moist, with a strong diesel odor.

A 1999 site visit by the IT Industrial Sites PA group revealed staining within a 17-m (56-ft) bermed area (IT, 2000). This area was approximately $3 \mathrm{~m} \mathrm{(10} \mathrm{ft)} \mathrm{high} \mathrm{and} \mathrm{was} \mathrm{lower} \mathrm{in} \mathrm{elevation}$ than the surrounding area. The stain measured $2 \mathrm{~m}(5 \mathrm{ft})$ in diameter. Approximately $10.7 \mathrm{~m}^{3}$ $\left.\left(14 \mathrm{yd}^{3}\right]\right)$ of stained soil was removed and disposed of before it was determined that additional excavation was beyond the scope of that project.

On May 25, 2001, BN collected nine samples from the surface and from a depth of up to $0.6 \mathrm{~m}$ $(2 \mathrm{ft}$ ) in the area of the impacted soil. The samples were analyzed for full-scan TPH and gamma spectroscopy. Sample results indicate the presence of TPH at concentrations above regulatory levels in the surface samples (Table 1).

\subsubsection{CAS 25-25-17, Subsurface Hydraulic Oil Spill}

CAS 25-25-17 (Figure 12) consists of a hydraulic oil spill identified on December 1, 1998, during the housekeeping closure of CAS 25-25-01 at CAU 297 (DOE/NV, 1999). Since the spill was more extensive than originally believed, CAS $25-25-17$ was created. The spill occurred as a result of the continual overfilling of two 38-L (10-gal) above-groud metal containers, which were used for recirculating oil in a vacuum pump oil recovery system at E-MAD Building 3900

(DOE/NV, 1999). 


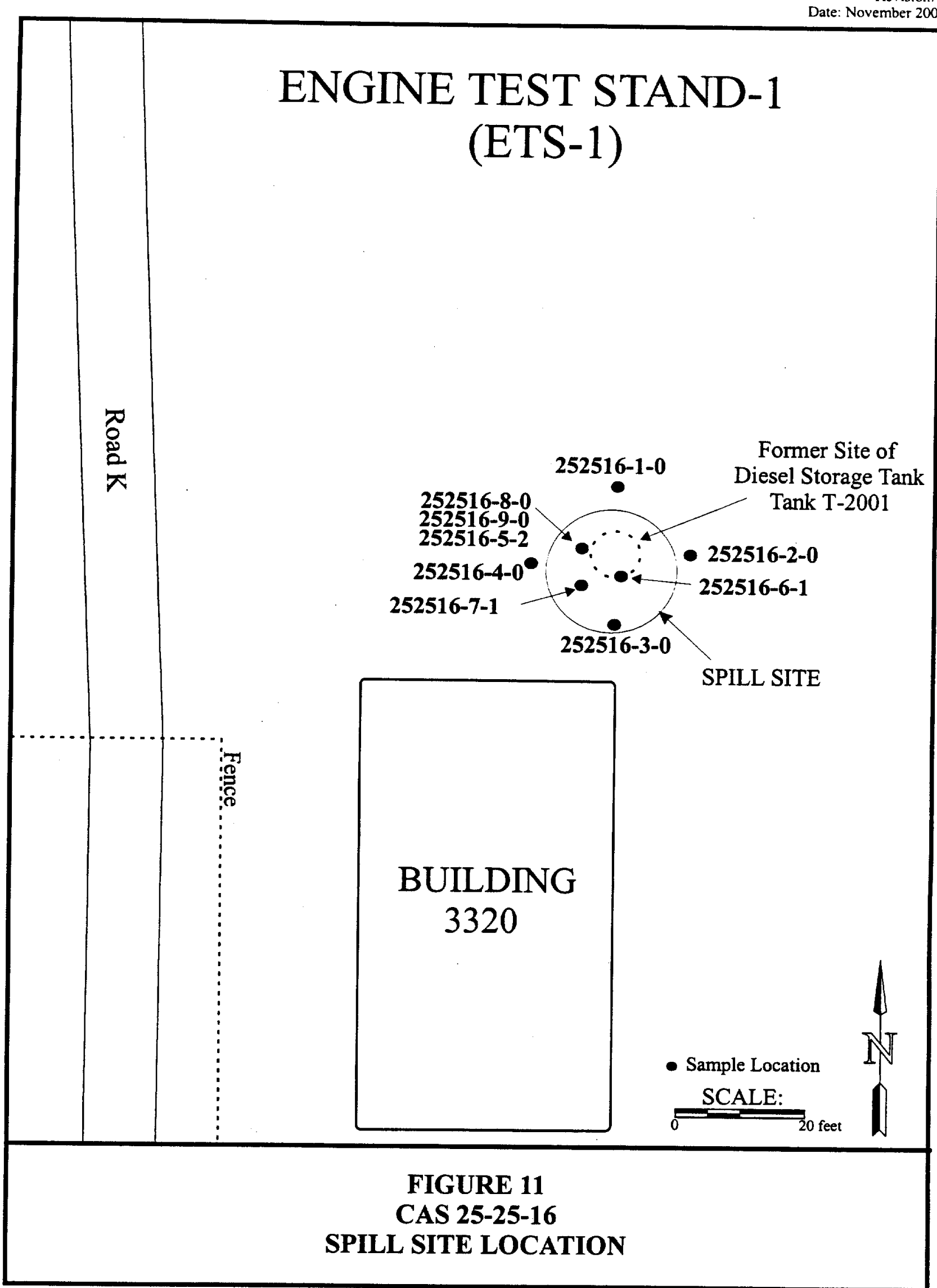


Date: November 200I

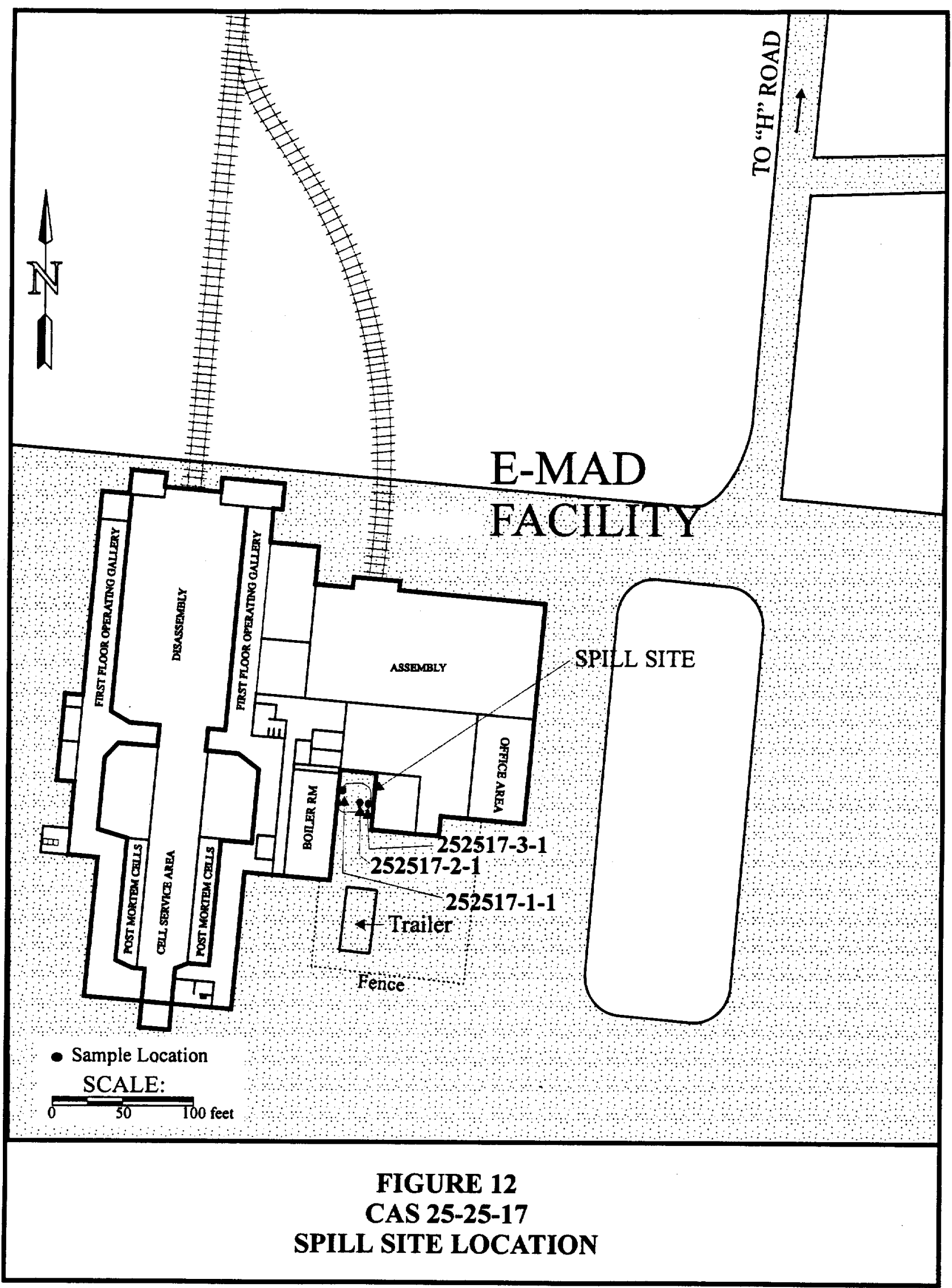


The vacuum oil recovery system was installed in approximately 1965 and was operational until the early to mid-1970s. BN completed closure of the vacuum oil recovery system on December 1, 1998. Disposal of the tanks, associated piping, and the first few inches of impacted soil was performed on January 14, 1999. This release was reported to the NDEM on January 21, 1999, as case number NDEM \#99-0120-3281 (DOE/NV, 1999b).

The spill is in a significant power/utility corridor feeding into Building 3900 . The quantity of oil released to the soil was estimated to be greater than $95 \mathrm{~L} \mathrm{(25}$ gal). While excavating, utilities

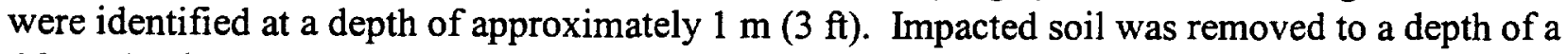
$46 \mathrm{~cm}$ (18 in). Work was discontinued due to the extent of the impacted area, confining work space limitations, and proximity to utilities. Clean soil was used as backfill over the excavated area.

Samples of the soil were collected for verification and waste disposition during CAU 297 closure activities. The samples were analyzed for TPH, TCLP VOCs, TCLP SVOCs, TCLP metals, PCBs, pesticides, and gamma spectroscopy. Sample results indicated the COPCs to be TPH and PCBs.

The site was later re-sampled in May 2001. Three soil samples were collected from a minimum depth of $0.3 \mathrm{~m}(1 \mathrm{ft})$ to ensure they were below the level of the backfill. The samples were analyzed for full-scan TPH, PCBs, and gamma spectroscopy. Results indicate the presence of TPH above regulatory limits (Table 1).

\subsection{SITE LOCATION AND DESCRIPTION}

\subsubsection{CAS 25-44-01, Fuel Spill}

CAS 25-44-01 is located near the northwest corner of the intersection of Roads C and D in Area 25 of the NTS on a concrete pad at what was known as the Fuel Storage Facility (FFACO, 1996). During site visits in 1996 and 1998, the spill was thought to be fuel spilled on soil that covers the concrete pad. The dimensions of the spill were measured to be 18 by $12 \mathrm{~m}$ ( 60 by $40 \mathrm{ft}$ ) with the depth ranging from 0 to $13 \mathrm{~cm}$ ( 0 to $5 \mathrm{in})(\mathrm{IT}, 2000)$. BN visited the site in May 2001. It was determined that the spill was not fuel but a dark-colored rock, probably used as railroad bedding.

\subsubsection{CAS 25-44-02, Spill}

CAS 25-44-02 is located on and near a concrete pad on the north side of Building 3117 at Test Cell A in Area 25 (FFACO, 1996). The site consists of a spill to the soil caused by leaking drums. A literature search and site visit by IT in 1993 determined that the drums had been removed and shipped out of state for disposal as hazardous liquid waste. The shipping manifest indicated that the drum contents contained various oils, lead, and chromium. BN visited the site in May, 2001 and collected samples from around the pad and from the suspected spill area. 


\subsubsection{CAS 25-44-03, Spill}

CAS 25-44-03 is located on a concrete pad on the southeast side of Test Cell C in Area 25 (FFACO, 1996). This site was a temporary drum storage area where the contents of two drums labeled as containing PCBs leaked onto the pad and surrounding soil. Soil staining was observed on the concrete pad extending $2 \mathrm{~m}(7 \mathrm{ft}$ ) east of the pad. The area was measured to be 4 by $3 \mathrm{~m}$ (12 by $10 \mathrm{ft})$.

\subsubsection{CAS 25-44-04, Acid Spill}

CAS 25-44-04 is located on a concrete pad on the east side of Building 3320 at the ETS-1 facility (FFACO, 1996). The site consists of spills from two tanks that were used for a water demineralization process. Tank T-2002 contained sodium hydroxide and Tank T-2003 contained sulfuric acid; each tank had an 18,927-L (5,000-gal) capacity. A site visit by IT on December 2, 1999, determined the size of the spill to be approximately 10 by $5 \mathrm{~m}$ ( 33 by $15 \mathrm{ft}$ ). The spill extended another $3 \mathrm{~m}(10 \mathrm{ft})$ east from the pad into a small gully. The the staining on the concrete pad was reddish-brown (believed to be sodium hydroxide) and yellow (believed to be sulfuric acid). The staining on the gully was reddish-brown (IT, 2000).

\subsubsection{CAS 25-25-02, Oil Spills}

CAS 25-25-02 is located on asphalt and nearby soil on the edge of the parking lot on the south side of E-MAD Building 3900 in Area 25 (FFACO, 1996). Since the loading ramp leads to the boiler room, the drums may have been waste oil or fuel from operations conducted there. The spill was described as 3 by $3 \mathrm{~m}$ ( 10 by $10 \mathrm{ft}$ ) and approximately $10 \mathrm{~cm}(4 \mathrm{in})$ below ground surface, and appeared to have been covered with soil (IT, 2000).

\subsubsection{CAS 25-25-03, Oil Spills}

CAS 25-25-03 is located $61 \mathrm{~m}$ (200 ft) south of Vehicle Maintenance Facility Building 4838 (i.e., the Gas Station) in Area 25 of the NTS (FFACO, 1996). The site was originally described as a spill adjacent to a tipped-over drum. In 1993 the spill was measured to be 2 by 1 by $1 \mathrm{~m}$ ( 6 by 3 by $3 \mathrm{ft}$ ).

\subsubsection{CAS 25-25-04, Oil Spills}

CAS 25-25-04 is located on the north side of the E-MAD facility near a building used to store flammable materials (FFACO, 1996). An estimated 133 to $151 \mathrm{~L}$ (35 to 40 gal) of waste liquids were discharged to the soil. The spill area is approximately 5 by $5 \mathrm{~m}$ ( 15 by $15 \mathrm{ft}$ ) (IT, 2000). On March 3, 1998, oil sludge was observed at one of the spills, and cement and metal debris remained in the surrounding area. One sample was collected from another location within the CAS, a small area on the surface of what appeared to be machine shop trash, a very oily, greasy absorbent material that contained metal turnings. 


\subsubsection{CAS 25-25-05, Oil Spills}

CAS 25-25-05 is located on the northeast side of the E-MAD facility in Area 25 (FFACO, 1996). The spills are oil and/or hydraulic fluid associated with heavy equipment that was once stored there. There are two larger and four smaller oil spills within the footprint of the heavy equipment. The spills are presumed to be from the same source (i.e, an equipment oil tank) but from different parts of the equipment. The discolored areas were described as black to dark brown to light brown.

\subsubsection{CAS 25-25-06, Oil Spills}

CAS 25-25-06 is located at the Drill Hole Wash Monitoring Station, which is west of the Yucca Mountain facility in Area 25 (FFACO, 1996). The site was described as diesel fuel stains underneath two generators. A January 1996 site visit by IT determined that the generators were gone and no soil staining was apparent. Two trailers remained. A later IT site visit on March 5, 1998, revealed that the larger of the two trailers was gone. However, dark-brown staining was evident on the soil where the larger trailer had been. The staining was approximately 9 by $2 \mathrm{~m}$ ( 28 by $8 \mathrm{ft}$ ). No samples were collected (IT, 2000). A later visit by BN revealed no indication of this staining.

\subsubsection{CAS 25-25-07, Hydraulic Oil Spill(s)}

CAS 25-25-07 is adjacent to a boring machine in an escape drift of X-Tunnel, which was left in place when X-Tunnel was placed on inactive status in 1982. It is estimated that approximately 1,514 L (400 gal) of hydraulic fluid may have been released from leaking hydraulic lines. The soil layer is estimated to be $0.3 \mathrm{~m}(1 \mathrm{ft})$ thick, over bedrock.

\subsubsection{CAS 25-25-08, Hydraulic Oil Spill(s)}

CAS 25-25-08 is adjacent to a boring machine in an escape drift of Y-Tunnel, which was left in place when Y-Tunnel was placed on inactive status in 1982. It is estimated that approximately $1,514 \mathrm{~L}$ (400 gal) of hydraulic fluid may have been released from leaking hydraulic lines. The soil layer is estimated to be $0.3 \mathrm{~m}(1 \mathrm{ft})$ thick, over bedrock.

\subsubsection{CAS 25-25-16, Diesel Spill}

CAS 25-25-26, east of the ETS-1 entrance, consists of soil impacted by a diesel release from an above-groud storage tank (i.e., Tank T-2001), which was a 79,000-L (21,000-gal) capacity steel tank used to store diesel fuel for a boiler in Building 3320. Soil surrounding a valve connected to the drain pipe was also moist with a strong diesel odor. 
A 1999 site visit by the IT Industrial Sites PA group revealed that staining was located within a bermed area $17 \mathrm{~m}(56 \mathrm{ft})$ in diameter (IT, 2000). This area was approximately $3 \mathrm{~m}(10 \mathrm{ft})$ high and was lower in elevation than the surrounding area.

\subsubsection{CAS 25-25-17, Subsurface Hydraulic Oil Spill}

CAS 25-25-17 is on the south side of the E-MAD facility. After completion of closure activities for CAU 297, a hydrocarbon release associated with historical operations of the oil recovery system was identified. The spill is located in a significant power/utility corridor feeding into the

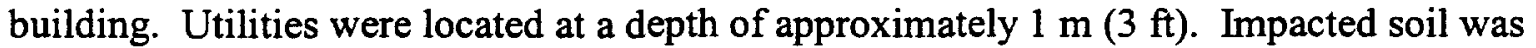
removed to a depth of $46 \mathrm{~cm}$ (18 in).

\subsection{PROCESS KNOWLEDGE}

This information was generated by the IT Industrial Sites PA group and compiled from interviews of personnel, review of historical records, and logs of field activities. The information can be obtained from the IT Las Vegas CAU 398 project files (IT, 2000).

\subsubsection{CAS 25-44-01, Fuel Spill}

Historical information about CAS 25-44-01 is limited. The origins, use, and chronological history of the spill material are unknown, although it is suspected to be railroad bedding material. There is no process knowledge of the area being used for fuel storage. The area surrounding the CAS is littered with various construction debris and concrete, which indicates that it may have been used to dump unused concrete and excess construction material.

\subsubsection{CAS 25-44-02, Spill}

Based on literature research and site investigations, it has been determined that the drums that are thought to have caused the spill at CAS 25-44-02 were removed. A uniform hazardous waste manifest from the State of Louisiana Department of Environmental Quality, Hazardous Waste Division, dated April 27, 1991, states that waste from this site, containing oil, hydraulic oil, and water, was shipped from the NTS to the state of Louisiana for disposal as hazardous liquid waste (IT, 2000). The shipping manifest indicated that the waste contained various oils, lead, and chromium. The source of the drums is unknown. Building 3117 was used to support activities at Test Cell A, but it is unclear whether these activities generated the waste in the drums.

\subsubsection{CAS 25-44-03, Spill}

CAS 25-44-03 was a temporary drum storage area where two drums labeled as containing PCBs leaked onto the pad and surrounding soil. On December 14, 1990, the two 55-gal drums were removed under on-site Manifest 91044 and transported to the Area 6 PCB storage facility. The drums were then combined with other material and shipped off site under Manifest 91014 (REECo, 1991a). The drums were characterized non-PCB, containing less than 50 parts per 
million (ppm) PCB oils (REECo, 1991b). The source of the drums and contents is unknown, but likely resulted from the draining of transformers or other electrical equipment. Available documentation indicates that the oil contained no PCBs (IT, 2000).

\subsubsection{CAS 25-44-04, Acid Spill}

There are two spills associated with this CAS, a sulfuric acid spill and a sodium hydroxide spill. Based on a site visit in December 1999, the size of the acid spill at CAS 25-44-04 was

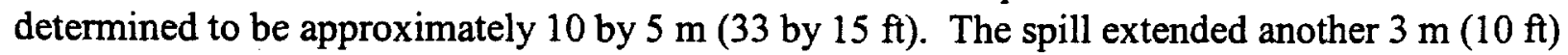
east of the pad into a small gully. The spill was identified by the reddish-brown and yellow staining. The spill from the sulfuric acid tank was believed to have occurred over a period of time due to corrosion of the tank bottom. The spill was reported to the NDEM on August 19, 1998 as NDEM \#980819-3014. The spill was estimated to be approximately $380 \mathrm{~L}$ (100 gal). The sodium hydroxide spill (NDEM \#980811-3001) occurred on August 10, 1998, during tank removal. Approximately $380 \mathrm{~L}$ (100 gal) were spilled and mixed with the residue from the sulfuric acid spill. According to the Spill Notification Notice for the sodium hydroxide release, it was assumed that the release consisted of a 50-percent solution of sodium hydroxide. A pH meter measured the $\mathrm{pH}$ of the standing liquid to be 8.5 . Approximately $2.3 \mathrm{~m}^{3}\left(3 \mathrm{yd}^{3}\right)$ of impacted soil were excavated and disposed of in the NTS U10c Construction Landfill on August 12, 1998 (BN, 1998a; 1998b).

\subsubsection{CAS 25-25-02, Oil Spills}

Available documentation indicates that the spills at CAS 25-25-02 were associated with leaking drums that were removed in 1992 and only oil spills from the leaking drums remain (REECo, 1992). The source of the drums is not listed in the documentation, although since the loading ramp leads to the boiler room, the drums may have been waste oil or fuel from operations conducted there. The spill was described as 3 by $3 \mathrm{~m}$ (10 by $10 \mathrm{ft}$ ) and approximately $10 \mathrm{~cm}$ (4 in) below ground surface, and appeared to have been covered with soil (IT, 2000).

\subsubsection{CAS 25-25-03, Oil Spills}

CAS 25-25-03 is described as a spill adjacent to a tipped-over drum. Documentation indicates that the drum was removed in July 1991, and in 1993 the spill was measured to be 2 by 1 by $1 \mathrm{~m}$ ( 6 by 3 by $3 \mathrm{ft}$ ) (REECo, 1991b). The source of the drum is not listed, although it is likely from vehicle maintenance operations at the gas station.

\subsubsection{CAS 25-25-04, Oil Spills}

CAS 25-25-04 was described by the E-MAD facility engineer as an area where used oils and cooling fluids from metal machining operations were poured directly onto the ground. An estimated 133 to $151 \mathrm{~L}$ ( 35 to $40 \mathrm{gal}$ ) of waste liquids were discharged to the soil. The spill area is approximately 5 by $5 \mathrm{~m}$ ( 15 by $15 \mathrm{ft}$ ). Cutting oils and coolants may also be present

(IT, 2000). 


\subsubsection{CAS 25-25-05, Oil Spills}

The spills at CAS 25-25-05 are composed of oil and/or hydraulic fluid associated with heavy equipment once stored there. There are four oil spills within the footprint of the heavy equipment. The spills are presumed to be from the same source (i.e., an equipment oil tank) but leaked from different parts of the equipment. The discolored areas were described as black to dark brown to light brown. The most apparent stain was measured to be 1 by $1 \mathrm{~m}$ ( 3 by $3 \mathrm{ft})$ (IT, 2000).

\subsubsection{CAS 25-25-06, Oil Spills}

CAS 25-25-06 is described as diesel fuel stains beneath two generators at the Area 25 Drill Hole Wash Monitoring Station (IT, 2000). The generators likely provided power for two trailers and monitoring equipment. A January 1996 site visit determined that the generators were gone and no soil staining was apparent. The two trailers remained. A site visit by IT in March 1998 revealed that the larger of the two trailers was gone. However, dark-brown staining was evident on the soil where the larger trailer had been. The staining was approximately 9 by $2 \mathrm{~m}$ ( 28 by $8 \mathrm{ft})(\mathrm{IT}, 2000)$.

\subsubsection{CAS 25-25-07, Hydraulic Oil Spill(s)}

At CAS 25-25-07, a boring machine was left in place when X-Tunnel was placed on inactive status in 1982. Over time, the hydraulic lines leaked fluid to the surrounding soil. The lines may also have leaked due to damage from the removal of salvageable parts from the boring machine. A sample of the hydraulic reservoir fluid was collected by IT on January 31,1997 , and analyzed for PCBs. Results indicated that PCBs were below regulatory levels (IT, 2000). The reservoir was later drained. An interview indicated that an estimated 1,514-L (400-gal) of hydraulic fluid may have been released. It was also stated that soil depth to bedrock is typically $30 \mathrm{~cm}$ (12 in) beneath the boring machine (IT, 2000).

\subsubsection{CAS 25-25-08, Hydraulic Oil Spill(s)}

At CAS 25-25-07, a boring machine was left in place when Y-Tunnel was placed on inactive status in 1982. Over time, the hydraulic lines leaked fluid to the surrounding soil. The lines may also have leaked due to damage from the removal of salvageable parts from the boring machine. It is estimated that approximately 1,514 L (400 gal) of hydraulic fluid may have been released. The soil layer is estimated to be $30 \mathrm{~cm}$ (1 ft) thick, over bedrock.

\subsubsection{CAS 25-25-16, Diesel Spill}

CAS 25-25-16 is east of the ETS-1 entrance in Area 25. The site consists of soil staining from a diesel release from an above-groud storage tank (Tank T-2001), a 79,000-L (21,000-gal) capacity steel tank used to store diesel fuel for a boiler located in Building 3320 . 


\subsubsection{CAS 25-25-17, Subsurface Hydraulic Oil Spill}

This spill was identified in December 1998 during the housekeeping closure of CAS 25-25-01 in CAU 297. Closure activities consisted of the removal of two 38-L (10-gal) metal containers used in a closed vacuum pump oil recovery system. Completion of the closure activities identified a hydrocarbon release associated with historical operations of the oil recovery system. The spill is located in a significant power/utility corridor feeding into the building. The first of the utilities was located at a depth of approximately $1 \mathrm{~m}(3 \mathrm{ft})$. Impacted soil was removed to a maximum depth of approximately $46 \mathrm{~cm}$ (18 in). The quantity of oil released to the soil was estimated to be greater than $95 \mathrm{~L}$ ( $25 \mathrm{gal})$ (IT, 2000). Work was discontinued due to the extent of the impacted area, confining work space limitations, and proximity to utilities. Clean soil was used as backfill over the excavated area.

\subsection{CLOSURE STANDARDS}

The clean-closure standards for the purposes of closure verification for this SAFER Plan are:

- $\quad$ EPA Region IX risk-based Preliminary Remediation Goals (PRG) for industrial soils (EPA, 1996a).

- $\quad$ Nevada State action level for petroleum hydrocarbons in soil (i.e., $100 \mathrm{mg} / \mathrm{kg}$ ) as stated in NAC Section 445A.2272 (NAC, 2000). 


\subsection{FIELD ACTIVITIES AND CLOSURE OBJECTIVES}

This section provides the framework and rationale for characterization, neutralization, removal, closure verification, site restoration, and waste disposal. The SAFER process is discussed in detail in the following subsections.

Before field activities begin, the following activities will have been completed:

- Development of an endangered species survey.

- Preparation of a National Environmental Policy Act documentation.

- Preparation of a Site-Specific Health \& Safety Plan.

- Cultural Resource Survey. Because of the potential to harm cultural resources, off-road driving in areas surrounding the sites is not allowed.

\subsection{CONSTITUENTS OF CONCERN}

Based on site process knowledge and the analytical results for site characterization samples collected by BN Environmental Restoration personnel during May 2001, the COCs for each CAS are listed in Table 2. Appendix A-2, DQOs, contains details about the analyses performed for $\mathrm{BN}$ site waste characterization samples.

\subsection{REMEDIATION}

\subsubsection{Closure by No Further Action}

Sample results for the following sites show no COCs present above regulatory levels. Therefore, these CASs will be closed by taking no further remedial action. However, as a best management practice, material and debris (e.g., the railroad bedding material and debris at CAS 25-44-01) will be removed and disposed of as sanitary waste.

- $\quad$ CAS 25-44-01, Fuel Spill

- $\quad$ CAS 25-44-04, Acid Spill

- CAS 25-25-06, Oil Spills

\subsubsection{Clean Closure by Excavation and Disposal}

Sample results for ten CASs show the presence of COCs above action levels. Seven of these ten sites will be closed by excavation and appropriate disposal of the impacted soil/material. The remaining three sites will be closed administratively (see Section 3.2.3). The extent of the excavation will be determined by TPH field-screening results, using detection kits such as PetroFLAG test kits. Once field-screening results indicate that all TPH-impacted soil has been removed (i.e., TPH levels for screening samples are less than $75 \mathrm{ppm}$ ), soil verification samples will be collected for analysis by an approved off-site contract laboratory. 


\section{TABLE 2 - CAU 398 CONSTITUENTS OF CONCERN}

\begin{tabular}{|c|c|c|c|}
\hline CAS & $\begin{array}{c}\text { CHARACTERIZATION SAMPLE } \\
\text { ANALYSES } \\
\end{array}$ & \multicolumn{2}{|c|}{$\begin{array}{l}\text { CONSTITUENTS OF } \\
\text { CONCERN }(\mathrm{mg} / \mathrm{kg})\end{array}$} \\
\hline $25=44-01-$ Fuel Spill & TCLP $^{\mathrm{b}}$ metals $^{\mathrm{c}}$, gamma spectroscopy ${ }^{\mathrm{d}}$ & None & \\
\hline 25-44-02 - Spill & $\begin{array}{l}\text { TPH }, \text { TCLP cadmium and lead, gamma } \\
\text { spectroscopy }\end{array}$ & TPH & 1,990 \\
\hline 25-44-03 - Spill & $\begin{array}{l}\text { TPH, PCBs }{ }^{f} \text {, TCLP metals, gamma } \\
\text { spectroscopy }\end{array}$ & TPH & 5,320 \\
\hline 25-44-04 - Acid Spill & $\mathrm{pH}^{\mathrm{g}}$, gamma spectroscopy & None & \\
\hline 25-25-02 - Oil Spills & $\begin{array}{l}\text { TPH, PCBs, TCLP SVOCs }{ }^{\mathrm{h}} \text {, TCLP metals, } \\
\text { gamma spectroscopy }\end{array}$ & $\begin{array}{l}\text { TPH } \\
\text { PCBs }\end{array}$ & $\begin{array}{r}3,440 \\
9.2\end{array}$ \\
\hline 25-25-03 - Oil Spills & TPH, gamma spectroscopy & $\mathrm{TPH}$ & 12,800 \\
\hline 25-25-04 - Oil Spills & $\begin{array}{l}\text { TPH, PCBs, TCLP VOCs', TCLP SVOCs, } \\
\text { TCLP metals, gamma spectroscopy }\end{array}$ & $\begin{array}{l}\text { TPH } \\
\text { PCBs } \\
\text { Lead } \\
\text { Cadmium }\end{array}$ & $\begin{array}{r}21,600 \\
920 \\
11(\mathrm{mg} / \mathrm{L}) \\
1.1(\mathrm{mg} / \mathrm{L})\end{array}$ \\
\hline 25-25-05 - Oil Spills & $\begin{array}{l}\text { TPH, PCBs, TCLP VOCs, TCLP SVOCs, } \\
\text { TCLP metal, pesticides', gamma } \\
\text { spectroscopy }\end{array}$ & $\begin{array}{l}\text { TPH } \\
\text { Cadmium }\end{array}$ & $\begin{array}{r}46,500 \\
1.1(\mathrm{mg} / \mathrm{L})\end{array}$ \\
\hline 25-25-06 - Oil Spills & TPH, gamma spectroscopy & None & \\
\hline $\begin{array}{l}\text { 25-25-07 - Hydraulic Oil } \\
\text { Spill(s) }\end{array}$ & TPH, PCBs, gamma spectroscopy & TPH & 105,000 \\
\hline $\begin{array}{l}\text { 25-25-08 - Hydraulic Oil } \\
\text { Spill(s) }\end{array}$ & TPH, PCBs, gamma spectroscopy & TPH & 140,000 \\
\hline 25-25-16 - Diesel Spill & TPH, gamma spectroscopy & $\mathrm{TPH}$ & 1,320 \\
\hline $\begin{array}{l}25-25-17 \text { - Subsurface } \\
\text { Hydraulic Oil Spill }\end{array}$ & $\begin{array}{l}\text { TPH, TCLP VOCs, TCLP SVOCs, } \\
\text { TCLP metals, PCBs, pesticides, gamma } \\
\text { spectroscopy }\end{array}$ & TPH & \\
\hline
\end{tabular}

\section{Notes:}

- All analyses were made by an off-site laboratory during 2001 using EPA Test Method for Evaluating Waste, $3^{\text {rd }}$ Edition, Parts 1-4, SW-846 (EPA, 1996b).

b Toxicity Characteristic Leaching Procedure (TCLP), sample preparation method SW-846 1311 (EPA, 1996b).

c Metals include arsenic, barium, cadmium, chromium, lead, mercury, selenium, and silver by method SW-846 6010 and 7471A (EPA, 1996b).

- Radionuclides analyzed by gamma spectroscopy.

- Total petroleum hydrocarbons, full-scan, gasoline, diesel, and waste oil carbon ranges, by SW-846 8015 modified (EPA, 1996b).

f Polychlorinated biphenyls by SW-846 8082 (EPA, 1996b).

Measure of acidity.

h Semivolatile organic constituents by SW-846 8270 (EPA, 1996b).

i Volatile organic constituents by SW-846 8260 (EPA, 1996b).

$j$ Pesticides by SW-846 8081 (EPA, 1996b). 
Analysis of all verification samples will be by standard EPA methods (EPA, 1996b). When analytical results for the verification samples confirm that no COCs are present above action levels, the excavations will be backfilled with clean fill material and graded approximate the original site contours. The excavated impacted soil will be disposed of at the NTS Area 6 Hydrocarbon Landfill, which accepts soils with PCB levels of less than $50 \mathrm{mg} / \mathrm{kg}$. Excavated soils with PCB levels greater than $50 \mathrm{mg} / \mathrm{kg}$ or other COCs above RCRA levels will be disposed as hazardous waste. The soil will be packaged appropriately and shipped to an approved off-site hazardous waste treatment and disposal facility by BN Hazardous Waste Operations. Copies of all shipping manifests will be included in the CAU 398 Closure Report (CR). The following seven CASs will be clean-closed by excavation and disposal of impacted soil:

- CAS 25-44-02, Spill. Approximately $3 \mathrm{~m}^{3}\left(4 \mathrm{yd}^{3}\right)$ of TPH-impacted soil will be excavated and disposed of in the NTS Area 6 Hydrocarbon Landfill.

- $\quad$ CAS 25-44-03, Spill. Approximately $2.3 \mathrm{~m}^{3}\left(3 \mathrm{yd}^{3}\right)$ of TPH-impacted soil will be excavated and disposed of in the NTS Area 6 Hydrocarbon Landfill.

- $\quad$ CAS 25-25-02, Oil Spill. Approximately $6 \mathrm{~m}^{3}\left(8 \mathrm{yd}^{3}\right)$ of TPH- and PCB-impacted soil will be excavated and disposed of in the NTS Area 6 Hydrocarbon Landfill if PCB concentrations are less than the land ban limit of $50 \mathrm{mg} / \mathrm{kg}$. Otherwise, soil will be disposed of as hazardous waste.

- $\quad$ CAS 25-25-03, Oil Spill. Approximately $0.76 \mathrm{~m}^{3}\left(1 \mathrm{yd}^{3}\right)$ of TPH-impacted soil will be excavated and disposed of in the NTS Area 6 Hydrocarbon Landfill.

- CAS 25-25-04, Oil Spill. Approximately $0.76 \mathrm{~m}^{3}\left(1 \mathrm{yd}^{3}\right)$ of TPH, PCB, and RCRA metals-impacted soil will be excavated and disposed of as hazardous waste. Waste material will containerized and stored on-site in a 90-day accumulation area. Waste will be characterized for disposal, and will be disposed of accordingly.

- CAS 25-25-05, Oil Spill. Approximately $34 \mathrm{~m}^{3}\left(45 \mathrm{yd}^{3}\right)$ of TPH and RCRA metalsimpacted soil will be excavated and disposed of as hazardous waste. Waste material will containerized and stored on-site in a 90-day accumulation area. Waste will be characterized for disposal, and will be disposed of accordingly.

- CAS 25-25-16, Diesel Spill. Approximately $0.9 \mathrm{~m}^{3}\left(1.2 \mathrm{yd}^{3}\right)$ of TPH-impacted soil will be excavated and disposed of in the NTS Area 6 Hydrocarbon Landfill.

Sufficient information exists for these CASs to be clean-closed by excavation and disposal of impacted soil. All sites contain TPH-impacted soil. Three sites contain COPCs in addition to TPH that are associated with the TPH. CAS 25-25-02 also contains PCBs below the land ban restrictions; CAS 25-25-05 also contains cadmium above land ban restrictions; and CAS 25-25-04 also contains lead, cadmium, and PCBs above land ban restrictions. Soil excavated from CASs with COPCs above the land ban restrictions must be sent to an off-site treatment and disposal facility. 


\subsubsection{Administrative Closure}

Three CASs will be administratively closed. Field work to close these sites either poses unacceptable health and safety risks to workers or is impractical (e.g., inside bedrock-lined tunnels). In addition, these sites currently pose a very low risk to the environment, groundwater, and off-site populations. For each of the three CASs, an "A through K" risk evaluation will be made following the NAC Section 445A.2272 guidelines (NAC, 2000). In addition, the sites will be surveyed to implement use restrictions. The following three CASs will be closed administratively:

- $\quad$ CAS 25-25-07, Hydraulic Oil Spill(s)

- $\quad$ CAS 25-25-08, Hydraulic Oil Spill(s)

- $\quad$ CAS 25-25-17, Subsurface Hydraulic Oil Spill

\subsection{VERIFICATION}

In order to assess the completeness of the remediation activities, biased soil samples will be collected to confirm that all materials exceeding the established clean-up criteria have been removed. The action level for TPH is $100 \mathrm{mg} / \mathrm{kg}$, based on NAC $445 \mathrm{~A} .2272$, and $1.0 \mathrm{mg} / \mathrm{kg}$ for PCBs, $750 \mathrm{mg} / \mathrm{kg}$ for lead, and $810 \mathrm{mg} / \mathrm{kg}$ for cadmium based on EPA Region IX PRGs for Industrial Soils (EPA, 1996a). Soil verification samples will be collected from each side and the bottom of the excavation. A minimum of one sample will be collected from each wall/floor. Additional samples may be required if the first set of verification samples indicates that COCs are still present. BN Organization Instruction OI-2152.108, "Soil Sampling," will be followed to collect all soil verification samples (BN, 2000). All samples will be labeled and sample jars will be sealed with custody tape, placed in individual zip-lock bags, and placed on ice in a cooler for transport to the NTS Analytical Services office. Each verification sample will be submitted for laboratory analysis using standard EPA methods (EPA, 1996b).

\subsection{DATA QUALITY OBJECTIVES}

DQOs are qualitative and quantitative statements that specify the quality of the data required to support potential closure alternatives for CAU 398. The DQOs were developed to clearly define the purposes for which environmental data will be used and to design a data-collection program that will satisfy these purposes. The formulation of a conceptual site model (CSM) is an aid to the development of DQOs for the site.

Details of the DQO process are presented in Appendix A-2. During the DQO discussions for CAU 398, data needed to resolve problem statements and decision statements were identified. Criteria for data collection and analysis were defined and agreed upon, and the appropriate quality assurance (QA)/quality control (QC) required for particular data collection activities was assigned. The analytical methods and reporting limits prescribed through the DQO process, and the data quality indicators (DQIs) for laboratory analysis such as a precision and accuracy requirements, are provided in more detail in Section 6.0 of this SAFER Plan. At the end of the investigation, resulting laboratory data will be evaluated to confirm or refute the CSM and 
determine whether the DQOs were met by using the DQIs of precision, accuracy, representativeness, completeness, and comparability. Other DQIs, such as sensitivity, may be used.

\subsection{CLOSURE}

Hold points and conditions that are outside the assumptions of this plan may impact the requirements for closure. The proposed activities for closure of CAU 398 include the following:

- The extent of soil with TPH and PCB concentrations greater than action levels will be determined in the field for each impacted CAS.

- All impacted soil with TPH and PCB concentrations greater than action levels will be excavated from each impacted CAS.

- All removed impacted soil will be loaded and transported to the NTS Area 6 Hydrocarbon Landfill or, if determined to be hazardous waste, transported to an approved permitted off-site hazardous waste treatment and storage facility.

- All excavations will be backfilled with clean fill.

- If necessary, an "A through $\mathrm{K}$ " evaluation of the site will be prepared.

- If necessary, the site will be surveyed and a Use Restriction will be implemented.

- The Closure Report will be submitted for approval by the NDEP.

\subsection{DURATION}

The schedule will require modifications if conditions exist that are outside the assumptions on which the schedule was developed. Flexibility has been placed in the project schedule to account for minor difficulties (e.g., weather, equipment breakdowns, personnel availability, NTS operational and security constraints, or operation and mobility in X and $\mathrm{Y}$ Tunnels and next to the E-MAD facility). NNSA/NV will keep the NDEP informed of any condition that may impact the project schedule. Figure 13 presents the proposed project schedule following submittal of the final SAFER Plan for CAU 398. 


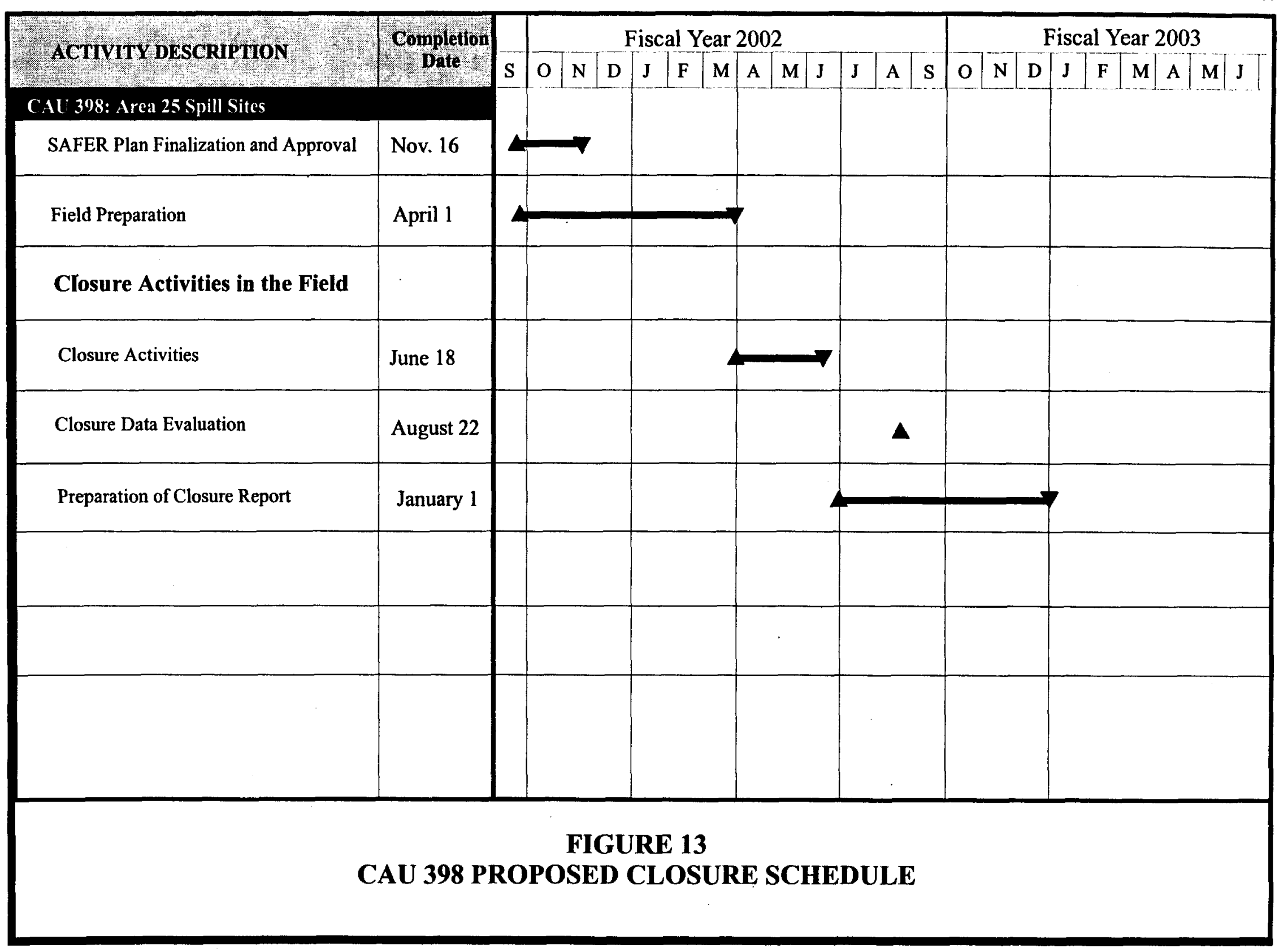

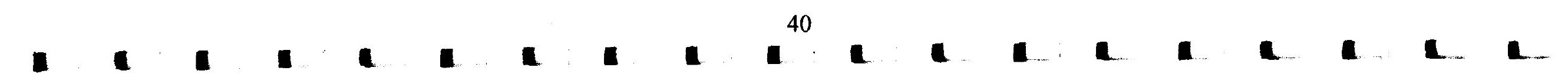




\subsection{REPORTS AND RECORDS AVAILABILITY}

A daily report will be prepared when field activities have started. The report will summarize the daily activities, site visitors, health and safety issues, and any relevant issues or problems. This report will be provided to the NNSA/NV Task Manager for submittal to the NDEP.

Upon completion of closure activities, a CR will be prepared and will include the following sections and subsections:

- Introduction (Purpose and Scope)

- Closure Activities (Description of Corrective Action Activities, Deviation from the SAFER Plan as Approved, Corrective Action Schedule as Completed, and Site Plan/Survey Plan)

- Waste Disposition

- Closure Verification Results (Data Quality Assessment, and Use Restrictions)

- $\quad$ References

- Supporting Documentation (Analytical Results, As-Built Documentation, Waste Disposition Documentation, and Modifications to the SAFER Plan)

The final CR will be submitted to NNSA/NV and NDEP for review and approval. This SAFER Work Plan and the subsequent CR will be available in the NNSA/NV Public Reading facilities in Las Vegas and Carson City, Nevada, or by contracting the NNSA/NV Project Manager. The NDEP maintains the official administrative record for all activities conducted under the auspices of the FFACO (FFACO, 1996) 
SAFER PLAN - CAU 398

Area 25 Spill Sites

Section: Reports \& Records

Revision: 0

Date: November 10, 2001

\section{THIS PAGE INTENTIONALLY LEFT BLANK}




\subsection{INVESTIGATION/REMEDIATION WASTE MANAGEMENT}

Waste fro m CAU 398 will be managed in accordance with all state and federal regulations, DOE orders, BN procedures, and the RCRA Operational Permit. Known waste types will include nonhazardous (e.g., sanitary/construction debris) waste, TPH-impacted soils, and hazardous waste.

\subsection{WASTE MINIMIZATION}

Waste generation will be minimized by limiting the number of people in the work area. For the duration of the project, site workers will adhere to the principles of the BN Waste Minimization and Pollution Prevention Program. Care will be taken to segregate waste from non-waste materials, if at all possible, and avoid cross contamination.

\subsection{POTENTIAL WASTE STREAMS}

Table 3 shows the potential waste streams by the CAS in CAU 398. Table 4 shows the types of waste management requirements. No waste is expected to be generated at the CASs to be closed by taking no further action or administratively.

\section{TABLE 3 - POTENTIAL WASTE STREAMS BY CAS AT CAU 398}

\begin{tabular}{|c|c|c|c||}
\hline CAS & Sanitary & $\begin{array}{c}\text { Hydrocarbon- } \\
\text { Impacted Soil }\end{array}$ & Hazardous \\
\hline \hline CAS 25-44-01 & -- & -- & - \\
\hline CAS 25-44-02 & $X$ & $X$ & $X$ \\
\hline CAS 25-44-03 & $X$ & $X$ & $X$ \\
\hline CAS 25-44-04 & - & - & $X$ \\
\hline CAS 25-25-02 & $X$ & $X$ & $X$ \\
\hline CAS 25-25-03 & $X$ & $X$ & $X$ \\
\hline CAS 25-25-04 & $X$ & $X$ & $X$ \\
\hline CAS 25-25-05 & $X$ & $X$ & - \\
\hline CAS 25-25-06 & -- & -- & - \\
\hline CAS 25-25-07 & -- & -- & $X$ \\
\hline CAS 25-25-08 & -- & $X$ & - \\
\hline CAS 25-52-16 & $X$ & - & $X$ \\
\hline CAS 25-25-17 & -- & $X$ & $X$ \\
\hline
\end{tabular}

Note: Dashes indicate no waste generated; $X$ indicates potential waste generation. 


\section{TABLE 4 - MANAGEMENT OF WASTE TYPES TO BE PRODUCED DURING CAU 398 CLOSURE}

\begin{tabular}{|c|c|c|}
\hline MEDIA & WASTE TYPE & WASTE MANAGEMENT \\
\hline \multirow{3}{*}{$\begin{array}{l}\text { PPE, } \\
\text { Sampling } \\
\text { Equipment, } \\
\text { and TPH Field } \\
\text { Screening } \\
\text { Waste }\end{array}$} & $\begin{array}{l}\text { Material with PCB }>50 \text { ppm } \\
\text { is toxic (Toxic Substance } \\
\text { Control Act }[\text { TSCA]) waste. }\end{array}$ & $\begin{array}{l}\text { Waste will be managed in accordance with BN Company } \\
\text { Directive CD-0442.007 "Management of Polychlorinated } \\
\text { Biphenyls". TSCA waste will be transported off-site to an } \\
\text { approved hazardous waste treatment and disposal facility. }\end{array}$ \\
\hline & $\begin{array}{l}\text { Material with } \mathrm{PCB}<50 \mathrm{ppm} \\
\text { is sanitary waste. }\end{array}$ & $\begin{array}{l}\text { Waste will be managed as sanitary waste and transported } \\
\text { to the Area 9, U10C Landfill or the Area } 23 \text { Sanitary } \\
\text { Landfill for disposal. }\end{array}$ \\
\hline & $\begin{array}{l}\text { Spent methanol from TPH } \\
\text { field screening is hazardous } \\
\text { waste. }\end{array}$ & $\begin{array}{l}\text { Material will be transported to and placed in the Area } 23 \\
\text { SAA Number NTS099. The SAA is managed in } \\
\text { accordance with NNSANV and DOE procedures } \\
\text { governing hazardous waste. }\end{array}$ \\
\hline \multirow{4}{*}{ Soil } & $\begin{array}{l}\text { Soil with } \mathrm{PCBs}<50 \mathrm{ppm} \text { and } \\
\mathrm{TPH}>100 \mathrm{mg} / \mathrm{kg} \text {. }\end{array}$ & $\begin{array}{l}\text { The soil will be transported to the Area } 6 \text { Hydrocarbon } \\
\text { Landfill for disposal. }\end{array}$ \\
\hline & $\begin{array}{l}\text { Soil with PCBs }>50 \mathrm{ppm} \text { and } \\
\text { any level of TPH. }\end{array}$ & $\begin{array}{l}\text { The soil is TSCA waste and will be transported off-site to } \\
\text { an approved hazardous waste treatment and disposal } \\
\text { facility. }\end{array}$ \\
\hline & $\begin{array}{l}\text { Soil with TPH }>100 \mathrm{mg} / \mathrm{kg} \\
\text { and no PCBs. }\end{array}$ & $\begin{array}{l}\text { The soil will be transported to the Area } 6 \text { Hydrocarbon } \\
\text { Landfill for disposal. }\end{array}$ \\
\hline & $\begin{array}{l}\text { Soil with } \mathrm{TPH}<100 \mathrm{mg} / \mathrm{kg} \\
\text { and PCBs }<1 \mathrm{ppm} \text {. }\end{array}$ & $\begin{array}{l}\text { The soil will be returned to the excavation and used as } \\
\text { backfill material. }\end{array}$ \\
\hline
\end{tabular}

\section{Note:}

The Area 6 Hydrocarbon Landfill and the Area 23 Sanitary Landfill permits allows disposal of waste having PCB levels less than or equal to $50 \mathrm{ppm}$. Waste with PCB levels greater than $50 \mathrm{ppm}$ are considered toxic waste (TSCA, 1976) and must be disposed of at an approved and permitted off-site facility. BN Hazardous Waste Operations will handle the disposal of any toxic waste generated during the closure of CAU 398.

\subsubsection{Nonhazardous Waste}

Nonhazardous waste (i.e., sanitary debris) will be generated during closure of CAU 398. Nonhazardous waste will consist primarily of used personal protective equipment (PPE) and housekeeping debris, such as paper, plastic, and wood. Where possible, this type of debris will be recycled. Non-recyclable materials will be disposed in the NTS Sanitary Landfill.

\subsubsection{TPH-Impacted Soil}

TPH-impacted soil will be excavated from seven CASs during closure of CAU 398. If no other COCs are present at concentrations greater than regulatory limits, the removed soil will be transported and disposed of in the NTS Area 6 Hydrocarbon Landfill. If COCs (e.g., PCBs) are present in the soil at concentrations greater than regulatory limits, the soil will be treated as hazardous waste (EPA, 1996c; 1996d). 


\subsubsection{Hazardous Waste}

Hazardous waste (e.g., spent methanol) will be produced by TPH field screening of soil during the closure of CAU 398. Spent methanol is classified by RCRA as a hazardous waste and will be managed as such. The waste will be transported to NTS Area 23 Satellite Accumulation Area (SAA) Number NTS099. The SAA is registered and serviced by the BN Hazardous Waste Operations group. The accumulated waste is collected from the SAA periodically and transported and disposed of off site at an approved, permitted hazardous waste treatment, storage, and disposal facility.

Excavated soil that contain PCBs and/or RCRA metals at concentrations greater than RCRA hazardous waste levels (EPA, 1996c) will be treated as hazardous waste. The waste will be placed in approved containers inside an established SAA. BN Hazardous Waste Operations will oversee the transport of the containers to the Area 5 Hazardous Waste Pad and the shipment of the waste off-site to an approved hazardous waste treatment and storage facility for disposal.

\subsection{CONTAINER MANAGEMENT}

All containers must be in good condition. If the container begins to leak, the contents must be transferred to a container that is in good condition without dents or significant rust. The containers must always be closed while stored unless waste is being added or removed. They must be handled in such a manner that will not jeopardize the integrity of the container.

Some 208-L (55-gal) drums may be used during this project. Containers will not be filled above their specified weight capacity. Compactable waste will then be placed in 208-L (55-gal) drums. After a container has been filled, the container will be locked. If a container is not completely filled to capacity at the end of a workday, it will be locked and tamper-resistant tape will be placed over the container's hinge. Additional precautions will include not filling 208-L (55-gal) drums more than 7/8 full and not mixing waste types (e.g., personal PPE and decontamination water).

Because the majority of the waste produced during this project is anticipated to be soil and debris, secondary containment will not be required. If, however, free liquids, such as decontamination water, are placed in containers, the containers will be placed on spill containment pallets or within a plastic-lined bermed area. 
Appropriate labels and relevant information will be marked on each container with an indelible marker and must be legible and clearly visible for inspections. Pertinent data will be written on duct tape or a blank adhesive label that is applied to the side of the container. The following information will be included:

- Waste-tracking label

- Type of waste in the container (e.g., marked "Hazardous Waste")

- Location where waste was derived

- Date that accumulation begins/ends

- If sampling is required, an "Awaiting Analysis" sticker after sampling has been completed 


\subsection{QUALITY ASSURANCE/QUALITY CONTROL}

The overall objective of the closure activities described in this plan is to collect accurate and defensible data to support the selection of and to implement a closure alternative for each CAS in CAU 398. The following sections discuss the collection of required QC samples in the field and QA requirements for laboratory/analytical data to achieve closure.

\subsection{PROPOSED FIELD SAMPLE COLLECTION ACTIVITIES}

Field QC samples will be collected in accordance with established procedures (BN, 2000). Field QC samples are collected and analyzed to aid in determining the validity of sample results. The number of required QC samples depends on the type and number of environmental samples collected. The minimum frequency of collecting and analyzing QC samples for this investigation, as determined in the DQO process, include:

- $\quad$ Equipment blanks (one per sampling event)

- Field duplicates (1 per 20 environmental samples or 1 if less than 20 are collected)

- Matrix spike/matrix spike duplicate (1 per 20 environmental samples or 1 if less than 20 are collected)

Additional QC samples may be substituted, based on site conditions, at the discretion of the Technical Lead. Field QC samples will be analyzed using the same analytical procedures used for environmental samples. The results of the QC sample analysis will be included in the CR.

\subsection{PROPOSED LABORATORY/ANALYTICAL DATA QUALITY INDICATORS}

Criteria for site closure requires laboratory analysis of samples used to provide a quantitative measurement of any COCs present. Rigorous $\mathrm{QA} / \mathrm{QC}$ will be implemented for all laboratory samples and will include documentation, data verification, validation of analytical results, and meeting the requirements of DQIs as they relate to laboratory analysis.

Data verification and validation will be performed in accordance with established procedures and with this SAFER Plan. All laboratory data from samples collected and analyzed will be evaluated for data quality according to EPA Functional Guidelines (EPA, 1994; 1999). The data will be reviewed to ensure that all critical samples were appropriately collected and analyzed and that the results passed data validation criteria. Validated data, whether estimated or not, will be assessed to determine whether they meet the DQOs of the investigation and the performance criteria for the DQIs. The results of this assessment will be documented in the CR. If the DQOs were not met, corrective actions will be evaluated, selected, and implemented (e.g., refine the CSM or resample to fill data gaps). 
DQIs are qualitative and quantitative statements that specify the data requirements of a project and include precision, accuracy, representativeness, completeness, and comparability. In addition, sensitivity has been included as a DQI for laboratory analysis. The performance criteria for each indicator has been selected based on the intended use of the data, current field and analytical procedures, and instrumentation. Precision and accuracy goals have been standardized for both organic and inorganic analytes for analytical laboratories under the EPA Contract Laboratory Program (EPA, 1988a; 1988b). Laboratory QC samples used to measure the precision and accuracy of analytical procedures will be analyzed using the same analytical procedures used for environmental samples.

Table 5 provides the established performance criteria for each of the DQIs and the impacts to the decision if the criteria are not met. Any deficiencies noted during the investigation that renders the data quality unacceptable will be documented in the CR. 
TABLE 5 - LABORATORY/ANALYTICAL DATA QUALITY INDICATORS

\begin{tabular}{|c|c|c|}
\hline $\begin{array}{l}\text { DATA QUALITY } \\
\text { INDICATOR }\end{array}$ & PERFORMANCE CRITERIA & $\begin{array}{l}\text { IMPACT ON DECISION IF } \\
\text { PERFORMANCE CRITERIA NOT MET }\end{array}$ \\
\hline Precision & $\begin{array}{l}\text { Variations between duplicates (field } \\
\text { and lab) and original sample should } \\
\text { not exceed analytical method-specific } \\
\text { criteria listed in Table } 6 \text {. }\end{array}$ & $\begin{array}{l}\text { Estimated data within samples delivery group } \\
\text { (SDG) will be evaluated for there usability. If } \\
\text { data are determined to be unusable, data will not } \\
\text { be used in decision and completeness criteria will } \\
\text { be assessed. }\end{array}$ \\
\hline Accuracy & $\begin{array}{l}\text { Laboratory control sample results and } \\
\text { matrix spike results should be within } \\
\text { analytical method-specific criteria } \\
\text { listed in Table } 6 \text {. }\end{array}$ & $\begin{array}{l}\text { Estimated data within SDG will be evaluated for } \\
\text { its usability. If estimated data are biased high or } \\
\text { conservative, the data may be used in decision. If } \\
\text { estimated data are biased low and below the } \\
\text { decision threshold, the data may not be used in } \\
\text { decision and completeness criteria will be } \\
\text { assessed. }\end{array}$ \\
\hline Sensitivity & $\begin{array}{l}\text { Detection limits of laboratory } \\
\text { instruments must be less than action } \\
\text { level for COC. }\end{array}$ & $\begin{array}{l}\text { Cannot determine if COCs are present at levels of } \\
\text { concern, thereby investigation objectives cannot } \\
\text { be met. }\end{array}$ \\
\hline Completeness & $\begin{array}{l}100 \% \text { of samples submitted to } \\
\text { laboratory } \\
100 \% \text { of requested analyses } \\
\text { performed } \\
100 \% \text { of critical analytes to be valid } \\
80 \% \text { of non-critical analytes to be } \\
\text { valid }\end{array}$ & $\begin{array}{l}\text { 1. Decision of whether extent of contamination } \\
\text { has been bounded cannot be determined. Impacts } \\
\text { to decisions will be assessed. } \\
\text { 2. Decision of whether COCs (TPH) above NAC } \\
\text { level remain in soil cannot be determined. } \\
\text { Impacts to decisions will be assessed. }\end{array}$ \\
\hline Comparability & $\begin{array}{l}\text { Equivalent samples analyzed using } \\
\text { same analytical methods, same units } \\
\text { of measurement, and detection limits } \\
\text { must be used for like analyses. }\end{array}$ & Inability to use data collected. \\
\hline $\begin{array}{l}\text { Representa- } \\
\text { tiveness }\end{array}$ & $\begin{array}{l}\text { Correct analytical method performed } \\
\text { for appropriate COPC: valid data } \\
\text { reflects appropriate target population. }\end{array}$ & $\begin{array}{l}\text { Cannot identify COC or estimate concentration of } \\
\text { COC; therefore, cannot make decision(s) on } \\
\text { target population. }\end{array}$ \\
\hline
\end{tabular}

${ }^{2} \mathrm{C}$ ritical analytes are those analytes most likely present in the target population at COCs that have been identified through process knowledge of similar sites and historical documentation. Critical analytes for samples are TPH and PCBs. 
TABLE 6 - LABORATORY TPH PROCEDURE FOR CAU 398

\begin{tabular}{|c|c|c|c|c|c|c|}
\hline $\begin{array}{c}\text { PARA- } \\
\text { METER } \\
\text { OR } \\
\text { ANALYTE } \\
\end{array}$ & $\begin{array}{l}\text { MEDIUM } \\
\text { OR } \\
\text { MATRIX }\end{array}$ & $\begin{array}{c}\text { ANALY- } \\
\text { TICAL } \\
\text { METHOD }\end{array}$ & $\begin{array}{c}\text { MINIMUM } \\
\text { REPORTING } \\
\text { LIMIT }\end{array}$ & $\begin{array}{l}\text { REGULATORY } \\
\text { LIMIT }\end{array}$ & $\begin{array}{c}\text { RELATIVE } \\
\text { PERCENT } \\
\text { DIFFERENCE } \\
(\text { RPD) } \\
\end{array}$ & $\begin{array}{c}\text { PERCENT } \\
\text { RECOVERY } \\
\text { (\%R) }^{\mathbf{b}}\end{array}$ \\
\hline \multirow{4}{*}{ TPH } & $\begin{array}{l}\text { Water } \\
\text { Gasoline }\end{array}$ & \multirow{4}{*}{$\begin{array}{c}\text { 8015B } \\
\text { modified }^{c}\end{array}$} & $\begin{array}{c}0.1 \text { milligrams } \\
\text { per liter }(\mathrm{mg} / \mathrm{L})^{\mathrm{d}}\end{array}$ & $100 \mathrm{mg} / \mathrm{L}^{\mathrm{e}}$ & \multirow{4}{*}{ Lab specific ${ }^{f}$} & \multirow{4}{*}{ Lab specific ${ }^{f}$} \\
\hline & $\begin{array}{l}\text { Soil } \\
\text { Gasoline }\end{array}$ & & $\begin{array}{l}0.5 \text { milligrams } \\
\text { per kilogram } \\
(\mathrm{mg} / \mathrm{kg})^{\mathrm{d}}\end{array}$ & $100 \mathrm{mg} / \mathrm{kg}^{\mathrm{e}}$ & & \\
\hline & $\begin{array}{l}\text { Water } \\
\text { Diesel }\end{array}$ & & $0.5 \mathrm{mg} / \mathrm{L}^{\mathrm{d}}$ & $100 \mathrm{mg} / \mathrm{L}^{\mathrm{e}}$ & & \\
\hline & Soil Diesel & & $25 \mathrm{mg} / \mathrm{kg}^{\mathrm{d}}$ & $100 \mathrm{mg} / \mathrm{kg}^{\mathrm{e}}$ & & \\
\hline \multirow[b]{2}{*}{ PCBs } & Water & \multirow[b]{2}{*}{$8082^{c}$} & $\begin{array}{c}0.54-0.90 \\
\text { micrograms per } \\
\text { liter }(\mu \mathrm{g} / \mathrm{L})^{\mathrm{c}}\end{array}$ & $1000 \mu \mathrm{g} / \mathrm{L}^{\mathrm{g}}$ & \multirow[b]{2}{*}{ Lab specific ${ }^{f}$} & \multirow[b]{2}{*}{ Lab specific $^{f}$} \\
\hline & Soil & & $\begin{array}{c}57-90 \\
\text { micrograms per } \\
\text { kilogram } \\
(\mu \mathrm{g} / \mathrm{kg})^{\mathrm{c}}\end{array}$ & $1000 \mu \mathrm{g} / \mathrm{kg}^{\mathrm{g}}$ & & \\
\hline
\end{tabular}

"RPD is used to calculate precision. Precision is estimated from the RPD of the concentration measured for the MS/MSD analyses of unspiked field samples, or field duplicates of unspiked samples. It is calculated by: RPD = $100 \times\left\{\left(\left|C_{1}-C_{2}\right|\right) /\left[\left(C_{1}+C_{2}\right) / 2\right]\right\}$, where $C_{1}=$ concentration of the analyte in the first aliquot, $C_{2}$ is the concentration of the analyte in the second sample aliquot.

${ }^{b} \% \mathrm{R}$ is used to calculate accuracy. Accuracy is assessed from the recovery of analytes spiked into a blank or sample matrix of interest, or from the recovery of surrogate compounds spiked into each sample. The recovery of each spiked analyte is calculated by: $\% R=100 \times\left(C_{s}-C_{u} / C_{n}\right)$, where $C_{s}=$ concentration of the analyte in the spiked sample, $\mathrm{C}_{u}=$ the concentration of the analyte in the unspiked sample, and $C_{n}=$ the concentration increase that should result from spiking the sample.

'U.S. Environmental Protection Agency's (EPA's) Test Methods for Evaluating Solid Waste, $3^{\text {rd }}$ Edition, Parts 1-4, SW-846 (EPA, 1996b)

'Industrial Sites Quality Assurance Project Plan (DOE/NV, 1996).

TPH regulatory limit set in Nevada Administrative Code, 445A.2272 (NAC, 2000).

In-house generated RPD and \%R Performance Criteria. It is necessary for laboratories to develop in-house performance criteria and compare them to those in the methods. The laboratory begins by analyzing 15-20 samples of each matrix and calculating the mean \%R for each analyte. The standard deviation (DS) of each \%R is then calculated, and the warning and control limits for each analyte are established at \pm 2 SD and \pm 3 SD fro the mean, respectively. If the warning limit is exceeded during the analysis of any SDG, the laboratory institutes corrective action to bring the analytical system back into control. If the control limit is exceeded, the sample results for that SDG are considered unacceptable. These limits are reviewed after every 20-30 samples of the same matrix and are updated at least semiannually. The laboratory tracks trends in both performance and control limits by the use of control charts. The laboratory's compliance with these requirements is confirmed as part of an annual laboratory audit. Similar procedures are followed in order to generate acceptance criteria for precision measurements. gPCB regulatory limit of $1 \mathrm{ppm}$ is the TSCA concentration for nonrestricted use established by Title 40 Code of Federal Regulations 761.61 (EPA, 2000b). 


\subsection{REFERENCES}

Bechtel Nevada. 1998a. 30-Day Report for Nevada Division of Emergency Management (NDEM) Case Number 98-0819-3014 - Above-groud Storage Tank (AST) T-2003, August 27.

Bechtel Nevada. 1998b. Final Report for Nevada Division of Emergency Management (NDEM) Case Number 98-0811-3001 - Abovegroud Storage Tank (AST) T-2002, August 27.

Bechtel Nevada. 2000. Company Directive CD-0442.007, "Management of Polychlorinated Biphenyls."

Bechtel Nevada. 2000. Organization Instruction OI-2152.106, “Soil Sampling."

BN, see Bechtel Nevada.

DOE/NV, see U.S. Department of Energy, Nevada Operations Office.

EPA, see U.S. Environmental Protection Agency.

FFACO, see Federal Facility Agreement and Consent Order

$\therefore \quad$ Federal Facility Agreement and Consent Order. 1996, as amended. Agreed to by the Nevada Division of Environmental Protection, U.S. Department of Energy, and U.S. Department of Defense, April 1996.

IT, see International Technology Corporation.

International Technology Corporation. 2000. CAU 398 Area 25 Spill Sites, NTS, Project Files, Las Vegas, NV.

NAC, see Nevada Administrative Code.

Nevada Administrative Code, Section 445A.2272. July 2000.

Occupational Safety and Health Administration. 2001. Title 29 Code of Federal Regulations 1910.120, Hazardous Waste Operations and Emergency Response, Washington, D.C.

OSHA, see Occupational Safety and Health Administration.

REECo, see Reynolds Electrical \& Engineering Co., Inc. 
Reynolds Electrical \& Engineering Co., Inc. 1991a. Inventory of Inactive and Abandoned Facilities and Waste Sites, Volume 5, April. Prepared for the DOE/NV. Las Vegas, NV.

Reynolds Electrical \& Engineering Co., Inc. 1991b. Health Physics Department Waste Management Support Report, 14 July. Prepared for the DOE/NV. Las Vegas, NV.

Reynolds Electrical \& Engineering Co., Inc. 1992. Detailed Site Activity Summary NTS Cleanup and Restoration, 14 May. Prepared for the DOE/NV. Las Vegas, NV.

Reynolds Electrical \& Engineering Co., Inc. 1993. Field Investigation Checklists, 23 August. Prepared for the DOE/NV. Las Vegas, NV.

Space Nuclear Propulsion Office. 1970. NRDS Master Plan 1969-1970, Nuclear Rocket Development Station, Jackass Flats, Nevada. Las Vegas, NV.

U.S. Department of Energy, Nevada Operations Office. 1994. Nevada Environmental Restoration Project, Project Management Plan, Revision 0.

U.S. Department of Energy, Nevada Operations Office. 1996. Industrial Sites Quality Assurance Project Plan, Nevada Test Site, Nevada, DOE/NV-372, Revision 1.

U.S. Department of Energy, Nevada Operations Office. 1999a. Closure Report For Housekeeping Category Corrective Action Unit 297 Nevada Test Site, Nevada, DOE/NV--289, Revision 0.

U.S. Department of Energy, Nevada Operations Office. 1999b. Spill Notification Report, 19 January, Las Vegas, NV.

U.S. Environmental Protection Agency. 1988a. Contract Laboratory Program Statement of Work for Inorganic Analysis, SOW No. 788, EPA/540/R-94/093. Washington, D.C.

U.S. Environmental Protection Agency. 1988b. Contract Laboratory Program Statement of Work for Organic Analysis, SOW No. 288, EPA/540/R-94/096. Washington, D.C.

U.S. Environmental Protection Agency. 1994. Contract Laboratory Program National Functional Guidelines for Inorganic Data Review, EPA/540/R-94/078. Washington, D.C.

U.S. Environmental Protection Agency. 1996a. Region LX Preliminary Remediation Goals (PRGs), San Francisco, CA.

U.S. Environmental Protection Agency. 1996b. Test Methods for Evaluating Solid Waste Physical/Chemical Methods (SW-846) Third Edition, Washington, D.C. 
U.S. Environmental Protection Agency. 1996c. Title 40 Code of Federal Regulations 761.24, Toxicity Characteristic, Washington, D.C.

U.S. Environmental Protection Agency. 1996d. Title 40 Code of Federal Regulations 761.61, PCB Remediation Waste, Washington, D.C.

U.S. Environmental Protection Agency. 1999. Contract Laboratory Program National Functional Guidelines for Organic Data Review, EPA/540/R-99/008. Washington, D.C.

U.S. Environmental Protection Agency. 2000. Guidance for the Data Quality Objective Process, EPA QA/G-4, EPA/600/R-96-055. Washington, D.C. 
THIS PAGE INTENTIONALLY LEFT BLANK

$\square$

$-$

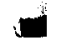

a

$-$

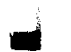

$-$

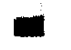

(1)

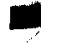

$-$

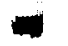

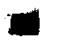

$-$

-

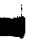

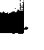




\section{APPENDIX A-1 \\ CHARACTERIZATION SAMPLE ANALYTICAL RESULTS}


THIS PAGE INTENTIONALLY LEFT BLANK

0
-
-
-
-
-

$-$

$\omega$

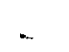

a 
CLIENT:

Bechtel Nevada

P.O. Box 98521, M/S NTS273

ATTN:

Las Vegas, NV 89193-8521

PROJECT NAME:

Ted Redding

NEL ORDER ID: L0105247

PROJECT NUMBER: 23081

Attached are the analytical results for samples in support of the above referenced project.

Samples submitted for this project were not sampled by NEL Laboratories. Samples were received by NEL in good condition, under chain of custody on 5/24/01.

Should you have any questions or comments, please feel free to contact our Client Services department at (702) 657-1010.

Some QA results have been flagged as follows:

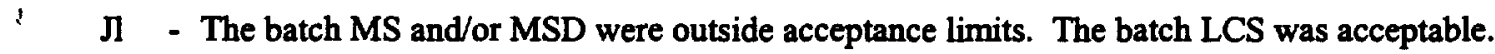
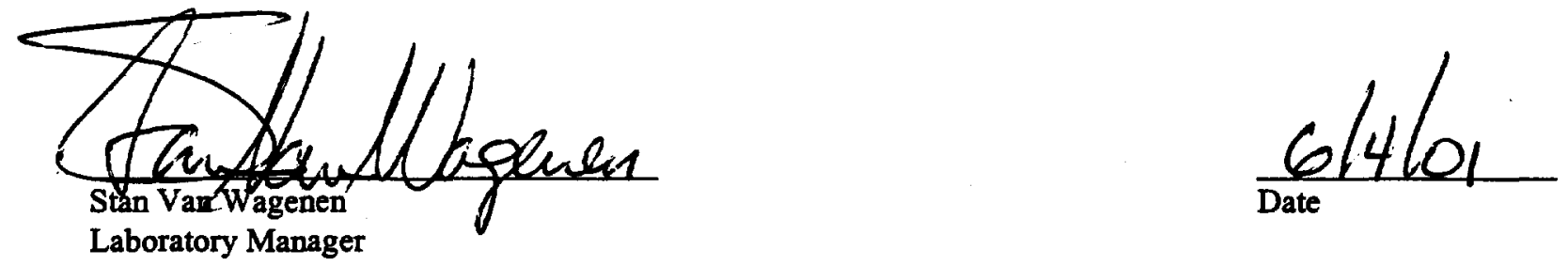

\section{CERTIFICATIONS:}

\begin{tabular}{llll}
\hline & Reno & Las Vegas & S. California \\
\cline { 2 - 4 } Arizona & AZ0520 & AZ0518 & AZ0605 \\
California & 1707 & 2002 & 2264 \\
US Army Corps & Certified & Certified & \\
of Engineers & & &
\end{tabular}

\begin{tabular}{llll} 
& Reno & Las Vegas & S. California \\
\cline { 2 - 4 } Idaho & Certified & Certified & \\
Montana & Certified & Certified & \\
Nevada & NV033 & NV052 & CA084 \\
L.A.C.S.D. & & & 10228
\end{tabular}




$\begin{array}{llll}\text { CLIENT: } & \text { Bechtel Nevada } & \text { CLIENT ID: } & \text { 254402-2-0 } \\ \text { PROJECT D: } & \text { V1119 } & \text { DATE SAMPLED: 5/23/01 } \\ \text { PROJECT \#: } & 23081 & \text { NEL SAMPLE ID: L0105247-02 }\end{array}$

TEST: $\quad$ Total Extractable Petroleum Hydrocarbons Fuel Finger Print by EPA Method 8015 M, July 1992

METHOD: $\quad$ EPA 8015M

MATRIX: Solid ANALYST: $\quad$ CCS - Las Vegas Division

DILUTION: 1

EXTRACTED: $\quad 5 / 30 / 01$

ANALYZED: $\quad 5 / 31 / 01$

\begin{tabular}{lll}
\hline PARAMETER & Result & Reporting \\
\cline { 2 - 3 } & ND & $\frac{\text { Limit }}{10 . \mathrm{mg} / \mathrm{kg}}$ \\
Giesel Range (C12-C22) & ND & $10 . \mathrm{mg} / \mathrm{kg}$ \\
Oil Range (C12-C34) & $\mathrm{ND}$ & $50 . \mathrm{mg} / \mathrm{kg}$ \\
Total & $\mathrm{ND}$ & $10 . \mathrm{mg} / \mathrm{kg}$ \\
\hline \hline
\end{tabular}

QUALITY CONTROL DATA:

Surrogate

$\%$ Recovery

Octacosane

92

Acceptable Range

$54-130$

ND - Not Detected

This report shall not be reproduced except in full, without the written approval of the laboratory. 


\begin{tabular}{|c|c|c|}
\hline $\begin{array}{l}\text { CLIENT: } \\
\text { PROJECT ID: } \\
\text { PROJECT \#: }\end{array}$ & $\begin{array}{l}\text { Bechtel Nevada } \\
\text { V1119 } \\
23081\end{array}$ & $\begin{array}{ll}\text { CLIENT ID: } & \mathbf{2 5 4 4 0 2 - 3 - 0} \\
\text { DATE SAMPLED: } & \text { 5/23/01 } \\
\text { NEL SAMPLE ID: } & \text { L0105247-03 }\end{array}$ \\
\hline
\end{tabular}

- TEST: $\quad$ Total Extractable Petroleum Hydrocarbons Fuel Finger Print by EPA Method $8015 M$, July 1992

METHOD: $\quad$ EPA 8015M ANALYST: CCS - Las Vegas Division

MATRIX: Solid $\quad$ EXTRACTED: $5 / 30 / 01$

c DILUTION: $1 \quad$ ANALYZED: 5/31/01

\begin{tabular}{|c|c|c|}
\hline PARAMETER & Result & $\begin{array}{l}\text { Reporting } \\
\text { Limit }\end{array}$ \\
\hline 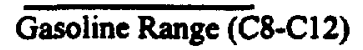 & ND & 10. $\mathrm{mg} / \mathrm{kg}$ \\
\hline Diesel Range (C12-C22) & ND & 10. $\mathrm{mg} / \mathrm{kg}$ \\
\hline Oil Range (C12-C34) & ND & 50. $\mathrm{mg} / \mathrm{kg}$ \\
\hline Total & ND & 10. $\mathrm{mg} / \mathrm{kg}$ \\
\hline
\end{tabular}

\section{- QUALITY CONTROL DATA:}

Surrogate

Octacosane

\section{\% Recovery}

86

\section{Acceptable Range}

$54-130$

- ND - Not Detected

This report shall not be reproduced except in full, without the written approval of the laboratory. 


$\begin{array}{lll}\text { CLIENT: } & \text { Bechtel Nevada } & \text { CLIENT ID: } \\ \text { PROJECT ID: } & \text { V1119 } & \text { DATE SAMPLED: } 5 / 23 / 01 \\ \text { PROJECT \#: } & 23081 & \text { NEL SAMPLE ID: L0105247-04 }\end{array}$

TEST: $\quad$ Total Extractable Petroleum Hydrocarbons Fuel Finger Print by EPA Method 8015M, July 1992

METHOD: EPA 8015M ANALYST: CCS - Las Vegas Division

MATRIX: Solid $\quad$ EXTRACTED: $5 / 30 / 01$

DIUUTION: $\quad 1$ -

\begin{tabular}{|c|c|c|}
\hline DILUTION: 1 & ANALYZED: & \\
\hline PARAMETER & Result & $\begin{array}{l}\text { Reporting } \\
\text { Limit }\end{array}$ \\
\hline 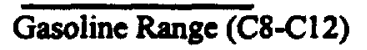 & ND & 10. $\mathrm{mg} / \mathrm{kg}$ \\
\hline Diesel Range (C12-C22) & ND & 10. $\mathrm{mg} / \mathrm{kg}$ \\
\hline Oil Range (C12-C34) & ND & 50. $\mathrm{mg} / \mathrm{kg}$ \\
\hline Total & ND & 10. $\mathrm{mg} / \mathrm{kg}$ \\
\hline
\end{tabular}

QUALITY CONTROL DATA:

Surrogate

\% Recovery

Octacosane

72

Acceptable Range

$54-130$

ND - Not Detected

This report shall not be reproduced except in full, without the written approval of the laboratory. 


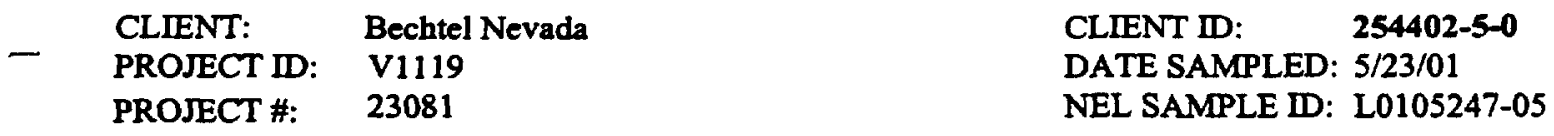

- TEST: $\quad$ Total Extractable Petroleum Hydrocarbons Fuel Finger Print by EPA Method 8015M, July 1992

METHOD: EPA 8015M ANALYST: CCS - Las Vegas Division

MATRIX: Solid $\quad$ EXTRACTED: $5 / 30 / 01$

DILUTION: 1 ANALYZED: 5/31/01

\begin{tabular}{|c|c|c|}
\hline PARAMITER & Result & $\begin{array}{c}\text { Reporting } \\
\text { Limit }\end{array}$ \\
\hline$\overline{\text { Gasoline Range (C8-C12) }}$ & $\mathrm{ND}$ & 10. $\mathrm{mg} / \mathrm{kg}$ \\
\hline Diesel Range (C12-C22) & ND & 10. $\mathrm{mg} / \mathrm{kg}$ \\
\hline Oil Range (Cl2-C34) & ND & 50. $\mathrm{mg} / \mathrm{kg}$ \\
\hline Total & ND & 10. $\mathrm{mg} / \mathrm{kg}$ \\
\hline
\end{tabular}

- QUALITY CONTROL DATA:

Surrogate

Octacosane

\section{$\%$ Recovery}

77
Acceptable Range

$54-130$

$\rightarrow \quad$ ND - Not Detected

This report shall not be reproduced except in full, without the written approval of the laboratory. 
CLIENT: Bechtel Nevada

PROJECT ID: V1119

PROJECT \#: 23081
CLIENT ID: $\quad$ 254402-6-0

DATE SAMPLED: 5/23/01

NEL SAMPLE ID: L0105247-06
TEST:

METHOD:

MATRIX:

DILUTION:

Total Extractable Petroleum Hydrocarbons Fuel Finger Print by EPA Method 8015M, July 1992

EPA 8015M

Solid ANALYST:

EXTRACTED:

CCS - Las Vegas Division

ANALYZED:

$5 / 30 / 01$

$5 / 31 / 01$

\section{PARAMETER}

Gasoline Range (C8-C12)

Diesel Range (C12-C22)

Oil Range (C12-C34)

Total

QUALITY CONTROL DATA:

Surrogate

Octacosane

\section{$\%$ Recovery}

89
Reporting

Limit

10. $\mathrm{mg} / \mathrm{kg}$

10. $\mathrm{mg} / \mathrm{kg}$

50. $\mathrm{mg} / \mathrm{kg}$

10. $\mathrm{mg} / \mathrm{kg}$

10. $\mathrm{mg} / \mathrm{kg}$

ND

\section{Acceptable Range}

$54-130$

ND - Not Detected

This report shall not be reproduced except in full, without the written approval of the laboratory. 
CLIENT: Bechtel Nevada

PROJECT ID: V1119

PROJECT \#: 23081
CLIENT ID: $\quad$ 254402-1-2

DATE SAMPLED: $5 / 23 / 01$

NEL SAMPLE ID: L0105247-07

$-$

TEST:

Total Extractable Petroleum Hydrocarbons Fuel Finger Print by EPA Method 8015M, July 1992

METHOD: EPA 8015M

MATRIX: Solid

DILUTION: 1 ANALYST: $\quad$ CCS - Las Vegas Division

EXTRACTED: $\quad 5 / 30 / 01$

ANALYZED: $\quad 5 / 31 / 01$

\section{PARAMETER}

Gasoline Range (C8-C12)

- Diesel Range (C12-C22)

Oil Range (C12-C34)

Total

\begin{tabular}{l} 
Result \\
\hline ND \\
ND \\
ND \\
ND
\end{tabular}

Reporting

$\frac{\text { Limit }}{10 . \mathrm{mg} / \mathrm{kg}}$

10. $\mathrm{mg} / \mathrm{kg}$

50. $\mathrm{mg} / \mathrm{kg}$

10. $\mathrm{mg} / \mathrm{kg}$

QUALITY CONTROL DATA:

Surrogate

$\%$ Recovery

Octacosane

70

Acceptable Range

$54-130$

- ND - Not Detected

This report shall not be reproduced except in full, without the written approval of the laboratory. 


$\begin{array}{llll}\text { CLIENT: } & \text { Bechtel Nevada } & \text { CLIENT ID: } & \text { Method Blank } \\ \text { PROJECT ID: } & \text { V1119 } & \text { DATE SAMPLED: } & \text { NA } \\ \text { PROJECT \#: } & 23081 & \text { NEL SAMPLE ID: } & \text { 010530TPHS-FP-BLK }\end{array}$

TEST: $\quad$ Total Extractable Petroleum Hydrocarbons Fuel Finger Print by EPA Method 8015M, July 1992

METHOD: EPA 8015M ANALYST: CCS - Las Vegas Division

MATRIX: Solid $\quad$ EXTRACTED: 5/30/01

ANALYZED: $\quad 5 / 31 / 01$

\begin{tabular}{lll}
\hline PARAMETER & Result & Reporting \\
\cline { 2 - 3 } Gasoline Range (C8-C12) & ND & $\frac{\text { Limit }}{10 . \mathrm{mg} / \mathrm{kg}}$ \\
Diesel Range (C12-C22) & ND & $10 . \mathrm{mg} / \mathrm{kg}$ \\
Oil Range (C12-C34) & ND & $50 . \mathrm{mg} / \mathrm{kg}$ \\
Total & ND & $10 . \mathrm{mg} / \mathrm{kg}$ \\
\hline \hline
\end{tabular}

QUALITY CONTROL DATA:

Surrogate

Octacosane
\% Recovery

81

\section{Acceptable Range} $54-130$

ND - Not Detected

This report shall not be reproduced except in full, without the written approval of the laboratory. 


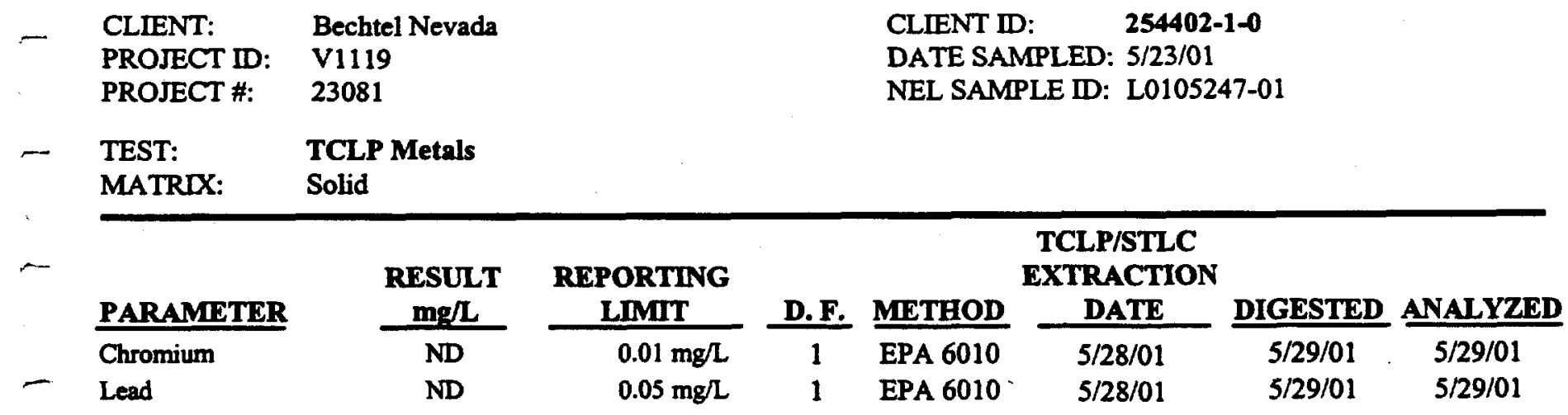

- D.F. - Dilution Factor

ND - Not Detected

This report shall not be reproduced except in full, without the written approval of the laboratory. 


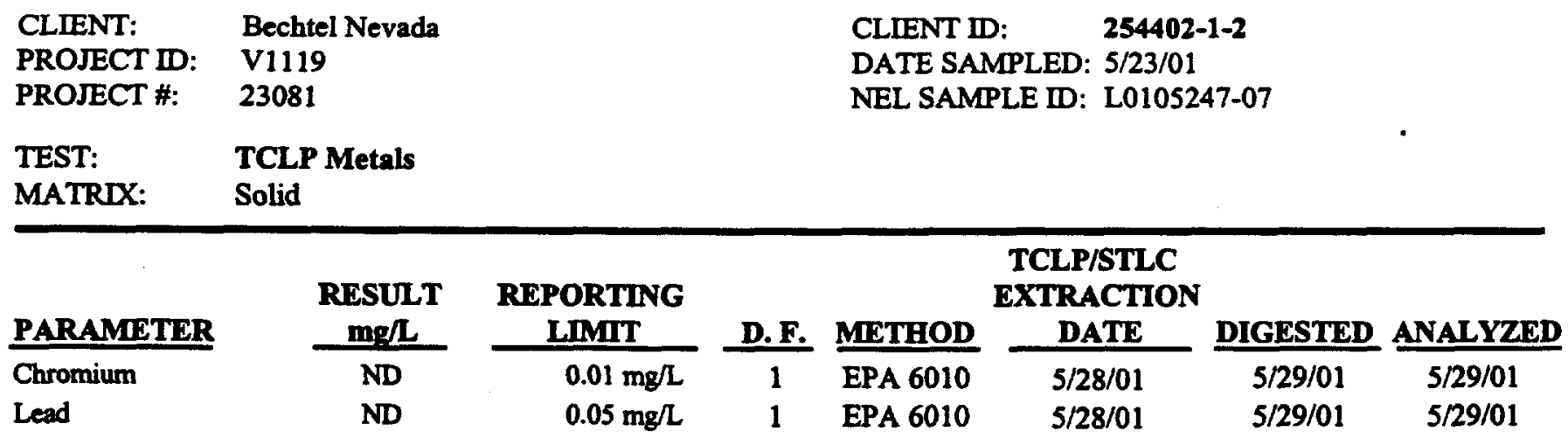

D.F. - Dilution Factor

ND - Not Detected

This report shall not be reproduced except in full, without the written approval of the laboratory. 


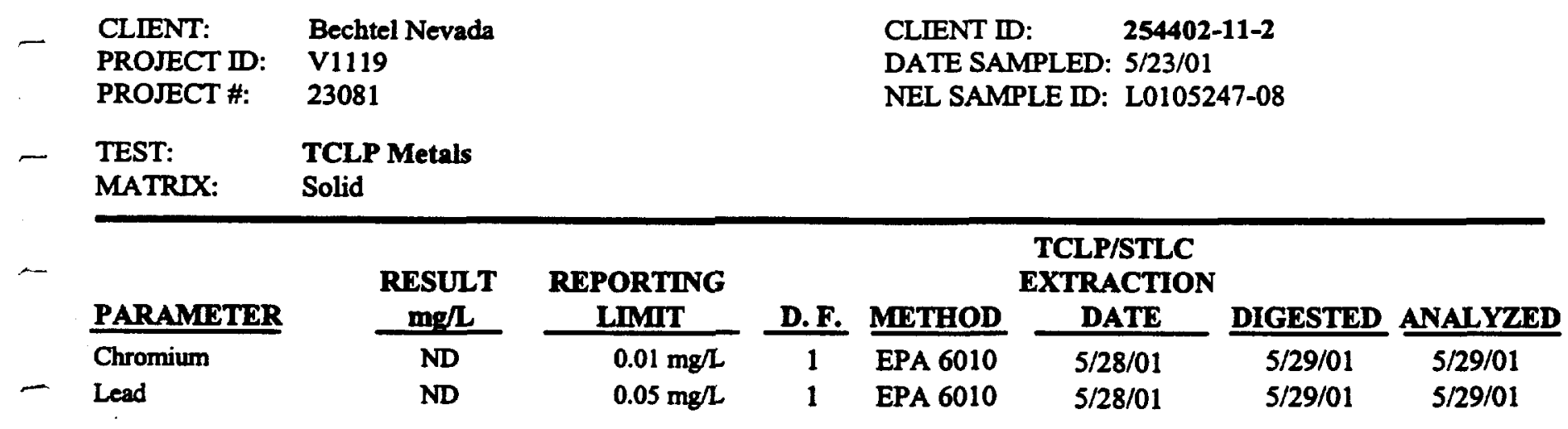

- D.F. - Dilution Factor ND - Not Detected

This report shall not be reproduced except in full, without the written approval of the laboratory. 


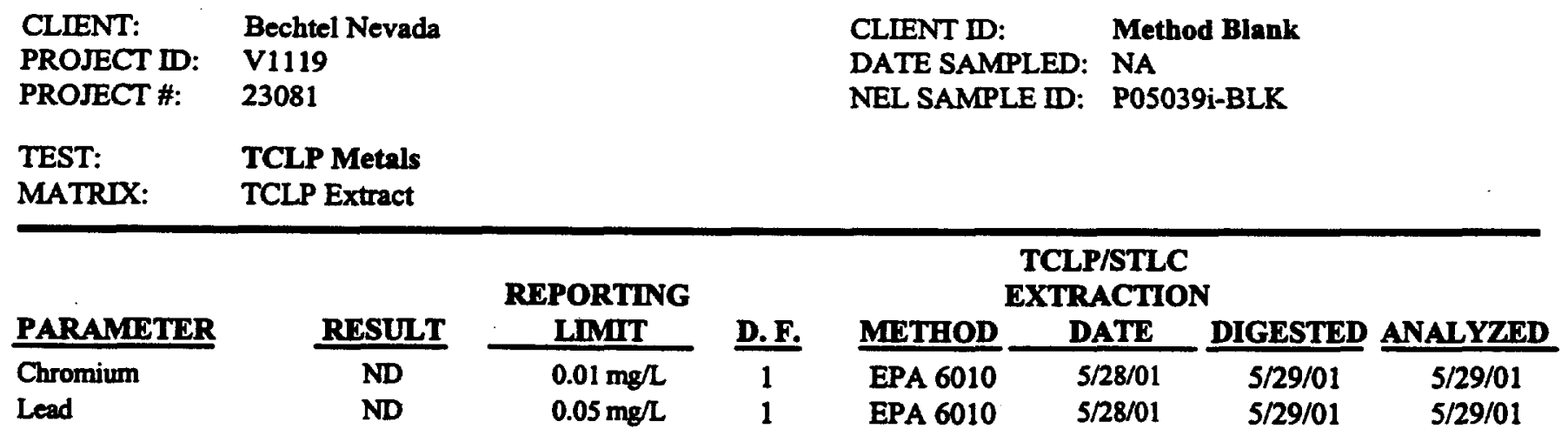

D.F. - Dilution Factor

ND - Not Detected

This report shall not be reproduced except in full, without the written approval of the laboratory. 


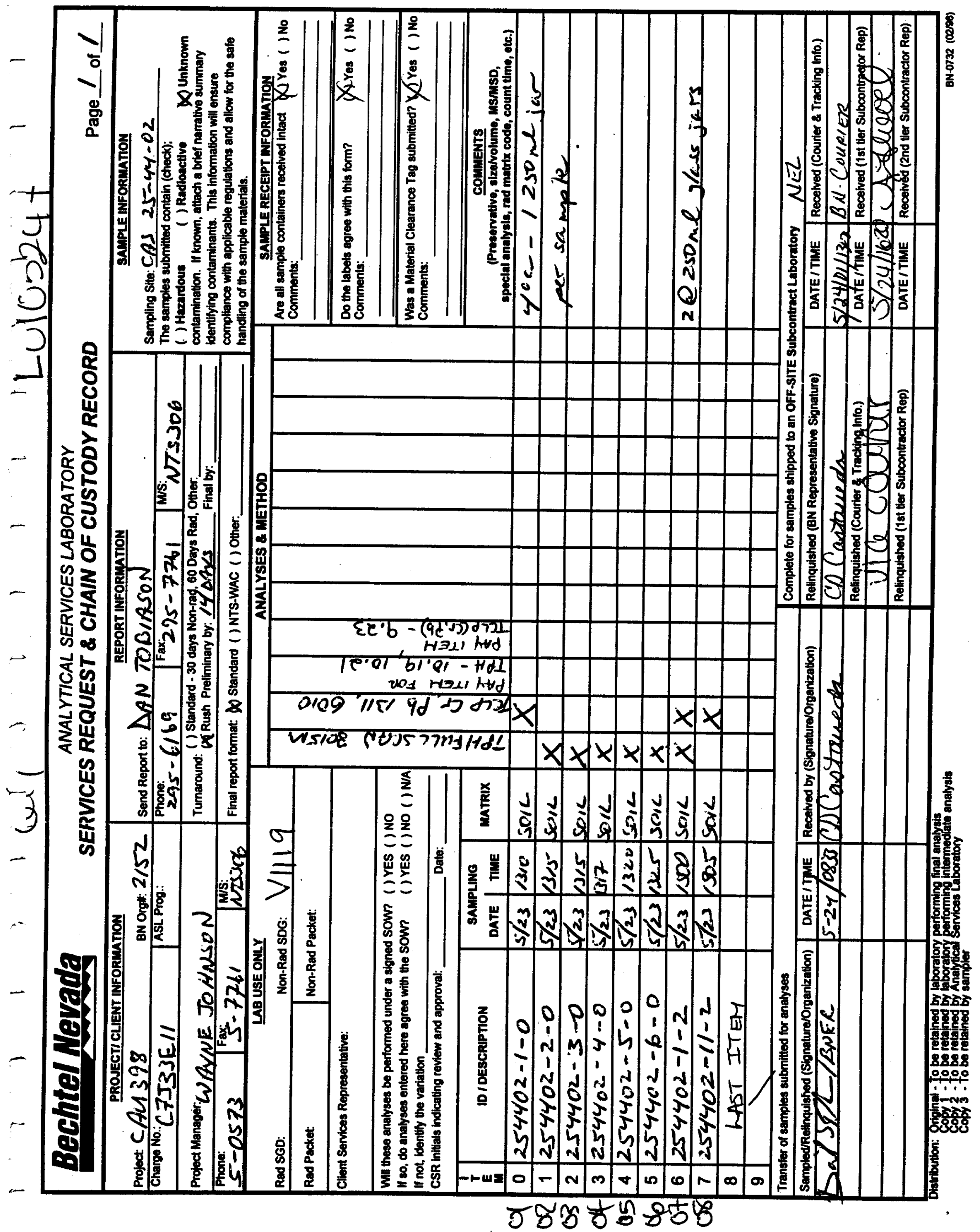




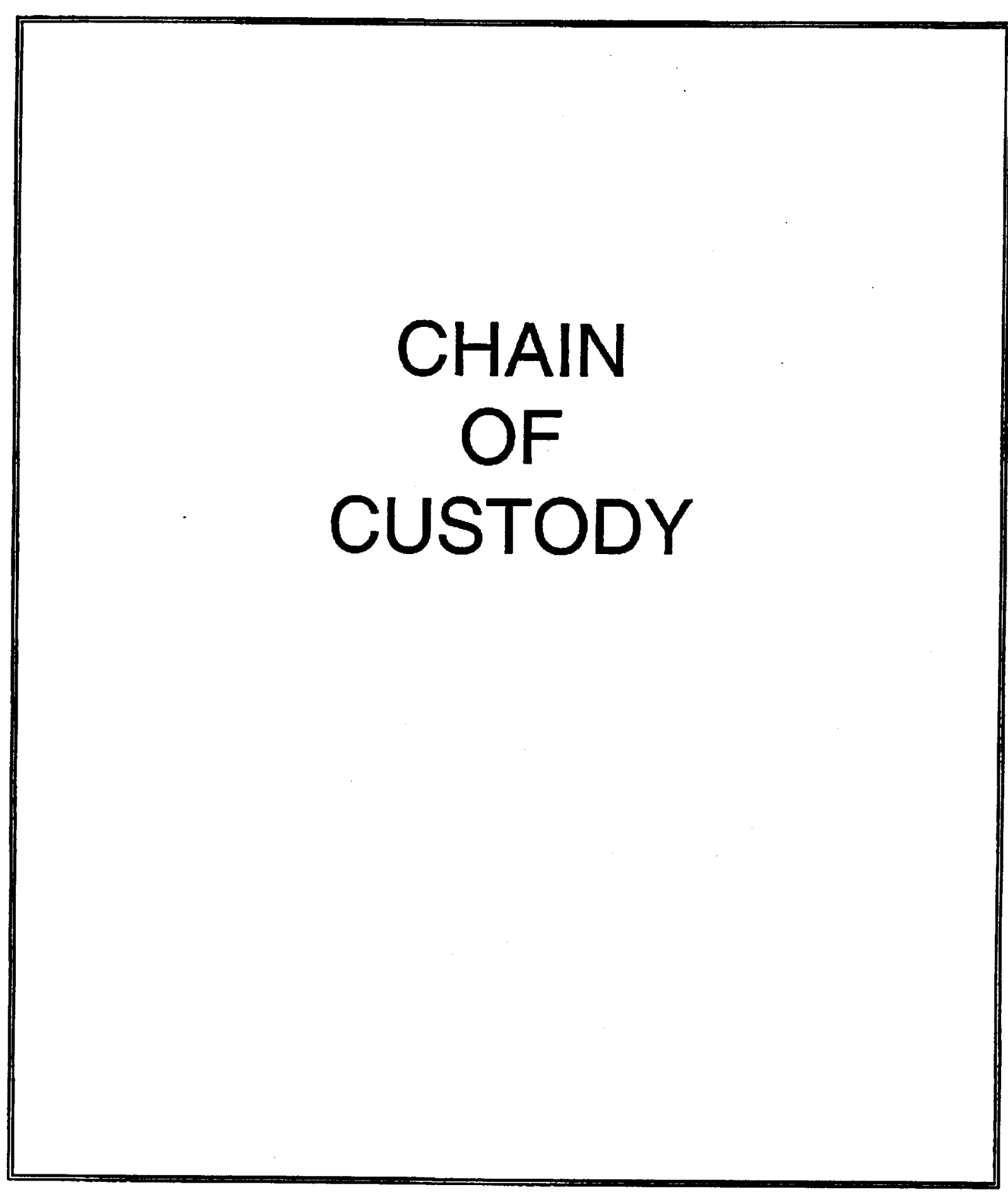


Project Manager:(NAFYNE TUY $/ A) \rightarrow O N$

\begin{tabular}{|c|c|c|}
\hline Phone: $5-0373$ & Fax: $5-276 ;$ & $\begin{array}{l}\text { M/S: } \\
\text { h/Sjot }\end{array}$ \\
\hline
\end{tabular}

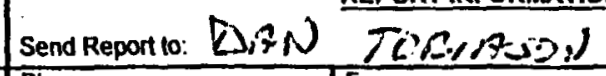

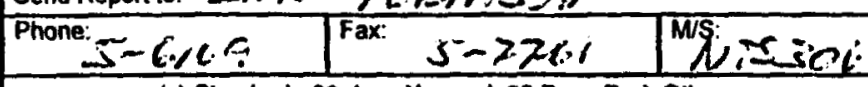
Turnaround: () Standard - 30 days Non-rad, 60 Days Rad, Other:Final report format: $f_{\text {II }}$ Standard ( ) NTS-WAC () Other:

al by:

ANALYSES \& METHOD

\begin{tabular}{l|l}
\hline Rad SGD: & \multicolumn{2}{l}{ LAB USE ONLY } \\
\hline Rad Packet: & Non-Rad SOag:
\end{tabular}

Client Services Representative:

Will these analyses be performed under a signed SOWn () YES () NO

If so, do analyses entered here agree with the sown

If not, identify the variation

CSR initials indicating review and approval: Date:

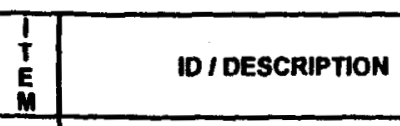

$0 \sin 402-1-0$

$\therefore 11254422-6-1)$

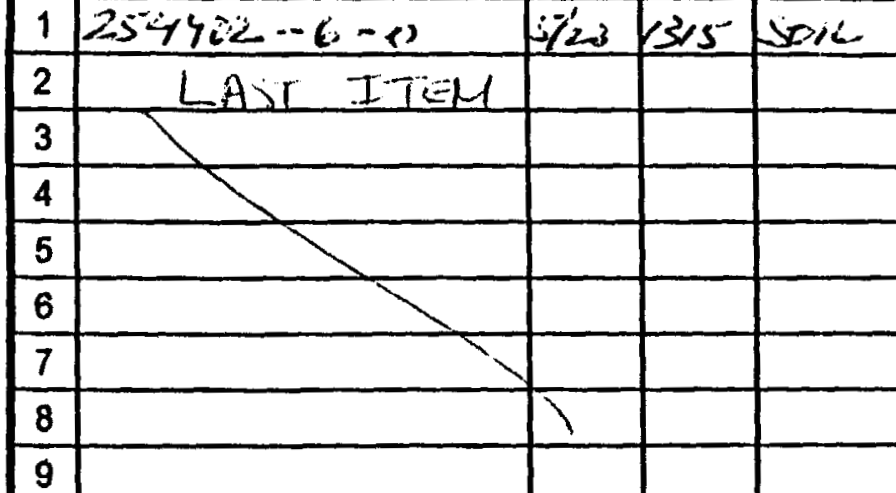

Transfer of samples submilted for analyses

\begin{tabular}{|l|l|l|l}
\hline Sampled/Relinquished (Signature/Organization) & DATE I TIME & Received by (Signature/Organization)
\end{tabular}

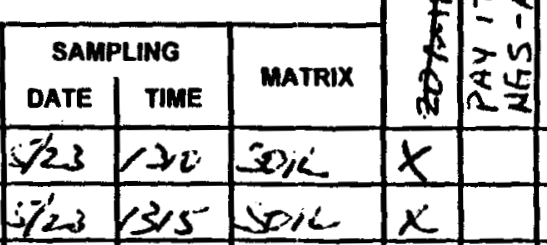

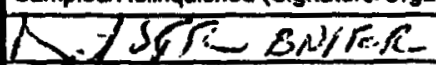
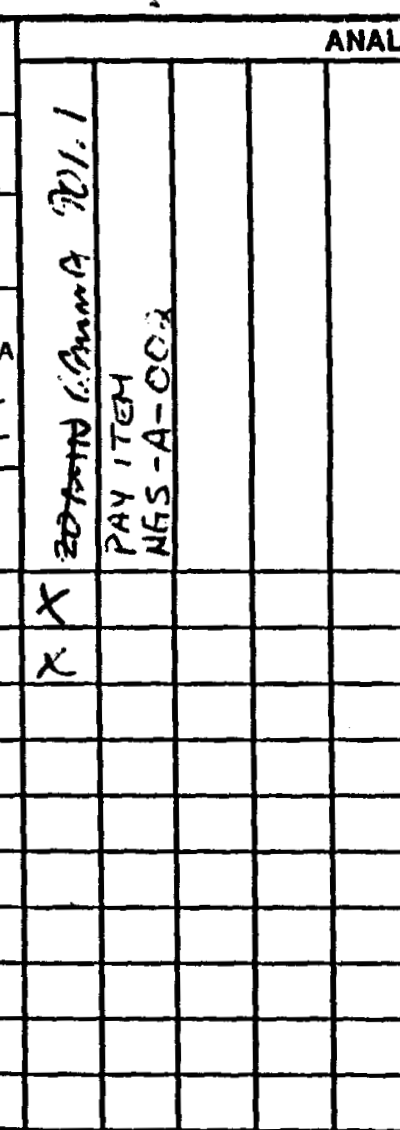

Complete lor samples shipped to an OFF-SITE Subcontract Laboratory $1 \mathrm{GC}$

\begin{tabular}{|c|c|c|c|c|c|}
\hline & $\bar{\pi}$ & - & 20 & 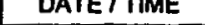 & 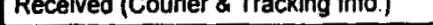 \\
\hline 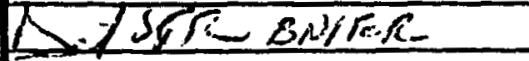 & $s^{-2}-2+k, 8 ;$ & (1) (laingriols & 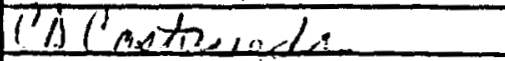 & 32461,90 & 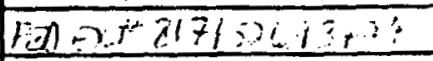 \\
\hline & & 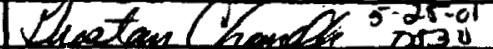 & Relinquished (Courier \& Tracking Info.) & OATE / TIME & Received (1st tier Subcontractor Rep) \\
\hline & & & Relinquished (1st tier Subcontractor Rep) & DATE / TMME & Received (2nd tier Subcontractor Rep) \\
\hline
\end{tabular}




\section{SAMPLE RECEIPT REVIEW}

Client Bectitel

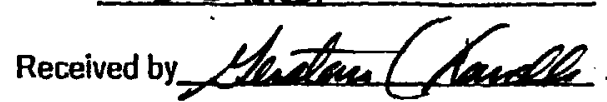

SAMPLE REVIEW CRITERIA

\begin{tabular}{|c|c|c|c|}
\hline & SAMPLEREVIEW CRIIERIA & YES NO NAA & COMMENISIQUALIFIERS \\
\hline 1 & Were shipping containers received intact and sealed? If no, notify the Project Manager & - & \\
\hline 2 & Were chain of cuslody documents included? & $\gamma$ & \\
\hline 3 & Shipping container temperature(s) checked: & $\dashv$ & 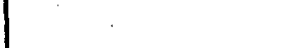 \\
\hline 4 & Is temperature documented on Chain of Custody & & \\
\hline 5 & Was shipping container temperature within specifications ( $4+1-2 \mathrm{C}$ ) If no, notify Project Manager & - & \\
\hline 6 & Are any of the samples identified by the client as radioactive? If yes, complele radioactive receipt form & & \\
\hline & Any samples not indentified by the client as radioactive must be screened for radioactiviliy. & +0 & Dbserved background CPM \\
\hline & If screening results indicate $>\times 2$ background inform the RSO. & 50 & obsreved sempla CPM \\
\hline 7 & Were chain of custody documents completed correctly? (lnk, signed, match containers) & - & \\
\hline 8 & Were sample containers received intact and sealed? If no, notify the Project Manager & -1 & \\
\hline $\mathbf{9}$ & Were all sample containers properly labeled? & & \\
\hline 10 & Were correct sample containers received? & & \\
\hline 11 & Preserved samples checked for $\mathrm{pH}$ ? & & aced \\
\hline 12 & Were samples preserved correclly? If no, notify Project Manager & - & \\
\hline 13 & Were samples recelved within holding time? If No, notify Project Manager & & \\
\hline 14 & Were VOA vials free of headspace? & & \\
\hline 15 & ARCOC\# & & . \\
\hline 16 & SDG\# & & \\
\hline
\end{tabular}

PM(A) Review: ENLKC

Date Reviewed:

$5 \longdiv { 2 9 1 0 }$

Additional Comments: 


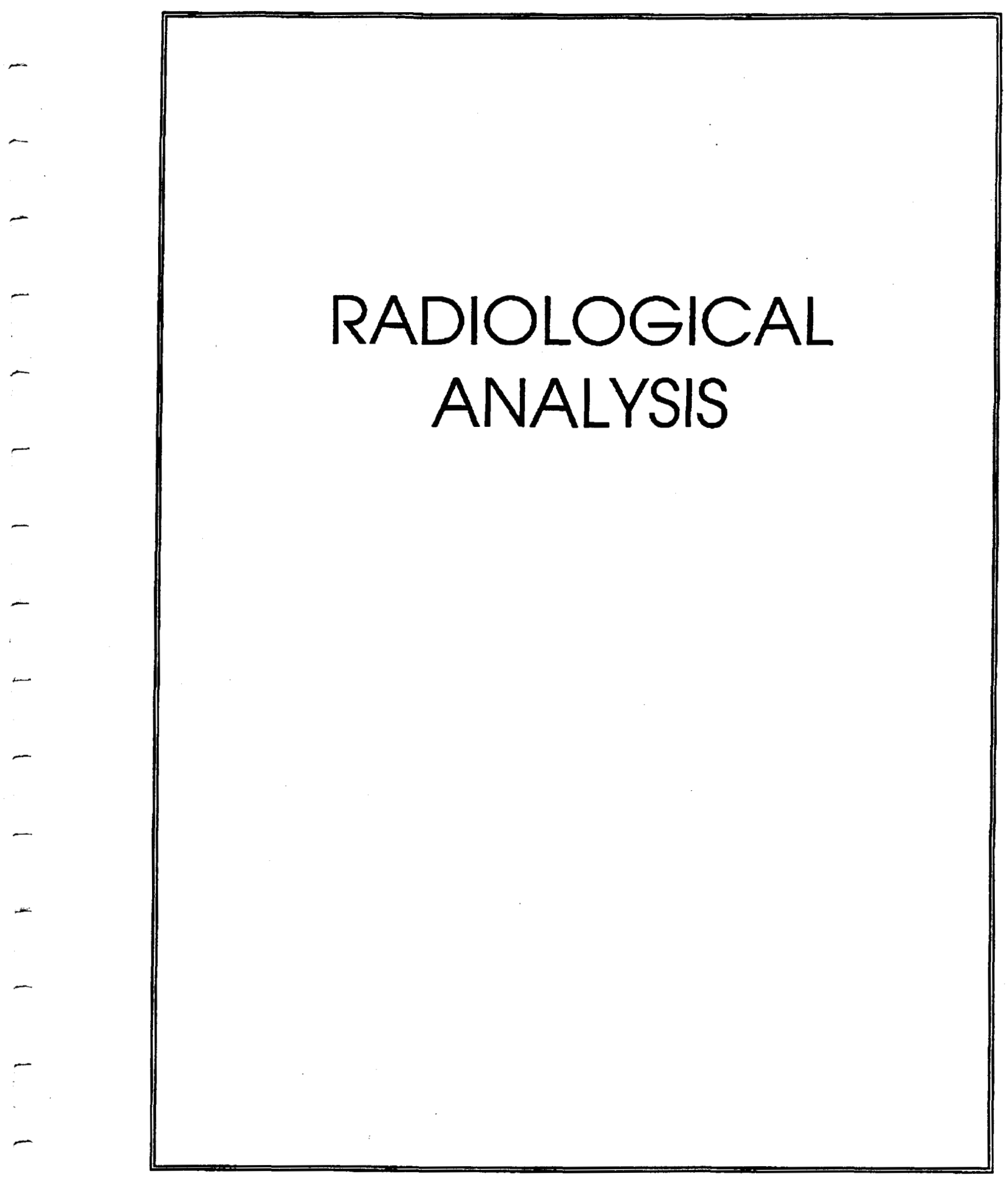




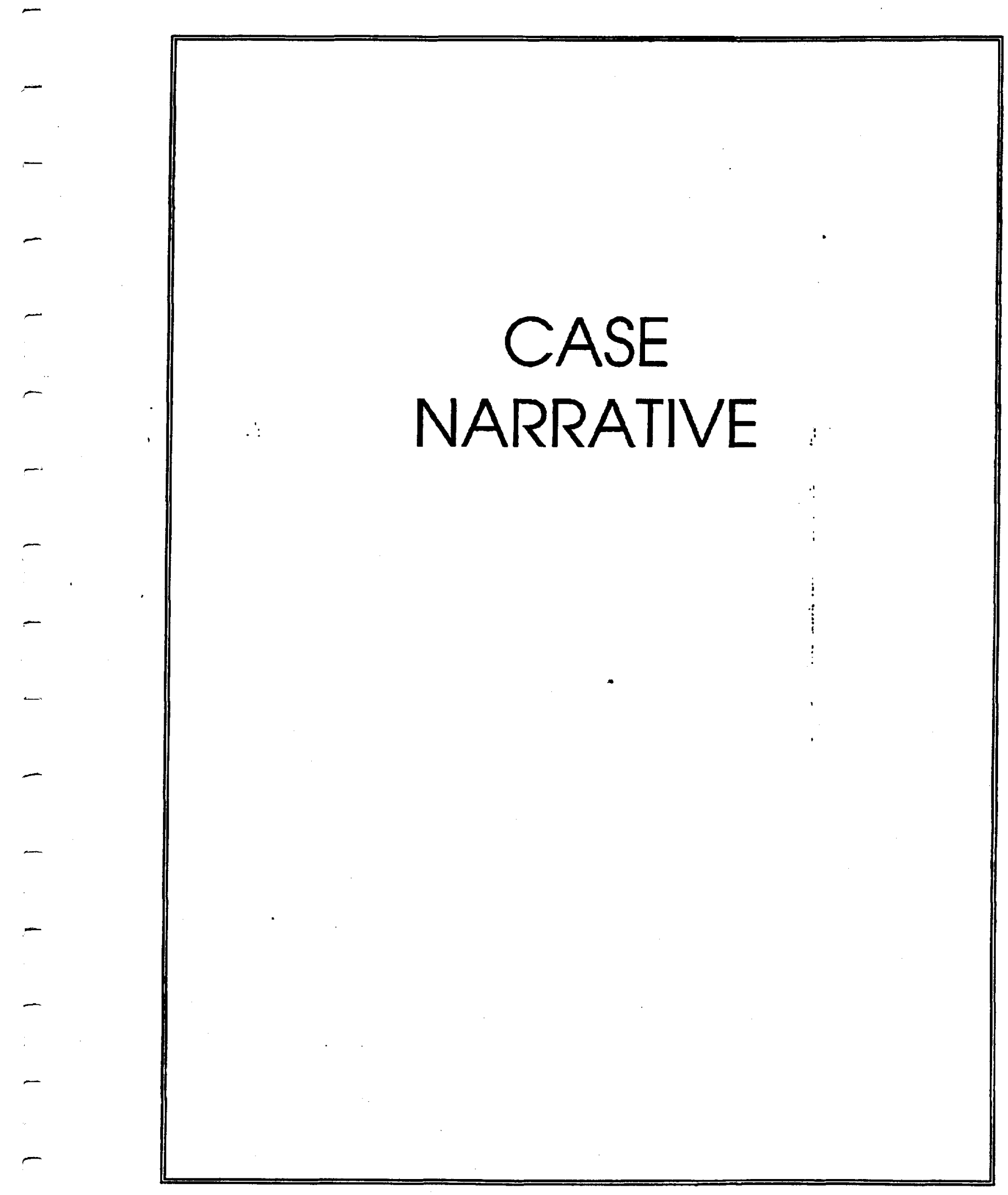




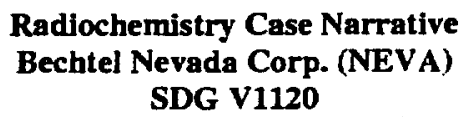

Method/Analysis Information

Batch Number:

Procedure:

Analytical Method:

$$
\begin{aligned}
& \text { Sample ID } \\
& 42918001 \\
& 42918002 \\
& 1200013917 \\
& 1200013918 \\
& 1200013919
\end{aligned}
$$

80749

Determination of Gamma Isotopes in Water and Soil

DOE EML HASL 300

Client ID

254402-1-0

254402-6-0

MB for batch 80749

254402-1-0(42918001DUP)

LCS for batch 80749

SOP Reference

Procedures for preparation, analysis and reporting of analytical data are controlled by General Engineering Laboratories, Inc. as Standard Operating Procedures (SOP). The data discussed in this narrative has been prepared and analyzed in accordance with GL-RAD-A-013.

\section{Calibration Information:}

Calibration Information

All initial and continuing calibration requirements have been met.

Standards Information

Standard solution(s) for these analyses are NIST traceable and used before the expiration date(s).

\section{Sample Geometry}

All counting sources were prepared in the same geometry as the calibration standards.

\section{Ouality Control (OC) Information:}

Blank Information

The blank volume is representative of the sample volume(s) in this batch.

Designated QC

The following sample(s) was used for QC: 42918001 .

QC Information

All of the QC samples met the required acceptance limits.

\section{Technical Information:}

Holding Time

All sample procedures for this sample set were performed within the required holding time.

Preparation Information*

All preparation criteria have been met for these analyses.

Sample Re-prep/Re-analysis

None of the samples in this sample set required reprep or reanalysis. 


\section{Miscellaneous Information:}

\section{NCR Documentation}

No NCR were generated for the preparation or analysis of this sample set.

\section{Manual Integration}

No manual integrations were performed on data in this batch.

Additional Comments

$\mathbf{K}-40$ is being reported in all samples in this batch.

\section{Revien Validation:}

GEL requires all analytical data to be verified by a qualified data validator. In addition, all data designated for CLP or CLP-like packaging will receive a third level validation upon completion of the data package.

The following data palidator verified the information presented in this case narrative:

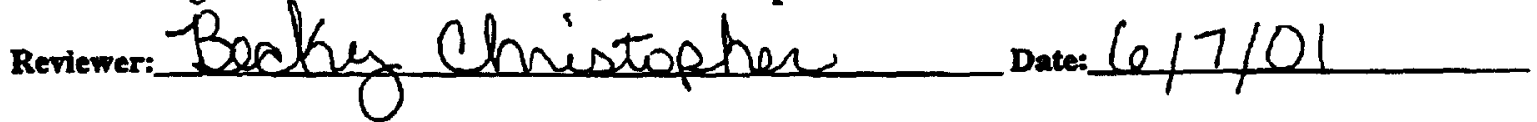




\section{GENERAL ENGINEERING LABORATORIES}

Meeting today's needs uith a virion for tomorrow.

\section{Certificate of Analysis}

Comprany : Bechtel Nevada Corp.

Address: 2621 Losee Road

North Las Vegas, NV 89030-4134

Contact: Ted Redding

Project: Enviornmental Rad Services

Report Date: June 7,2001

Page 1 of 1

\begin{tabular}{|c|c|c|c|c|c|c|c|c|c|c|c|}
\hline \multirow[b]{2}{*}{ Parameter } & \multicolumn{2}{|c|}{$\begin{array}{l}\text { Client Sample ID: } \\
\text { Sample D: } \\
\text { Matrix: } \\
\text { Collect Date: } \\
\text { Receive Date: } \\
\text { Collector: }\end{array}$} & & \multicolumn{2}{|c|}{$\begin{array}{l}254402-1-0 \\
42918001 \\
\text { Soil } \\
23-\mathrm{MAY}=01 \\
25-\mathrm{MAY}-01 \\
\text { Client }\end{array}$} & \multirow[b]{2}{*}{$\mathbf{R I}$} & \multirow{2}{*}{$\begin{array}{l}\text { Proiect: } \\
\text { Client iD: } \\
\text { Units }\end{array}$} & \multicolumn{3}{|c|}{$\begin{array}{l}\text { NEVA102000 } \\
\text { NEVADO1 }\end{array}$} & \multirow{2}{*}{ Bateh Mitd } \\
\hline & Qualifier & Result & & DL & TPU & & & DF & AnalystDote & Time & \\
\hline \multicolumn{12}{|l|}{ Rad Gamma Spee } \\
\hline
\end{tabular}

The following Prep Methods were performed

\begin{tabular}{llllll}
\hline Method & Deseription & Andyst & Date & Time & Prep Batch \\
\hline Dry Soil Prep & Dry Soil Prep RAD A-021,A-021B,A-026 & CCMI & 05/29/01 & 1358 & 80126 \\
The following Andytical Methods were performed & & & $\cdot$ & \\
\hline Method & & & & \\
\hline
\end{tabular}

1 DOE EML HASL 300

Notes:

The Qualifiers in this report are defined as follows:

* Indicates the analyte is a surrogate compound.

< Actual result is less than amount reported

$>$ Actual result is greater than amount reported

J Indicates an estimated value. The result was greater than the detection limit, but less than the reporting limit.

U Indicates the compound was analyzed for but not detected above the detection limit

The above sample is reported on an "as received" basis.

This data report has been prepared and reviewed in accordance with General Engineering Laboratories, Inc.

standard operating procedures. Please direct any questions to your Project Manager, Stacy L. Griffin at 843-556-8171 Ext. 4264.

Reviewed by

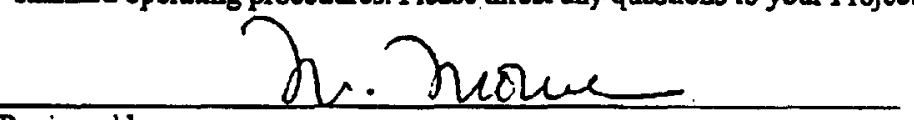




\section{GENERAL ENGINEERING LABORATORIES}

'Meeting roday's needs with a virion for romorrow.

\section{Certificate of Analysis}

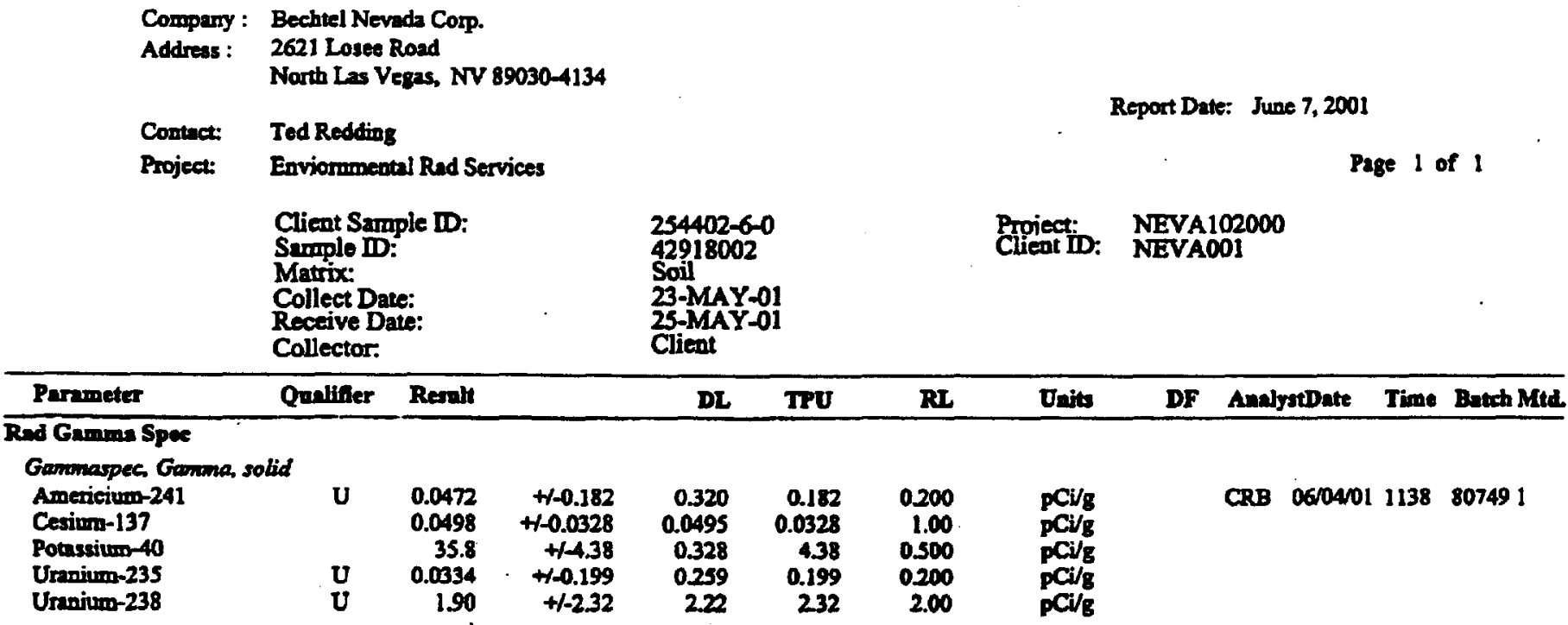

The following Prep Metbods were performed

\begin{tabular}{llllll}
\hline Method & Deacription & Analyst & Date & Tine & Frep Barel \\
\hline Dry Soil Prep & Dry Soil Prep RAD A-021,A-021B,A-026 & CCMI & 05/29/01 & 1358 & 80126
\end{tabular}

The following Andiytical Methods were performed

$\frac{\text { Method }}{1}$ Deseription

Notes:

The Qualifiers in this report are defined as follows:

* Indicates the analyte is a surrogate compound.

$<$ Actual result is less than amount reported

$>$ Actual result is greater than amount reported

J Indicates an estimated value. The result was greater than the detection limit, but less than the reporting limit.

U Indicates the compound was analyzed for but not detected above the detection limit

The above sample is reported on an "as received" basis.

This data report has been prepared and reviewed in accordance with General Engineering Laboratories, Inc.

standard operating procedures. Please direct any questions to your Project Manager, Stacy L. Griffin at 843-556-8171 Ext. 4264.

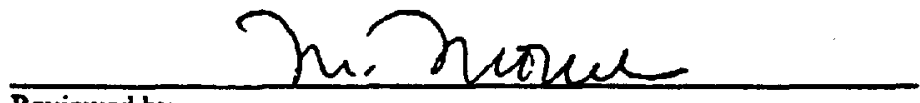

Reviewed by 


\section{GENERAL ENGINEERING LABORATORIES}

Meeting roday's needs with a vision for tomormw.

\section{QC Summary}

Report Date: June 7, 2001

Page 1 of 2

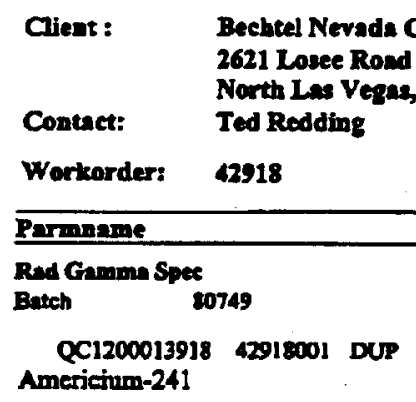

Cesium-137

NOM Somple Qunal

QC
Units $\mathbf{R P D} \%$ REC\% Range Anlat Dare Tine

QC1200013918 42918001 DUP Amerieium-241

Potassium-40

Uranium-235

Uranium-238

QC1200013919 LCS Americium-241

Uncent:
TPU:
0.00041 $1-0.0574$

$\mathbf{U}$

0.0291
+-0.115

pCis N/A

(+/-0.200) CRB 06/05/01 01:04

0.048

Uncert: $\quad+-0.023$

TPU: $\quad 0.023$

Uneert $\quad 35.2$

noert $\quad+.3 .91$

IPU: $\quad 3.91$

3.91

Unoert: $\quad+1-0.159$

TPU: $\quad 0.159$

$\begin{array}{lr}\text { U } & 0.752 \\ \text { Uneert } & +-0.975\end{array}$

TPU:

0.975

TPU.

Cesium-137

441

Uncert:

IPU:

Ponssium-40

Uncert:

TPU:

Uranium-235

Uncert:

TPU:

Uranium-238

Uncert:

TPU:

QC1200013917 MB

Ameticium-241

Uncert:
TPU:

Cesiun-137

Uncert:

IPU:

Potassium-40

Uranium-235

\section{Uncert:}

TPU:

Uncert:

$\mathrm{U}$

0.115

0.0378

$+-0.0292$

0.0292

35.6

$+\$ 4.21$

4.21

0.056 PCi/g 10 ค

$+1-0.172$

0.172

1.90

$+1-1.56$

$\mathrm{pCi} / 8$ 87

1.56

$\mathrm{pCV}_{8} 24^{\wedge}$

$(+1-1.00)$

$\mathrm{pCi} / \mathrm{s} \quad 1$

$(+1-0.200)$

$(+/-2.00)$

$1220 \mathrm{pCi} / \mathrm{g}$

$+\gamma-132$

132

486

$+1-61.1$

61.1

U

$-0.383$

-0.383
$+/-2.19$

219

U $\quad 0.74$

$+.1 .81$

1.81

$\mathbf{U}$

$-1.93$

$+1-6.52$

6.52

$\mathrm{pCv} / \mathrm{g}$

110

(75\%-125\%)

pCi/g

pCi/g

$\mathrm{pCi} / \mathrm{g}$

$\begin{array}{lll}\text { U } & 0.030 & \mathrm{pCi} / \mathrm{g}\end{array}$

06/05/01 07:08
U $\quad 0.0211$ $+1-0.0117$

0.0117

U $\quad 0.253$

$++-0.181$

0.181
$\quad \quad 0.0501$

$+1-0.102$

$\mathrm{pCi} / \mathrm{g}$

pCi/g

$\mathrm{pCi} / \mathrm{g}$

PO Box 30712 - Charleston, SC 29417 - 2040 Savage Road - 29407

(843) 546-8171 • Fax (RA3) 766-1178 


\section{GENERAL ENGINEERING LABORATORIES}

Meeting today's needs with a vision for tomorrow.

Workorder:

42918

\section{QC Summary}

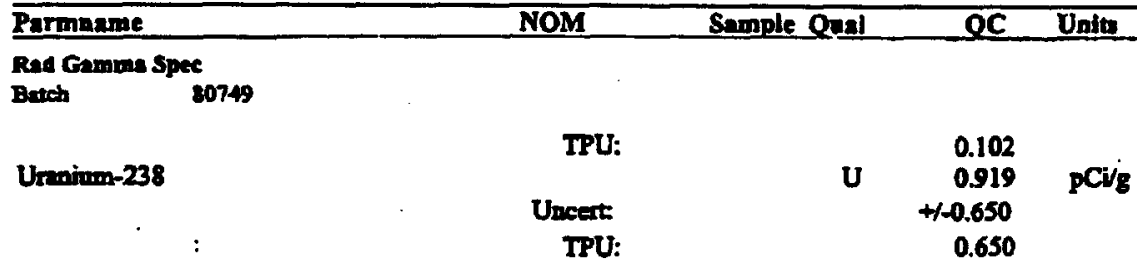

Notes:

The Qualifiess in this report are defined as followe:

- Indientes the analyte is a surogrte compound

< Actud rovult is less than monint reported

$>\quad$ Actoul result is greater then mount reported

J Indicates en estimated value. The result was greater then the detection limil but less then the roportiog limit.

U Indicates the compound was antyred for but not detected above the detection limit

N/A indieates that spike recovery limits do not apply when sample concentution exceeds spike conc. by a factor of 4 or more.

A The Relative Percent Difference (RPD) obtuined from the sample duplieate (DUP) is evalunted against the aceeplesce criteria when the sample is greater than five times (5X) the contrect required detection linit (RU). In erses where either the sumple or duplicate value is less than $5 X$ the RI, a coutrol limit of $+\%$ the $R L$ is used to cvahute the DUP result.

For PS, PSD, and SDIIT reoults, the vahes listed are the measured amonats, not finel concentrations. 
CLIENT: Bechtel Nevada

P.O. Box 98521 , M/S NTS273

Las Vegas, NV 89193-8521

ATTN: $\quad$ Ted Redding

PROJECT NAME: V1122

NEL ORDER ID: L0105275

PROJECT NUMBER: 23081

Attached are the analytical results for samples in support of the above referenced project.

Samples submitted for this project were not sampled by NEL Laboratories. Samples were received by NEL in good condition, under chain of custody on 5/29/01.

Should you have any questions or comments, please feel free to contact our Client Services department at (702) 657-1010.

Some QA results have been flagged as follows:

J1 - The batch MS and/or MSD were outside acceptance limits. The batch LCS was acceptable.

Some surrogate results have been flagged as follows:

D - Sample required dilution. Sample QC results were diluted outside the calibrated range.
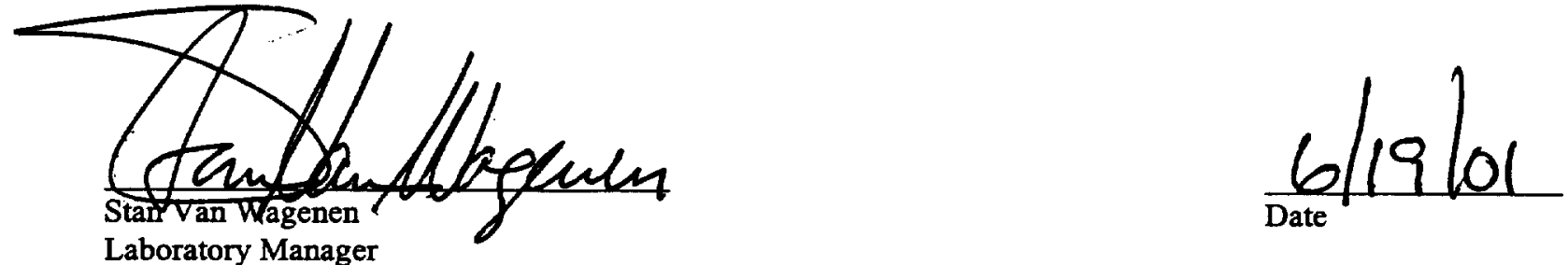

\section{CERTIFICATIONS:}

\begin{tabular}{llll}
\hline & Reno & Las Vegas & S. California \\
\cline { 2 - 4 } Arizona & AZ0520 & AZ0518 & AZ0605 \\
California & 1707 & 2002 & 2264 \\
US Army Corps & Certified & Certified &
\end{tabular}

Idaho

Montana

Nevada

L.A.C.S.D.
Reno Las Vegas S. California

Certified Certified

Certified Certified

NV033 NV052

CA084

10228 
NEL LABORATORIES

$\begin{array}{llll}\text { CLIENT: } & \text { Bechtel Nevada } & \text { CLIENT ID: } & 254404-1-0 \\ \text { PROJECT ID: } & \text { V1122 } & \text { DATE SAMPLED: } & 5 / 24 / 01 \\ \text { PROJECT \#: } & 23081 & \text { NEL SAMPLE ID: } & \text { L0105275-04 }\end{array}$

TEST: Inorganic Non-Metals

MATRIX: $\quad$ Solid

\begin{tabular}{|c|c|c|c|c|c|c|}
\hline PARAMETER & RESULT & R. L. & D. F. & METHOD & UNITS & ANALYZED \\
\hline & 8.85 & 2 & 1 & EPA 9045C & pH Units & $6 / 1 / 01$ \\
\hline pH Temperature & 26.8 & 1. & 1 & EPA $9045 C$ & ${ }^{\circ} \mathrm{C}$ & $6 / 1 / 01$ \\
\hline
\end{tabular}

R.L. - Reporting Limit

D.F. - Dilution Factor

ND - Not Detected

This report shall not be reproduced except in full, without the written approval of the laboratory. 
NEL LABORATORIES

\begin{tabular}{llll}
\hline CLIENT: & Bechtel Nevada & CLIENT ID: & 254404-2-0 \\
PROJECT ID: & V1122 & DATE SAMPLED: 5/24/01 \\
PROJECT \#: & 23081 & NEL SAMPLE ID: & L0105275-05 \\
TEST: & Inorganic Non-Metals & & \\
MATRIX: & Solid & & \\
\hline
\end{tabular}

-

- $\overline{\mathrm{pH}} \frac{\text { RESULT }}{7.48}$

$\begin{array}{ll}\mathrm{pH} \text { Temperature } & \mathbf{7 6} \\ & 26.6\end{array}$

R. L.

D. F.

\begin{tabular}{l} 
METHOD \\
\hline EPA $9045 C$ \\
EPA 9045C
\end{tabular}

\begin{tabular}{|c|c|}
\hline UNITS & ANALYZED \\
\hline $\mathrm{pH}$ Units & 6 \\
\hline
\end{tabular}

-

-

$-$

- 
NEL LABORATORIES

\begin{tabular}{llll}
\hline CLIENT: & Bechtel Nevada & CLIENT ID: & 254404-3-0 \\
PROJECT D: & V1122 & DATE SAMPLED: $5 / 24 / 01$ \\
PROJECT \#: & 23081 & NEL SAMPLE ID: & L0105275-06 \\
TEST: & Inorganic Non-Metals & & \\
MATRIX: & Solid & \\
\hline
\end{tabular}

\begin{tabular}{|c|c|c|c|c|c|c|}
\hline PARAMETER & RESULT & R. L. & D. F. & METHOD & UNITS & ANALYZED \\
\hline $\begin{array}{l}\text { pH } \\
\text { pH Temperature }\end{array}$ & $\begin{array}{l}9.77 \\
26.3\end{array}$ & $\begin{array}{l}2 . \\
1 .\end{array}$ & $\begin{array}{l}1 \\
1\end{array}$ & $\begin{array}{l}\text { EPA } 9045 C \\
\text { EPA } 9045 C\end{array}$ & $\begin{array}{l}\mathrm{pH} \text { Units } \\
{ }^{\circ} \mathrm{C}\end{array}$ & $\begin{array}{l}6 / 1 / 01 \\
6 / 1 / 01\end{array}$ \\
\hline
\end{tabular}

R.L. - Reporting Limit

D.F. - Dilution Factor

ND - Not Detected

This report shall not be reproduced except in full, without the written approval of the laboratory. 
NEL LABORATORIES

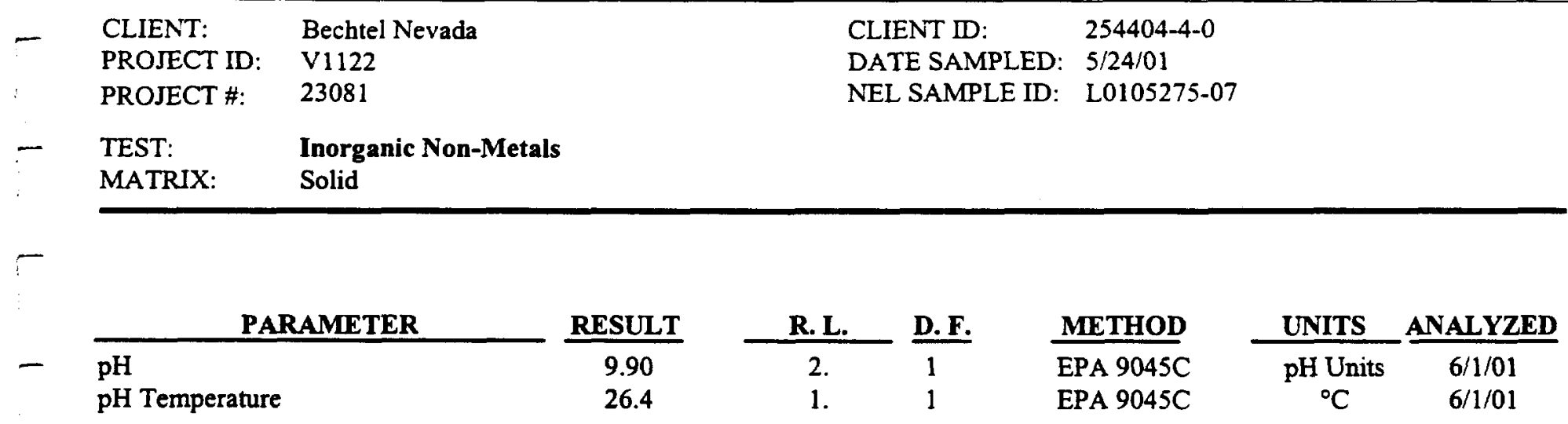


NEL LABORATORIES

\begin{tabular}{|c|c|c|}
\hline CLIENT: & Bechtel Nevada & CLIENT ID: \\
\hline PROJECT ID: & V1122 & DATE SAMPLED: 5/24/01 \\
\hline PROJECT \#: & 23081 & NEL SAMPLE ID: L0105275-12 \\
\hline
\end{tabular}

TEST: $\quad$ PCB's (Polychlorinated Biphenyls) by EPA 8082, Dec. 1996

$\begin{array}{llll}\text { METHOD: } & \text { EPA } 8082 & \text { ANALYST: } & \text { JRW - Las Vegas Division } \\ \text { MATRIX: } & \text { Solid } & \text { EXTRACTED: } & 5 / 30 / 01\end{array}$

MATRIX: Solid

DILUTION: 5

ANALYZED: $\quad 6 / 6 / 01$

\begin{tabular}{|c|c|c|}
\hline PARAMETER & Result & $\begin{array}{c}\text { Reporting } \\
\text { Limit }\end{array}$ \\
\hline Aroclor-1016 & ND & $100 . \mu \mathrm{g} / \mathrm{kg}$ \\
\hline Aroclor- 1221 & ND & 100. $\mu \mathrm{g} / \mathrm{kg}$ \\
\hline Aroclor- 1232 & ND & 100. $\mu \mathrm{g} / \mathrm{kg}$ \\
\hline Aroclor- 1242 & ND & 100. $\mu \mathrm{g} / \mathrm{kg}$ \\
\hline Aroclor- 1248 & ND & 100. $\mu \mathrm{g} / \mathrm{kg}$ \\
\hline Aroclor -1254 & ND & 100. $\mu \mathrm{g} / \mathrm{kg}$ \\
\hline Arocior- 1260 & ND & 100. $\mu \mathrm{g} / \mathrm{kg}$ \\
\hline
\end{tabular}

Surrogate

Decachlorobiphenyl

Tetrachloro-m-xylene
\% Recovery

133

127

\section{Acceptable Range}

$46-155$

$49-140$

ND - Not Detected

This report shall not be reproduced except in full, without the written approval of the laboratory. 
NEL LABORATORIES

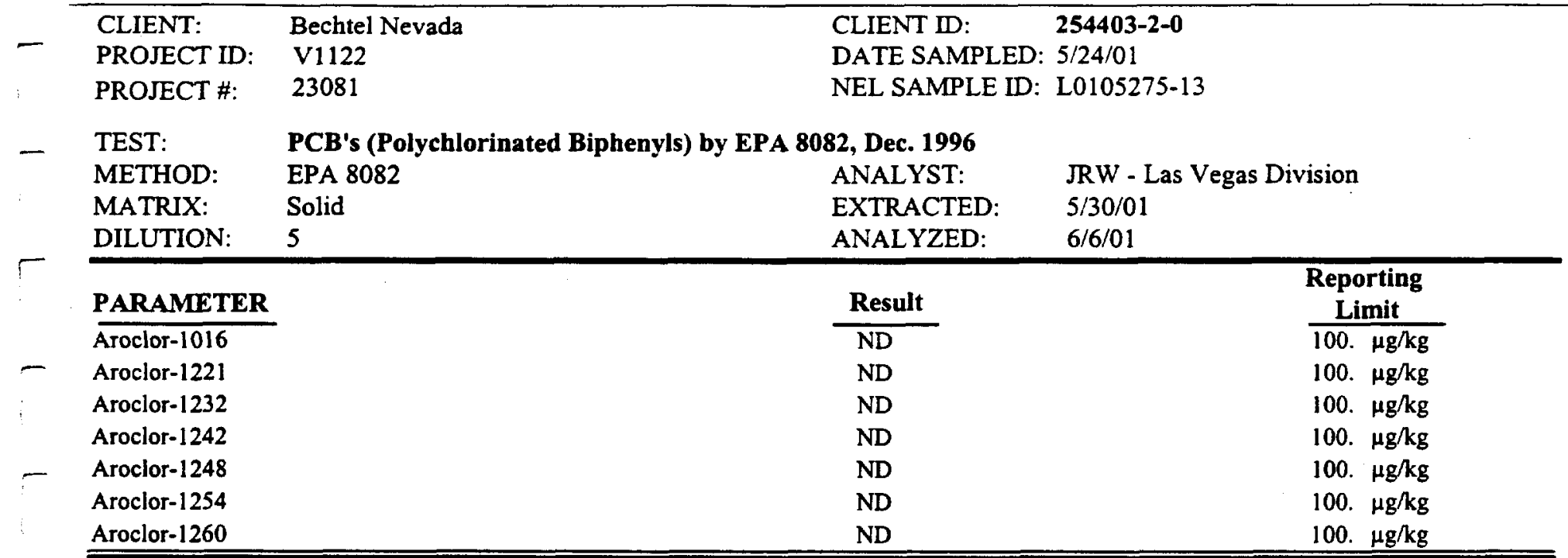

- QUALITY CONTROL DATA:

Surrogate

Decachlorobiphenyl

- Tetrachloro-m-xylene

\section{$\%$ Recovery}

155

133

\section{Acceptable Range}

$46-155$

$49-140$

- ND - Not Detected

This report shall not be reproduced except in full, without the written approval of the laboratory. 
NEL LABORATORIES

\begin{tabular}{|c|c|c|}
\hline & Bechtel Nevada & CLIENT ID: \\
\hline PROJECT ID: & V1122 & DATE SAMPLED: $5 / 24 / 01$ \\
\hline PROJECT \#: & 23081 & NEL SAMPLE ID: L0105275-08 \\
\hline
\end{tabular}

TEST: $\quad$ Total Extractable Petroleum Hydrocarbons Fuel Finger Print by EPA Method 8015 M, July 1992

METHOD: EPA 8015M ANALYST: CCS - Las Vegas Division

MATRIX: Solid $\quad$ EXTRACTED: 5/30/01

DILUTION: $1 \quad$ ANALYZED: $\quad 5 / 31 / 01$

\begin{tabular}{lll}
\hline PARAMETER & Result & Reporting \\
\cline { 2 - 3 } & $\mathrm{ND}$ & $\frac{\text { Limit }}{10 . \mathrm{mg} / \mathrm{kg}}$ \\
Gasoline Range (C8-C12) & $\mathrm{ND}$ & $10 . \mathrm{mg} / \mathrm{kg}$ \\
Diesel Range (C12-C22) & $\mathrm{ND}$ & $50 . \mathrm{mg} / \mathrm{kg}$ \\
Oil Range (C12-C34) & $\mathrm{ND}$ & $10 . \mathrm{mg} / \mathrm{kg}$ \\
\hline Total & & 1 \\
\hline \hline
\end{tabular}

QUALITY CONTROL DATA:

Surrogate

Octacosane

\section{\% Recovery}

89

\section{Acceptable Range}

$54-130$

ND - Not Detected

This report shall not be reproduced except in full, without the written approval of the laboratory. 
NEL LABORATORIES

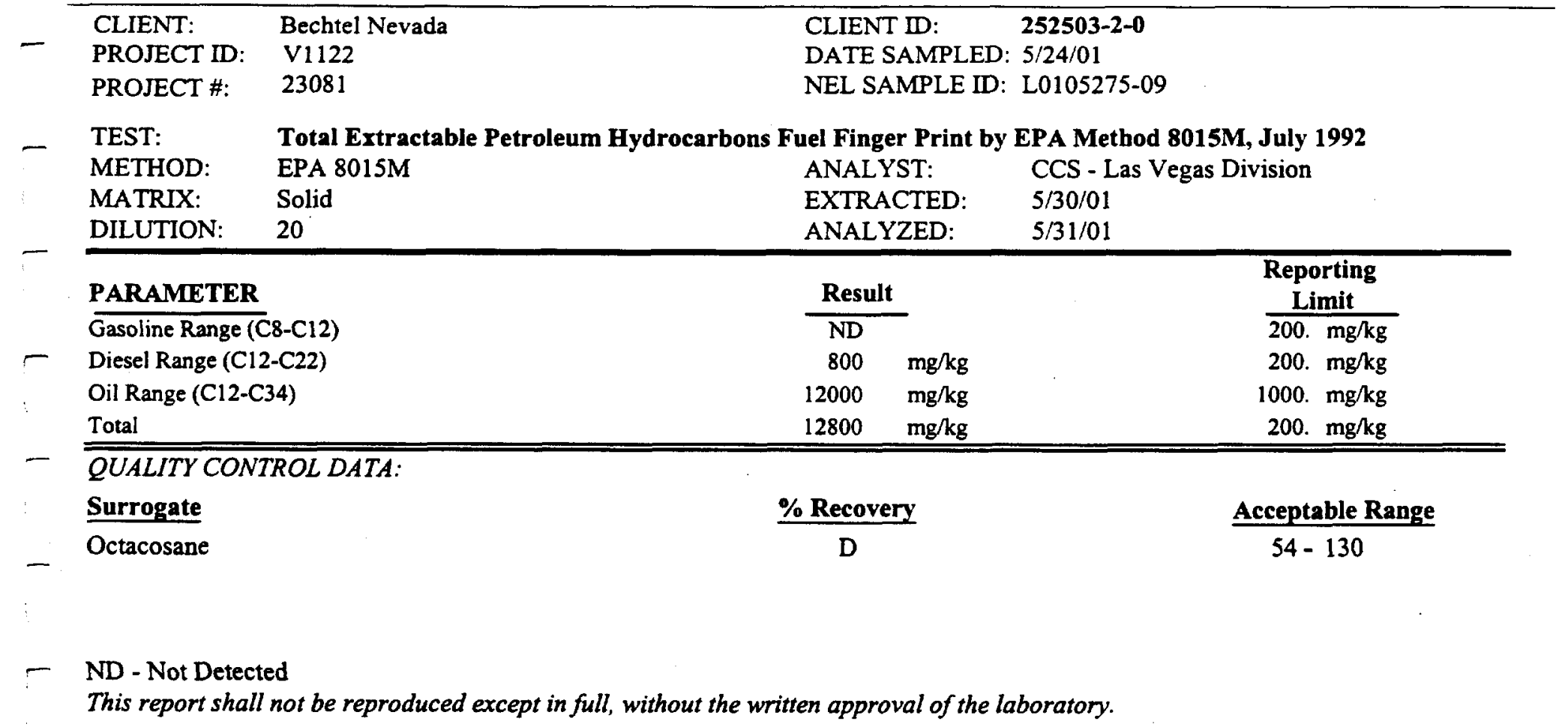


NEL LABORATORIES

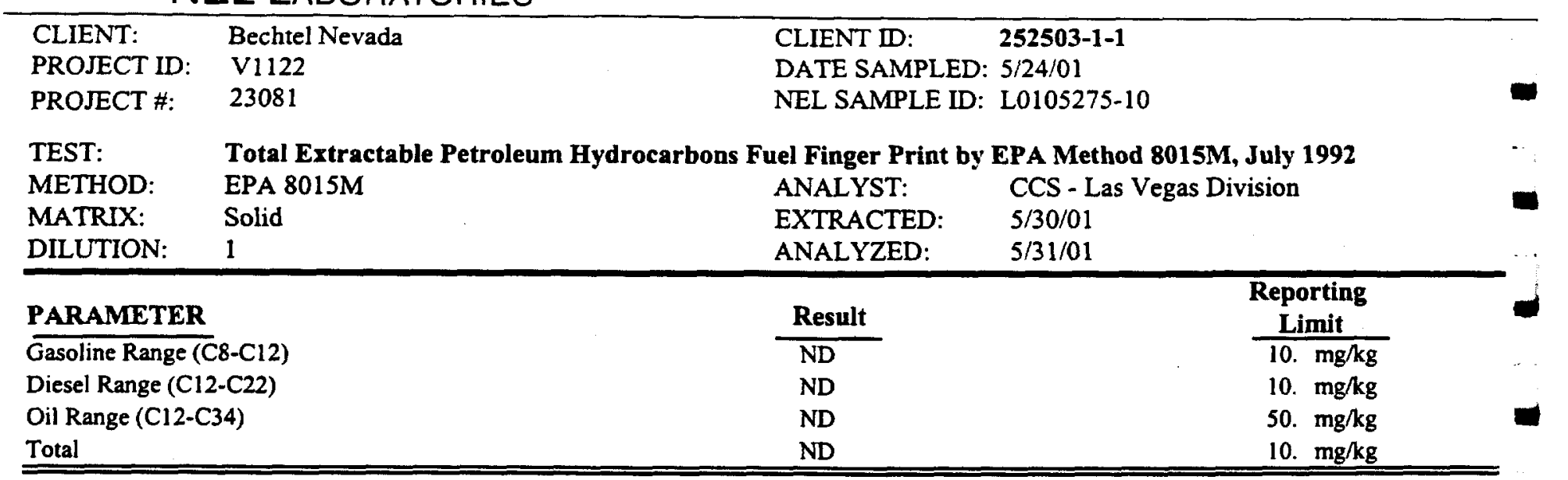

QUALITY CONTROL DATA:

Surrogate

\% Recovery

70

\section{Acceptable Range}

$54-130$

ND - Not Detected

This report shall not be reproduced except in full, without the written approval of the laboratory. 
NEL LABORATORIES

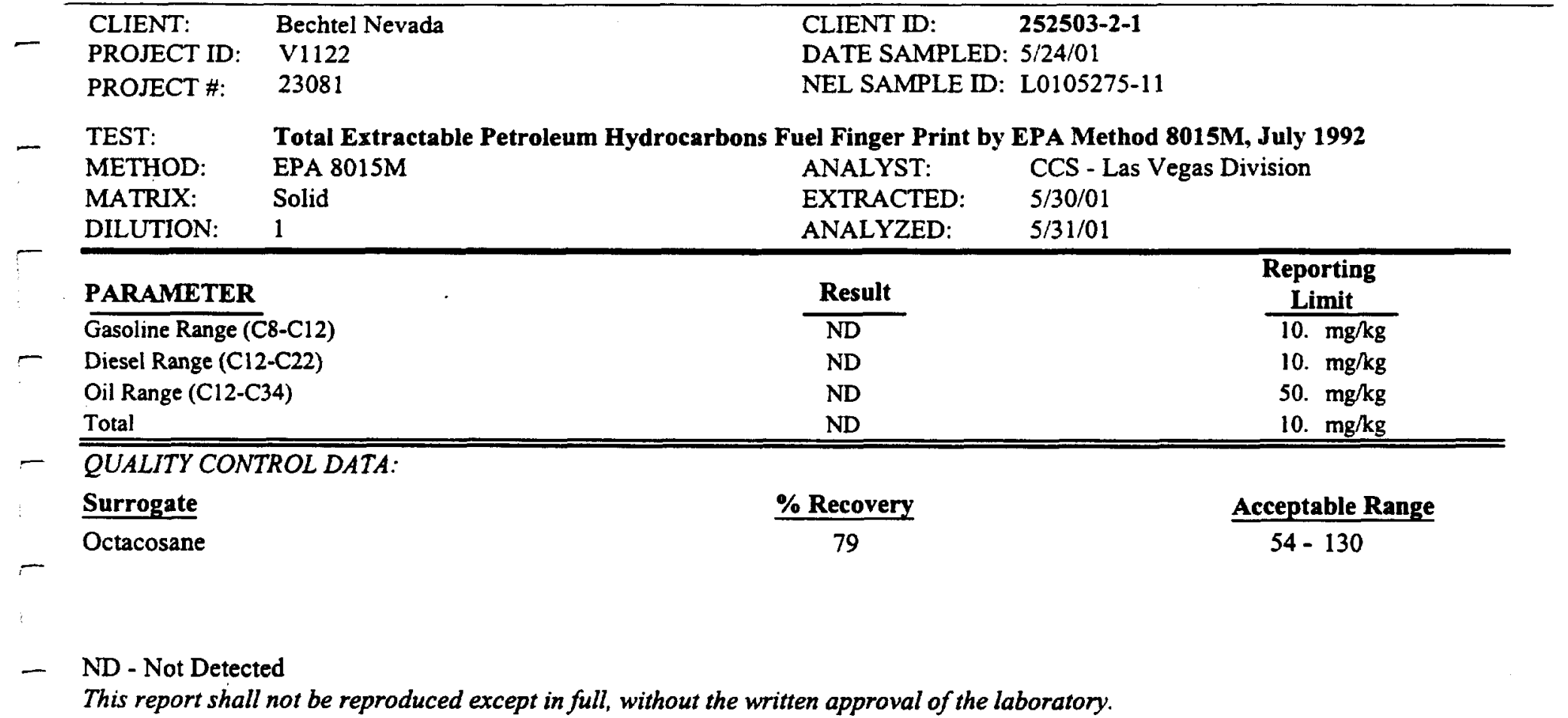


NEL LABORATORIES

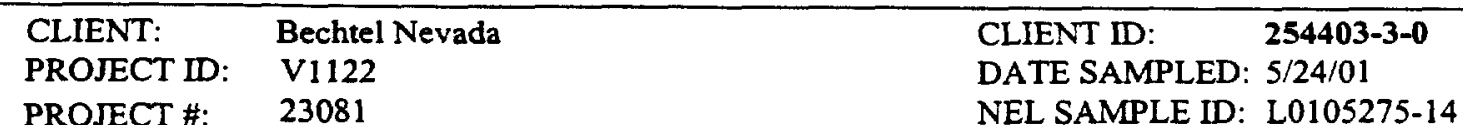

TEST: $\quad$ Total Extractable Petroleum Hydrocarbons Fuel Finger Print by EPA Method 8015M, July 1992

METHOD: EPA 8015M ANALYST: CCS - Las Vegas Division

MATRIX: Solid $\quad$ EXTRACTED: 5/30/01

DILUTION: $5 \quad$ ANALYZED: $5 / 31 / 01$

\begin{tabular}{lcc}
\hline PARAMETER & Result & Reporting \\
\cline { 2 - 3 } & Lasoline Range (C8-C12) & Limit \\
Diesel Range (C12-C22) & $520 \quad \mathrm{mg} / \mathrm{kg}$ & $50 . \mathrm{mg} / \mathrm{kg}$ \\
Oil Range (C12-C34) & $4800 \quad \mathrm{mg} / \mathrm{kg}$ & $50 . \mathrm{mg} / \mathrm{kg}$ \\
Total & $5320 \quad \mathrm{mg} / \mathrm{kg}$ & $250 . \mathrm{mg} / \mathrm{kg}$ \\
\hline \hline
\end{tabular}

QUALITY CONTROL DATA:

Surrogate

\% Recovery

Octacosane

D

Acceptable Range

$54-130$

ND - Not Detected

This report shall not be reproduced except in full, without the written approval of the laboratory. 
NEL LABORATORIES

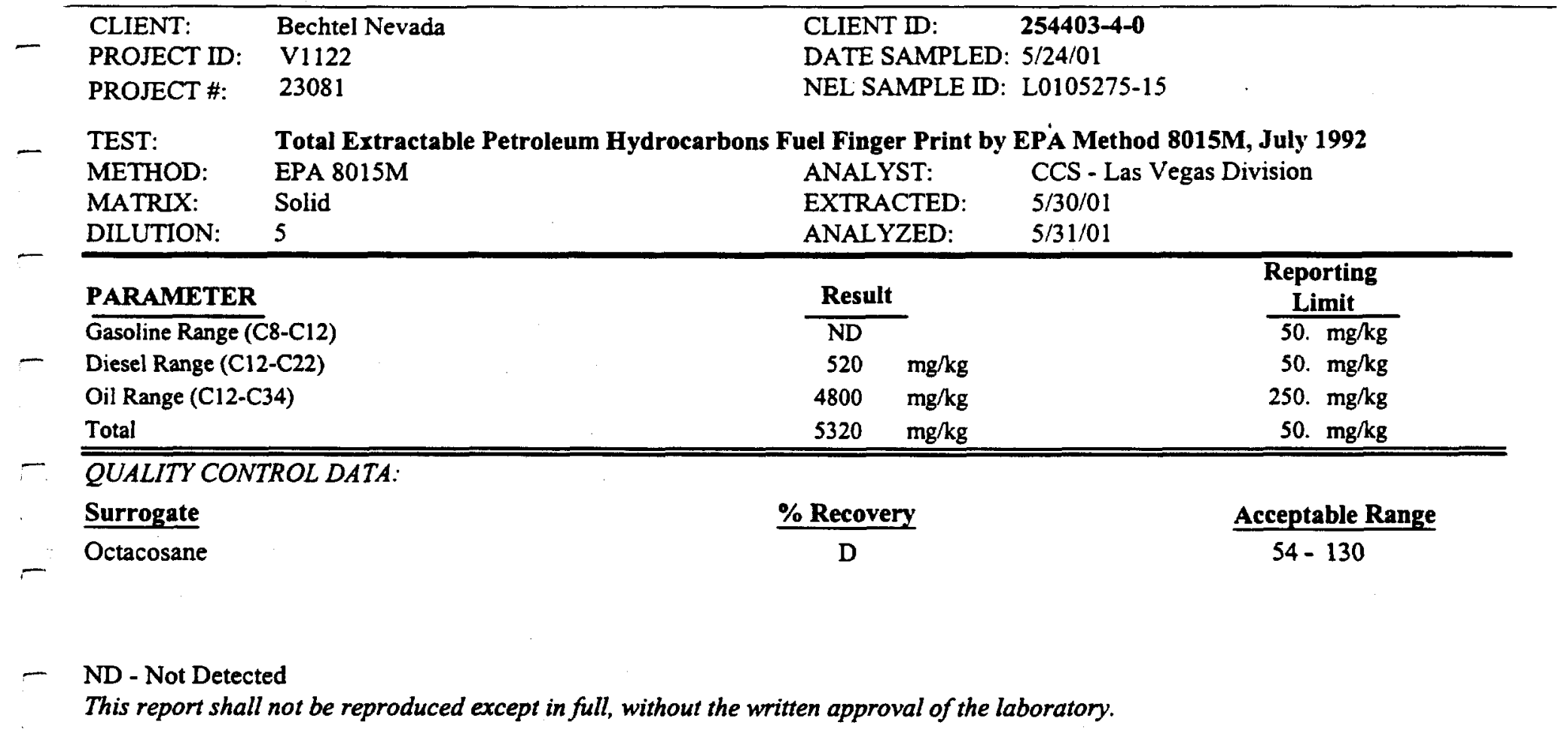


NEL LABORATORIES

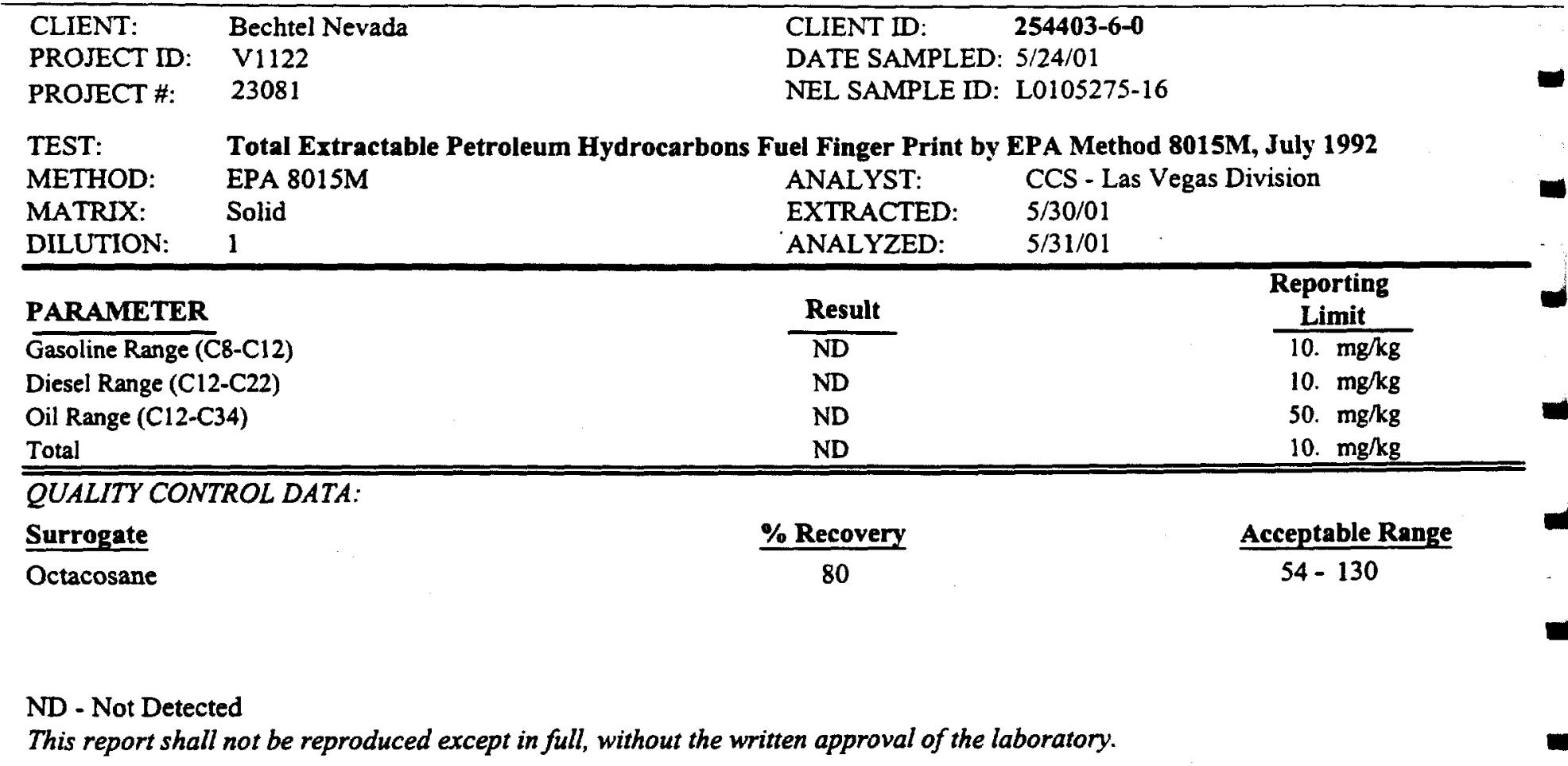


NEL LABORATORIES

\begin{tabular}{lll}
\hline CLIENT: & Bechtel Nevada & CLIENT ID: \\
PROJECT ID: & V1122 & DATE S53-3-1 \\
PROJECT \#: & 23081 & NEL SAMPLE ID: L0105275-17
\end{tabular}

- TEST: Total Extractable Petroleum Hydrocarbons Fuel Finger Print by EPA Method 8015M, July 1992

METHOD: $\quad$ EPA 8015M ANALYST: CCS - Las Vegas Division

MATRIX: Solid

EXTRACTED: $\quad 5 / 30 / 01$

DILUTION: 1 ANALYZED: $5 / 31 / 01$

\section{$-$ \\ PARAMETER \\ Gasoline Range ( $\mathrm{C} 8-\mathrm{Cl} 2)$ \\ - Diesel Range (C12-C22) \\ Oil Range (C12-C34) \\ Total \\ - QUALITY CONTROL DATA:}

Surrogate

Octacosane

-

- ND - Not Detected

This report shall not be reproduced except in full, without the written approval of the laboratory.

\begin{tabular}{l} 
Result \\
\hline ND \\
ND \\
ND \\
ND
\end{tabular}

\% Recovery

88
Reporting

Limit

$10 . \mathrm{mg} / \mathrm{kg}$

10. $\mathrm{mg} / \mathrm{kg}$

50. $\mathrm{mg} / \mathrm{kg}$

10. $\mathrm{mg} / \mathrm{kg}$

\section{Acceptable Range}

$54-130$ 
NEL LABORATORIES

\begin{tabular}{|c|c|c|c|}
\hline $\begin{array}{l}\text { CLIENT: } \\
\text { PROJECT ID: } \\
\text { PROJECT \#: }\end{array}$ & $\begin{array}{l}\text { Bechtel Nevada } \\
\text { V1122 } \\
23081\end{array}$ & $\begin{array}{l}\text { CLIENT ID: } \\
\text { DATE SAMPLED: } \\
\text { NEL SAMPLE ID: }\end{array}$ & $\begin{array}{l}\text { Method Blank } \\
\text { NA } \\
\text { 010530PCBS-BLK }\end{array}$ \\
\hline $\begin{array}{l}\text { TEST: } \\
\text { METHOD: } \\
\text { MATRIX: }\end{array}$ & $\begin{array}{l}\text { PCB's (Polychlo } \\
\text { EPA } 8082 \\
\text { Solid }\end{array}$ & $\begin{array}{l}\text { 8082, Dec. } 1996 \\
\text { ANALYST: } \\
\text { EXTRACTED: } \\
\text { ANALYZED: }\end{array}$ & $\begin{array}{l}\text { JRW - Las Vegas Division } \\
5 / 30 / 01 \\
6 / 6 / 01\end{array}$ \\
\hline $\begin{array}{l}\text { PARAMETER } \\
\text { Aroclor-1016 } \\
\text { Aroclor-1221 } \\
\text { Aroclor- } 1232 \\
\text { Aroclor-1242 } \\
\text { Aroclor- } 1248 \\
\text { Aroclor- } 1254 \\
\text { Aroclor- } 1260 \\
\end{array}$ & & $\begin{array}{l}\text { Result } \\
\text { ND } \\
\text { ND } \\
\text { ND } \\
\text { ND } \\
\text { ND } \\
\text { ND } \\
\text { ND } \\
\end{array}$ & \begin{tabular}{cc} 
Reporting \\
\multicolumn{2}{c}{ Limit } \\
20. & $\mu \mathrm{g} / \mathrm{kg}$ \\
20. & $\mu \mathrm{g} / \mathrm{kg}$ \\
20. & $\mu \mathrm{g} / \mathrm{kg}$ \\
20. & $\mu \mathrm{g} / \mathrm{kg}$ \\
20. & $\mu \mathrm{g} / \mathrm{kg}$ \\
20. & $\mu \mathrm{g} / \mathrm{kg}$ \\
20. & $\mu \mathrm{g} / \mathrm{kg}$ \\
\end{tabular} \\
\hline $\begin{array}{l}\text { QUALITY CON } \\
\text { Surrogate } \\
\text { Decachlorobiph } \\
\text { Tetrachloro-m-x }\end{array}$ & $\begin{array}{l}\text { ROL DATA: } \\
\text { anyl } \\
\text { ylene }\end{array}$ & $\frac{\% \text { Recovery }}{82}$ & $\begin{array}{l}\text { Acceptable Range } \\
\begin{array}{l}46-155 \\
49-140\end{array}\end{array}$ \\
\hline $\begin{array}{l}\text { ND - Not Detec } \\
\text { This report shal } \\
\text { CLIENT: } \\
\text { PROJECT ID: } \\
\text { PROJECT \#: }\end{array}$ & $\begin{array}{l}\text { ed } \\
\text { not be reproduce } \\
\text { Bechtel Nevada } \\
\text { V1122 } \\
23081\end{array}$ & $\begin{array}{l}\text { itten approval of the lo } \\
\text { CLIENT ID: } \\
\text { DATE SAMPLED: } \\
\text { NEL SAMPLE ID: }\end{array}$ & $\begin{array}{l}\text { boratory. } \\
\text { Method Blank } \\
\text { NA } \\
\text { 010530TPHS-FP-BLK }\end{array}$ \\
\hline $\begin{array}{l}\text { TEST: } \\
\text { METHOD: } \\
\text { MATRIX: }\end{array}$ & \multicolumn{3}{|c|}{$\begin{array}{lll}\text { Total Extractable Petroleum Hydrocarbons Fuel Finger Print by EPA Method } 8015 \mathrm{M}, \text { July } 1992 \\
\text { EPA } 8015 \mathrm{M} & \text { ANALYST: } & \text { CCS - Las Vegas Division } \\
\text { Solid } & \text { EXTRACTED: } & 5 / 30 / 01 \\
& \text { ANALYZED: } & 5 / 31 / 01\end{array}$} \\
\hline $\begin{array}{l}\text { PARAMETER } \\
\text { Gasoline Range (C } \\
\text { Diesel Range (Cl } \\
\text { Oil Range (C12-C } \\
\text { Total } \\
\end{array}$ & $\begin{array}{l}(8-C 12) \\
(-C 22) \\
34)\end{array}$ & $\begin{array}{l}\text { Result } \\
\text { ND } \\
\text { ND } \\
\text { ND } \\
\text { ND } \\
\end{array}$ & $\begin{array}{c}\begin{array}{c}\text { Reporting } \\
\text { Limit }\end{array} \\
10 . \mathrm{mg} / \mathrm{kg} \\
10 . \mathrm{mg} / \mathrm{kg} \\
50 . \mathrm{mg} / \mathrm{kg} \\
10 . \mathrm{mg} / \mathrm{kg} \\
\end{array}$ \\
\hline $\begin{array}{l}\text { QUALITY CON } \\
\text { Surrogate } \\
\text { Octacosane }\end{array}$ & TROL DATA: & $\frac{\text { \% Recovery }}{81}$ & $\frac{\text { Acceptable Range }}{54-130}$ \\
\hline
\end{tabular}


NEL LABORATORIES

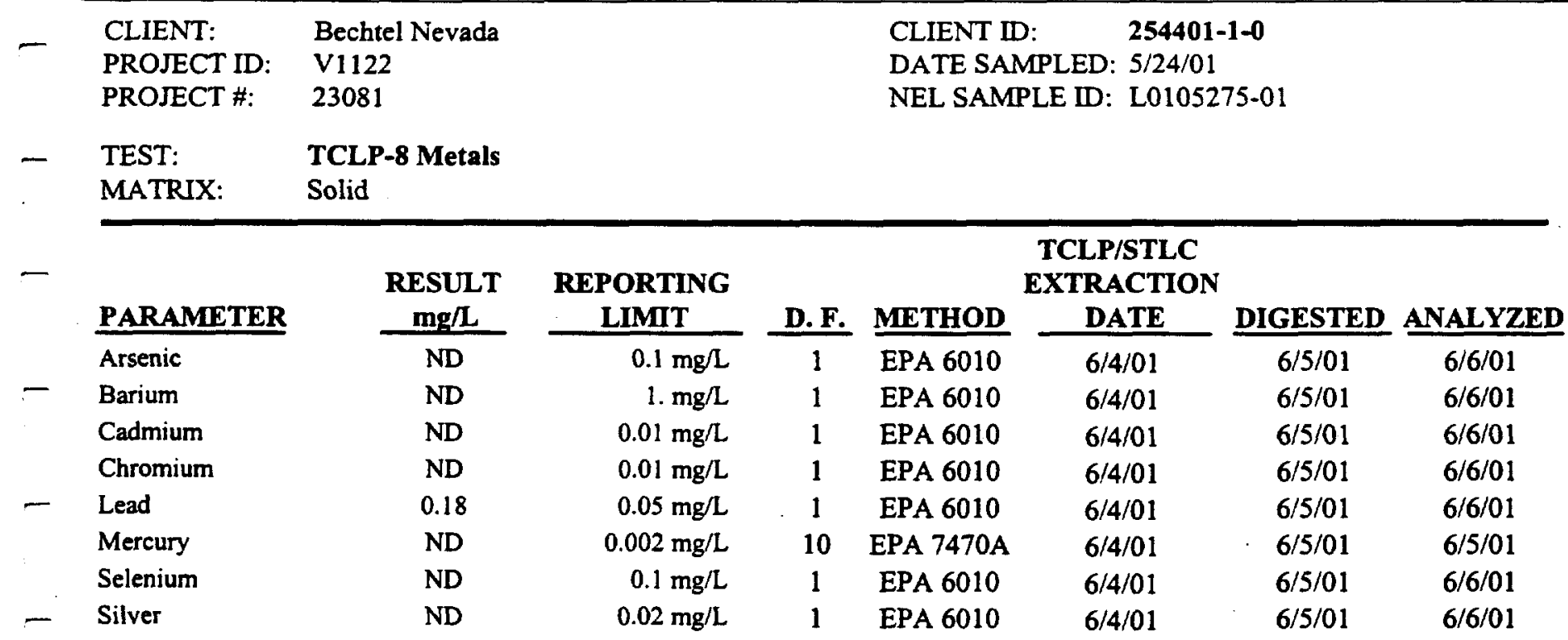

- D.F. - Dilution Factor

ND - Not Detected

This report shall not be reproduced except in full, without the written approval of the laboratory. 
NEL LABORATORIES

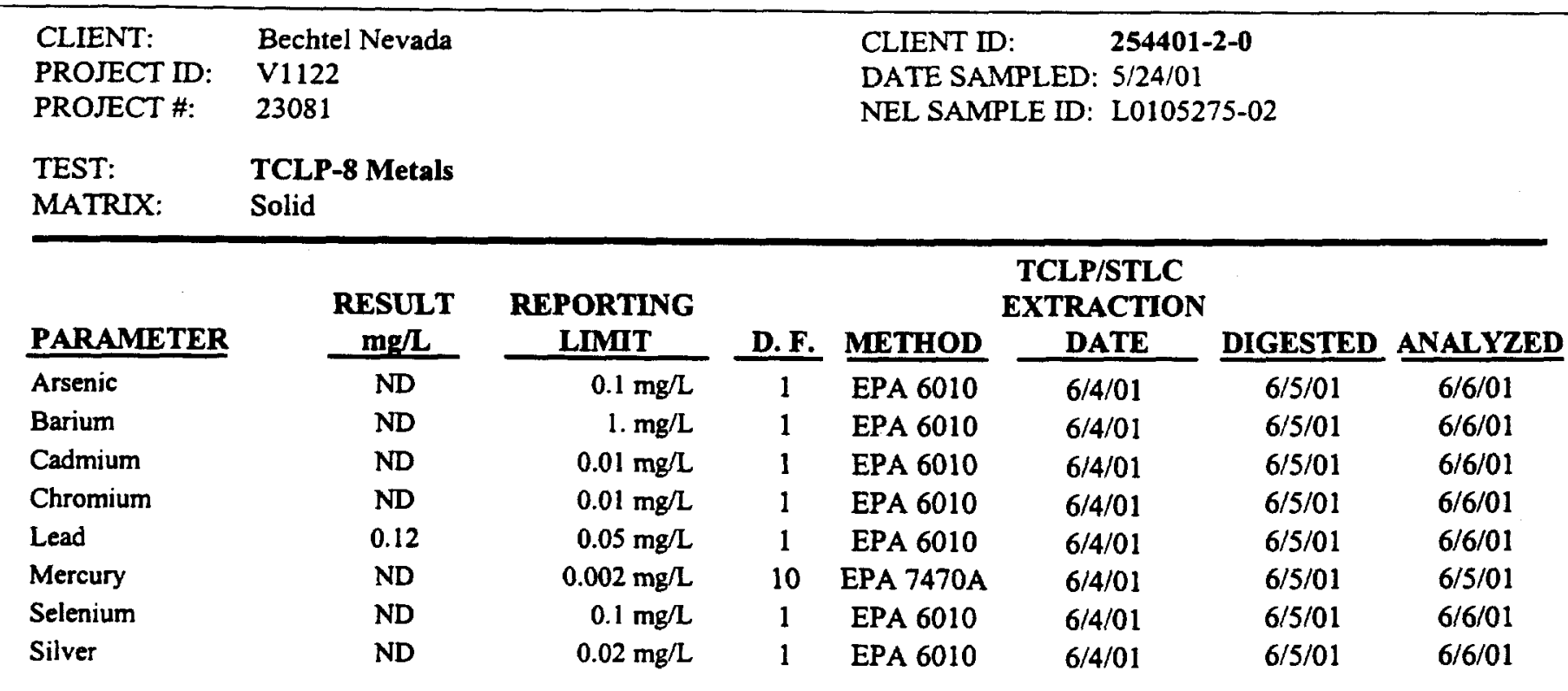

D.F. - Dilution Factor

ND - Not Detected

This report shall not be reproduced except in full, without the written approval of the laboratory. 
NEL LABORATORIES

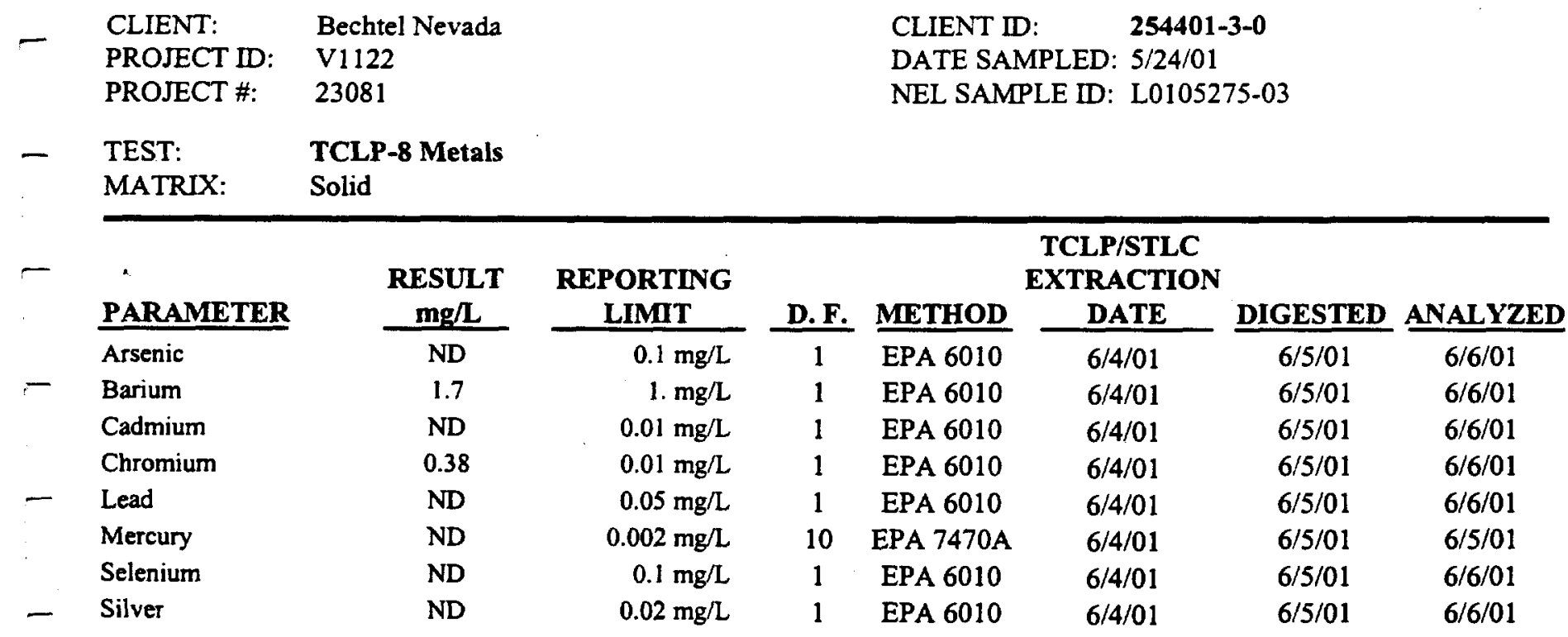

- D.F. - Dilution Factor

ND - Not Detected

This report shall not be reproduced except in full, without the written approval of the laboratory. 
NEL LABORATORIES

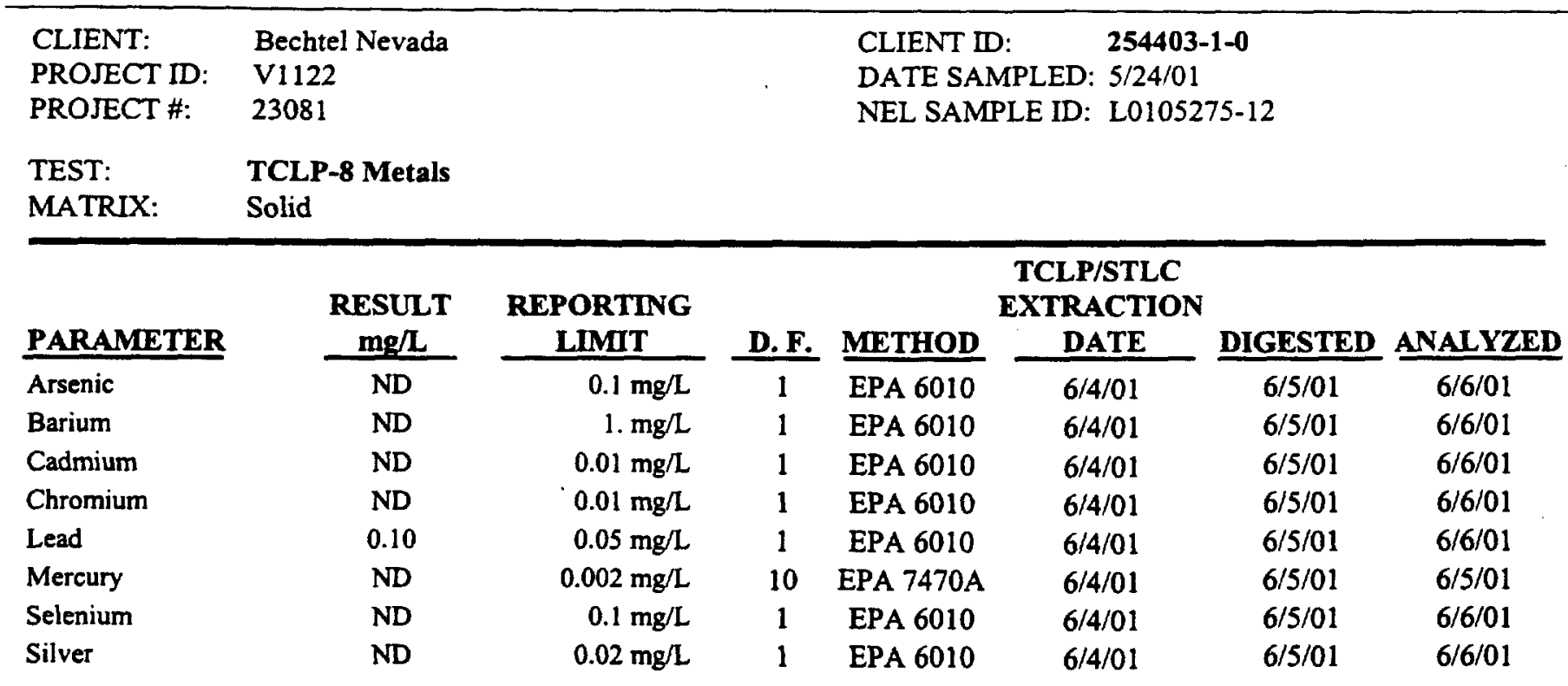

D.F. - Dilution Factor

ND - Not Detected

This report shall not be reproduced except in full, without the written approval of the laboratory. 
NEL LABORATORIES

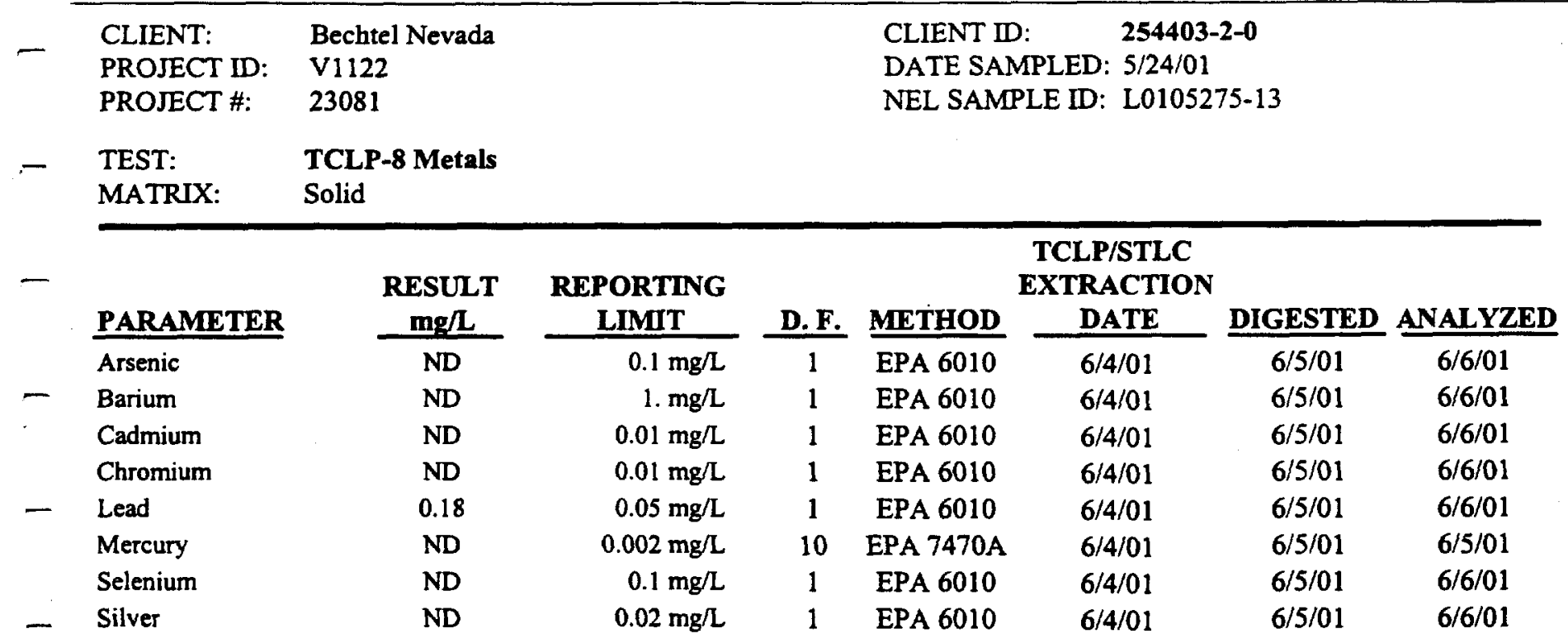

- D.F. - Dilution Factor ND - Not Detected

This report shall not be reproduced except in full, without the written approval of the laboratory. 
NEL LABORATORIES

$\begin{array}{llll}\text { CLIENT: } & \text { Bechtel Nevada } & \text { CLIENT ID: } & \text { Method Blank } \\ \text { PROJECT ID: } & \text { V1122 } & \text { DATE SAMPLED: } & \text { NA } \\ \text { PROJECT \#: } & 23081 & \text { NEL SAMPLE ID: } & \text { L5275HGTCLP-BLK }\end{array}$

TEST: $\quad$ TCLP by EPA 1311, July 1992 \& Mercury by EPA 7470A, July 1992

MATRIX: $\quad$ TCLP Extract

\begin{tabular}{|c|c|c|c|c|c|c|c|}
\hline \multirow[b]{2}{*}{ PARAMETER } & \multirow[b]{2}{*}{ RESULT } & \multirow{2}{*}{$\begin{array}{l}\text { REPORTING } \\
\text { LIMTT }\end{array}$} & \multicolumn{4}{|c|}{$\begin{array}{c}\text { TCLP/STLC } \\
\text { EXTRACTION }\end{array}$} & \\
\hline & & & D. F. & METHOD & DATE & DIGESTED & ANALYZED \\
\hline Mercury & ND & $0.002 \mathrm{mg} / \mathrm{L}$ & 10 & EPA 7470A & $6 / 4 / 01$ & $6 / 5 / 01$ & $6 / 5 / 01$ \\
\hline
\end{tabular}

D.F. - Dilution Factor

ND - Not Detected

This report shall not be reproduced except in full, without the written approval of the laboratory. 
NEL LABORATORIES

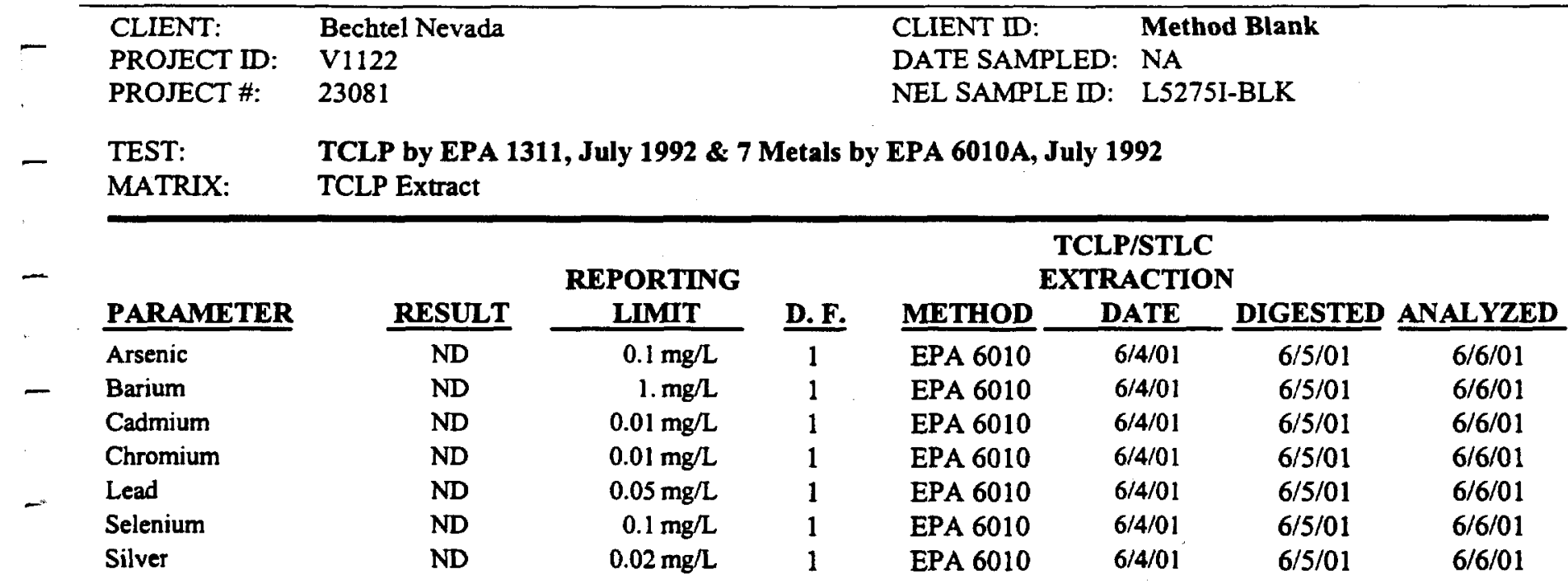

D.F. - Dilution Factor

ND - Not Detected

- This report shall not be reproduced except in full, without the written approval of the laboratory. 


\section{NEL LABORATORIES}

$\begin{array}{ll}\text { CLIENT: } & \text { Bechtel Nevada } \\ \text { PROJECT ID: } & \text { V1122 } \\ \text { PROJECT \#: } & 23081 \\ \text { TEST: } & \text { PCB's (Polychlorinated Biphenyls) by EPA 8082, Dec. } 1996 \\ \text { MATRIX: } & \text { Solid }\end{array}$

\begin{tabular}{|c|c|c|c|c|c|c|c|}
\hline PARAMETER & NEL Sample ID & $\frac{\text { Spike }}{\text { Amount }}$ & $\begin{array}{l}\text { Spike } \\
\underline{\text { Result }}\end{array}$ & $\frac{\text { Percent }}{\text { Recovery }}$ & & $\frac{\text { Acceptable }}{\underline{\text { Range }}}$ & $\underline{\mathbf{R P D}}$ \\
\hline Aroclor-1016 & $\overline{010530 P C B S-L C S}$ & 333 & 262 & 79 & & $63-127$ & \\
\hline Aroclor-1016 & 010530PCBS-LCSD & 333 & 258 & 77 & & $63-127$ & 1.5 \\
\hline Aroclor-1016 & L0105277-01-MS & 333 & 0 & 0 & Л & $55-142$ & \\
\hline Aroclor-1016 & L0105277-01-MSD & 333 & 0 & 0 & $\mathrm{Jl}$ & $55-142$ & 0 . \\
\hline Aroclor -1260 & 010530PCBS-LCS & 333 & 274 & 82 & & $57-138$ & \\
\hline Aroclor -1260 & 010530PCBS-LCSD & 333 & 244 & 73 & & $57-138$ & 11.6 \\
\hline Aroclor -1260 & L0105277-01-MS & 333 & 340 & 102 & & $48-129$ & \\
\hline Aroclor -1260 & L0105277-01-MSD & 333 & 337 & 101 & & $48-129$ & 0.9 \\
\hline
\end{tabular}


NEL LABORATORIES

CLIENT: Bechtel Nevada

PROJECT ID: V1122

PROJECT \#: 23081

TEST: $\quad$ Total Extractable Petroleum Hydrocarbons Fuel Finger Print by EPA Method 8015M, July 1992 MATRIX: Solid

- PARAMETER

Diesel Range (C12-C22)

Diesel Range (C12-C22)

- Diesel Range (C12-C22)

Diesel Range (C12-C22)

Total

Total

Total

- Total

\section{NEL Sample ID}

010530TPHS-FP-LCS

010530TPHS-FP-LCSD

L0105247-02-MS

L0105247-02-MSD

010530TPHS-FP-LCS

010530TPHS-FP-LCSD

L0105247-02-MS

L0105247-02-MSD

\begin{tabular}{|c|c|c|c|c|}
\hline$\frac{\text { Spike }}{\text { Amount }}$ & $\begin{array}{l}\text { Spike } \\
\text { Result }\end{array}$ & $\frac{\text { Percent }}{\text { Recovery }}$ & $\frac{\text { Acceptable }}{\text { Range }}$ & RPD \\
\hline 166.7 & 128 & 77 & $53-91$ & \\
\hline 166.7 & 133 & 80 & $53-91$ & 3.8 \\
\hline 166.7 & 135 & 81 & $34-114$ & \\
\hline 166.7 & 142 & 85 & $34-114$ & 5.1 \\
\hline 166.7 & 128 & 77 & $53-91$ & \\
\hline 166.7 & 133 & 80 & $53-91$ & 3.8 \\
\hline 166.7 & 135 & 81 & $34-114$ & \\
\hline 166.7 & 142 & 85 & $34-114$ & 5.1 \\
\hline
\end{tabular}

ND - Not Detected

This report shall not be reproduced except in full, without the written approval of the laboratory. 
NEL LABORATORIES

CLIENT: Bechtel Nevada

PROJECT ID: V1122

PROJECT \#: 23081

TEST: Inorganic Non-Metals

MATRIX: Solid

PARAMETER

7.00 Buffer
NEL Sample ID

010601PH2-LCS
Spike

Amount

7
Spike

Result

6.98
Percent

Recovery

100
Acceptable

Range

$99-101$ 
NEL LABORATORIES

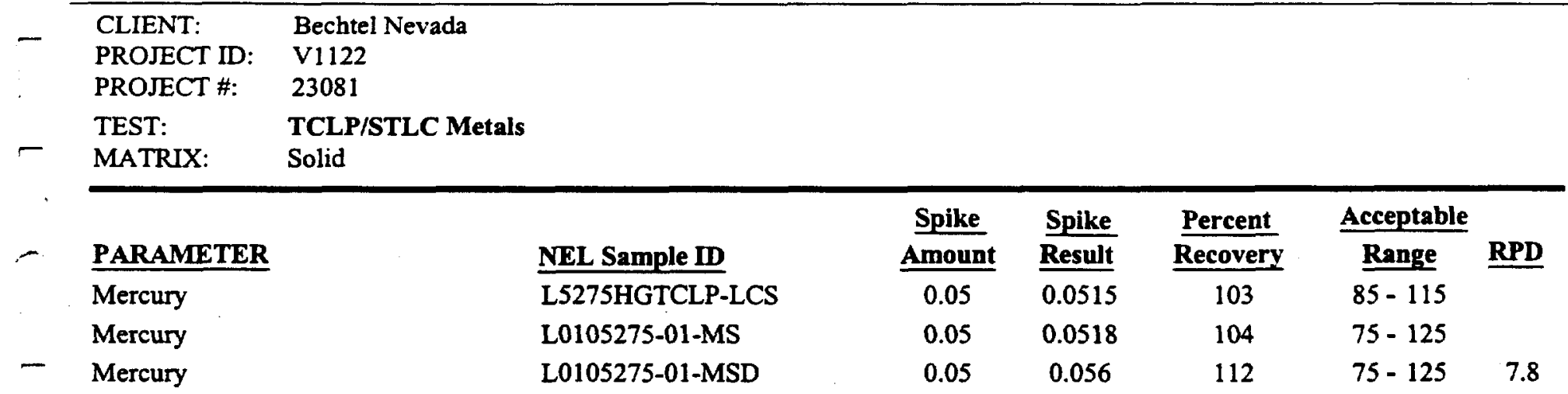




$\begin{array}{ll}\text { CLIENT: } & \text { Bechtel Nevada } \\ \text { PROJECT ID: } & \text { V1122 } \\ \text { PROJECT \#: } & 23081 \\ \text { TEST: } & \text { TCLP/STLC Metals } \\ \text { MATRIX: } & \text { Solid }\end{array}$

\begin{tabular}{|c|c|c|c|c|c|c|}
\hline PARAMETER & NEL Sample ID & $\frac{\text { Spike }}{\text { Amount }}$ & $\frac{\text { Spike }}{\text { Result }}$ & $\frac{\text { Percent }}{\text { Recovery }}$ & $\frac{\text { Acceptable }}{\text { Range }}$ & $\underline{\mathbf{R P D}}$ \\
\hline Arsenic & L5275I-LCS & 0.5 & 0.529 & 106 & $85-115$ & \\
\hline Arsenic & L0105275-01-MS & 0.5 & 0.553 & 111 & $75-125$ & \\
\hline Arsenic & L0105275-01-MSD & 0.5 & 0.547 & 109 & $75-125$ & 1.1 \\
\hline Silver & L5275I-LCS & 0.5 & 0.434 & 87 & $85-115$ & \\
\hline Silver & L0105275-01-MS & 0.5 & 0.45 & 90 & $75-125$ & \\
\hline Silver & L0105275-01-MSD & 0.5 & 0.455 & 91 & $75-125$ & 1.1 \\
\hline Barium & L5275I-LCS & 1 & 0.99 & 99 & $85-115$ & \\
\hline Barium & L0105275-01-MS & 1 & 1.2 & 120 & $75-125$ & \\
\hline Barium & L0105275-01-MSD & 1 & 1.19 & 119 & $75-125$ & 0.8 \\
\hline Cadmium & L5275I-LCS & 0.5 & 0.472 & 94 & $85-115$ & \\
\hline Cadmium & L0105275-01-MS & 0.5 & 0.484 & 97 & $75-125$ & \\
\hline Cadmium & L0105275-01-MSD & 0.5 & 0.505 & 101 & $75-125$ & 4.2 \\
\hline Chromium & L5275I-LCS & 0.5 & 0.478 & 96 & $85-115$ & \\
\hline Chromium & L0105275-01-MS & 0.5 & 0.504 & 101 & $75-125$ & \\
\hline Chromium & L0105275-01-MSD & 0.5 & 0.513 & 103 & $75-125$ & 1.8 \\
\hline Lead & L5275I-LCS & 1 & 0.928 & 93 & $85-115$ & \\
\hline Lead & L0105275-01-MS & 1 & 1.11 & 93 & $75-125$ & \\
\hline Lead & L0105275-01-MSD & 1 & 1.17 & 99 & $75-125$ & 6.2 \\
\hline Selenium & L5275I-LCS & 0.5 & 0.507 & 101 & $85-115$ & \\
\hline Selenium & L0105275-01-MS & 0.5 & 0.48 & 96 & $75-125$ & \\
\hline Selenium & L0105275-01-MSD & 0.5 & 0.485 & 97 & $75-125$ & 1. \\
\hline
\end{tabular}




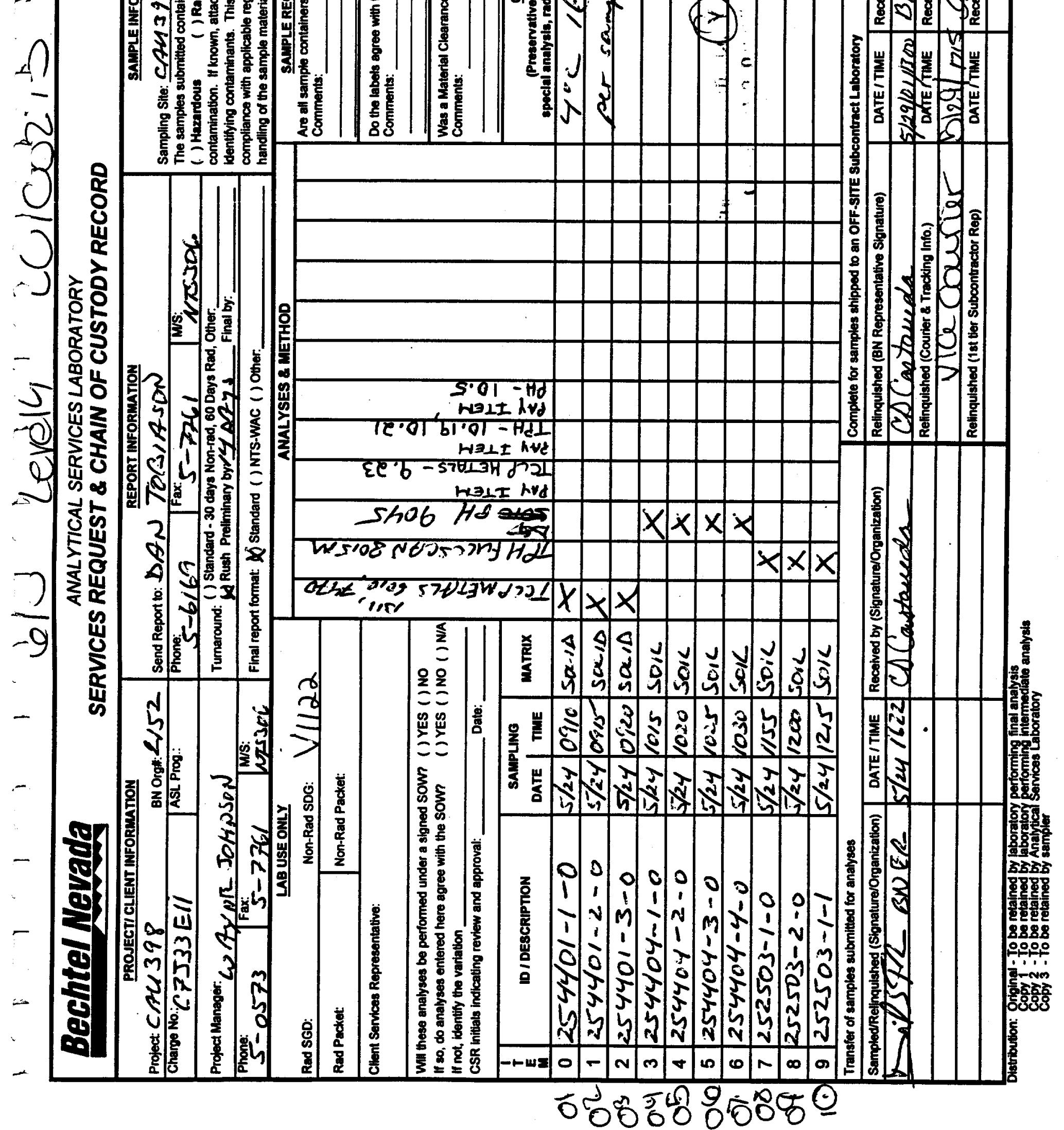




\section{5 cerel4 colos 275}

Bechtel Nevada

ANALYTICAL SERVICES LABORATORY

SERVICES REQUEST \& CHAIN OF CUSTODY RECORD

Page 2 of 2

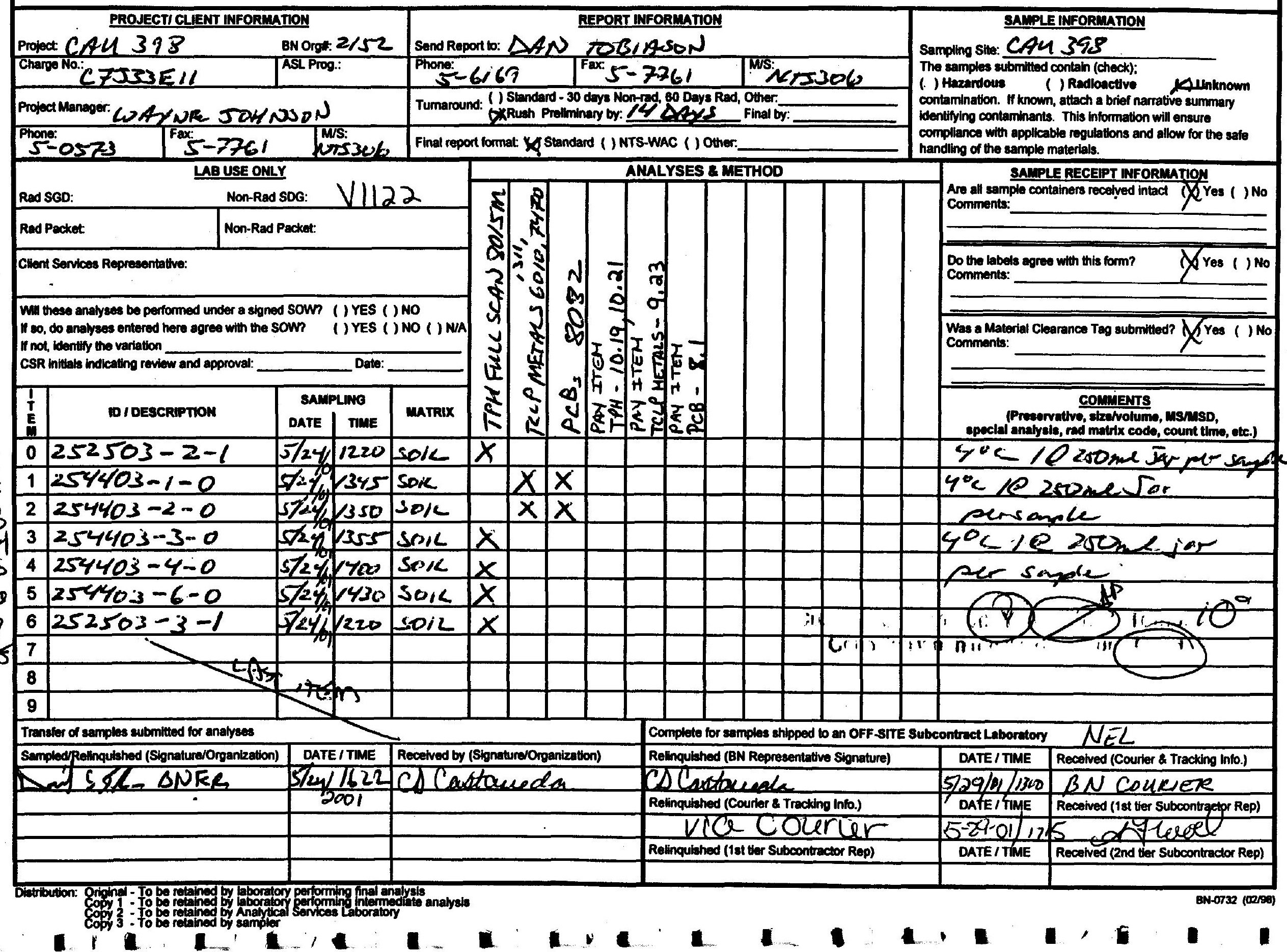




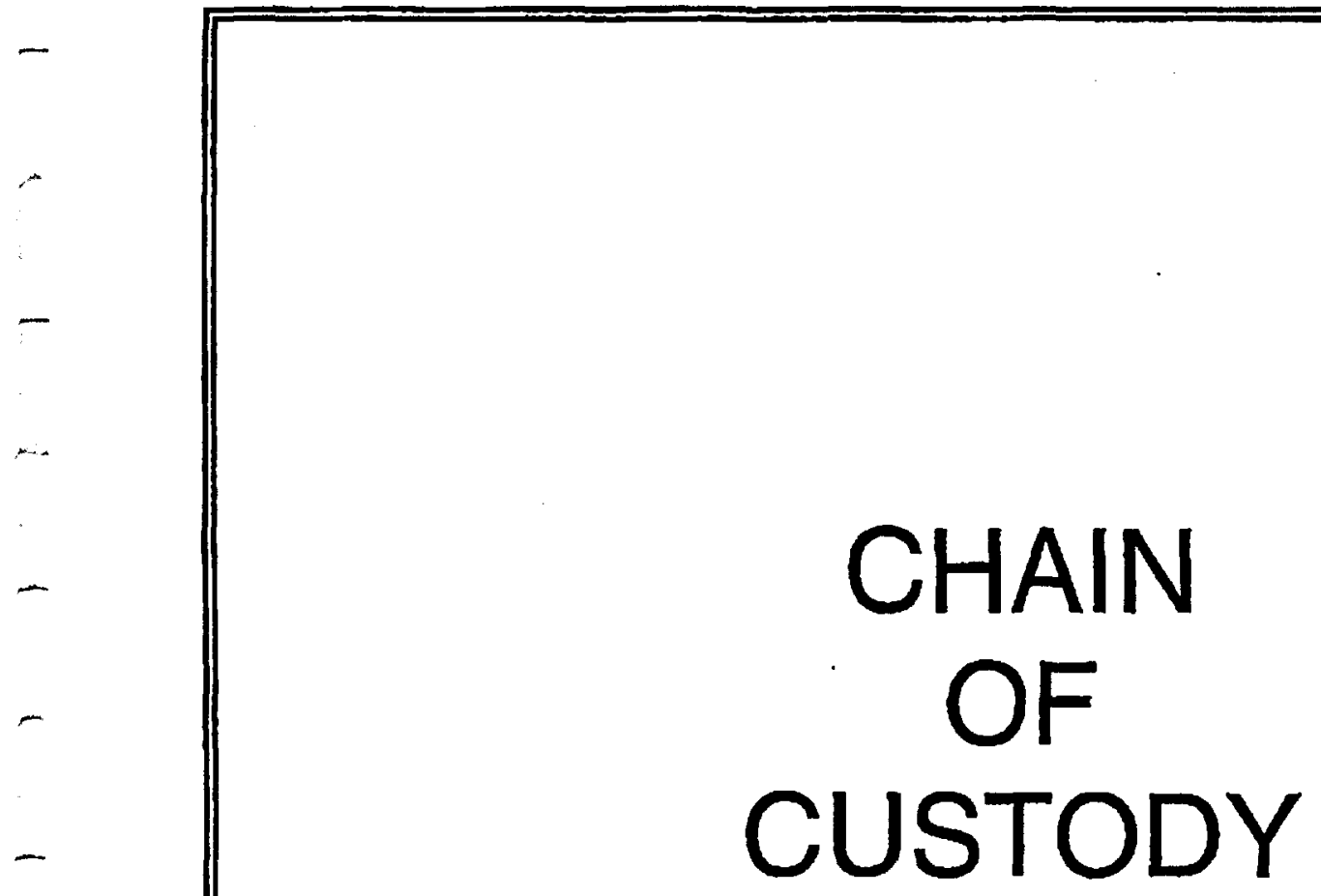

$-$

$\leftrightarrow$

$-$ 


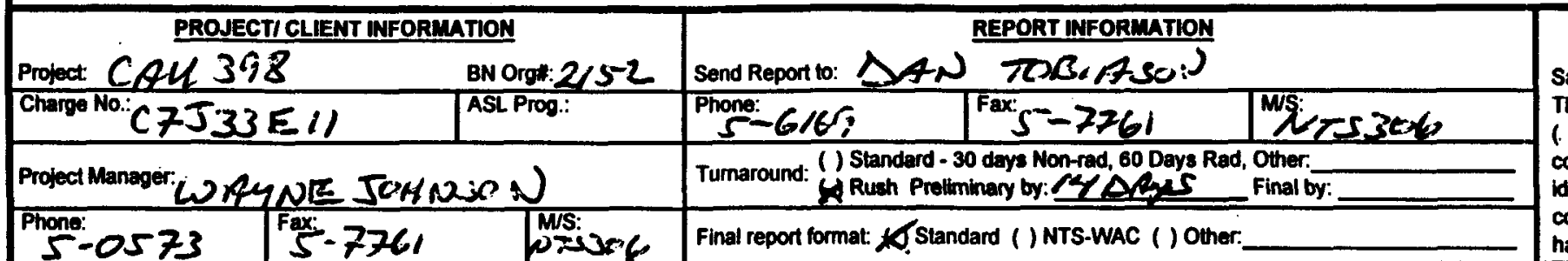

SAMPLE INFORMATION
Sampling Site:
The samples submitted contain (check);
(.) Harandous ( ) Radlosctive
contamination. If known. attach a brief narrative summary
identifying contaminants. This information will ensure
complianco with applicable regulations and allow for the safo
handling of the sample materials.

\begin{tabular}{|c|c|}
\hline \multicolumn{2}{|c|}{ LAB USE ONLY } \\
\hline Rad SGD: & Non-Rad SDG: \\
\hline Rad Packet: & Non-Rad Packet: \\
\hline
\end{tabular}

Client Services Representative:

Will these analyses be performed under a signed SOW? () YES () NO

If so, do analyses entered here agree with the SOW? () YES () NO () N/A

If not, identify the variation

CSR initials indicating review and approval:__ Date:

\begin{tabular}{|l|l}
\hline $\mathbf{I}$ & ID I DESCRIPTION \\
E &
\end{tabular}

$0254401-3-0$

. $1254404-2-0$

$2252503-2-0$
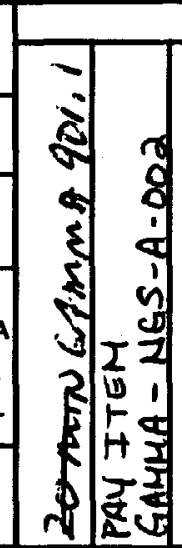

ANALYSES \& METHOD

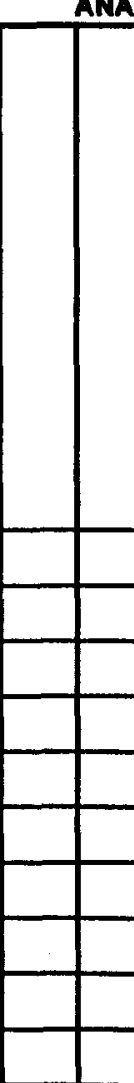

9

Transfer of samples submitted for analyses

\begin{tabular}{|l|l|l|l|l}
\hline Sampled/Relinquished (Signature/Organization) & DATE / TIME & Recelved by (Signature/Organization)
\end{tabular}

\begin{tabular}{|c|c|c|}
\hline \multicolumn{2}{|c|}{ SAMPLING } & MatRIX \\
DATE & TMME & MAT \\
\hline
\end{tabular}

$5 / 24$ 0\%20 $5 / 24$ 1020 50,2 $\sqrt{24}(20 x)$ sare

$3254403-1-0$

4

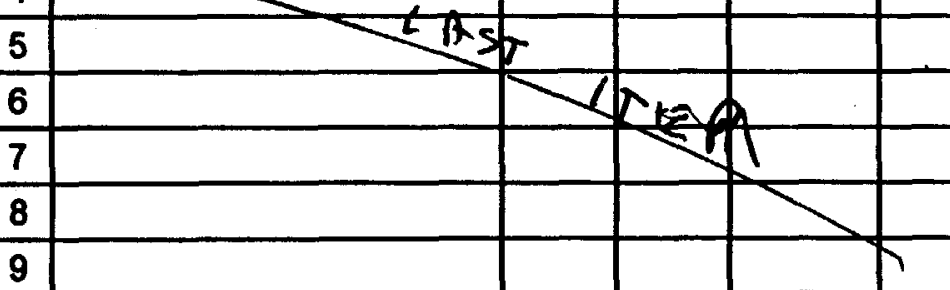
$5 / 2 y+345$ jork $\triangle A 7 S$ PQ BNEK Complete for samples shipped to an OFF-sITE Subcontract Laboratory

GEL

\begin{tabular}{|l|l|l}
\hline Relinquished (BN Representative Signature) & DATE / TIME & Received (Courier \& Tracking Info.)
\end{tabular} (1) Casthereh

Relinquished (Courier \& Tracking Info.)

Relinquished (1st ther Subcontractor Rep)
SAMPLE RECEIPT INFORMATION' Are all sample containers received intact (XYYes ( ) No Comments:

Do the labels agree with this form? (XYes ( ) No Comments:

Was a Material Clearance Tag submitted? Wuyes ( I No Comments:

COMMENTS

Procervatho slzetrolume nsmsp speclal analysis, rad matrix code, count time, otc.)

12500 w Nalsen in

per bample eres 


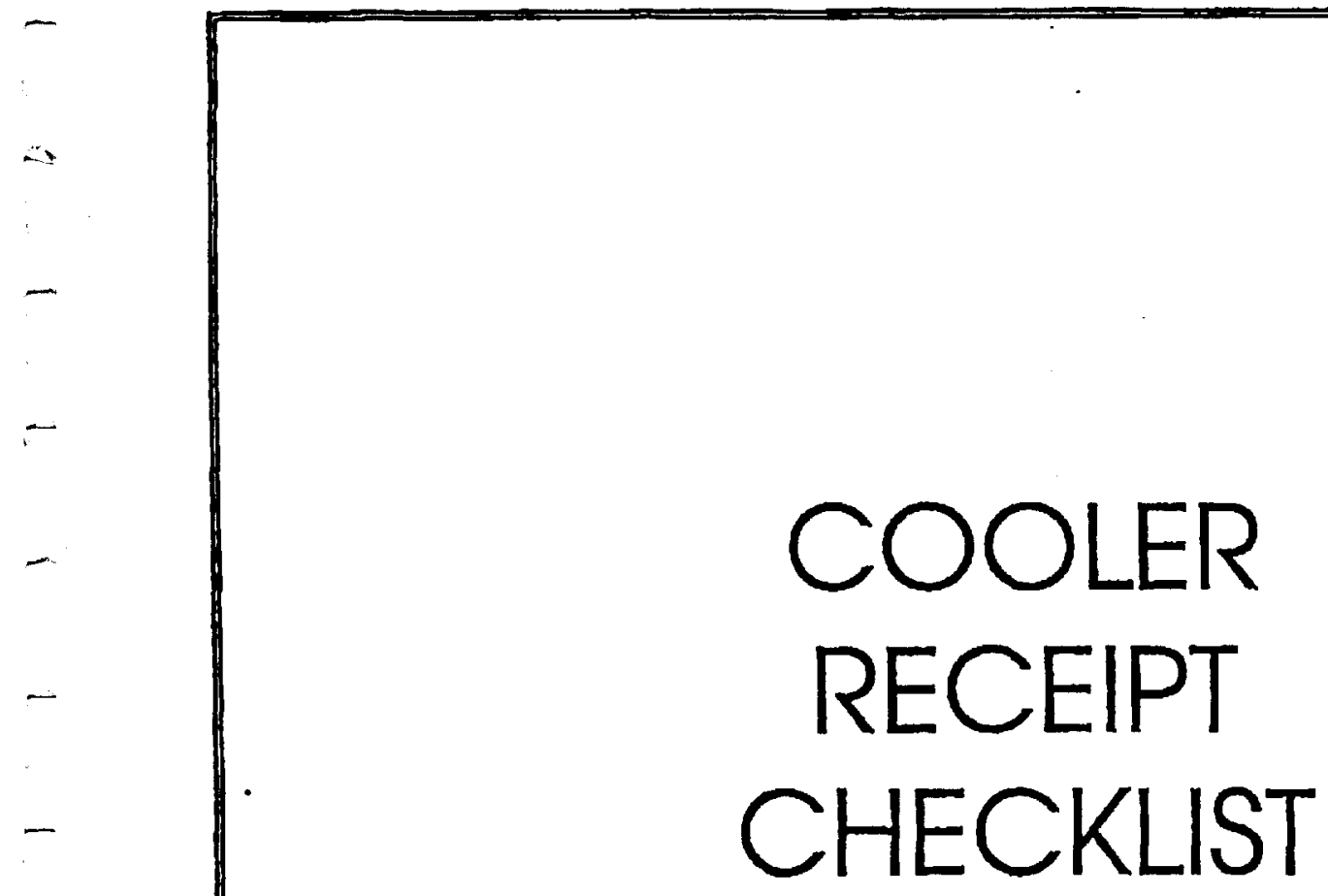

$-$

in

-

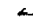

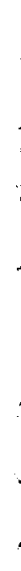

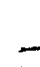

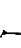




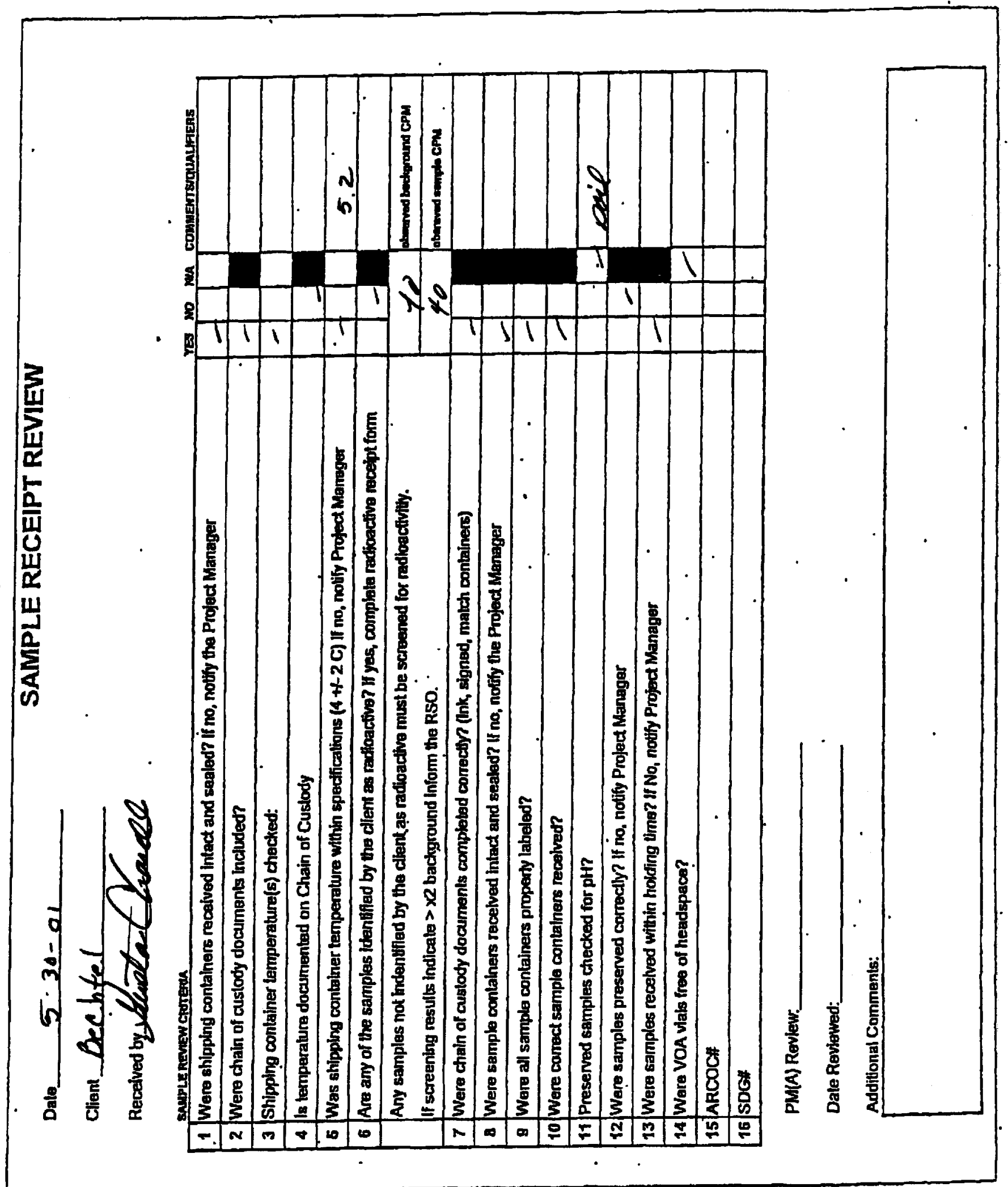


-

RADIOLOGICAL ANALYSIS 


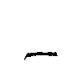

NARRATIVE :

$\ldots$

CASE

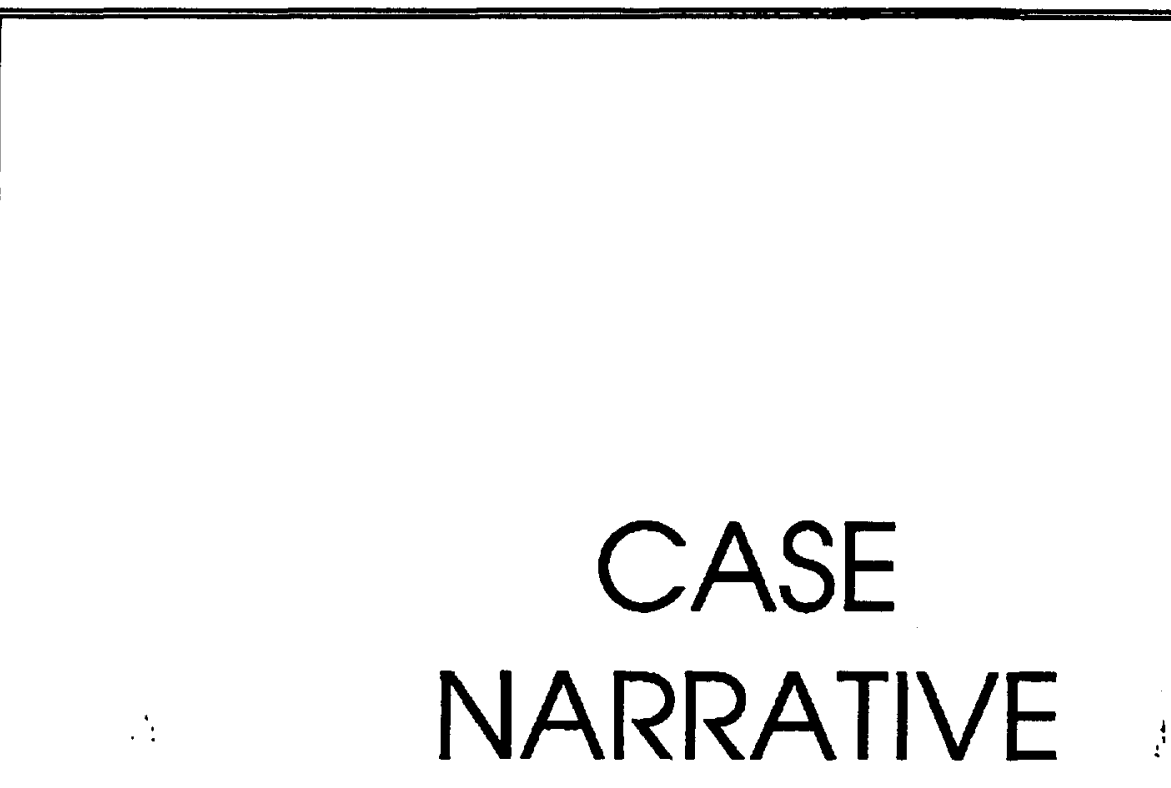




\section{Radiochemistry Case Narrative \\ Bechtel Nevada Corp. (NEVA) \\ SDG V1123}

Method/Analysis Information

Batch Number:

Procedure:

Analytical Method:

Sample ID
43010001
43010002
43010003
43010004
1200013917
1200013918
1200013919

80749

Determination of Gamma Isotopes in Water and Soil

DOE EML HASL 300

\author{
Client ID \\ 254401-3-0 \\ 254404-2-0 \\ 252503-2-0 \\ 254403-1-0 \\ MB for batch 80749 \\ 254402-1-0(42918001DUP) \\ LCS for batch 80749
}

SOP Reference

Procedures for preparation, analysis and reporting of analytical data are controlled by General Engineering Laboratories, Inc. as Standard Operating Procedures (SOP). The data discussed in this narrative has been prepared and analyzed in accordance with GL-RAD-A-013.

\section{Calibration Information:}

Calibration Information

All initial and continuing calibration requirements have been met.

\section{Standards Information}

Standard solution(s) for these analyses are NIST traceable and used before the expiration date(s).

\section{Sample Geometry}

All counting sources were prepared in the same geometry as the calibration standards.

\section{Quality Control (OC) Information:}

Blank Information

The blank volume is representative of the sample volume(s) in this batch.

Designated QC

The following sample(s) was used for QC: 42918001.

QC Information

All of the QC samples met the required acceptance limits.

\section{Technical Information:}

\section{Holding Time}

All sample procedures for this sample set were performed within the required holding time.

\section{Preparation Information}

All preparation criteria have been met for these analyses.

Sample Re-prep/Re-analysis

None of the samples in this sample set required reprep or reanalysis. 


\section{Miscellaneous Information:}

NCR Documentation

No NCR were generated for the preparation or analysis of this sample set.

Manual Integration

No manual integrations were performed on data in this batch.

\section{Additional Comments}

$\mathbf{K}-40$ is being reported in all samples in this batch.

Reviev. Valldation:

GEL requires all analytical data to be verified by a qualified data validator. In addition, all data designated for CLP or CLP-like packaging will receive a third level validation upon completion of the data package.

The following data validator vertifed the information presented in this case narrative:

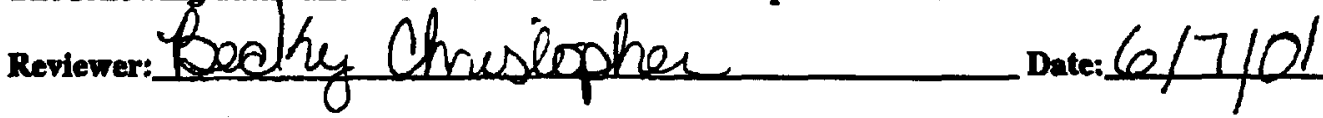




\section{GENERAL ENGINEERING LABORATORIES}

Meeting loday's needs with a vision for tomorrow.

\section{Certificate of Analysis}

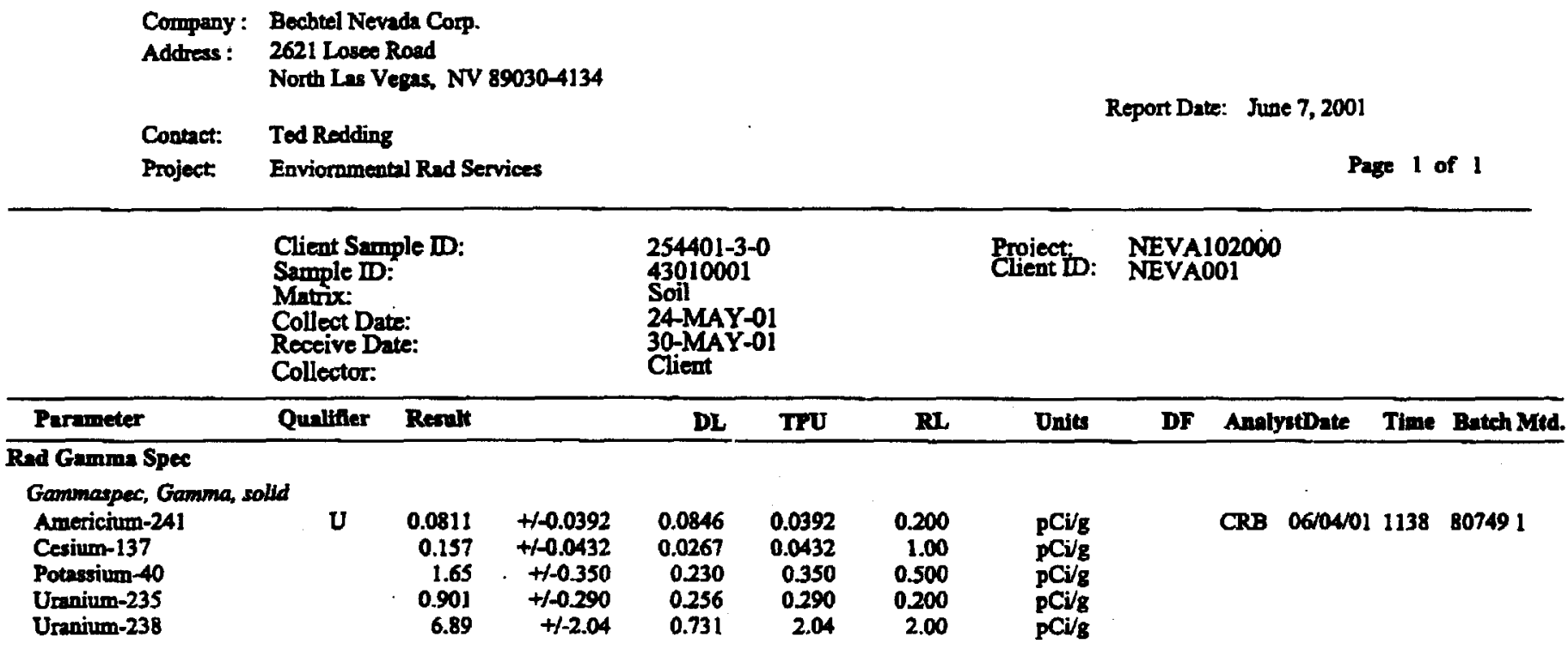

The following Prep Methods were performed

\begin{tabular}{llllll}
\hline Method & Description & Analyst & Date & Thme & Prep Bateh \\
\hline Dry Soil Prep & Dry Soil Prep RAD A-021,A-021B,A-026 & CCM1 & 05/31/01 & 1534 & 80663
\end{tabular}

The following Ansiytical Methods were performed

$\frac{\text { Method }}{1}$ Description

Notes:

The Qualifiers in this report are defined as follows :

** Indicates the analyte is a surrogate compound.

$<$ Actual result is less than amount reported

$>$ Actual result is greater than amount reported

- J Indicates an estimated vahue. The result was greater than the detection'limit, but less than the reporting limit

U Indicates the compound was annlyzed for but not detected above the detection linit

The above sample is reported on an "as received" basis.

This data report has been prepared and reviewed in accordance with General Engineering Laboratories, Inc.

standard operating procedures. Please direct any questions to your Project Manager, Stacy L. Griffin at 843-556-8171 Ext. 4264.

Reviewed by

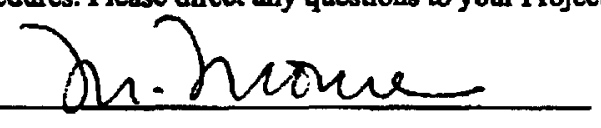




\section{GENERAL ENGINEERING LABORATORIES}

Meeting today's needs with a vision for tomorrow:

\section{Certificate of Analysis}

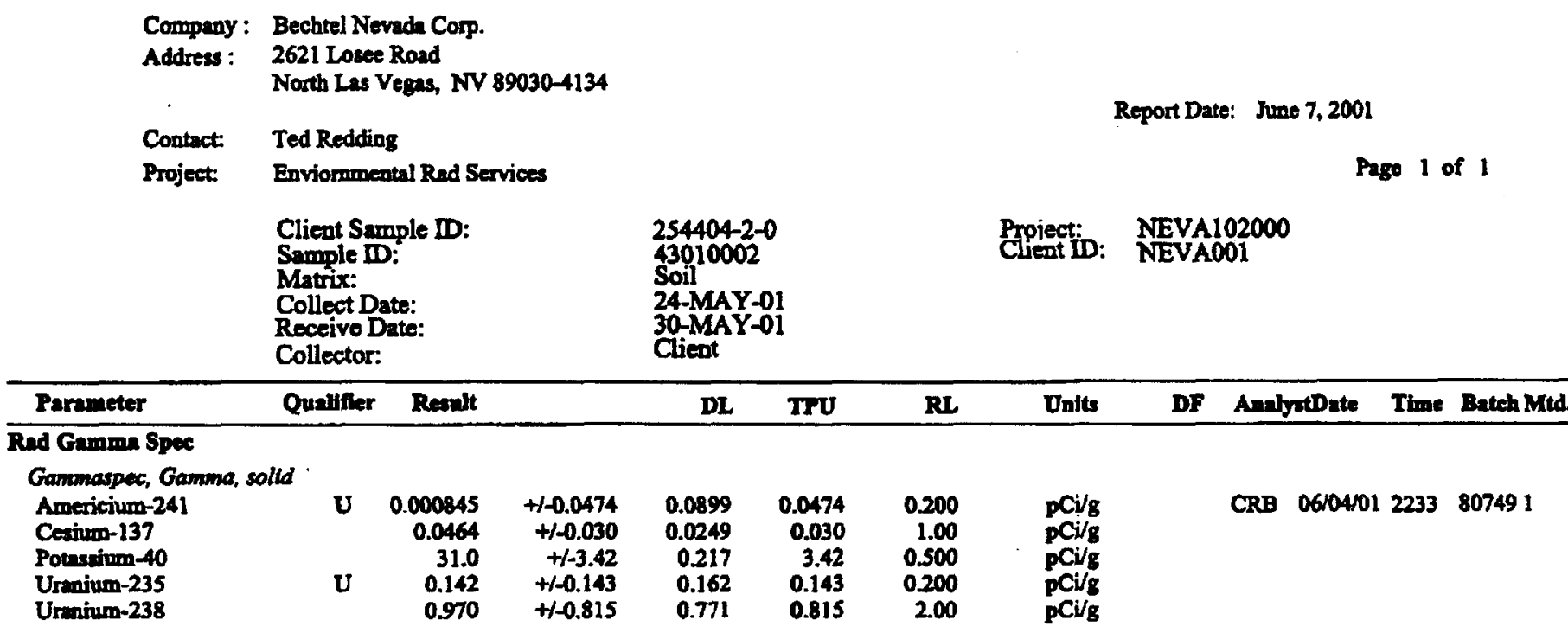

The followin Prep Methods rere performed

\begin{tabular}{llllll}
\hline Method & Dereription & Analyit & Date & Time & Prep Batch \\
\hline Dry Soil Prep & Dry Soil Prep RAD A-021,A-021B,A-026 & CCM1 & 05/31/01 & 1534 & 80663 \\
The followiag Ainalytical Methods were performed & & & & \\
\hline Method & Deacription & & & \\
\hline 1 & DOE EMI HASL 300
\end{tabular}

Notes:

The Qualifiers in this report are defined as follows :

** Indicates the analyte is a surrogato compound.

$<$ Actual result is less than amount reported

$>$ Actual result is greater than amount reported

I Indicates an estimated value. The result was greater than the detection limit, but less than the reporting limit

U Indicates the compound was analyzed for but not detected above the detection limit

The above sample is reported on an "as received" basis.

This data report has been prepared and reviewed in accordance with General Engineering Laboratories, Inc.

standard operating procedures. Please direct any questions to your Project Manager, Stacy L. Griffin at 843-556-8171 Ext. 4264.

Reviewed by

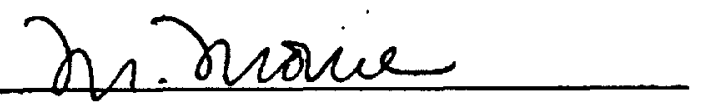




\section{GENERAL ENGINEERING LABORATORIES}

Meeting today's needs with a vision-for tomorrow.

\section{Certificate of Analysis}

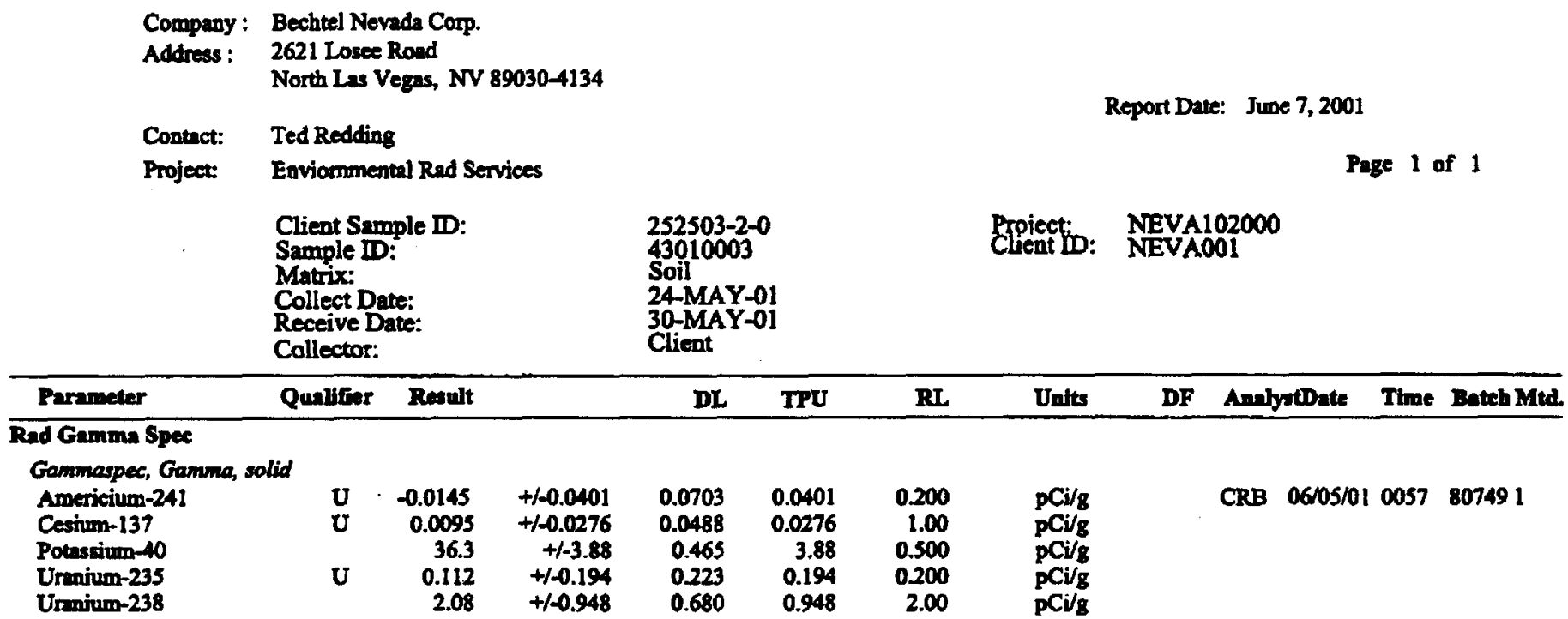

The followine Prep Methods were perforned

\begin{tabular}{|c|c|c|c|c|c|}
\hline Method & Deseription & Analyat & Date & Time & Prep Batch \\
\hline$\overline{\text { Dry Soil Prep }}$ & Dry Soil Pxep RAD A-021,A-021B,A-026 & $\mathrm{CCMI}$ & $05 / 31 / 01$ & 1534 & 80663 \\
\hline \multicolumn{6}{|c|}{ The followis Annlytical Methods were performed } \\
\hline Method & Deserlption & & & & \\
\hline
\end{tabular}

Notes:

The Qualifiers in this report are defined as follows :

* Indicates the analyte is a surrogate compound.

$<$ Actual result is less than amount reported

$>$ Actual result is greater than amount reported

J Indicates an estimated value. The rosult was greater than the detection limit, but less than the reporting limit.

$\mathrm{U}$ Indicates the compound was analyzed for but not detected above the detection limit

The above sample is reported on an "as received" basis.

This data report has been prepared and reviewed in accordance with General Engineering Laboratories, Inc. standard operating procedures. Please direct any questions to your Project Manager, Stacy L. Griffin at 843-556-8171 Ext. 4264.

Reviewed by

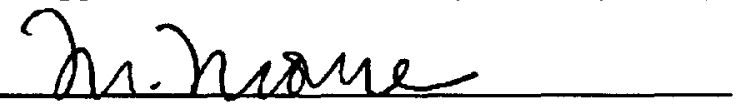




\section{GENERAL ENGINEERING LABORATORIES}

Meeting today's needs with a vision for tomorrow.

\section{Certificate of Analysis}

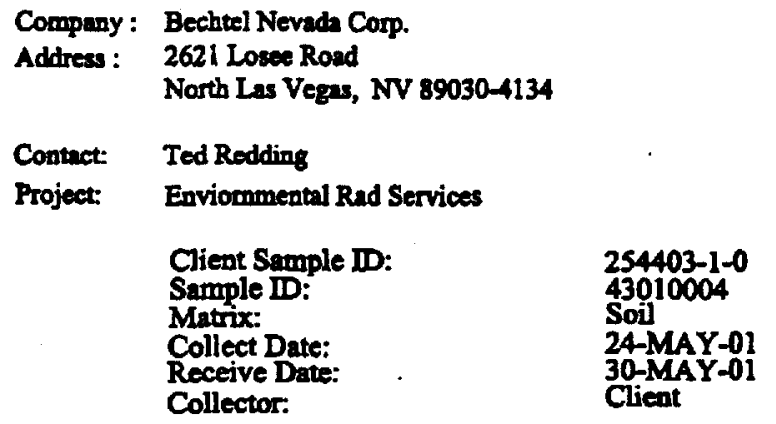

\begin{tabular}{|c|c|c|c|c|c|c|c|c|c|c|c|}
\hline Parameter & Quallifer & Reauk & & $\mathbf{D L}$ & TPU & $\mathbf{R L}$ & Units & DF & AnslyatDate & Thine & Batch Mtd. \\
\hline \multicolumn{12}{|l|}{ Rad Ganma Spec } \\
\hline $\begin{array}{l}\text { Gammaspec, Gam } \\
\text { Americium-241 } \\
\text { Cesium-137 } \\
\text { Pocassium-40 } \\
\text { Uranium-235 } \\
\text { Uravium-238 }\end{array}$ & $\begin{array}{l}\mathbf{U} \\
\mathbf{U}\end{array}$ & $\begin{array}{r}0.0796 \\
0.188 \\
30.6 \\
0.000765 \\
0.772\end{array}$ & $\begin{array}{r}+-0.121 \\
+/-0.0538 \\
+/-3.68 \\
+-0.144 \\
+/-1.50\end{array}$ & $\begin{array}{r}0.161 \\
0.0318 \\
0.308 \\
0.214 \\
1.42\end{array}$ & $\begin{array}{r}0.121 \\
0.0538 \\
3.68 \\
0.144 \\
1.50\end{array}$ & $\begin{array}{r}0.200 \\
1.00 \\
0.500 \\
0.200 \\
2.00\end{array}$ & $\begin{array}{l}\mathrm{pCi} / \mathrm{g} \\
\mathrm{pCi} / \mathrm{g} \\
\mathrm{pCi} / \mathrm{g} \\
\mathrm{pCi} / \mathrm{g} \\
\mathrm{pCi} / \mathrm{g}\end{array}$ & & CRB 06104/01 & 1144 & 807491 \\
\hline
\end{tabular}

The following Prep Methods were performed

\begin{tabular}{llllll}
\hline Method & Description & Analyat & Date & Time & Prep Bateh \\
\hline Dry Soil Prep $\quad$ Dry Soil Prep RAD A-021,A-021B,A-026 & CCM1 & 05/31/01 & 1534 & 80663 \\
The following Analytical Methods were performed & & & & \\
\hline Method $\quad$ Deceription & & & & \\
\hline 1 & DOE EML HASL 300
\end{tabular}

Notes:

The Qualifiers in this report are defined as follows :

* Indicates the analyte is a surrogate compound.

$<$ Actual result is less than amount reported

$>$ Actual result is greater than amount reported

J Indicates an estimated value. The result was greater than the detection limit, but less than the reporting limit.

U Indicates the compound was analyzed for but not detected above the detection limit

The above sample is reported on an "as received" basis.

This data report bas been prepared and reviewed in accordunce with General Engineering Laboratories, Inc.

standard operatiog procedures. Please direct any questions to your Project Manager, Stacy L. Griffin at 843-556-8171 Ext. 4264.

Reviewed by

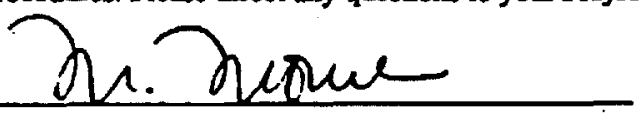




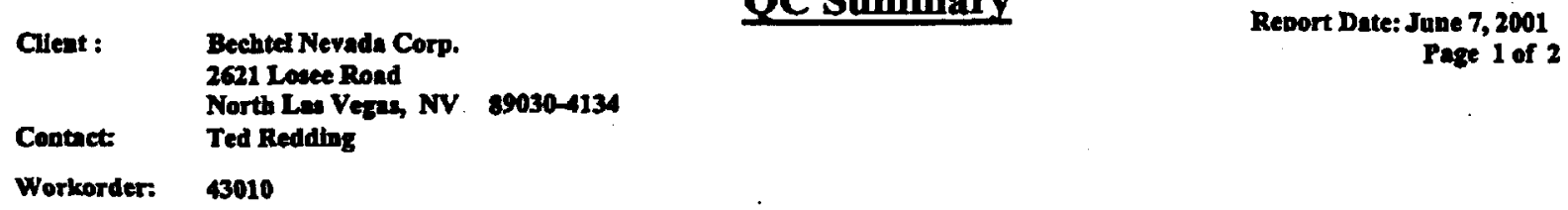

Page 1 of 2

QC1200013917 MB

Uraniom-235

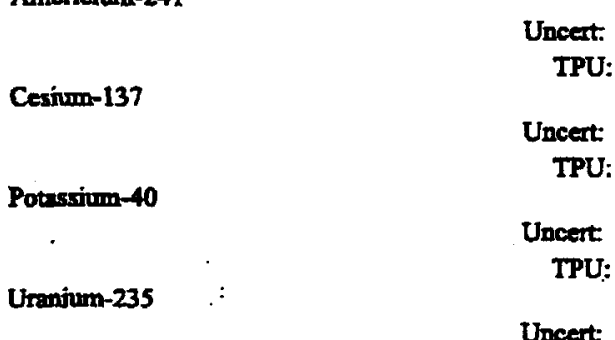$$
0.030
$$$$
+1-0.0852
$$$$
0.0852
$$

U 0.0211 $+-0.0117$ 0.0117

U $\quad 0.253$ $+-0.181$

0.181

U $\quad 0.0501$ Defts 


\section{GENERAL ENGINEERING LABORATORIES}

Meeting today's needs with a vision for tomorrow.

\section{QC Summary}

Workorder:

43010

Parmane

NOM Sample Qual

Page 2 of 2

Rad Gamma Spee

Batch

80749

Uramium-238

TPU:

0.102
0.919
++0.650 $\quad$ PCi/g

Uncert:

0.650

TPU:

OC Unit RPD\% REC\%

Range Anlot

Dace Time

U:

0.650

Notes:

The Qualifiers in this report are defined as follows:

* Indicates the analyte is a surrogate compound.

< Actud reoult is less thin moum reported

$>$ Actud result is greater then emount reported

J Indicates an eatimated value. The result was greater then the detection limit, but less then the reporting limit

U Indicates the compound was antyed for but not detected above the detection limit

N/A indicates the spile recovery limits do not epply when semple cencentention exceeds ppike cone. by a factor of 4 or more.

A The Relative Pexcent Difference (RPD) obtained from the somple tuplieate (DUP) is evilunted agrinst the acceptence criteria when the sample is greater then five times (SX) the contruct required detestion limit (RL). In osses where either the snmple or duplicate value is less then $5 X$ the RL, a control limit of $+f$ - the $R L$ is used to evaluate the DUP realt.

For PS, PSD, and SDILT results, the values listed are the measured anounts, not final concentrations. 
CLIENT: $\quad$ Bechtel Nevada

P.O. Box 98521 , M/S NTS273

Las Vegas, NV 89193-8521

ATTN:

Ted Redding

PROJECT NAME: V1124

NEL ORDER ID: L0105291

PROJECT NUMBER: 23081

Attached are the analytical results for samples in support of the above referenced project.

Samples submitted for this project were not sampled by NEL Laboratories. Samples were received by NEL in good condition, under chain of custody on 5/30/01.

Should you have any questions or comments, please feel free to contact our Client Services department at (702) 657-1010.

Pesticide samples were originally extracter within hold but had failling surrogates. Sample were reextracted out of hold and those results are reported. The LCS suffered spillage during the re-extract.

Some results have been flagged as follows:

$\mathrm{Di}$ - Results reported from analysis at a higher dilution.

ת - The batch MS and/or MSD were outside acceptance limits. The batch LCS was acceptable.

Some QA results have been flagged as follows:

J - This concentration should be considered an estimate due laboratory control sample failure.

J1 - The batch MS and/or MSD were outside acceptance limits. The batch LCS was acceptable.

Some surrogate results have been flagged as follows:

D - Sample required dilution. Sample QC results were diluted outside the calibrated range.

Jc - This concentration may be biased because the continuing calibration verification (CCV) standard did not meet $\mathrm{QC}$ requirements for this analyte. However, overall CCV standard recoveries meet method requirements and analytical results are in control.

Sf - This surrogate was outside acceptance limits.
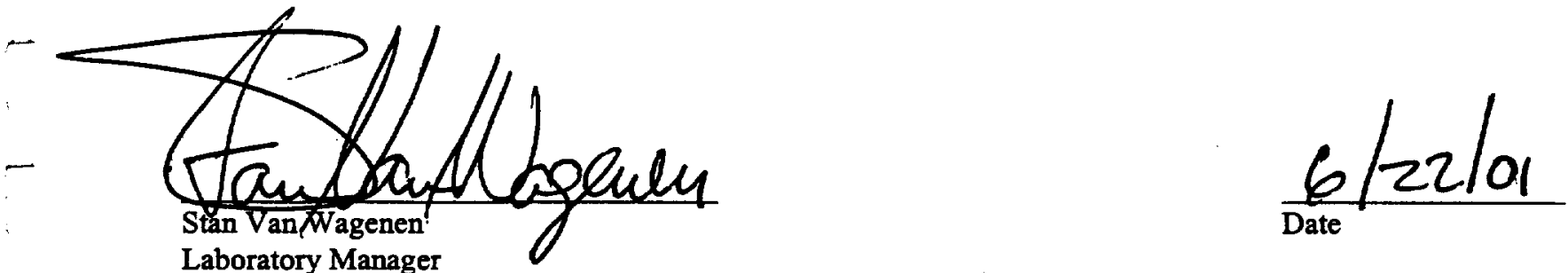

\section{CERTIFICATIONS:}

\begin{tabular}{llll}
\hline & Reno & Las Vegas & S. Califormia \\
\cline { 2 - 4 } Arizona & AZ0520 & AZ0518 & AZ0605 \\
Califomia & 1707 & 2002 & 2264 \\
US Army Corps & Certified & Certified &
\end{tabular}

of Engineers

\begin{tabular}{llll} 
& Reno & Las Vegas & S. California \\
\cline { 2 - 4 } Idaho & Certified & Certified & \\
Montana & Certified & Certified & \\
Nevada & NV033 & NV052 & CA084 \\
L.A.C.S.D. & & & 10228
\end{tabular}




\begin{tabular}{lll}
\hline CLIENT: & Bechtel Nevada & CLIENT ID: 2 252505-1-0 \\
PROJECT ID: & V1124 & DATE SAMPLED: 5/25/01 \\
PROJECT \#: & 23081 & NEL SAMPLE ID: L0105291-10
\end{tabular}

TEST: Organochlorine Pesticides by EPA 8081A, Dec. 1996

METHOD: $\quad$ EPA 8081

MATRIX: Solid

DILUTION: 1

ANALYST: JRW - Las Vegas Division

EXTRACTED: $\quad 6 / 8 / 01$

\begin{tabular}{|c|c|c|c|}
\hline PARAMETER & Result & & $\begin{array}{l}\text { Reporting } \\
\text { Limit }\end{array}$ \\
\hline Aldrin & ND & & 5. $\mu \mathrm{g} / \mathrm{kg}$ \\
\hline alpha-BHC & 14 & $\mu \mathrm{g} / \mathrm{kg}$ & 5. $\mu \mathrm{g} / \mathrm{kg}$ \\
\hline beta-BHC & 52 & $\mu \mathrm{g} / \mathrm{kg}$ & 5. $\mu \mathrm{g} / \mathrm{kg}$ \\
\hline delta-BHC & 11 & $\mu g / \mathrm{kg}$ & 5. $\mu \mathrm{g} / \mathrm{kg}$ \\
\hline gamma-BHC (Lindane) & 21 & $\mu \mathrm{g} / \mathrm{kg}$ & 5. $\mu g / \mathrm{kg}$ \\
\hline Alpha-chlordane & ND & & 5. $\mu g / \mathrm{kg}$ \\
\hline Gamma-chlordane & ND & & 5. $\mu \mathrm{g} / \mathrm{kg}$ \\
\hline Chlordane & ND & & 20. $\mu g / \mathrm{Kg}$ \\
\hline 4,4-DDD & ND & & 5. $\mu \mathrm{g} / \mathrm{kg}$ \\
\hline 4,4-DDE & ND & & 5. $\mu \mathrm{g} / \mathrm{kg}$ \\
\hline 4,4-DDT & $210 \mathrm{JI}$ & $\mu g / \mathrm{kg}$ & 5. $\mu g / \mathbf{k g}$ \\
\hline Dieldrin & $31 \mathrm{~J}$ & $\mu g / \mathrm{kg}$ & 5. $\mu \mathrm{g} / \mathrm{kg}$ \\
\hline Endosulfan I & ND & & 5. $\mu \mathrm{g} / \mathrm{kg}$ \\
\hline Endosulfan II & ND & & 5. $\mu \mathrm{g} / \mathrm{kg}$ \\
\hline Endosulfan sulfate & ND & & 5. $\mu g / \mathrm{kg}$ \\
\hline Endrin & ND & & 5. $\mu \mathrm{g} / \mathrm{kg}$ \\
\hline Endrin aldehyde & ND & & 5. $\mu \mathrm{g} / \mathrm{kg}$ \\
\hline Endrin ketone & ND & & 5. $\mu g / \mathrm{kg}$ \\
\hline Heptachlor & ND & & 5. $\mu \mathrm{g} / \mathrm{kg}$ \\
\hline Heptachlor epoxide & ND & & 5. $\mu g / \mathrm{kg}$ \\
\hline Methoxychlor & ND & & 10. $\mu \mathrm{g} / \mathrm{kg}$ \\
\hline Toxaphene & ND & & $600 \mu \mathrm{g} / \mathrm{kg}$ \\
\hline
\end{tabular}

QUALITY CONTROL DATA:

Surrogate

Decachlorobiphenyl

Tetrachloro-m-xylene

\section{\% Recovery}

42

98
Sf

Acceptable Range

$45-149$

$58-146$

ND - Not Detected

This report shall not be reproduced except in full, without the written approval of the laboratory. 
NEL LABORATORIES

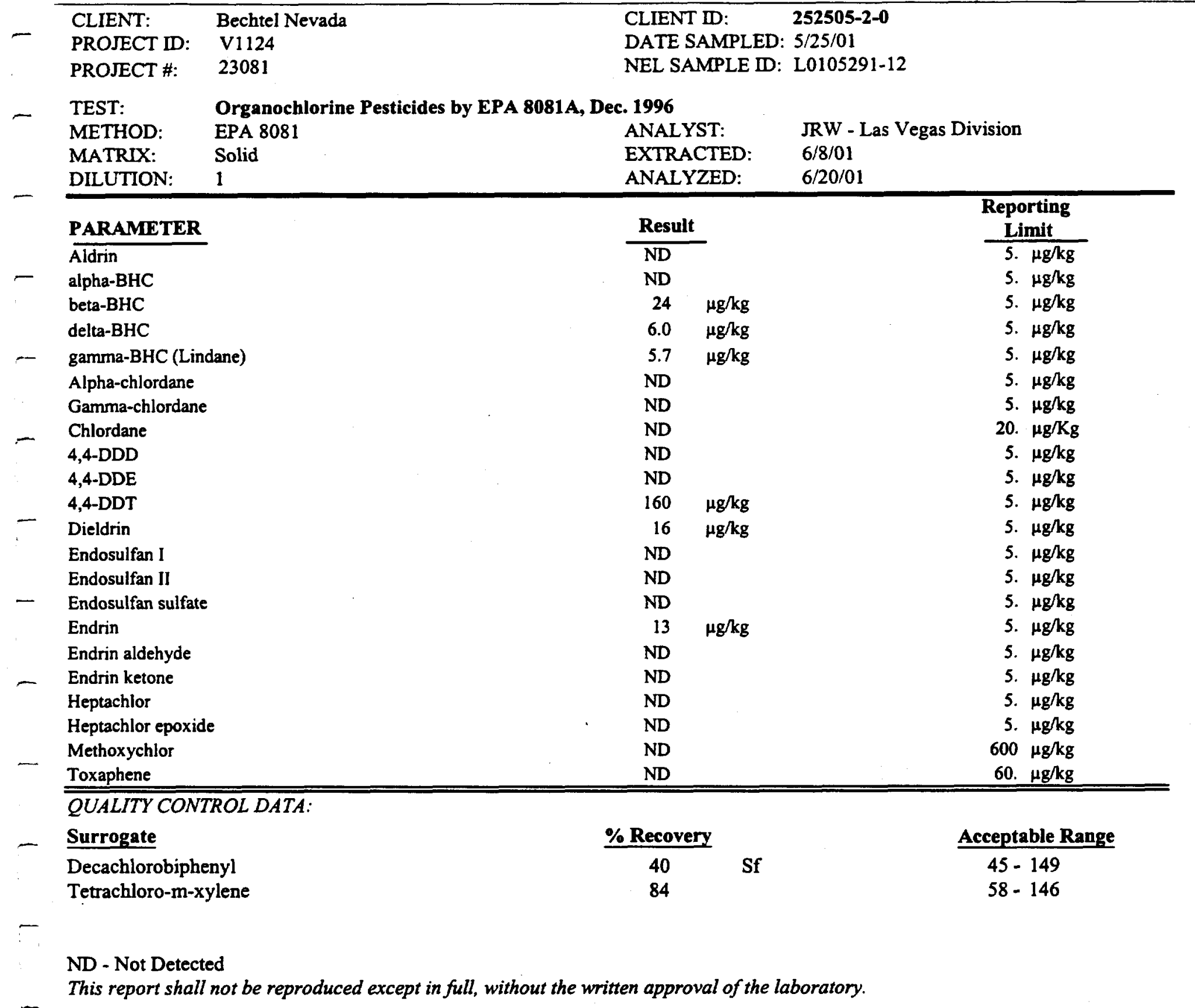


NEL LABORATORIES

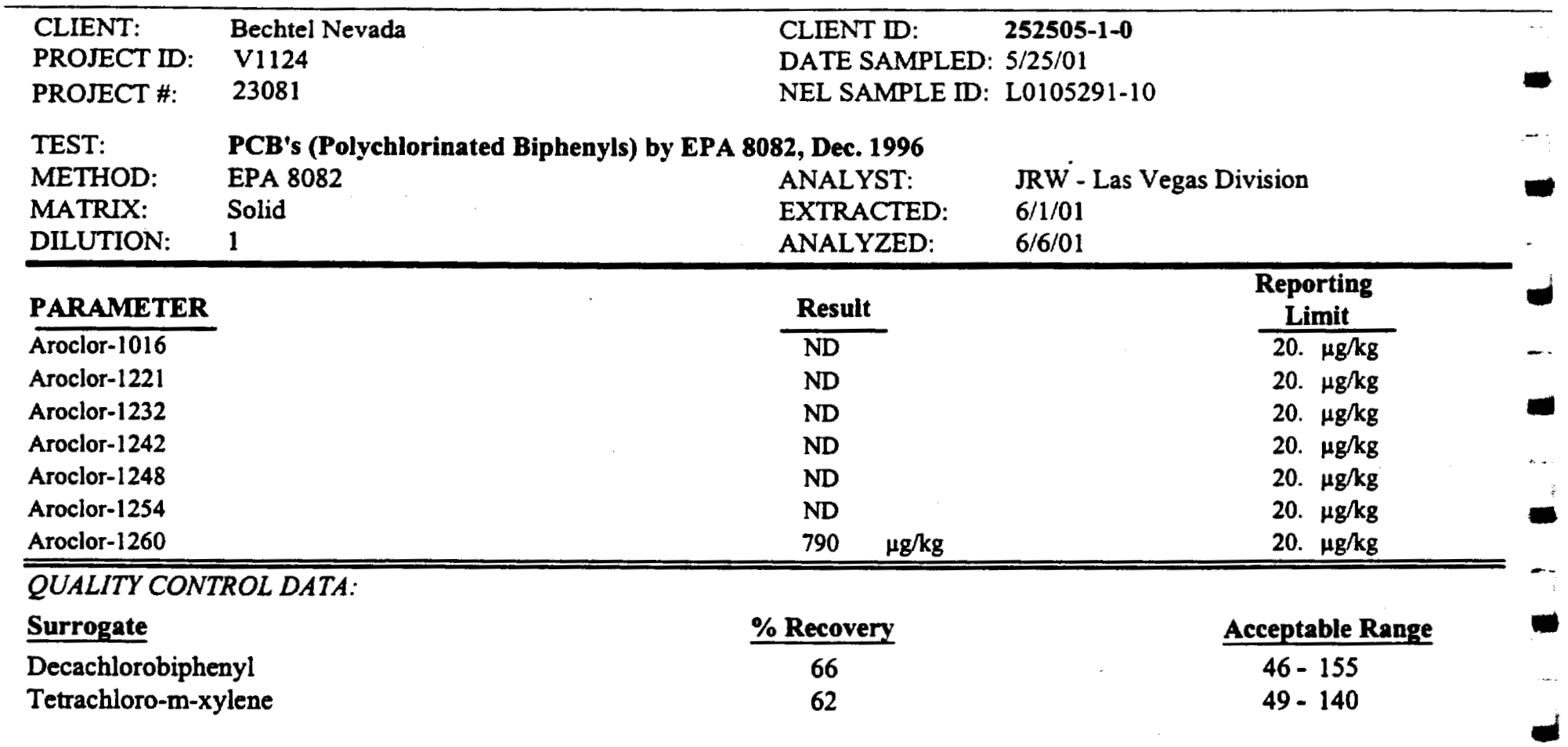

ND - Not Detected

This report shall not be reproduced except in full, without the written approval of the laboratory. 
NEL LABORATORIES

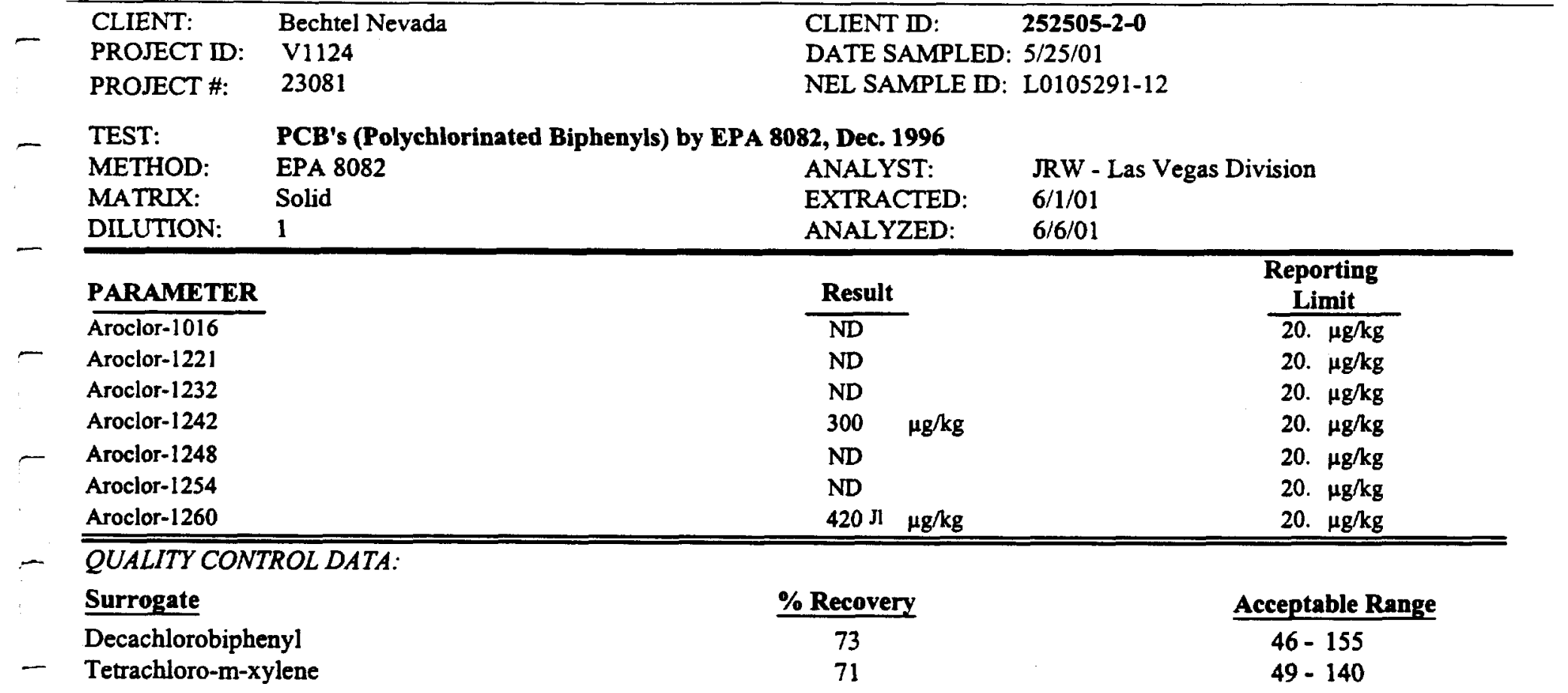

- ND - Not Detected

This report shall not be reproduced except in full, without the written approval of the laboratory. 
NEL LABORATORIES

\begin{tabular}{|c|c|c|}
\hline $\begin{array}{l}\text { CLIENT: } \\
\text { PROJECT ID: }\end{array}$ & $\begin{array}{l}\text { Bechtel Nevada } \\
\text { V1124 }\end{array}$ & $\begin{array}{ll}\text { CLIENT ID: } & \mathbf{2 5 2 5 1 6 - 1 - 0} \\
\text { DATE SAMPLED: } & 5 / 25 / 01\end{array}$ \\
\hline PROJECT \#: & 23081 & NEL SAMPLE ID: L0105291-0 \\
\hline
\end{tabular}

TEST: $\quad$ Total Extractable Petroleum Hydrocarbons Fuel Finger Print by EPA Method 8015M, July 1992

METHOD: $\quad$ EPA $8015 \mathrm{M}$

MATRIX: Solid

DILUTION: 1 ANALYST: $\quad$ CCS - Las Vegas Division EXTRACTED: $\quad 5 / 31 / 01$ ANALYZED: $\quad 6 / 1 / 01$

\begin{tabular}{llc}
\hline PARAMETER & Result & Reporting \\
\cline { 2 - 3 } Gasoline Range (C8-C12) & $\mathrm{ND}$ & $\frac{\text { Limit }}{10 . \mathrm{mg} / \mathrm{kg}}$ \\
Diesel Range (C12-C22) & $\mathrm{ND}$ & $10 . \mathrm{mg} / \mathrm{kg}$ \\
Oil Range (C12-C34) & $\mathrm{ND}$ & $50 . \mathrm{mg} / \mathrm{kg}$ \\
Total & $\mathrm{ND}$ & $10 . \mathrm{mg} / \mathrm{kg}$ \\
\hline \hline
\end{tabular}

QUALITY CONTROL DATA:

Surrogate

Octacosane

\% Recovery

61

\section{Acceptable Range}

$54-130$

ND - Not Detected

This report shall not be reproduced except in full, without the written approval of the laboratory. 
NEL LABORATORIES

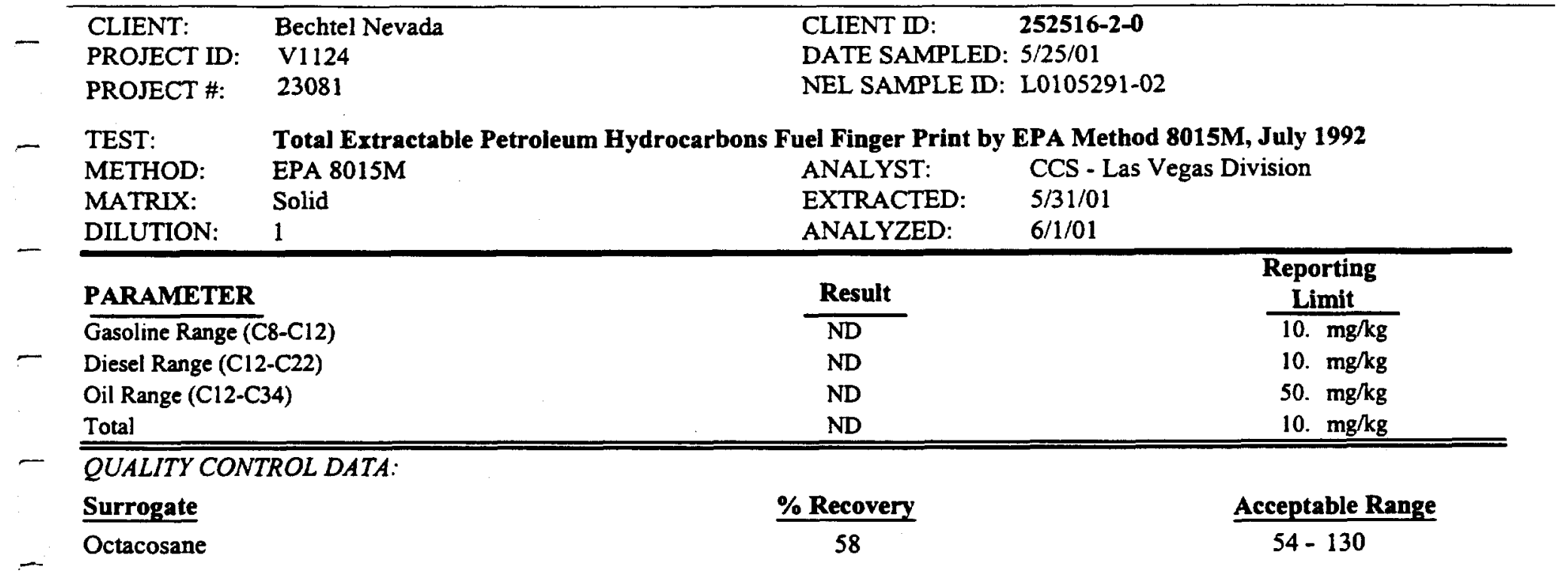

- ND - Not Detected

This report shall not be reproduced except in full, without the written approval of the laboratory. 
NEL LABORATORIES

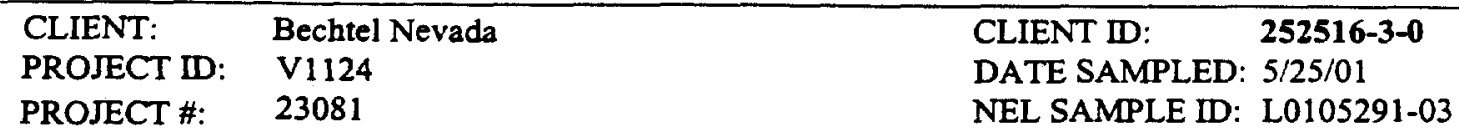

TEST:

Total Extractable Petroleum Hydrocarbons Fuel Finger Print by EPA Method 8015M, July 1992

METHOD: $\quad$ EPA 8015M

MATRIX: Solid ANALYST: $\quad$ CCS - Las Vegas Division

DILUTION

1

EXTRACTED: $\quad 5 / 31 / 01$

ANALYZED: $\quad 6 / 1 / 01$

\section{PARAMETER}

Gasoline Range (C8-C12)

Diesel Range (C12-C22)

Oil Range (C12-C34)

Total

\begin{tabular}{l} 
Result \\
\hline ND \\
ND \\
ND \\
ND
\end{tabular}

QUALITY CONTROL DATA:

Surrogate

Octacosane
\% Recovery

58
Reporting

Limit

10. $\mathrm{mg} / \mathrm{kg}$

10. $\mathrm{mg} / \mathrm{kg}$

50. $\mathrm{mg} / \mathrm{kg}$

10. $\mathrm{mg} / \mathrm{kg}$

\section{Acceptable Range}

$54-130$

ND - Not Detected

This report shall not be reproduced except in full, without the written approval of the laboratory. 
NEL LABORATORIES

\begin{tabular}{lll}
\hline CLIENT: & Bechtel Nevada & CLIENT D: \\
PROJECT ID: & V11242516-4-0 \\
PROJECT \#: & 23081 & DATE SAMPLED: 5/25/01 \\
& NEL SAMPLE ID: L0105291-04
\end{tabular}

$\begin{array}{ll}\text { TEST: } & \text { Total Extractab } \\ \text { METHOD: } & \text { EPA 8015M }\end{array}$

MATRIX: Solid

DILUTION: 1

Fuel Finger Print by EPA Method 8015M, July 1992

ANALYST: $\quad$ CCS - Las Vegas Division

EXTRACTED: $\quad 5 / 31 / 01$

ANALYZED: $\quad 6 / 1 / 01$

\begin{tabular}{lll}
\hline PARAMETER & Result & Reporting \\
\cline { 2 - 3 } & GD & $\frac{\text { Limit }}{10 . \mathrm{mg} / \mathrm{kg}}$ \\
Gasoline Range (C8-C12) & $\mathrm{ND}$ & $10 . \mathrm{mg} / \mathrm{kg}$ \\
Diesel Range (C12-C22) & $\mathrm{ND}$ & $50 . \mathrm{mg} / \mathrm{kg}$ \\
Oil Range (C12-C34) & $\mathrm{ND}$ & $10 . \mathrm{mg} / \mathrm{kg}$ \\
\hline
\end{tabular}

- QUALITY CONTROL DATA:

Surrogate

\% Recovery

58

Acceptable Range

Octacosane

$54-130$

-

- ND - Not Detected

This report shall not be reproduced except in full, without the written approval of the laboratory. 


$\begin{array}{llll}\text { CLIENT: } & \text { Bechtel Nevada } & \text { CLIENT D: } & \text { 252516-5-2 } \\ \text { PROJECT ID: } & \text { V1124 } & \text { DATE SAMPLED: } 5 / 25 / 01 \\ \text { PROJECT \#: } & 23081 & \text { NEL SAMPLE ID: L0105291-05 }\end{array}$

TEST:

METHOD

Total Extractable Petroleum Hydrocarbons Fuel Finger Print by EPA Method 8015M, July 1992

EPA 8015M ANALYST:

CCS - Las Vegas Division

MATRIX:

Solid

EXTRACTED:

$5 / 31 / 01$

DILUTION: 1

ANALYZED:

$6 / 1 / 01$

\section{PARAMETER}

Gasoline Range (C8-C12)

Diesel Range (C12-C22)

\begin{tabular}{l} 
Result \\
\hline ND \\
ND \\
ND \\
ND
\end{tabular}

Total

QUALITY CONTROL DATA:

Surrogate

Octacosane
$\%$ Recovery

66

\section{Reporting}

Limit

10. $\mathrm{mg} / \mathrm{kg}$

10. $\mathrm{mg} / \mathrm{kg}$

50. $\mathrm{mg} / \mathrm{kg}$

10. $\mathrm{mg} / \mathrm{kg}$

Acceptable Range

$54-130$

ND - Not Detected

This report shall not be reproduced except in full, without the written approval of the laboratory. 
NEL LABORATORIES

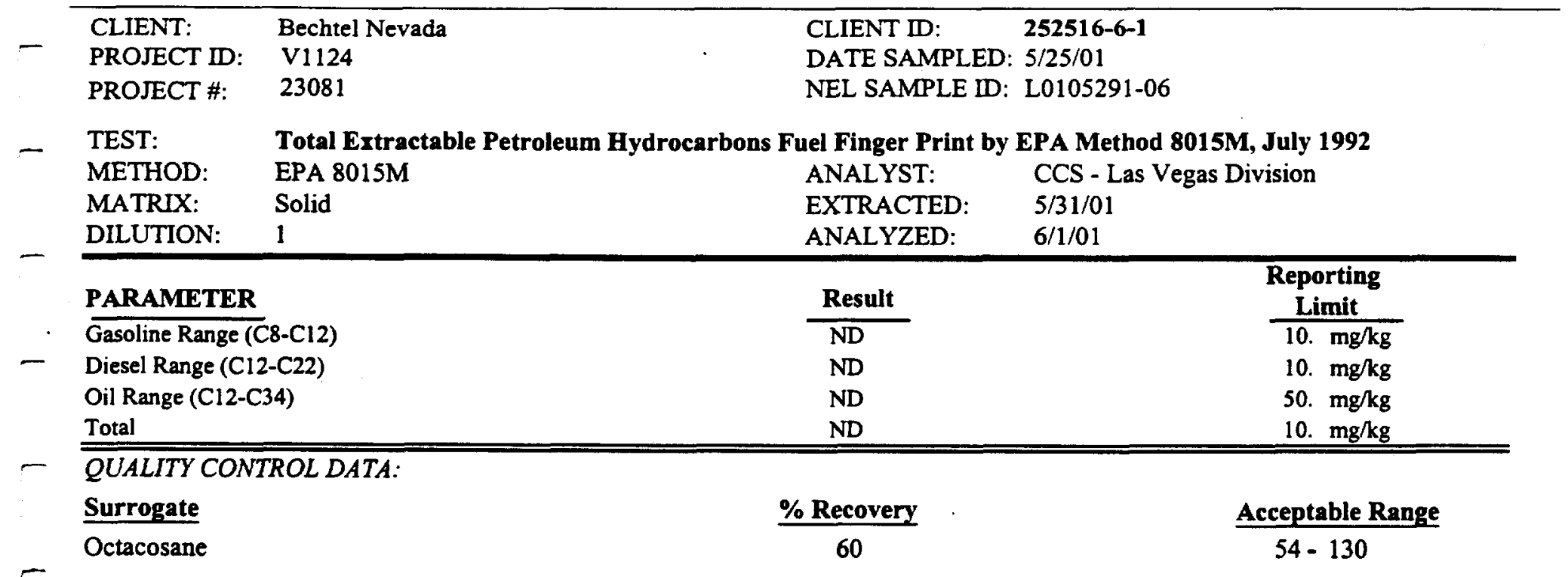

- ND - Not Detected

This report shall not be reproduced except in full, without the written approval of the laboratory. 
NEL LABORATORIES

$\begin{array}{llll}\text { CLIENT: } & \text { Bechtel Nevada } & \text { CLIENT ID: } & \text { 252516-7-1 } \\ \text { PROJECT ID: } & \text { V1124 } & \text { DATE SAMPLED: 5/25/01 } \\ \text { PROJECT \#: } & 23081 & \text { NEL SAMPLE ID: L0105291-07 }\end{array}$

TEST: $\quad$ Total Extractable Petroleum Hydrocarbons Fuel Finger Print by EPA Method 8015M, July 1992

METHOD: EPA 8015M ANALYST: CCS - Las Vegas Division

MATRIX: Solid $\quad$ EXTRACTED: $5 / 31 / 01$

DILUTION: $1 \quad$ ANALYZED: $6 / 1 / 01$

\begin{tabular}{lll}
\hline PARAMETER & ANALYZED & Reporting \\
Gasoline Range (C8-C12) & Result & $\frac{\text { Limit }}{10 . \mathrm{mg} / \mathrm{kg}}$ \\
\cline { 2 - 3 } Diesel Range (C12-C22) & ND & $10 . \mathrm{mg} / \mathrm{kg}$ \\
Oil Range (C12-C34) & ND & $50 . \mathrm{mg} / \mathrm{kg}$ \\
Total & ND & $10 . \mathrm{mg} / \mathrm{kg}$ \\
\hline \hline
\end{tabular}

QUALITY CONTROL DATA:

Surrogate

Octacosane

ND - Not Detected

This report shall not be reproduced except in full, without the written approval of the laboratory.

\section{Acceptable Range}

54 - 130 
NEL LABORATORIES

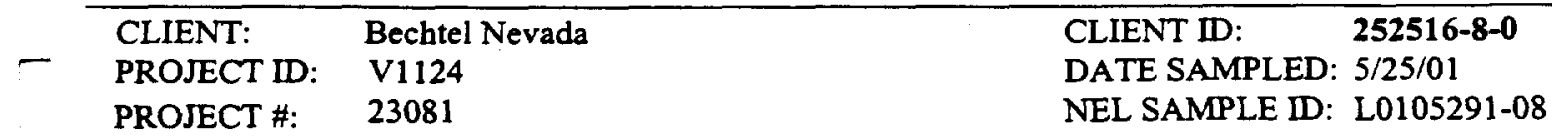

- TEST: $\quad$ Total Ertractable Petroleum Hydrocarbons Fuel Finger Print by EPA Method 8015M, July 1992

METHOD: EPA 8015M ANALYST: CCS - Las Vegas Division

MATRIX: $\quad$ Solid

EXTRACTED: $\quad 5 / 31 / 01$

DILUTION: $1 \quad$ ANALYZED: $\quad 6 / 1 / 01$

\begin{tabular}{|c|c|c|}
\hline PARAMETER & Result & $\begin{array}{l}\text { Reporting } \\
\text { Limit } \\
\end{array}$ \\
\hline$\overline{\text { Gasoline Range ( }}$ $8-C 12)$ & ND & 10. $\mathrm{mg} / \mathrm{kg}$ \\
\hline Diesel Range (C12-C22) & $\mathrm{mg} / \mathrm{kg}$ & 10. $\mathrm{mg} / \mathrm{kg}$ \\
\hline Oil Range (C12-C34) & $\mathrm{mg} / \mathrm{kg}$ & 50. $\mathrm{mg} / \mathrm{kg}$ \\
\hline Total & $\mathrm{mg} / \mathrm{kg}$ & 10. $\mathrm{mg} / \mathrm{kg}$ \\
\hline
\end{tabular}

- QUALITY CONTROL DATA:

Surrogate

- Octacosane

$\%$ Recovery

$58 \quad \mathrm{Jc}$

Acceptable Range

$54-130$

- ND - Not Detected

This report shall not be reproduced except in full, without the written approval of the laboratory. 
NEL LABORATORIES

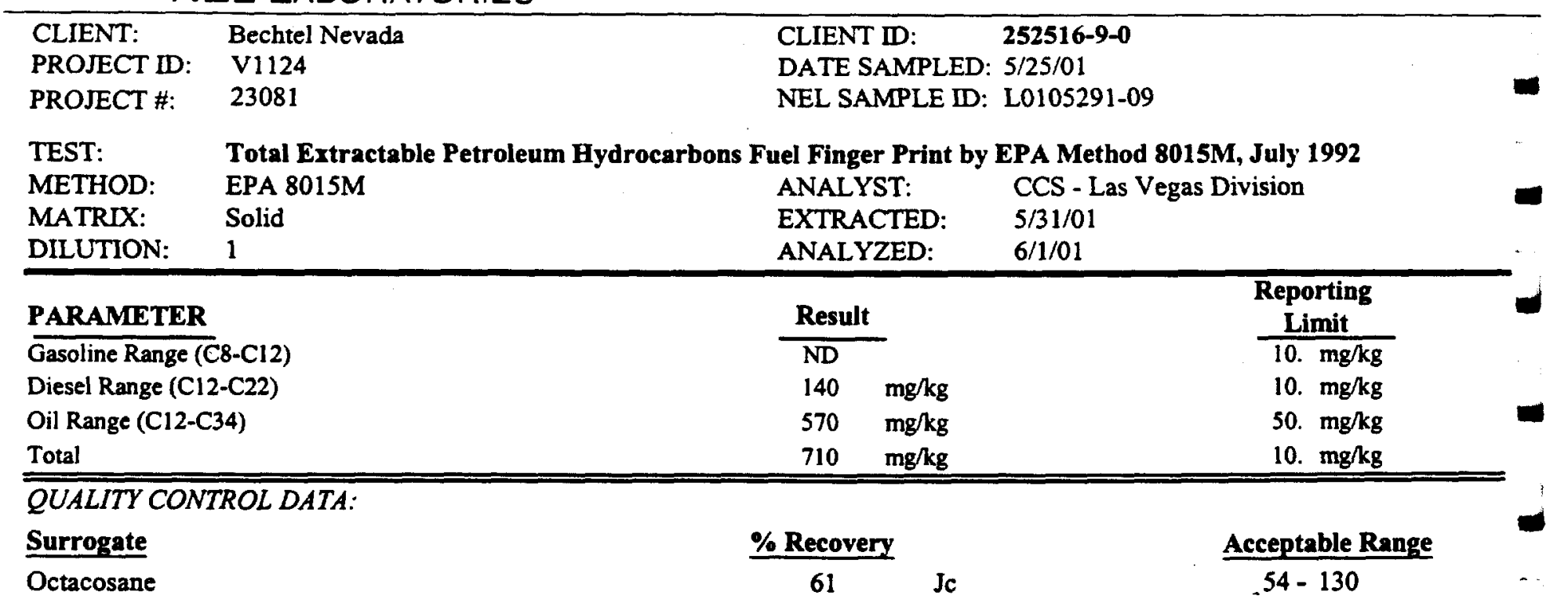

ND - Not Detected

This report shall not be reproduced except in full, without the written approval of the laboratory. 
NEL LABORATORIES

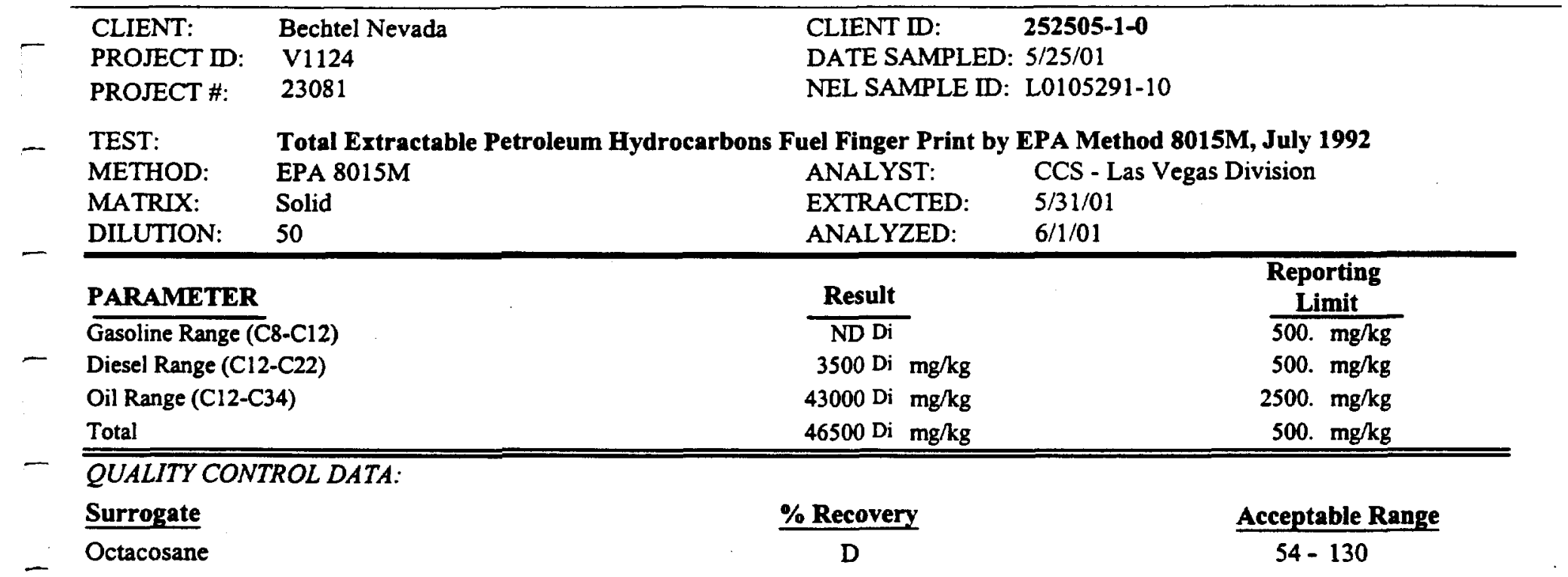

- ND - Not Detected

This report shall not be reproduced except in full, without the written approval of the laboratory. 
NEL LABORATORIES

$\begin{array}{llll}\text { CLIENT: } & \text { Bechtel Nevada } & \text { CLIENT ID: } & \text { 252505-2-2 } \\ \text { PROJECT ID: } & \text { V1124 } & \text { DATE SAMPLED: 5/25/01 } \\ \text { PROJECT \#: } & 23081 & \text { NEL SAMPLE ID: L0105291-11 }\end{array}$

TEST: $\quad$ Total Extractable Petroleum Hydrocarbons Fuel Finger Print by EPA Method 8015M, July 1992

METHOD: EPA 8015M ANALYST: CCS - Las Vegas Division

MATRIX: $\quad$ Solid

DILUTION: $\quad 1$

EXTRACTED: $\quad 5 / 31 / 01$

ANALYZED: $\quad 6 / 1 / 01$

\begin{tabular}{lcc}
\hline PARAMETER & Result & Reporting \\
\cline { 2 - 3 } Gasoline Range (C8-C12) & $\mathrm{ND}$ & Limit \\
Diesel Range (C12-C22) & $22 \quad \mathrm{mg} / \mathrm{kg}$ & $10 . \mathrm{mg} / \mathrm{kg}$ \\
Oil Range (C12-C34) & $\mathrm{ND} \quad$ & $10 . \mathrm{mg} / \mathrm{kg}$ \\
Total & $22 \quad \mathrm{mg} / \mathrm{kg}$ & $50 . \mathrm{mg} / \mathrm{kg}$ \\
\hline \hline
\end{tabular}

QUALITY CONTROL DATA:

Surrogate

Octacosane
\% Recovery

69

\section{Acceptable Range} $54-130$

ND - Not Detected

This report shall not be reproduced except in full, without the written approval of the laboratory. 
NEL LABORATORIES

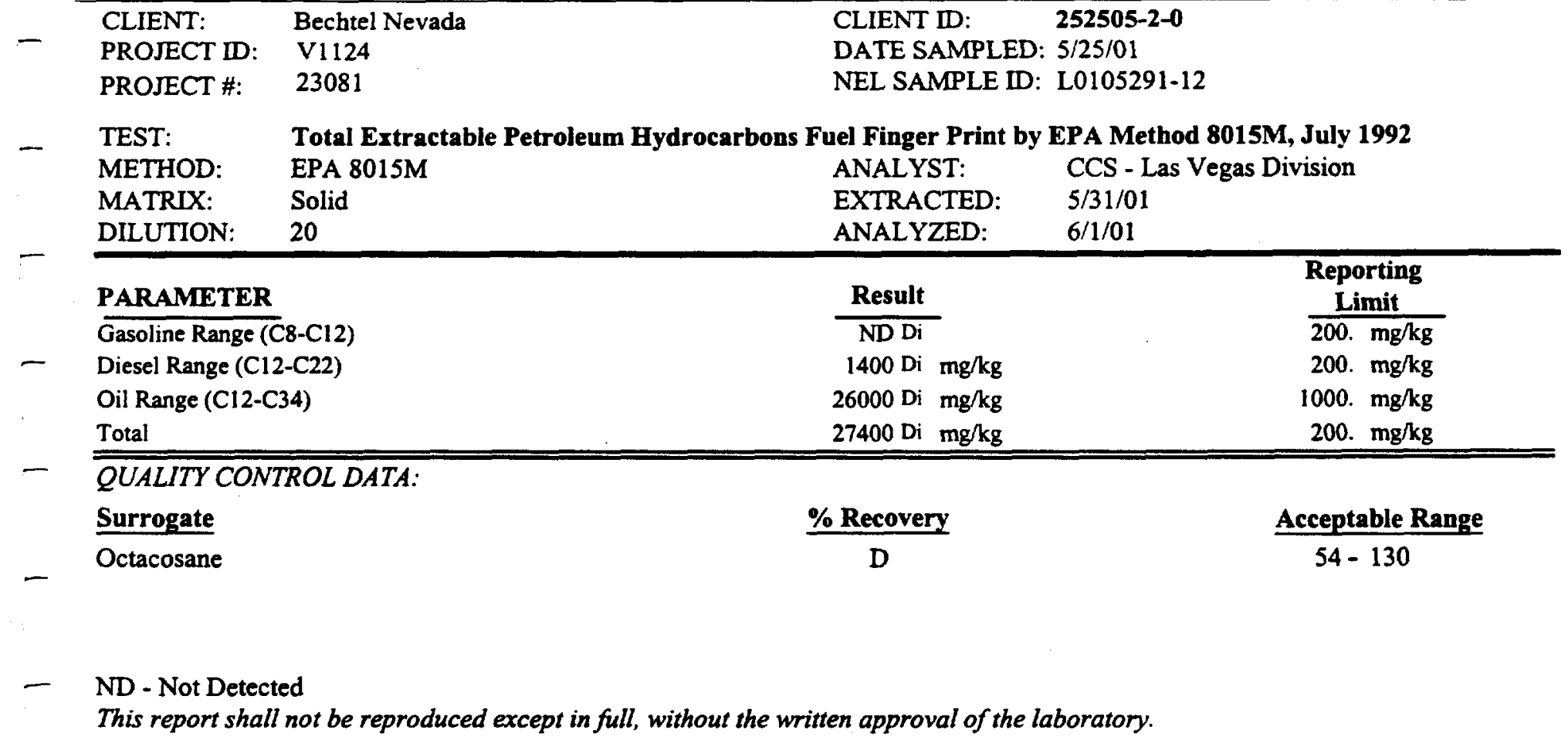


NEL LABORATORIES

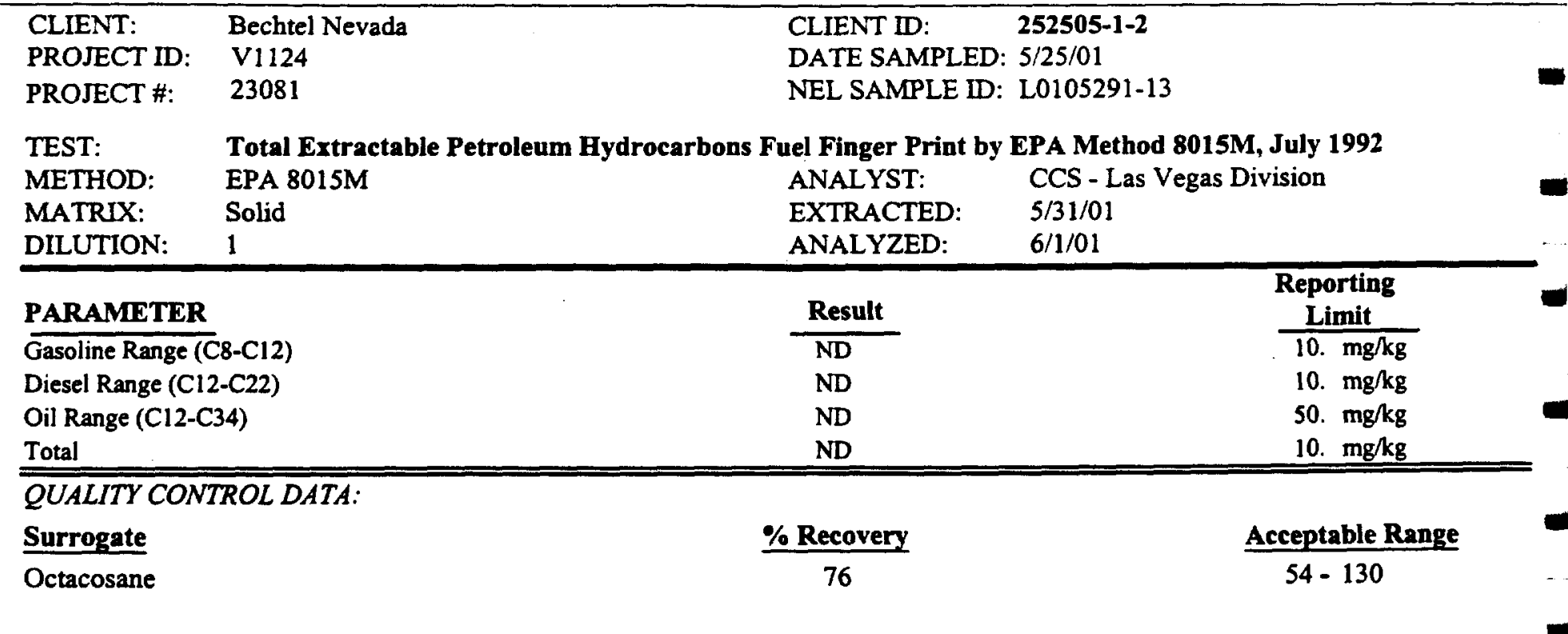

ND - Not Detected

This report shall not be reproduced except in full, without the written approval of the laboratory. 
NEL LABORATORIES

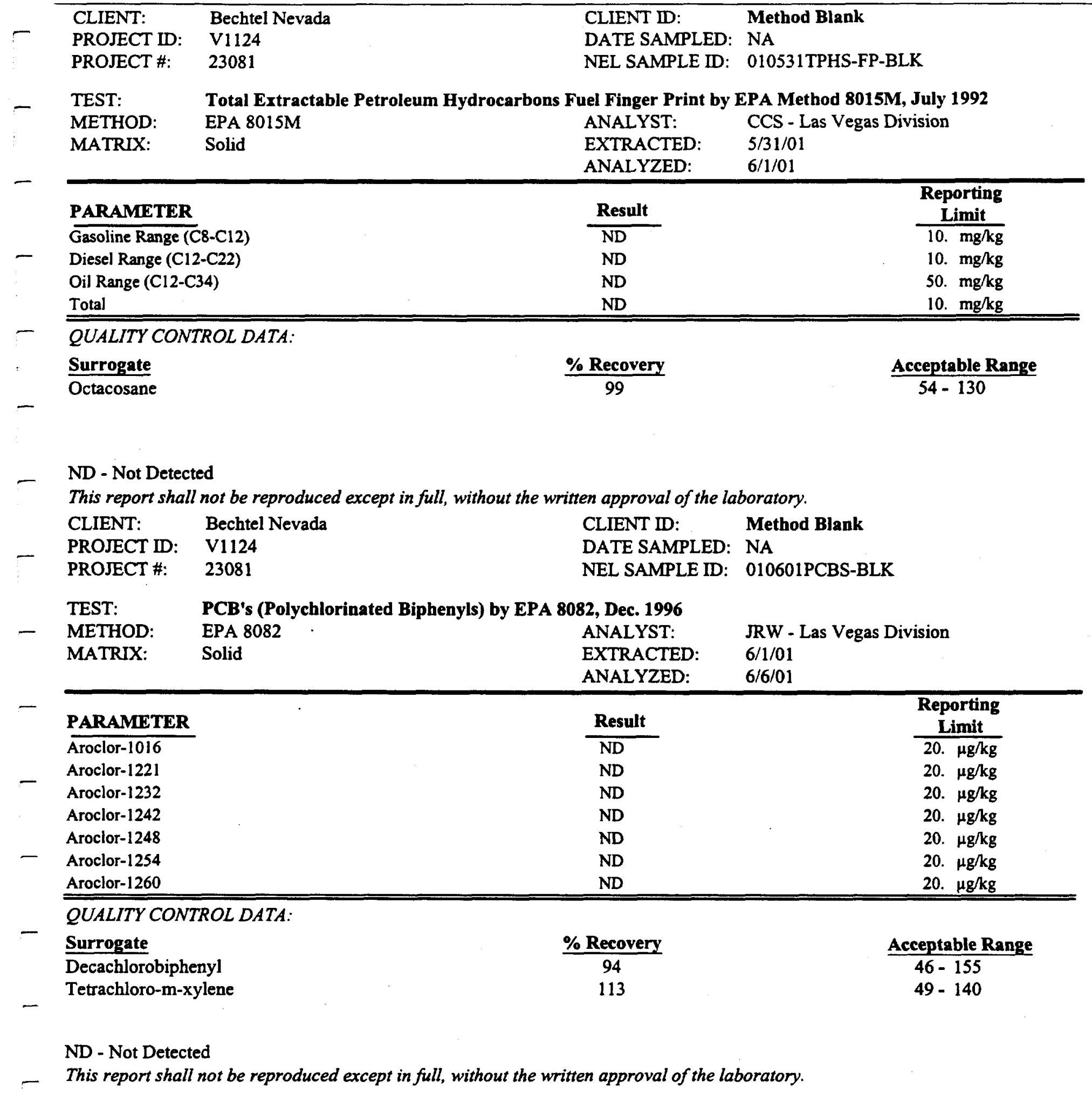




$\begin{array}{llll}\text { CLIENT: } & \text { Bechtel Nevada } & \text { CLIENT ID: } & \text { Method Blank } \\ \text { PROJECT ID: } & \text { V1124 } & \text { DATE SAMPLED: } & \text { NA } \\ \text { PROJECT \#: } & 23081 & \text { NEL SAMPLE ID: } & 010608 \text { PSTS-BLK }\end{array}$

TEST:

METHOD:

MATRIX:

Organochlorine Pesticides by EPA 8081A, Dec. 1996

EPA 8081

Solid
ANALYST:

EXTRACTED

ANALYZED:
JRW - Las Vegas Division $6 / 8 / 01$ $6 / 20 / 01$

\begin{tabular}{llc}
\hline PARAMETER & Result & Reporting \\
\cline { 2 - 3 } Aldrin & $\mathrm{Limit}$ \\
\cline { 2 - 3 } alpha-BHC & $\mathrm{ND}$ & $5 . \mu \mathrm{g} / \mathrm{kg}$ \\
beta-BHC & $\mathrm{ND}$ & $5 . \mu \mathrm{g} / \mathrm{kg}$ \\
delta-BHC & $\mathrm{ND}$ & $5 . \mu \mathrm{g} / \mathrm{kg}$ \\
gamma-BHC (Lindane) & $\mathrm{ND}$ & $5 . \mu \mathrm{g} / \mathrm{kg}$ \\
Alpha-chlordane & $\mathrm{ND}$ & $5 . \mu \mathrm{\mu g} / \mathrm{kg}$ \\
Gamma-chlordane & $\mathrm{ND}$ & $5 . \mu \mathrm{g} / \mathrm{kg}$ \\
Chlordane & $\mathrm{ND}$ & $5 . \mu \mathrm{g} / \mathrm{kg}$ \\
4,4-DDD & $\mathrm{ND}$ & $20 . \mu \mathrm{gg} / \mathrm{kg}$ \\
4,4-DDE & $\mathrm{ND}$ & $5 . \mu \mathrm{g} / \mathrm{kg}$ \\
4,4-DDT & $\mathrm{ND}$ & $5 . \mu \mathrm{g} / \mathrm{kg}$ \\
Dieldrin & $\mathrm{ND}$ & $5 . \mu \mathrm{\mu g} / \mathrm{kg}$ \\
Endosulfan I & $\mathrm{ND}$ & $5 . \mu \mathrm{g} / \mathrm{kg}$ \\
Endosulfan II & $\mathrm{ND}$ & $5 . \mu \mathrm{\mu} / \mathrm{kg}$ \\
Endosulfan sulfate & $\mathrm{ND}$ & $5 . \mu \mathrm{g} / \mathrm{kg}$ \\
Endrin & $\mathrm{ND}$ & $5 . \mu \mathrm{g} / \mathrm{kg}$ \\
Endrin aldehyde & $\mathrm{ND}$ & $5 . \mu \mathrm{g} / \mathrm{kg}$ \\
Endrin ketone & $\mathrm{ND}$ & $5 . \mu \mathrm{g} / \mathrm{kg}$ \\
Heptachlor & $\mathrm{ND}$ & $5 . \mu \mathrm{g} / \mathrm{kg}$ \\
Heptachlor epoxide & $\mathrm{ND}$ & $5 \mathrm{gg} / \mathrm{kg}$ \\
Methoxychlor & $\mathrm{ND}$ & $5 . \mu \mathrm{g} / \mathrm{kg}$ \\
Toxaphene & $\mathrm{ND}$ & $10 . \mu \mathrm{g} / \mathrm{kg}$ \\
\hline \hline
\end{tabular}

QUALITY CONTROL DATA:

Surrogate

Decachlorobiphenyl

Tetrachloro-m-xylene
$\%$ Recovery

94

89
Acceptable Range

45- 149

$58-146$

ND - Not Detected

This report shall not be reproduced except in full, without the written approval of the laboratory. 
NEL LABORATORIES

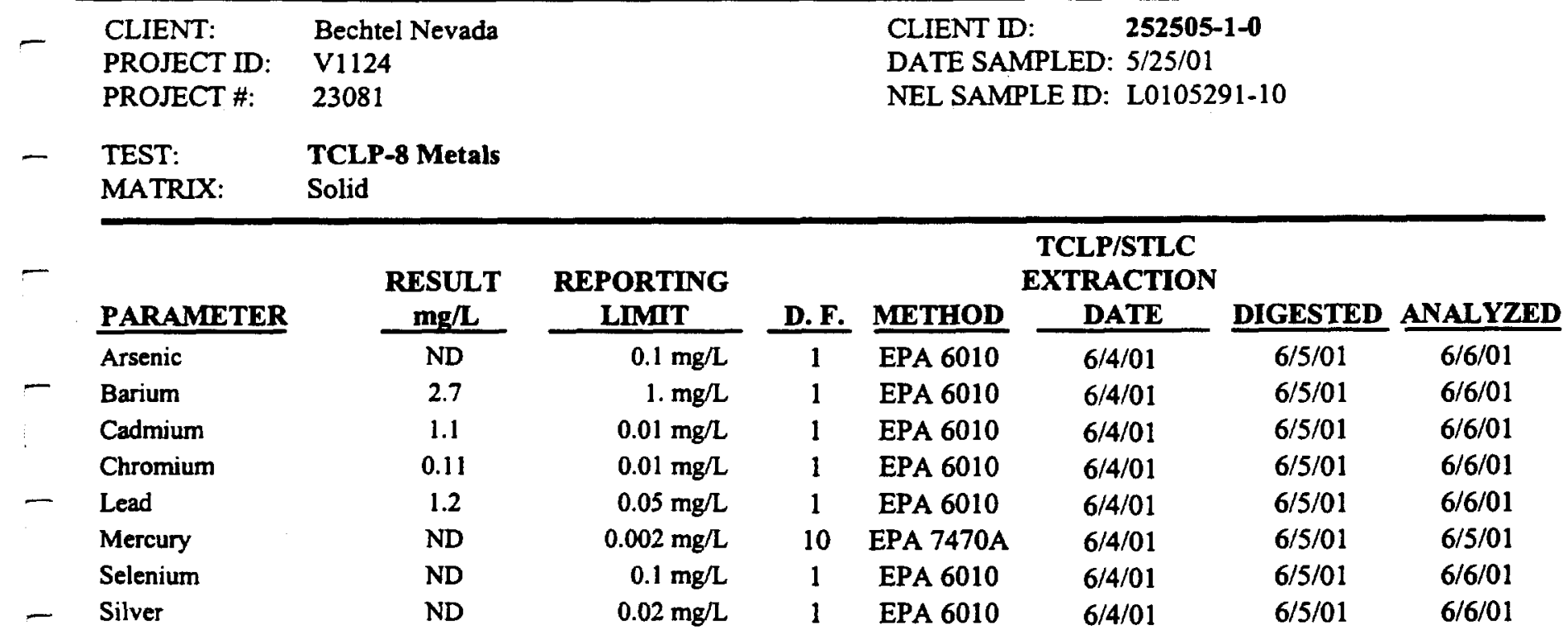

- D.F. - Dilution Factor ND - Not Detected

This report shall not be reproduced except in full, without the written approval of the laboratory. 
NEL LABORATORIES

\begin{tabular}{|c|c|c|c|c|c|c|c|}
\hline $\begin{array}{l}\text { CLIENT: } \\
\text { PROJECT ID: } \\
\text { PROJECT \#: }\end{array}$ & $\begin{array}{l}\text { Bechtel Nevada } \\
\text { V1124 } \\
23081\end{array}$ & & & $\begin{array}{l}\text { CLIENT ID } \\
\text { DATE SAI } \\
\text { NEL SAMI }\end{array}$ & $\begin{array}{l}\text { MPLED: } \\
\text { M/25/01 } \\
\text { PLE ID: } \\
\text { L01052 }\end{array}$ & $\begin{array}{l}-2-0 \\
91-12\end{array}$ & \\
\hline $\begin{array}{l}\text { TEST: } \\
\text { MATRIX: }\end{array}$ & $\begin{array}{l}\text { TCLP-8 Metals } \\
\text { Solid }\end{array}$ & & & & & & \\
\hline PARAMETER & $\begin{array}{c}\text { RESULT } \\
\text { mg/L } \\
\end{array}$ & $\begin{array}{c}\text { REPORTING } \\
\text { LIMIT }\end{array}$ & D. F. & METHOD & $\begin{array}{c}\text { TCLP/STLC } \\
\text { EXTRACTION } \\
\text { DATE } \\
\end{array}$ & & ANALYZED \\
\hline Arsenic & ND & $0.1 \mathrm{mg} / \mathrm{L}$ & 1 & EPA 6010 & $6 / 4 / 01$ & $6 / 5 / 01$ & $6 / 6 / 01$ \\
\hline Barium & ND & 1. $\mathrm{mg} / \mathrm{L}$ & 1 & EPA 6010 & $6 / 4 / 01$ & $6 / 5 / 01$ & $6 / 6 / 01$ \\
\hline Cadmium & 0.13 & $0.01 \mathrm{mg} / \mathrm{L}$ & 1 & EPA 6010 & $6 / 4 / 01$ & $6 / 5 / 01$ & $6 / 6 / 01$ \\
\hline Chromium & ND & $0.01 \mathrm{mg} / \mathrm{L}$ & 1 & EPA 6010 & $6 / 4 / 01$ & $6 / 5 / 01$ & $6 / 6 / 01$ \\
\hline Lead & 0.21 & $0.05 \mathrm{mg} / \mathrm{L}$ & 1 & EPA 6010 & $6 / 4 / 01$ & $6 / 5 / 01$ & $6 / 6 / 01$ \\
\hline Mercury & ND & $0.002 \mathrm{mg} / \mathrm{L}$ & 10 & EPA $7470 \mathrm{~A}$ & $6 / 4 / 01$ & $6 / 5 / 01$ & $6 / 5 / 01$ \\
\hline Selenium & ND & $0.1 \mathrm{mg} / \mathrm{L}$ & 1 & EPA 6010 & $6 / 4 / 01$ & $6 / 5 / 01$ & $6 / 6 / 01$ \\
\hline Silver & ND & $0.02 \mathrm{mg} / \mathrm{L}$ & 1 & EPA 6010 & $6 / 4 / 01$ & $6 / 5 / 01$ & $6 / 6 / 01$ \\
\hline
\end{tabular}

D.F. - Dilution Factor

ND - Not Detected

This report shall not be reproduced except in full, without the written approval of the laboratory. 
NEL LABORATORIES

$\begin{array}{llll}\text { CLIENT: } & \text { Bechtel Nevada } & \text { CLIENT ID: } & \text { Method Blank } \\ \text { PROJECT ID: } & \text { V1124 } & \text { DATE SAMPLED: NA } \\ \text { PROJECT \#: } & 23081 & \text { NEL SAMPLE ID: } & \text { L5275HGTCLP-BLK }\end{array}$

- TEST: $\quad$ TCLP by EPA 1311, July 1992 \& Mercury by EPA 7470A, July 1992

MATRIX: TCLP EXtract

\begin{tabular}{lcccc}
\hline & REPORTING & \multicolumn{2}{c}{ TCLP/STLC } \\
PARAMETER \\
Mercury & $\frac{\text { RESULT }}{\text { ND }}$ & $\frac{\text { LIMT }}{0.002 \mathrm{mg} / \mathrm{L}}$ & $\frac{\text { D. F. }}{10}$ & $\frac{\text { METHOD }}{\text { EPA } 7470 \mathrm{~A}} \frac{\text { DATE }}{6 / 4 / 01} \frac{\text { DIGESTED ANALYZED }}{6 / 5 / 01}$
\end{tabular}

D.F. - Dilution Factor

- ND - Not Detected

This report shall not be reproduced except in full, without the written approval of the laboratory. 
NEL LABORATORIES

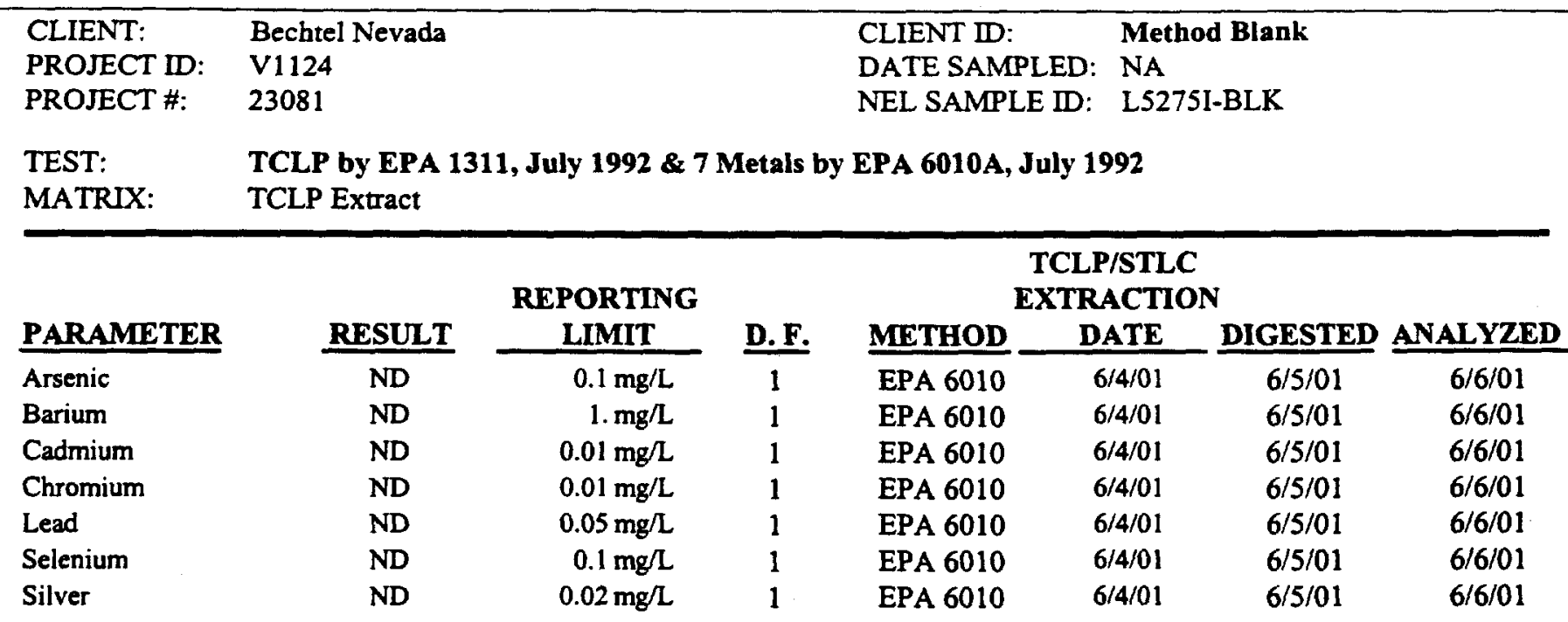

D.F. - Dilution Factor

ND - Not Detected

This report shall not be reproduced except in full, without the written approval of the laboratory. 
NEL LABORATORIES

\begin{tabular}{llll}
\hline CLIENT: & Bechtel Nevada & CLIENT ID: & 252505-1-0 \\
PROJECT ID: & V1124 & DATE SAMPLED: & $5 / 25 / 01$ \\
PROJECT \#: & 23081 & NEL SAMPLE ID: L0105291-10 \\
TEST: & TCLP by EPA 1311, July 1992 \& Semivolatile Organics by EPA Method 8270C, Dec. 1996 \\
METHOD: & EPA 8270 & TCLP EXTRACT DATE: 6/4/01 \\
MATRIX: & Solid & EXTRACTED & $6 / 6 / 01$ \\
DILUTION: & 1 & ANALYZED: & $6 / 7 / 01$
\end{tabular}

PARAMETER

1,4-Dichlorobenzene ( $\mathrm{p}-\mathrm{DCB}$ )

2,4-Dinitrotoluene (DNT)

Hexachlorobenzene

Hexachlorobutadiene

Hexachloroethane

2-Methylphenol

- 3,4-Methylphenol (isomeric pair)

Nitrobenzene

Pentachlorophenol

Pyridine

2,4,5-Trichlorophenol

2,4,6-Trichlorophenol

\begin{tabular}{c}
$\begin{array}{c}\text { Result } \\
\text { mg/L }\end{array}$ \\
\hline ND \\
ND \\
ND \\
ND \\
ND \\
ND \\
ND \\
ND \\
ND \\
ND \\
ND \\
ND
\end{tabular}

Reporting

Limit

$0.1 \mathrm{mg} / \mathrm{L}$

$0.1 \mathrm{mg} / \mathrm{L}$

$0.1 \mathrm{mg} / \mathrm{L}$

$0.1 \mathrm{mg} / \mathrm{L}$

$0.1 \mathrm{mg} / \mathrm{L}$

$0.1 \mathrm{mg} / \mathrm{L}$

$0.1 \mathrm{mg} / \mathrm{L}$

$0.1 \mathrm{mg} / \mathrm{L}$

$0.1 \mathrm{mg} / \mathrm{L}$

$0.1 \mathrm{mg} / \mathrm{L}$

$0.1 \mathrm{mg} / \mathrm{L}$

$0.1 \mathrm{mg} / \mathrm{L}$

- $\overline{\text { QUALITY CONTROL DATA: }}$

\section{Surrogate}

- 2,4,6-Tribromophenol

2-Fluorobiphenyl

2-Fluorophenol

Nitrobenzene-d5

p-Terphenyl-d14

Phenol-d5

\section{$\%$ Recovery}

41

20

21

20

27

22
Acceptable Range

0 - 161

$16-127$

0 - 88

$9-132$

$16-163$

0.63

-

ND - Not Detected

This report shall not be reproduced except in full, without the written approval of the laboratory. 
NEL Laboratories

\begin{tabular}{llll}
\hline CLIENT: & Bechtel Nevada & CLIENT ID: & 252505-2-0 \\
PROJECT ID: & V1124 & DATE SAMPLED: $5 / 25 / 01$ \\
PROJECT \#: & 23081 & NEL SAMPLE ID: & L0105291-12 \\
TEST: & TCLP by EPA 1311, July 1992 \& Semivolatile Organics by EPA Method 8270C, Dec. 1996 \\
METHOD: & EPA 8270 & TCLP EXTRACT DATE: 6/4/01 \\
MATRIX: & Solid & EXTRACTED & $6 / 6 / 01$ \\
DILUTION: & 1 & ANALYZED: & $6 / 7 / 01$
\end{tabular}

\begin{tabular}{lcc}
\hline PARAMETER & $\begin{array}{c}\text { Result } \\
\text { mg/L }\end{array}$ & $\begin{array}{c}\text { Reporting } \\
\text { Limit }\end{array}$ \\
\cline { 2 - 3 } 1,4-Dichlorobenzene (p-DCB) & ND & $0.1 \mathrm{mg} / \mathrm{L}$ \\
2,4-Dinitrotoluene (DNT) & ND & $0.1 \mathrm{mg} / \mathrm{L}$ \\
Hexachlorobenzene & ND & $0.1 \mathrm{mg} / \mathrm{L}$ \\
Hexachlorobutadiene & ND & $0.1 \mathrm{mg} / \mathrm{L}$ \\
Hexachloroethane & ND & $0.1 \mathrm{mg} / \mathrm{L}$ \\
2-Methylphenol & ND & $0.1 \mathrm{mg} / \mathrm{L}$ \\
3,4-Methylphenol (isomeric pair) & ND & $0.1 \mathrm{mg} / \mathrm{L}$ \\
Nitrobenzene & ND & $0.1 \mathrm{mg} / \mathrm{L}$ \\
Pentachlorophenol & ND & $0.1 \mathrm{mg} / \mathrm{L}$ \\
Pyridine & ND & $0.1 \mathrm{mg} / \mathrm{L}$ \\
2,4,5-Trichlorophenol & ND & $0.1 \mathrm{mg} / \mathrm{L}$ \\
2,4,6-Trichlorophenol & ND & $0.1 \mathrm{mg} / \mathrm{L}$
\end{tabular}

\section{QUALITY CONTROL DATA:}

\section{Surrogate}

2,4,6-Tribromophenol

2-Fluorobiphenyl

2-Fluorophenol

Nitrobenzene-d5

p-Terphenyl-d14

Phenol-d5
\% Recovery

37

18

19

18

24

16
Acceptable Range

$0-161$

$16-127$

$0-88$

$9-132$

$16-163$

$0-63$

ND - Not Detected

This report shall not be reproduced except in full, without the written approval of the laboratory. 


$\begin{array}{llll}\text { CLIENT: } & \text { Bechtel Nevada } & \text { CLIENT ID: } & \text { Method Blank } \\ \text { PROJECT ID: } & \text { V1124 } & \text { DATE SAMPLED: } & \text { NA } \\ \text { PROJECT \#: } & 23081 & \text { NEL SAMPLE ID: } 0606 E 1 \text { BLK-BLK } \\ \text { TEST: } & \text { TCLP by EPA 1311, July 1992 \& Semivolatile Organics by EPA Method 8270C, Dec. } 1996 \\ \text { METHOD: } & \text { EPA 8270 } & \text { TCLP EXTRACT DATE: 6/4/01 } \\ \text { MATRIX: } & \text { TCLP Extract } & \text { EXTRACTED } & 6 / 6 / 01 \\ & & \text { ANALYZED: } & 6 / 7 / 01\end{array}$

\begin{tabular}{lcc}
\hline & $\begin{array}{c}\text { Result } \\
\text { mg/L }\end{array}$ & $\begin{array}{c}\text { Reporting } \\
\text { Limit }\end{array}$ \\
\cline { 2 - 3 }$-\quad$ & ND & $0.1 \mathrm{mg} / \mathrm{L}$ \\
1,4-Dichlorobenzene (p-DCB) & ND & $0.1 \mathrm{mg} / \mathrm{L}$ \\
2,4-Dinitrotoluene (DNT) & ND & $0.1 \mathrm{mg} / \mathrm{L}$ \\
Hexachlorobenzene & ND & $0.1 \mathrm{mg} / \mathrm{L}$ \\
Hexachlorobutadiene & ND & $0.1 \mathrm{mg} / \mathrm{L}$ \\
Hexachloroethane & ND & $0.1 \mathrm{mg} / \mathrm{L}$ \\
2-Methylphenol & ND & $0.1 \mathrm{mg} / \mathrm{L}$ \\
3,4-Methylphenol (isomeric pair) & ND & $0.1 \mathrm{mg} / \mathrm{L}$ \\
Nitrobenzene & ND & $0.1 \mathrm{mg} / \mathrm{L}$ \\
Pentachlorophenol & ND & $0.1 \mathrm{mg} / \mathrm{L}$ \\
Pyridine & ND & $0.1 \mathrm{mg} / \mathrm{L}$ \\
2,4,5-Trichlorophenol & ND & $0.1 \mathrm{mg} / \mathrm{L}$
\end{tabular}

QUALITY CONTROL DATA:

\section{Surrogate}

- 2,4,6-Tribromophenol

2-Fluorobiphenyl

2-Fluorophenol

- Nitrobenzene-d5

p-Terphenyl-dl4

Phenol-d5
\% Recovery

74

73

36

76

122

25
Acceptable Range

$0-161$

$16-127$

0 - 88

9- 132

$16-163$

0- 63

This report shall not be reproduced except in full, without the written approval of the laboratory. 
CLIENT: Bechtel Nevada

PROJECT ID: V1124

PROJECT \#: 23081

TEST:

Total Extractable Petroleum Hydrocarbons Fuel Finger Print by EPA Method 8015M, July 1992

MATRIX: $\quad$ Solid

\section{PARAMETER}

Diesel Range (C12-C22)

Diesel Range (C12-C22)

Total

Total

$\frac{\text { NEL Sample ID }}{\text { 010531TPHS-FP-LCS }}$
010531TPHS-FP-LCSD
010531TPHS-FP-LCS
010531TPHS-FP-LCSD

NEL Sample ID 010531TPHS-FP-LCSD

010531TPHS-FP-LCSD

$\begin{array}{ccc}\frac{\text { Spike }}{\text { Amount }} & & \text { Spike } \\ 166.7 & & 143 \\ 166.7 & 148 \\ 166.7 & 143 \\ 166.7 & 148\end{array}$

\begin{tabular}{c}
$\frac{\text { Percent }}{\text { Recovery }}$ \\
\hline 86 \\
89 \\
86 \\
89
\end{tabular}

Acceptable

Range RPD

$53-91$

$53-91$

3.4

$53-91$

$53-91$ 
PROJECT \#: 23081

TEST: $\quad$ PCB's (Polychlorinated Biphenyls) by EPA 8082, Dec. 1996

MATRLX: Solid

- PARAMETER

Aroclor-1016

Aroclor-1016

- Aroclor-1016

Aroclor-1016

Aroclor- 1260

Aroclor-1260

Aroclor-1260

Aroclor-1260

\section{NEL Sample ID}

010601PCBS-LCS

010601PCBS-LCSD

L0105291-12-MS

L0105291-12-MSD

010601 PCBS-LCS

010601PCBS-LCSD

L0105291-12-MS

L0105291-12-MSD

\begin{tabular}{|c|c|}
\hline Spike & Spike \\
\hline Amount & Result \\
\hline 333 & 367 \\
\hline 333 & 374 \\
\hline 333 & 193 \\
\hline 333 & 197 \\
\hline 333 & 352 \\
\hline 333 & 372 \\
\hline 333 & 872 \\
\hline 333 & 741 \\
\hline
\end{tabular}

\begin{tabular}{cccc}
$\frac{\text { Percent }}{\text { Recovery }}$ & & $\begin{array}{c}\text { Acceptable } \\
\text { Range }\end{array}$ & RPD \\
\cline { 1 - 1 } 110 & & $63-127$ & \\
112 & & $63-127$ & 1.9 \\
58 & & $55-142$ & \\
59 & $55-142$ & 2.1 \\
106 & $57-138$ & \\
112 & & $57-138$ & 5.5 \\
136 & $\mathrm{~J}$ & $48-129$ & \\
96 & & $48-129$ & 33.9
\end{tabular}




$\begin{array}{ll}\text { CLIENT: } & \text { Bechtel Nevada } \\ \text { PROJECT ID: } & \text { V1124 } \\ \text { PROJECT \#: } & 23081 \\ \text { TEST: } & \text { Organochlorine Pesticides by EPA 8081A, Dec. } 1996 \\ \text { MATRIX: } & \text { Solid }\end{array}$

\begin{tabular}{|c|c|c|c|c|c|c|c|}
\hline PARAMETER & NEL Sample ID & $\underline{\text { Spike }}$ & $\frac{\text { Spike }}{\text { Result }}$ & $\underline{\text { Percent }}$ & & $\frac{\text { Acceptable }}{\text { Range }}$ & $\underline{\mathbf{R P D}}$ \\
\hline Aldrin & 010608PSTS-LCS & 33.3 & 19.91 & 60 & & $63-131$ & \\
\hline Aldrin & 010608PSTS-LCSD & 33.3 & 28.5 & 86 & & $63-131$ & 35.5 \\
\hline Aldrin & L0105291-12-MS & 33.3 & 41.3 & 124 & & $63-131$ & \\
\hline Aldrin & L0105291-12-MSD & 33.3 & 45 & 135 & Л & $63-131$ & 8.6 \\
\hline alpha-BHC & 010608PSTS-LCS & 33.3 & 18.95 & 57 & & $55-127$ & \\
\hline alpha-BHC & 010608PSTS-LCSD & 33.3 & 29.42 & 88 & & $55-127$ & 43.3 \\
\hline beta-BHC & 010608PSTS-LCS & 33.3 & 19.62 & 59 & & $55-134$ & \\
\hline beta-BHC & 010608PSTS-LCSD & 33.3 & 29.07 & 87 & & $55-134$ & 38.8 \\
\hline delta-BHC & 010608PSTS-LCS & 33.3 & 18.88 & 57 & & $58-122$ & \\
\hline delta-BHC & 010608PSTS-LCSD & 33.3 & 25.73 & 77 & & $58-122$ & 30.7 \\
\hline gamma-BHC (Lindane) & 010608PSTS-LCS & 33.3 & 18.88 & 57 & & $58-132$ & \\
\hline gamma-BHC (Lindane) & 010608PSTS-LCSD & 33.3 & 29.57 & 89 & & $58-132$ & 44.1 \\
\hline gamma-BHC (Lindane) & L0105291-12-MS & 33.3 & 37.3 & 95 & & $58-132$ & \\
\hline gamma-BHC (Lindane) & L0105291-12-MSD & 33.3 & 44.3 & 116 & & $58-132$ & 19.9 \\
\hline Alpha-chlordane & 010608PSTS-LCS & 33.3 & 19.31 & 58 & & $65-129$ & \\
\hline Alpha-chlordane & 010608PSTS-LCSD & 33.3 & 28.07 & 84 & & $65-129$ & 37. \\
\hline Gamma-chlordane & 010608PSTS-LCS & 33.3 & 20.65 & 62 & & $63-132$ & \\
\hline Gamma-chlordane & 010608PSTS-LCSD & 33.3 & 29.62 & 89 & & $63-132$ & 35.7 \\
\hline 4,4-DDD & 010608PSTS-LCS & 33.3 & 23.4 & 70 & & $64-131$ & \\
\hline 4,4-DDD & 010608PSTS-LCSD & 33.3 & 31.5 & 95 & & $64-131$ & 29.5 \\
\hline 4,4-DDD & L0105291-12-MS & 33.3 & 30 & 90 & & $64-131$ & \\
\hline 4,4-DDD & L0105291-12-MSD & 33.3 & 36.3 & 109 & & $64-131$ & 19. \\
\hline 4,4-DDE & 010608PSTS-LCS & 33.3 & 24.26 & 73 & & $67-140$ & \\
\hline 4,4-DDE & 010608PSTS-LCSD & 33.3 & 29.42 & 88 & & $67-140$ & 19.2 \\
\hline 4,4-DDE & L0105291-12-MS & 33.3 & 36.7 & 110 & & $67-140$ & \\
\hline 4,4-DDE & L0105291-12-MSD & 33.3 & 39 & 117 & & $67-140$ & 6.1 \\
\hline 4,4-DDT & 010608PSTS-LCS & 33.3 & 27.36 & 82 & & $65-138$ & \\
\hline 4,4-DDT & 010608PSTS-LCSD & 33.3 & 33.58 & 101 & & $65-138$ & 20.4 \\
\hline 4,4-DDT & L0105291-12-MS & 33.3 & 156 & -12 & Л & $65-138$ & \\
\hline 4,4-DDT & L0105291-12-MSD & 33.3 & 136 & -72 & $\boldsymbol{\Omega}$ & $65-138$ & -142.9 \\
\hline Dieldrin & 010608PSTS-LCS & 33.3 & 25.21 & 76 & & $70-137$ & \\
\hline Dieldrin & 010608PSTS-LCSD & 33.3 & 34.12 & 102 & & $70-137$ & 30. \\
\hline Dieldrin & L0105291-12-MS & 33.3 & 41.3 & 76 & & $70-137$ & \\
\hline Dieldrin & L0105291-12-MSD & 33.3 & 55.7 & 119 & $\boldsymbol{\jmath}$ & $70-137$ & 44.3 \\
\hline Endosulfan I & 010608PSTS-LCS & 33.3 & 20.61 & 62 & & $62-127$ & \\
\hline Endosulfan I & 010608PSTS-LCSD & 33.3 & 29.54 & 89 & & $62-127$ & 35.6 \\
\hline Endosulfan II & 010608PSTS-LCS & 33.3 & 23.8 & 71 & & $64-131$ & \\
\hline Endosulfan II & 010608PSTS-LCSD & 33.3 & 31.08 & 93 & & $64-131$ & 26.5 \\
\hline
\end{tabular}

ND - Not Detected

This report shall not be reproduced except in full, without the written approval of the laboratory. 
NEL LABORATORIES

CLIENT: Bechtel Nevada

PROJECT ID: V1124

PROJECT \#: 23081

TEST: $\quad$ Organochlorine Pesticides by EPA 8081A, Dec. 1996

MATRIX: Solid

$\hookrightarrow \quad$ PARAMETER

Endosulfan sulfate

Endosulfan sulfate

- Endrin

Endrin

Endrin

Endrin

Endrin aldehyde

Endrin aldehyde

Endrin ketone

Endrin ketone

- Heptachlor

Heptachlor

Heptachlor

- Heptachlor

Heptachlor epoxide

Heptachlor epoxide

Methoxychlor

Methoxychlor

- Toxaphene

Toxaphene
NEL Sample ID

010608PSTS-LCS

010608PSTS-LCSD

010608PSTS-LCS

010608PSTS-LCSD

L0105291-12-MS

L0105291-12-MSD

010608PSTS-LCS

010608PSTS-LCSD

010608PSTS-LCS

010608PSTS-LCSD

010608PSTS-LCS

010608PSTS-LCSD

L0105291-12-MS

L0105291-12-MSD

010608PSTS-LCS

010608PSTS-LCSD

010608PSTS-LCS

010608PSTS-LCSD

010608PSTS-LCS

010608PSTS-LCSD

\begin{tabular}{|c|c|}
\hline$\underline{\text { Spike }}$ & $\frac{\text { Spike }}{\text { Result }}$ \\
\hline 33.3 & 24.46 \\
\hline 33.3 & 31.89 \\
\hline 33.3 & 26.44 \\
\hline 33.3 & 35.99 \\
\hline 33.3 & 35.3 \\
\hline 33.3 & 37.7 \\
\hline 33.3 & 34.92 \\
\hline 33.3 & 48.06 \\
\hline 33.3 & 23.48 \\
\hline 33.3 & 29.63 \\
\hline 33.3 & 23.79 \\
\hline 33.3 & 35.54 \\
\hline 33.3 & 34.7 \\
\hline 33.3 & 39 \\
\hline 33.3 & 20.5 \\
\hline 33.3 & 30.74 \\
\hline 33.3 & 47.99 \\
\hline 33.3 & 57.24 \\
\hline 333 & 361 \\
\hline 333 & 307 \\
\hline
\end{tabular}

307

\begin{tabular}{c}
$\frac{\text { Percent }}{\text { Recovery }}$ \\
\hline 73 \\
96 \\
79 \\
108 \\
67 \\
74 \\
105 \\
144 \\
71 \\
89 \\
71 \\
107 \\
104 \\
117 \\
62 \\
92 \\
144 \\
172 \\
108 \\
92
\end{tabular}

Acceptable

Range RPD

$68-128$

$68-128$

26.4

$70-140$

$70-140$

30.6

$70-140$

$70-140$

10.2

$73-221$

$73-221$

$65-133$

$65-133$

$55-127$

$55-127$

$55-127$

$55-127$

$62-127$

$62-127$

$38-168$

$38-168$

17.6

$68-113$

$68-113$

16.2

ND - Not Detected

This report shall not be reproduced except in full, without the written approval of the laboratory. 


$\begin{array}{ll}\text { CLIENT: } & \text { Bechtel Nevada } \\ \text { PROJECT ID: } & \text { V1124 } \\ \text { PROJECT \#: } & 23081 \\ \text { TEST: } & \text { TCLP by EPA 1311, July } 1992 \text { \& Semivolatile Organics by EPA Method 8270C, Dec. } 1996 \\ \text { MATRIX: } & \text { Solid }\end{array}$

\begin{tabular}{|c|c|c|c|c|c|c|}
\hline PARAMETER & NEL Sample ID & $\begin{array}{c}\text { Spike } \\
\text { Amount }\end{array}$ & $\frac{\text { Spike }}{\text { Result }}$ & $\underline{\text { Percent }}$ & $\frac{\text { Acceptable }}{\underline{\text { Range }}}$ & $\underline{\mathbf{R P D}}$ \\
\hline Pyridine & 0606E1 BLK-LCS & 80 & 43.5 & 54 & $10-130$ & \\
\hline Pyridine & 0606E1 BLK-LCSD & 80 & 39.4 & 49 & $10-130$ & 9.9 \\
\hline Pyridine & L0105291-12-MS & 80 & 37.9 & 47 & $10-130$ & \\
\hline 1,4-Dichlorobenzene (p-DCB) & 0606E1 BLK-LCS & 80 & 58.1 & 73 & $7-105$ & \\
\hline 1,4-Dichlorobenzene ( $\mathrm{p}-\mathrm{DCB}$ ) & 0606E1 BLK-LCSD & 80 & 49.8 & 62 & $7-105$ & 15.4 \\
\hline 1,4-Dichlorobenzene (p-DCB) & L0105291-12-MS & 80 & 45 & 56 & $7-105$ & \\
\hline Hexachloroethane & 0606E1 BLK-LCS & 80 & 49.6 & 62 & $43-104$ & \\
\hline Hexachloroethane & 0606E1 BLK-LCSD & 80 & 34.1 & 43 & $43-104$ & 37. \\
\hline Hexachloroethane & L0105291-12-MS & 80 & 36.9 & 46 & $43-104$ & \\
\hline Nitrobenzene & 0606E1 BLK-LCS & 80 & 66.1 & 83 & $28-124$ & \\
\hline Nitrobenzene & 0606E1 BLK-LCSD & 80 & 60.8 & 76 & $28-124$ & 8.4 \\
\hline Nitrobenzene & L0105291-12-MS & 80 & 53.4 & 67 & $28-124$ & \\
\hline Hexachlorobutadiene & 0606E1 BLK-LCS & 80 & 57.7 & 72 & $39-111$ & \\
\hline Hexachlorobutadiene & 0606E1 BLK-LCSD & 80 & 34.3 & 43 & $39-111$ & 50.9 \\
\hline Hexachlorobutadiene & L0105291-12-MS & 80 & 42.6 & 53 & $39-111$ & \\
\hline 2-Methylphenol & 0606E1 BLK-LCS & 80 & 55.6 & 70 & $30-130$ & \\
\hline 2-Methylphenol & 0606E1 BLK-LCSD & 80 & 53.4 & 67 & $30-130$ & 4. \\
\hline 2-Methylphenol & L0105291-12-MS & 80 & 46.6 & 58 & $30-130$ & \\
\hline 3,4-Methylphenol (isomeric pair) & 0606E1 BLK-LCS & 80 & 49.8 & 62 & $30-130$ & \\
\hline 3,4-Methylphenol (isomeric pair) & 0606E1 BLK-LCSD & 80 & 48.1 & 60 & $30-130$ & 3.5 \\
\hline 3,4-Methylphenol (isomeric pair) & L0105291-12-MS & 80 & 40.8 & 51 & $30-130$ & \\
\hline 2,4,6-Trichlorophenol & 0606El BLK-LCS & 80 & 75.1 & 94 & $43-110$ & \\
\hline 2,4,6-Trichlorophenol & 0606E1 BLK-LCSD & 80 & 76.5 & 96 & $43-110$ & 1.8 \\
\hline 2,4,6-Trichlorophenol & L0105291-12-MS & 80 & 69.2 & 87 & $43-110$ & \\
\hline 2,4,5-Trichlorophenol & 0606E1 BLK-LCS & 80 & 76.3 & 95 & $30-130$ & \\
\hline 2,4,5-Trichlorophenol & 0606E1 BLK-LCSD & 80 & 77.3 & 97 & $30-130$ & 1.3 \\
\hline 2,4,5-Trichlorophenol & L0105291-12-MS & 80 & 70.3 & 88 & $30-130$ & \\
\hline 2,4-Dinitrotoluene (DNT) & 0606E1 BLK-LCS & 80 & 92.2 & 115 & $50-111$ & \\
\hline 2,4-Dinitrotoluene (DNT) & 0606E1 BLK-LCSD & 80 & 88.5 & 111 & $50-111$ & 4.1 \\
\hline 2,4-Dinitrotoluene (DNT) & L0105291-12-MS & 80 & 65.4 & 82 & $50-111$ & \\
\hline Hexachlorobenzene & 0606E1 BLK-LCS & 80 & 83.5 & 104 & $41-125$ & \\
\hline Hexachlorobenzene & 0606E1 BLK-LCSD & 80 & 79.2 & 99 & $41-125$ & 5.3 \\
\hline Hexachlorobenzene & L0105291-12-MS & 80 & 61.6 & 77 & $41-125$ & \\
\hline Pentachlorophenol & 0606E1 BLK-LCS & 80 & 74 & 93 & $47-127$ & \\
\hline Pentachlorophenol & 0606E1 BLK-LCSD & 80 & 72.7 & 91 & $47-127$ & 1.8 \\
\hline Pentachlorophenol & L0105291-12-MS & 80 & 66.5 & 83 & $47-127$ & \\
\hline
\end{tabular}


CLIENT: Bechtel Nevada

PROJECT ID: V1124

PROJECT \#: 23081

TEST: $\quad$ TCLP/STLC Metals

MATRIX: Solid

$\triangle \quad$ PARAMETER

Mercury

Mercury

- Mercury
NEL Sample ID L5275HGTCLP-LCS

L0105275-01-MS

L0105275-01-MSD

\section{Spike \\ Spike}

Amount

0.05

0.05

0.05
Result

0.0515

0.0518

0.056

\section{Percent}

Recovery

103

104

112
Acceptable

Range RPD

$85-115$

$75-125$

$75-125$ 


$\begin{array}{ll}\text { CLIENT: } & \text { Bechtel Nevada } \\ \text { PROJECT ID: } & \text { V1124 } \\ \text { PROJECT \#: } & 23081 \\ \text { TEST: } & \text { TCLP/STLC Metals } \\ \text { MATRIX: } & \text { Solid }\end{array}$

\begin{tabular}{|c|c|c|c|c|c|c|}
\hline PARAMETER & NEL Sample ID & $\begin{array}{c}\text { Spike } \\
\text { Amount }\end{array}$ & $\frac{\text { Spike }}{\text { Result }}$ & $\frac{\text { Percent }}{\text { Recovery }}$ & $\frac{\text { Acceptable }}{\text { Range }}$ & $\underline{\text { RPD }}$ \\
\hline Arsenic & L5275I-LCS & 0.5 & 0.529 & 106 & $85-115$ & \\
\hline Arsenic & L0105275-01-MS & 0.5 & 0.553 & 111 & $75-125$ & \\
\hline Arsenic & L0105275-01-MSD & 0.5 & 0.547 & 109 & $75-125$ & 1.1 \\
\hline Silver & L5275I-LCS & 0.5 & 0.434 & 87 & $85-115$ & \\
\hline Silver & L0105275-01-MS & 0.5 & 0.45 & 90 & $75-125$ & \\
\hline Silver & L0105275-01-MSD & 0.5 & 0.455 & 91 & $75-125$ & 1.1 \\
\hline Barium & L5275I-LCS & 1 & 0.99 & 99 & $85-115$ & \\
\hline Barium & L0105275-01-MS & 1 & 1.2 & 120 & $75-125$ & \\
\hline Barium & L0105275-01-MSD & 1 & 1.19 & 119 & $75-125$ & 0.8 \\
\hline Cadmium & L5275I-LCS & 0.5 & 0.472 & 94 & $85-115$ & \\
\hline Cadmium & L0105275-01-MS & 0.5 & 0.484 & 97 & $75-125$ & \\
\hline Cadmium & L0105275-01-MSD & 0.5 & 0.505 & 101 & $75-125$ & 4.2 \\
\hline Chromium & L5275I-LCS & 0.5 & 0.478 & 96 & $85-115$ & \\
\hline Chromium & L0105275-01-MS & 0.5 & 0.504 & 101 & $75-125$ & \\
\hline Chromium & L0105275-01-MSD & 0.5 & 0.513 & 103 & $75-125$ & 1.8 \\
\hline Lead & L5275I-LCS & 1 & 0.928 & 93 & $85-115$ & \\
\hline Lead & L0105275-01-MS & 1 & 1.11 & 93 & $75-125$ & \\
\hline Lead & L0105275-01-MSD & 1 & 1.17 & 99 & $75-125$ & 6.2 \\
\hline Selenium & L5275I-LCS & 0.5 & 0.507 & 101 & $85-115$ & \\
\hline Selenium & L0105275-01-MS & 0.5 & 0.48 & 96 & $75-125$ & \\
\hline Selenium & L0105275-01-MSD & 0.5 & 0.485 & 97 & $75-125$ & 1. \\
\hline
\end{tabular}




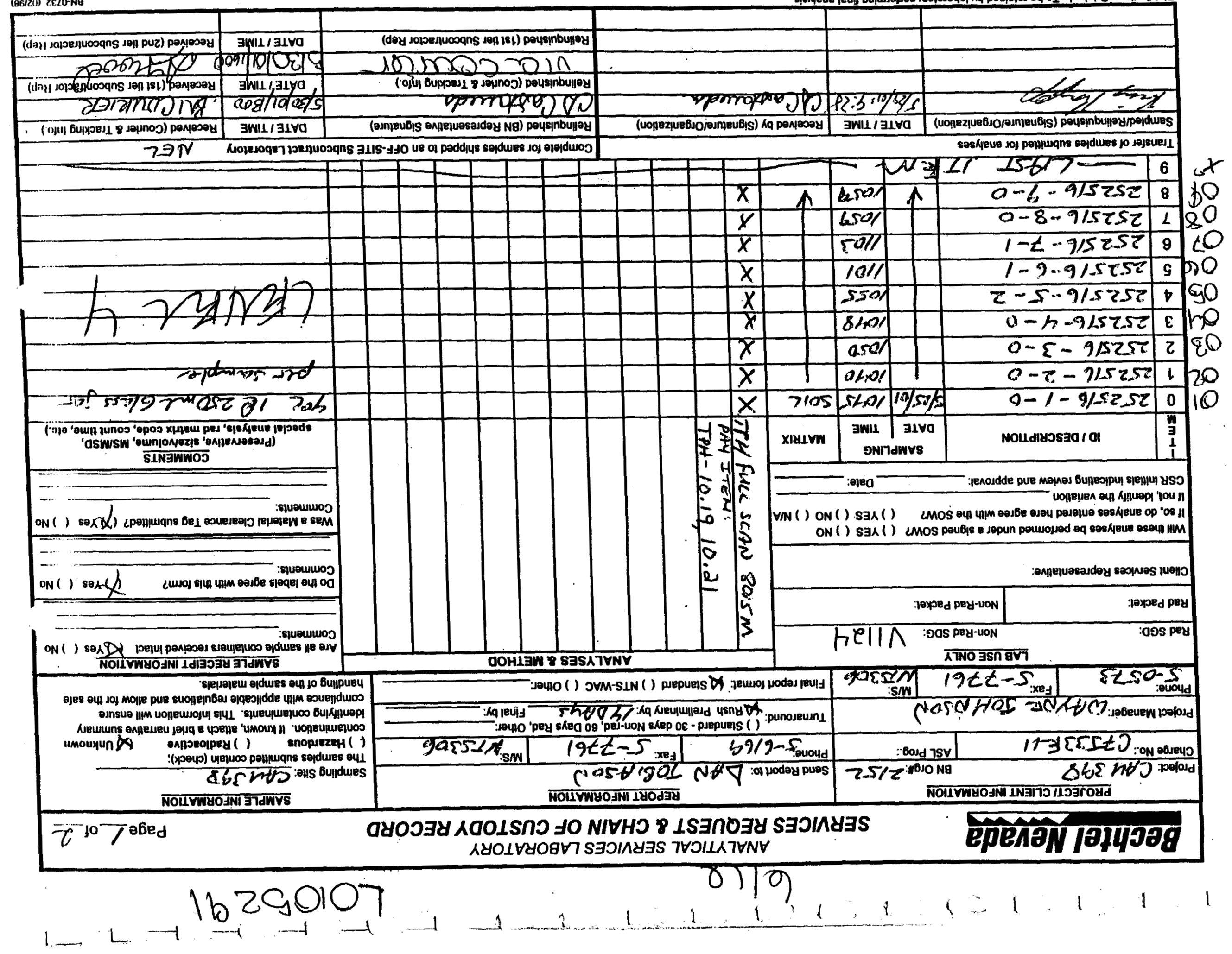


Cel6

\section{Beghtel I'evada ANALYTICAL SERVICES LABORATORY \\ SERVICES REQUEST \& CHAIN OF CUSTODY RECORD}

Paged of 2

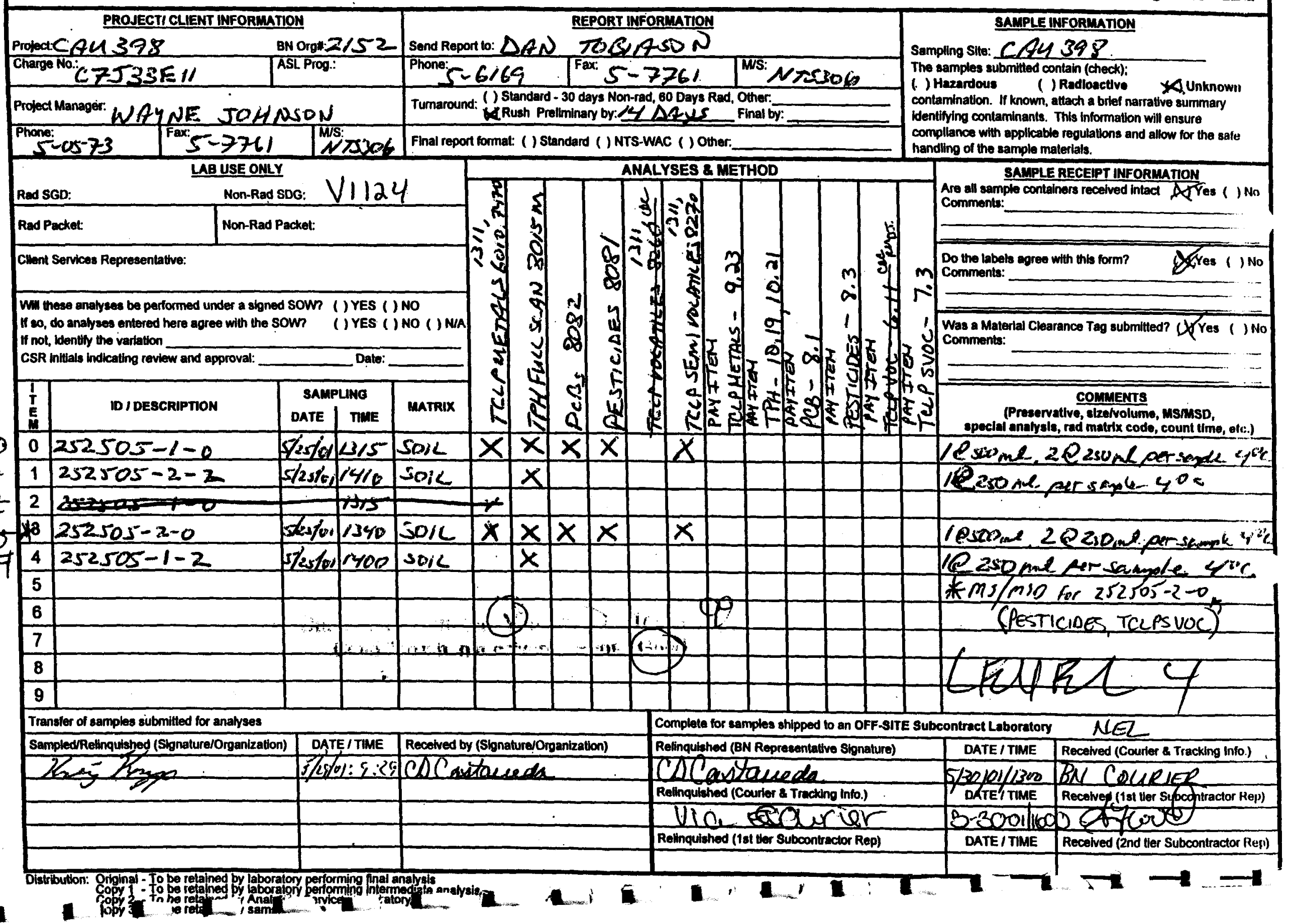




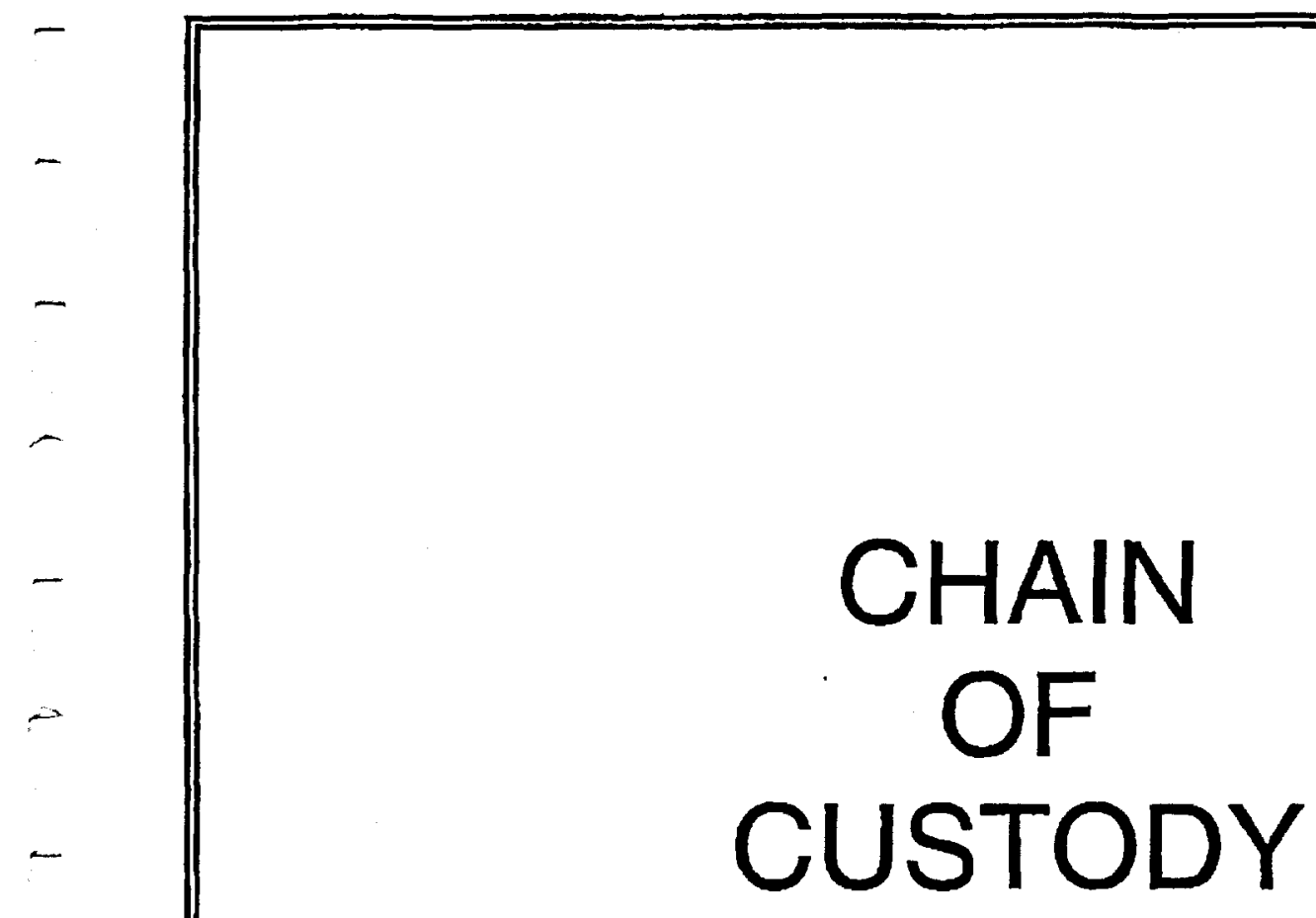

-

$-$

-

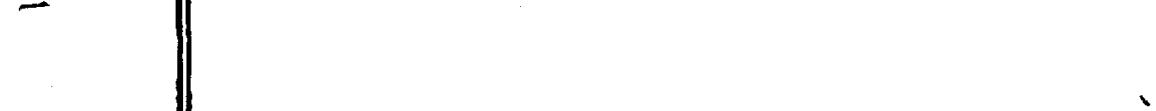




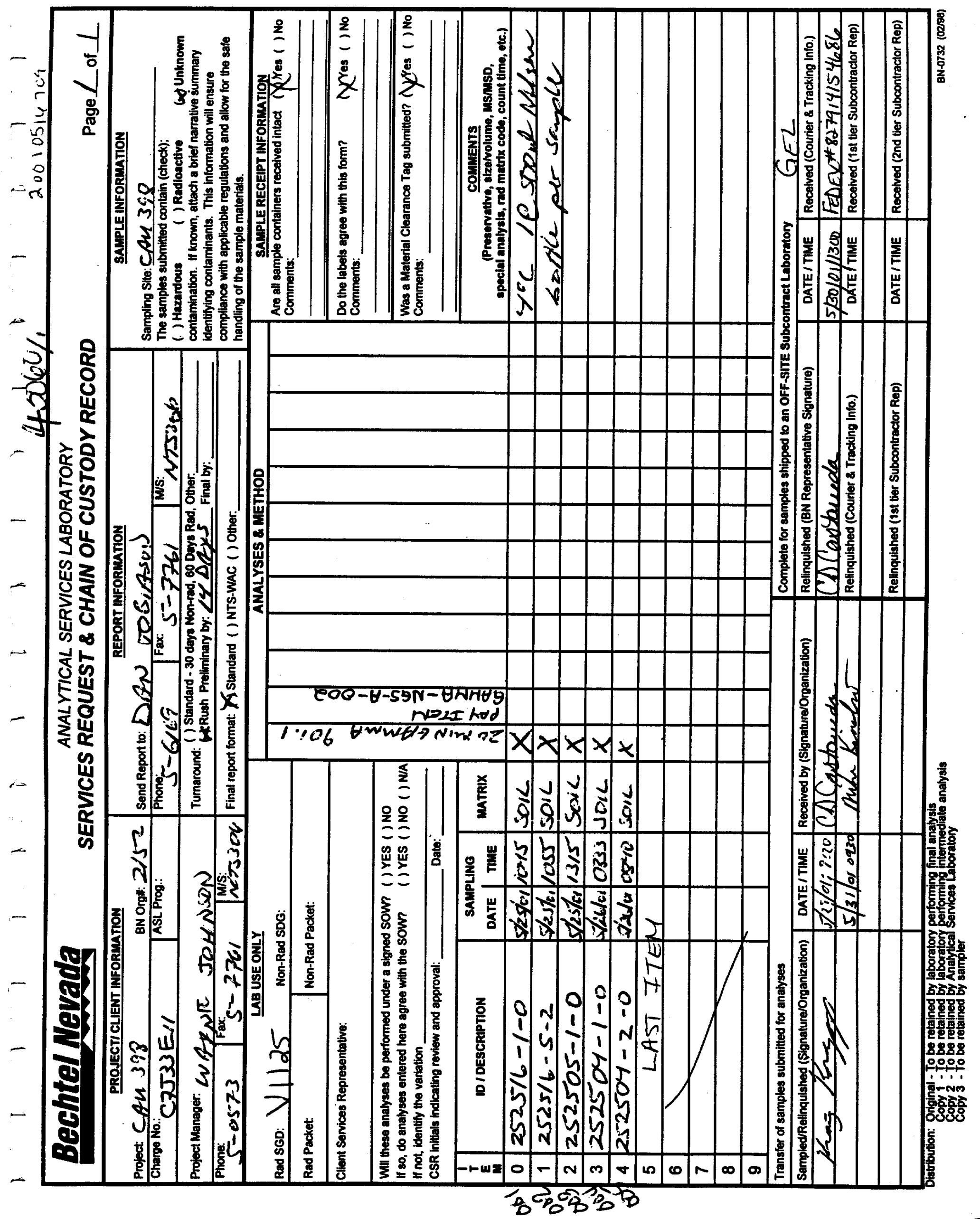




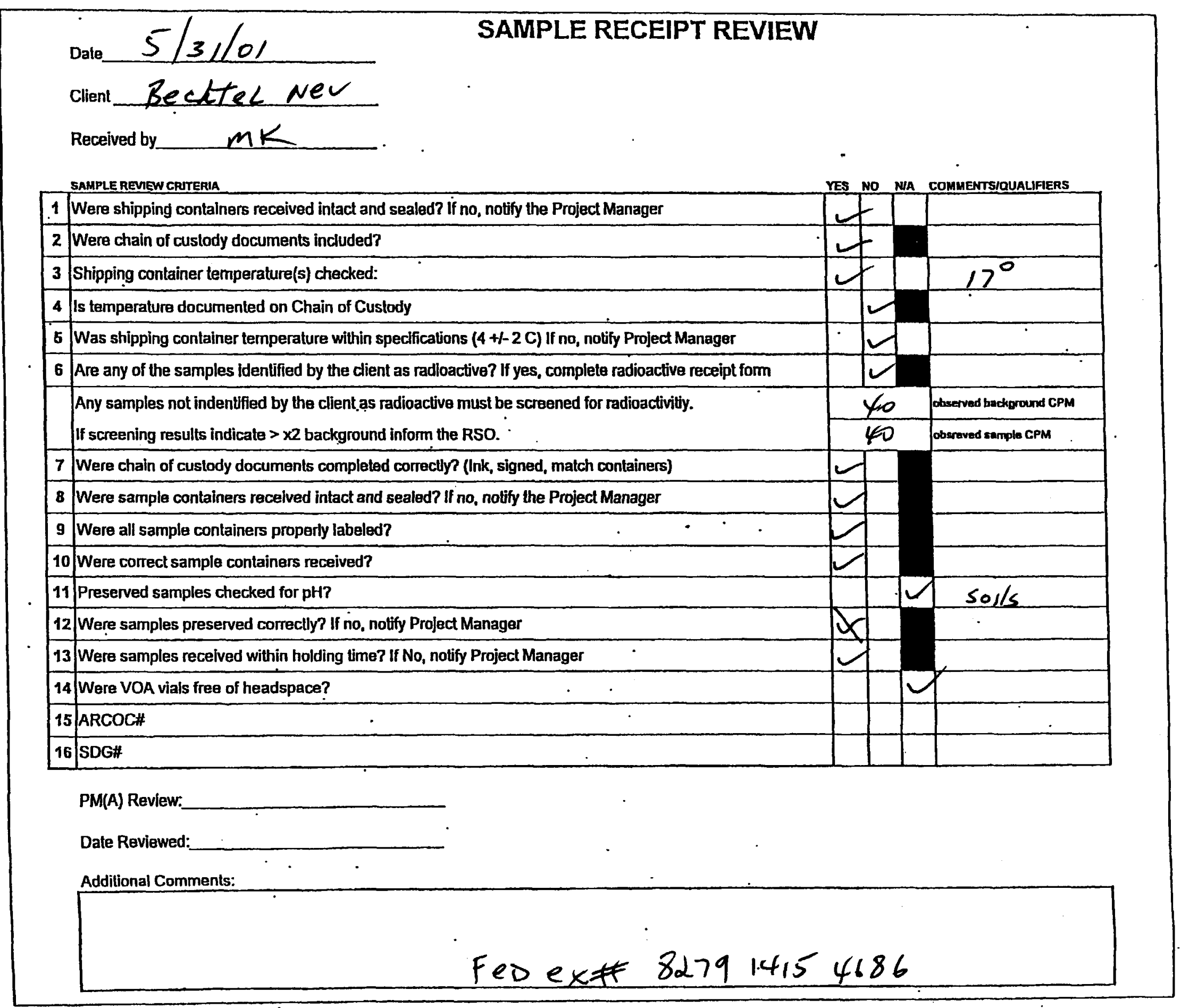


$-$

RADIOLOGICAL ANALYSIS 
-

$-$

$-$

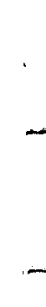

CASE 


\section{Radiochemistry Case Narrative \\ Bechtel Nevada Corp. (NEVA) \\ Workorder $\mathbf{4 3 0 6 0}$}

Method/Analysis Information

Batch Number:

Procedure:

Analytical Method:

Sample ID
43060001
43060002
43060003
43060004
43060005
1200015733
1200015734
1200015735

81497

Determination of Gamma Isotopes in Water and Soil

DOE EML HASL 300

\section{SOP Reference}

Procedures for preparation, analysis and reporting of analytical data are controlled by General Engineering Laboratories, Inc. as Standard Operating Procedures (SOP). The data discussed in this narrative has been prepared and analyzed in accordance with GL-RAD-A-013.

\section{Calibration Information:}

\section{Calibration Information}

All initial and continuing calibration requirements have been met.

\section{Standards Information}

Standard solution(s) for these analyses are NIST traceable and used before the expiration date(s).

\section{Sample Geometry}

All counting sources were prepared in the same geometry as the calibration standards.

\section{Quality Control (OC) Information:}

Blank Information

The blank volume is representative of the sample volume(s) in this batch.

\section{Designated QC}

The following sample(s) was used for QC: 43060001 .

QC Information

All of the QC samples met the required acceptance limits.

\section{Technical Information:}

Holding Time

All sample procedures for this sample set were performed within the required holding time.

Preparation Information

All preparation criteria have been met for these analyses.

Sample Re-prep/Re-analysis 
None of the samples in this sample set required reprep or reanalysis.

\section{Miscellaneons Information:}

NCR Documentation

No NCR were generated for the preparation or analysis of this sample set.

\section{Manual Integration}

No manual integrations were performed on data in this batch.

\section{Additional Comments}

The following data was rejected due to low abundance: Sample 1200015733; U-238, K-40.

The following was rejected due to no valid peak: Sample 1200015734; Cs-137.

$\mathrm{K}-40$ is being reported in all samples in batch due to activities above $5 \mathrm{pCi} / \mathrm{g}$ and $\mathrm{MDA}$.

\section{Review Vallidation:}

GEL requires all analytical data to be verified by a qualified data validator. In addition, all data designated for CLP or CLP-like packaging will receive a third level validation upon completion of the data package.

The following data validator verified the information presented in this case narrative:

Reviewer:

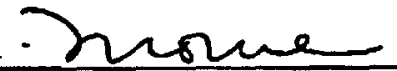

Date: 


\title{
GENERAL ENGINEERING LABORATORIES
}

Meeting today's needs with a vision for tomornow.

\section{Certificate of Analysis}

\author{
Company : Bechtel Nevada Corp. \\ Aditress: 2621 Losee Road \\ North Las Vegas, NV 89030-4134 \\ Contact: Ted Redding \\ Project: Enviormmental Rad Services
}

Repon Date: June 13, 2001

Page 1 of 1

\begin{tabular}{|c|c|c|c|c|c|c|c|c|c|c|c|c|}
\hline \multirow[b]{2}{*}{ Parmmeter } & \multicolumn{2}{|c|}{$\begin{array}{l}\text { Client Sample D: } \\
\text { Sample ID: } \\
\text { Matrix: } \\
\text { Collect Date: } \\
\text { Receive Date: } \\
\text { Collector: }\end{array}$} & & \multicolumn{2}{|c|}{$\begin{array}{l}252516-1-0 \\
43060001 \\
\text { Sol } \\
25-M A Y-01 \\
31-M A Y-01 \\
\text { Client }\end{array}$} & \multirow[b]{2}{*}{$\mathbf{R}$} & \multirow{2}{*}{$\begin{array}{c}\text { Proiect: } \\
\text { Client D: } \\
\text { Units }\end{array}$} & \multicolumn{3}{|c|}{$\begin{array}{l}\text { NEVA102000 } \\
\text { NEVA001 }\end{array}$} & \multirow[b]{2}{*}{ Time } & \multirow[b]{2}{*}{ Bateh Mid } \\
\hline & Qualiner & Resuls & & $\mathbf{D L}$ & TPU & & & $\mathbf{D F}$ & Anal & yot Date & & \\
\hline \multicolumn{13}{|l|}{ Bed Garame Spec } \\
\hline \multicolumn{13}{|c|}{ Gammaspec, Gamma, solid } \\
\hline $\begin{array}{l}\text { Americium-241 } \\
\text { Cesium-137 } \\
\text { Potassium-40 } \\
\text { Uranium-235 } \\
\text { Uranium-238 }\end{array}$ & $\begin{array}{l}\mathbf{U} \\
\mathbf{U} \\
\mathbf{U} \\
\mathbf{U}\end{array}$ & $\begin{array}{r}0.0284 \\
0.0255 \\
35.3 \\
0.0139 \\
0.0424\end{array}$ & $\begin{array}{r}+/-0.0917 \\
+/-0.020 \\
+-4.15 \\
+/-0.105 \\
+/-1.77\end{array}$ & $\begin{array}{r}0.150 \\
0.0263 \\
0.194 \\
0.181 \\
1.24\end{array}$ & $\begin{array}{r}0.0917 \\
0.020 \\
4.15 \\
0.105 \\
1.77\end{array}$ & $\begin{array}{r}0.200 \\
1.00 \\
0.200 \\
2.00\end{array}$ & $\begin{array}{l}\mathrm{pCi} / \mathrm{g} \\
\mathrm{pCi} / \mathrm{g} \\
\mathrm{pCi} / \mathrm{g} \\
\mathrm{pCi} / \mathrm{g} \\
\mathrm{pCi} / \mathrm{g}\end{array}$ & & CRB & 061110 & 12342 & 814971 \\
\hline
\end{tabular}

The following Prep Methods were performed

\begin{tabular}{llllll}
\hline Method & Description & Analyst & Date & Time & Prep Batch \\
\hline Dry Soil Prep & Dry Soil Prep RAD A-021,A-021B,A-026 & CCM1 & 06/01/01 & 2038 & 81015
\end{tabular}

The following Analytical Methods were performed

\begin{tabular}{ll} 
Method & Deserption \\
\hline 1 & DOE EML HASL 300
\end{tabular}

Notes:

The Qualifiers in this report are defined as follows :

** Indicates the analyte is a surrogate compound.

$<$ Actual result is less than amount reported

$>$ Actual result is greater than amoint reported

J Indicates an estimated value. The result was greater than the detection limit, but less than the reporting limit.

U Indicates the compound was analyzed for but not detected above the detection limit

The above sample is reported on an "as received" basis.

- This data report has been prepared and reviewed in accordance with General Engineering Laboratories, Inc. standard operating procedures. Please direct any questions to your Project Manager, Stacy L. Griffin at 843-556-8171 Ext. 4264.

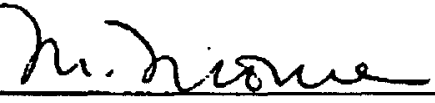

Reviewed by 


\section{GENERAL ENGINEERING LABORATORIES}

Meeing today's needs with a vision for tomorrow.

\section{Certificate of Analysis}

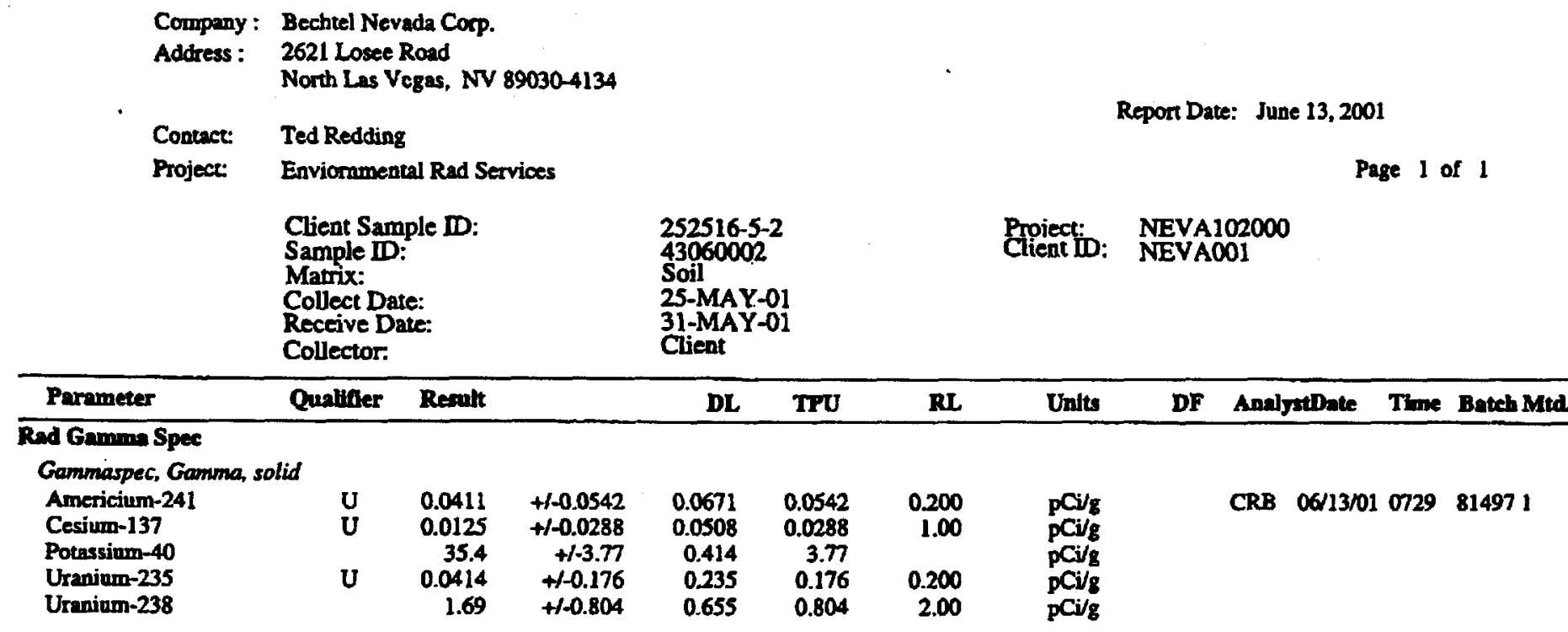

The following Prep Methods were performed

\begin{tabular}{|c|c|c|c|c|c|}
\hline Method & Description & Andyt & Date & Thre & Prep Batch \\
\hline Dry Soil Prep & Dry Soil Prep RAD A-021,A-021B,A-026 & CCM1 & $06 / 01 / 01$ & 2038 & 81015 \\
\hline \multicolumn{6}{|c|}{ The following Analytical Methods vere performed } \\
\hline Method & Description & & & & \\
\hline
\end{tabular}

1 DOE EML HASL 300

Notes:

The Qualifiers in this report are defined as follows :

** Indicates the analyte is a surrogate compound.

$<$ Actual result is less than amount reported

$>$ Actual result is greater than amount reported

J Indicates an estimated value. The result was greater than the detection limit, but less than the reporting limit.

$U$ Indicates the compound was analyzed for but not detected above the detection limit

The above sample is reported on an "as received" basis.

This data report has been prepared and reviewed in accordance with General Engineering Laboratories, Inc.

standard operating procedures. Please direct any questions to your Project Manager, Stacy L. Griffin at 843-556-8171 ExL 4264.

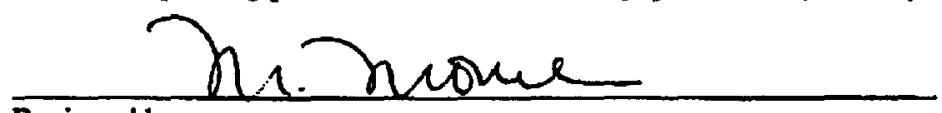

Reviewed by 


\section{GENERAL ENGINEERING LABORATORIES}

Meeting today's needs with a vision for tomorrow.

\section{Certificate of Analysis}

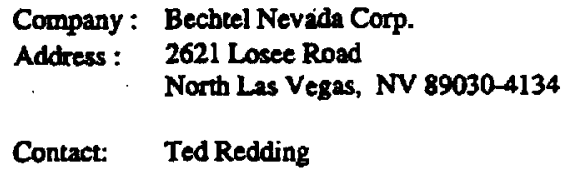

Report Date: June 13, 2001

Page 1 of 1

$\begin{array}{ll}\text { Client Sample ID: } & 252505-1-0 \\ \text { Sample ID: } & 43060003 \\ \text { Matrix: } & \text { Soil } \\ \text { Collect Date: } & 25-\mathrm{MAY}-01 \\ \text { Receive Date: } & 31-\mathrm{MAY}-01 \\ \text { Collector: } & \text { Client }\end{array}$

\begin{tabular}{|c|c|c|c|c|c|c|c|c|c|c|c|}
\hline Parameter & Quallitier & Resplt & & DL & TPU & $\mathbf{R L}$ & Untts & $\mathbf{D F}$ & Analyst Date & Time & Batch Mid. \\
\hline \multicolumn{12}{|l|}{ Rad Gamma Spec } \\
\hline \multicolumn{12}{|c|}{ Gammaspec, Gamma, solid } \\
\hline $\begin{array}{l}\text { Americium-241 } \\
\text { Cesium-137 } \\
\text { Potassium-40 } \\
\text { Uranium-235 } \\
\text { Uranium-238 }\end{array}$ & $\mathbf{U}$ & $\begin{array}{r}-0.0166 \\
0.0437 \\
31.4 \\
0.128 \\
1.50\end{array}$ & $\begin{array}{r}+-0.105 \\
+/-0.0237 \\
+/-3.72 \\
+/-0.189 \\
+/-1.31\end{array}$ & $\begin{array}{r}0.171 \\
0.0305 \\
0.220 \\
0.195 \\
1.36\end{array}$ & $\begin{array}{r}0.105 \\
0.0237 \\
3.72 \\
0.189 \\
1.31\end{array}$ & $\begin{array}{r}0.200 \\
1.00 \\
\\
0.200 \\
2.00\end{array}$ & $\begin{array}{l}\mathrm{pCi} / \mathrm{g} \\
\mathrm{pCi/g} \\
\mathrm{pCi/g} \\
\mathrm{pCi} / \mathrm{g} \\
\mathrm{pCi} / \mathrm{g}\end{array}$ & & $06 / 13 / 0$ & 0730 & 814971 \\
\hline
\end{tabular}

The following Prep Methods were performed

$\begin{array}{llllll}\text { Method } & \text { Description } & \text { Analyst } & \text { Date } & \text { Time } & \text { Prep Batch } \\ \text { Dry Soil Prep } & \text { Dry Soil Prep RAD A-021,A-021B,A-026 } & \text { CCM1 } & 06 / 01 / 01 & 2038 & 81015\end{array}$

The following Analytical Methods were performed Method Description

1 DOE EML HASL 300

Notes:

The Qualifiers in this report are defined as follows :

** Indicates the analyte is a surrogate compound.

$<$ Actual result is less than amount reported

$>$ Actual result is greater than amount reponted

J Indicates an estimated value. The result was greater than the detection limit, but less than the reporting limit.

U Indicates the compound was analyzed for but not detected above the detection limit

The above sample is reported on an "as received" basis.

This data report has been prepared and reviewed in accordance with General Engineering Laboratories, Inc.

standard operating procedures. Please direct any questions to your Project Manager, Stacy L. Griffin at 843-556-8171 Ext. 4264.

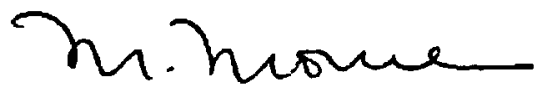

- Reviewed by 


\section{GENERAL ENGINEERING LABORATORIES}

Meeting today'sneeds with a vision for tomorrow.

\section{Certificate of Analysis}

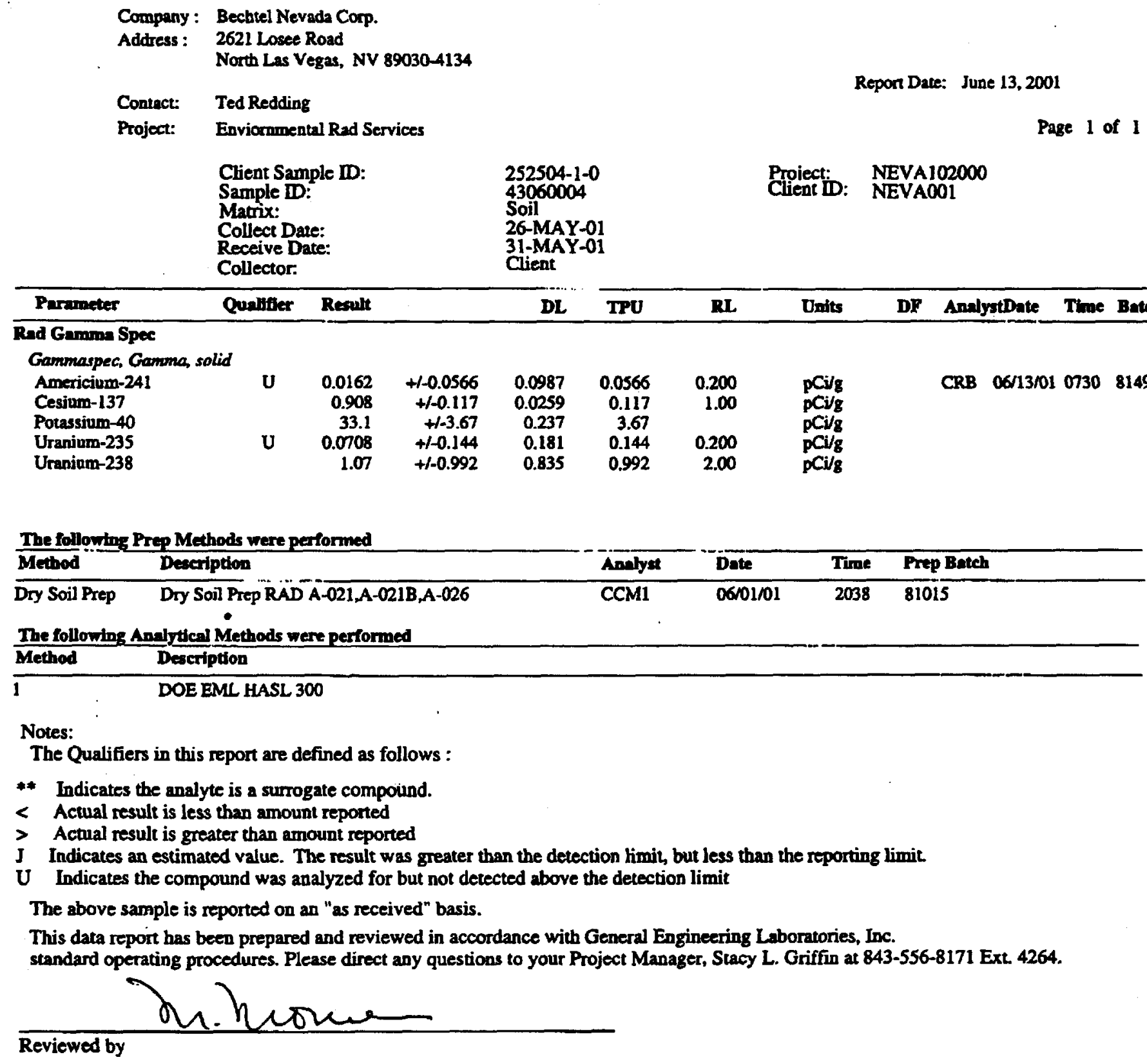




\title{
GENERAL ENGINEERING LABORATORIES
}

Mecting today's needs with a vision for tomorrow.

\section{Certificate of Analysis}

\author{
Company : Bechtel Nevada Corp. \\ Address : 2621 Losee Road \\ North Las Vegas, NV $89030-4134$ \\ Contact: Ted Redding \\ Project: Enviommental Rad Services
}

Report Date: June 13, 2001

Page 1 of 1

Client Sample ID:

Sample ID:

Matrix:

Collect Date:

Receive Date:

Collector:
252504-2-0 Proiect: NEVA102000

43060005 Client ID: NEVAO01

26-MAY-01

31-MAY-01

Client

\begin{tabular}{|c|c|c|c|c|c|c|c|c|c|c|c|c|}
\hline Parameter & Qustiffer & Rewalt & & DL & TPU & $\mathbf{R I}$ & Units & $\mathbf{D r}$ & Anal & yetbate & Thine & Betoh Mt \\
\hline \multicolumn{13}{|c|}{ Rad Gamman Spec. } \\
\hline \multicolumn{13}{|c|}{ Gammaspec, Ganma, solid } \\
\hline $\begin{array}{l}\text { Americium-241 } \\
\text { Cesium-137 } \\
\text { Potassium-40 } \\
\text { Uranium-235 } \\
\text { Uranium-238 }\end{array}$ & $\underset{\mathbf{U}}{\mathbf{U}}$ & $\begin{array}{r}0.0343 \\
0.122 \\
29.6 \\
0.040 \\
1.01\end{array}$ & $\begin{array}{r}+/-0.151 \\
+/-0.0497 \\
+/-3.65 \\
+/-0.113 \\
+/-1.73\end{array}$ & $\begin{array}{r}0.250 \\
0.0377 \\
0.338 \\
0.212 \\
1.78\end{array}$ & $\begin{array}{r}0.151 \\
0.0497 \\
3.65 \\
0.113 \\
1.73\end{array}$ & $\begin{array}{r}0.200 \\
1.00 \\
0.200 \\
2.00\end{array}$ & $\begin{array}{l}\mathrm{pCi} / \mathrm{g} \\
\mathrm{pCi} / \mathrm{g} \\
\mathrm{pCi} / \mathrm{g} \\
\mathrm{pCi} / \mathrm{g} \\
\mathrm{pCi} / \mathrm{g}\end{array}$ & & CRB & $06 / 13 / 01$ & 0730 & 814971 \\
\hline
\end{tabular}

The following: Prep Methods were performed

\begin{tabular}{llllll} 
Method & Description & Analgst & Date & Time & Prep Batch \\
\hline Dry Soil Prep & Dry Soil Prep RAD A-021,A-021B,A-026 & CCM1 & $06101 / 01$ & 2038 & 81015
\end{tabular}

The following Anglytical Methods were performed

Method Description

- DOE EML HASL 300

Notes:

The Qualifiers in this report are defined as follows:

- Indicates the analyte is a surrogate compound.

$<$ Actual result is less than amount reported

$>$ Actual result is greater than amount reported

J Indicates an estimated value. The result was greater than the detection limit, but less than the reporting limit

- U Indicates the compound was analyzed for but not detected above the detection limit

The above sample is reported on an "as received" basis.

This data report has been prepared and reviewed in accordance with General Engineering Laboratories, Inc.

standard operating procedures. Please direct any questions to your Project Manager, Stacy L. Griffin at 843-556-8171 Ext. 4264.

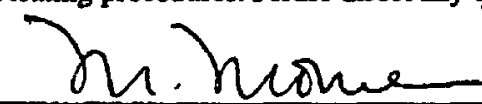

- Reviewed by. 


\section{GENERAL ENGINEERING LABORATORIES}

Meeting today's reeds with a vision for romornow.

QC Summary

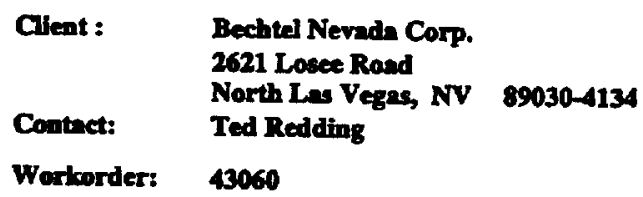

Report Date: June 13, 2001

Page 1 of 2

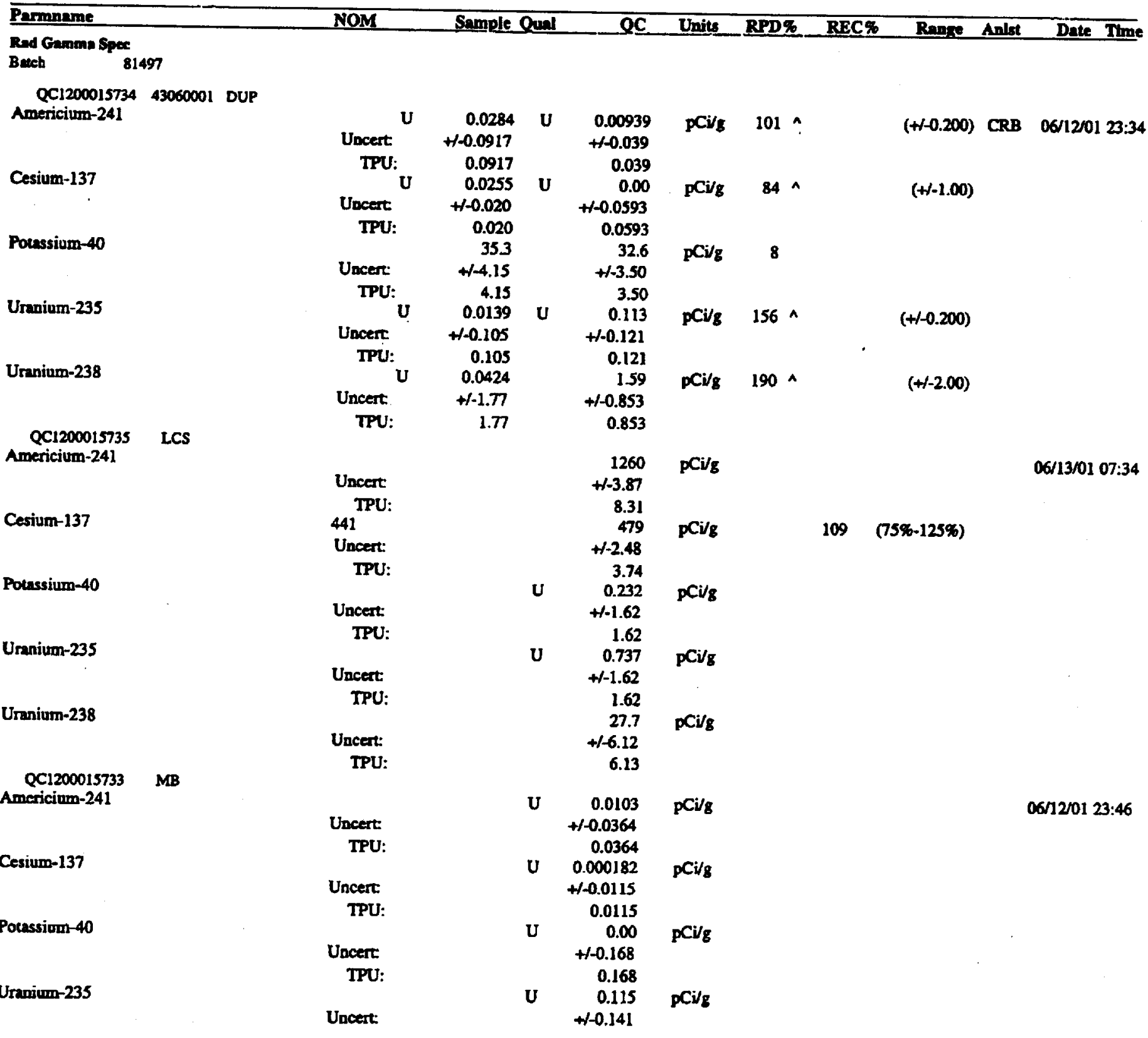

PO Box 30712 • Charleston, SC 29417 • 2040 Savage Road 29407

(843) 556-8171 • Fax (843) 766-1178 


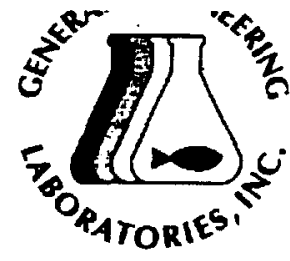

Wortzorder: 43060

\section{GENERAL ENGINEERING LABORATORUES}

Meeting loday:s needs with a-vision for tomormw.

\section{QC Summary}

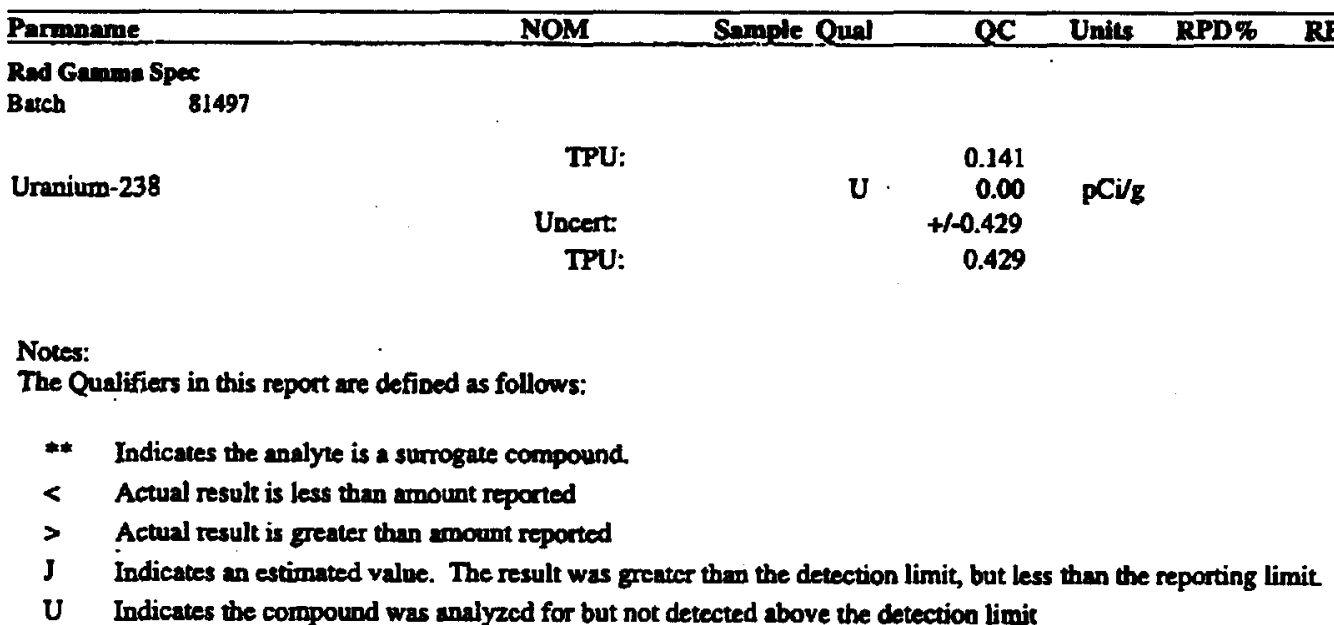

N/A indicates that spike recovery limits do not apply when sample concentration exceeds spike conc. by a factor of 4 or more.

n The Relative Percent Difference (RPD) obtained from the sample duplieate (DUP) is evaluated against the acceptence criteria when the ample is greater than five times (5X) the contract required detection limit (RL). In cases where either the sample or duplicate value is less than $5 X$ the $R L$, a control limit of $+\%$ - the $R L$ is used to evaluate the DUP result.

For PS, PSD, and SDIIT results, the values listed are the measured amounts, not finvl concentrations. 


$\begin{array}{ll}\text { CLIENT: } & \text { Bechtel Nevada } \\ & \text { P.O. Box 98521, M/S NTS273 } \\ & \text { Las Vegas, NV 89193-8521 } \\ & \text { Ted Redding }\end{array}$

PROJECT NAME: $\quad$ V1126

NEL ORDER ID: L0105290

PROJECT NUMBER: 23081

Attached are the analytical results for samples in support of the above referenced project.

Samples submitted for this project were not sampled by NEL Laboratories. Samples were received by NEL in good condition, under chain of custody on 5/30/01.

Should you have any questions or comments, please feel free to contact our Client Services department at (702) $657-1010$.

\section{Some QA results have been flagged as follows:}

C - Sample concentration is a least 5 times greater than spike contribution. Spike recovery criteria do not apply.

ת1 - The batch MS and/or MSD were outside acceptance limits. The batch LCS was acceptable.

Some surrogate results have been flagged as follows:

D - Sample required dilution. Sample QC results were diluted outside the calibrated range.
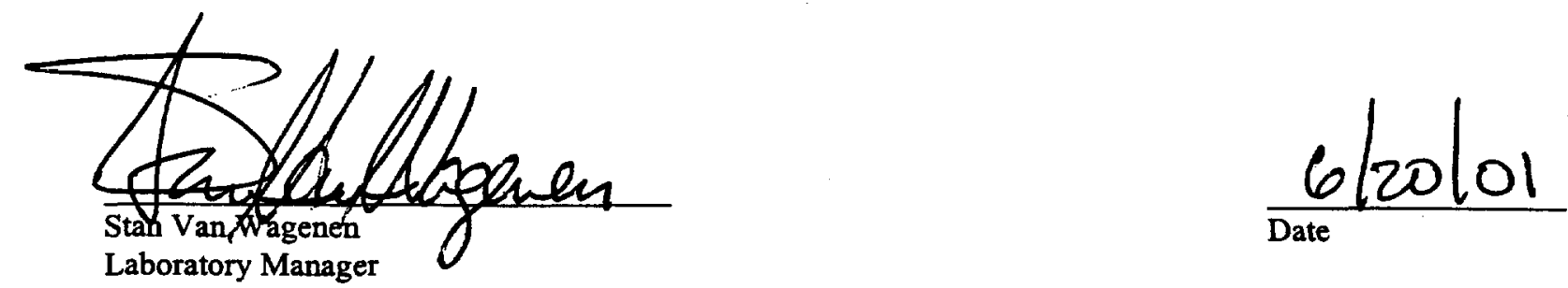

CERTIFICATIONS:

\begin{tabular}{lllllllll}
\hline & Reno & Las Vegas & S. Califormia & & \multicolumn{2}{c}{ Reno } & Las Vegas & S. California \\
\cline { 2 - 4 } & AZizona & AZ0518 & AZ0605 & & Idaho & Certified & Certified & \\
California & 1707 & 2002 & 2264 & & Montana & Certified & Certified & \\
US Army Corps & Certified & Certified & & Nevada & NV033 & NV052 & CA084 \\
of Engineers & & & & L.A.C.S.D. & & & 10228
\end{tabular}


NEL LABORATORIES

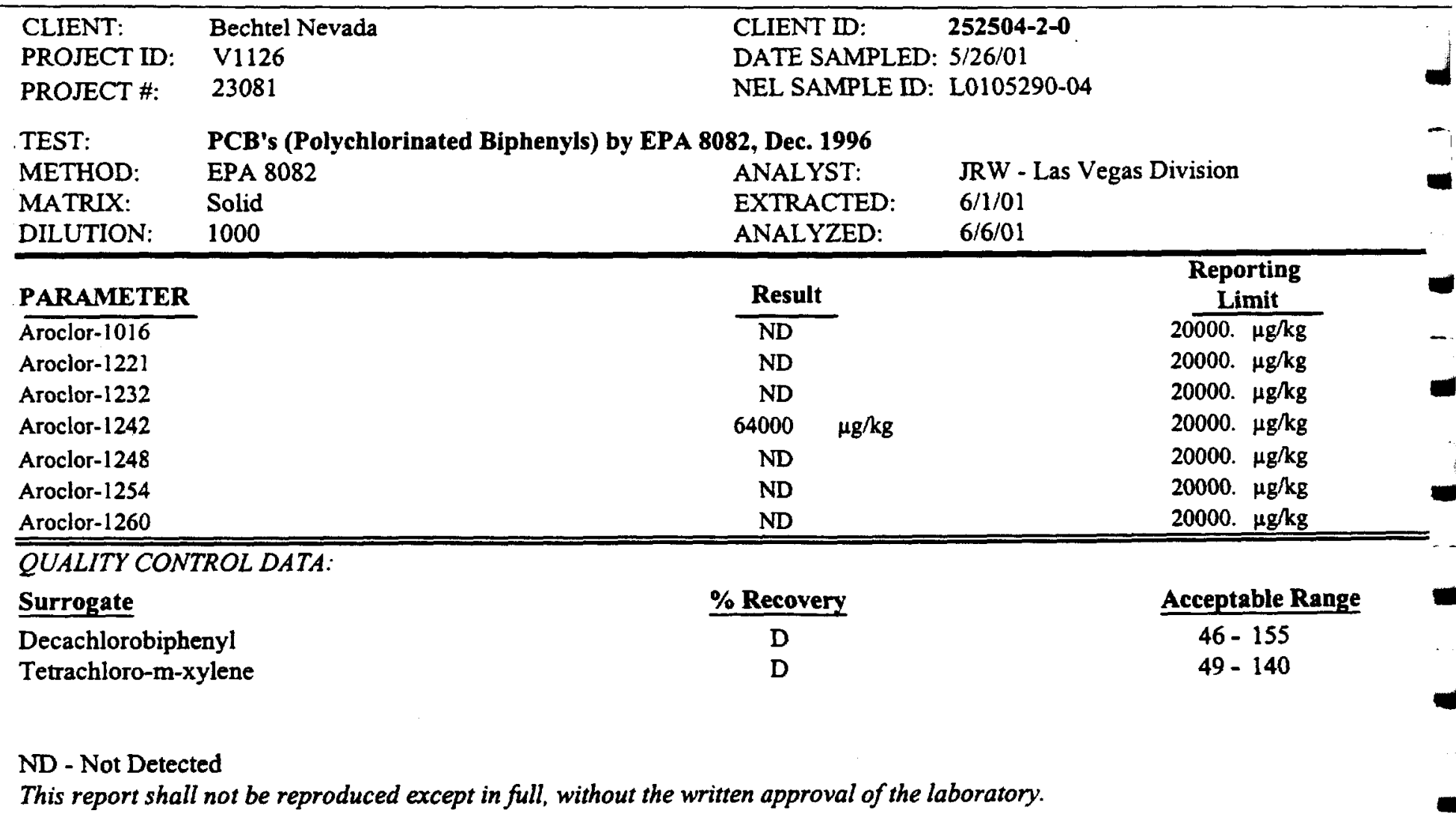


NEL LABORATORIES

CLIENT: Bechtel Nevada

PROJECT ID: V1126

PROJECT \#: 23081
CLIENT ID: 252504-3-0

DATE SAMPLED: $5 / 26 / 01$

NEL SAMPLE ID: L0105290-05
- TEST:

METHOD: MATRIX: DILUTION:
PCB's (Polychlorinated Biphenyls) by EPA 8082, Dec. 1996

\section{EPA 8082}

Solid

1000
ANALYST:

EXTRACTED:

ANALYZED:
JRW - Las Vegas Division 6/1/01 $6 / 6 / 01$

\begin{tabular}{cc} 
Result & $\begin{array}{c}\text { Reporting } \\
\text { Limit }\end{array}$ \\
\cline { 1 - 1 } ND & $20000 . \mu \mathrm{g} / \mathrm{kg}$ \\
ND & $20000 . \mu \mathrm{g} / \mathrm{kg}$ \\
ND & $20000 . \mu \mathrm{g} / \mathrm{kg}$ \\
ND & $20000 . \mu \mathrm{g} / \mathrm{kg}$ \\
ND & $20000 . \mu \mathrm{g} / \mathrm{kg}$ \\
$920000 \quad \mu \mathrm{g} / \mathrm{kg}$ & $20000 . \mu \mathrm{g} / \mathrm{kg}$ \\
ND & $20000 . \mu \mathrm{g} / \mathrm{kg}$ \\
\hline
\end{tabular}

- $\overline{\text { QUALITY CONTROL DATA: }}$

\section{Surrogate}

Decachlorobiphenyl

Tetrachloro-m-xylene

\section{$\%$ Recovery}

D

D

\section{Acceptable Range}

$46-155$

$49-140$

\section{ND - Not Detected}

This report shall not be reproduced except in full, without the written approval of the laboratory. 
NEL LABORATORIES

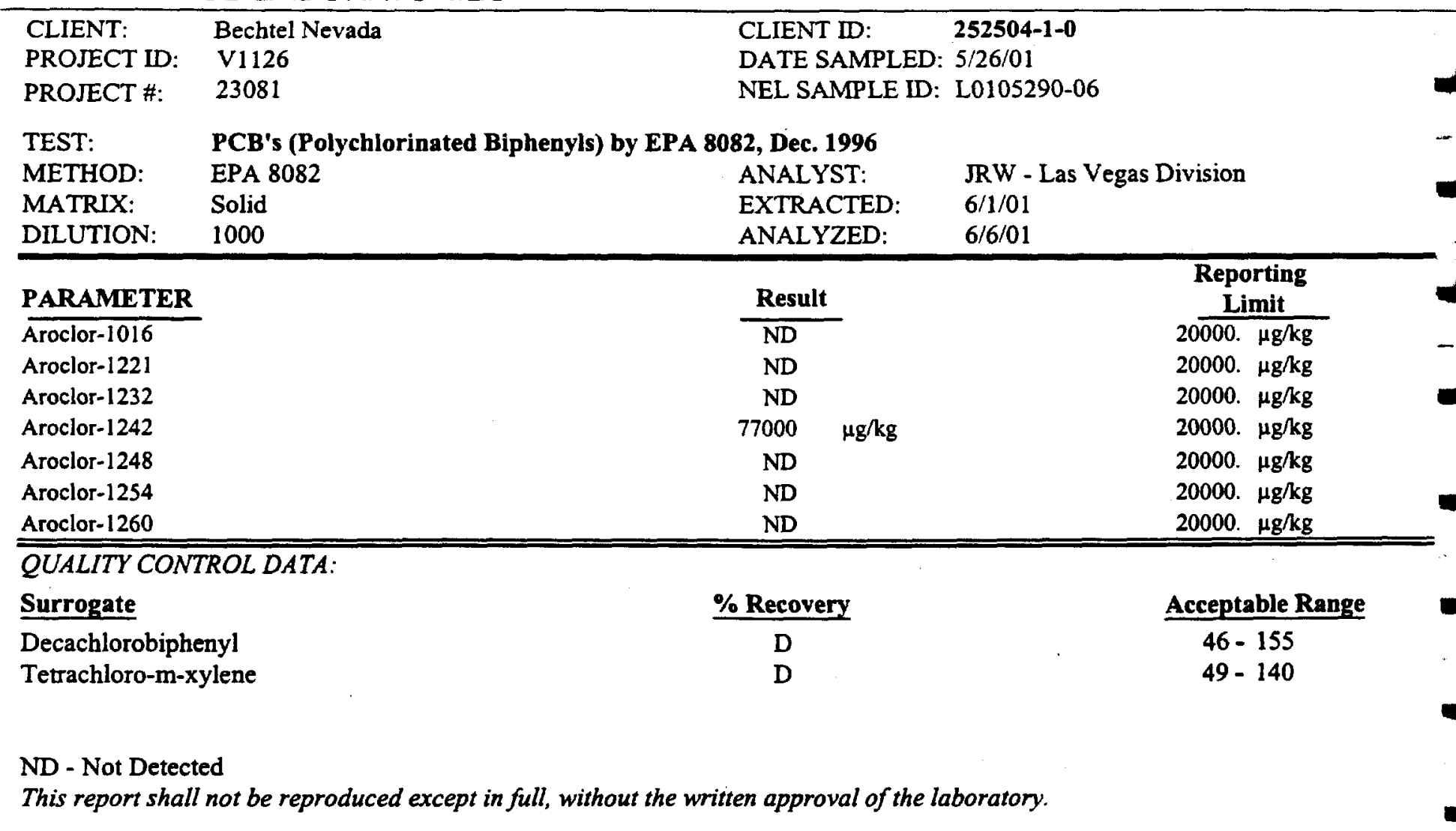


NEL LABORATORIES

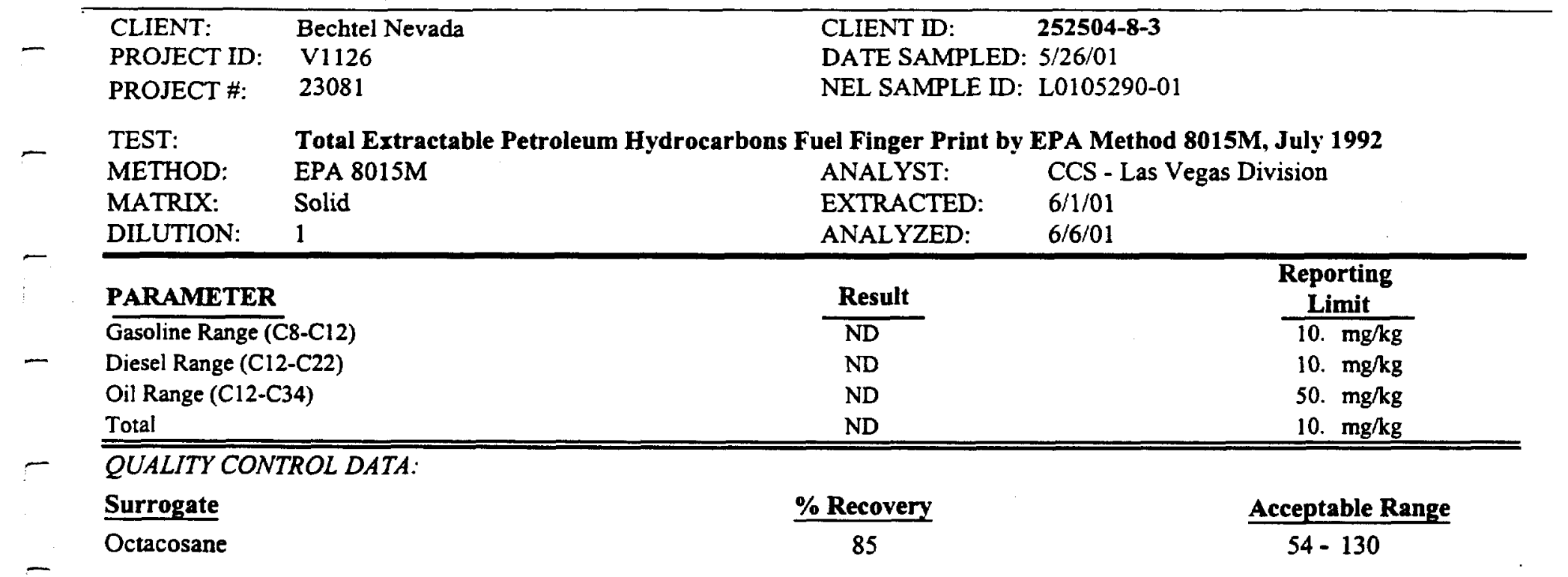

- ND - Not Detected

This report shall not be reproduced except in full, without the written approval of the laboratory. 
NEL LABORATORIES

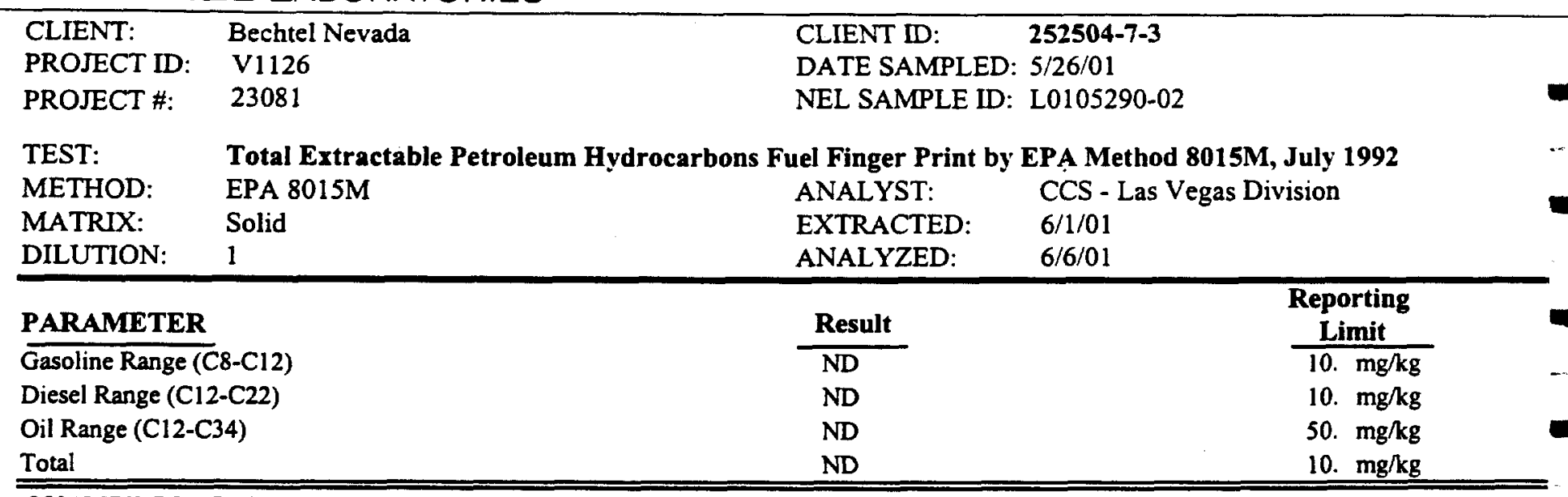

QUALITY CONTROL DATA:

Surrogate

Octacosane
\% Recovery

87

\section{Acceptable Range}

$54-130$

ND - Not Detected

This report shall not be reproduced except in full, without the written approval of the laboratory. 
NEL LABORATORIES

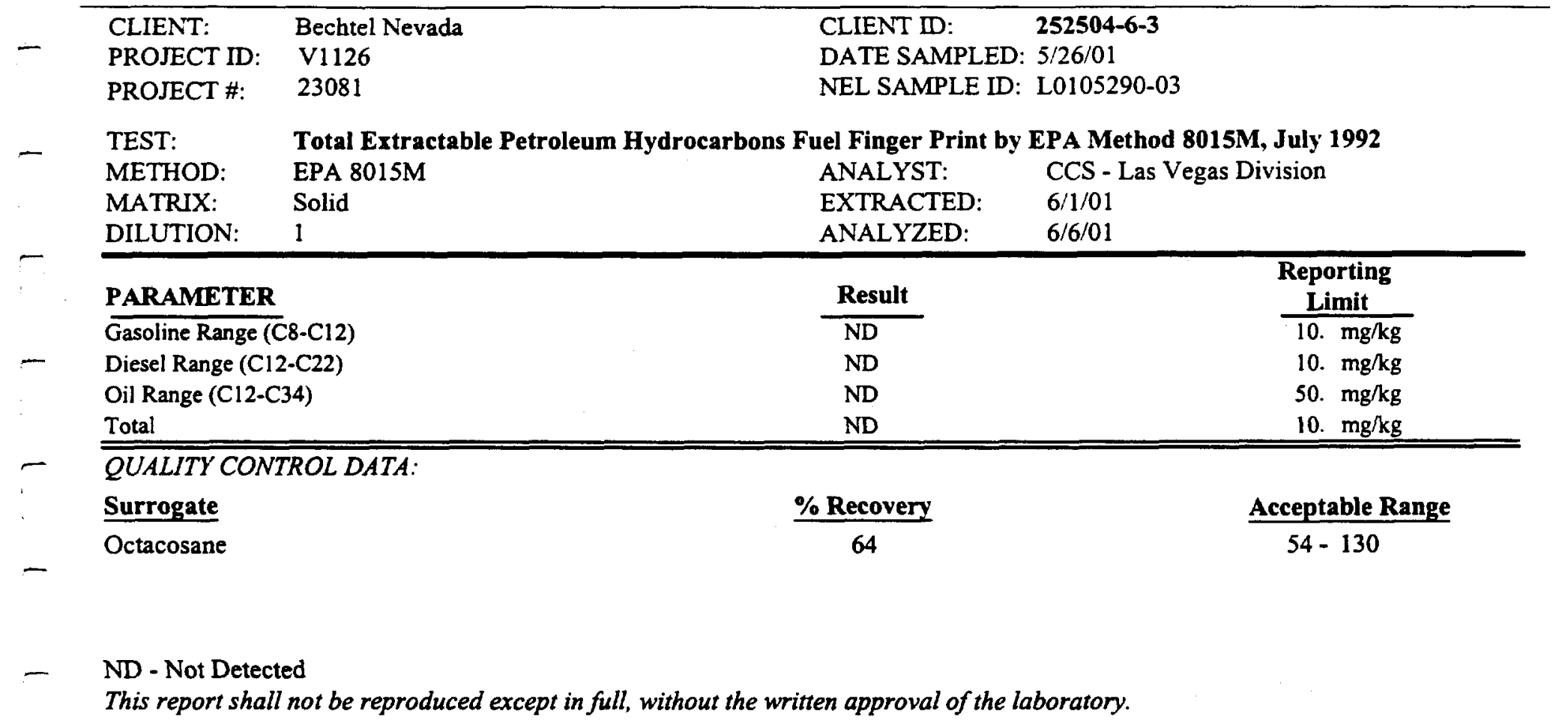


NEL LABORATORIES

$\begin{array}{llll}\text { CLIENT: } & \text { Bechtel Nevada } & \text { CLIENT ID: } & \mathbf{2 5 2 5 0 4 - 2 - 0} \\ \text { PROJECT ID: } & \text { V1126 } & \text { DATE SAMPLED: } 5 / 26 / 01 \\ \text { PROJECT \#: } & 23081 & \text { NEL SAMPLE ID: } & \text { L0105290-04 }\end{array}$

TEST: $\quad$ Total Extractable Petroleum Hydrocarbons Fuel Finger Print by EPA Method 8015M, July 1992

METHOD: $\quad$ EPA 8015M

MATRIX: Solid

ANALYST: $\quad$ CCS - Las Vegas Division

DILUTION: $\quad 2$

EXTRACTED: $\quad 6 / 1 / 01$

ANALYZED: $\quad 6 / 6 / 01$

\begin{tabular}{|c|c|c|}
\hline PARAMETER & Result & $\begin{array}{l}\text { Reporting } \\
\text { Limit }\end{array}$ \\
\hline 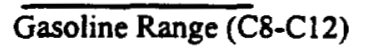 & ND & $\overline{20 . \mathrm{mg} / \mathrm{kg}}$ \\
\hline Diesel Range (C12-C22) & $\mathrm{mg} / \mathrm{kg}$ & 20. $\mathrm{mg} / \mathrm{kg}$ \\
\hline Oil Range (Cl2-C34) & $\mathrm{mg} / \mathrm{kg}$ & 100. $\mathrm{mg} / \mathrm{kg}$ \\
\hline Total & $\mathrm{mg} / \mathrm{kg}$ & 20. $\mathrm{mg} / \mathrm{kg}$ \\
\hline \multicolumn{3}{|c|}{$\overline{Q U A L I T Y ~ C O N T R O L ~ D A T A:}$} \\
\hline Surrogate & $\%$ Recovery & Acceptable Range \\
\hline$\overline{\text { Octacosane }}$ & $\mathrm{D}$ & $54-130$ \\
\hline
\end{tabular}

ND - Not Detected

This report shall not be reproduced except in full, without the written approval of the laboratory. 
NEL LABORATORIES

PROJECT ID: V1126

PROJECT \#: 23081
CLIENT ID: $\quad$ 252504-3-0

DATE SAMPLED: $5 / 26 / 01$

NEL SAMPLE ID: L0105290-05

TEST:

Total Extractable Petroleum Hydrocarbons Fuel Finger Print by EPA Method 8015M, July 1992

METHOD: $\quad$ EPA $8015 \mathrm{M}$

MATRIX: $\quad$ Solid

DILUTION: $\quad 20$

ANALYST: $\quad$ CCS - Las Vegas Division

EXTRACTED: $\quad 6 / 1 / 01$

ANALYZED: $\quad 6 / 6 / 01$

$-$

PARAMETER

Gasoline Range (C8-C12)

- Diesel Range (C12-C22)

Oil Range (C12-C34)

\section{Result}

ND

$3600 \mathrm{mg} / \mathrm{kg}$

$18000 \mathrm{mg} / \mathrm{kg}$

$21600 \mathrm{mg} / \mathrm{kg}$
Reporting

Limit

200. $\mathrm{mg} / \mathrm{kg}$

200. $\mathrm{mg} / \mathrm{kg}$

1000. $\mathrm{mg} / \mathrm{kg}$

200. $\mathrm{mg} / \mathrm{kg}$

\section{- $\overline{\text { QUALITY CONTROL DATA: }}$}

Surrogate

Octacosane

\% Recovery

D
Acceptable Range

$54-130$

ND - Not Detected

This report shall not be reproduced except in full, without the written approval of the laboratory. 
NEL LABORATORIES

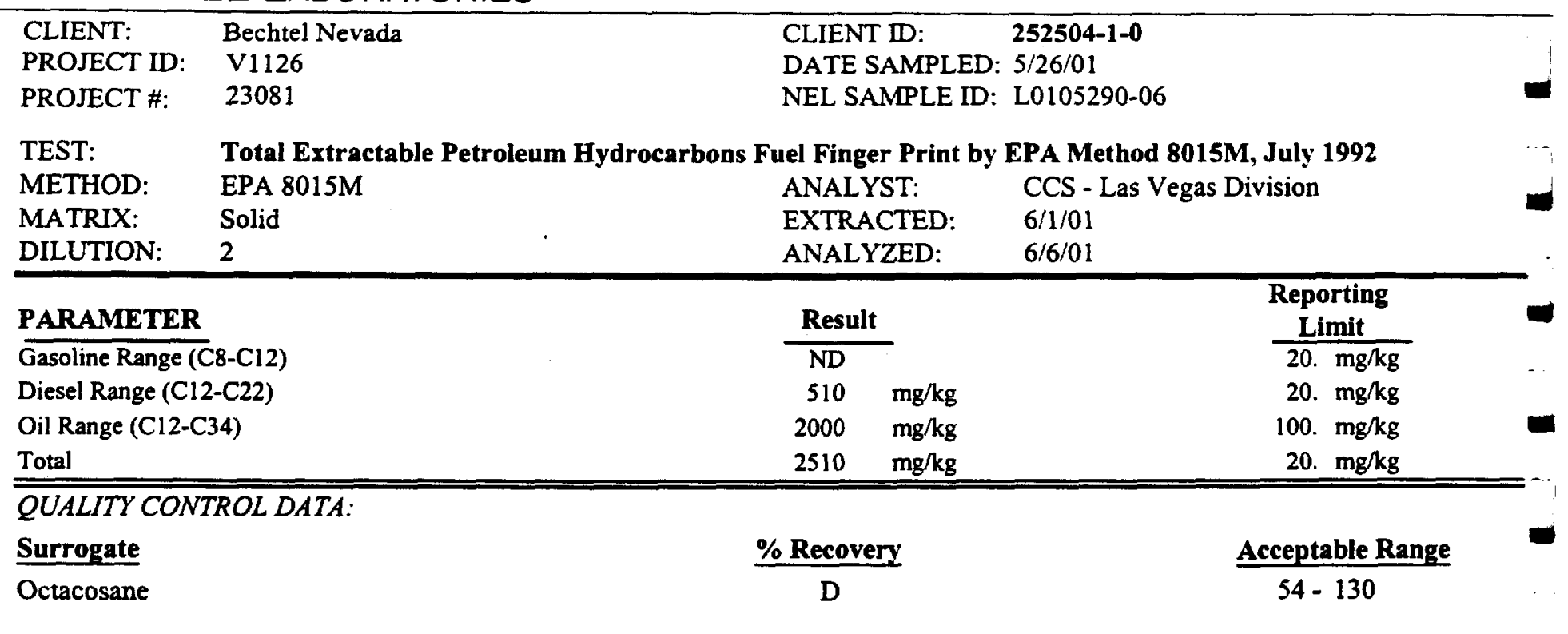

ND - Not Detected

This report shall not be reproduced except in full, without the written approval of the laboratory. 
NEL LABORATORIES

\begin{tabular}{llll}
\hline CLIENT: & Bechtel Nevada & CLIENT ID: & Method Blank \\
PROJECT ID: & V1126 & DATE SAMPLED: & NA \\
PROJECT \#: & 23081 & NEL SAMPLE ID: 010601 PCBS-BLK
\end{tabular}

- TEST:

PCB's (Polychlorinated Biphenyls) by EPA 8082, Dec. 1996

METHOD: $\quad$ EPA 8082

MATRIX: Solid

ANALYST: JRW - Las Vegas Division

EXTRACTED: $\quad 6 / 1 / 01$

ANALYZED: $\quad 6 / 6 / 01$

\section{PARAMETER}

Aroclor-1016

- Aroclor-1221

Aroclor- 1232

Aroclor- 1242

- Aroclor- 1248

Aroclor-1254

Aroclor-1260

QUALITY CONTROL DATA:

Surrogate

Decachlorobiphenyl

Tetrachloro-m-xylene

\begin{tabular}{l} 
Result \\
\hline ND \\
ND \\
ND \\
ND \\
ND \\
ND \\
ND
\end{tabular}

$\%$ Recovery

94

113
Reporting

Limit

20. $\mu \mathrm{g} / \mathrm{kg}$

20. $\mu \mathrm{g} / \mathrm{kg}$

20. $\mu g / \mathrm{kg}$

20. $\mu \mathrm{g} / \mathrm{kg}$

20. $\mu \mathrm{g} / \mathrm{kg}$

20. $\mu \mathrm{g} / \mathrm{kg}$

20. $\mu \mathrm{g} / \mathrm{kg}$

ND - Not Detected

This report shall not be reproduced except in full, without the written approval of the laboratory.

CLIENT: Bechtel Nevada CLIENT ID: Method Blank

PROJECT ID: V1126

DATE SAMPLED: NA

PROJECT \#: 23081

NEL SAMPLE ID: 010601TPHS-FP-BLK

TEST: $\quad$ Total Extractable Petroleum Hydrocarbons Fuel Finger Print by EPA Method 8015 M, July 1992

METHOD: EPA 8015M ANALYST: CCS - Las Vegas Division

MATRIX: Solid $\quad$ EXTRACTED: 6/1/01

ANALYZED: $\quad 6 / 6 / 01$

- PARAMETER

Gasoline Range (C8-C12)

Diesel Range (C12-C22)

\begin{tabular}{l} 
Result \\
\hline ND \\
ND \\
ND \\
ND
\end{tabular}

Reporting

Oil Range (C12-C34)

Total

QUALITY CONTROL DATA:

\section{Surrogate}

Octacosane
\% Recovery

67
Acceptable Range

$46-155$

$49-140$

ND - Not Detected

This report shall not be reproduced except in full, without the written approval of the laboratory. 
NEL LABORATORIES

\begin{tabular}{|c|c|c|c|c|c|c|c|}
\hline $\begin{array}{l}\text { CLIENT: } \\
\text { PROJECT ID: } \\
\text { PROJECT \#: }\end{array}$ & $\begin{array}{l}\text { Bechtel Nevada } \\
\text { V1126 } \\
23081\end{array}$ & & & $\begin{array}{l}\text { CLIENT ID } \\
\text { DATE SAN } \\
\text { NEL SAMF }\end{array}$ & $\begin{array}{ll} & \mathbf{2 5 2 5 0 4 - 2} \\
\text { MPLED: } & 5 / 26 / 01 \\
\text { PLE ID: } & \text { L010529 }\end{array}$ & $10-04$ & \\
\hline $\begin{array}{l}\text { TEST: } \\
\text { MATRIX: }\end{array}$ & $\begin{array}{l}\text { TCLP-8 Metals } \\
\text { Solid }\end{array}$ & & & & & & \\
\hline PARAMETER & $\begin{array}{c}\text { RESULT } \\
\mathrm{mg} / \mathrm{L} \\
\end{array}$ & $\begin{array}{c}\text { REPORTING } \\
\text { LIMIT } \\
\end{array}$ & D. F. & METHOD & $\begin{array}{c}\text { TCLP/STLC } \\
\text { EXTRACTION } \\
\text { DATE } \\
\end{array}$ & DIGESTED & ANALYZED \\
\hline Arsenic & ND & $0.1 \mathrm{mg} / \mathrm{L}$ & 1 & EPA 6010 & $6 / 5 / 01$ & $6 / 6 / 01$ & $6 / 7 / 01$ \\
\hline Barium & ND & 1. $\mathrm{mg} / \mathrm{L}$ & 1 & EPA 6010 & $6 / 5 / 01$ & $6 / 6 / 01$ & $6 / 7 / 01$ \\
\hline Cadmium & ND & $0.01 \mathrm{mg} / \mathrm{L}$ & 1 & EPA 6010 & $6 / 5 / 01$ & $6 / 6 / 01$ & $6 / 7 / 01$ \\
\hline Chromium & ND & $0.01 \mathrm{mg} / \mathrm{L}$ & 1 & EPA 6010 & $6 / 5 / 01$ & $6 / 6 / 01$ & $6 / 7 / 01$ \\
\hline Lead & 10 & $0.05 \mathrm{mg} / \mathrm{L}$ & 1 & EPA 6010 & $6 / 5 / 01$ & $6 / 6 / 01$ & $6 / 7 / 01$ \\
\hline Mercury & ND & $0.002 \mathrm{mg} / \mathrm{L}$ & 10 & EPA $7470 A$ & $6 / 5 / 01$ & $6 / 6 / 01$ & $6 / 6 / 01$ \\
\hline Selenium & ND & $0.1 \mathrm{mg} / \mathrm{L}$ & 1 & EPA 6010 & $6 / 5 / 01$ & $6 / 6 / 01$ & $6 / 7 / 01$ \\
\hline Silver & ND & $0.02 \mathrm{mg} / \mathrm{L}$ & 1 & EPA 6010 & $6 / 5 / 01$ & $6 / 6 / 01$ & $6 / 7 / 01$ \\
\hline
\end{tabular}

D.F. - Dilution Factor

ND - Not Detected

This report shall not be reproduced except in full, without the written approval of the laboratory. 
NEL LABORATORIES

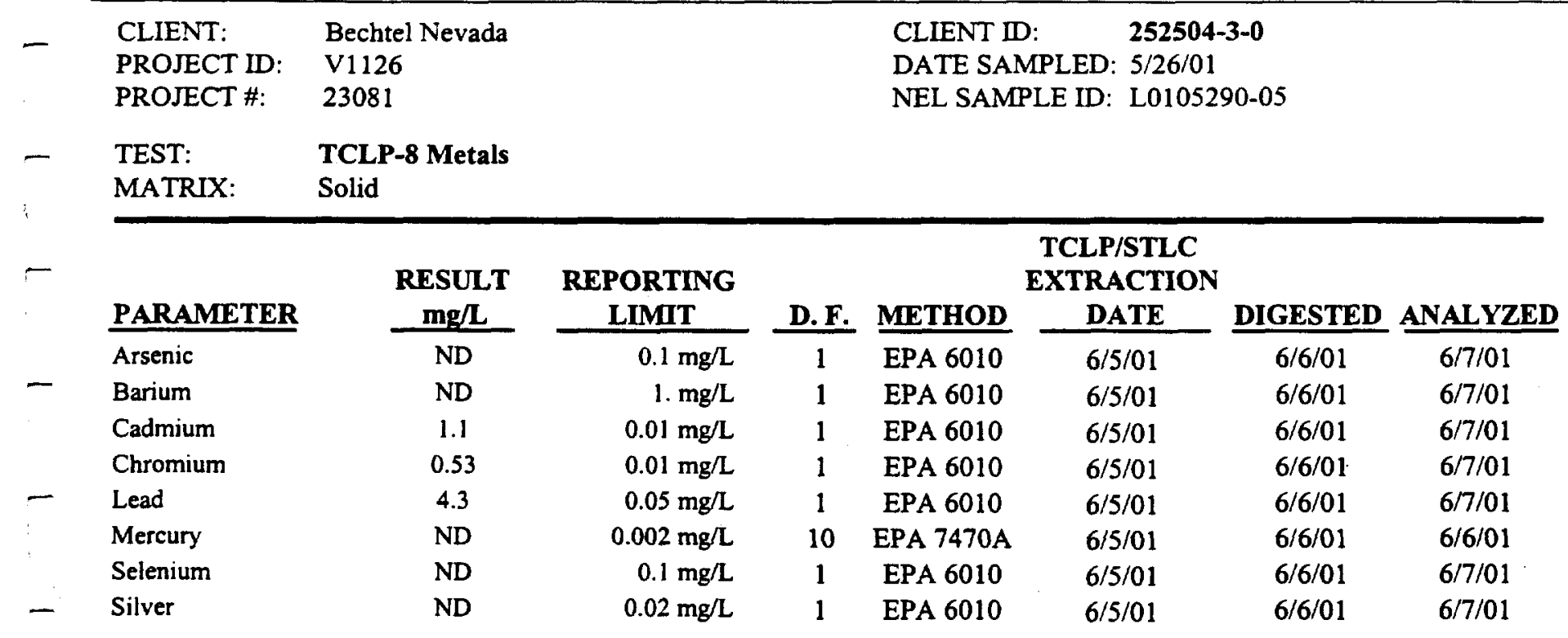

- D.F. - Dilution Factor

ND - Not Detected

This report shall not be reproduced except in full, without the written approval of the laboratory. 
NEL LABORATORIES

\begin{tabular}{|c|c|c|c|c|c|c|c|}
\hline $\begin{array}{l}\text { CLIENT: } \\
\text { PROJECT ID: } \\
\text { PROJECT \#: }\end{array}$ & $\begin{array}{l}\text { Bechtel Nevada } \\
\text { V1126 } \\
23081\end{array}$ & & & $\begin{array}{l}\text { CLIENT II } \\
\text { DATE SAI } \\
\text { NEL SAMI }\end{array}$ & $\begin{array}{ll}\text { D: } & \mathbf{2 5 2 5 0 4 -} \\
\text { MPLED: } & 5 / 26 / 01 \\
\text { PLE ID: } & \text { L01052 }\end{array}$ & $90-06$ & \\
\hline $\begin{array}{l}\text { TEST: } \\
\text { MATRIX: }\end{array}$ & $\begin{array}{l}\text { TCLP-8 Metals } \\
\text { Solid }\end{array}$ & & & & & & \\
\hline PARAMETER & $\begin{array}{c}\text { RESULT } \\
\mathrm{mg} / \mathrm{L} \\
\end{array}$ & $\begin{array}{c}\text { REPORTING } \\
\text { LIMIT } \\
\end{array}$ & D. $\mathbf{F}$. & METHOD & $\begin{array}{c}\text { TCLP/STLC } \\
\text { EXTRACTION } \\
\text { DATE } \\
\end{array}$ & DIGESTED & ANAL YZED \\
\hline Arsenic & ND & $0.1 \mathrm{mg} / \mathrm{L}$ & 1 & EPA 6010 & $6 / 5 / 01$ & $6 / 6 / 01$ & $6 / 7 / 01$ \\
\hline Barium & ND & 1. $\mathrm{mg} / \mathrm{L}$ & 1 & EPA 6010 & $6 / 5 / 01$ & $6 / 6 / 01$ & $6 / 7 / 01$ \\
\hline Cadmium & ND & $0.01 \mathrm{mg} / \mathrm{L}$ & 1 & EPA 6010 & $6 / 5 / 01$ & $6 / 6 / 01$ & $6 / 7 / 01$ \\
\hline Chromium & ND & $0.01 \mathrm{mg} / \mathrm{L}$ & 1 & EPA 6010 & $6 / 5 / 01$ & $6 / 6 / 01$ & $6 / 7 / 01$ \\
\hline Lead & 11 & $0.05 \mathrm{mg} / \mathrm{L}$ & 1 & EPA 6010 & $6 / 5 / 01$ & $6 / 6 / 01$ & $6 / 7 / 01$ \\
\hline Mercury & ND & $0.002 \mathrm{mg} / \mathrm{L}$ & 10 & EPA 7470A & $6 / 5 / 01$ & $6 / 6 / 01$ & $6 / 6 / 01$ \\
\hline Selenium & ND & $0.1 \mathrm{mg} / \mathrm{L}$ & 1 & EPA 6010 & $6 / 5 / 01$ & $6 / 6 / 01$ & $6 / 7 / 01$ \\
\hline Silver & ND & $0.02 \mathrm{mg} / \mathrm{L}$ & 1 & EPA 6010 & $6 / 5 / 01$ & $6 / 6 / 01$ & $6 / 7 / 01$ \\
\hline
\end{tabular}

D.F. - Dilution Factor

ND - Not Detected

This report shall not be reproduced except in full, without the written approval of the laboratory. 
NEL LABORATORIES

$\begin{array}{llll}\text { CLIENT: } & \text { Bechtel Nevada } & \text { CLIENT ID: } & \text { Method Blank } \\ \text { PROJECT ID: } & \text { V1126 } & \text { DATE SAMPLED: } & \text { NA } \\ \text { PROJECT \#: } & 23081 & \text { NEL SAMPLE ID: } & \text { L05290-HG-BLK }\end{array}$

TEST: $\quad$ TCLP by EPA 1311, July 1992 \& Mercury by EPA 7470A, July 1992

MATRIX: TCLP Extract

$-$

\section{PARAMETER}

Mercury

RESULT
ND $\frac{\begin{array}{c}\text { REPORTING } \\ \text { LIMIT }\end{array}}{0.002 \mathrm{mg} / \mathrm{L}}$

$\frac{\text { D. F. }}{10}$

TCLP/STLC
EXTRACTION
METHOD DATE

10 EPA 7470A

6/5/01

DIGESTED ANALYZED

$-$

D.F. - Dilution Factor

ND - Not Detected

This report shall not be reproduced except in full, without the written approval of the laboratory. 
NEL LABORATORIES

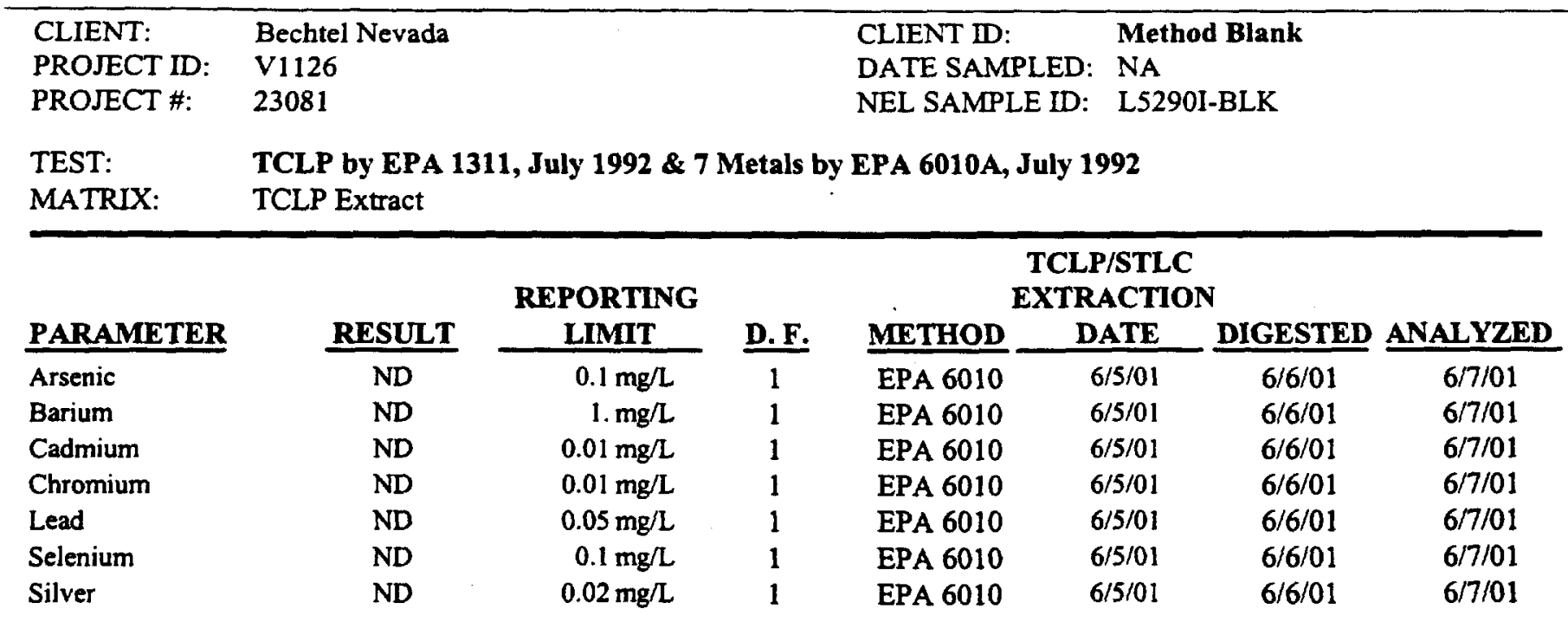

D.F. - Dilution Factor

ND - Not Detected

This report shall not be reproduced except in full, without the written approval of the laboratory. 
NEL LABORATORIES

\begin{tabular}{llll}
\hline CLIENT: & Bechtel Nevada & CLIENT ID: & 252504-2-0 \\
PROJECT ID: & V1126 & DATE SAMPLED: $5 / 26 / 01$ \\
PROJECT \#: & 23081 & NEL SAMPLE ID: & L0105290-04 \\
& & & \\
TEST: & TCLP by EPA 1311, July 1992 & \& Semivolatile Organics by EPA Method 8270C, Dec. 1996 \\
METHOD: & EPA 8270 & TCLP EXTRACT DATE: 6/5/01 \\
MATRIX: & Solid & EXTRACTED & $6 / 6 / 01$ \\
DILUTION: & 1 & ANALYZED: & $6 / 7 / 01$
\end{tabular}

\section{PARAMETER}

- $\overline{1,4-D i c h l o r o b e n z e n e ~(p-D C B) ~}$

2,4-Dinitrotoluene (DNT)

Hexachlorobenzene

- Hexachlorobutadiene

Hexachloroethane

2-Methylphenol

3,4-Methylphenol (isomeric pair)

Nitrobenzene

Pentachlorophenol

Pyridine

2,4,5-Trichlorophenol

2,4,6-Trichlorophenol

\begin{tabular}{c}
$\begin{array}{c}\text { Result } \\
\mathbf{m g} / \mathbf{L}\end{array}$ \\
\hline ND \\
ND \\
ND \\
ND \\
ND \\
ND \\
ND \\
ND \\
ND \\
ND \\
ND \\
ND
\end{tabular}

Reporting Limit

$0.1 \mathrm{mg} / \mathrm{L}$

$0.1 \mathrm{mg} / \mathrm{L}$

$0.1 \mathrm{mg} / \mathrm{L}$

$0.1 \mathrm{mg} / \mathrm{L}$

$0.1 \mathrm{mg} / \mathrm{L}$

$0.1 \mathrm{mg} / \mathrm{L}$

$0.1 \mathrm{mg} / \mathrm{L}$

$0.1 \mathrm{mg} / \mathrm{L}$

$0.1 \mathrm{mg} / \mathrm{L}$

$0.1 \mathrm{mg} / \mathrm{L}$

$0.1 \mathrm{mg} / \mathrm{L}$

$0.1 \mathrm{mg} / \mathrm{L}$

QUALITY CONTROL DATA:

\section{Surrogate}

2,4,6-Tribromophenol

2-Fluorobiphenyl

2-Fluorophenol

Nitrobenzene-d5

p-Terphenyl-d14

Phenol-d5
\% Recovery

27

13

13

14

18

8
Acceptable Range

$0-161$

$16-127$

$0-88$

9- 132

$16-163$

$0-63$

ND - Not Detected

This report shall not be reproduced except in full, without the written approval of the laboratory. 
NEL LABORATORIES

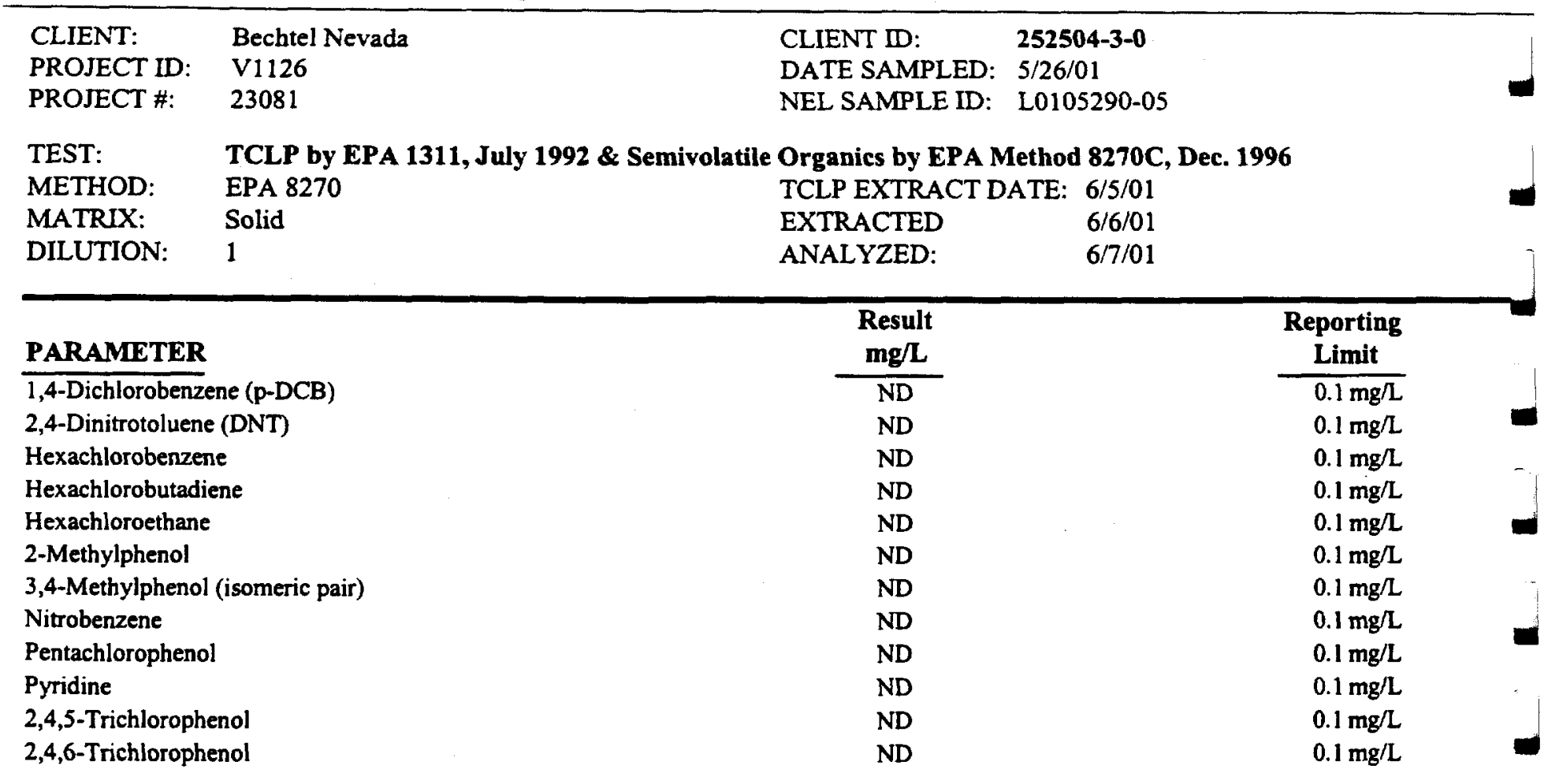

\section{QUALITY CONTROL DATA:}

Surrogate

2,4,6-Tribromophenol

2-Fluorobiphenyl

2-Fluorophenol

Nitrobenzene-d5

p-Terphenyl-d14

Phenol-d5
$\%$ Recovery

40

20

21

19

25

16
Acceptable Range

0 - 161

$16-127$

0 - 88

$9-132$

$16-163$

$0-63$

ND - Not Detected

This report shall not be reproduced except in full, without the written approval of the laboratory. 
NEL LABORATORIES

$\begin{array}{llll}\text { CLIENT: } & \text { Bechtel Nevada } & \text { CLIENT ID: } & \text { 252504-1-0 } \\ \text { PROJECT ID: } & \text { V1126 } & \text { DATE SAMPLED: } & \text { 5/26/01 } \\ \text { PROJECT \#: } & 23081 & \text { NEL SAMPLE ID: } & \text { L0105290-06 }\end{array}$

- TEST:

METHOD: $\quad$ EPA 8270

MATRIX: Solid

DILUTION: 1

Organies by EPA Method 8270C, Dec. 1996

TCLP EXTRACT DATE: $6 / 5 / 01$

EXTRACTED $\quad 6 / 6 / 01$

ANALYZED: $\quad 6 / 7 / 01$

\section{PARAMETER}

1,4-Dichlorobenzene (p-DCB)

2,4-Dinitrotoluene (DNT)

Hexachlorobenzene

Hexachlorobutadiene

Hexachloroethane

2-Methylphenol

3,4-Methylphenol (isomeric pair)

Nitrobenzene

Pentachlorophenol

Pyridine

2,4,5-Trichlorophenol

2,4,6-Trichlorophenol

\begin{tabular}{c}
$\begin{array}{c}\text { Result } \\
\text { mg/L }\end{array}$ \\
\hline ND \\
ND \\
ND \\
ND \\
ND \\
ND \\
ND \\
ND \\
ND \\
ND \\
ND \\
ND
\end{tabular}

Result

$\mathrm{mg} / \mathrm{L}$

ND

ND

ND

ND

ND

ND

ND
Reporting Limit

$0.1 \mathrm{mg} / \mathrm{L}$

$0.1 \mathrm{mg} / \mathrm{L}$

$0.1 \mathrm{mg} / \mathrm{L}$

$0.1 \mathrm{mg} / \mathrm{L}$

$0.1 \mathrm{mg} / \mathrm{L}$

$0.1 \mathrm{mg} / \mathrm{L}$

$0.1 \mathrm{mg} / \mathrm{L}$

$0.1 \mathrm{mg} / \mathrm{L}$

$0.1 \mathrm{mg} / \mathrm{L}$

$0.1 \mathrm{mg} / \mathrm{L}$

$0.1 \mathrm{mg} / \mathrm{L}$

$0.1 \mathrm{mg} / \mathrm{L}$

QUALITY CONTROL DATA:

\section{Surrogate}

2,4,6-Tribromophenol

2-Fluorobiphenyl

2-Fluorophenol

Nitrobenzene-d5

p-Terphenyl-d14

Phenol-d5
\% Recovery

38

18

19

19

27

14

\section{Acceptable Range}

$0-161$

$16-127$

0 - 88

$9-132$

$16-163$

$0-63$

\section{ND - Not Detected}

This report shall not be reproduced except in full, without the written approval of the laboratory. 
NEL LABORATORIES

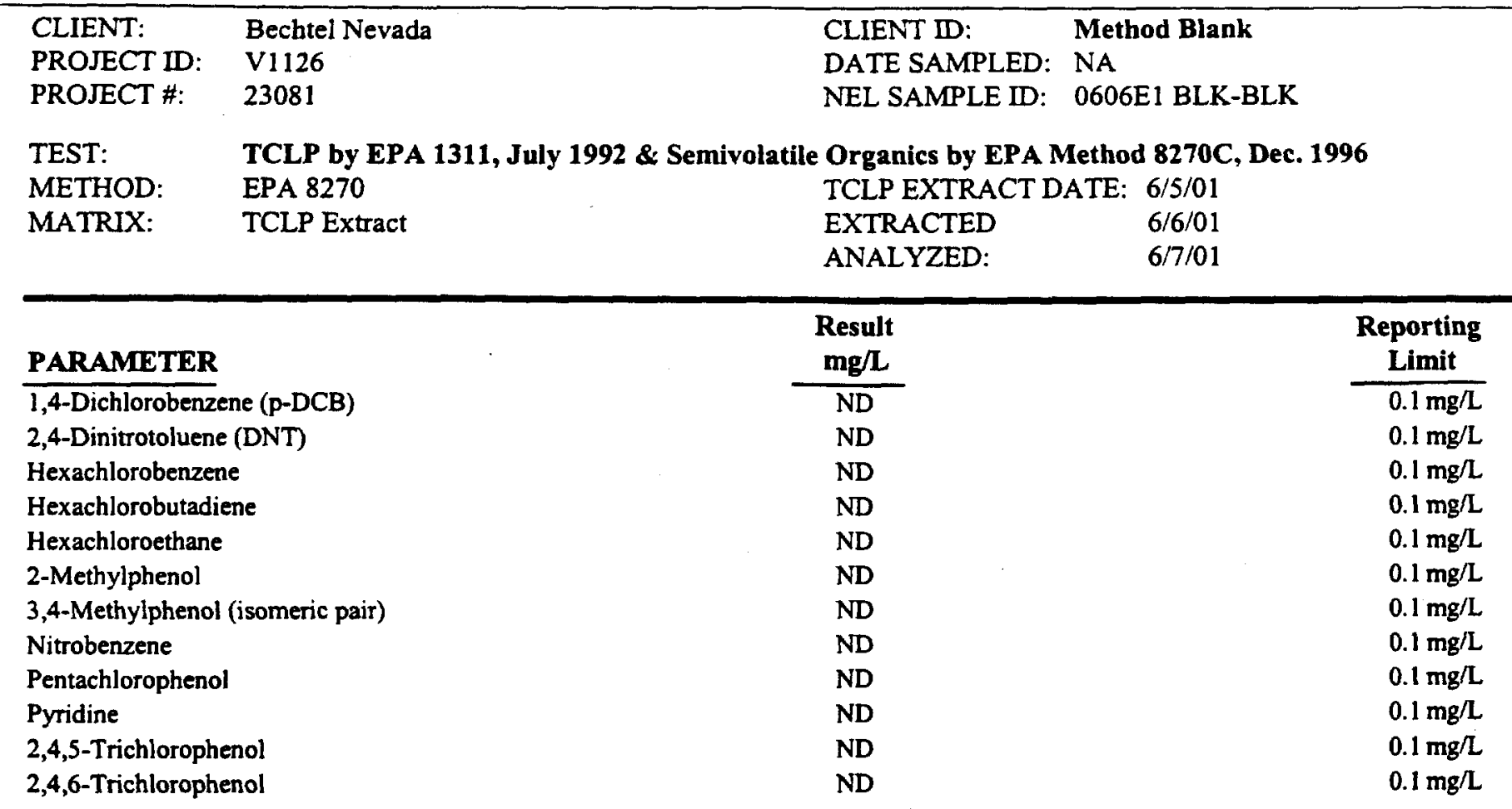

QUALITY CONTROL DATA:

\section{Surrogate}

2,4,6-Tribromophenol

2-Fluorobiphenyl

2-Fluorophenol

Nitrobenzene-d5

p-Terphenyl-d14

Phenol-d5
\% Recovery

74

73

36

76

122

25
Acceptable Range

0 - 161

$16-127$

$0-88$

9- 132

$16-163$

$0-63$

ND - Not Detected

This report shall not be reproduced except in full, without the written approval of the laboratory. 
NEL LABORATORIES

- CLIENT: Bechtel Nevada

PROJECT ID: V1126

PROJECT \#: 23081

TEST: $\quad$ PCB's (Polychlorinated Biphenyls) by EPA 8082, Dec. 1996

-MATRIX: Solid

\section{- PARAMETER}

Aroclor-1016

Aroclor-1016

- Aroclor-1016

Aroclor-1016

Aroclor-1260

Aroclor- 1260

Aroclor- 1260

Aroclor-1260

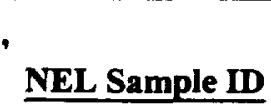

010601PCBS-LCS

010601PCBS-LCSD

L0105291-12-MS

L0105291-12-MSD

010601PCBS-LCS

010601PCBS-LCSD

L0105291-12-MS

L0105291-12-MSD

\section{Spike \\ Amount}

333

333

333

333

333

333

333

333

\begin{tabular}{c}
$\frac{\text { Spike }}{\text { Result }}$ \\
\hline 367 \\
374 \\
193 \\
197 \\
352 \\
372 \\
872 \\
741
\end{tabular}

\begin{tabular}{c}
$\frac{\text { Percent }}{\text { Recovery }}$ \\
\hline 110 \\
112 \\
58 \\
59 \\
106 \\
112 \\
136 \\
96
\end{tabular}

Acceptable

Range RPD

$63-127$

$63-127$

$55-142$

$55-142$

$57-138$

$57-138$

$48-129$

$48-129$

1.9

2.1

5.5

33.9 
NEL LABORATORIES

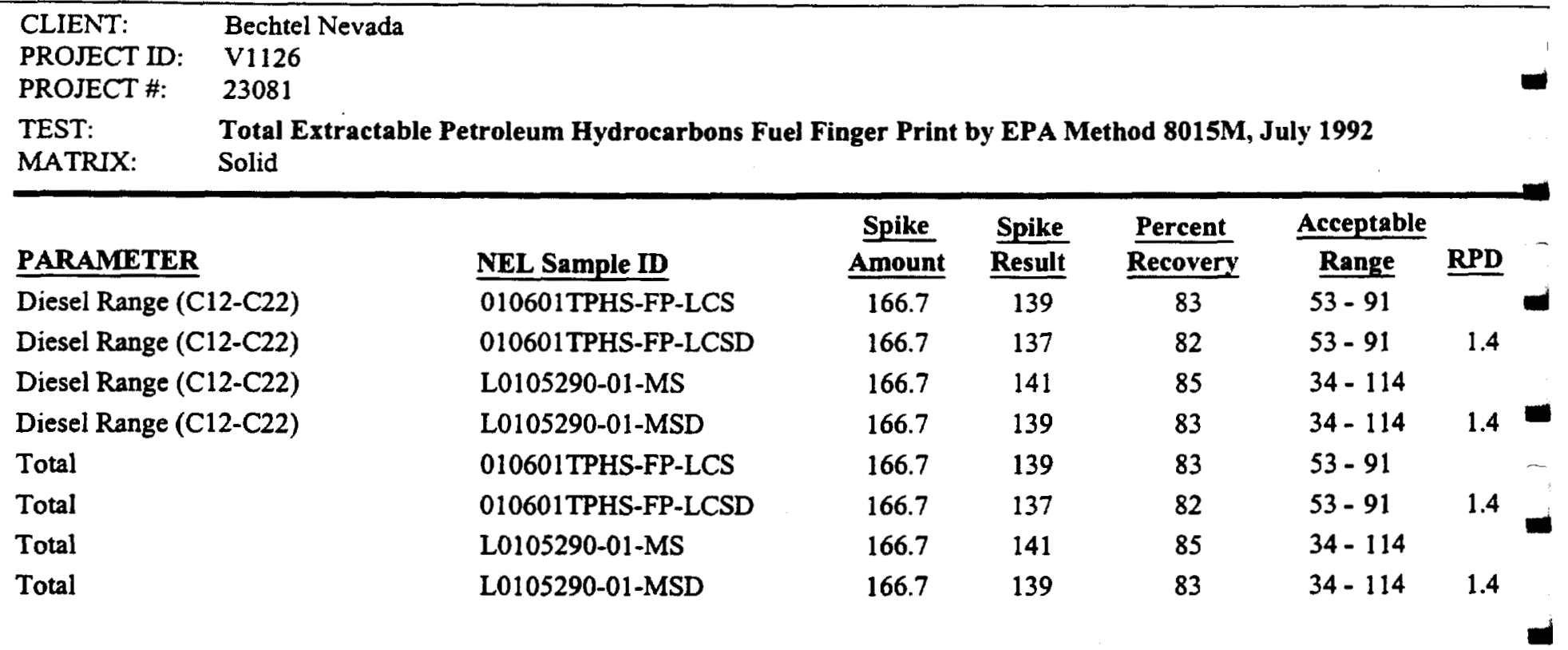

ND - Not Detected

This report shall not be reproduced except in full, without the written approval of the laboratory. 


\section{CLIENT: Bechtel Nevada}

PROJECT ID: V1126

PROJECT \#: 23081

TEST: $\quad$ TCLP by EPA 1311, July 1992 \& Semivolatile Organics by EPA Method 8270C, Dec. 1996

MATRIX: $\quad$ Solid

\begin{tabular}{|c|c|c|c|c|c|c|}
\hline PARAMETER & NEL Sample ID & $\begin{array}{c}\text { Spike } \\
\text { Amount }\end{array}$ & $\frac{\text { Spike }}{\underline{\text { Result }}}$ & $\frac{\text { Percent }}{\text { Recovery }}$ & $\frac{\text { Acceptable }}{\text { Range }}$ & $\underline{\text { RPD }}$ \\
\hline Pyridine & 0606E1 BLK-LCS & 80 & 43.5 & 54 & $10-130$ & \\
\hline Pyridine & 0606E1 BLK-LCSD & 80 & 39.4 & 49 & $10-130$ & 9.9 \\
\hline Pyridine & L0105291-12-MS & 80 & 37.9 & 47 & $10-130$ & \\
\hline 1,4-Dichlorobenzene (p-DCB) & 0606E1 BLK-LCS & 80 & 58.1 & 73 & $7-105$ & \\
\hline 1,4-Dichlorobenzene ( $\mathrm{p}-\mathrm{DCB}$ ) & 0606E1 BLK-LCSD & 80 & 49.8 & 62 & $7 \cdot 105$ & 15.4 \\
\hline 1,4-Dichlorobenzene (p-DCB) & L0105291-12-MS & 80 & 45 & 56 & $7-105$ & \\
\hline Hexachloroethane & 0606E1 BLK-LCS & 80 & 49.6 & 62 & $43-104$ & \\
\hline Hexachloroethane & 0606E1 BLK-LCSD & 80 & 34.1 & 43 & $43-104$ & 37. \\
\hline Hexachloroethane & L0105291-12-MS & 80 & 36.9 & 46 & $43-104$ & \\
\hline Nitrobenzene & 0606E1 BLK-LCS & 80 & 66.1 & 83 & $28-124$ & \\
\hline Nitrobenzene. & 0606E1 BLK-LCSD & 80 & 60.8 & 76 & $28-124$ & 8.4 \\
\hline Nitrobenzene & L0105291-12-MS & 80 & 53.4 & 67 & $28-124$ & \\
\hline Hexachlorobutadiene & 0606E1 BLK-LCS & 80 & 57.7 & 72 & $39-111$ & \\
\hline Hexachlorobutadiene & 0606E1 BLK-LCSD & 80 & 34.3 & 43 & $39-111$ & 50.9 \\
\hline Hexachlorobutadiene & L0105291-12-MS & 80 & 42.6 & 53 & $39-111$ & \\
\hline 2-Methylphenol & 0606E1 BLK-LCS & 80 & 55.6 & 70 & $30-130$ & \\
\hline 2-Methylphenol & 0606E1 BLK-LCSD & 80 & 53.4 & 67 & $30-130$ & 4. \\
\hline 2-Methylphenol & L0105291-12-MS & 80 & 46.6 & 58 & $30-130$ & \\
\hline 3,4-Methylphenol (isomeric pair) & 0606E1 BLK-LCS & 80 & 49.8 & 62 & $30-130$ & \\
\hline 3,4-Methylphenol (isomeric pair) & 0606E1 BLK-LCSD & 80 & 48.1 & 60 & $30-130$ & 3.5 \\
\hline 3,4-Methylphenol (isomeric pair) & L0105291-12-MS & 80 & 40.8 & 51 & $30-130$ & \\
\hline 2,4,6-Trichlorophenol & 0606E1 BLK-LCS & 80 & 75.1 & 94 & $43-110$ & \\
\hline 2,4,6-Trichlorophenol & 0606E1 BLK-LCSD & 80 & 76.5 & 96 & $43-110$ & 1.8 \\
\hline 2,4,6-Trichlorophenol & L0105291-12-MS & 80 & 69.2 & 87 & $43-110$ & \\
\hline 2,4,5-Trichlorophenol & 0606E1 BLK-LCS & 80 & 76.3 & 95 & $30-130$ & \\
\hline 2,4,5-Trichlorophenol & 0606E1 BLK-LCSD & 80 & 77.3 & 97 & $30-130$ & 1.3 \\
\hline 2,4,5-Trichlorophenol & L0105291-12-MS & 80 & 70.3 & 88 & $30-130$ & \\
\hline 2,4-Dinitrotoluene (DNT) & 0606E1 BLK-LCS & 80 & 92.2 & 115 & $50-111$ & \\
\hline 2,4-Dinitrotoluene (DNT) & 0606E1 BLK-LCSD & 80 & 88.5 & 111 & $50-111$ & 4.1 \\
\hline 2,4-Dinitrotoluene (DNT) & L0105291-12-MS & 80 & 65.4 & 82 & $50-111$ & \\
\hline Hexachlorobenzene & 0606E1 BLK-LCS & 80 & 83.5 & 104 & $41-125$ & \\
\hline Hexachlorobenzene & 0606E1 BLK-LCSD & 80 & 79.2 & 99 & $41-125$ & 5.3 \\
\hline Hexachlorobenzene & L0105291-12-MS & 80 & 61.6 & 77 & $41-125$ & \\
\hline Pentachlorophenol & 0606E1 BLK-LCS & 80 & 74 & 93 & $47-127$ & \\
\hline Pentachlorophenol & 0606E1 BLK-LCSD & 80 & 72.7 & 91 & $47-127$ & 1.8 \\
\hline Pentachlorophenol & L0105291-12-MS & 80 & 66.5 & 83 & $47-127$ & \\
\hline
\end{tabular}

ND - Not Detected

This report shall not be reproduced except in full, without the written approval of the laboratory. 
NEL LABORATORIES

$\begin{array}{ll}\text { CLIENT: } & \text { Bechtel Nevada } \\ \text { PROJECT ID: } & \text { V1126 } \\ \text { PROJECT \#: } & 23081 \\ \text { TEST: } & \text { TCLP/STLC Metals } \\ \text { MATRIX: } & \text { Solid }\end{array}$

\begin{tabular}{ll}
\hline & \\
PARAMETER & NEL Sample ID \\
\cline { 2 - 2 } Mercury & L05290-HG-LCS \\
Mercury & L0105290-04-MS \\
Mercury & L0105290-04-MSD
\end{tabular}

\begin{tabular}{cc}
$\frac{\text { Spike }}{\text { Amount }}$ & $\frac{\text { Spike }}{\text { Result }}$ \\
\hline 0.05 & 0.0492 \\
0.05 & 0.0497 \\
0.05 & 0.0499
\end{tabular}

\begin{tabular}{c}
$\frac{\text { Percent }}{\text { Recovery }}$ \\
\hline 98 \\
99 \\
100
\end{tabular}

\begin{tabular}{cc}
$\frac{\text { Acceptable }}{\text { Range }}$ & RPD \\
\hline $85-115$ & \\
$75-125$ & \\
$75-125$ & 0.4
\end{tabular}

ND - Not Detected

This report shall not be reproduced except in full, without the written approval of the laboratory. 


\section{CLIENT: Bechtel Nevada}

PROJECT ID: V1126

PROJECT \#: 23081

TEST: $\quad$ TCLP/STLC Metals

MATRIX: Solid

\section{- PARAMETER}

Arsenic

Arsenic

- Arsenic

Silver

Silver

Silver

Barium

Barium

Barium

Cadmium

Cadmium

Cadmium

Chromium

Chromium

Chromium

Lead

Lead

Lead

Selenium

Selenium

Selenium
NEL Sample ID

L5290I-LCS

L0105290-04-MS

L0105290-04-MSD

L5290I-LCS

L0105290-04-MS

L0105290-04-MSD

L5290I-LCS

L0105290-04-MS

L0105290-04-MSD

L52901-LCS

L0105290-04-MS

L0105290-04-MSD

L5290I-LCS

L0105290-04-MS

L0105290-04-MSD

L5290I-LCS

L0105290-04-MS

L0105290-04-MSD

L5290I-LCS

L0105290-04-MS

L0105290-04-MSD

\section{Spike Spike Percent}

Amount Result Recovery Range RPD

$\begin{array}{lll}0.5 & 0.552 & 110\end{array}$

112

108

95

101

95

98

105

96

102

108

101

99

106

100

103

$170 \mathrm{C}$

120

98

111

109
$85-115$

$75-125$

$75-125$

$85-115$

$75-125$

$75-125$

$85-115$

$75-125$

$75-125$

$85-115$

$75-125$

$75-125$

$85-115$

$75-125$

$75-125$

$85-115$

$75-125$

$75-125$

$85-115$

$75-125$

$75-125$
4.

ND - Not Detected

This report shall not be reproduced except in full, without the written approval of the laboratory. 


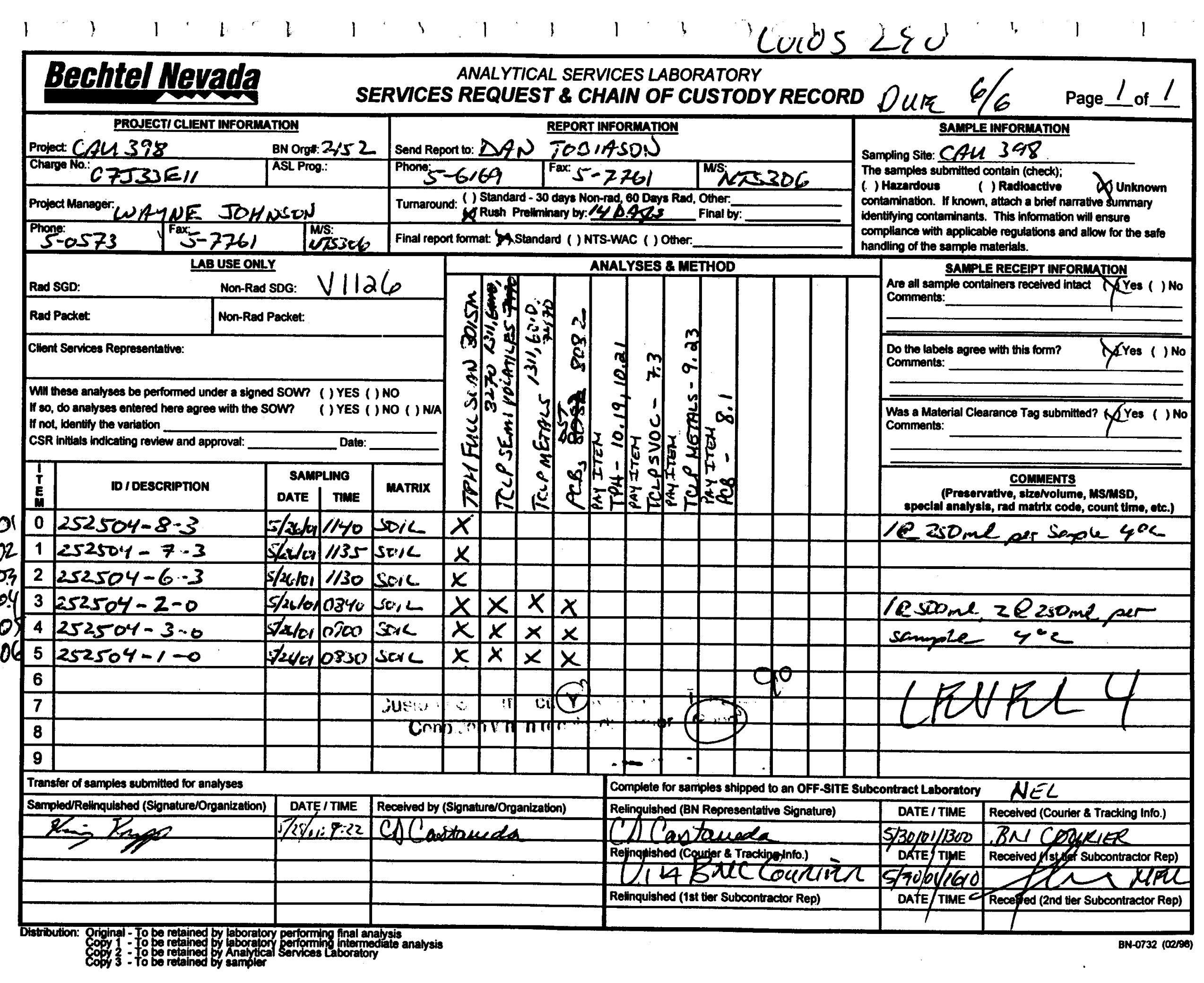


CLIENT: $\quad$ Bechtel Nevada P.O. Box 98521, M/S NTS273

Las Vegas, NV 89193-8521

ATTN:

Ted Redding

PROJECT NAME: V1136

NEL ORDER ID: L0105303

PROJECT NUMBER: 23081

Attached are the analytical results for samples in support of the above referenced project.

Samples submitted for this project were not sampled by NEL Laboratories. Samples were received by NEL in good condition, under chain of custody on 5/31/01.

Should you have any questions or comments, please feel free to contact our Client Services department at (702) 657-1010.
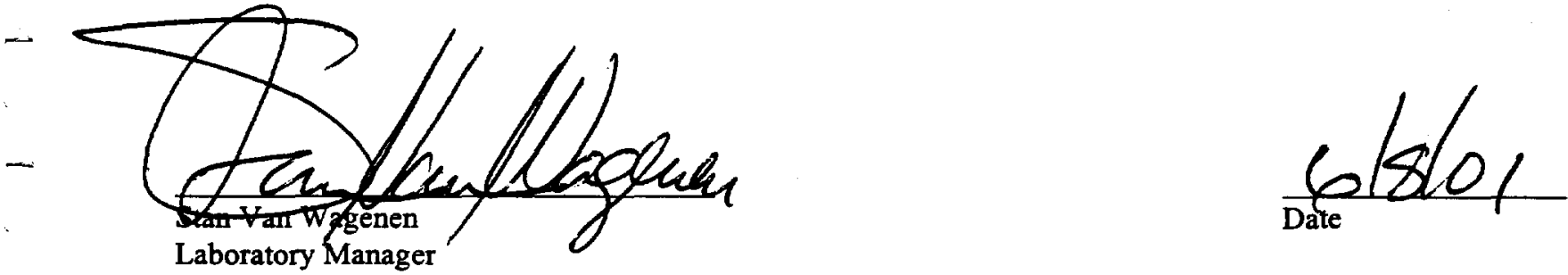

CERTIFICATIONS:

\begin{tabular}{llll}
\hline & Reno & Las Vegas & S. Califormia \\
\cline { 2 - 4 } Arizona & AZ0520 & AZ0518 & AZ0605 \\
California & 1707 & 2002 & 2264 \\
$\begin{array}{l}\text { US Army Corps } \\
\text { of Engineers }\end{array}$ & Certified & Certified & \\
& & &
\end{tabular}

Idaho

Montana

Nevada

L.A.C.S.D.

\begin{tabular}{lll} 
Reno & Las Vegas & S. California \\
\hline Certified & Certified & \\
Certified & Certified & \\
NV033 & NV052 & CA084 \\
& & 10228
\end{tabular}




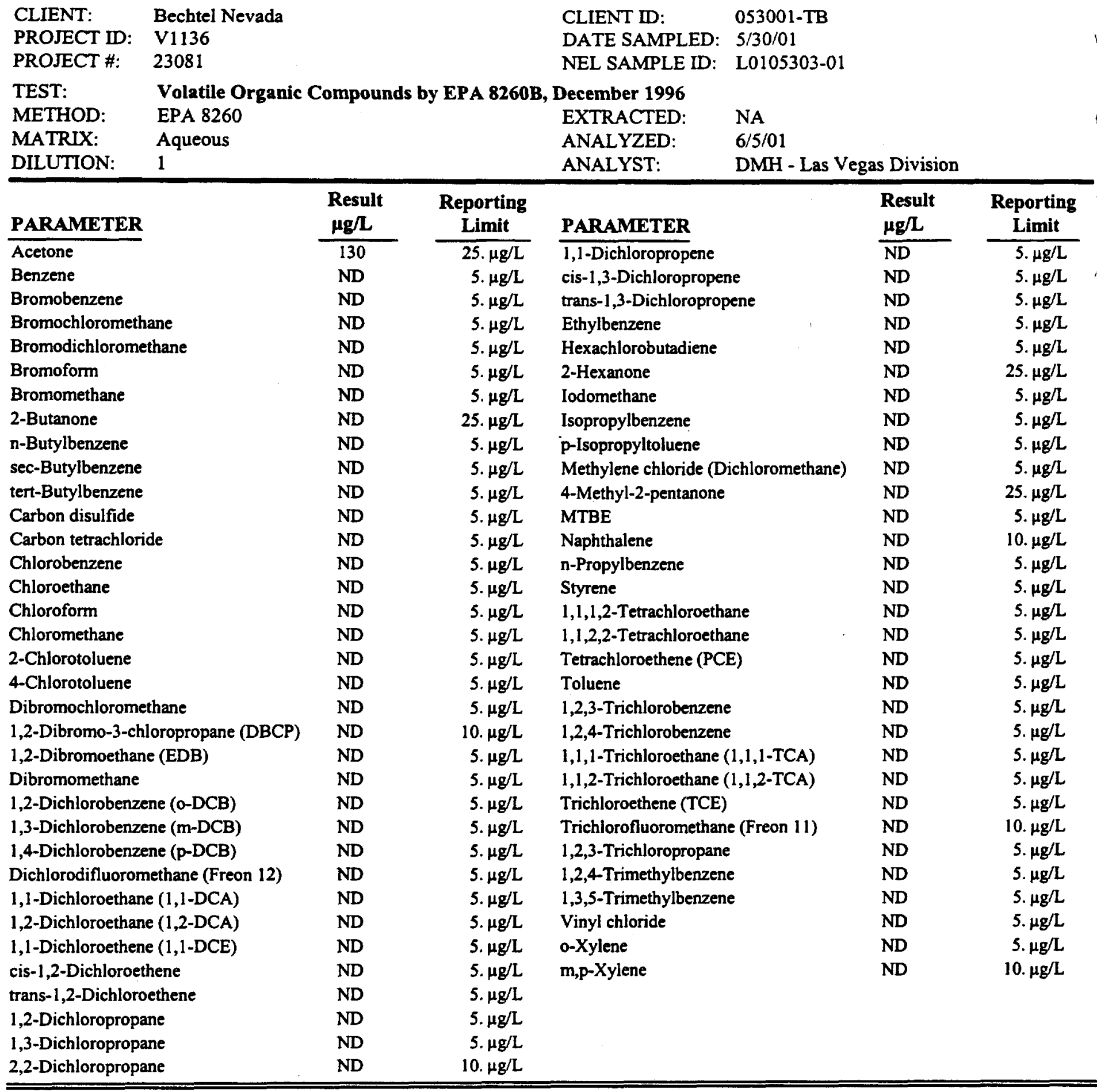

QUALITY CONTROL DATA:

Surrogate

4-Bromofluorobenzene

Dibromofluoromethane

Toluene-d8
$\%$ Recovery

99

103

100
Acceptable Range

$83-112$

$78-109$

$88-113$

ND - Not Detected

This report shall not be reproduced except in full, without the written approval of the laboratory. 


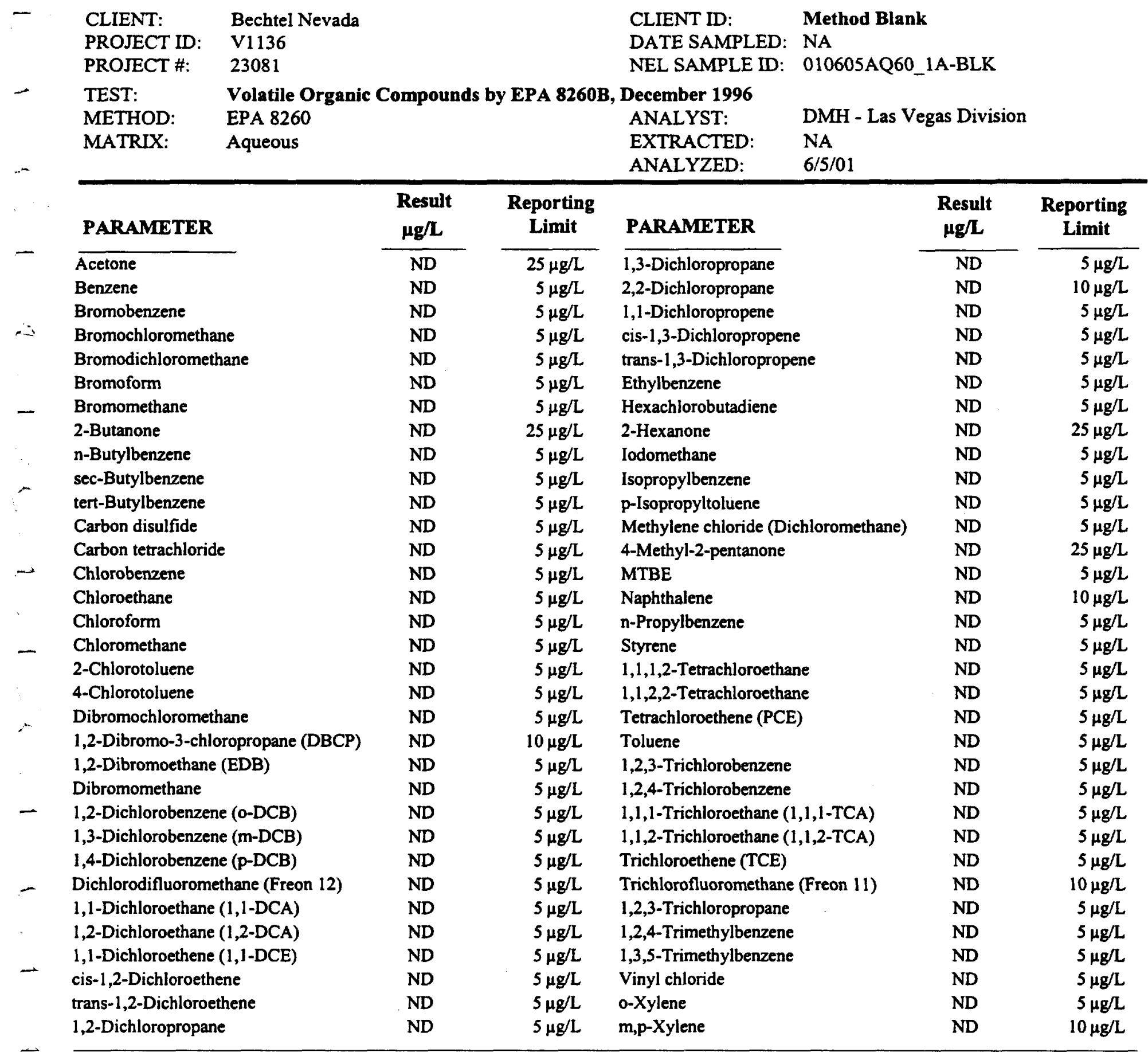

QUALITY CONTROL DATA:

Surrogate

4-Bromofluorobenzene

Dibromofluoromethane

Toluene-d8
$\%$ Recovery

100

105

100
Acceptable Range

$83-112$

$78-109$

$88-113$

ND - Not Detected

This report shall not be reproduced except in full, without the written approval of the laboratory. 


$\begin{array}{llll}\text { CLIENT: } & \text { Bechtel Nevada } & \text { CLIENT ID: } & \text { 252505-1-0 } \\ \text { PROJECT ID: } & \text { V1136 } & \text { DATE SAMPLED: } & 5 / 30 / 01 \\ \text { PROJECT \#: } & 23081 & \text { NEL SAMPLE ID: } & \text { L0105303-02 }\end{array}$

TEST: $\quad$ TCLP by EPA 1311, July 1992 \& Volatile Organics by EPA 8260B, Dec. 1996

METHOD: EPA 8260 TCLP EXTRACT DATE: 6/4/01

MATRIX: Solid EXTRACTED NA

$\begin{array}{llll}\text { DILUTION: } & 1 & \text { ANALYZED: } & 6 / 5 / 01\end{array}$

\begin{tabular}{lcc}
\hline PARAMETER & $\begin{array}{c}\text { Result } \\
\text { mg/L }\end{array}$ & $\begin{array}{c}\text { Reporting } \\
\text { Limit }\end{array}$ \\
\cline { 2 - 3 } Benzene & ND & $0.1 \mathrm{mg} / \mathrm{L}$ \\
Carbon tetrachloride & ND & $0.1 \mathrm{mg} / \mathrm{L}$ \\
Chlorobenzene & ND & $0.1 \mathrm{mg} / \mathrm{L}$ \\
Chloroform & ND & $0.1 \mathrm{mg} / \mathrm{L}$ \\
1,4-Dichlorobenzene (p-DCB) & ND & $0.1 \mathrm{mg} / \mathrm{L}$ \\
1,2-Dichloroethane (1,2-DCA) & ND & $0.1 \mathrm{mg} / \mathrm{L}$ \\
1,1-Dichloroethene (1,1-DCE) & ND & $0.1 \mathrm{mg} / \mathrm{L}$ \\
Methyl Ethyl Ketone & ND & $2 . \mathrm{mg} / \mathrm{L}$ \\
Tetrachloroethene (PCE) & ND & $0.1 \mathrm{mg} / \mathrm{L}$ \\
Trichloroethene (TCE) & ND & $0.1 \mathrm{mg} / \mathrm{L}$ \\
Vinyl chloride & ND & $0.1 \mathrm{mg} / \mathrm{L}$
\end{tabular}

QUALITY CONTROL DATA:

Surrogate

4-Bromofluorobenzene

Dibromofluoromethane

Toluene-d8

\section{\% Recovery}

99

102

99
Acceptable Range

$74-121$

$78-120$

$81-117$

ND - Not Detected

This report shall not be reproduced except in full, without the written approval of the laboratory. 


$\begin{array}{llll}\text { CLIENT: } & \text { Bechtel Nevada } & \text { CLIENT ID: } & \text { 252505-2-0 } \\ \text { PROJECT ID: } & \text { V1136 } & \text { DATE SAMPLED: } & \text { 5/30/01 } \\ \text { PROJECT \#: } & 23081 & \text { NEL SAMPLE ID: } & \text { L0105303-03 }\end{array}$

- TEST: $\quad$ TCLP by EPA 1311, July 1992 \& Volatile Organies by EPA 8260B, Dec. 1996 METHOD: EPA 8260 TCLP EXTRACT DATE: $6 / 4 / 01$ MATRIX: Solid EXTRACTED NA

$\begin{array}{llll}\text { - DILUTION: } 1 & \text { ANALYZED: } & \text { 6/5/01 }\end{array}$

\section{PARAMETER}

Benzene

Carbon tetrachloride

Chlorobenzene

Chloroform

1,4-Dichlorobenzene ( $p$-DCB)

1,2-Dichloroethane (1,2-DCA)

1,1-Dichloroethene (1,1-DCE)

Methyl Ethyl Ketone

Tetrachloroethene (PCE)

Trichloroethene (TCE)

Vinyl chloride

\begin{tabular}{c}
$\begin{array}{c}\text { Result } \\
\mathbf{m g} / \mathbf{L}\end{array}$ \\
\hline $\mathrm{ND}$ \\
$\mathrm{ND}$ \\
$\mathrm{ND}$ \\
$\mathrm{ND}$ \\
$\mathrm{ND}$ \\
$\mathrm{ND}$ \\
$\mathrm{ND}$ \\
$\mathrm{ND}$ \\
$\mathrm{ND}$ \\
$\mathrm{ND}$ \\
$\mathrm{ND}$
\end{tabular}

\section{Reporting}

Limit

$0.1 \mathrm{mg} / \mathrm{L}$

$0.1 \mathrm{mg} / \mathrm{L}$

$0.1 \mathrm{mg} / \mathrm{L}$

$0.1 \mathrm{mg} / \mathrm{L}$

$0.1 \mathrm{mg} / \mathrm{L}$

$0.1 \mathrm{mg} / \mathrm{L}$

$0.1 \mathrm{mg} / \mathrm{L}$

2. $\mathrm{mg} / \mathrm{L}$

$0.1 \mathrm{mg} / \mathrm{L}$

$0.1 \mathrm{mg} / \mathrm{L}$

$0.1 \mathrm{mg} / \mathrm{L}$

QUALITY CONTROL DATA:

\section{Surrogate}

4-Bromofluorobenzene

Dibromofluoromethane

Toluene-d8
\% Recovery

101

105

99
Acceptable Range

$74-121$

$78-120$

$81-117$

ND - Not Detected

This report shall not be reproduced except in full, without the written approval of the laboratory. 
CLIENT: Bechtel Nevada

PROJECT ID: V1136

PROJECT \#: 23081
CLIENT ID: 252504-1-0

DATE SAMPLED: 5/30/01

NEL SAMPLE ID: L0105303-04

TEST: $\quad$ TCLP by EPA 1311, July 1992 \& Volatile Organics by EPA 8260B, Dec. 1996

METHOD: $\quad$ EPA 8260

MATRIX: Solid

DILUTION: 1

TCLP EXTRACT DATE: $6 / 4 / 01$

EXTRACTED NA

ANALYZED: $\quad 6 / 5 / 01$

\section{PARAMETER}

Benzene

Carbon tetrachloride

Chlorobenzene

Chloroform

1,4-Dichlorobenzene ( $p$-DCB)

1,2-Dichloroethane (1,2-DCA)

1,1-Dichloroethene (1,1-DCE)

Methyl Ethyl Ketone

Tetrachloroethene (PCE)

Trichloroethene (TCE)

Vinyl chloride

\begin{tabular}{c}
$\begin{array}{c}\text { Result } \\
\text { mg/L }\end{array}$ \\
\hline ND \\
ND \\
ND \\
ND \\
ND \\
ND \\
ND \\
ND \\
ND \\
ND \\
ND
\end{tabular}

\section{Reporting} Limit

$0.1 \mathrm{mg} / \mathrm{L}$

$0.1 \mathrm{mg} / \mathrm{L}$

$0.1 \mathrm{mg} / \mathrm{L}$

$0.1 \mathrm{mg} / \mathrm{L}$

$0.1 \mathrm{mg} / \mathrm{L}$

$0.1 \mathrm{mg} / \mathrm{L}$

$0.1 \mathrm{mg} / \mathrm{L}$

2. $\mathrm{mg} / \mathrm{L}$

$0.1 \mathrm{mg} / \mathrm{L}$

$0.1 \mathrm{mg} / \mathrm{L}$

$0.1 \mathrm{mg} / \mathrm{L}$

\section{QUALITY CONTROL DATA:}

Surrogate

4-Bromofluorobenzene

Dibromofluoromethane

Toluene-d8
$\%$ Recovery

102

104

99
Acceptable Range

$74-121$

$78-120$

$81-117$

ND - Not Detected

This report shall not be reproduced except in full, without the written approval of the laboratory. 


$\begin{array}{llll}\text { CLIENT: } & \text { Bechtel Nevada } & \text { CLIENT D: } & \text { 252504-2-0 } \\ \text { PROJECT ID: } & \text { V1136 } & \text { DATE SAMPLED: } & \text { 5/30/01 } \\ \text { PROJECT \#: } & 23081 & \text { NEL SAMPLE ID: } & \text { L0105303-05 }\end{array}$

- TEST: $\quad$ TCLP by EPA 1311, July 1992 \& Volatile Organics by EPA $8260 B$, Dec. 1996

METHOD: $\quad$ EPA 8260 TCLP EXTRACT DATE: $6 / 5 / 01$

MATRIX: Solid EXTRACTED NA

$\begin{array}{llll}\text { DILUTION: } & 1 & \text { ANALYZED: } & 6 / 6 / 01\end{array}$

PARAMETER

Benzene

Carbon tetrachloride

Reporting

\begin{tabular}{cc}
$\begin{array}{c}\text { Result } \\
\text { mg/L }\end{array}$ & $\begin{array}{c}\text { Reporting } \\
\text { Limit }\end{array}$ \\
\cline { 2 - 2 } ND & $0.1 \mathrm{mg} / \mathrm{L}$ \\
ND & $0.1 \mathrm{mg} / \mathrm{L}$ \\
ND & $0.1 \mathrm{mg} / \mathrm{L}$ \\
ND & $0.1 \mathrm{mg} / \mathrm{L}$ \\
ND & $0.1 \mathrm{mg} / \mathrm{L}$ \\
ND & $0.1 \mathrm{mg} / \mathrm{L}$ \\
ND & $0.1 \mathrm{mg} / \mathrm{L}$ \\
ND & $2 . \mathrm{mg} / \mathrm{L}$ \\
ND & $0.1 \mathrm{mg} / \mathrm{L}$ \\
ND & $0.1 \mathrm{mg} / \mathrm{L}$ \\
ND & $0.1 \mathrm{mg} / \mathrm{L}$
\end{tabular}

QUALITY CONTROL DATA:

Surrogate

4-Bromofluorobenzene

\% Recovery

Dibromofluoromethane

101

103

Toluene-d8

99

$74-121$

$78-120$

$81-117$

ND - Not Detected

This report shall not be reproduced except in full, without the written approval of the laboratory. 

CLIENT: Bechtel Nevada
CLIENT ID:
252504-3-0
PROJECT ID: V1136
DATE SAMPLED: $5 / 30 / 01$
PROJECT \#: 23081
NEL SAMPLE ID: L0105303-06

TEST: $\quad$ TCLP by EPA 1311, July 1992 \& Volatile Organies by EPA 8260B, Dec. 1996

METHOD: $\quad$ EPA 8260

MATRIX: Solid

DILUTION: 1

TCLP EXTRACT DATE: $6 / 5 / 01$

EXTRACTED NA

ANALYZED: $\quad 6 / 6 / 01$

PARAMETER

Benzene

Carbon tetrachloride

Chlorobenzene

Chloroform

1,4-Dichlorobenzene ( $p-D C B$ )

1,2-Dichloroethane (1,2-DCA)

1,1-Dichloroethene (1,1-DCE)

Methyl Ethyl Ketone

Tetrachloroethene (PCE)

Trichloroethene (TCE)

Vinyl chloride

\begin{tabular}{c}
$\begin{array}{c}\text { Result } \\
\text { mg/L }\end{array}$ \\
\hline ND \\
ND \\
ND \\
ND \\
ND \\
ND \\
ND \\
ND \\
ND \\
ND \\
ND
\end{tabular}

Reporting

Limit

$0.1 \mathrm{mg} / \mathrm{L}$

$0.1 \mathrm{mg} / \mathrm{L}$

$0.1 \mathrm{mg} / \mathrm{L}$

$0.1 \mathrm{mg} / \mathrm{L}$

$0.1 \mathrm{mg} / \mathrm{L}$

$0.1 \mathrm{mg} / \mathrm{L}$

$0.1 \mathrm{mg} / \mathrm{L}$

2. $\mathrm{mg} / \mathrm{L}$

$0.1 \mathrm{mg} / \mathrm{L}$

$0.1 \mathrm{mg} / \mathrm{L}$

$0.1 \mathrm{mg} / \mathrm{L}$

QUALITY CONTROL DATA:

\section{Surrogate}

4-Bromofluorobenzene

Dibromofluoromethane

Toluene-d8
$\%$ Recovery

100

103

99
Acceptable Range

$74-121$

$78-120$

$81-117$

ND - Not Detected

This report shall not be reproduced except in full, without the written approval of the laboratory. 

CLIENT:
Bechtel Nevada
CLIENT ID:
Method Blank
PROJECT ID: V1136
DATE SAMPLED: NA
PROJECT \#: 23081
NEL SAMPLE ID: 010605TCLP_1A-BLK

- TEST:

TCLP by EPA 1311, July 1992 \& Volatile Organics by EPA 8260B, Dec. 1996

METHOD: $\quad$ EPA 8260

MATRIX: TCLP Extract

TCLP EXTRACT DATE: 6/4/01

EXTRACTED NA

ANALYZED: $\quad 6 / 5 / 01$

PARAMETER

- Benzene

Carbon tetrachloride

Chlorobenzene

Chloroform

1,4-Dichlorobenzene (p-DCB)

1,2-Dichloroethane (1,2-DCA)

1,1-Dichloroethene (1,1-DCE)

Methyl Ethyl Ketone

Tetrachloroethene (PCE)

Trichloroethene (TCE)

- Vinyl chloride

\section{Result}

$\mathrm{mg} / \mathrm{L}$

ND

$\mathrm{ND}$

ND

$\mathrm{ND}$

ND

ND

ND

ND

ND

ND

ND
Reporting Limit

$0.1 \mathrm{mg} / \mathrm{L}$

$0.1 \mathrm{mg} / \mathrm{L}$

$0.1 \mathrm{mg} / \mathrm{L}$

$0.1 \mathrm{mg} / \mathrm{L}$

$0.1 \mathrm{mg} / \mathrm{L}$

$0.1 \mathrm{mg} / \mathrm{L}$

$0.1 \mathrm{mg} / \mathrm{L}$

$2 \mathrm{mg} / \mathrm{L}$

$0.1 \mathrm{mg} / \mathrm{L}$

$0.1 \mathrm{mg} / \mathrm{L}$

$0.1 \mathrm{mg} / \mathrm{L}$

QUALITY CONTROL DATA:

Surrogate

4-Bromofluorobenzene

$\rightarrow$ Dibromofluoromethane

Toluene-d8
\% Recovery

99

102

100
Acceptable Range

$74-121$

$78-120$

$81-117$

- ND - Not Detected

This report shall not be reproduced except in full, without the written approval of the laboratory. 


$\begin{array}{llll}\text { CLIENT: } & \text { Bechtel Nevada } & \text { CLIENT ID: } & \text { Method Blank } \\ \text { PROJECT ID: } & \text { V1136 } & \text { DATE SAMPLED: } & \text { NA } \\ \text { PROJECT \#: } & 23081 & \text { NEL SAMPLE ID: } & \text { 010606TCLP_1A-BLK }\end{array}$

TEST: $\quad$ TCLP by EPA 1311, July 1992 \& Volatile Organics by EPA 8260B, Dec. 1996

METHOD: EPA 8260 TCLP EXTRACT DATE: $6 / 5 / 01$

MATRIX: TCLP EXtract EXTRACTED NA

ANALYZED: $\quad 6 / 6 / 01$

\begin{tabular}{lcc}
\hline PARAMETER & $\begin{array}{c}\text { Result } \\
\text { mg/L }\end{array}$ & $\begin{array}{c}\text { Reporting } \\
\text { Limit }\end{array}$ \\
\cline { 2 - 3 } Benzene & ND & $0.1 \mathrm{mg} / \mathrm{L}$ \\
Carbon tetrachloride & $\mathrm{ND}$ & $0.1 \mathrm{mg} / \mathrm{L}$ \\
Chlorobenzene & $\mathrm{ND}$ & $0.1 \mathrm{mg} / \mathrm{L}$ \\
Chloroform & $\mathrm{ND}$ & $0.1 \mathrm{mg} / \mathrm{L}$ \\
1,4-Dichlorobenzene (p-DCB) & $\mathrm{ND}$ & $0.1 \mathrm{mg} / \mathrm{L}$ \\
1,2-Dichloroethane (1,2-DCA) & $\mathrm{ND}$ & $0.1 \mathrm{mg} / \mathrm{L}$ \\
1,1-Dichloroethene (1,1-DCE) & $\mathrm{ND}$ & $0.1 \mathrm{mg} / \mathrm{L}$ \\
Methyl Ethyl Ketone & $\mathrm{ND}$ & $2 \mathrm{mg} / \mathrm{L}$ \\
Tetrachloroethene (PCE) & $\mathrm{ND}$ & $0.1 \mathrm{mg} / \mathrm{L}$ \\
Trichloroethene (TCE) & $\mathrm{ND}$ & $0.1 \mathrm{mg} / \mathrm{L}$ \\
Vinyl chloride & $\mathrm{ND}$ & $0.1 \mathrm{mg} / \mathrm{L}$
\end{tabular}

QUALITY CONTROL DATA:

\section{Surrogate}

4-Bromofluorobenzene

Dibromofluoromethane

Toluene-d8
$\%$ Recovery

102

104

100
Acceptable Range

$74-121$

$78-120$

$81-117$

ND - Not Detected

This report shall not be reproduced except in full, without the written approval of the laboratory. 


\begin{tabular}{|c|c|c|c|c|c|c|c|}
\hline - & $\begin{array}{l}\text { Bechtel Nevada } \\
\text { V1136 } \\
23081\end{array}$ & & & & & & \\
\hline - & $\begin{array}{l}\text { Volatile Organic } \\
\text { Aqueous }\end{array}$ & mpounds by EPA 8260B, & nber 1996 & & & & \\
\hline- & PARAMETER & NEL Sample ID & $\begin{array}{c}\text { Spike } \\
\text { Amount }\end{array}$ & $\frac{\text { Spike }}{\text { Result }}$ & $\frac{\text { Percent }}{\text { Recovery }}$ & $\frac{\text { Acceptable }}{\text { Range }}$ & $\underline{\text { RPD }}$ \\
\hline & Acetone & 010605AQ60_1A-LCS & 50 & 75.45 & $151 \mathrm{~J}$ & $70-130$ & \\
\hline$\Rightarrow$ & Acetone & P0105052-01-MS & 50 & 50.25 & 100 & $70-130$ & \\
\hline & Acetone & P0105052-01-MSD & 50 & 44.81 & 90 & $70-130$ & 11.4 \\
\hline & Benzene & 010605AQ60_1A-LCS & 50 & 48.26 & 97 & $70-130$ & \\
\hline - & Benzene & P0105052-01-MS & 50 & 51.32 & 103 & $70-130$ & \\
\hline & Benzene & P0105052-01-MSD & 50 & 51.34 & 103 & $70-130$ & 0. \\
\hline & Bromobenzene & 010605AQ60_1A-LCS & 50 & 51.24 & 102 & $70-130$ & \\
\hline - & Bromobenzene & P0105052-01-MS & 50 & 54.33 & 109 & $70-130$ & \\
\hline & Bromobenzene & P0105052-01-MSD & 50 & 55.03 & 110 & $70-130$ & 1.3 \\
\hline - & Bromochloromethane & 010605AQ60_1A-LCS & 50 & 48.49 & 97 & $70-130$ & \\
\hline & Bromochloromethane & P0105052-01-MS & 50 & 52.57 & 105 & $70-130$ & \\
\hline & Bromochloromethane & P0105052-01-MSD & 50 & 54.22 & 108 & $70-130$ & 3.1 \\
\hline - & Bromodichloromethane & 010605AQ60_1A-LCS & 50 & 50.37 & 101 & $70-130$ & \\
\hline & Bromodichloromethane & P0105052-01-MS & 50 & 53.63 & 107 & $70-130$ & \\
\hline & Bromodichloromethane & P0105052-01-MSD & 50 & 54.36 & 109 & $70-130$ & 1.4 \\
\hline - & Bromoform & 010605AQ60_1A-LCS & 50 & 45.84 & 92 & $70-130$ & \\
\hline & Bromoform & P0105052-01-MS & 50 & 53.18 & 106 & $70-130$ & \\
\hline & Bromoform & P0105052-01-MSD & 50 & 52.63 & 105 & $70-130$ & 1. \\
\hline- & Bromomethane & 010605AQ60_1A-LCS & 50 & 56.67 & 113 & $70-130$ & \\
\hline & Bromomethane & P0105052-01-MS & 50 & 56.02 & 112 & $70-130$ & \\
\hline[ & Bromomethane & P0105052-01-MSD & 50 & 57.05 & 114 & $70-130$ & 1.8 \\
\hline- & 2-Butanone & 010605AQ60_1A-LCS & 50 & 43.56 & 87 & $70-130$ & \\
\hline & 2-Butanone & P0105052-01-MS & 50 & 40.5 & 81 & $70-130$ & \\
\hline r & 2-Butanone & P0105052-01-MSD & 50 & 39.36 & 79 & $70-130$ & 2.9 \\
\hline & n-Butylbenzene & 010605AQ60_1A-LCS & 50 & 48.51 & 97 & $70-130$ & \\
\hline & n-Butylbenzene & P0105052-01-MS & 50 & 54.48 & 109 & $70-130$ & \\
\hline - & n-Butylbenzene & P0105052-01-MSD & 50 & 51.73 & 103 & $70-130$ & 5.2 \\
\hline & sec-Butylbenzene & 010605AQ60_1A-LCS & 50 & 51.87 & 104 & $70-130$ & \\
\hline & sec-Butylbenzene & P0105052-01-MS & 50 & 53.79 & 108 & $70-130$ & \\
\hline 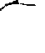 & sec-Butylbenzene & P0105052-01-MSD & 50 & 54.34 & 109 & $70-130$ & 1. \\
\hline & tert-Butylbenzene & 010605AQ60_1A-LCS & 50 & 50.17 & 100 & $70-130$ & \\
\hline$\Rightarrow$ & tert-Butylbenzene & P0105052-01-MS & 50 & 49.13 & 98 & $70-130$ & \\
\hline & tert-Butylbenzene & P0105052-01-MSD & 50 & 50.15 & 100 & $70-130$ & 2.1 \\
\hline & Carbon disulfide & 010605AQ60_1A-LCS & 50 & 49.67 & 99 & $70-130$ & \\
\hline - & Carbon disulfide & P0105052-01-MS & 50 & 50.43 & 101 & $70-130$ & \\
\hline & Carbon disulfide & P0105052-01-MSD & 50 & 50.99 & 102 & $70-130$ & 1.1 \\
\hline & Carbon tetrachloride & 010605AQ60_1A-LCS & 50 & 52.09 & 104 & $70-130$ & \\
\hline - & Carbon tetrachloride & P0105052-01-MS & 50 & 51.95 & 104 & $70-130$ & \\
\hline
\end{tabular}

ND - Not Detected

- This report shall not be reproduced except in full, without the written approval of the laboratory. 


$\begin{array}{ll}\text { CLIENT: } & \text { Bechtel Nevada } \\ \text { PROJECT ID: } & \text { V1136 } \\ \text { PROJECT \#: } & 23081 \\ \text { TEST: } & \text { Volatile Organic Compounds by EPA 8260B, December } 1996 \\ \text { MATRIX: } & \text { Aqueous }\end{array}$

\begin{tabular}{|c|c|c|c|c|c|c|}
\hline PARAMETER & NEL Sample ID & $\begin{array}{l}\text { Spike } \\
\text { Amount }\end{array}$ & $\begin{array}{l}\text { Spike } \\
\text { Result }\end{array}$ & $\begin{array}{l}\text { Percent } \\
\text { Recovery }\end{array}$ & $\frac{\text { Acceptable }}{\text { Range }}$ & $\underline{\text { RPD }}$ \\
\hline Carbon tetrachloride & P0105052-01-MSD & 50 & 52.57 & 105 & $70-130$ & 1.2 \\
\hline Chlorobenzene & 010605AQ60_1A-LCS & 50 & 51.01 & 102 & $70-130$ & \\
\hline Chlorobenzene & P0105052-01-MS & 50 & 53.39 & 107 & $70-130$ & \\
\hline Chlorobenzene & P0105052-01-MSD & 50 & 54 & 108 & $70-130$ & 1.1 \\
\hline Chloroethane & 010605AQ60_1A-LCS & 50 & 49.23 & 98 & $70-130$ & \\
\hline Chloroethane & P0105052-01-MS & 50 & 52.17 & 104 & $70-130$ & \\
\hline Chloroethane & P0105052-01-MSD & 50 & 52.58 & 105 & $70-130$ & 0.8 \\
\hline Chloroform & 010605AQ60_1A-LCS & 50 & 45.56 & 91 & $70-130$ & \\
\hline Chloroform & P0105052-01-MS & 50 & 48.56 & 97 & $70-130$ & \\
\hline Chloroform & P0105052-01-MSD & 50 & 48.98 & 98 & $70-130$ & 0.9 \\
\hline Chloromethane & 010605AQ60_1A-LCS & 50 & 45.68 & 91 & $70-130$ & \\
\hline Chloromethane & P0105052-01-MS & 50 & 58.75 & 118 & $70-130$ & \\
\hline Chloromethane & P0105052-01-MSD & 50 & 56.34 & 113 & $70-130$ & 4.2 \\
\hline 2-Chlorotoluene & 010605AQ60_1A-LCS & 50 & 52.29 & 105 & $70-130$ & \\
\hline 2-Chlorotoluene & P0105052-01-MS & 50 & 53.4 & 107 & $70-130$ & \\
\hline 2-Chlorotoluene & P0105052-01-MSD & 50 & 56.43 & 113 & $70-130$ & 5.5 \\
\hline 4-Chlorotoluene & 010605AQ60_1A-LCS & 50 & 47.51 & 95 & $70-130$ & \\
\hline 4-Chlorotoluene & P0105052-01-MS & 50 & 48.21 & 96 & $70-130$ & \\
\hline 4-Chlorotoluene & P0105052-01-MSD & 50 & 46.15 & 92 & $70-130$ & 4.4 \\
\hline Dibromochloromethane & 010605AQ60_1A-LCS & 50 & 48.75 & 98 & $70-130$ & \\
\hline Dibromochloromethane & P0105052-01-MS & 50 & 52.45 & 105 & $70-130$ & \\
\hline Dibromochloromethane & P0105052-01-MSD & 50 & 52.87 & 106 & $70-130$ & 0.8 \\
\hline 1,2-Dibromo-3-chloropropane (DBCP) & 010605AQ60_1A-LCS & 50 & 31.1 & 62 & $70-130$ & \\
\hline 1,2-Dibromo-3-chloropropane (DBCP) & P0105052-01-MS & 50 & 40.94 & 82 & $70-130$ & \\
\hline 1,2-Dibromo-3-chloropropane (DBCP) & P0105052-01-MSD & 50 & 38.91 & 78 & $70-130$ & 5.1 \\
\hline 1,2-Dibromoethane (EDB) & 010605AQ60_1A-LCS & 50 & 43.65 & 87 & $70-130$ & \\
\hline 1,2-Dibromoethane (EDB) & P0105052-01-MS & 50 & 50.09 & 100 & $70-130$ & \\
\hline 1,2-Dibromoethane (EDB) & P0105052-01-MSD & 50 & 49.99 & 100 & $70-130$ & 0.2 \\
\hline Dibromomethane & 010605AQ60_1A-LCS & 50 & 45.88 & 92 & $70-130$ & \\
\hline Dibromomethane & P0105052-01-MS & 50 & 52.44 & 105 & $70-130$ & \\
\hline Dibromomethane & P0105052-01-MSD & 50 & 51.93 & 104 & $70-130$ & 1. \\
\hline 1,2-Dichlorobenzene (o-DCB) & 010605AQ60_1A-LCS & 50 & 48.76 & 98 & $70-130$ & \\
\hline 1,2-Dichlorobenzene (o-DCB) & P0105052-01-MS & 50 & 51.94 & 104 & $70-130$ & \\
\hline 1,2-Dichlorobenzene (o-DCB) & P0105052-01-MSD & 50 & 52.7 & 105 & $70-130$ & 1.5 \\
\hline 1,3-Dichlorobenzene (m-DCB) & 010605AQ60_1A-LCS & 50 & 50.79 & 102 & $70-130$ & \\
\hline 1,3-Dichlorobenzene (m-DCB) & P0105052-01-MS & 50 & 53.28 & 107 & $70-130$ & \\
\hline 1,3-Dichlorobenzene (m-DCB) & P0105052-01-MSD & 50 & 53.42 & 107 & $70-130$ & 0.3 \\
\hline 1,4-Dichlorobenzene (p-DCB) & 010605AQ60_1A-LCS & 50 & 55 & 110 & $70-130$ & \\
\hline
\end{tabular}


CLIENT: Bechtel Nevada

PROJECT ID: V1136

PROJECT \#: 23081

TEST: $\quad$ Volatile Organic Compounds by EPA 8260B, December 1996

MATRIX: Aqueous

\section{PARAMETER}

1,4-Dichlorobenzene ( $\mathrm{p}-\mathrm{DCB}$ )

1,4-Dichlorobenzene (p-DCB)

Dichlorodifluoromethane (Freon 12)

Dichlorodifluoromethane (Freon 12)

Dichlorodifluoromethane (Freon 12)

1,1-Dichloroethane (1,1-DCA)

1,1-Dichloroethane (1,1-DCA)

1,1-Dichloroethane (1,1-DCA)

1,2-Dichloroethane (1,2-DCA)

1,2-Dichloroethane (1,2-DCA)

1,2-Dichloroethane (1,2-DCA)

1,1-Dichloroethene (1,1-DCE)

1,1-Dichloroethene (1,1-DCE)

1,1-Dichloroethene (1,1-DCE)

cis-1,2-Dichloroethene

cis-1,2-Dichloroethene

cis-1,2-Dichloroethene

trans-1,2-Dichloroethene

trans-1,2-Dichloroethene

trans-1,2-Dichloroethene

1,2-Dichloropropane

1,2-Dichloropropane

1,2-Dichloropropane

1,3-Dichloropropane

1,3-Dichloropropane

1,3-Dichloropropane

2,2-Dichloropropane

2,2-Dichloropropane

2,2-Dichloropropane

1,1-Dichloropropene

1,1-Dichloropropene

1,1-Dichloropropene

cis-1,3-Dichloropropene

cis-1,3-Dichloropropene

cis-1,3-Dichloropropene

trans-1,3-Dichloropropene

trans-1,3-Dichloropropene

trans-1,3-Dichloropropene
NEL Sample ID

P0105052-01-MS

P0105052-01-MSD

010605AQ60_1A-LCS

P0105052-01-MS

P0105052-01-MSD

010605AQ60_1A-LCS

P0105052-01-MS

P0105052-01-MSD

010605AQ60_1A-LCS

P0105052-01-MS

P0105052-01-MSD

010605AQ60_1A-LCS

P0105052-01-MS

P0105052-01-MSD

010605AQ60_1A-LCS

P0105052-01-MS

P0105052-01-MSD

010605AQ60_1A-LCS

P0105052-01-MS

P0105052-01-MSD

010605AQ60_1A-ICS

P0105052-01-MS

P0105052-01-MSD

010605AQ60_1A-LCS

P0105052-01-MS

P0105052-01-MSD

010605AQ60_1A-LCS

P0105052-01-MS

P0105052-01-MSD

010605AQ60_1A-LCS

P0105052-01-MS

P0105052-01-MSD

010605AQ60_1A-LCS

P0105052-01-MS

P0105052-01-MSD

010605AQ60_1A-LCS

P0105052-01-MS

P0105052-01-MSD
Spike Amount

50

50

50

50

50

50

50

50

50

50

50

50

50

50

50

50

50

50

50

50

50

50

50

50

50

50

50

50

50

50

50

50

50

50

50

50

50

50
Spike

Result

58.13

58.7

62.41

61.34

60.03

46.62

51.23

51.7

46.47

50.69

50.88

46.87

50.87

50.74

46.44

50.58

51.05

46.49

49.97

49.98

50.18

53.9

54.17

45.48

52.25

51.84

62.03

56.95

57.32

50.86

52.73

52.52

43.73

46.66

47.03

43.73

46.66

47.03

\begin{tabular}{c}
$\frac{\text { Percent }}{\text { Recovery }}$ \\
\hline 116 \\
117 \\
125
\end{tabular}

123

120

93

102

103

93

101

102

94

102

101

93

101

102

93

100

100

100

108

108

91

105

104

124

114

115

102

105

105

87

93

94

87

93

94
Acceptable

Range

RPD

$70-130$

$70-130 \quad 1$

$70-130$

$70-130$

$70-130$

$70-130$

$70-130$

$70-130$

0.9

$70-130$

$70-130$

$70-130$

0.4

$70-130$

$70-130$

$70-130$

0.3

$70-130$

$70-130$

$70-130$

0.9

$70-130$

$70-130$

$70-130$

0.

$70-130$

$70-130$

$70-130$

0.5

$70-130$

$70-130$

$70-130$

0.8

$70-130$

$70-130$

$70-130$

0.6

$70-130$

$70-130$

$70-130$

0.4

$70-130$

$70-130$

$70-130$

$70-130$

$70-130$

$70-130$

.

ND - Not Detected

- This report shall not be reproduced except in full, without the written approval of the laboratory. 


$\begin{array}{ll}\text { CLIENT: } & \text { Bechtel Nevada } \\ \text { PROJECT ID: } & \text { V1136 } \\ \text { PROJECT \#: } & 23081 \\ \text { TEST: } & \text { Volatile Organic Compounds by EPA 8260B, December } 1996 \\ \text { MATRIX: } & \text { Aqueous }\end{array}$

Aqueous

\section{PARAMETER}

Ethylbenzene

Ethylbenzene

Ethylbenzene

Hexachlorobutadiene

Hexachlorobutadiene

Hexachlorobutadiene

2-Hexanone

2-Hexanone

2-Hexanone

Iodomethane

Iodomethane

Iodomethane

Isopropylbenzene

Isopropylbenzene

Isopropylbenzene

p-Isopropyitoluene

p-Isopropyltoluene

p-Isopropyltoluene

Methylene chloride (Dichloromethane)

Methylene chloride (Dichloromethane)

Methylene chloride (Dichloromethane)

4-Methyl-2-pentanone

4-Methyl-2-pentanone

4-Methyl-2-pentanone

MTBE

MTBE

MTBE

Naphthalene

Naphthalene

Naphthalene

n-Propylbenzene

n-Propylbenzene

n-Propylbenzene

Styrene

Styrene

Styrene

1,1,1,2-Tetrachloroethane

1,1,1,2-Tetrachloroethane
NEL Sample ID

010605AQ60_1A-LCS

P0105052-01-MS

P0105052-01-MSD

010605AQ60_1A-LCS

P0105052-01-MS

P0105052-01-MSD

010605AQ60_1A-LCS

P0105052-01-MS

P0105052-01-MSD

010605AQ60_1A-LCS

P0105052-01-MS

P0105052-01-MSD

010605AQ60_1A-LCS

P0105052-01-MS

P0105052-01-MSD

010605AQ60_1A-LCS

P0105052-01-MS

P0105052-01-MSD

010605AQ60_1A-LCS

P0105052-01-MS

P0105052-01-MSD

010605AQ60_1A-LCS

P0105052-01-MS

P0105052-01-MSD

010605AQ60_1A-LCS

P0105052-01-MS

P0105052-01-MSD

010605AQ60_1A-LCS

P0105052-01-MS

P0105052-01-MSD

010605AQ60_1A-LCS

P0105052-01-MS

P0105052-01-MSD

010605AQ60_1A-LCS

P0105052-01-MS

P0105052-01-MSD

010605AQ60_1A-LCS

P0105052-01-MS

\section{Spike}

Amount

50

50

50

50

50

50

50

50

50

50

50

50

50

50

50

50

50

50

50

50

50

50

50

50

50

50

50

50

50

50

50

50

50

50

50

50

50

50
Resul

48.01

50.6

51.12

51.5

52.59

54.28

45.3

46.83

42.88

54.87

21.75

30.98

49.45

51.62

51.72

50.81

52.68

52.91

45.62

55.53

56.39

34.7

44.83

42.51

35.58

41.78

41.43

36.72

42.25

44.17

48.59

50.99

50.94

51.28

1.35

1.6

51.04

53.83
Recover

96

101

102

103

105

109

91

94

86

110

$44 \pi$

$62 \pi$

99

103

103

102

105

106

91

111

113

69

90

85

71

84

83

73

85

88

97

102

102

103

$3 \mathrm{~J}$

3 Л

102

108
Acceptable Range

RPD

$70-130$

$70-130$

$70-130$

1.

$70-130$

$70-130$

$70-130$

3.2

$70-130$

$70-130$

$70-130$

8.8

$70-130$

$70-130$

$70-130$

$70-130$

$70-130$

$70-130$

0.2

$70-130$

$70-130$

$70-130$

0.4

$70-130$

$70-130$

$70-130$

1.5

$70-130$

$70-130$

$70-130$

$70-130$

$70-130$

$70-130$

$70-130$

$70-130$

$70-130$

$70-130$

$70-130$

$70-130$

$70-130$

$70-130$

$70-130$

$70-130$

$70-130$

ND - Not Detected

This report shall not be reproduced except in full, without the written approval of the laboratory. 


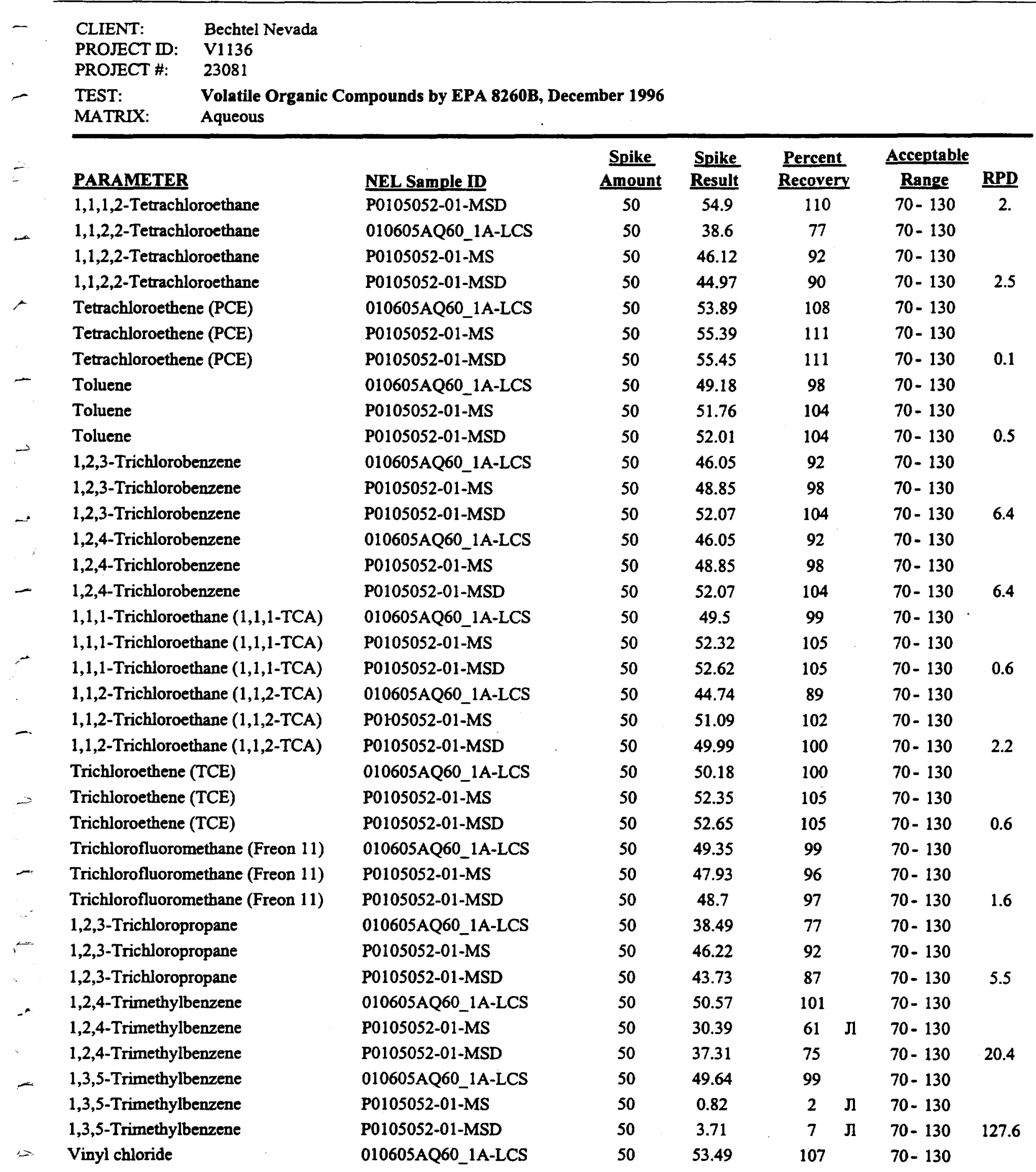

ND - Not Detected

This report shall not be reproduced except in full, without the written approval of the laboratory. 
CLIENT: Bechtel Nevada

PROJECT ID: V1136

PROJECT \#: 23081

TEST: $\quad$ Volatile Organic Compounds by EPA 8260B, December 1996

MATRIX: Aqueous

\section{PARAMETER}

Vinyl chloride

Vinyl chloride

o-Xylene

o-Xylene

o-Xylene

m,p-Xylene

m,p-Xylene

m,p-Xylene
NEL Sample ID

P0105052-01-MS

P0105052-01-MSD

010605AQ60_1A-LCS

P0105052-01-MS

P0105052-01-MSD

010605AQ60_1A-LCS

P0105052-01-MS

P0105052-01-MSD

\section{Spike} Amoun

50

50

50

50

50

100

100

100
Spike

Result

56

56.64

50.53

53.23

52.89

102.22

93.91

99.21
Percent Recovery

112

113

101

106

106

102

94

99
Acceptable

Range RPD

$70-130$

$70-130$

$70-130$

$70-130$

$70-130$

$70-130$

$70-130$

$70-130$

0.6

0.6

5.5

$1.1-$ 
- CLIENT: Bechtel Nevada

PROJECT ID: V1136

PROJECT \#: 23081

- TEST: $\quad$ TCLP by EPA 1311, July 1992 \& Volatile Organics by EPA 8260B, Dec. 1996

MATRIX: $\quad$ Solid

PARAMETER

1,1-Dichloroethene (1,1-DCE)

- Benzene

Chlorobenzene

Toluene

Trichloroethene (TCE)

1,1-Dichloroethene (1,1-DCE)

1,1-Dichloroethene (1,1-DCE)

- Benzene

Benzene

Chlorobenzene

Chlorobenzene

Trichloroethene (TCE)

Trichloroethene (TCE)

\section{NEL Sample ID}

010606TCLP_1A1-LCS

010606TCLP_1A1-LCS

010606TCLP_1A1-LCS

010606TCLP_1A1-LCS

010606TCLP_1A1-LCS

L0105306-04-MS

L0105306-04-MSD

L0105306-04-MS

L0105306-04-MSD

L0105306-04-MS

L0105306-04-MSD

L0105306-04-MS

L0105306-04-MSD

\begin{tabular}{ccc}
$\frac{\text { Spike }}{\text { Amount }}$ & $\frac{\text { Spike }}{\text { Result }}$ \\
\cline { 1 - 1 } 50 & & 50.34 \\
50 & 52.13 \\
50 & 53.3 \\
50 & 52.67 \\
50 & 53.72 \\
50 & 51.91 \\
50 & 53.2 \\
50 & 116.92 \\
50 & 120.82 \\
50 & 55.77 \\
50 & 56.81 \\
50 & 54.22 \\
50 & 55.44
\end{tabular}

\begin{tabular}{c}
$\frac{\text { Percent }}{\text { Recovery }}$ \\
\hline 101 \\
104 \\
107 \\
105 \\
107 \\
104 \\
106 \\
233 \\
240 \\
112 \\
114 \\
108 \\
111
\end{tabular}

Acceptable

Range RPD

$70-130$

$70-130$

$70-130$

$70-130$

$70-130$

$61-145$

$61-145$

$76-127$

$76-127$

$75-130$

$75-130$

1.8

$71-120$

$71-120$

2.2 


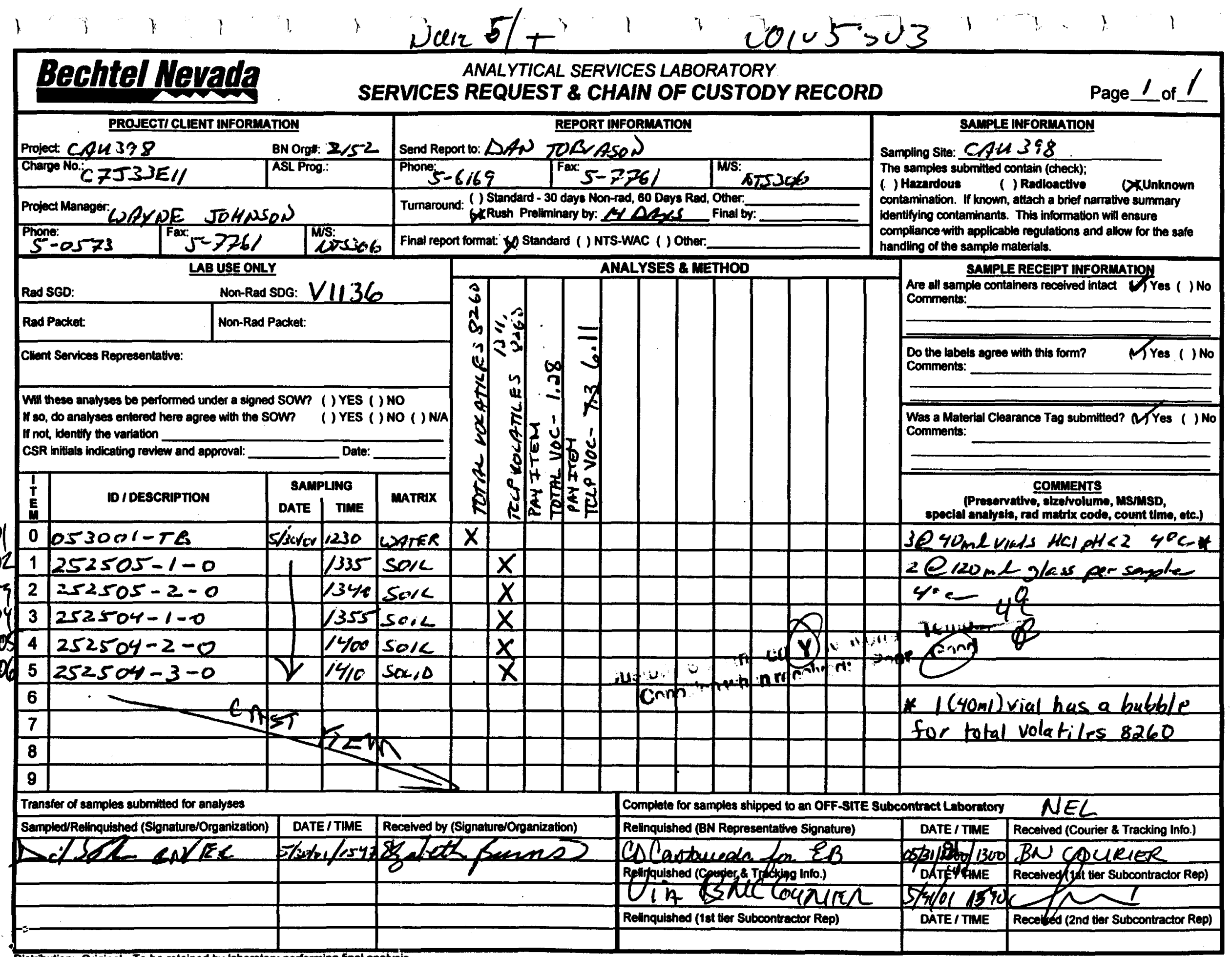




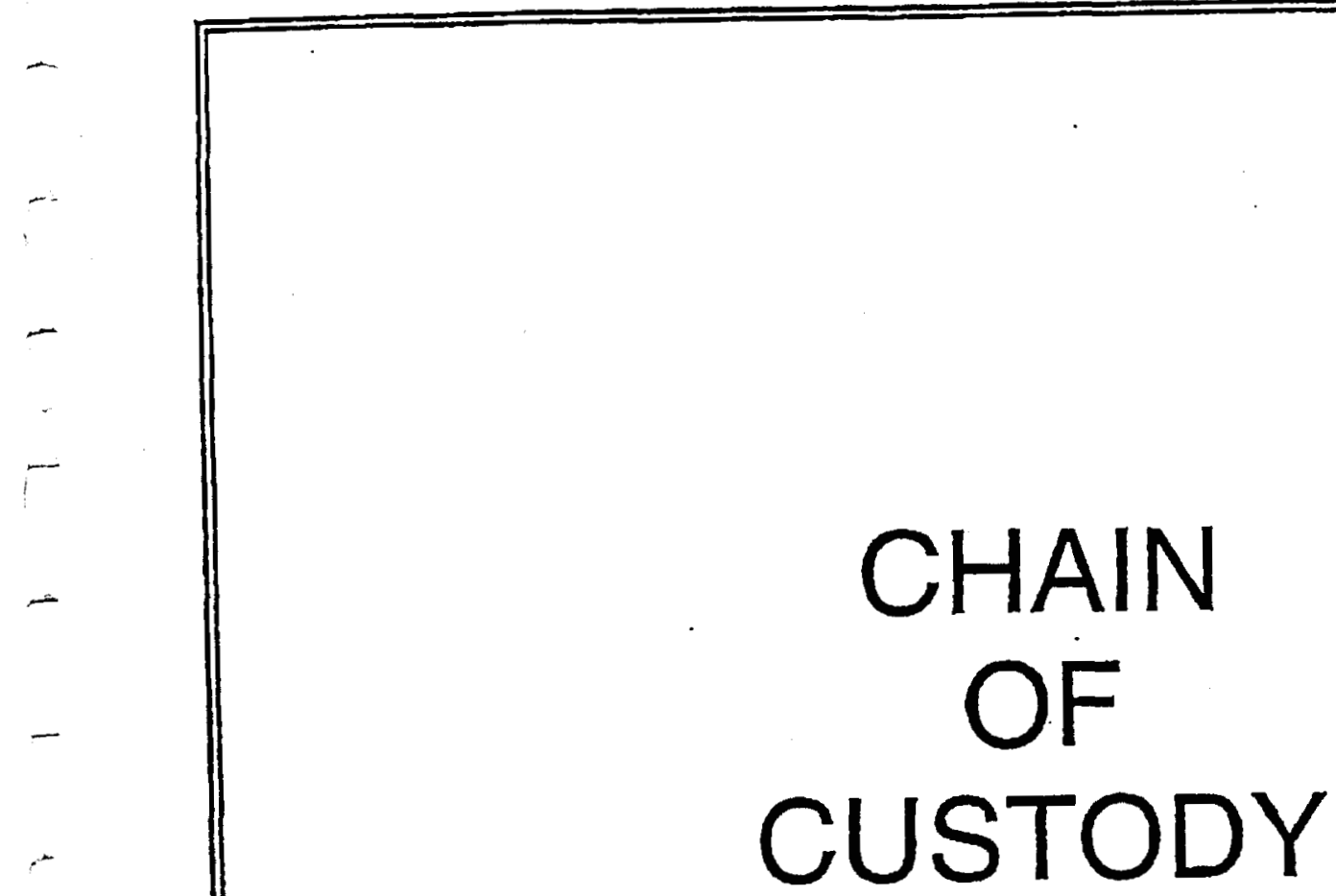

-

-

$-$

$-$

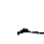

$-$

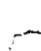




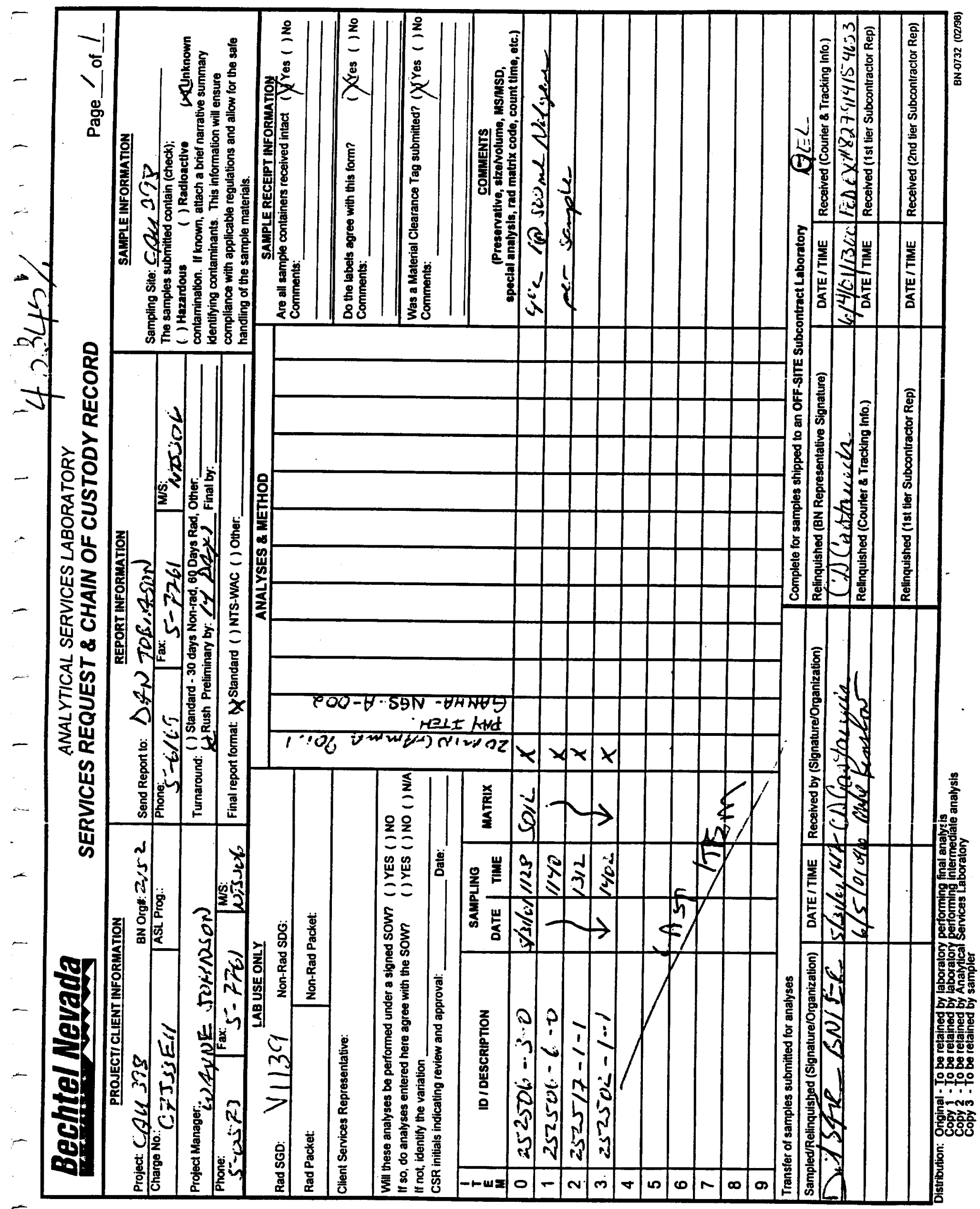


imapay (V)Wd

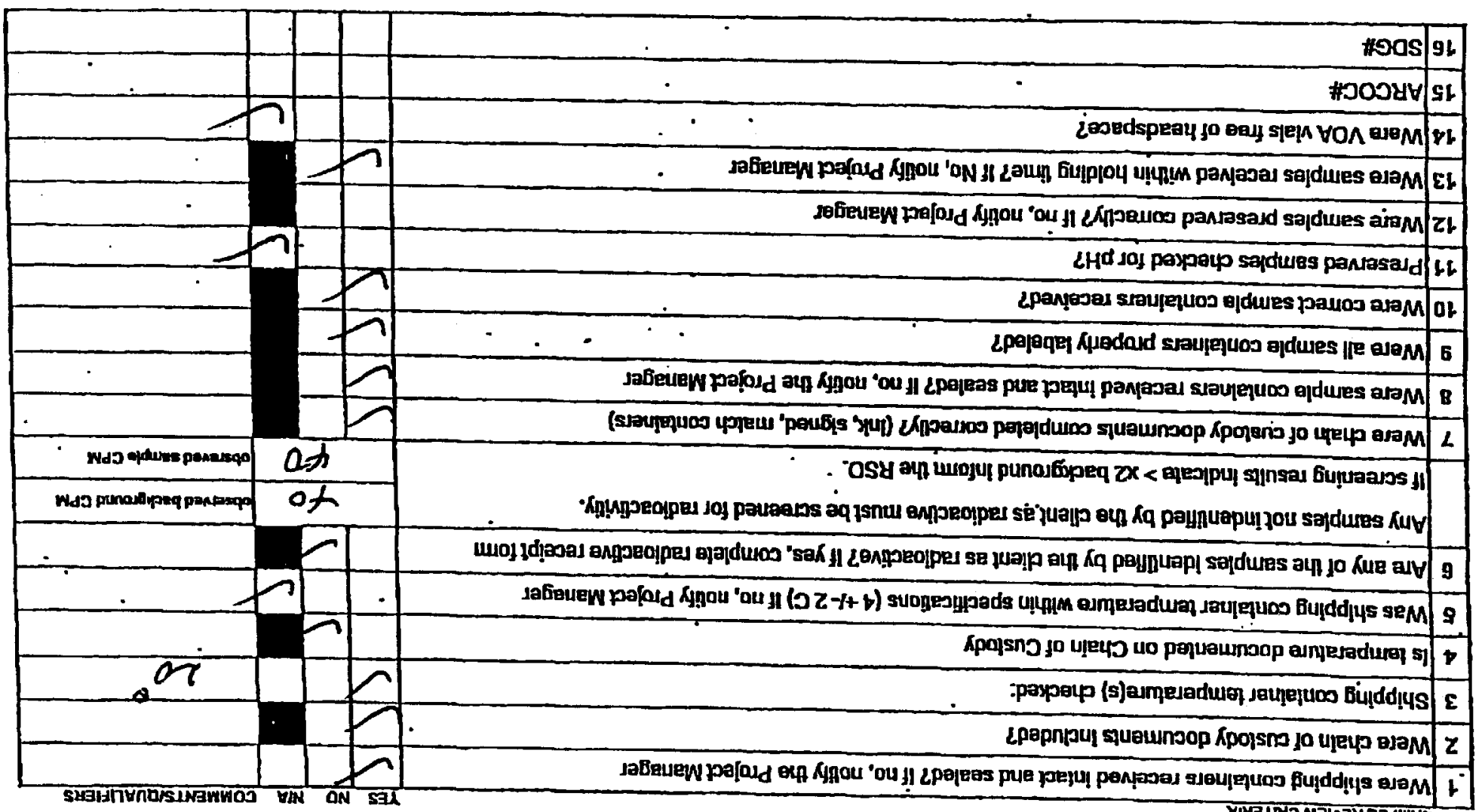

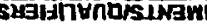

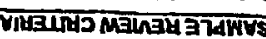

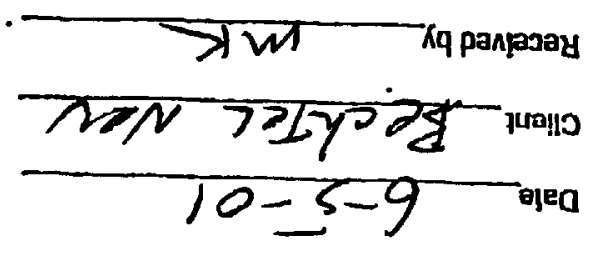

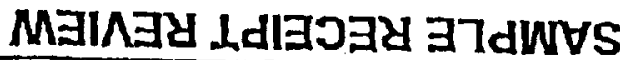




\section{DATA REVIEW QUALIFIER FLAG DEFINITION SHEET}




\section{General Engineering Laibrarories. Inc. DÄTA QUALIFIERS FOR INORGANTC ANALYSES}

Daca Qualiniess used on Form Is or Cerriñcares of Analysis (C of $A$ ) follow the specinicarions se: forth in the technical specificarions of the mosi currear CLP Siaremeat of Work and are deāned as follows.

\begin{tabular}{|c|c|c|}
\hline Secion & Exolanarion & Locarion \\
\hline$E$ & 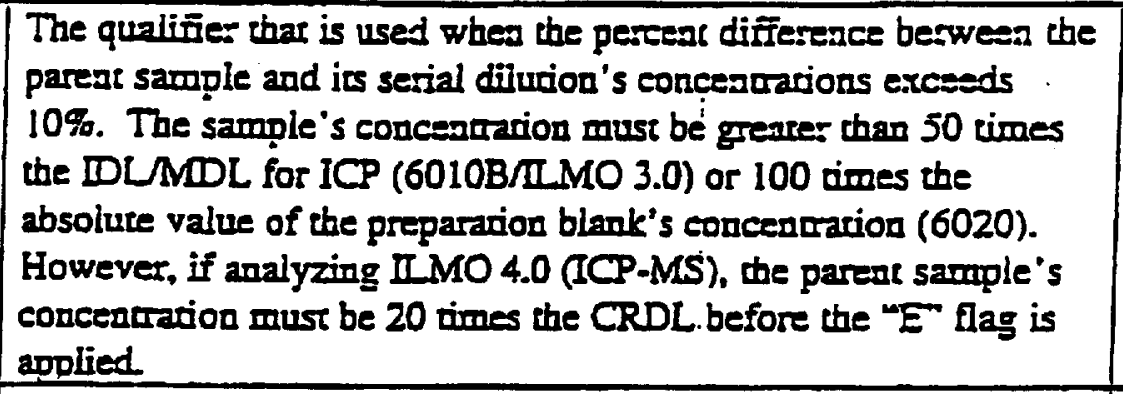 & Form 1 \\
\hline 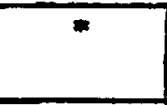 & $\begin{array}{l}\text { The qualifier that is used to indicare thar the duplicare sample } \\
\text { analysis for an anaiyre is our of contol. }\end{array}$ & $\begin{array}{l}\text { Form } 1 . \\
\text { and EDD }\end{array}$ \\
\hline$\div$ & $\begin{array}{l}\text { Correlation coefficieat the Mechod of Standard Addition (MSA) } \\
\text { is less than } 0.095 \text {. }\end{array}$ & $\begin{array}{l}\text { Form } 2 \\
\text { and EDD }\end{array}$ \\
\hline B & $\begin{array}{l}\text { The qualifier is used to indicate that the reported result fell above } \\
\text { the IDLMDL bus below the CRDL }\end{array}$ & $\begin{array}{l}\text { Form } 1 . \\
\text { and EDD }\end{array}$ \\
\hline $\bar{M}$ & $\begin{array}{l}\text { The qualifier is used to indicate that the replicare injecrion } \\
\text { readings of the GFAA sample analysis do pot agree within } 20 \% \\
\text { relarive scandard deviation (RSD) or coefficient of variacion (CV). }\end{array}$ & Form 1. \\
\hline $\mathbf{N}$ & $\begin{array}{l}\text { This qualifier is used to indicate thar the macrix or pre-digested } \\
\text { spike sample recovery for an analyte is not within the specified } \\
\text { control limit. }\end{array}$ & Form l. \\
\hline $\bar{S}$ & $\begin{array}{l}\text { The reported value was determined by the Mechod of Standard } \\
\text { Addicion (MSA). }\end{array}$ & $\begin{array}{l}\text { Form } 1 . \\
\text { and EDD }\end{array}$ \\
\hline$U$ & The analyte's result was less than the IDLMDL. & $\begin{array}{l}\text { C of } A \text { Form } 1 . \\
\text { and EDD }\end{array}$ \\
\hline W & $\begin{array}{l}\text { Post-digestion spike for GFAA analysis is out of control limits } \\
\text { (85\%-115\%), while sample results are less than } 50 \% \text { of the spike } \\
\text { absorioance. }\end{array}$ & $\begin{array}{l}\text { EDD, and } \\
\text { Form } 5, \text { part ? }\end{array}$ \\
\hline $\bar{x}$ & Other reporing flag as defined in repor narrave. & $\begin{array}{l}\text { Form } 1 \text {, } \\
\text { and EDD }\end{array}$ \\
\hline$=$ & $\begin{array}{l}\text { Thus qualifier is used to indicate that the Laborarory Control } \\
\text { Sample (LCS) recovery for an analyre is outside of the specified } \\
\text { limics. }\end{array}$ & $\begin{array}{l}\text { QC Summary } \\
\text { Repor }\end{array}$ \\
\hline
\end{tabular}

All surrogalle recoveries and uesspanes ranges are reported af the bonom of Form 2 or $C$ of A. Any recoveries fulling outside the aceeptance range will be flagged with a $* 0$. All flags do noe apply to QC Summary and Cerriñcare of Analysis packages. 
RADIOLOGICAL

ANALYSIS 
CASE NARRATIVE 


\section{Radiochemistry Case Narrative \\ Bechtel Nevada Corp. (NEVA) \\ SDG V1139}

Method/Analvsis Information

Batch Number:

Procedure:

82420

Analytical Method:

Determination of Gamma Isotopes in Water and Soil

DOE EML HASL 300

Sample ID
43345001
43345002
43345003
43345004
1200018241
1200018242
1200018243

Client ID

252506-3-0

252506-6-0

252517-1-1

252502-1-1

MB for batch 82420

252506-3-0(43345001DUP)

LCS for batch 82420

\section{SOP Reference}

Procedures for preparation, analysis and reporting of analytical data are controlled by General Engineering Laboratories, Inc, as Standard Operating Procedures (SOP). The data discussed in this narrative has been prepared and analyzed in accordance with GL-RAD-A-013.

\section{Calibration Information:}

\section{Calibration Information}

All initial and continuing calibration requirements have been met.

\section{Standards Information}

Standard solution(s) for these analyses are NIST traceable and used before the expiration date(s).

\section{Sample Geometry}

All counting sources were prepared in the same geometry as the calibration standards.

\section{Quality Control (OC) Information:}

\section{Blank Information}

The blank volume is representative of the sample volume(s) in this batch.

\section{Designated QC}

The following sample(s) was used for QC: 43345001 .

QC Information

All of the QC samples met the required acceptance limits.

\section{Technical Information:}

\section{Holding Time}

All sample procedures for this sample set were performed within the required holding time.

Preparation Information

All preparation criteria have been met for these analyses.

Sample Re-prep/Re-analysis 
None of the samples in this sample set required reprep or reanalysis.

Miscellaneous Information:

NCR Documentation

No NCR were generated for the preparation or analysis of this sample set.

\section{Manual Integration}

No manual integrations were performed on data in this batch.

\section{Review Validation:}

GEL requires all analytical data to be verified by a qualified data validator. In addition, all data designated for CLP or CLP-like packaging will receive a third level validution upon completion of the data package.

The following data validator verifed the information presented in this case narrative:

Reviewer:Du. nusure

Date:

$12 \ln 2001$ 


\title{
GENERAL ENGINEERING LABORATORIES
}

Meeting today's needs with a vision for tomorrow.

\section{Certificate of Analysis}

\author{
Company: Bechtel Nevada Corp. \\ Address: 2621 Losee Road \\ North Las Vegas, NV 89030-4134 \\ Contact: Ted Redding \\ Project: Enviommental Rad Services
}

Report Date: June 20, 2001

Page 1 of 1

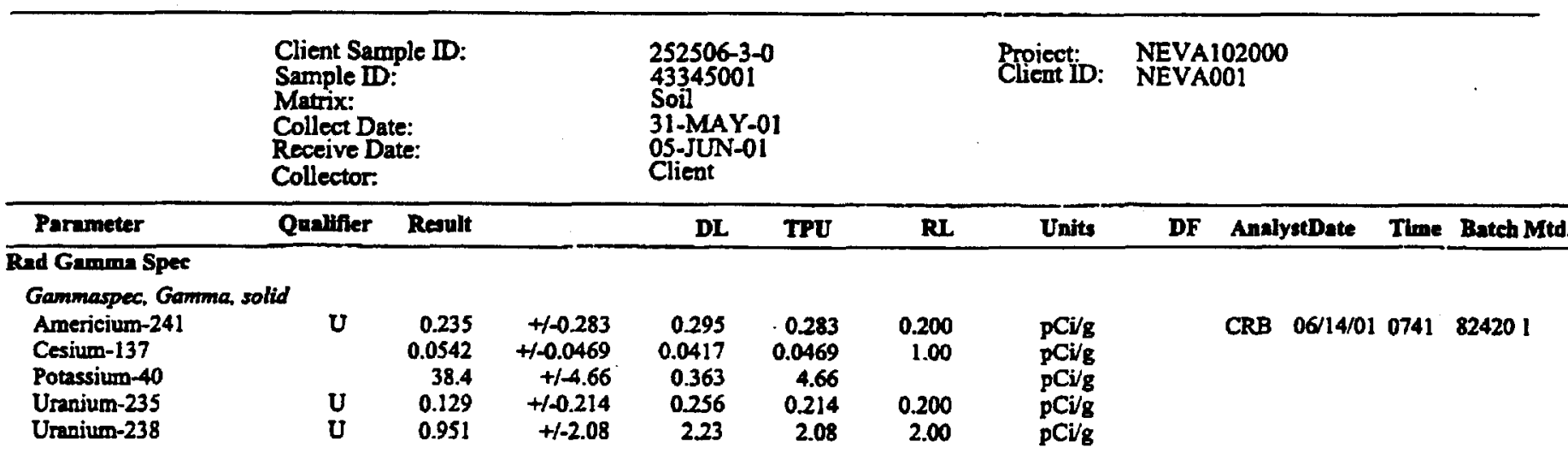

The following Prep Methods were performed

\begin{tabular}{llllll}
\hline Method & Deteription & Anolyst & Date & Time & Prep Batch \\
\hline Dry Soil Prep & Dry Soil Prep RAD A-021, A-021B,A-026 & WEO & $06 / 06 / 01$ & 1126 & 81601
\end{tabular}

The following Analytical Methods were performed

Method Description

1 DOE EML HASL 300

Notes:

The Qualifiers in this report are defined as follows :

** Indicates the analyte is a surrogate compound.

$<$ Actual result is less than amount reported

$>$ Actual result is greater than amount reported

$\mathrm{J}$ Indicates an estimated value. The result was greater than the detection limit, but less than the reporting linit.

$U$ Indicates the compound was analyzed for but not detected above the detection limit

The above sample is reported on an "as received" basis.

This data report has been prepared and reviewed in accordance with General Engineering Laboratories, Inc.

standard operating procedures. Please direct any questions to your Project Manager, Stacy L. Griffin at 843-556-8171 Ext. 4264.

Reviewed by

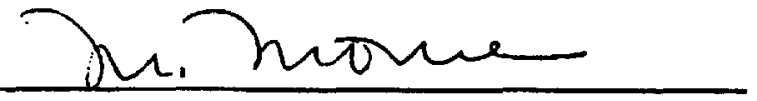




\section{GENERAL ENGINEERING LABORATORIES}

Meeting roday's needs with a vision for tomorrow.

\section{Certificate of Analysis}

$\begin{array}{ll}\text { Company : } & \text { Bechtel Nevada Corp. } \\ \text { Address : } & \text { 2621 Losee Road } \\ & \text { North Las Vegas, NV 89030-4134 } \\ \text { Contact: } & \text { Ted Redding } \\ \text { Project: } & \text { Enviommental Rad Services }\end{array}$
Report Date: June 20, 2001

Page 1 of 1

Client Sample ID: 252506-6-0 Proiect: NEVA102000

Sample D: $\quad 43345002$

Client DD: NEVA001

Soil

31-MAY-01

Collect Date:

Receive Date:

Collector:

$05-\pi N=01$

Client

\begin{tabular}{|c|c|c|c|c|c|c|c|c|c|c|c|}
\hline Parnmeter & Qualifier & Reault & & DL & IPU & $\mathbf{R}$ & Units & DF & AndystDate & Time & Butch Mtd \\
\hline \multicolumn{12}{|l|}{ Rad Gamma Spee } \\
\hline \multicolumn{12}{|c|}{ Gammaspec. Gamma, solid } \\
\hline $\begin{array}{l}\text { Americium-241 } \\
\text { Cesium-137 } \\
\text { Potassium-40 } \\
\text { Uranium-235 } \\
\text { Uranium-238 }\end{array}$ & $\begin{array}{l}U \\
U \\
U\end{array}$ & $\begin{array}{r}0.0681 \\
-0.023 \\
34.8 \\
0.172 \\
2.20\end{array}$ & $\begin{array}{r}+/-0.0855 \\
+/ 0.0327 \\
+1-1.47 \\
+1-0.256 \\
+/-1.13\end{array}$ & $\begin{array}{r}0.093 \\
0.0565 \\
0.385 \\
0.299 \\
0.908\end{array}$ & $\begin{array}{r}0.0855 \\
0.0327 \\
1.48 \\
0.256 \\
1.13\end{array}$ & $\begin{array}{r}0.200 \\
1.00 \\
0.200 \\
2.00\end{array}$ & $\begin{array}{l}\mathrm{pCi} / \mathrm{g} \\
\mathrm{pCi} / \mathrm{g} \\
\mathrm{pCi} / \mathrm{g} \\
\mathrm{pCi} / \mathrm{g} \\
\mathrm{pCi} / \mathrm{g}\end{array}$ & & CRB $06 / 140$ & 0742 & 824201 \\
\hline
\end{tabular}

The followine Prep Methods werc performed

\begin{tabular}{llllll}
\hline Method & Description & Anabyst & Date & Time & Prep Batch \\
\hline Dry Soil Prep & Dry Soil Prep RAD A-021,A-021B,A-026 & WEO & 06/06/01 & 1126 & 81601 \\
The following Analytical Methods were performed & Deseription & & & & \\
\hline Method & DOE EMIL HASL 300
\end{tabular}

Notes:

The Qualifiers in this report are defined as follows :

* Indicates the analyte is a surrogate compound.

$<$ Actual result is less than amount reported

$>$ Actual result is greater than amount reported

J Indicates an estimated value. The result was greater than the detection limit, but less than the reporting limit.

$\mathrm{U}$ Indicates the compound was analyzed for but not detected above the detection limit

The above sample is reported on an "as received" basis.

This data report has been prepared and reviewed in accordance with General Engineering Laboratories, Inc.

standard operating procedures. Please direct any questions to your Project Manager, Stacy L. Griffin at 843-556-8171 Ext. 4264.

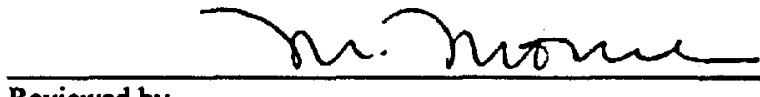

Reviewed by 


\section{GENERAL ENGINEERING LABORATORIES}

Meering todoy's needs with a vision for comorrow:

\section{Certificate of Analysis}

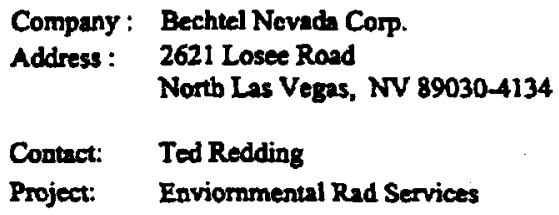

Report Date: June 20, 2001

Page 1 of 1

Client Sample ID:

Sample ID:

Matrix:

Collect Date:

Receive Date:

Collector:

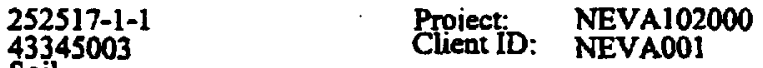

31-MAY-01

OS-JUN-01

Client

\begin{tabular}{|c|c|c|c|c|c|c|c|c|c|c|c|c|}
\hline Parameter & Quolifier & Result & & DL & TPU & $\mathbf{R L}$ & Units & DF & Ansl & ystDate & Time & Batch Mtd \\
\hline \multicolumn{13}{|l|}{ Rad Gammin Spee } \\
\hline $\begin{array}{l}\text { Gammaspec, Gam } \\
\text { Americium-241 } \\
\text { Cesium-137 } \\
\text { Potzssium-40 } \\
\text { Uranium-235 } \\
\text { Uranium-238 }\end{array}$ & $\begin{array}{l}\mathbf{U} \\
\mathbf{U} \\
\mathbf{U}\end{array}$ & $\begin{array}{r}0.00649 \\
-0.00384 \\
29.4 \\
0.0621 \\
1.45\end{array}$ & $\begin{array}{r}+-0.0389 \\
++0.0288 \\
+/-3.17 \\
+/-0.238 \\
+/-0.873\end{array}$ & $\begin{array}{r}0.0686 \\
0.0495 \\
0.385 \\
0.236 \\
0.666\end{array}$ & \begin{tabular}{r|}
0.0389 \\
0.0288 \\
3.17 \\
0.238 \\
0.873
\end{tabular} & $\begin{array}{r}0.200 \\
1.00 \\
0.200 \\
2.00\end{array}$ & $\begin{array}{l}\mathrm{pCi} / \mathrm{g} \\
\mathrm{pCi} / \mathrm{g} \\
\mathrm{pCi} / \mathrm{g} \\
\mathrm{pCi} / \mathrm{g} \\
\mathrm{pCi} / \mathrm{g}\end{array}$ & & CRB & $06 / 14 / 01$ & 10749 & 824201 \\
\hline
\end{tabular}

The following Prep Methods were performed

\begin{tabular}{|c|c|c|c|c|c|}
\hline Method & Description & Analyst & Date & Thre & Prep Bateh \\
\hline Dry Soil Prep & Dry Soil Prep RAD A-021,A-021B,A-026 & WEO & $06 / 06 / 01$ & 1126 & 81601 \\
\hline
\end{tabular}

The following Anslytical Methods were performed

Method Description

1 DOE EMIL HASL 300

Notes:

The Qualifiers in this report are defined as follows :

* Indicates the analyte is a surrogate compound

$<$ Actual result is less than amount reported

$>$ Actual result is greater than amount reported

I Indicates an estimated value. The result was greater than the detection limit, but less than the reporting limit.

$U$ Indicates the compound was analyzed for but not detected above the detection limit

The above sample is reported on an "as received" basis.

This data report has been prepared and reviewed in accordance with General Engineering Laboratories, Inc.

standard operating procedures. Please direct any questions to your Project Manager, Stacy L. Griffin at 843-556-8171 Ext. 4264.

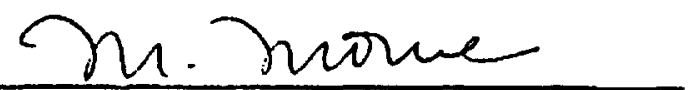

Reviewed by 


\section{GENERAL ENGINEERING LABORATORIES}

Meeting today's needs with a vision for tomorrow.

\section{Certificate of Analysis}

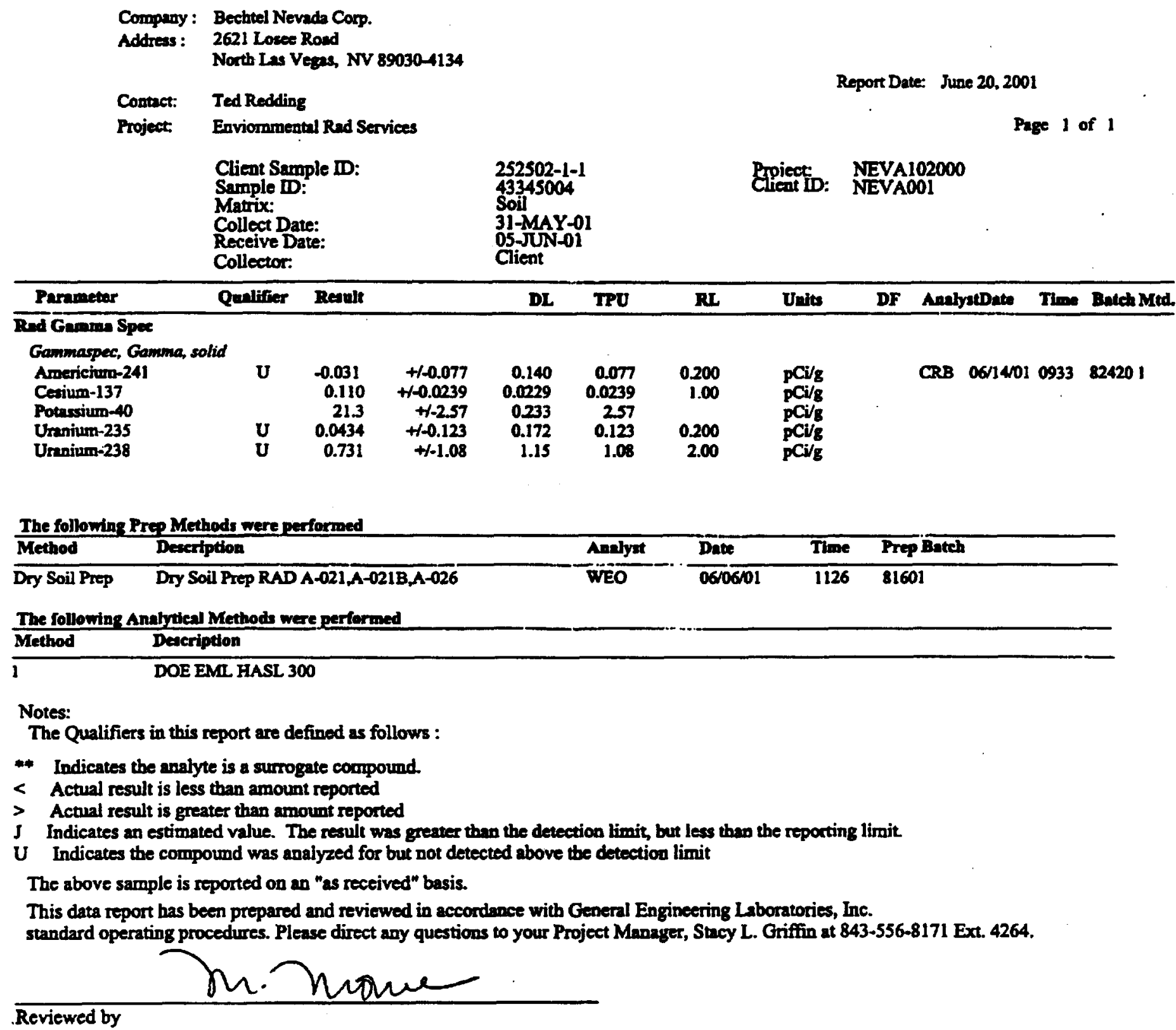

P OBox 30712 - Charieston, SC 29417 - 2040 Savage Road - 29407

(843) $556-8171 \cdot \operatorname{Fax}(843) 766-1.178$ 


\section{GENERAL ENGINEERING LABORATORIES}

Meeting today's needs with a vision for tomorrow.

\section{OC Summary}

\begin{abstract}
Cient : 2621 Losee Raed

Contact: Ted Redding

Workorder: 43345

Parminane
Rad Gamma Spec
Batch $\quad 82420$
QC1200018242 43345001 DUP
Americium-241
\end{abstract}

Bechtel Nevada Corp. North Las Veges, NV
Cesium-137

Potassium-40

Uranium-235

Uranium-238

QC1200018243 LCS

Americium-241

Cesium-137

Potassium -40

Uranium-235

Uranium-238

OC1200018241 MB

Americium-241

Cesium-137

Potassium-40

Uranium-235
NOM Sample Oual

QC
QC Units RPD\% REC\%

Rewort Date: July 12, 2001

Page 1 of 2

$89030-4134$

anl

Range Anlst

Date Time

\begin{tabular}{|c|c|c|c|c|c|c|c|c|c|}
\hline $\mathbf{U}$ & 0.235 & $\mathbf{U}$ & 0.0596 & $\mathrm{pCi} / \mathrm{g}$ & 119 & $\wedge$ & $(+1-0.200)$ & CRB & $06 / 14 / 0113: 52$ \\
\hline Uncert: & $+1-0.283$ & & +-0.120 & & & & & & 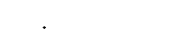 \\
\hline TPU: & 0.283 & & 0.120 & & & & & & \\
\hline & 0.0542 & $\mathbf{U}$ & 0.0269 & $\mathrm{pCi} / \mathrm{g}$ & 67 & $\wedge$ & $(t-1.00)$ & & \\
\hline Uncert & +-0.0469 & & $+1-0.0251$ & & & & & & \\
\hline TPU: & $\begin{array}{r}0.0469 \\
38.4\end{array}$ & & $\begin{array}{r}0.0251 \\
38.6\end{array}$ & $\mathrm{pCi} / \mathrm{g}$ & 1 & & & & \\
\hline Uncert: & +4.66 & & $+4-4.55$ & & & & & & \\
\hline TPU: $_{U}$ & $\begin{array}{r}4.66 \\
0.129\end{array}$ & $\mathbf{U}$ & $\begin{array}{r}4.55 \\
0.00889\end{array}$ & $\mathrm{pCi} / \mathrm{g}$ & 174 & $\wedge$ & $(+/-0.200)$ & & \\
\hline Uncert: & +-0.214 & & +-0.133 & & & & & & \\
\hline $\mathbf{T P U}_{\mathbf{U}}$ & $\begin{array}{l}0.214 \\
0.951\end{array}$ & & $\begin{array}{r}0.133 \\
1.93\end{array}$ & $\mathrm{pCi} / \mathrm{g}$ & 68 . & n & $(t+2.00)$ & & \\
\hline Uncert & $+1-2.08$ & & +-1.69 & & & & & & \\
\hline
\end{tabular}

$06 / 14 / 0113: 53$

Uncert:

$1250 \quad \mathrm{pCi} / \mathrm{g}$

TPU:

Uncert:

TPU:

135

487

$\mathrm{pCi} / \mathrm{g}$

110 (75\%-125\%)

$+-61.1$

61.1
$\quad 0.923$

Uncert:

TPU:

Uncert:
TPU:

1.55

0.966

$+1-1.29$

1.29

U $\begin{array}{r}-1.17 \\ +1-4.64\end{array} \quad \mathrm{pCi} / \mathrm{g}$

Uncert:

TPU:

4.64

$\mathrm{pCi} / \mathrm{g}$

pCi $y_{g}$

U $\quad-0.00539 \quad \mathrm{pCi} / \mathrm{g}$

06/14/01 09:33 


\section{GENERAL ENGINEERING. LABORATORIES}

Meeting today's needs with a vision for tomorrow.

\section{QC Summary}

Wortorder: 43345

Peroneme

Barch

82420

Uranium-238

IPU:

Uncert

NOW Semple Oagl

Page 2 of 2

Penge Ania Date Time

TPU:

0.140
0.0446
+-0.651
0.651 $\quad$

Notes:

The Qualifiers in this report are defined as follows:

* Indicates the andyte is a sumogate compound

$<\quad$ Actunl result is less than amount reported

$>$ Actual result is greater than amount reported

J Indicates an estimated value. The result was greater then the detection limit, but lass than the reporting limit.

U Indicates the compound was analyzed for but not detecied above the detection limit

N/A indicates thut spike recovery limits do not apply when sample concestration exceeds spiks conc. by a factor of 4 or more.

^ The Relavive Percem Difference (RPD) obtained from the sample duplicare (DUP) is evaluated againat the acceptence criteris when the sumple is greater than five times (5X) the contract required detection linit (RI). In cases where either the sample or duplicate value is less then $5 X$ the $R L$, control limit of + - the $R$ is used to evaluate the DUP result.

For PS, PSD, and SDITT results, the values listed are the mensured amonnts, not final concentrations. 


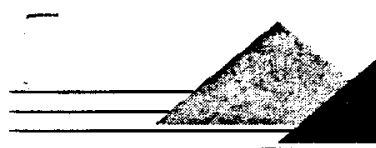

CLIENT:

Bechtel Nevada

-

P.O. Box 98521, M/S NTS273

Las Vegas, NV 89193-8521

ATTN:

Ted Redding

PROJECT NAME: $\quad$ V1140

NEL ORDER ID: L0106022

PROJECT NUMBER: 23081

Attached are the analytical results for samples in support of the above referenced project.

Samples submitted for this project were not sampled by NEL Laboratories. Samples were received by NEL in good condition, under chain of custody on 6/4/01.

Should you have any questions or comments, please feel free to contact our Client Services department at (702) 657-1010.
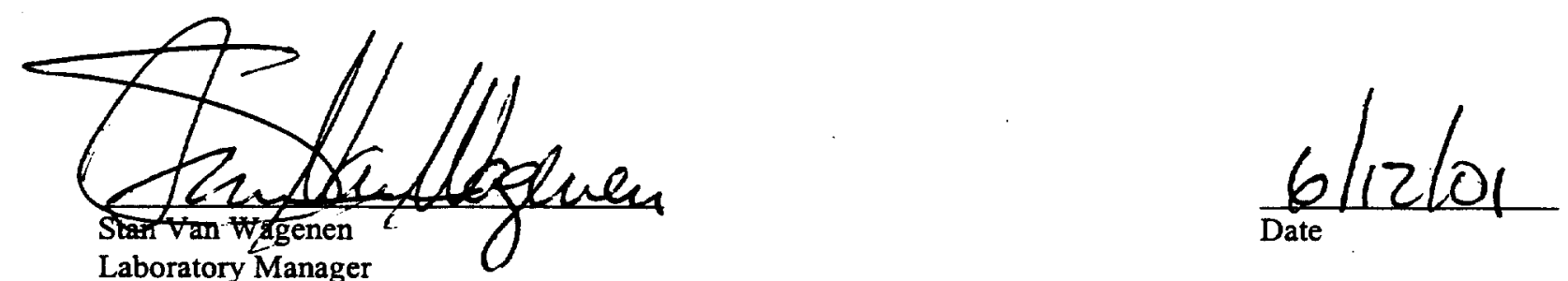

CERTIFICATIONS:

\begin{tabular}{llll}
\hline & Reno & Las Vegas & S. Califomia \\
\cline { 2 - 4 } Arizona & AZ0520 & AZ0518 & AZ0605 \\
California & 1707 & 2002 & 2264 \\
$\begin{array}{l}\text { US Army Corps } \\
\text { of Engineers }\end{array}$ & Certified & Certified & \\
& & &
\end{tabular}

Montana

Reno Las Vegas S. California 
$\begin{array}{llll}\text { CLIENT: } & \text { Bechtel Nevada } & \text { CLIENT ID: } & \text { 252506-1-0 } \\ \text { PROJECT ID: } & \text { V1140 } & \text { DATE SAMPLED: 5/31/01 } \\ \text { PROJECT \#: } & 23081 & \text { NEL SAMPLE ID: } \text { L0106022-01 }\end{array}$

TEST: $\quad$ Total Extractable Petroleum Hydrocarbons Fuel Finger Print by EPA Method 8015M, July 1992

METHOD: $\quad$ EPA 8015M

MATRIX: Solid

ANALYST: $\quad$ CCS - Las Vegas Division

DILUTION: 1

EXTRACTED: $\quad 6 / 5 / 01$

ANALYZED: $\quad 6 / 6 / 01$

PARAMETER

Gasoline Range (C8-C12)

Diesel Range (C12-C22)

Oil Range (C12-C34)

Total

QUALITY CONTROL DATA:

Surrogate

Octacosane
$\%$ Recovery

72
Reporting

Limit

10. $\mathrm{mg} / \mathrm{kg}$

10. $\mathrm{mg} / \mathrm{kg}$

50. $\mathrm{mg} / \mathrm{kg}$

10. $\mathrm{mg} / \mathrm{kg}$

ND - Not Detected

This report shall not be reproduced except in full, without the written approval of the laboratory. 
CLIENT: Bechtel Nevada PROJECT ID: V1140

PROJECT \#: 23081
CLIENT ID: $\quad$ 252506-2-0

DATE SAMPLED: $5 / 31 / 01$

NEL SAMPLE ID: L0106022-02

$-$

TEST:

Total Extractable Petroleum Hydrocarbons Fuel Finger Print by EPA Method 8015M, July 1992

METHOD: EPA 8015M

MATRIX: Solid ANALYST: $\quad$ CCS - Las Vegas Division

DILUTION: EXTRACTED: $\quad 6 / 5 / 01$ ANALYZED: $\quad 6 / 6 / 01$

\section{$-$ \\ PARAMETER \\ Gasoline Range (C8-C12) \\ - Diesel Range (C12-C22) \\ Oil Range (C12-C34) \\ Total \\ QUALITY CONTROL DATA:}

Surrogate

Octacosane
$\%$ Recovery

79
Reporting

\begin{tabular}{l} 
Result \\
\hline ND \\
ND \\
ND \\
ND
\end{tabular}

ND
Limit

10. $\mathrm{mg} / \mathrm{kg}$

10. $\mathrm{mg} / \mathrm{kg}$

50. $\mathrm{mg} / \mathrm{kg}$

10. $\mathrm{mg} / \mathrm{kg}$

$-$

- ND - Not Detected

This report shall not be reproduced except in full, without the written approval of the laboratory.

Acceptable Range

$54-130$ 
$\begin{array}{llll}\text { CLIENT: } & \text { Bechtel Nevada } & \text { CLIENT ID: } & \text { 252506-3-0 } \\ \text { PROJECT ID: } & \text { V1140 } & \text { DATE SAMPLED: } 5 / 31 / 01 \\ \text { PROJECT \#: } & 23081 & \text { NEL SAMPLE ID: } \text { L0106022-03 }\end{array}$

TEST: $\quad$ Total Extractable Petroleum Hydrocarbons Fuel Finger Print by EPA Method 8015 M, July 1992

METHOD: EPA 8015M ANALYST: CCS - Las Vegas Division

MATRIX: Solid $\quad$ EXTRACTED: $6 / 5 / 01$

DILUTION: 1 ANALYZED: $6 / 6 / 01$

\begin{tabular}{llc}
\hline PARAMETER & Result & Reporting \\
\cline { 2 - 3 } Gasoline Range (C8-C12) & $\mathrm{ND}$ & $\frac{\text { Limit }}{10 . \mathrm{mg} / \mathrm{kg}}$ \\
Diesel Range (C12-C22) & $\mathrm{ND}$ & $10 . \mathrm{mg} / \mathrm{kg}$ \\
Oil Range (C12-C34) & $\mathrm{ND}$ & $50 . \mathrm{mg} / \mathrm{kg}$ \\
Total & $\mathrm{ND}$ & $10 . \mathrm{mg} / \mathrm{kg}$ \\
\hline \hline
\end{tabular}

QUALITY CONTROL DATA:

Surrogate

\% Recovery

Acceptable Range

Octacosane

84

$54-130$

ND - Not Detected

This report shall not be reproduced except in full, without the written approval of the laboratory. 


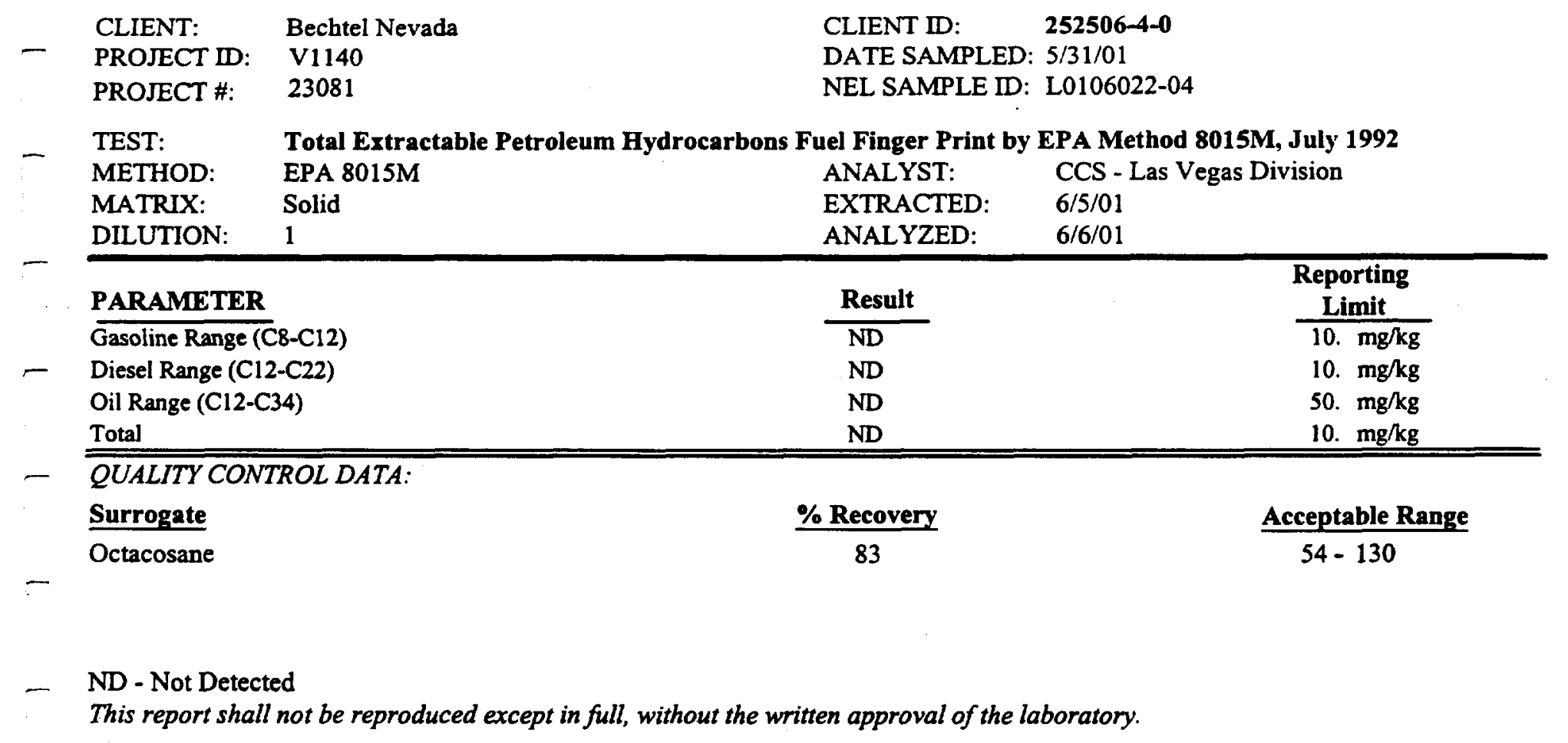


$\begin{array}{llll}\text { CLIENT: } & \text { Bechtel Nevada } & \text { CLIENT ID: } & \text { 252506-5-0 } \\ \text { PROJECT ID: } & \text { V1140 } & \text { DATE SAMPLED: 5/31/01 } \\ \text { PROJECT \#: } & 23081 & \text { NEL SAMPLE ID: L0106022-05 }\end{array}$

TEST: $\quad$ Total Extractable Petroleum Hydrocarbons Fuel Finger Print by EPA Method 8015 M, July 1992

METHOD: EPA 8015M ANALYST: CCS - Las Vegas Division

MATRIX: Solid $\quad$ EXTRACTED: $6 / 5 / 01$

DILUTION: 1 ANALYZED: $\quad 6 / 6 / 01$

\begin{tabular}{lcc}
\hline PARAMETER & Result & $\begin{array}{c}\text { Reporting } \\
\text { Limit }\end{array}$ \\
\cline { 2 - 3 } Gasoline Range (C8-C12) & $\mathrm{ND}$ & $10 . \mathrm{mg} / \mathrm{kg}$ \\
Diesel Range (C12-C22) & $\mathrm{ND}$ & $10 . \mathrm{m} / \mathrm{kg}$ \\
Oil Range (C12-C34) & $\mathrm{ND}$ & $50 . \mathrm{mg} / \mathrm{kg}$ \\
Total & $\mathrm{ND}$ & $10 . \mathrm{mg} / \mathrm{kg}$ \\
\hline \hline
\end{tabular}

QUALITY CONTROL DATA:

Surrogate

Octacosane
$\%$ Recovery

83
Acceptable Range

$54-130$

ND - Not Detected

This report shall not be reproduced except in full, without the written approval of the laboratory. 


$\begin{array}{llll}\text { CLIENT: } & \text { Bechtel Nevada } & \text { CLIENT ID: } & \text { 252506-6-0 } \\ \text { PROJECT ID: } & \text { V1140 } & \text { DATE SAMPLED: 5/31/01 } \\ \text { PROJECT \#: } & 23081 & \text { NEL SAMPLE D: } \text { L0106022-06 }\end{array}$

- TEST: $\quad$ Total Extractable Petroleum Hydrocarbons Fuel Finger Print by EPA Method $8015 M$, July 1992

METHOD: EPA 8015M ANALYST: CCS - Las Vegas Division

MATRIX: Solid

EXTRACTED: $\quad 6 / 5 / 01$

DILUTION: $1 \quad$ ANALYZED: $\quad$ 6/6/01

\begin{tabular}{|c|c|c|}
\hline PARAMETER & Result & $\begin{array}{c}\text { Reporting } \\
\text { Limit }\end{array}$ \\
\hline Gasoline Range (C $8-\mathrm{C} 12)$ & $\mathrm{ND}$ & $10 . \mathrm{mg} / \mathrm{kg}$ \\
\hline Diesel Range (C12-C22) & ND & 10. $\mathrm{mg} / \mathrm{kg}$ \\
\hline Oil Range (C12-C34) & $\mathrm{ND}$ & 50. $\mathrm{mg} / \mathrm{kg}$ \\
\hline Total & ND & 10. $\mathrm{mg} / \mathrm{kg}$ \\
\hline
\end{tabular}

- QUALITY CONTROL DATA:

Surrogate

$\%$ Recovery

79

Acceptable Range

Octacosane

$54-130$

- ND - Not Detected

This report shall not be reproduced except in full, without the written approval of the laboratory. 


\begin{tabular}{|c|c|c|}
\hline CLIENT: & Bechtel Nevada & CLIENT ID: $\quad 252506-7-0$ \\
\hline PROJECT ID: & V1140 & DATE SAMPLED: $5 / 31 / 01$ \\
\hline PROJECT \#: & 23081 & NEL SAMPLE ID: L0106022-07 \\
\hline
\end{tabular}

TEST: $\quad$ Total Extractable Petroleum Hydrocarbons Fuel Finger Print by EPA Method 8015M, July 1992

METHOD: $\quad$ EPA 8015M

MATRIX: Solid ANALYST: $\quad$ CCS - Las Vegas Division

DILUTION: 1

EXTRACTED: $\quad 6 / 5 / 01$

ANALYZED: $\quad 6 / 6 / 01$

\section{PARAMETER}

Gasoline Range (C8-C12)

Diesel Range (C12-C22)

Oil Range (C12-C34)

Total

\begin{tabular}{l} 
Result \\
\hline ND \\
ND \\
ND \\
ND
\end{tabular}

Reporting

QUALITY CONTROL DATA:

\section{Surrogate}

Octacosane

\section{\% Recovery}

84
Limit

10. $\mathrm{mg} / \mathrm{kg}$

10. $\mathrm{mg} / \mathrm{kg}$

50. $\mathrm{mg} / \mathrm{kg}$

10. $\mathrm{mg} / \mathrm{kg}$

ND - Not Detected

This report shall not be reproduced except in full, without the written approval of the laboratory. 
- CLIENT: Bechtel Nevada

PROJECT ID: V1140

PROJECT \#: 23081
CLIENT ID: $\quad$ 252506-8-0

DATE SAMPLED: 5/31/01

NEL SAMPLE ID: L0106022-08

- TEST: $\quad$ Total Extractable Petroleum Hydrocarbons Fuel Finger Print by EPA Method $8015 M$, July 1992

METHOD: $\quad$ EPA 8015M

MATRIX: Solid ANALYST: $\quad$ CCS - Las Vegas Division

DILUTION: $\quad 1$

EXTRACTED: $\quad 6 / 5 / 01$

ANALYZED: $\quad 6 / 6 / 01$

\begin{tabular}{lll}
\hline PARAMETER & Result & \multicolumn{1}{c}{ Reporting } \\
\cline { 2 - 3 } Limit & ND & mg/kg \\
Gasoline Range (C8-C12) & ND & 10. mg/kg \\
Oill Range (C12-C34) & ND & 50. mg/kg \\
Total & ND & 10. mg/kg \\
\hline
\end{tabular}

QUALITY CONTROL DATA:

Surrogate

Octacosane
$\%$ Recovery

77
Acceptable Range

$54-130$

- ND - Not Detected

This report shall not be reproduced except in full, without the written approval of the laboratory. 


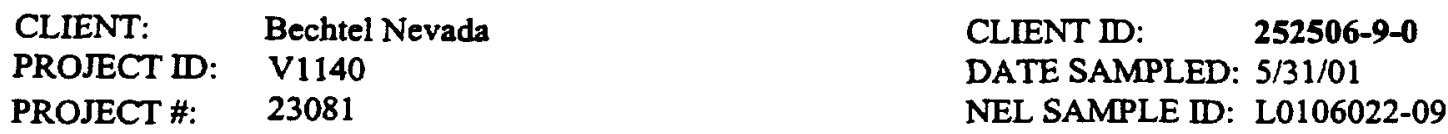

TEST: $\quad$ Total Extractable Petroleum Hydrocarbons Fuel Finger Print by EPA Method 8015M, July 1992

METHOD: $\quad$ EPA 8015M ANALYST: CCS - Las Vegas Division

$\begin{array}{llll}\text { MATRIX: } & \text { Solid } & \text { EXTRACTED: } & 6 / 5 / 01 \\ & 1 & \end{array}$

DILUTION: 1 ANALYZED: $6 / 5 / 01$

\begin{tabular}{lll}
\hline PARAMETER & Result & Reporting \\
\cline { 2 - 3 } & Limit \\
Gasoline Range $(\mathrm{C} 8-\mathrm{Cl} 2)$ & $\mathrm{ND}$ & $10 . \mathrm{mg} / \mathrm{kg}$ \\
Diesel Range $(\mathrm{Cl2}-\mathrm{C22})$ & $\mathrm{ND}$ & $10 . \mathrm{mg} / \mathrm{kg}$ \\
Oil Range $(\mathrm{C12}-\mathrm{C} 34)$ & $\mathrm{ND}$ & $50 . \mathrm{mg} / \mathrm{kg}$ \\
Total & $10 . \mathrm{mg} / \mathrm{kg}$ \\
\hline \hline
\end{tabular}

QUALITY CONTROL DATA:

Surrogate

Octacosane

\section{\% Recovery}

89

\section{Acceptable Range}

$54-130$

ND - Not Detected

This report shall not be reproduced except in full, without the written approval of the laboratory. 

CLIENT: Bechtel Nevada
CLIENT ID: $\quad$ Method Blank
PROJECT ID: V1140
DATE SAMPLED: NA
PROJECT \#: $\quad 23081$
NEL SAMPLE ID: 010605TPHS-FP1-BLK

- TEST:

METHOD:

MATRIX:

Total Extractable Petroleum Hydrocarbons Fuel Finger Print by EPA Method 8015M, July 1992

EPA 8015M

Solid
ANALYST:

EXTRACTED:

ANALYZED:

\begin{tabular}{l} 
Result \\
\hline ND \\
ND \\
ND \\
ND
\end{tabular}

CCS - Las Vegas Division $6 / 5 / 01$

$6 / 5 / 01$

$-$

PARAMETER

Gasoline Range (C8-C12)

- Diesel Range (C12-C22)

Oil Range (C12-C34)

Total

QUALITY CONTROL DATA:

Surrogate

Octacosane

\% Recovery

93
Reporting

Limit

10. $\mathrm{mg} / \mathrm{kg}$

10. $\mathrm{mg} / \mathrm{kg}$

50. $\mathrm{mg} / \mathrm{kg}$

10. $\mathrm{mg} / \mathrm{kg}$

$-$

ND - Not Detected

This report shall not be reproduced except in full, without the written approval of the laboratory. 
NEL LABORATORIES

CLIENT: Bechtel Nevada

PROJECT ID: V1140

PROJECT \#: 23081

TEST: $\quad$ Total Extractable Petroleum Hydrocarbons Fuel Finger Print by EPA Method 8015M, July 1992

MATRIX: Solid

\begin{tabular}{|c|c|c|c|c|c|c|}
\hline PARAMETER & NEL Sample ID & $\frac{\text { Spike }}{\text { Amount }}$ & $\begin{array}{l}\text { Spike } \\
\text { Result }\end{array}$ & $\frac{\text { Percent }}{\text { Recovery }}$ & $\frac{\text { Acceptable }}{\text { Range }}$ & $\underline{\text { RPD }}$ \\
\hline Diesel Range (C12-C22) & 010605TPHS-FP1-LCS & 166.7 & 154 & 92 & $53-91$ & \\
\hline Diesel Range (C12-C22) & 010605TPHS-FP1-LCSD & 166.7 & 160 & 96 & $53-91$ & 3.8 \\
\hline Total & 010605TPHS-FP1-LCS & 166.7 & 154 & 92 & $53-91$ & \\
\hline Total & 010605TPHS-FP1-LCSD & 166.7 & 160 & 96 & $53-91$ & 3.8 \\
\hline
\end{tabular}

ND - Not Detected

This report shall not be reproduced except in full, without the written approval of the laboratory. 


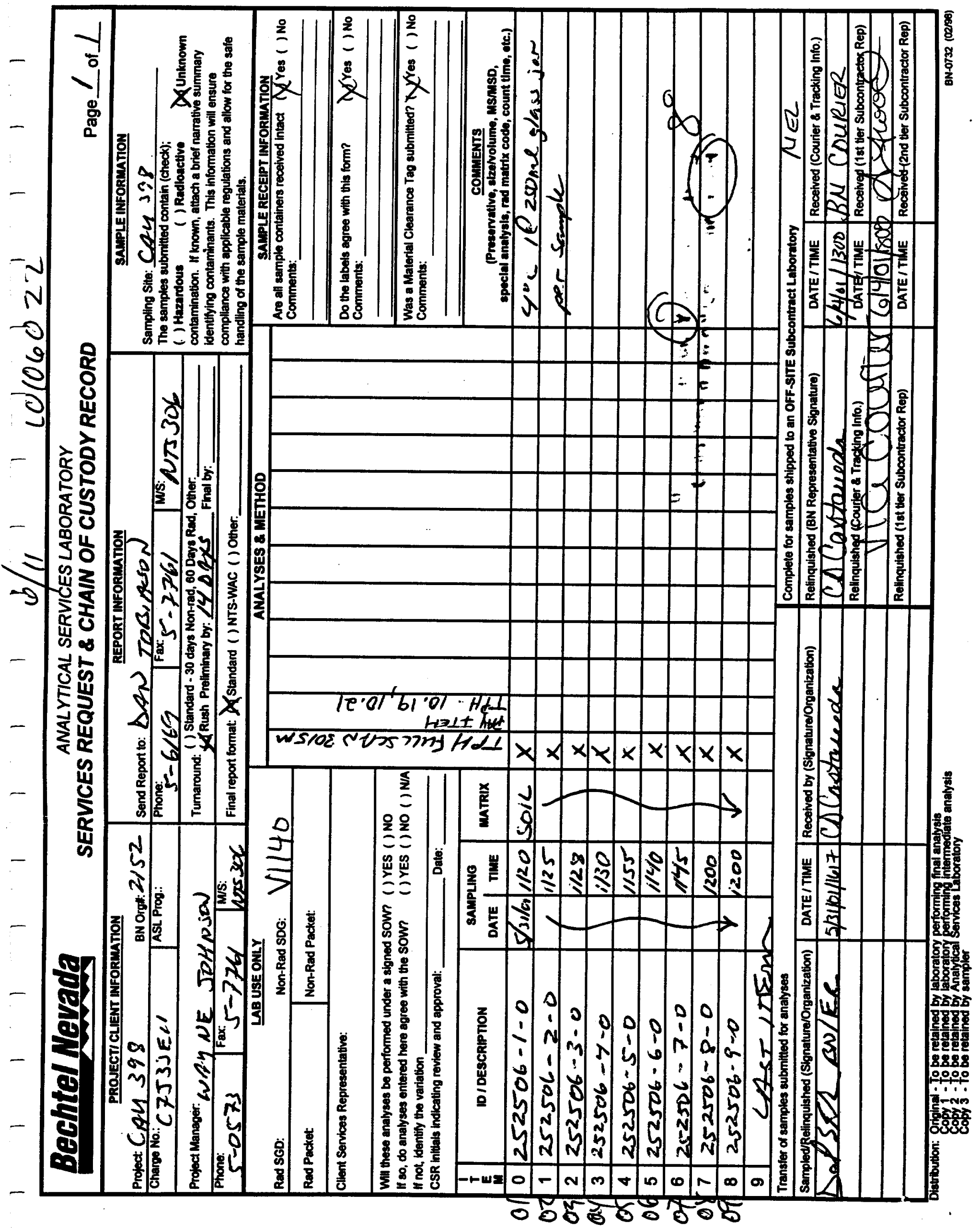


CLIENT:

Bechtel Nevada

P.O. Box $98521, \mathrm{M} / \mathrm{S}$ NTS273

Las Vegas, NV 89193-8521

ATTN:

Ted Redding

PROJECT NAME: $\quad$ V1140

NEL ORDER ID: L0106023

PROJECT NUMBER: 23081

Attached are the analytical results for samples in support of the above referenced project.

Samples submitted for this project were not sampled by NEL Laboratories. Samples were received by NEL in good condition, under chain of custody on $6 / 4 / 01$.

Should you have any questions or comments, please feel free to contact our Client Services department at (702) 657-1010.

\section{Some results have been flagged as follows:}

Di - Results reported from analysis at a higher dilution.

Some QA results have been flagged as follows:

C - Sample concentration is a least 5 times greater than spike contribution. Spike recovery criteria do not apply. Some surrogate results have been flagged as follows:

D - Sample required dilution. Sample QC results were diluted outside the calibrated range.

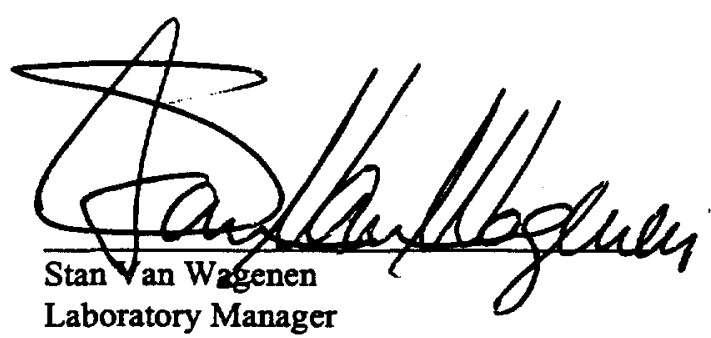

CERTIFICATIONS:

\begin{tabular}{llll}
\hline & Reno & Las Vegas & S. Califomia \\
\cline { 2 - 4 } Arizona & AZ0520 & AZ0518 & AZ0605 \\
California & 1707 & 2002 & 2264 \\
US Army Corps & Certified & Certified & \\
of Engineers & & &
\end{tabular}

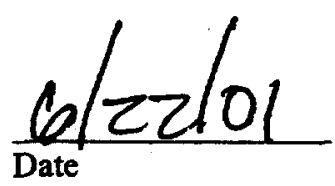

Idaho Montana Nevada L.A.C.S.D.

\begin{tabular}{lll} 
Reno & Las Vegas & S. California \\
\hline Certified & Certified & \\
Certified & Certified & \\
NV033 & NV052 & CA084 \\
& & 10228
\end{tabular}


NEL LABORATORIES

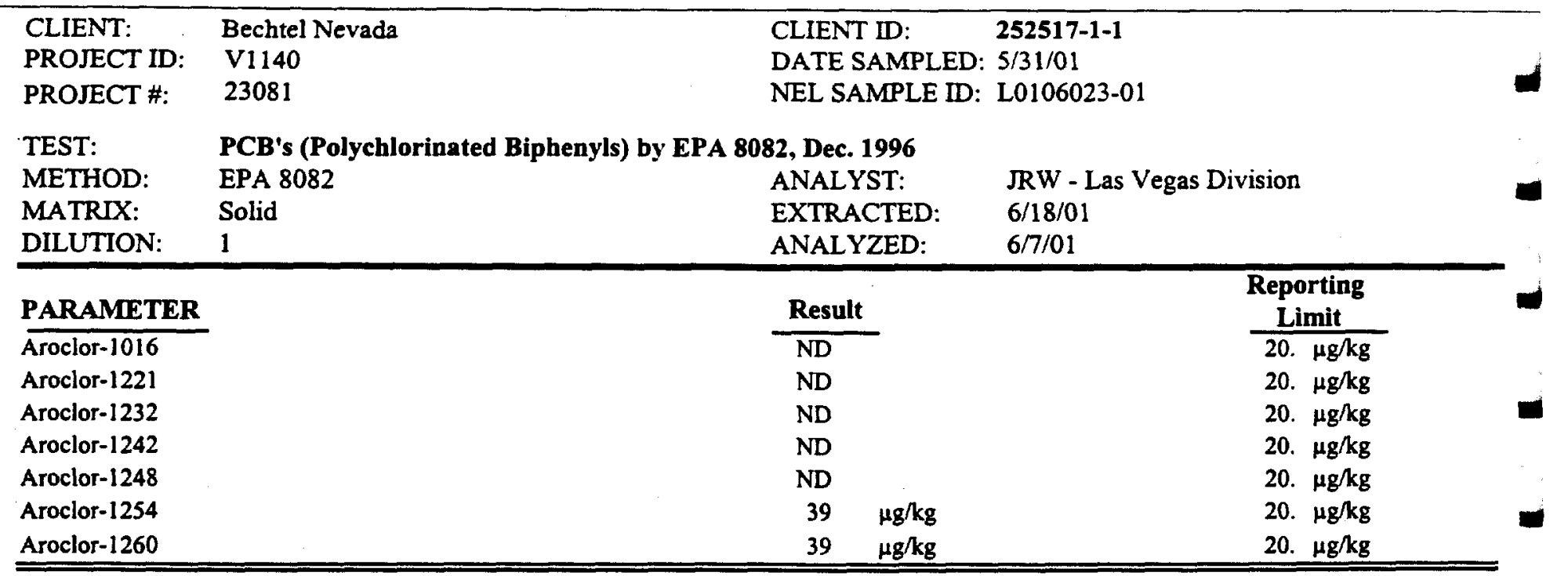

QUALITY CONTROL DATA:

Surrogate

Decachlorobiphenyl

Tetrachioro-m-xylene

\section{\% Recovery}

75

75

\section{Acceptable Range}

$46-155$

$49-140$

ND - Not Detected

This report shall not be reproduced except in full, without the written approval of the laboratory. 
NEL LABORATORIES

\begin{tabular}{lll}
\hline CLIENT: & Bechtel Nevada & CLIENT ID: \\
PROJECT ID: & V11402517-2-1 \\
PROJECT \#: & 23081 & DATE SAMPLED: 5/31/01 \\
& & NEL SAMPLE ID: L0106023-02
\end{tabular}

- TEST: $\quad$ PCB's (Polychlorinated Biphenyls) by EPA 8082, Dec. 1996

METHOD: EPA 8082 ANALYST: JRW - Las Vegas Division

MATRIX: Solid

EXTRACTED: $\quad 6 / 18 / 01$

DILUTION: 1 ANALYZED: $\quad$ 6/7/01

\section{PARAMETER}

Aroclor-1016

- Aroclor-1221

Aroclor-1232

Aroclor-1242

- Aroclor-1248

Aroclor-1254

Aroclor- 1260

\begin{tabular}{ll}
\multicolumn{2}{l}{ Result } \\
\cline { 1 - 1 } ND \\
ND \\
ND \\
ND \\
ND & \\
310 & $\mu g / k g$ \\
310 & $\mu \mathrm{g} / \mathrm{kg}$ \\
\hline
\end{tabular}

Reporting

Limit

20. $\mu \mathrm{g} / \mathrm{kg}$

20. $\mu \mathrm{g} / \mathrm{kg}$

20. $\mu \mathrm{g} / \mathrm{kg}$

20. $\mu \mathrm{g} / \mathrm{kg}$

20. $\mu \mathrm{g} / \mathrm{kg}$

20. $\mu \mathrm{g} / \mathrm{kg}$

20. $\mu \mathrm{g} / \mathrm{kg}$

- QUALITY CONTROL DATA:

\section{Surrogate}

Decachlorobiphenyl

- Tetrachloro-m-xylene

\section{$\%$ Recovery}

90

105

Acceptable Range

$46-155$

$49-140$

- ND - Not Detected

This report shall not be reproduced except in full, without the written approval of the laboratory. 
NEL LABORATORIES

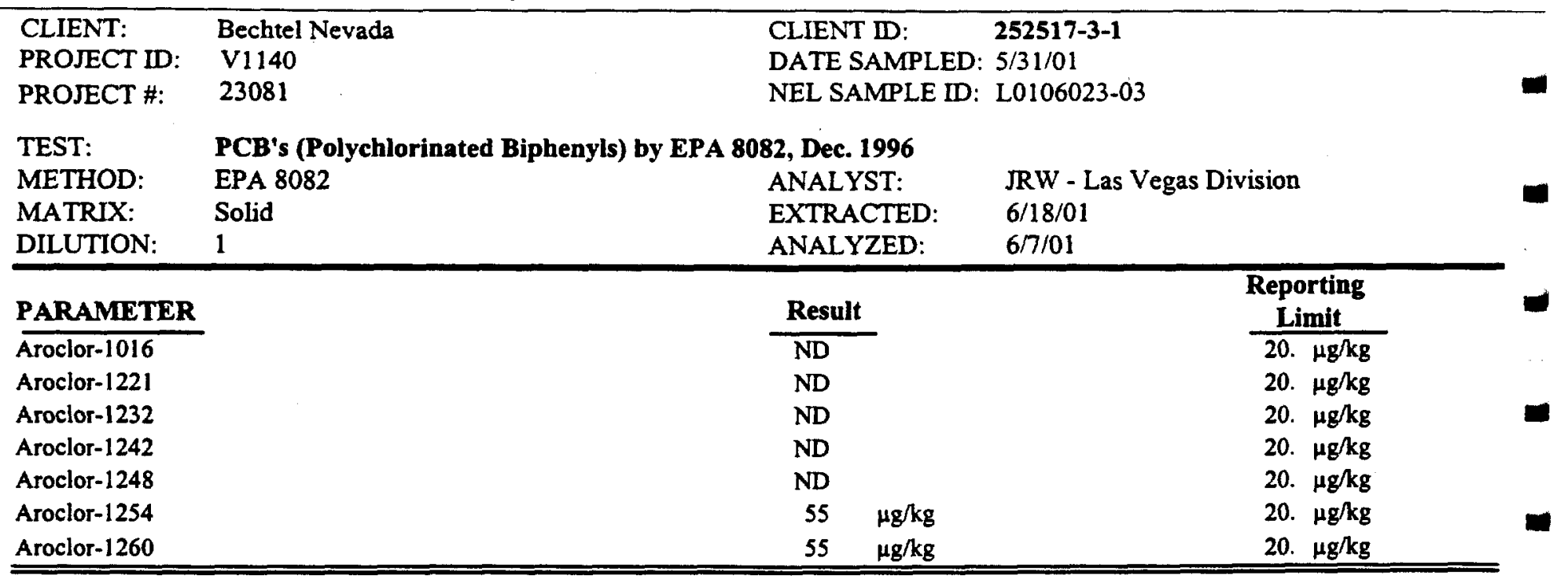

QUALITY CONTROL DATA:

Surrogate

Decachlorobiphenyl

Tetrachloro-m-xylene

\section{\% Recovery}

77

91

\section{Acceptable Range}

$46-155$

$49-140$

ND - Not Detected

This report shall not be reproduced except in full, without the written approval of the laboratory. 
NEL LABORATORIES

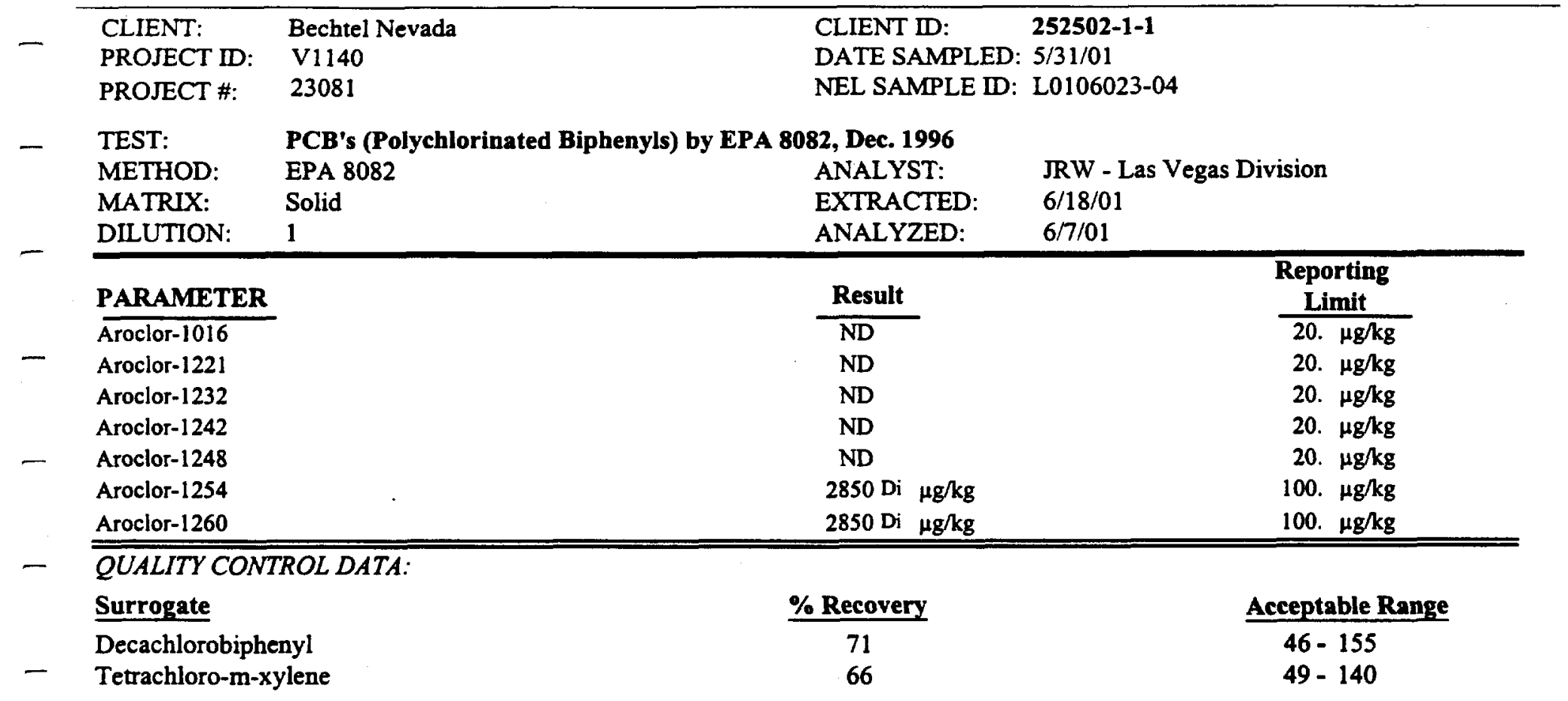

- ND - Not Detected

This report shall not be reproduced except in full, without the written approval of the laboratory. 
NEL LABORATORIES

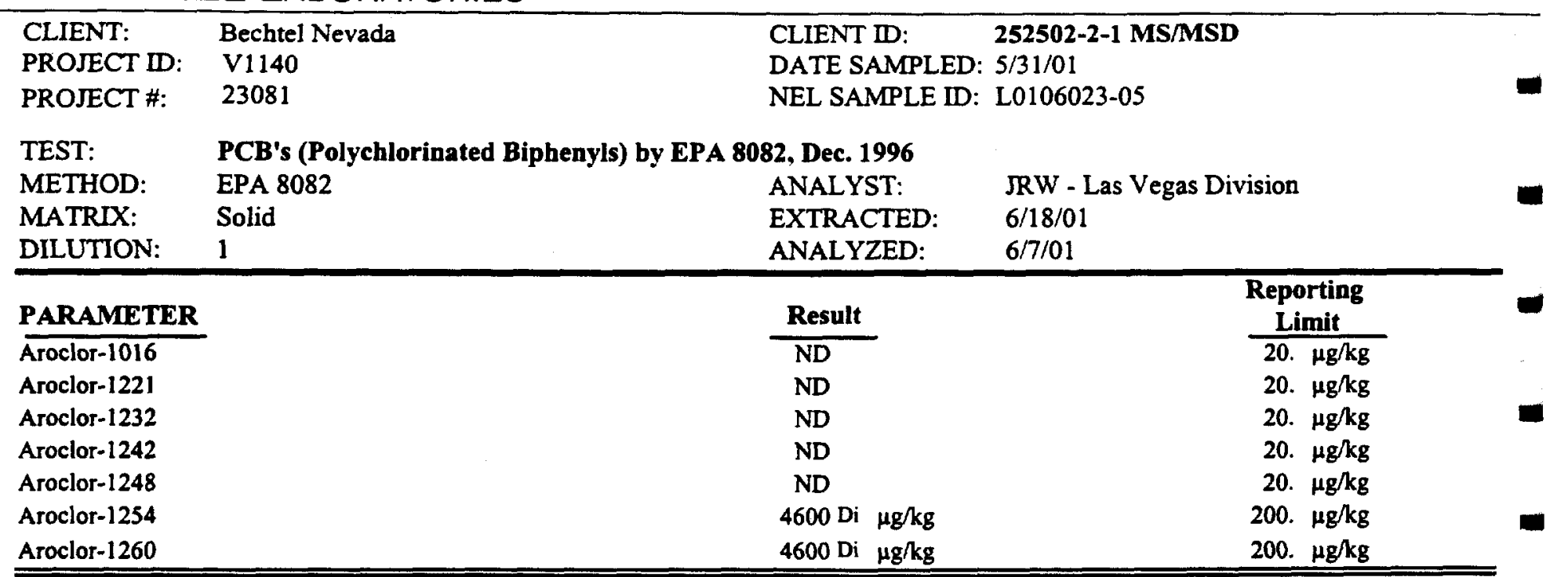

QUALITY CONTROL DATA:

\section{Surrogate}

Decachlorobiphenyl

Tetrachloro-m-xylene

\section{$\%$ Recovery}

98

83
Acceptable Range

$46-155$

$49-140$

ND - Not Detected

This report shall not be reproduced except in full, without the written approval of the laboratory. 
NEL LABORATORIES

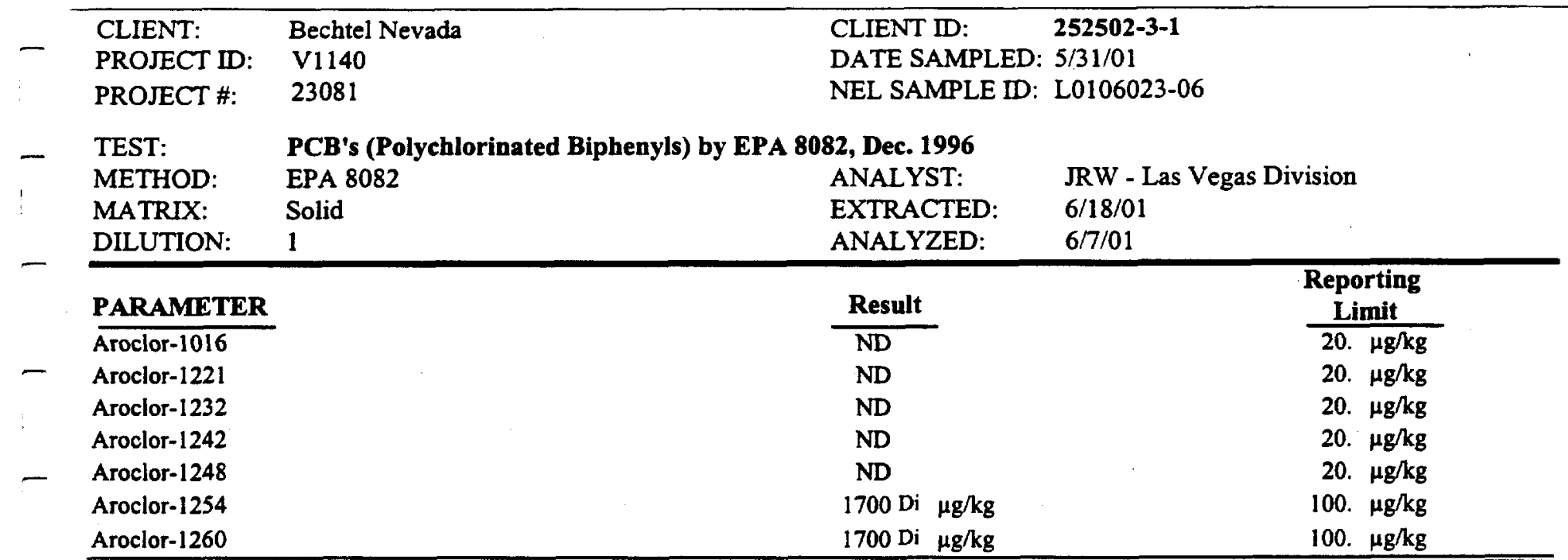

- QUALITY CONTROL DATA:

Surrogate

Decachlorobiphenyl

- Tetrachloro-m-xylene

\section{\% Recovery}

99

72

\section{Acceptable Range}

$46-155$

$49-140$

- ND - Not Detected

This report shall not be reproduced except in full, without the written approval of the laboratory. 
NEL LABORATORIES

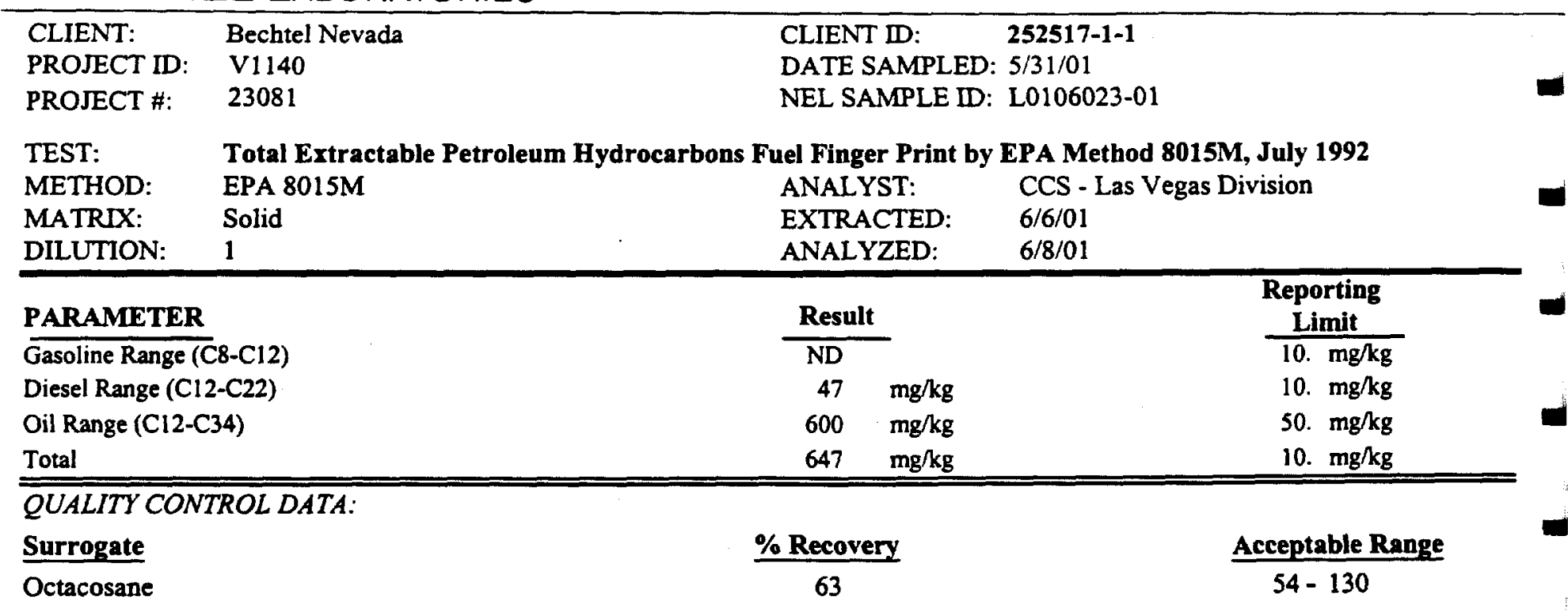

ND - Not Detected

This report shall not be reproduced except in full, without the written approval of the laboratory. 
NEL LABORATORIES

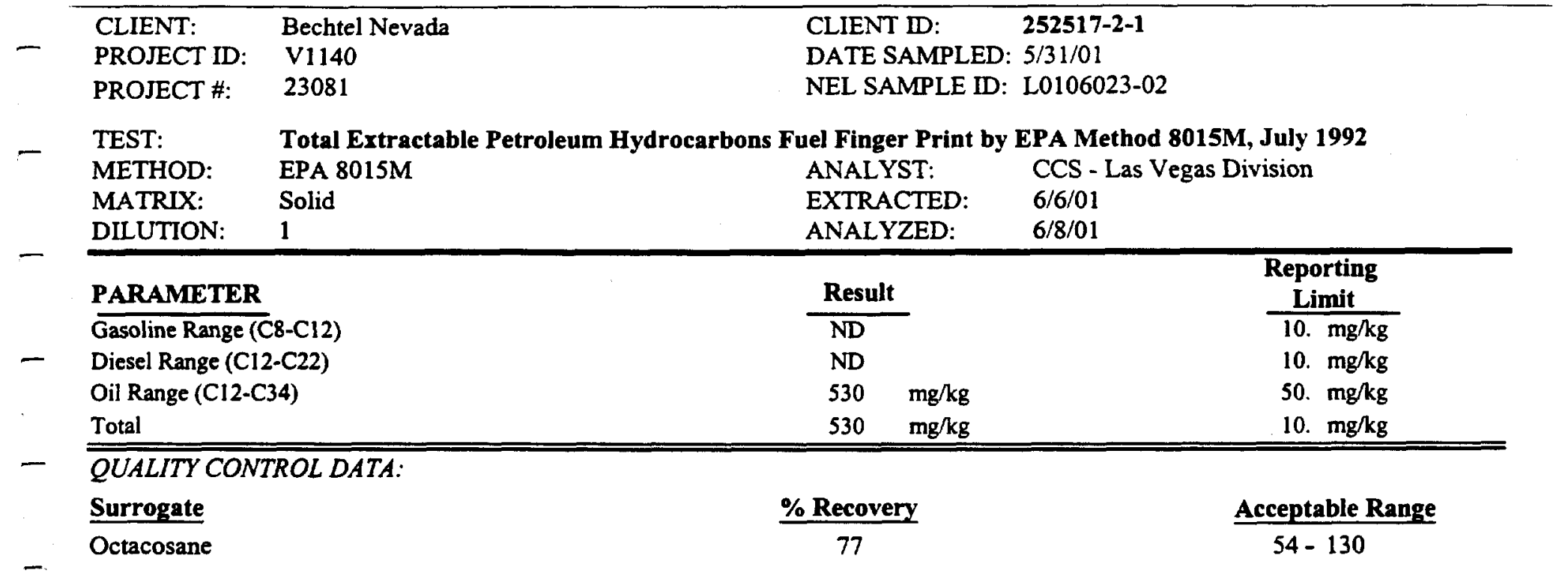

- ND - Not Detected

This report shall not be reproduced except in full, without the written approval of the laboratory. 
NEL LABORATORIES

$\begin{array}{llll}\text { CLIENT: } & \text { Bechtel Nevada } & \text { CLIENT ID: } & \text { 252517-3-1 } \\ \text { PROJECT ID: } & \text { V1140 } & \text { DATE SAMPLED: 5/31/01 } \\ \text { PROJECT \#: } & 23081 & \text { NEL SAMPLE ID: } \text { L0106023-03 }\end{array}$

TEST: $\quad$ Total Extractable Petroleum Hydrocarbons Fuel Finger Print by EPA Method 8015M, July 1992

METHOD: EPA 8015M ANALYST: CCS - Las Vegas Division

MATRIX: Solid . $\quad$ EXTRACTED: $6 / 6 / 01$

DILUTION: 1 ANALYZED: $6 / 8 / 01$

\begin{tabular}{lcc}
\hline PARAMETER & Result & Reporting \\
\cline { 2 - 3 } Gasoline Range (C8-C12) & $\mathrm{ND}$ & Limit \\
Diesel Range (C12-C22) & $25 \mathrm{mg} / \mathrm{kg}$ & $10 . \mathrm{mg} / \mathrm{kg}$ \\
Oil Range (C12-C34) & $\mathrm{ND}$ & $10 . \mathrm{mg} / \mathrm{kg}$ \\
Total & $25 \mathrm{mg} / \mathrm{kg}$ & $50 . \mathrm{mg} / \mathrm{kg}$ \\
\hline \hline
\end{tabular}

QUALITY CONTROL DATA:

Surrogate

Octacosane

ND - Not Detected

This report shall not be reproduced except in full, without the written approval of the laboratory.
Acceptable Range

$54-130$ 
NEL LABORATORIES

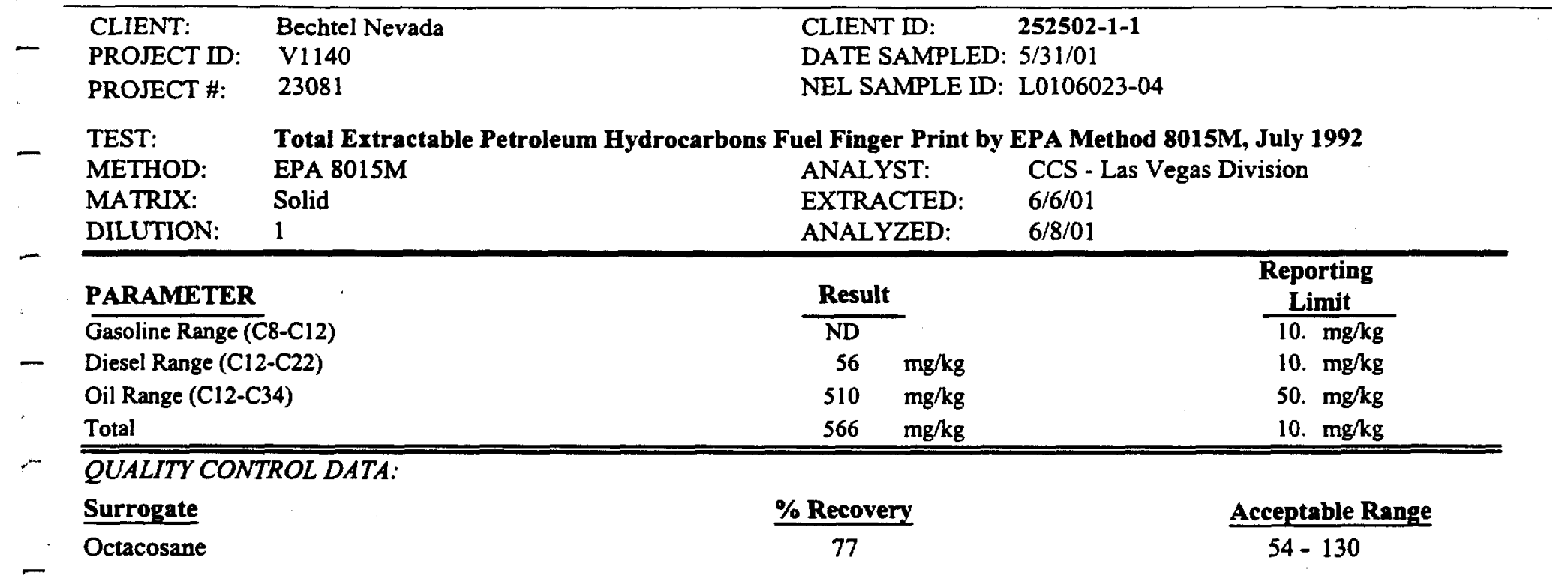

- ND - Not Detected

This report shall not be reproduced except in full, without the written approval of the laboratory. 
NEL LABORATORIES

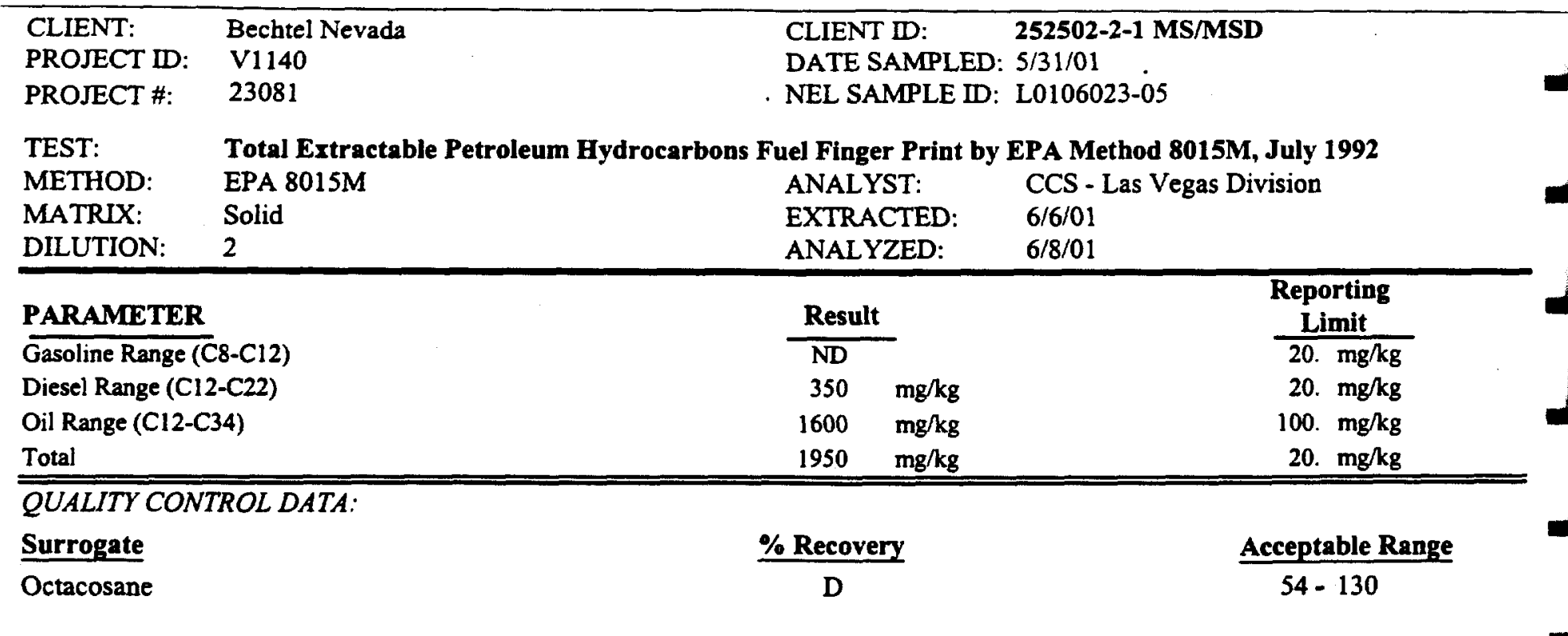

ND - Not Detected

This report shall not be reproduced except in full, without the written approval of the laboratory. 
NEL LABORATORIES

\begin{tabular}{lll}
\hline CLIENT: & Bechtel Nevada & CLIENT ID: \\
PROJECT ID: 252502-3-1 & V1140 & DATE SAMPLED: 5/31/01 \\
PROJECT \#: & 23081 & NEL SAMPLE ID: L0106023-06
\end{tabular}

- TEST: Total Extractable Petroleum Hydrocarbons Fuel Finger Print by EPA Method 8015M, July 1992

METHOD: EPA 8015M ANALYST: CCS - Las Vegas Division

MATRIX: Solid $\quad$ EXTRACTED: 6/6/01

DILUTION: 5 ANALYZED: $6 / 8 / 01$

\section{PARAMETER}

Gasoline Range (C8-C12)

- Diesel Range (C12-C22)

Oil Range (C12-C34)

Total

QUALITY CONTROL DATA:

Surrogate

\% Recovery

Octacosane

D

\begin{tabular}{|c|c|c|}
\hline Result & & $\begin{array}{l}\text { Reporting } \\
\text { Limit }\end{array}$ \\
\hline ND & & $50 . \mathrm{mg} / \mathrm{kg}$ \\
\hline 640 & $\mathrm{mg} / \mathrm{kg}$ & so. $\mathrm{mg} / \mathrm{kg}$ \\
\hline 2800 & $\mathrm{mg} / \mathrm{kg}$ & 250. $\mathrm{mg} / \mathrm{kg}$ \\
\hline 3440 & $\mathrm{mg} / \mathrm{kg}$ & 50. $\mathrm{mg} / \mathrm{kg}$ \\
\hline
\end{tabular}

- ND - Not Detected

This report shall not be reproduced except in full, without the written approval of the laboratory. 
NEL LABORATORIES

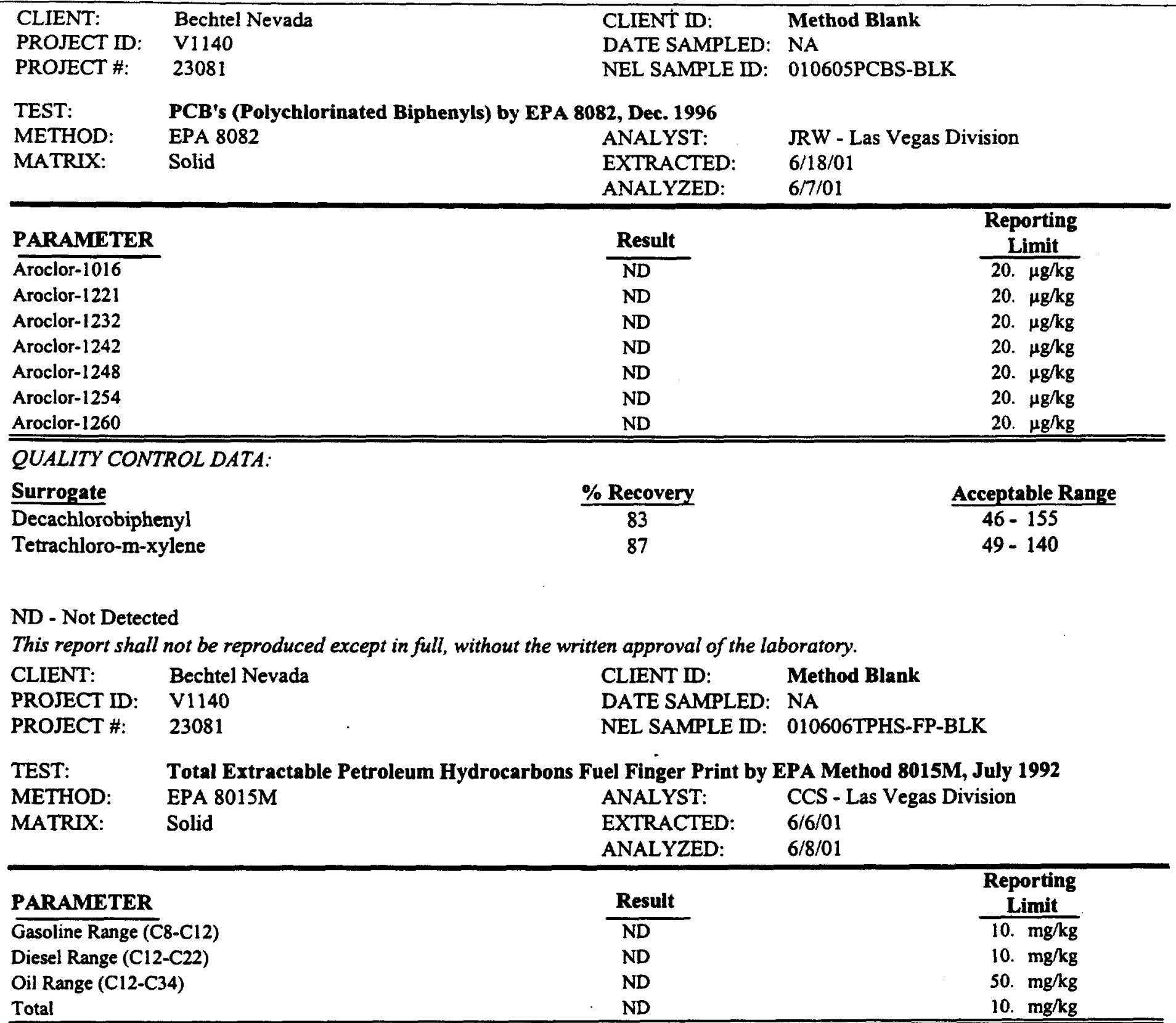

QUALITY CONTROL DATA:

Surrogate

Octacosane
\% Recovery

80
Acceptable Range

$54-130$

ND - Not Detected

This report shall not be reproduced except in full, without the written approval of the laboratory. 
NEL LABORATORIES

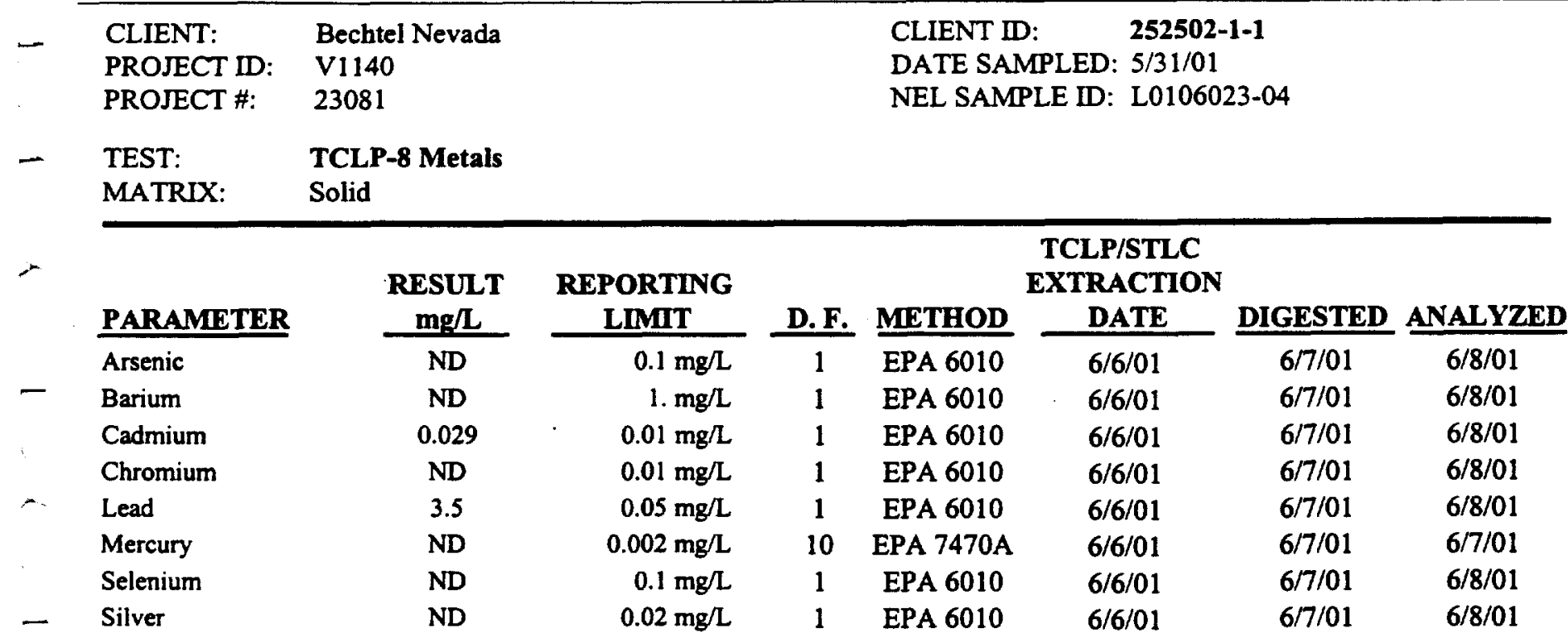

D.F. - Dilution Factor

ND - Not Detected

This report shall not be reproduced except in full, without the written approval of the laboratory. 
NEL LABORATORIES

\begin{tabular}{|c|c|c|c|c|c|c|c|}
\hline $\begin{array}{l}\text { CLIENT: } \\
\text { PROJECT ID: } \\
\text { PROJECT \#: }\end{array}$ & $\begin{array}{l}\text { Bechtel Nevada } \\
\text { V1140 } \\
23081\end{array}$ & & & $\begin{array}{l}\text { CLIENT ID } \\
\text { DATE SAN } \\
\text { NEL SAMF }\end{array}$ & $\begin{array}{l}\text { D: } \\
\text { MPLED: } 5 / 31 / 01 \\
\text { PLE ID: } \\
\text { L010602 }\end{array}$ & $\begin{array}{l}\text { 2-1 MS/MSD } \\
23-05\end{array}$ & \\
\hline $\begin{array}{l}\text { TEST: } \\
\text { MATRIX: }\end{array}$ & $\begin{array}{l}\text { TCLP-8 Metals } \\
\text { Solid }\end{array}$ & & & & & & \\
\hline PARAMETER & $\begin{array}{c}\text { RESULT } \\
\mathrm{mg} / \mathrm{L} \\
\end{array}$ & $\begin{array}{c}\text { REPORTING } \\
\text { LIMIT } \\
\end{array}$ & D. F. & METHOD & $\begin{array}{c}\text { TCLP/STLC } \\
\text { EXTRACTION } \\
\text { DATE } \\
\end{array}$ & DIGESTED & ANALYZED \\
\hline Arsenic & ND & $0.1 \mathrm{mg} / \mathrm{L}$ & 1 & EPA 6010 & $6 / 6 / 01$ & $6 / 7 / 01$ & $6 / 8 / 01$ \\
\hline Barium & ND & 1. $\mathrm{mg} / \mathrm{L}$ & 1 & EPA 6010 & $6 / 6 / 01$ & $6 / 7 / 01$ & $6 / 8 / 01$ \\
\hline Cadmium & 0.017 & $0.01 \mathrm{mg} / \mathrm{L}$ & 1 & EPA 6010 & $6 / 6 / 01$ & $6 / 7 / 01$ & $6 / 8 / 01$ \\
\hline Chromium & 0.015 & $0.01 \mathrm{mg} / \mathrm{L}$ & 1 & EPA 6010 & $6 / 6 / 01$ & $6 / 7 / 01$ & $6 / 8 / 01$ \\
\hline Lead & 0.11 & $0.05 \mathrm{mg} / \mathrm{L}$ & 1 & EPA 6010 & $6 / 6 / 01$ & $6 / 7 / 01$ & $6 / 8 / 01$ \\
\hline Mercury & ND & $0.002 \mathrm{mg} / \mathrm{L}$ & 10 & EPA 7470A & $6 / 6 / 01$ & $6 / 7 / 01$ & $6 / 7 / 01$ \\
\hline Selenium & ND & $0.1 \mathrm{mg} / \mathrm{L}$ & 1 & EPA 6010 & $6 / 6 / 01$ & $6 / 7 / 01$ & $6 / 8 / 01$ \\
\hline Silver & ND & $0.02 \mathrm{mg} / \mathrm{L}$ & 1 & EPA 6010 & $6 / 6 / 01$ & $6 / 7 / 01$ & $6 / 8 / 01$ \\
\hline
\end{tabular}

D.F. - Dilution Factor

ND - Not Detected

This report shall not be reproduced except in full, without the written approval of the laboratory. 
NEL LABORATORIES

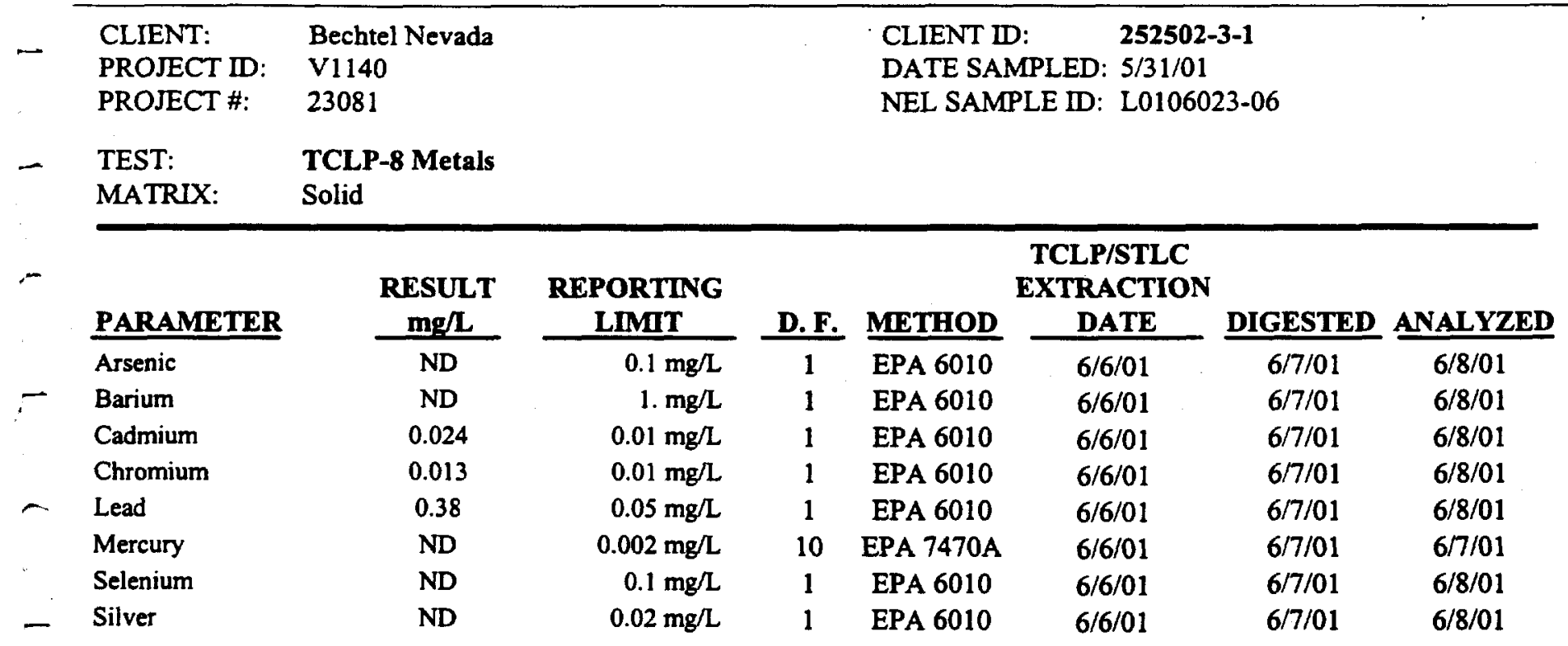

- D.F. - Dilution Factor ND - Not Detected

This report shall not be reproduced except in full, without the written approval of the laboratory. 
NEL LABORATORIES

$\begin{array}{llll}\text { CLIENT: } & \text { Bechtel Nevada } & \text { CLIENT ID: } & \text { Method Blank } \\ \text { PROJECT ID: } & \text { V1140 } & \text { DATE SAMPLED: } & \text { NA } \\ \text { PROJECT \#: } & 23081 & \text { NEL SAMPLE ID: } & \text { L06016-THg-BLK }\end{array}$

TEST: $\quad$ TCLP by EPA 1311, July 1992 \& Mercury by EPA 7470A, July 1992

MATRIX: TCLP Extract

\begin{tabular}{|c|c|c|c|c|c|c|c|}
\hline \multirow[b]{2}{*}{ PARAMETER } & \multirow[b]{2}{*}{ RESULT } & \multirow{2}{*}{$\begin{array}{c}\text { REPORTING } \\
\text { LIMIT } \\
\end{array}$} & \multicolumn{5}{|c|}{$\begin{array}{l}\text { TCLP/STLC } \\
\text { EXTRACTION }\end{array}$} \\
\hline & & & D. $\mathbf{F}$. & METHOD & DATE & DIGESTED & ANALYZED \\
\hline Mercury & ND & $0.002 \mathrm{mg} / \mathrm{L}$ & 10 & EPA $7470 A$ & $6 / 6 / 01$ & $6 / 7 / 01$ & $6 / 7 / 01$ \\
\hline
\end{tabular}

D.F. - Dilution Factor

ND - Not Detected

This report shall not be reproduced except in full, without the written approval of the laboratory. 
NEL LABORATORIES

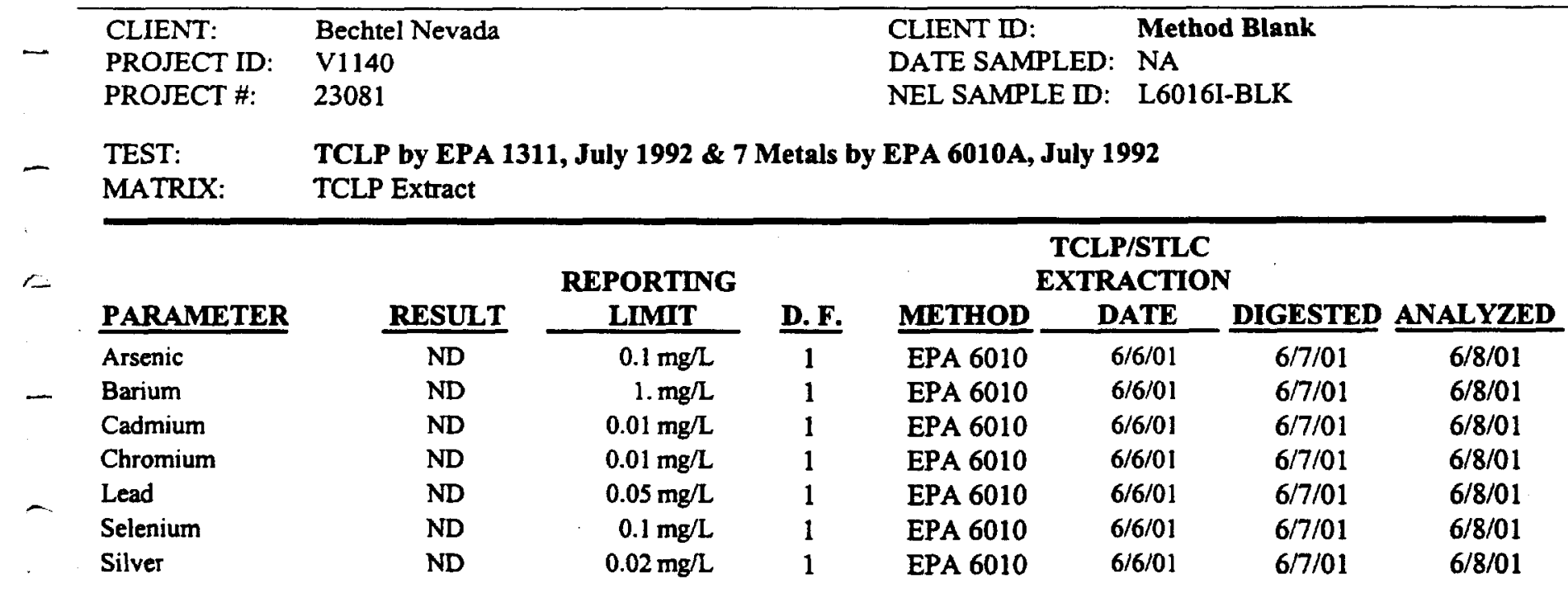

D.F. - Dilution Factor

ND - Not Detected

- This report shall not be reproduced except in full, without the written approval of the laboratory. 


$\begin{array}{llll}\text { CLIENT: } & \text { Bechtel Nevada } & \text { CLIENT ID: } & \text { 252502-1-1 } \\ \text { PROJECT ID: } & \text { V1140 } & \text { DATE SAMPLED: } & 5 / 31 / 01 \\ \text { PROJECT \#: } & 23081 & \text { NEL SAMPLE ID: } & \text { L0106023-04 }\end{array}$

TCLP by EPA 1311, July 1992 \& Semivolatile Organics by EPA Method 8270C, Dec. 1996

METHOD: $\quad$ EPA 8270

MATRIX: Solid

DILUTION: 1

TCLP EXTRACT DATE: $6 / 6 / 01$

EXTRACTED $\quad 6 / 15 / 01$

ANALYZED: $\quad 6 / 19 / 01$

\section{PARAMETER}

1,4-Dichlorobenzene (p-DCB)

2,4-Dinitrotoluene (DNT)

Hexachlorobenzene

Hexachlorobutadiene

Hexachloroethane

2-Methylphenol

3,4-Methylphenol (isomeric pair)

Nitrobenzene

Pentachlorophenol

Pyridine

2,4,5-Trichlorophenol

2,4,6-Trichlorophenol

\begin{tabular}{c}
$\begin{array}{c}\text { Result } \\
\text { mg/L }\end{array}$ \\
\hline ND \\
ND \\
ND \\
ND \\
ND \\
ND \\
ND \\
ND \\
ND \\
ND \\
ND \\
ND
\end{tabular}

Reporting Limit

$0.1 \mathrm{mg} / \mathrm{L}$

$0.1 \mathrm{mg} / \mathrm{L}$

$0.1 \mathrm{mg} / \mathrm{L}$

$0.1 \mathrm{mg} / \mathrm{L}$

$0.1 \mathrm{mg} / \mathrm{L}$

$0.1 \mathrm{mg} / \mathrm{L}$

$0.1 \mathrm{mg} / \mathrm{L}$

$0.1 \mathrm{mg} / \mathrm{L}$

$0.1 \mathrm{mg} / \mathrm{L}$

$0.1 \mathrm{mg} / \mathrm{L}$

$0.1 \mathrm{mg} / \mathrm{L}$

$0.1 \mathrm{mg} /$

QUALITY CONTROL DATA:

Surrogate

2,4,6-Tribromophenol

2-Fluorobiphenyl

2-Fluorophenol

Nitrobenzene-d5

p-Terphenyl-d14

Phenol-d5
$\%$ Recovery

36

19

18

23

34

14
Acceptable Range

0 - 161

$16-127$

0 - 88

9- 132

$16-163$

$0-63$

ND - Not Detected

This report shall not be reproduced except in full, without the written approval of the laboratory. 
NEL LABORATORIES

\begin{tabular}{llll}
\hline CLIENT: & Bechtel Nevada & CLIENT ID: & 252502-2-1 MS/MSD \\
PROJECT ID: & V1140 & DATE SAMPLED: $5 / 31 / 01$ \\
PROJECT \#: & 23081 & NEL SAMPLE ID: L0106023-05 \\
& & & \\
TEST: & TCLP by EPA 1311, July 1992 \& Semivolatile & Organics by EPA Method 8270C, Dec. 1996 \\
METHOD: & EPA 8270 & TCLP EXTRACT DATE: $6 / 6 / 01$ \\
MATRIX: & Solid & EXTRACTED & $6 / 15 / 01$ \\
DILUTION: & 1 & ANALYZED: & $6 / 19 / 01$
\end{tabular}

\section{PARAMETER}

- 1,4-Dichlorobenzene (p-DCB)

2,4-Dinitrotoluene (DNT)

Hexachlorobenzene

Hexachlorobutadiene

Hexachloroethane

2-Methylphenol

3,4-Methylphenol (isomeric pair)

Nitrobenzene

Pentachlorophenol

Pyridine

2,4,5-Trichlorophenol

2,4,6-Trichlorophenol

\begin{tabular}{c}
$\begin{array}{c}\text { Result } \\
\mathbf{m g} / \mathbf{L}\end{array}$ \\
\hline $\mathrm{ND}$ \\
$\mathrm{ND}$ \\
$\mathrm{ND}$ \\
$\mathrm{ND}$ \\
$\mathrm{ND}$ \\
$\mathrm{ND}$ \\
$\mathrm{ND}$ \\
$\mathrm{ND}$ \\
$\mathrm{ND}$ \\
$\mathrm{ND}$ \\
$\mathrm{ND}$ \\
$\mathrm{ND}$
\end{tabular}

Reporting

Limit

$0.1 \mathrm{mg} / \mathrm{L}$

$0.1 \mathrm{mg} / \mathrm{L}$

$0.1 \mathrm{mg} / \mathrm{L}$

$0.1 \mathrm{mg} / \mathrm{L}$

$0.1 \mathrm{mg} / \mathrm{L}$

$0.1 \mathrm{mg} / \mathrm{L}$

$0.1 \mathrm{mg} / \mathrm{L}$

$0.1 \mathrm{mg} / \mathrm{L}$

$0.1 \mathrm{mg} / \mathrm{L}$

$0.1 \mathrm{mg} / \mathrm{L}$

$0.1 \mathrm{mg} / \mathrm{L}$

$0.1 \mathrm{mg} / \mathrm{L}$

\section{Surrogate}

- 2,4,6-Tribromophenol

2-Fluorobiphenyl

2-Fluorophenol

Nitrobenzene-d5

p-Terphenyl-d14

Phenol-d5
$\%$ Recovery

44

22

18

24

32

16
Acceptable Range

0 - 161

$16-127$

$0-88$

$9-132$

$16-163$

0 - 63

ND - Not Detected

This report shall not be reproduced except in full, without the written approval of the laboratory. 


$\begin{array}{llll}\text { CLIENT: } & \text { Bechtel Nevada } & \text { CLIENT ID: } & \text { 252502-3-1 } \\ \text { PROJECT ID: } & \text { V1140 } & \text { DATE SAMPLED: } & \text { 5/31/01 } \\ \text { PROJECT \#: } & 23081 & \text { NEL SAMPLE ID: } & \text { L0106023-06 }\end{array}$

TEST: $\quad$ TCLP by EPA 1311, July 1992 \& Semivolatile Organics by EPA Method 8270C, Dec. 1996

METHOD: $\quad$ EPA 8270

MATRIX: Solid

DILUTION: 1

TCLP EXTRACT DATE: $6 / 6 / 01$

EXTRACTED $6 / 15 / 01$

ANALYZED: $\quad 6 / 19 / 01$

\section{PARAMETER}

1,4-Dichlorobenzene (p-DCB)

2,4-Dinitrotoluene (DNT)

Hexachlorobenzene

Hexachlorobutadiene

Hexachloroethane

2-Methylphenol

3,4-Methylphenol (isomeric pair)

Nitrobenzene

Pentachlorophenol

Pyridine

2,4,5-Trichlorophenol

2,4,6-Trichlorophenol

\begin{tabular}{c}
$\begin{array}{c}\text { Result } \\
\text { mglL }\end{array}$ \\
\hline ND \\
ND \\
ND \\
ND \\
ND \\
ND \\
ND \\
ND \\
ND \\
ND \\
ND \\
ND
\end{tabular}

Reporting Limit

$0.1 \mathrm{mg} / \mathrm{L}$

$0.1 \mathrm{mg} / \mathrm{L}$

$0.1 \mathrm{mg} / \mathrm{L}$

$0.1 \mathrm{mg} / \mathrm{L}$

$0.1 \mathrm{mg} / \mathrm{h}$

$0.1 \mathrm{mg} / \mathrm{L}$

$0.1 \mathrm{mg} / \mathrm{L}$

$0.1 \mathrm{mg} / \mathrm{L}$

$0.1 \mathrm{mg} / \mathrm{L}$

$0.1 \mathrm{mg} / \mathrm{L}$

$0.1 \mathrm{mg} / \mathrm{L}$

$0.1 \mathrm{mg} / \mathrm{L}$

\section{QUALITY CONTROL DATA:}

Surrogate

2,4,6-Tribromophenol

2-Fluorobiphenyl

2-Fluorophenol

Nitrobenzene- $d 5$

p-Terphenyl-d14

Phenol-d5
$\%$ Recovery

47

22

19

24

32

17
Acceptable Range

0 - 161

$16-127$

$0-88$

$9-132$

$16-163$

0 - 63

ND - Not Detected

This report shall not be reproduced except in full, without the written approval of the laboratory. 
NEL LABORATORIES

$\begin{array}{llll}\text { CLIENT: } & \text { Bechtel Nevada } & \text { CLIENT ID: } & \text { Method Blank } \\ \text { PROJECT ID: } & \text { V1140 } & \text { DATE SAMPLED: } & \text { NA } \\ \text { PROJECT \#: } & 23081 & \text { NEL SAMPLE ID: } & \text { 0615E1 TCLP8270-BLK }\end{array}$

- TEST: $\quad$ TCLP by EPA 1311, July 1992 \& Semivolatile Organics by EPA Method 8270C, Dec. 1996

METHOD: EPA 8270 TCLP EXTRACT DATE: 6/6/01

MATRIX: TCLP Extract $\quad$ EXTRACTED $6 / 15 / 01$

ANALYZED: $\quad 6 / 19 / 01$

PARAMETER

1,4-Dichlorobenzene (p-DCB)

2,4-Dinitrotoluene (DNT)

Hexachlorobenzene

$=\quad$ Hexachlorobutadiene

Hexachloroethane

2-Methylphenol

Result

mg/L

ND

ND

ND

ND

ND

ND

ND

$\begin{array}{ll}\text { Nitrobenzene } & \text { ND } \\ \text { Pentachlorophenol } & \text { ND }\end{array}$

$\begin{array}{ll}\text { Nitrobenzene } & \text { ND } \\ \text { Pentachlorophenol } & \text { ND }\end{array}$

Pyridine

$\mathrm{ND}$

ND

2,4,5-Trichlorophenol

ND

\section{Reporting}

Limit

$0.1 \mathrm{mg} / \mathrm{L}$

$0.1 \mathrm{mg} / \mathrm{L}$

$0.1 \mathrm{mg} / \mathrm{L}$

$0.1 \mathrm{mg} / \mathrm{L}$

$0.1 \mathrm{mg} / \mathrm{L}$

$0.1 \mathrm{mg} / \mathrm{L}$

$0.1 \mathrm{mg} / \mathrm{L}$

$0.1 \mathrm{mg} / \mathrm{L}$

$0.1 \mathrm{mg} / \mathrm{L}$

$0.1 \mathrm{mg} / \mathrm{L}$

$0.1 \mathrm{mg} / \mathrm{L}$

$0.1 \mathrm{mg} / \mathrm{L}$

QUALITY CONTROL DATA:

Surrogate

- 2,4,6-Tribromophenol

2-Fluorobiphenyl

2-Fluorophenol

- Nitrobenzene-d5

p-Terphenyl-d14

Phenol-d5
$\%$ Recovery

$$
\begin{aligned}
& 32 \\
& 18 \\
& 20 \\
& 22 \\
& 30 \\
& 16
\end{aligned}
$$

Acceptable Range

$0-161$

$16-127$

0 - 88

$9-132$

$16-163$

0 - 63

L. ND - Not Detected

This report shall not be reproduced except in full, without the written approval of the laboratory. 
CLIENT: Bechtel Nevada

PROJECT ID: V1140

PROJECT \#: 23081

TEST: $\quad$ PCB's (Polychlorinated Biphenyls) by EPA 8082, Dec. 1996

MATRIX: $\quad$ Solid

\begin{tabular}{|c|c|c|c|c|c|c|c|}
\hline PARAMETER & NEL Sample ID & $\begin{array}{c}\text { Spike } \\
\text { Amount }\end{array}$ & $\underline{\text { Spike }}$ & $\underline{\text { Percent }}$ & & $\frac{\text { Acceptable }}{\text { Range }}$ & RPD \\
\hline Aroclor-1016 & 010605 PCBS-LCS & 333 & 283 & 85 & & $63-127$ & \\
\hline Aroclor-1016 & 010605PCBS-LCSD & 333 & 226 & 68 & & $63-127$ & 22.4 \\
\hline Aroclor-1016 & L0106023-06-MS & 333 & 355 & 107 & & $55-142$ & \\
\hline Aroclor -1016 & L0106023-06-MSD & 333 & 306 & 92 & & $55-142$ & 14.8 \\
\hline Aroclor- 1260 & 010605PCBS-LCS & 333 & 332 & 100 & & $57-138$ & \\
\hline Aroclor -1260 & 010605PCBS-LCSD & 333 & 264 & 79 & & $57-138$ & 22.8 \\
\hline Aroclor- 1260 & L0106023-06-MS & 333 & 1810 & 33 & $\mathrm{C}$ & $48-129$ & \\
\hline Aroclor- 1260 & L0106023-06-MSD & 333 & 2040 & 102 & $\mathrm{C}$ & $48-129$ & 102.2 \\
\hline
\end{tabular}

ND - Not Detected

This report shall not be reproduced except in full, without the written approval of the laboratory. 
CLIENT: $\quad$ Bechtel Nevada

- PROJECT ID: V1140

PROJECT \#: 23081

TEST: $\quad$ Total Extractable Petroleum Hydrocarbons Fuel Finger Print by EPA Method 8015M, July 1992 MATRIX: $\quad$ Solid

\begin{tabular}{|c|c|c|c|c|c|c|}
\hline PARAMETER & NEL Sample ID & $\frac{\text { Spike }}{\text { Amount }}$ & Spike & $\frac{\text { Percent }}{\text { Recovery }}$ & $\frac{\text { Acceptable }}{\text { Range }}$ & $\underline{\text { RPD }}$ \\
\hline Diesel Range (C12-C22) & 010606TPHS-FP-LCS & 166.7 & 138 & 83 & $53-91$ & \\
\hline Diesel Range (C12-C22) & 010606TPHS-FP-LCSD & 166.7 & 145 & 87 & $53-91$ & 4.9 \\
\hline Total & 010606TPHS-FP-LCS & 166.7 & 138 & 83 & $53-91$ & \\
\hline Total & 010606TPHS-FP-LCSD & 166.7 & 145 & 87 & $53-91$ & 4.9 \\
\hline
\end{tabular}


CLIENT:

PROJECT ID:

PROJECT \#:

TEST:

MATRIX:
Bechtel Nevada

V1140

23081

TCLP by EPA 1311, July 1992 \& Semivolatile Organics by EPA Method 8270C, Dec. 1996

\section{PARAMETER}

Pyridine

Pyridine

Pyridine

1,4-Dichlorobenzene ( $p-D C B$ )

1,4-Dichlorobenzene (p-DCB)

1,4-Dichlorobenzene (p-DCB)

Hexachloroethane

Hexachloroethane

Hexachloroethane

Nitrobenzene

Nitrobenzene

Nitrobenzene

Hexachlorobutadiene

Hexachlorobutadiene

Hexachlorobutadiene

2-Methylphenol

2-Methylphenol

2-Methylphenol

3,4-Methylphenol (isomeric pair)

3,4-Methylphenol (isomeric pair)

3,4-Methylphenol (isomeric pair)

2,4,6-Trichlorophenol

2,4,6-Trichlorophenol

2,4,6-Trichlorophenol

2,4,5-Trichlorophenol

2,4,5-Trichlorophenol

2,4,5-Trichlorophenol

2,4-Dinitrotoluene (DNT)

2,4-Dinitrotoluene (DNT)

2,4-Dinitrotoluene (DNT)

Hexachlorobenzene

Hexachlorobenzene

Hexachlorobenzene

Pentachlorophenol

Pentachlorophenol

Pentachlorophenol
NEL Sample ID

0615E1 TCLP8270-LCS

0615E1 TCLP8270-LCSD

L0106023-04-MS

0615E1 TCLP8270-LCS

0615E1 TCLP8270-LCSD

L0106023-04-MS

0615E1 TCLP8270-LCS

0615E1 TCLP8270-LCSD

L0106023-04-MS

0615E1 TCLP8270-LCS

0615E1 TCLP8270-LCSD

L0106023-04-MS

0615E1 TCLP8270-LCS

0615E1 TCLP8270-LCSD

L0106023-04-MS

0615E1 TCLP8270-LCS

0615E1 TCLP8270-LCSD

L0106023-04-MS

0615E1 TCLP8270-LCS

0615E1 TCLP8270-LCSD

L0106023-04-MS

0615E1 TCLP8270-LCS

0615E1 TCLP8270-LCSD

L0106023-04-MS

0615E1 TCLP8270-LCS

0615E1 TCLP8270-LCSD

L0106023-04-MS

0615E1 TCLP8270-LCS

0615E1 TCLP8270-LCSD

L0106023-04-MS

0615E1 TCLP8270-LCS

0615E1 TCLP8270-LCSD

L0106023-04-MS

0615E1 TCLP8270-LCS

0615E1 TCLP8270-LCSD

L0106023-04-MS

\section{Spike}

Amount

80

80

80

80

80

80

80

80

80

80

80

80

80

80

80

80

80

80

80

80

80

80

80

80

80

80

80

80

80

80

80

80

80

80

80

80
Spike

Result

56.5

72.8

61.8

67.4

74.5

56

50.6

54

43.5

96.4

82.9

84.4

53

57

46.6

76.8

82.9

64.5

67.6

71.8

56.3

102.7

93.8

88.4

70.1

97.5

97.4

101.8

95.8

86.1

96.7

92.1

76.8

63.1

73.1

67.7

\section{Percent \\ Recovery}

71

91

77

84

93

70

63

68

54

121

104

106

66

71

58

96

104

81

85

90

70

128

117

111

88

122

122

127

120

108

121

115

96

79

91

85
Acceptable

Range RPD

$10-130$

$10-130$

25.2

$10-130$

$7-105$

$7-105$

$7-105$

$43-104$

$43-104$

$43-104$

$28-124$

$28-124$

28 - 124

$39-111$

$39-111$

$39-111$

$30-130$

$30-130$

$30-130$

$30-130$

$30-130$

$30-130$

$43-110$

$43-110$

$43-110$

$30-130$

$30-130$

32.7

$30-130$

$50-111$

$50-111$

$50-111$

$41-125$

$41-125$

$41-125$

$47-127$

$47-127$

$47-127$

10. 
NEL LABORATORIES

CLIENT: Bechtel Nevada

- PROJECT ID: V1140

PROJECT \#: 23081

TEST: $\quad$ TCLP/STLC Metals

- MATRIX: Solid

- PARAMETER

Mercury

Mercury

- Mercury

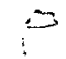

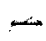

$\cdots$

$-$

$-$

$-$

$-$

$-$

$-$

ND - Not Detected

This report shall not be reproduced except in full, without the written approval of the laboratory. 


$\begin{array}{ll}\text { CLIENT: } & \text { Bechtel Nevada } \\ \text { PROJECT ID: } & \text { V1140 } \\ \text { PROJECT \#: } & 23081 \\ \text { TEST: } & \text { TCLP/STLC Metals } \\ \text { MATRIX: } & \text { Solid }\end{array}$

\begin{tabular}{|c|c|c|c|c|c|c|c|}
\hline PARAMETER & & NEL Sample ID & $\frac{\text { Spike }}{\text { Amount }}$ & $\frac{\text { Spike }}{\text { Result }}$ & $\frac{\text { Percent }}{\text { Recovery }}$ & $\frac{\text { Acceptable }}{\text { Range }}$ & $\underline{\text { RPD }}$ \\
\hline Arsenic & & L6016I-LCS & 0.5 & 0.498 & 100 & $85-115$ & \\
\hline Arsenic & & L0106016-01-MS & 0.5 & 0.505 & 101 & $75-125$ & \\
\hline Arsenic & * & L0106016-01-MSD & 0.5 & 0.508 & 102 & $75-125$ & 0.6 \\
\hline Silver & & L6016I-LCS & 0.5 & 0.45 & 90 & $85-115$ & \\
\hline Silver & & L0106016-01-MSD & 0.5 & 0.466 & 93 & $75-125$ & 22.2 \\
\hline Barium & & L6016I-LCS & 1 & 0.991 & 99 & $85-115$ & \\
\hline Barium & & L0106016-01-MS & 1 & 0.97 & -43 & $75-125$ & \\
\hline Barium & & L0106016-01-MSD & 1 & 2.91 & 151 & $75-125$ & 359.3 \\
\hline Cadmium & & L6016I-LCS & 0.5 & 0.504 & 101 & $85-115$ & \\
\hline Cadmium & & L0106016-01-MS & 0.5 & 0.587 & 87 & $75-125$ & \\
\hline Cadmium & & L0106016-01-MSD & 0.5 & 0.58 & 86 & $75-125$ & 1.6 \\
\hline Chromium & & L6016I-LCS & 0.5 & 0.496 & 99 & $85-115$ & \\
\hline Chromium & & L0106016-01-MS & 0.5 & 0.554 & 99 & $75-125$ & \\
\hline Chromium & & L0106016-01-MSD & 0.5 & 0.551 & 98 & $75-125$ & 0.6 \\
\hline Lead & & L6016I-LCS & 1 & 1.03 & 103 & $85-115$ & \\
\hline Lead & & L0106016-01-MS & 1 & 1.65 & 104 & $75-125$ & \\
\hline Lead & & L0106016-01-MSD & 1 & 1.67 & 106 & $75-125$ & 1.9 \\
\hline Selenium & & L6016I-LCS & 0.5 & 0.553 & 111 & $85-115$ & \\
\hline Selenium & & L0106016-01-MS & 0.5 & 0.463 & 93 & $75-125$ & \\
\hline Selenium & & L0106016-01-MSD & 0.5 & 0.417 & 83 & $75-125$ & 10.5 \\
\hline
\end{tabular}




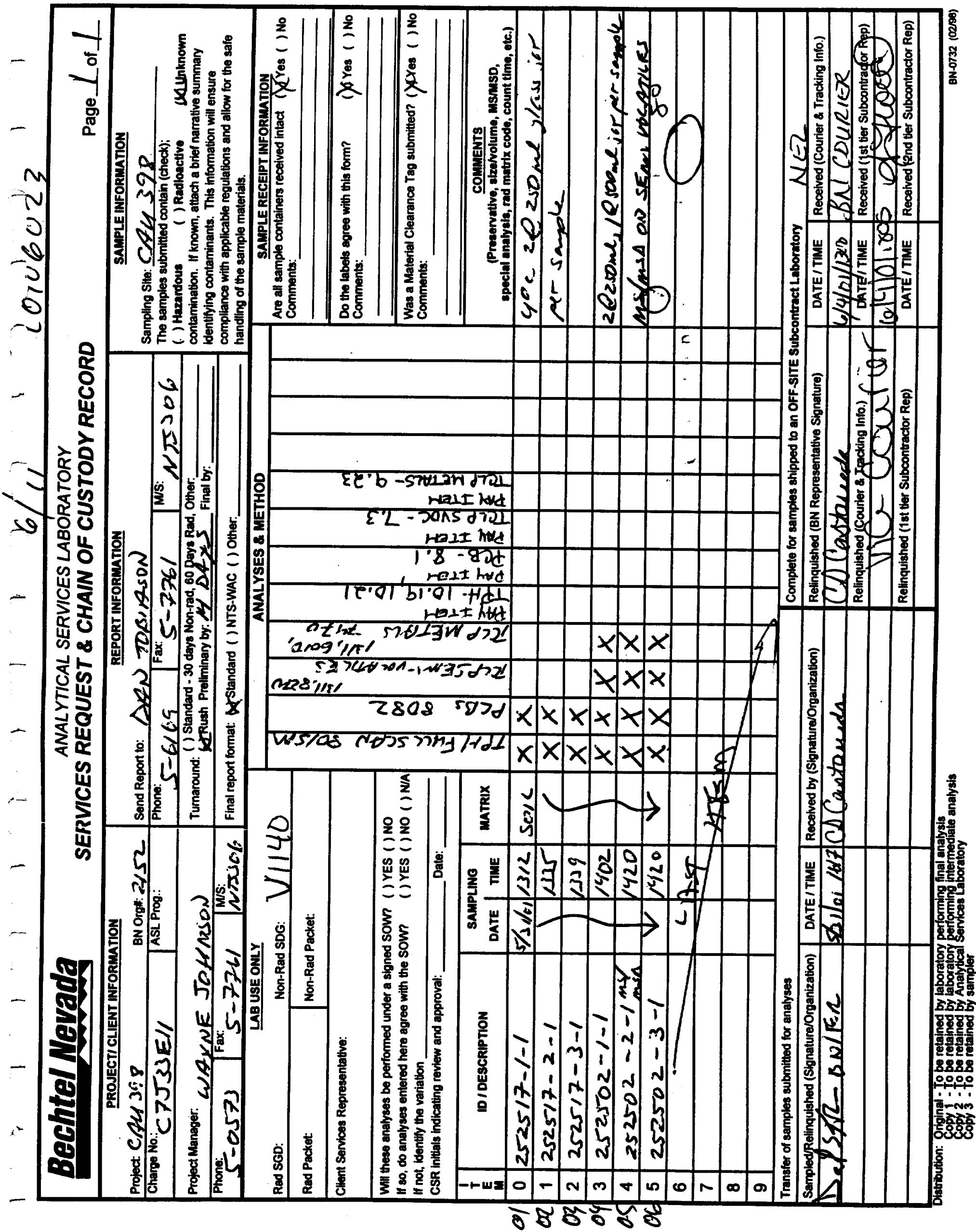




\section{NEL Laboratories \\ CHAIN OF CUSTODY \\ CHANGE ORDER FORM}

- Date: $6-12-01 \quad$ NEL Order ID: 20106023

Matrix: SO

Client Name / Contact: $13 \mathrm{NCC}$

Date Contacted:

$r$

\begin{tabular}{|c|c|}
\hline Please Mark All That Apply & Required Information \\
\hline New Project Due Date & \\
\hline Analysis Added & TCCP \\
\hline Anälysis Deleted & \\
\hline Samples Added & \\
\hline Samples Deleted & \\
\hline Samples Placed On-Hold & \\
\hline Samples Taken Off-Hold & \\
\hline
\end{tabular}

\section{Additional Requests or Comments}

- Did mot set Hi-litad ou COC That cane up to you. Please da tCLP.8270

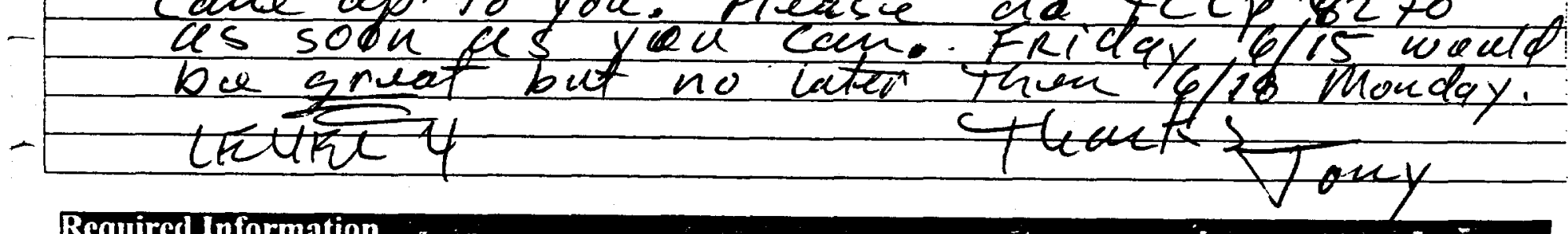

Required Information

Is there sufficient sample volume for the analysis or sample added?

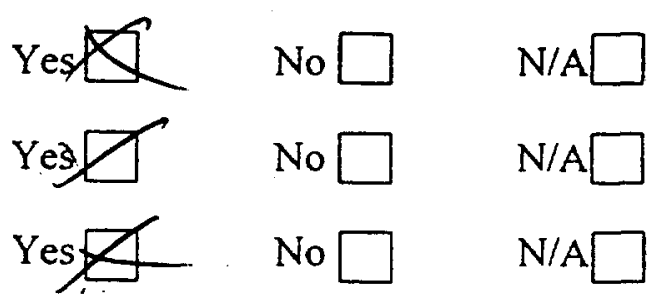

Can the sample(s) be analyzed within hold time?

Nosta

Change Order Faxed To

Boise $\square \quad$ Las Vegas $\square \quad$ Phoenix $\square$

Reno

Date Faxed: $6 / 12$ Time Faxed: $1635 \%$

Sacramento

Change Requested By: Tory Suarcligo 



\section{GENERAL ENGINEERING LABORATORIES}

Meeting today's needs with a vision for tomorrow:

\section{CASE NARRATIVE \\ for \\ Bechtel Nevada \\ Clark County, Nevada \\ SDG\# V1169}

July 21, 2001

Laboratory Identification:

General Engineering Laboratories, Inc. (GEL)

Mailing Address:

P.O. Box 30712

Charleston, SC 29414

Express Mail Delivery and Shipping Address:

2040 Savage Road

Charleston, SC 29414

Telephone Number:

(843) 556-8171

Summary:

\section{Sample Receipt}

The sample for SDG\# V1169 arrived at GEL located in Charleston, South Carolina on June 22, 2001 for environmental analyses.

The sample containers arrived without any visible signs of tampering or breakage. The samples were delivered with chain of custody documentation and signatures.

The laboratory received the following samples:

\begin{tabular}{ll}
$\begin{array}{l}\text { Laboratory } \\
\text { Identification }\end{array}$ & $\begin{array}{l}\text { Sample } \\
\text { Description }\end{array}$ \\
\hline 44601001 & $252507-1-0$ \\
44601002 & $252507-2-0$ \\
44601003 & $252507-3-0$ \\
44601004 & $252508-1-0$ \\
44601005 & $252508-2-0$
\end{tabular}




\section{Case Narrative}

Sample analyses were conducted using methodology as outlined in GEL Standard Operating Procedures. Any technical or administrative problems during analysis, data review and reduction are listed by analytical parameter in the applicable case narrative.

\section{Internal Chain of Custody:}

Custody was maintained for all samples.

\section{Items of Note:}

There are no items of note.

\section{Data Package:}

The enclosed data package contains the following sections: Case Narrative, Chain of Custody, Cooler Receipt Checklist, Data Review Definition Sheet, and Radiological parameters.

This data package, to the best of my knowledge, is in compliance with technical and administrative requirements.

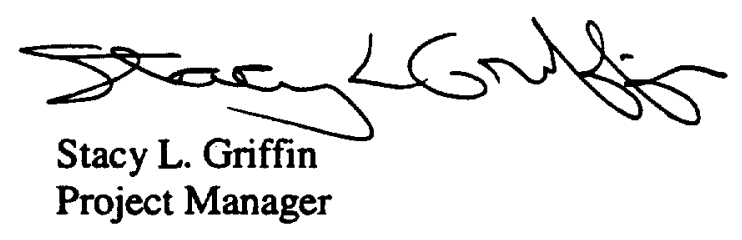



o

(1)

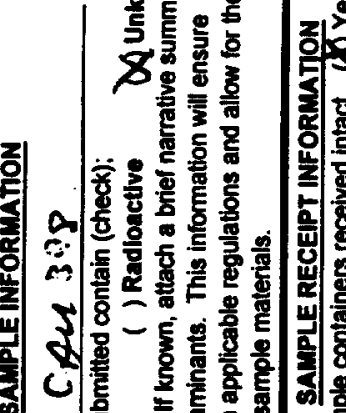

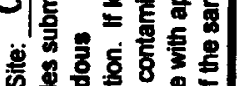
울

言

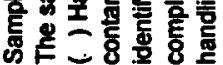

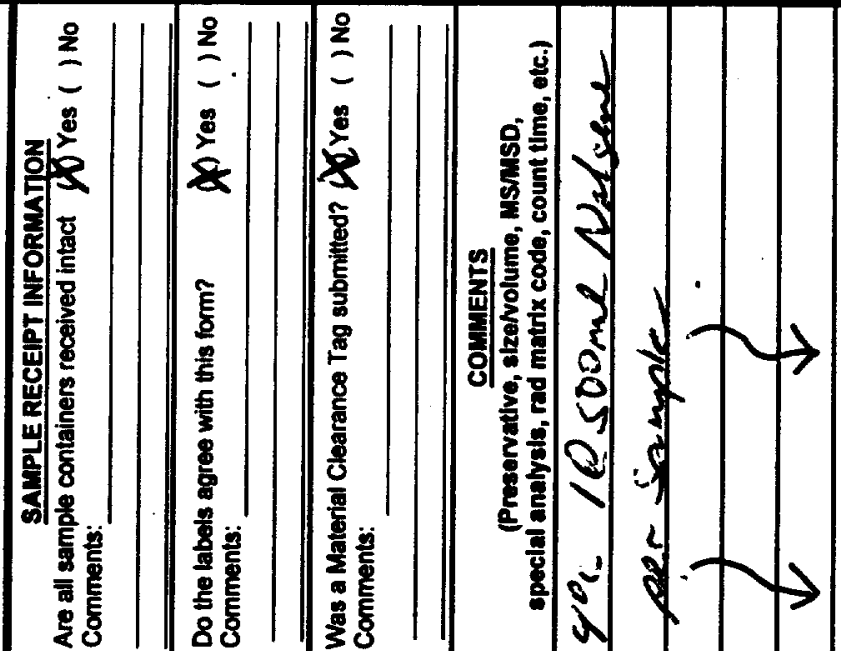

\& $\frac{8}{8}$

-

ํํㅇㅇำ

-

岁范

出?

-

它 $\frac{1}{0}$

战

$\lambda \cdot \frac{1}{4}$

j)

T雬岇

$f<x$

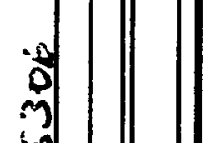

2

is

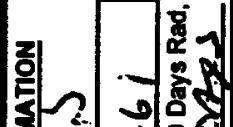

영 에워

흔 1 है

जी 1 पे

웝 d

옹

送

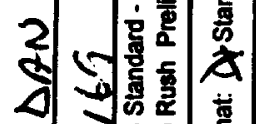

을

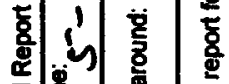

总兽

点

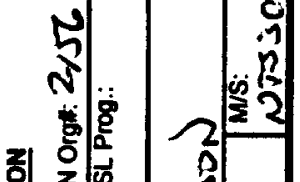

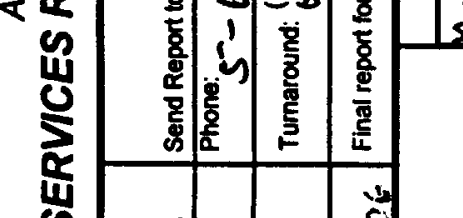

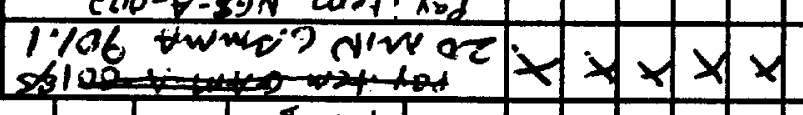

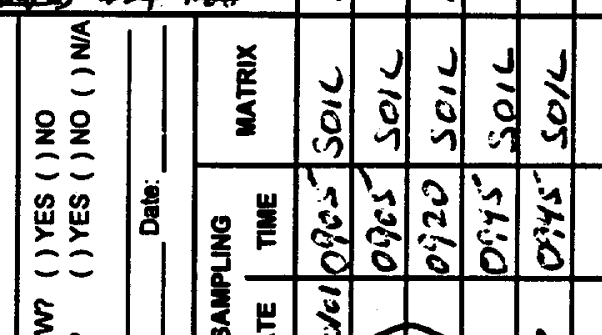

年这全

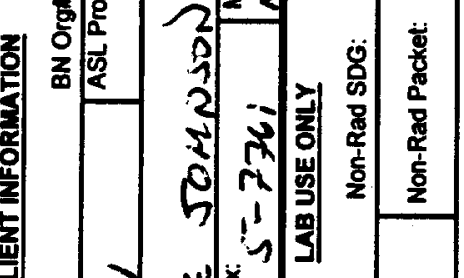

$\frac{1}{20}$

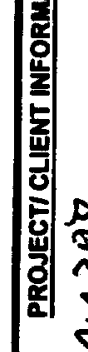

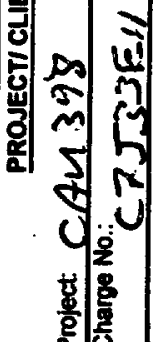

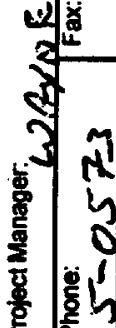

है

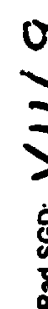

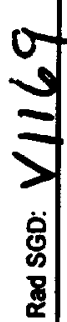

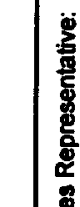

营

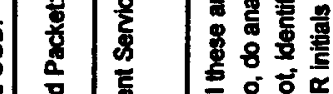

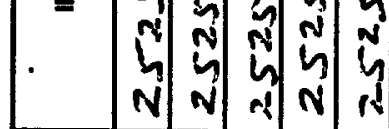

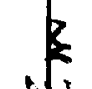

号号

产

要

空

密密

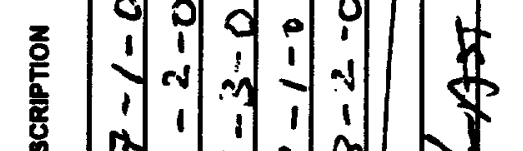

8 迸

8
8
8

要

政 $N$ m

番

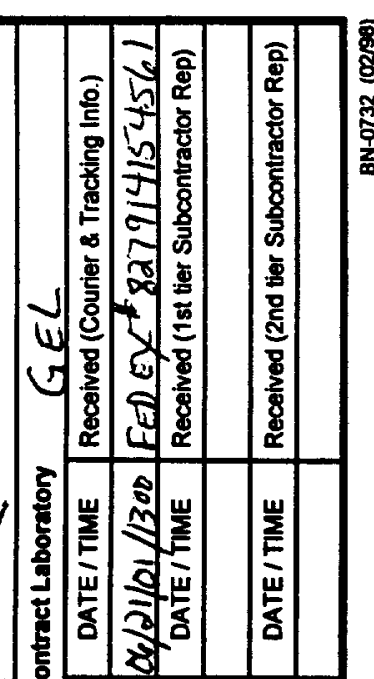

总
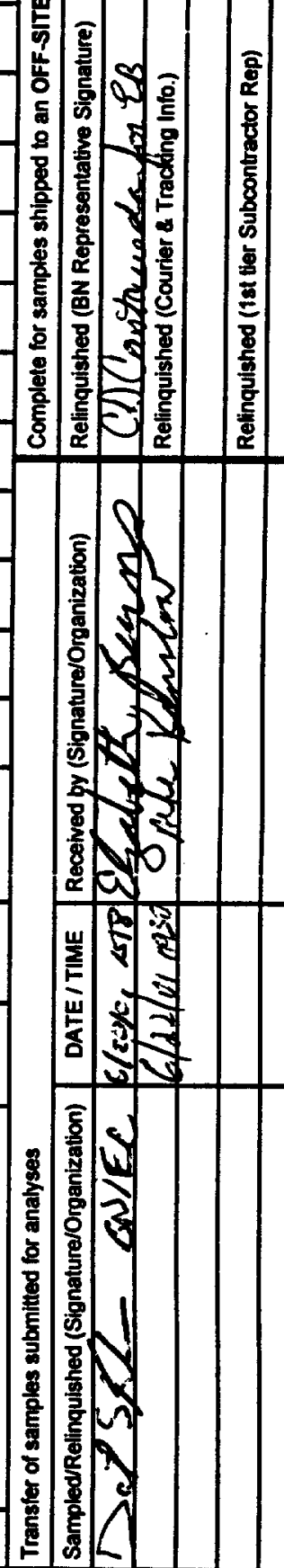

密 


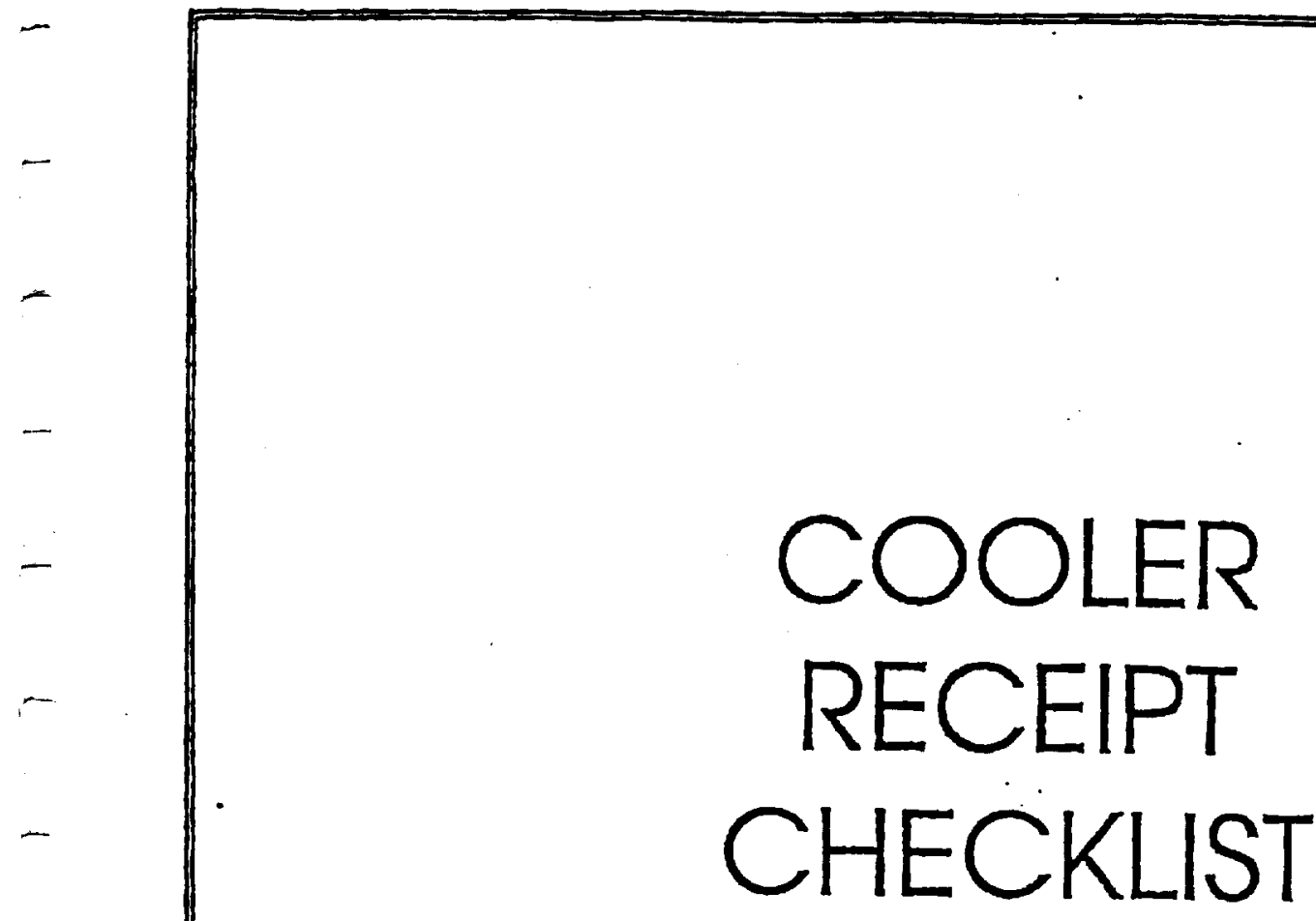


Dale $\quad 6-\alpha \alpha-01$

SAMPLE RECEIPT REVIEW

client_Becktel NeN

Recelved by $m k$

SAMPLEREMENGRMERTR

1 Were shipping containars received intact and sealed? if no, notify the Project Manlager

2 Were chain of custody documents included?

3 Shipping container temperature(s) checked:

4 Is temperature documented on Chain of Cuslody

5 Was shipping contalner temperature within specifications (4+1-2C) if no, notify Project Manager

6 Are any of the samples idenllfed by the dient as radioactive? if yes, complete radioactiva raceipt form

Any samples not indentffed by the client.as radioaclive must be screened for radioactivitiy.

If scresning results indicate $>\times 2$ background inforn the RSO."

7 Were chain of cuslody dacuments campleled correclly? (link, signed, match containers)

8 Were sample conlainers racelved intact and sealed? If no, nolify the Project Manager

9 Were all sample containers properly labeled?

10 Were conect sampla containers reciived?

11 Preserved samples checked for pH?

12 Were samples preserved comethly? If no, notify Projact Manager

13 Were samples recelved within holding time? If No, notify Project Manager

14 Were VDA vials free of headspace?

15 ARCOCH

16 SDG\#

-

PM(A) Review:

Date Reviewed:

Additionel Comments:

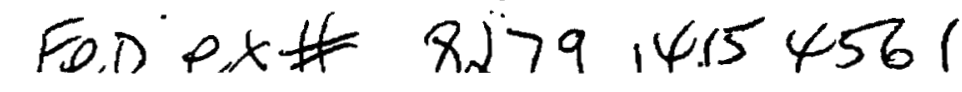

YES NO NA COMMENTSIOUALIFERS

res NO NA
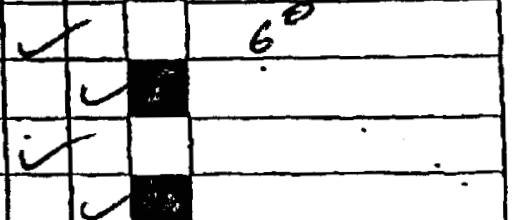

$Y_{0}$ observed bachordand CPM

oberoved somple CPM
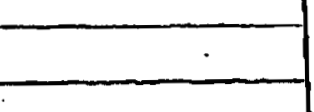

en

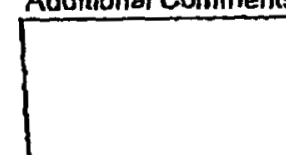




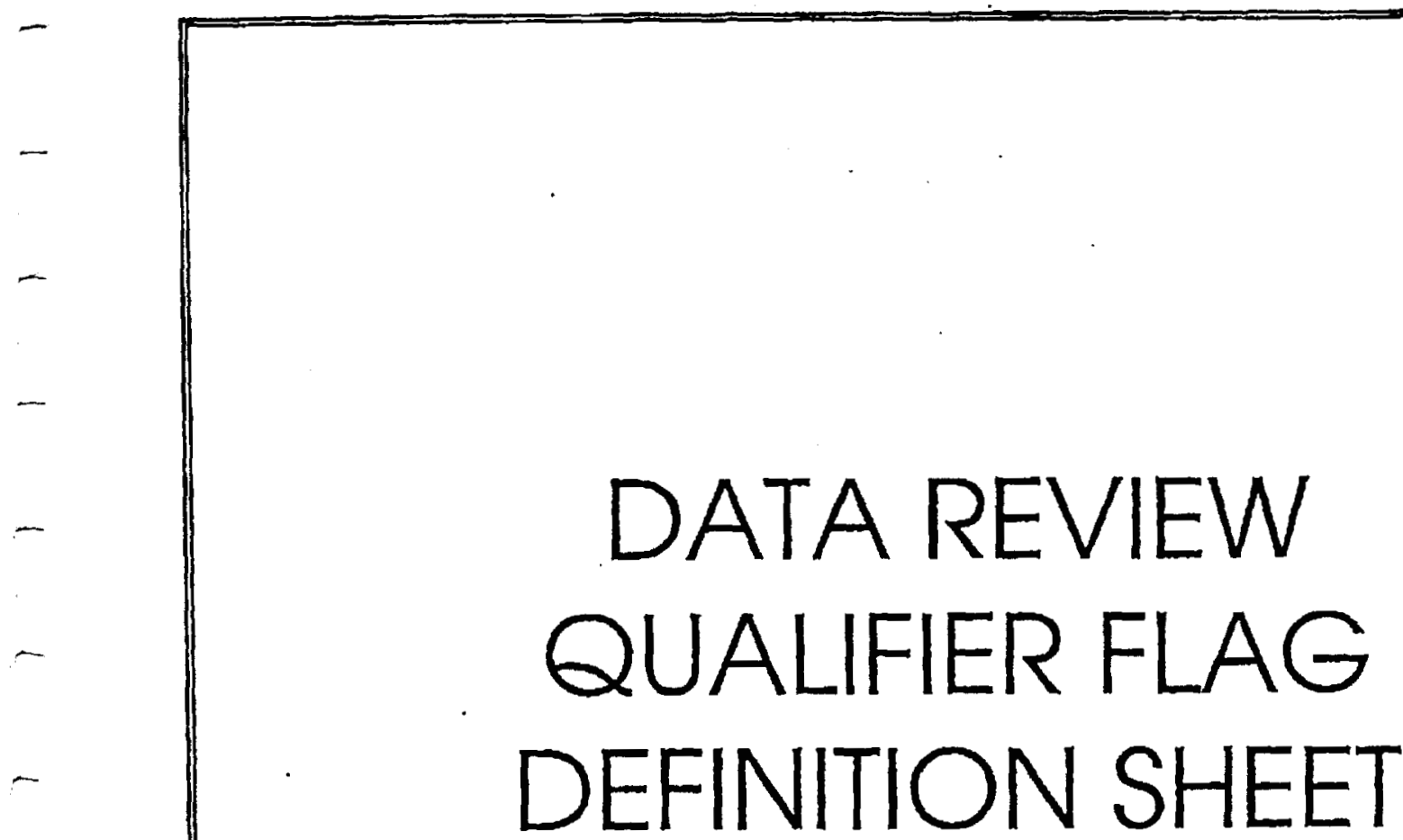

$-$

$-$

-

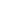




\section{General Engineering Laborarories. Inc. \\ DATA QUALIFIERS FOR INORGANIC ANALYSES}

Dara Qualiñess used on Form is or Certiñentes of Anaiysis ( $C$ of $A$ ) follow the spesinicarions set forth in the textnical specirications of the most currear CLP Statement of Work and are derined as follows.

\begin{tabular}{|c|c|c|}
\hline Sescion & Exolanation & Locarion \\
\hline$E$ & 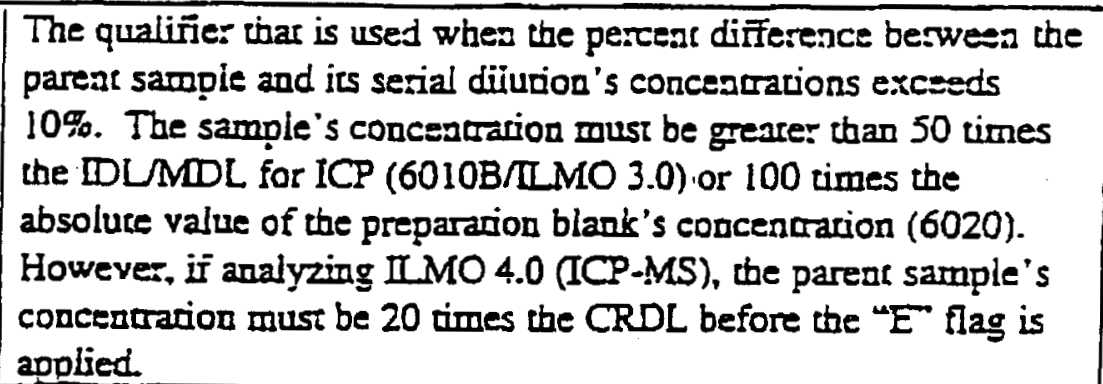 & $\begin{array}{l}\text { Form 1. } \\
\text { and EDD }\end{array}$ \\
\hline * & $\begin{array}{l}\text { The qualifier thar is used to indicare thar the duplicare sample } \\
\text { analysis for an anaivre is out of control. }\end{array}$ & $\begin{array}{l}\text { Form I, } \\
\text { and EDD }\end{array}$ \\
\hline$\div$ & $\begin{array}{l}\text { Correlation coefficient the Mechod of Standard Addition (MSA) } \\
\text { is less than } 0.095 \text {. }\end{array}$ & $\begin{array}{l}\text { Form 2, } \\
\text { and EDD }\end{array}$ \\
\hline B & $\begin{array}{l}\text { The qualifier is used to indicare thar the reported result fell above } \\
\text { the DLMDL bur below the CRDL. }\end{array}$ & $\begin{array}{l}\text { Form I, } \\
\text { and EDD }\end{array}$ \\
\hline M & $\begin{array}{l}\text { The qualifier is used to indicare thar the replicate injection } \\
\text { readings of the GFAA sample analysis do pot agree within } 20 \% \\
\text { relarive standard deviarion (RSD) or coefficient of variarion (CV). }\end{array}$ & $\begin{array}{l}\text { Form } 1 \text {. } \\
\text { and EDD }\end{array}$ \\
\hline $\bar{N}$ & $\begin{array}{l}\text { This qualifier is used to indicate thar the marrix or pre-digested } \\
\text { spike sample recovery for an analyce is not within the specified } \\
\text { conerol limait }\end{array}$ & $\begin{array}{l}\text { Form } 1 . \\
\text { and EDD }\end{array}$ \\
\hline 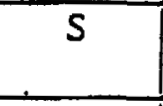 & $\begin{array}{l}\text { The reported value was determined by the Method of Standard } \\
\text { Addition (MSA). }\end{array}$ & $\begin{array}{l}\text { Form l. } \\
\text { and EDD }\end{array}$ \\
\hline $\mathrm{U}$ & The analyze's result was less than the IDLMDL. & $\begin{array}{l}\text { C of } A \text { Form } 1 . \\
\text { and EDD }\end{array}$ \\
\hline $\bar{W}$ & $\begin{array}{l}\text { Posi-digestion spike for GFAA, analysis is out of concrol limits } \\
\text { (85\%-115\%). while sample results are less than } 50 \% \text { of the spike } \\
\text { absorbance. }\end{array}$ & $\begin{array}{l}\text { EDD. and } \\
\text { Form 5. par ? }\end{array}$ \\
\hline $\mathrm{x}$ & Othe: reporing flag as defined in report narrave. & $\begin{array}{l}\text { Form } 1 . \\
\text { and EDD }\end{array}$ \\
\hline$=$ & $\begin{array}{l}\text { Thus qualifier is used to indicate that the Laboratory Concrol } \\
\text { Sample (LCS) recovery for an analyre is outside of the specified } \\
\text { limics. }\end{array}$ & $\begin{array}{l}\text { QC Summary } \\
\text { Report }\end{array}$ \\
\hline
\end{tabular}

All surogate recoveries and acceptance ranges are reported at the boctom of Form 2 or $C$ of $A$. Any recoveries falling outside the accepance range will be flagged with a $=$ ". All flags do not apply to QC Summary and Cerrificate of Anadysis packenges. 

$-$

CASE

NARRATIVE

-

-

$-$

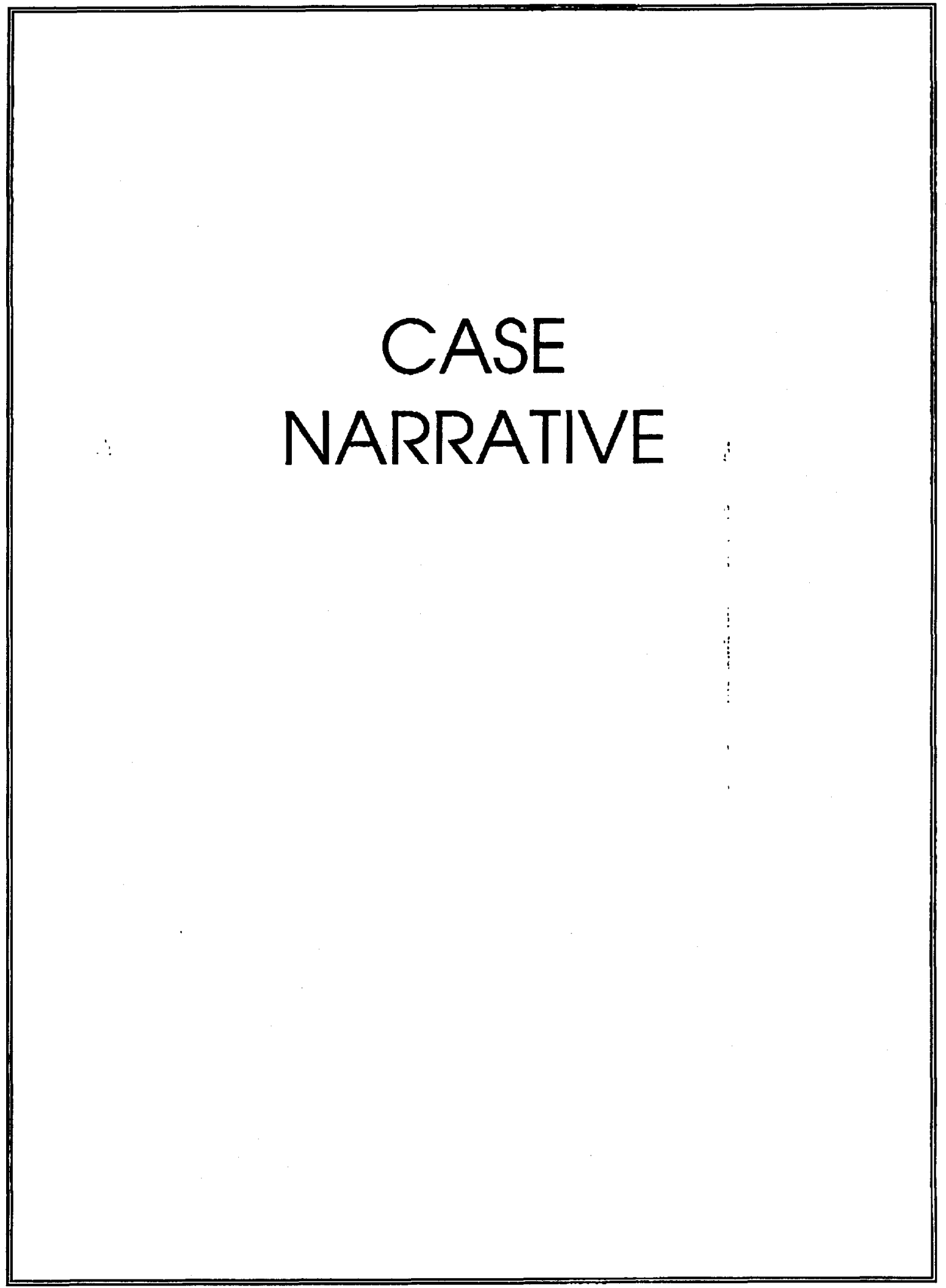




\section{Radiochemistry Case Narrative \\ Bechtel Nevada (NEVA) \\ SDG V1169}

Method/Analysis Information

Batch Number:

Procedure:

Analytical Method:
86288

Determination of Gamma Isotopes in Water and Soil

DOE EML HASL 300

Sample ID
44601001
44601002
44601003
44601004
44601005
1200027924
1200027925
1200027926

\section{Client ID}

252507-1-0

$252507-2-0$

252507-3-0

$252508-1-0$

252508-2-0

MB for batch 86288

044403-0-1(44379001DUP)

LCS for batch 86288

SOP Reference

Procedures for preparation, analysis and reporting of analytical data are controlled by General Engineering Laboratories, Inc. as Standard Operating Procedures (SOP). The data discussed in this narrative has been prepared and analyzed in accordance with GL-RAD-A-013.

\section{Calibration Information:}

Calibration Information

All initial and continuing calibration requirements have been met.

Standards Information

Standard solution(s) for these analyses are NIST traceable and used before the expiration date(s).

\section{Sample Geometry}

All counting sources were prepared in the same geometry as the calibration standards.

\section{Quality Control (OC) Information:}

\section{Blank Information}

The blank volume is representative of the sample volume(s) in this batch.

Designated QC

The following sample(s) was used for QC: 44379001.

\section{QC Information}

All of the $Q C$ samples met the required acceptance limits.

\section{Technical Information:}

\section{Holding Time}

All sample procedures for this sample set were performed within the required holding time.

\section{Preparation Information}

All preparation criteria have been met for these analyses. 
Sample Re-prep/Re-analysis

None of the samples in this sample set required reprep or reanalysis.

Miscellaneous Information:

NCR Documentation

No NCR were generated for the preparation or analysis of this sample set.

Manual Integration

No manual integrations were performed on data in this batch.

Additional Comments

The following data was rejected due to low abundance:

Sample 1200027924; U-238.

The following data was rejected due to no valid peak:

Sample 1200027924; U-235,

Sample 44601003; U-235.

Review Validation:

GEL requires all analytical data to be verified by a qualified data validator. In addition, all data designated for CLP or CLP-like packaging will receive a third level validation upon completion of the data package.

The following data validator verified the information presented in this case narrative:

Reviewer: Date:

20 Lul2001 


\title{
GENERAL ENGINEERING LABORATORIES
}

Meeting today's needs with a vision for tomormw:

\section{Certificate of Analysis}

\author{
Company : Bechtel Nevada \\ Address: 2621 Losee Road \\ North Las Vegas, Nevada B9030-4129 \\ Contact: Theodore Redding \\ Project: Eavironmental Rad Analysis
}

Report Date: July 20, 2001

Page 1 of 1

\begin{tabular}{|c|c|c|c|c|c|c|c|c|c|c|c|}
\hline \multirow[b]{2}{*}{ Parameter } & \multicolumn{2}{|c|}{$\begin{array}{l}\text { Client Sample ID: } \\
\text { Sample ID: } \\
\text { Matrix: } \\
\text { Collect Date: } \\
\text { Receive Date: } \\
\text { Collector: }\end{array}$} & & \multicolumn{2}{|c|}{$\begin{array}{l}252507-1-0 \\
44601001 \\
\text { Soil } \\
20-J U N-01 \\
\text { 22-JUN-01 } \\
\text { Client }\end{array}$} & \multicolumn{2}{|r|}{$\begin{array}{l}\text { Proiect: } \\
\text { Client ID: }\end{array}$} & \multicolumn{2}{|c|}{$\begin{array}{l}\text { NEVAO0101 } \\
\text { NEVA002 }\end{array}$} & \multirow[b]{2}{*}{ Time } & \multirow[b]{2}{*}{ Batch Mtd. } \\
\hline & Qualiner & Result & & DL. & TPU & $\mathbf{R L}$ & Units & DF & Analyst Date & & \\
\hline \multicolumn{12}{|c|}{ Rad Gamma Spec } \\
\hline \multicolumn{12}{|c|}{ Gammaspec, Gamma, solid } \\
\hline $\begin{array}{l}\text { Americium-241 } \\
\text { Cesium-137 } \\
\text { Potassium-40 } \\
\text { Uranium-235 } \\
\text { Uranium-238 }\end{array}$ & $\begin{array}{l}\mathbf{U} \\
\mathbf{U} \\
\mathbf{U}\end{array}$ & $\begin{array}{r}-0.0144 \\
0.023 \\
27.9 \\
0.054 \\
2.20\end{array}$ & $\begin{array}{r}+1-0.135 \\
+1-0.0192 \\
+1-3.26 \\
+1-0.130 \\
+1-1.89\end{array}$ & $\begin{array}{r}0.243 \\
0.0462 \\
0.332 \\
0.237 \\
1.92\end{array}$ & $\begin{array}{r}0.135 \\
0.0192 \\
3.26 \\
0.130 \\
1.89\end{array}$ & $\begin{array}{r}0.200 \\
0.050 \\
0.200 \\
1.00\end{array}$ & $\begin{array}{l}\mathrm{pCi} / \mathrm{g} \\
\mathrm{pCi} / \mathrm{g} \\
\mathrm{pCi} / \mathrm{g} \\
\mathrm{pCi} / \mathrm{g} \\
\mathrm{pCi} / \mathrm{g}\end{array}$ & & CRB $\quad 06 / 29 / 01$ & 1222 & 862881 \\
\hline
\end{tabular}

The following Prep Methods were performed

\begin{tabular}{llllll}
\hline Method & Description & Analyst & Date & Time & Prep Batch \\
\hline Dry Soil Prep & Dry Soil Prep RAD A-021,A-021B,A-026 & WEO & $06 / 25 / 01$ & 1110 & 85627 \\
The following Analytical Methods were performed & & & & \\
\hline Method & Descrtption & & & \\
\hline 1 & DOE EML HASL 300 & & \\
\hline
\end{tabular}

Notes:

The Qualifiers in this report are defined as follows :

** Indicates the analyte is a surrogate compound.

$<$ Actual result is less than amount reported

$>$ Actual result is greater than amount reported

- I Indicates an estimated value. The result was greater than the detection limit, but less than the reporting limit.

$\mathrm{U}$ Indicates the compound was analyzed for but not detected above the detection limit

The above sample is reported on an "as received" basis.

This data report has been prepared and reviewed in accordance with General Engineering Laboratories, Inc.

standard operating procedures. Please direct any questions to your Project Manager, Stacy Griffin.

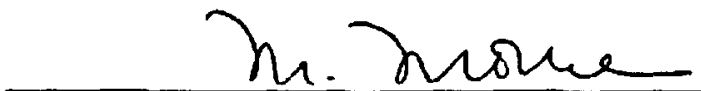

Reviewed by 


\title{
GENERAL ENGINEERING LABORATORIES
}

Meeting today's needs with a vision for tomorrow.

\section{Certificate of Analysis}

\author{
Company: Bechtel Nevada \\ Address: 2621 Losee Road
North Les Vegas, Nevada 89030-4129
Contact: Theodore Redding \\ Project: Environmental Rad Analysis
}

Report Date: July 20, 2001

$\begin{array}{ll}\text { Client Sample ID: } & 252507-2-0 \\ \text { Sample ID: } & 44601002 \\ \text { Matrix: } & \text { Soil } \\ \text { Collect Date: } & \text { 20-JUN-01 } \\ \text { Receive Date: } & \text { 22-JUN-01 } \\ \text { Collector: } & \text { Client }\end{array}$

\begin{tabular}{|c|c|c|c|c|c|c|c|c|c|c|c|c|}
\hline Parameter & Qualifier & Result & & $\mathbf{D L}$ & TPU & $\mathbf{R L}$ & Units & DF & Anal & yatDate & Time & Batch Mtc \\
\hline \multicolumn{13}{|l|}{ Rad Gomma Spec } \\
\hline \multicolumn{13}{|c|}{ Ganmaspec, Ganma, solid } \\
\hline $\begin{array}{l}\text { Americium-241 } \\
\text { Cesium-137 } \\
\text { Potassium-40 } \\
\text { Uranium-235 } \\
\text { Uranium-238 }\end{array}$ & $\begin{array}{l}\mathbf{U} \\
\mathbf{U} \\
\mathbf{U}\end{array}$ & $\begin{array}{r}0.0515 \\
0.0144 \\
26.0 \\
0.0143 \\
1.26\end{array}$ & $\begin{array}{r}+1-0.040 \\
+/-0.0265 \\
+/-1.07 \\
+/-0.186 \\
+-0.814\end{array}$ & $\begin{array}{r}0.0675 \\
0.0423 \\
0.370 \\
0.212 \\
0.641\end{array}$ & $\begin{array}{r}0.040 \\
0.0265 \\
1.09 \\
0.186 \\
0.814\end{array}$ & $\begin{array}{r}0.200 \\
0.050 \\
0.200 \\
1.00\end{array}$ & $\begin{array}{l}\mathrm{pCi} / \mathrm{g} \\
\mathrm{pCi} / \mathrm{g} \\
\mathrm{pCi} / \mathrm{g} \\
\mathrm{pCi} / \mathrm{g} \\
\mathrm{pCi} / \mathrm{g}\end{array}$ & & CRB & 06/28/01 & 2352 & 862881 \\
\hline
\end{tabular}

The following Prep Methods were performed

\begin{tabular}{llllll}
\hline Method & Description & Anslyst & Date & The & Prep Batch \\
\hline Dry Soil Prep & Dry Soil Prep RAD A-021,A-021B,A-026 & WEO & 06/25/01 & 1110 & 85627 \\
The following Analytical Methods were performed & & & & \\
\hline Method & Description & & & & \\
\hline 1 & DOE EML HASL 300
\end{tabular}

Notes:

The Qualifiers in this report are defined as follows:

** Indicates the analyte is a surrogate compound.

$<$ Actual result is less than amount reported

$>$ Actual result is greater than amount reported

J Indicates an estimated value. The result was greater than the detection limit, but less than the reporting limit.

$U$ Indicates the compound was analyzed for but not detected above the detection limit

The above sample is reported on an "as received" basis.

This data report has been prepared and reviewed in accordance with General Engineering Laboratories, Inc. standard operating procedures. Please direct any questions to your Project Manager, Stacy Griffin.

Reviewed by

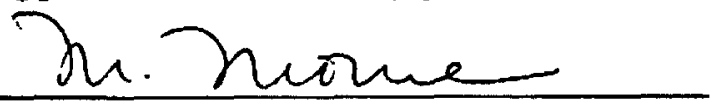




\section{GENERAL ENGINEERING LABORATORIES}

Meeting today's needs with a vision for tomorrow.

\section{Certificate of Analysis}

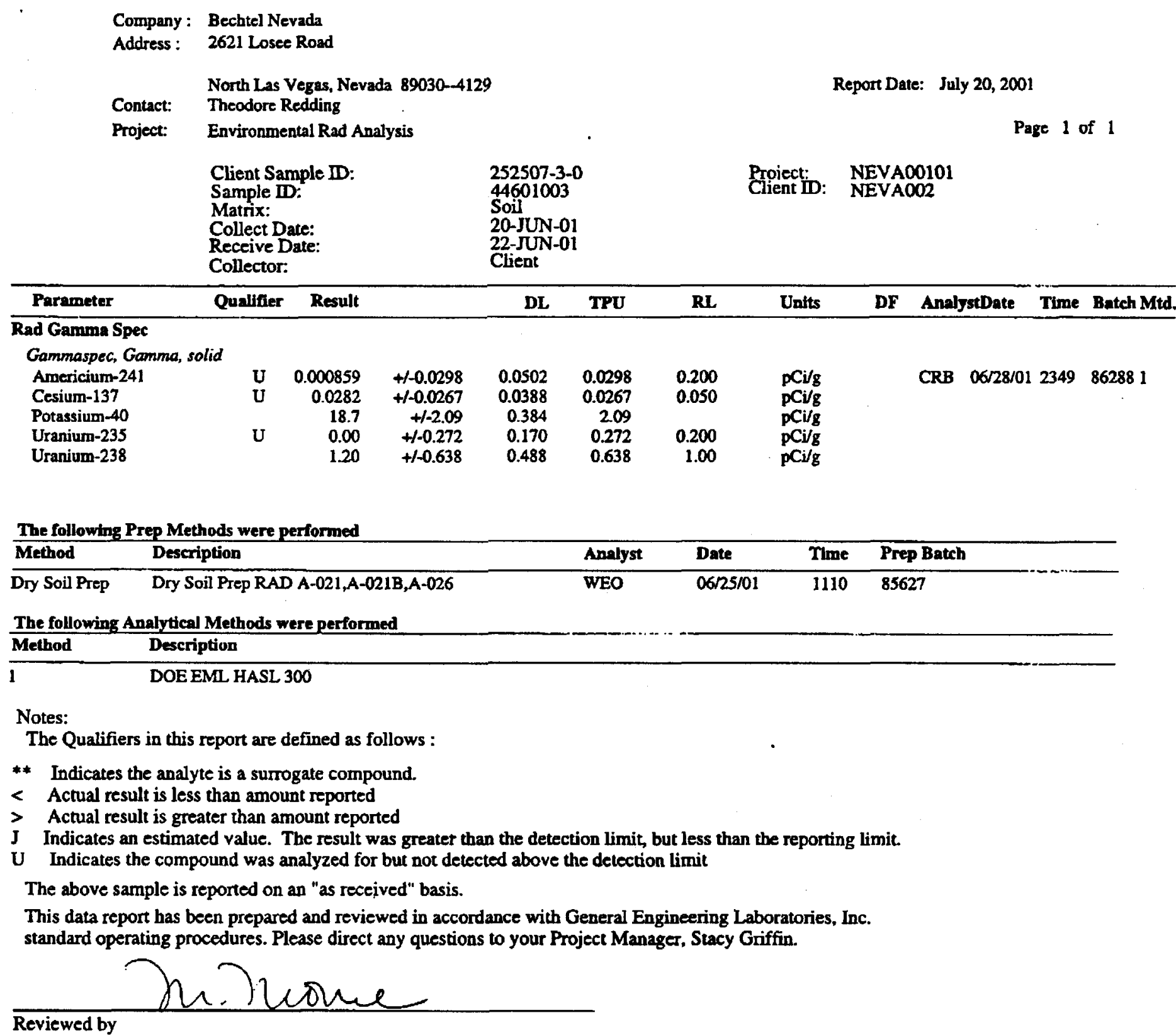




\title{
GENERAL ENGINEERING LABORATORIES
}

Meeting today's needs with a vision for tomorrow:

\section{Certificate of Analysis}

\author{
Company: Bechtel Nevada \\ Address : 2621 Losee Road

$\begin{array}{ll} & \text { North Las Vegas, Nevada } 89030-4129 \\ \text { Contact: } & \text { Theodore Redding } \\ \text { Project: } & \text { Environmental Rad Analysis }\end{array}$

Report Date: July 20, 2001

Page 1 of 1

$\begin{array}{ll}\text { Client Sample ID: } & 252508-1-0 \\ \text { Sample ID: } & 44601004 \\ \text { Matrix: } & \text { Soil } \\ \text { Collect Date: } & \text { 20-JUN-01 } \\ \text { Receive Date: } & \text { 22-JUN-01 } \\ \text { Collector: } & \text { Client }\end{array}$

\begin{tabular}{|c|c|c|c|c|c|c|c|c|c|c|c|}
\hline Parameter & Qualifier & Result & & DL & TPU & $\mathbf{R L}$ & Units & $\mathbf{D F}$ & AnslystDate & Time & Batch Mtd. \\
\hline \multicolumn{12}{|l|}{ Rad Gamma Spec } \\
\hline \multicolumn{12}{|c|}{ Cammaspec, Gamna, solid } \\
\hline $\begin{array}{l}\text { Americium-241 } \\
\text { Cesium-137 } \\
\text { Potassium-40 } \\
\text { Uranium-235 } \\
\text { Uranium-238 }\end{array}$ & $\begin{array}{l}\mathbf{U} \\
\mathbf{U}\end{array}$ & $\begin{array}{r}0.0159 \\
-0.00148 \\
21.1 \\
0.182 \\
1.76\end{array}$ & $\begin{array}{r}+/ 0.0898 \\
+-0.0181 \\
+/-2.41 \\
+/-0.173 \\
+/-1.12\end{array}$ & $\begin{array}{r}0.169 \\
0.0278 \\
0.252 \\
0.164 \\
1.28\end{array}$ & $\begin{array}{r}0.0898 \\
0.0181 \\
2.41 \\
0.173 \\
1.12\end{array}$ & $\begin{array}{r}0.200 \\
0.050 \\
\\
0.200 \\
1.00\end{array}$ & $\begin{array}{l}\mathrm{pCi} / \mathrm{g} \\
\mathrm{pCi} / \mathrm{g} \\
\mathrm{pCi} / \mathrm{g} \\
\mathrm{pCi} / \mathrm{g} \\
\mathrm{pCi} / \mathrm{g}\end{array}$ & & CRB $07 / 01 / 0$ & 12202 & 862881 \\
\hline
\end{tabular}

The following Prep Methods were performed

\begin{tabular}{llllll}
\hline Method & Description & Analyst & Date & Time & Prep Batch \\
\hline Dry Soil Prep & Dry Soil Prep RAD A-021,A-021B,A-026 & WEO & $06 / 25 / 01$ & 1110 & 85627 \\
The following Analytical Methods were performed & & & & \\
\hline Method & Description & & & & \\
\hline 1 & DOE EML HASL 300
\end{tabular}

Notes:

The Qualifiers in this report are defined as follows :

** Indicates the analyte is a surrogate compound.

$<$ Actual result is less than amount reported

$>$ Actual result is greater than amount repiorted

J Indicates an estimated value. The result was greater than the detection limit, but less than the reporting limit.

$U$ Indicates the compound was analyzed for but not detected above the detection limit

The above sample is reported on an "as received" basis.

This data report has been prepared and reviewed in accordance with General Engineering Laboratories, Inc. standard operating procedures. Please direct any questions to your Project Manager, Stacy Griffin.

Reviewed by

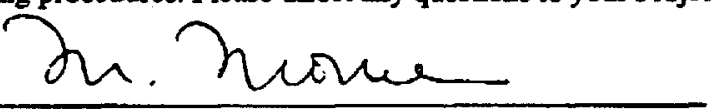




\section{GENERAL ENGINEERING LABORATORIES}

Meeting loday's needs with a vision for tomorrow.

\section{Certificate of Analysis}

Company: Bechlel Nevada

Address : 2621 Losee Road

North Las Vegas, Nevada 89030-4129

Reporn Date: July 20, 2001

Contact: Theodore Redding

Project: Environmental Rad Analysis

Page 1 of 1

Client Sample ID:

Sample ID:

Matrix:

Collect Date:

Receive Date:

Collector:

252508-2-0

44601005

Soil

20-JUN-01

22-JUN-01

Client

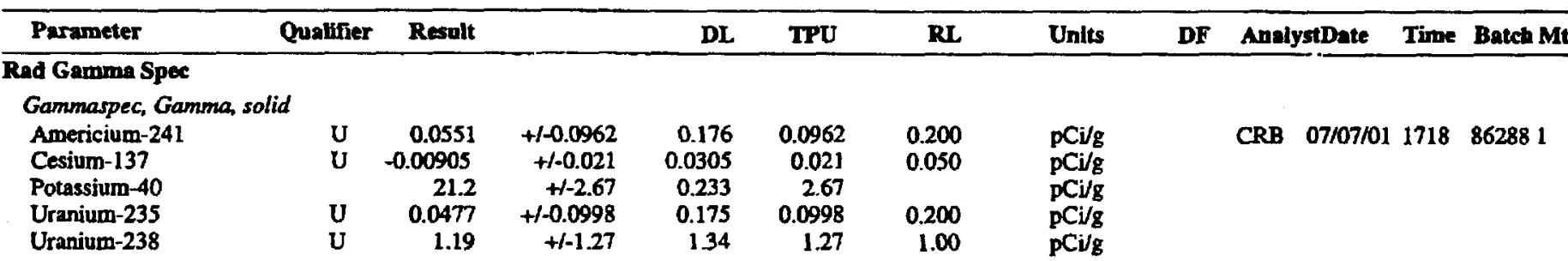

The following Prep Methods were performed

\begin{tabular}{l}
\begin{tabular}{lllllll}
\hline Method & Description & Analyst & Date & Time & Prep Batch \\
\hline Dry Soil Prep & Dry Soil Prep RAD A-021,A-021B,A-026 & WEO & 06/25/01 & 1110 & 85627 \\
The following Analytical Methods were performed & Description & & & \\
\hline Method & DOE EML HASL 300
\end{tabular} \\
\hline 1
\end{tabular}

Notes:

The Qualifiers in this repart are defined as follows:

** Indicates the analyte is a surrogate compound.

$<$ Actual result is less than amount reported

$>$ Actual result is greater than amount reported

J Indicates an estimated value. The result was greater than the detection limit, but less than the reporting limit.

$\mathrm{U}$ Indicates the compound was analyzed for but not detected above the detection limit

The above sample is reported on an "as received" basis.

This data report has been prepared and reviewed in accordance with General Engineering Laboratories, Inc. standard operating procedures. Please direct any questions to your Project Manager, Stacy Griffin.

Reviewed by

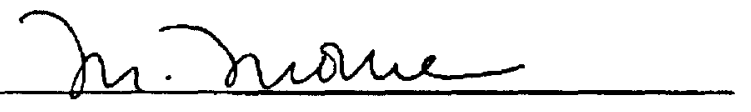




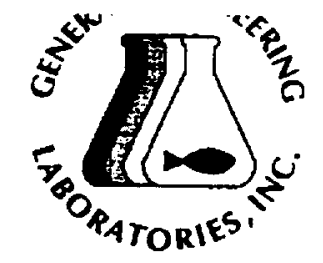

Bechtel Nevada

2621 Losee Road

North Las Vegas, Nevada

Contact:

Theodore Redding

Workorder:

44601
Client :

\section{GENERAL ENGINEERING LABORATORIES}

Meeting today's needs with a vision for tomorrow:

\section{QC Summary}

\begin{tabular}{l}
\hline Parmmame \\
\hline Rad Gamman Spec \\
Batch $\quad 86288$ \\
QC1200027925 $44379001 \quad$ DUP \\
Americium-241
\end{tabular}

Cesium-137

Potassium -40

Uranium-235

Uranium-238

QC1200027926 LCS

Americium-241

Cesium-137

Potassium-40

Uranium-235

Uranium-238

QC1200027924 MB

Americium-241

Cesium-137

Potassium-40

Uranium-235

NOM

Somple Oan QC Units RPD\% REC\% Range Anlst Report Date: July 20, 2001
Page 1 of 2 Nom Sample oal

Qc

\begin{tabular}{|c|c|c|c|c|c|c|c|}
\hline U & 0.0157 & $\mathbf{U}$ & 0.0548 & $\mathrm{pCi} / g$ & $111 \wedge$ & $(+/-0.200) \quad C R B$ & $06 / 30 / 0114: 49$ \\
\hline Uncert: & $+1-0.105$ & & $+/-0.0717$ & & & & \\
\hline TPU: $_{\mathbf{U}}$ & $\begin{array}{r}0.105 \\
0.0266\end{array}$ & $\mathbf{U}$ & $\begin{array}{l}0.0717 \\
0.0133\end{array}$ & $\mathrm{pCi} / \mathrm{g}$ & $67 \wedge$ & $(+(-0.050)$ & \\
\hline Uncert: & $+1-0.0243$ & & $+1-0.0237$ & & & & \\
\hline TPU: & $\begin{array}{r}0.0243 \\
14.7\end{array}$ & & $\begin{array}{r}0.0237 \\
16.0\end{array}$ & $\mathrm{pCi} / \mathrm{g}$ & 8 & & \\
\hline Uncert: & $+\ell-1.85$ & & $+/-1.93$ & & & & \\
\hline${ }^{\text {TPU: }}$ & $\begin{array}{r}1.85 \\
-0.0239\end{array}$ & $\mathbf{U}$ & $\begin{array}{r}1.93 \\
0.008\end{array}$ & $\mathrm{pCi} / \mathrm{g}$ & N/A & $(+1-0.200)$ & \\
\hline Uncert: & $+/-0.106$ & & $+1-0.114$ & & & & \\
\hline $\begin{array}{r}\text { TPU: } \\
\mathrm{U}\end{array}$ & $\begin{array}{r}0.106 \\
1.04\end{array}$ & $\mathbf{U}$ & $\begin{array}{l}0.114 \\
0.286\end{array}$ & $\mathrm{pCi} / \mathrm{g}$ & $114^{\wedge}$ & $(+t-1.00)$ & \\
\hline Uncert: & $+/-1.26$ & & $+1-0.905$ & & & & \\
\hline TPU: & 1.26 & & 0.905 & & & & \\
\hline
\end{tabular}

07/03/01 08:52

$\begin{array}{crlll} & 1260 & \mathrm{pCi} / \mathrm{g} & & \\ \text { Uncert: } & +/-135 & & & \\ \text { TPU: } & 135 & & & \\ 441 & 485 & \mathrm{pCi} / \mathrm{g} & 110 & (75 \%-125 \%) \\ \text { Uncert: } & +1-61.0 & & & \\ \text { TPU: } & 61.0 & & & \end{array}$

Uncert:

$\mathrm{U} \quad 1.12 \quad \mathrm{pCi} \mathrm{g}$

TPU:

$\mathbf{U}$

$+-2.00$

2.00

0.254

$+1-1.46$

Uncert:

TPU:

Uncert:

$\mathbf{U}$

1.46

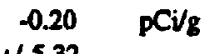

TPU:

5.32

PCi/g

U $\quad 0.0164 \quad \mathrm{pCi} / \mathrm{g}$

$06 / 30 / 01$ 14:48

Uncert:

$+1-0.0224$

0.0224

U

0.0296

$+-0.0234$

Uncert:

0.0234

TPU:

U

0.0829

$+1-0.305$

Uncert:

TPU:

U

0.305

$0.00 \quad \mathrm{pCi} / \mathrm{g}$ 


\section{GENERAL ENGINEERING LABORATORIES}

Meeting today's needs with a vision for tomorrow:

\section{QC Summary}

Workorder: 44601

Page 2 of 2

Parmaname

NOM
Sample Oual

QC Units RPD\% REC\%

\section{Rad Gamma Spec}

Batch

86288

Uranium-238

Uncert:

IPU:

Uncert:

TPU:

$+1-0.0975$

0.0975

U $\quad 0.00$

$\mathrm{pCi} / \mathrm{g}$

$+/-0.907$

0.907

Notes:

The Qualifiers in this report are defined as follows:

* Indicates the analyte is a surrogate compound.

< Actual result is less than amount reported

$>\quad$ Actual result is greater than amount reported

J Indicates an estimated value. The result was greater than the detection limic, but less than the reporting limit.

$\mathrm{U}$ Indicates the compound was analyzed for but not detected above the detection limit

N/A indicates that spike recovery limits do not apply when sample concentration exceeds spike conc. by a factor of 4 or more.

^ The Relative Percent Difference (RPD) obtained from the sample duplicate (DUP) is evaluated against the acceptence criteria when the sample is greater than five times ( $5 X)$ the contract required detection limit $(R L)$. In cases where either the sample or duplicate value is less than $5 X$ the $\mathbf{R L}$, a control limit of $+/$ the $\mathbf{R L}$ is used to evaluate the DUP result.

For PS, PSD, and SDLT results, the values listed are the measured amounts, not final concentrations. 
California

US Army Corps of Engineers 657-1010.

CERTIFICATIONS:
Bechtel Nevada

P.O. Box 98521, M/S NTS273

Las Vegas, NV 89193-8521

Ted Redding

PROJECT NAME: $\quad$ V1170

NEL ORDER ID: L0106266

PROJECT NUMBER: 23081

Attached are the analytical results for samples in support of the above referenced project.

Samples submitted for this project were not sampled by NEL Laboratories. Samples were received by NEL in good condition, under chain of custody on $6 / 21 / 01$.

Should you have any questions or comments, please feel free to contact our Client Services department at (702)

\section{Some results have been flagged as follows:}

Jc - This concentration may be biased because the continuing calibration verification (CCV) standard did not meet $\mathrm{QC}$ requirements for this analyte. However, overall CCV standard recoveries meet method requirements and analytical results are in control.

Some surrogate results have been flagged as follows:

D - Sample required dilution. Sample QC results were diluted outside the calibrated range.
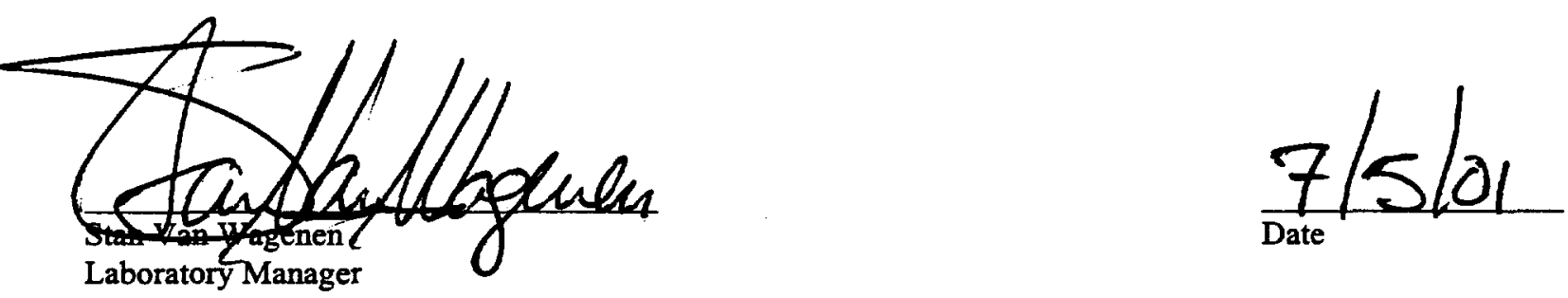

\begin{tabular}{|c|c|c|c|c|c|c|c|}
\hline & Reno & Las Vegas & S. California & & Reno & Las Vegas & S. California \\
\hline Arizona & AZ0520 & AZ0518 & AZ0605 & Idaho & Certified & Certified & \\
\hline California & 1707 & 2002 & 2264 & Montana & Certified & Certified & \\
\hline $\begin{array}{l}\text { US Army Corps } \\
\text { of Engineers }\end{array}$ & Certified & Certified & & $\begin{array}{l}\text { Nevada } \\
\text { L.A.C.S.D. }\end{array}$ & NV033 & NV052 & $\begin{array}{l}\text { CA084 } \\
10228\end{array}$ \\
\hline
\end{tabular}


NEL LABORATORIES

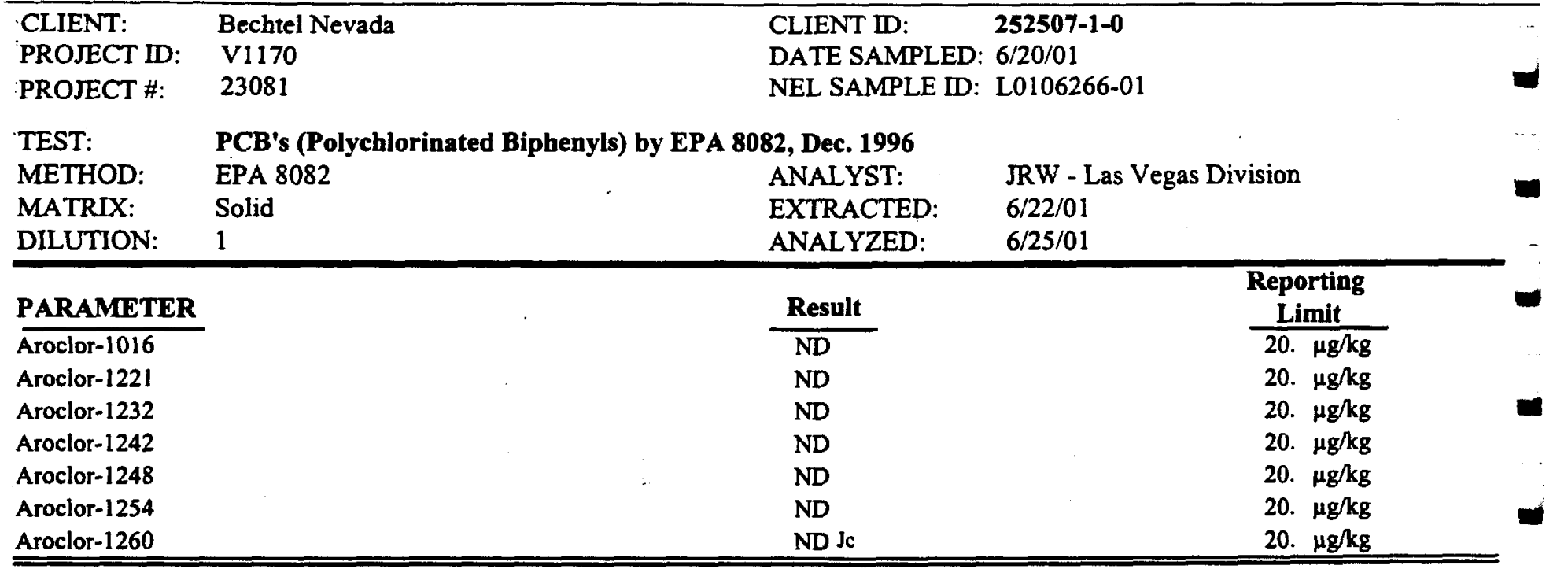

QUALITY CONTROL DATA:

\section{Surrogate}

Decachlorobiphenyl

Tetrachloro-m-xylene

\section{$\%$ Recovery}

68

87

\section{Acceptable Range}

$46-155$

$49-140$

ND - Not Detected

This report shall not be reproduced except in full, without the written approval of the laboratory. 
NNEL LABORATORIES

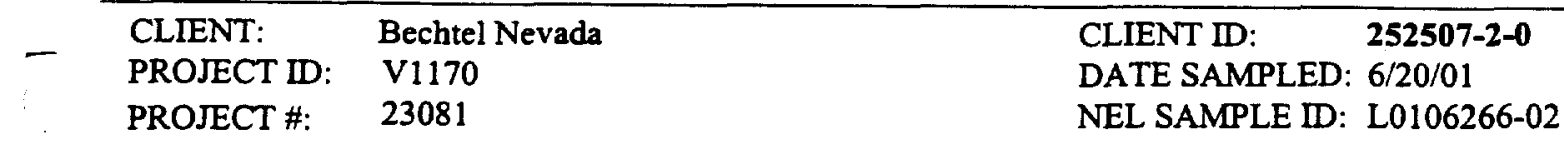

- TEST: $\quad$ PCB's (Polychlorinated Biphenyls) by EPA 8082, Dec. 1996

METHOD: EPA 8082 ANALYST: JRW - Las Vegas Division

MATRIX: Solid $\quad$ EXTRACTED: $6 / 22 / 01$

DILUTION: 1 ANALYZED: $6 / 25 / 01$
PARAMETER

Aroclor-1016

- Aroclor-1221

Aroclor-1232

Aroclor-1242

- Aroclor-1248

Aroclor- 1254

Aroclor-1 260

QUALITY CONTROL DATA:

Surrogate

Decachlorobiphenyl

- Tetrachloro-m-xylene

\begin{tabular}{l} 
Result \\
\hline ND \\
ND \\
ND \\
ND \\
ND \\
ND \\
ND Jc
\end{tabular}

$\%$ Recovery

65

85
Reporting

Limit

20. $\mu \mathrm{g} / \mathrm{kg}$

20. $\mu \mathrm{g} / \mathrm{kg}$

20. $\mu \mathrm{g} / \mathrm{kg}$

20. $\mu \mathrm{g} / \mathrm{kg}$

20. $\mu \mathrm{g} / \mathrm{kg}$

20. $\mu \mathrm{g} / \mathrm{kg}$

20. $\mu \mathrm{g} / \mathrm{kg}$
- ND - Not Detected

This report shall not be reproduced except in full, without the written approval of the laboratory.

\section{Acceptable Range}

$46-155$

$49-140$ 
NEL LABORATORIES

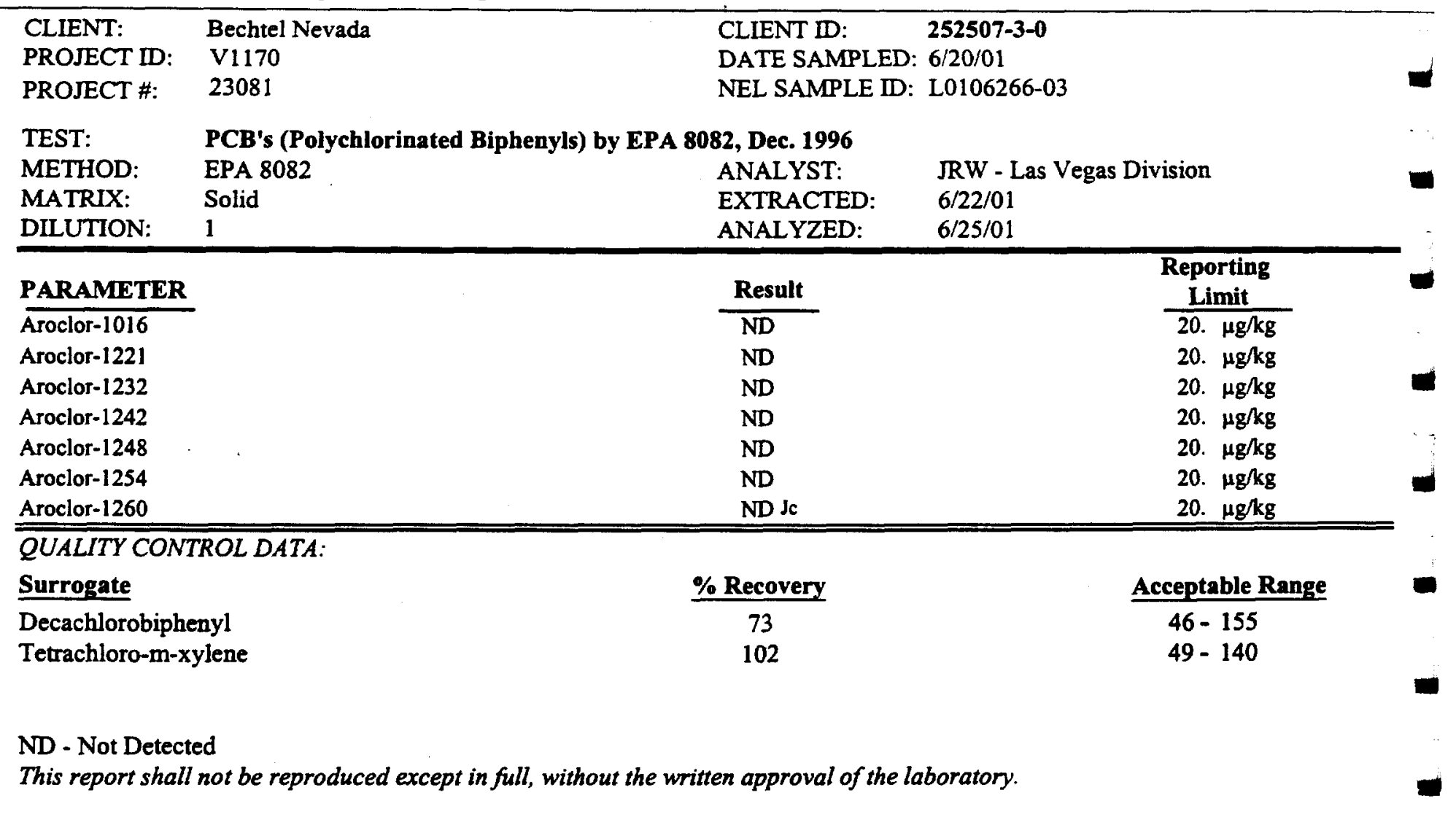


NEL LABORATORIES

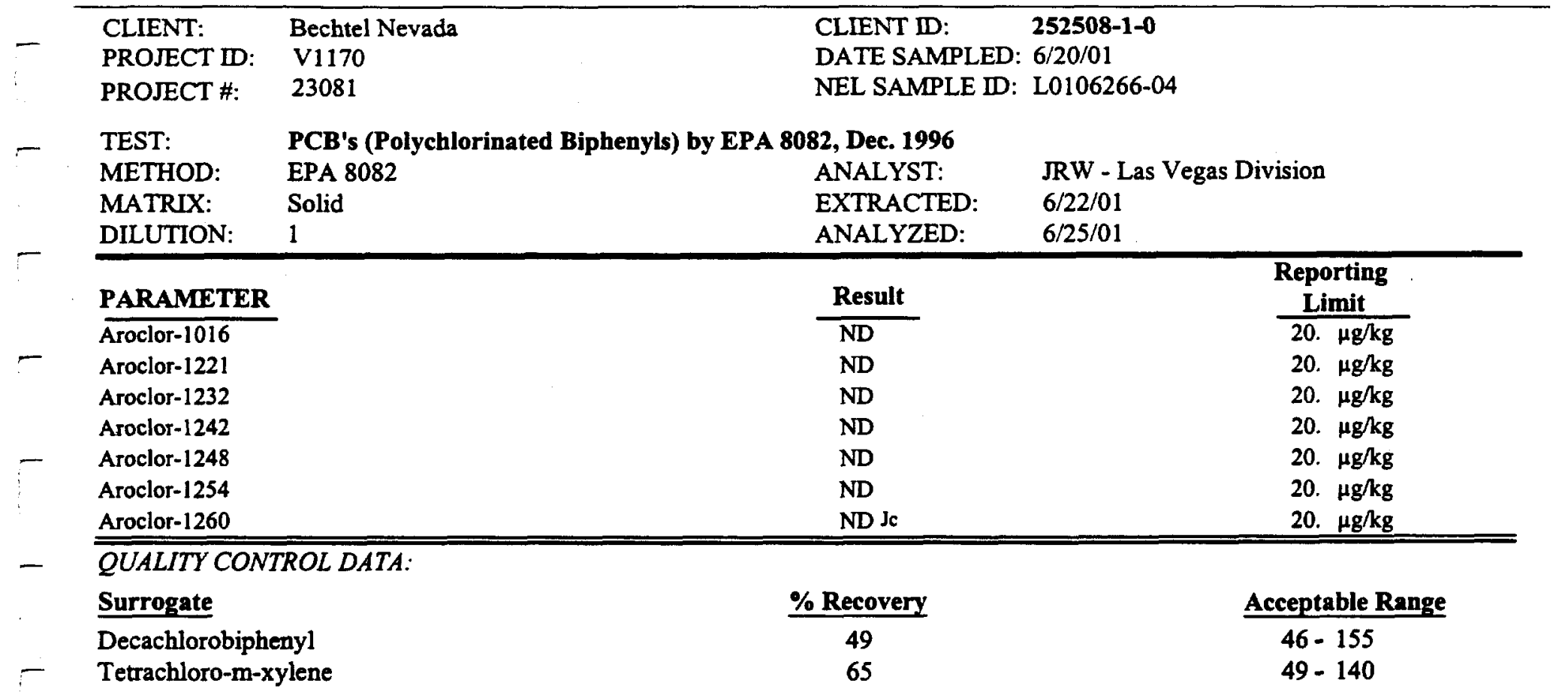

- ND - Not Detected

This report shall not be reproduced except in full, without the written approval of the laboratory. 
NEL LABORATORIES

$\begin{array}{llll}\text { CLIENT: } & \text { Bechtel Nevada } & \text { CLIENT ID: } & \text { 252508-2-0 } \\ \text { PROJECT ID: } & \text { V1170 } & \text { DATE SAMPLED: } 6 / 20 / 01 \\ \text { PROJECT \#: } & 23081 & \text { NEL SAMPLE ID: } \text { L0106266-05 }\end{array}$

TEST: $\quad$ PCB's (Polychlorinated Biphenyls) by EPA 8082, Dec. 1996

METHOD: $\quad$ EPA 8082

MATRIX: Solid

ANALYST: JRW - Las Vegas Division

DILUTION: 1

EXTRACTED: $\quad 6 / 22 / 01$

ANALYZED: $\quad 6 / 25 / 01$

\begin{tabular}{lll}
\hline PARAMETER & Result & \multicolumn{2}{c}{ Reporting } \\
\cline { 2 - 3 } \\
Aroclor-1016 & $\mathrm{ND}$ & $\frac{\text { Limit }}{20 . \mu \mathrm{kg}}$ \\
Aroclor-1221 & $\mathrm{ND}$ & $20 . \mu \mathrm{g} / \mathrm{kg}$ \\
Aroclor-1232 & $\mathrm{ND}$ & $20 . \mu \mathrm{g} / \mathrm{kg}$ \\
Aroclor-1242 & $\mathrm{ND}$ & $20 . \mu \mathrm{g} / \mathrm{kg}$ \\
Aroclor-1248 & $\mathrm{ND}$ & $20 . \mu \mathrm{g} / \mathrm{kg}$ \\
Aroclor-1254 & $\mathrm{ND}$ & $20 . \mu \mathrm{g} / \mathrm{kg}$ \\
Aroclor-1260 & $\mathrm{ND} \mathrm{Jc}$ & $20 . \mu \mathrm{g} / \mathrm{kg}$ \\
\hline \hline
\end{tabular}

QUALITY CONTROL DATA:

Surrogate

$\%$ Recovery

64

72

\section{Acceptable Range}

$46-155$

$49-140$

Tetrachloro-m-xylene

ND - Not Detected

This report shall not be reproduced except in full, without the written approval of the laboratory. 
NEL LABORATORIES

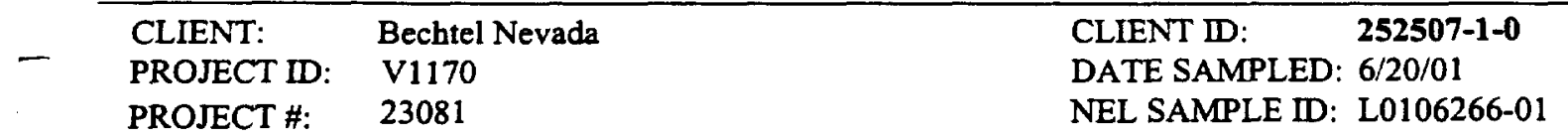

TEST: $\quad$ Total Extractable Petroleum Hydrocarbons Fuel Finger Print by EPA Method 8015M, July 1992 METHOD: EPA 8015M ANALYST: CCS - Las Vegas Division MATRIX: Solid $\quad$ EXTRACTED: $6 / 22 / 01$

DILUTION: $\quad 50 \quad$ ANALYZED: $6 / 27 / 01$

\begin{tabular}{|c|c|c|c|}
\hline PARAMETER & Result & & $\begin{array}{l}\text { Reporting } \\
\text { Limit }\end{array}$ \\
\hline$\overline{\text { Gasoline Range (C } 8-C 12)}$ & ND & & $500 . \mathrm{mg} / \mathrm{kg}$ \\
\hline Diesel Range (C12-C22) & 1300 & $\mathrm{mg} / \mathrm{kg}$ & 500. $\mathrm{mg} / \mathrm{kg}$ \\
\hline Oil Range (C22-C34) & 34000 & $\mathrm{mg} / \mathrm{kg}$ & 2500. $\mathrm{mg} / \mathrm{kg}$ \\
\hline Total & 35300 & $\mathrm{mg} / \mathrm{kg}$ & $500 . \mathrm{mg} / \mathrm{kg}$ \\
\hline QUALITY CONTROL D & \multirow{2}{*}{\multicolumn{2}{|c|}{ \% Recovery }} & \\
\hline Surrogate & & & Acceptable Range \\
\hline Octacosane & $\mathrm{D}$ & & $54-130$ \\
\hline
\end{tabular}

This report shall not be reproduced except in full, without the written approval of the laboratory. 
NEL LABORATORIES

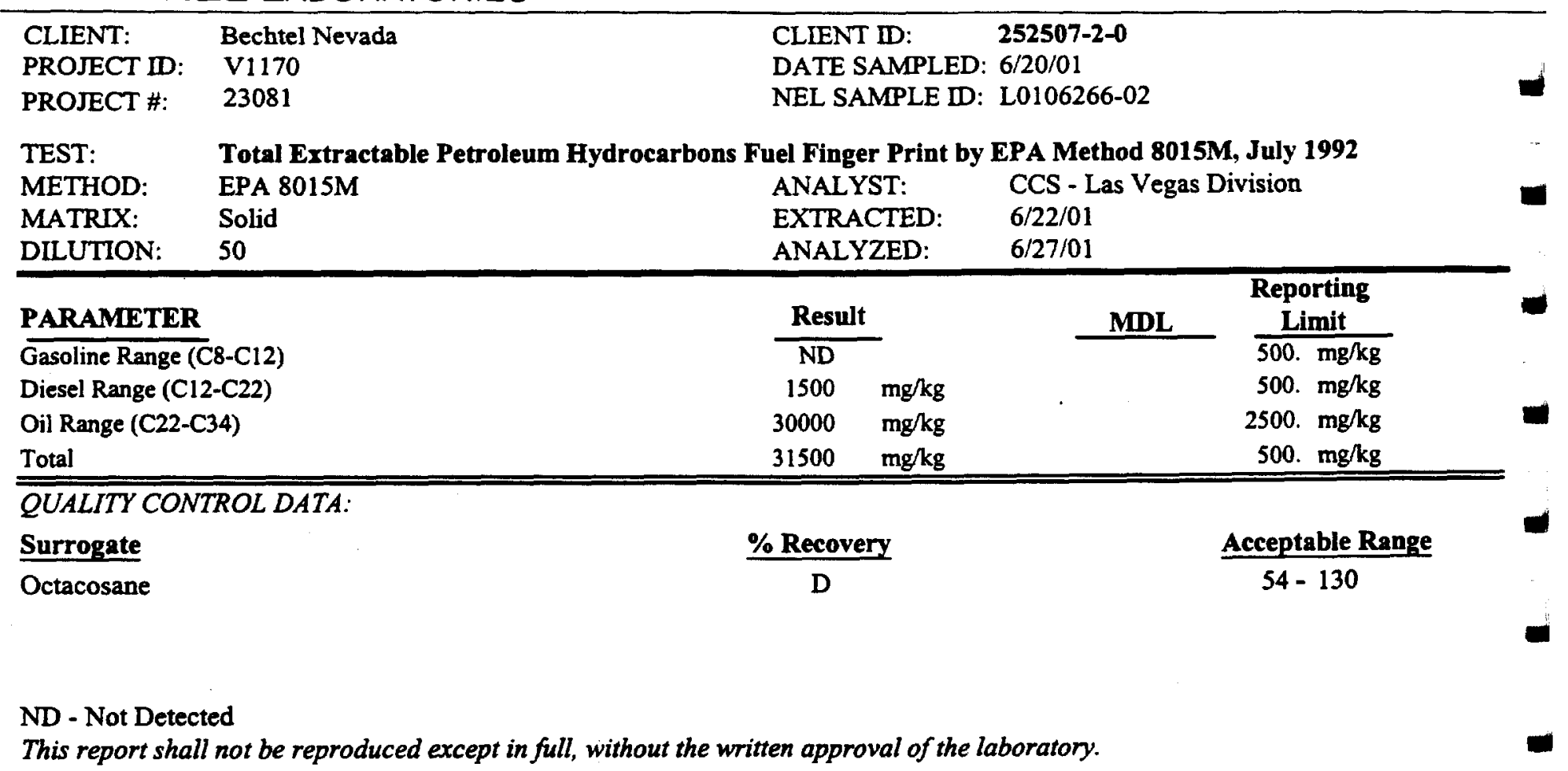

This report shall not be reproduced except in full, without the written approval of the laboratory. 
NEL LABORATORIES

\begin{tabular}{lll}
\hline CLIENT: & Bechtel Nevada & CLIENT ID: \\
PROJECT ID: & V1170 & DATE SAMPL-3-0 \\
PROJECT \#: & 23081 & NEL SAMPLE ID: L0106266-03
\end{tabular}

- TEST: $\quad$ Total Ertractable Petroleum Hydrocarbons Fuel Finger Print by EPA Method 8015M, July 1992

METHOD: EPA 8015M ANALYST: CCS - Las Vegas Division

MATRIX: Solid $\quad$ EXTRACTED: $6 / 22 / 01$

\begin{tabular}{lllll} 
DILUTION: & 100 & & EXTRACTED: & $6 / 22 / 01$ \\
\hline
\end{tabular}

-

PARAMETER

Gasoline Range (C8-C12)

- Diesel Range (C12-C22)

Oil Range (C22-C34)

Total

QUALITY CONTROL DATA:

Surrogate

\%ecovery

D

Octacosane

$\begin{array}{ll}\frac{2}{\text { Result }} & \\ \text { ND } & \\ 5000 & \mathrm{mg} / \mathrm{kg} \\ 100000 & \mathrm{mg} / \mathrm{kg} \\ 105000 & \mathrm{mg} / \mathrm{kg}\end{array}$

MDL

Limit

$1000 . \mathrm{mg} / \mathrm{kg}$

1000. $\mathrm{mg} / \mathrm{kg}$

$5000 . \mathrm{mg} / \mathrm{kg}$

1000. $\mathrm{mg} / \mathrm{kg}$

-

Octacosane

Acceptable Range

$54-130$

ND - Not Detected

This report shall not be reproduced except in full, without the written approval of the laboratory. 
NEL LABORATORIES

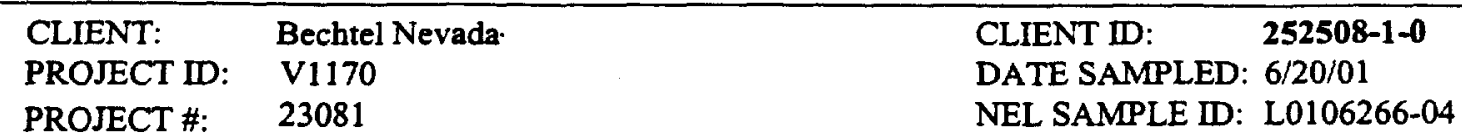

TEST: $\quad$ Total Extractable Petroleum Hydrocarbons Fuel Finger Print by EPA Method 8015M, July 1992

METHOD: EPA 8015M ANALYST: CCS - Las Vegas Division

MATRIX: Solid $\quad$ EXTRACTED: $6 / 22 / 01$

DILUTION: $\quad 100 \quad$ ANALYZED: $\quad 6 / 27 / 01$

\begin{tabular}{|c|c|c|c|c|}
\hline PARAMETER & Result & & MDL & $\begin{array}{c}\text { Reporting } \\
\text { Limit }\end{array}$ \\
\hline Gasoline Range (C8-C12) & ND & & & $1000 . \mathrm{mg} / \mathrm{kg}$ \\
\hline Diesel Range (C12-C22) & 9500 & $\mathrm{mg} / \mathrm{kg}$ & & 1000. $\mathrm{mg} / \mathrm{kg}$ \\
\hline Oil Range (C22-C34) & 110000 & $\mathrm{mg} / \mathrm{kg}$ & & 5000. $\mathrm{mg} / \mathrm{kg}$ \\
\hline Total & 119500 & $\mathrm{mg} / \mathrm{kg}$ & & $1000 . \mathrm{mg} / \mathrm{kg}$ \\
\hline
\end{tabular}

QUALITY CONTROL DATA

Surrogate

\% Recovery

Octacosane

D

Acceptable Range

$54-130$

ND - Not Detected

This report shall not be reproduced except in full, without the written approval of the laboratory. 
NEL LABORATORIES

\begin{tabular}{|c|c|c|}
\hline $\begin{array}{l}\text { CLIENT: } \\
\text { PROJECT ID: } \\
\text { PROJECT \#: }\end{array}$ & $\begin{array}{l}\text { Bechtel Nevada } \\
\text { V1170 } \\
23081\end{array}$ & $\begin{array}{ll}\text { CLIENT ID: } & \mathbf{2 5 2 5 0 8 - 2 - 0} \\
\text { DATE SAMPLED: } & \text { 6/20/01 } \\
\text { NEL SAMPLE ID: } & \text { L0106266-05 }\end{array}$ \\
\hline
\end{tabular}

- TEST: $\quad$ Total Extractable Petroleum Hydrocarbons Fuel Finger Print by EPA Method 8015M, July 1992 METHOD: EPA 8015M ANALYST: CCS - Las Vegas Division MATRIX: Solid $\quad$ EXTRACTED: $6 / 22 / 01$

DILUTION: $\quad 100 \quad$ ANALYZED: $6 / 27 / 01$

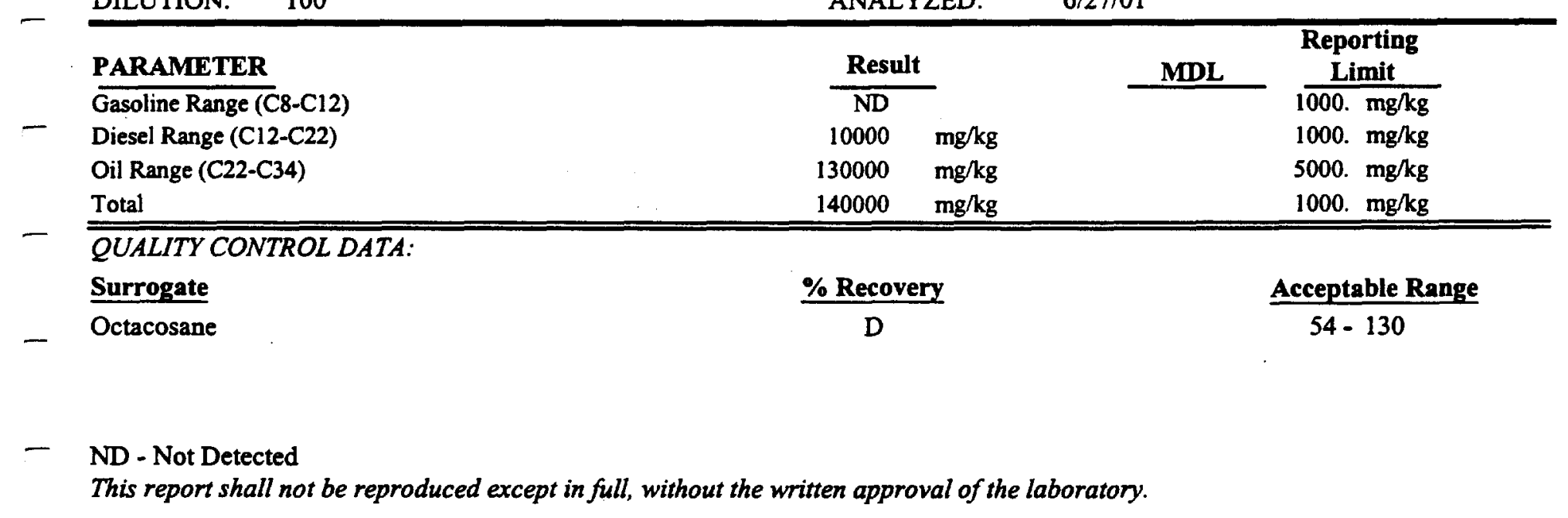


NEL LABORATORIES

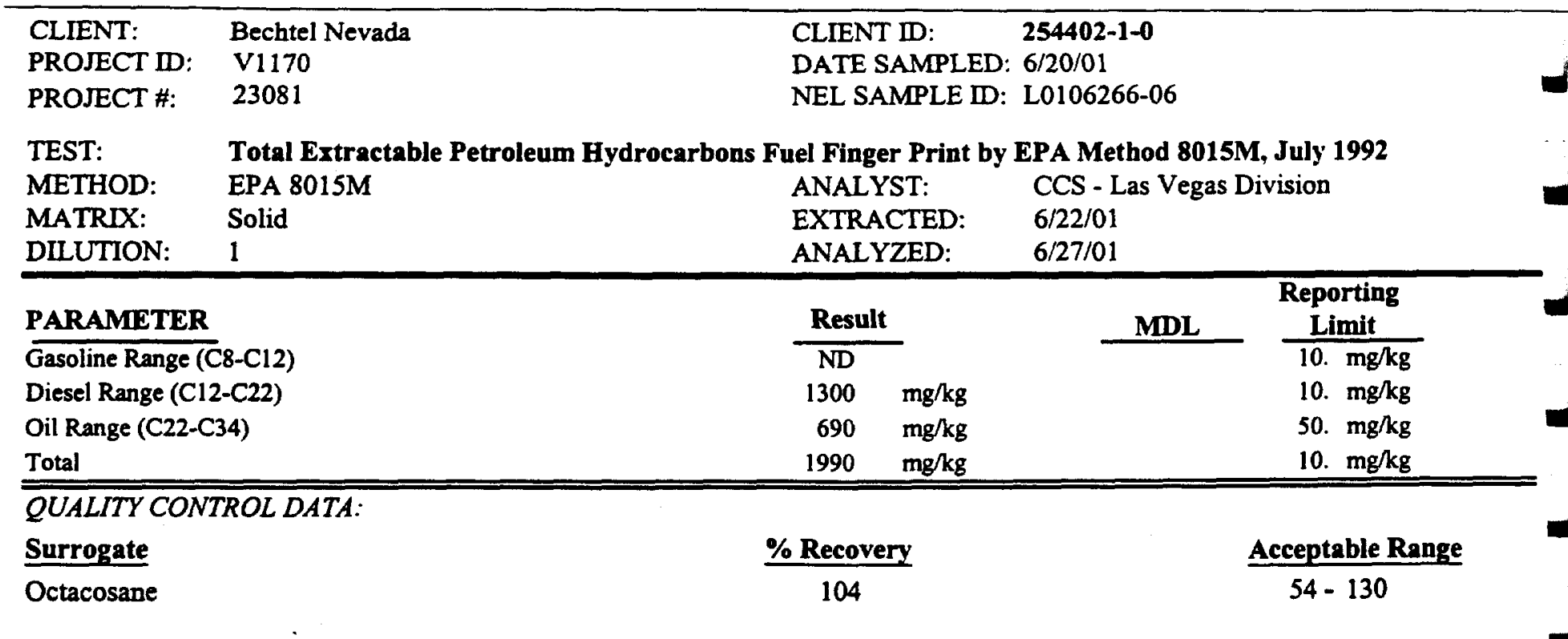

ND - Not Detected

This report shall not be reproduced except in full, without the written approval of the laboratory. 
NEL LABORATORIES

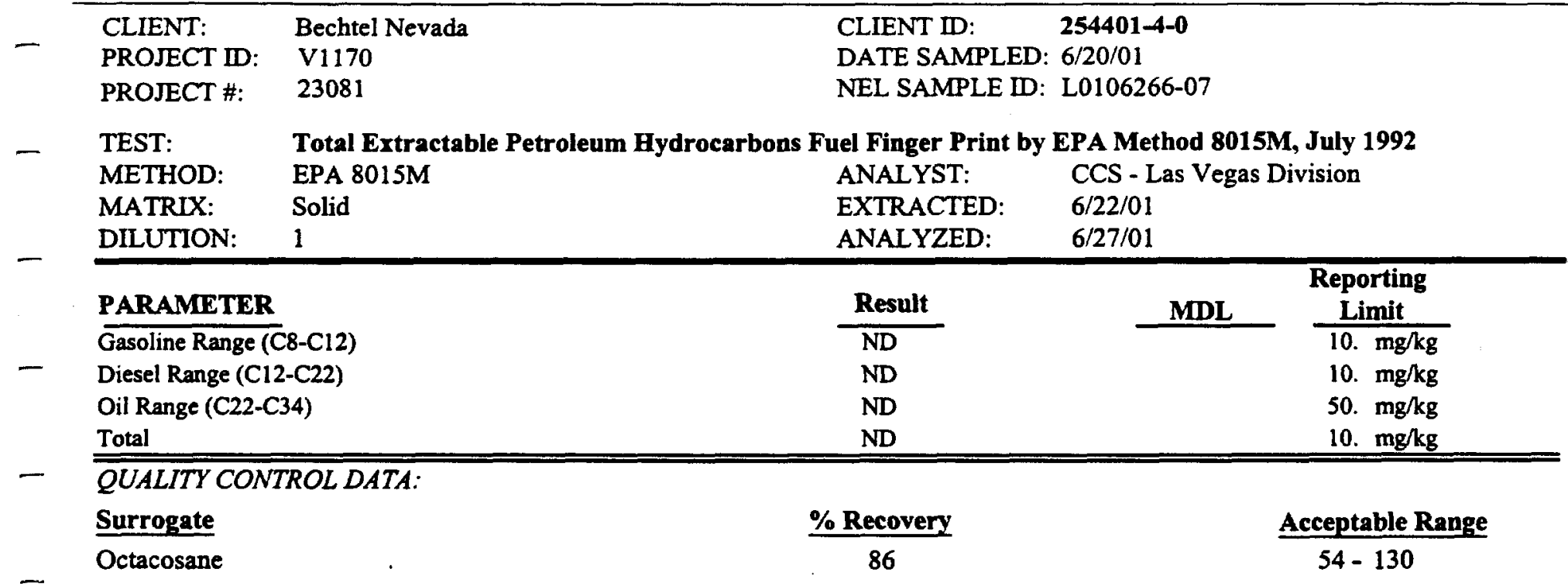

ND - Not Detected

This report shall not be reproduced except in full, without the written approval of the laboratory. 
NEL LABORATORIES

\begin{tabular}{llll}
\hline CLIENT: & Bechtel Nevada & CLIENT ID: & 254401-5-0 \\
PROJECT D: & V1170 & DATE SAMPLED: $6 / 20 / 01$ \\
PROJECT \#: & 23081 & NEL SAMPLE ID: L0106266-08
\end{tabular}

TEST: $\quad$ Total Extractable Petroleum Hydrocarbons Fuel Finger Print by EPA Method 8015M, July 1992

METHOD: EPA 8015M ANALYST: CCS - Las Vegas Division

MATRIX: Solid EXTRACTED: $\quad 6 / 22 / 01$

DILUTION: 1 ANALYZED: $6 / 27 / 01$

\begin{tabular}{lccc}
\hline PARAMETER & Result & MDL & $\begin{array}{c}\text { Reporting } \\
\text { Limit }\end{array}$ \\
${$\cline { 2 - 4 }$} }$ & $\mathrm{ND}$ & $10 . \mathrm{mg} / \mathrm{kg}$ \\
Diesel Range (C12-C22) & $\mathrm{ND}$ & $10 . \mathrm{mg} / \mathrm{kg}$ \\
Oil Range (C22-C34) & $\mathrm{ND}$ & $50 . \mathrm{mg} / \mathrm{kg}$ \\
Total & $\mathrm{ND}$ & $10 . \mathrm{mg} / \mathrm{kg}$ \\
\hline \hline
\end{tabular}

QUALITY CONTROL DATA:

Surrogate

Octacosane
\% Recovery

80
Acceptable Range

$54-130$

ND - Not Detected

This report shall not be reproduced except in full, without the written approval of the laboratory. 
NEL LABORATORIES

\begin{tabular}{llll}
\hline CLIENT: & Bechtel Nevada & CLIENT ID: & 254401-6-0 \\
PROJECT ID: & V1170 & DATE SAMPLED: 6/20/01 \\
PROJECT \#: & 23081 & NEL SAMPLE ID: L0106266-09
\end{tabular}

- TEST: $\quad$ Total Extractable Petroleum Hydrocarbons Fuel Finger Print by EPA Method 8015 M, July 1992

METHOD: EPA 8015M ANALYST: CCS - Las Vogas Division

MATRIX: $\quad$ Solid

EXTRACTED: $\quad 6 / 22 / 01$

DILUTION: $1 \quad$ ANALYZED: $6 / 27 / 01$

-

\section{PARAMETER}

Gasoline Range (C8-C12)

Diesel Range (C12-C22)

Oil Range (C22-C34)

Total

ANALYZED:

\begin{tabular}{l} 
Result \\
\hline ND \\
ND \\
ND \\
ND
\end{tabular}

\% Recovery

79

9

\begin{tabular}{ll} 
MDL & $\begin{array}{l}\text { Reporting } \\
\text { Limit }\end{array}$ \\
\cline { 1 - 1 } & $10 . \mathrm{mg} / \mathrm{kg}$ \\
$10 . \mathrm{mg} / \mathrm{kg}$ \\
$50 . \mathrm{mg} / \mathrm{kg}$ \\
$10 . \mathrm{mg} / \mathrm{kg}$
\end{tabular}

Surrogate

Octacosane
Acceptable Range

$54-130$

- ND - Not Detected

This report shall not be reproduced except in full, without the written approval of the laboratory. 


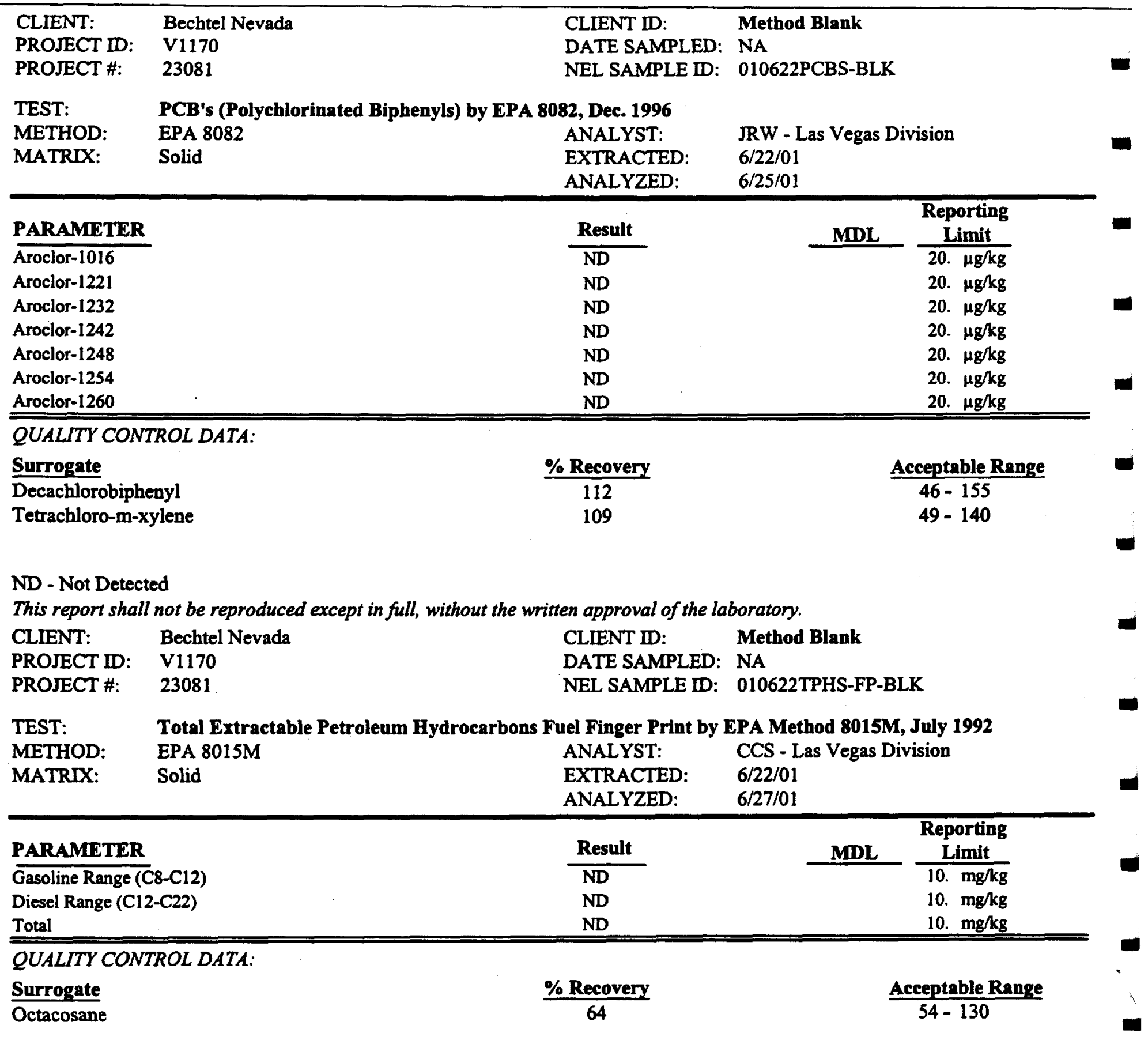

ND - Not Detected

This report shall not be reproduced except in full, without the written approval of the laboratory. 


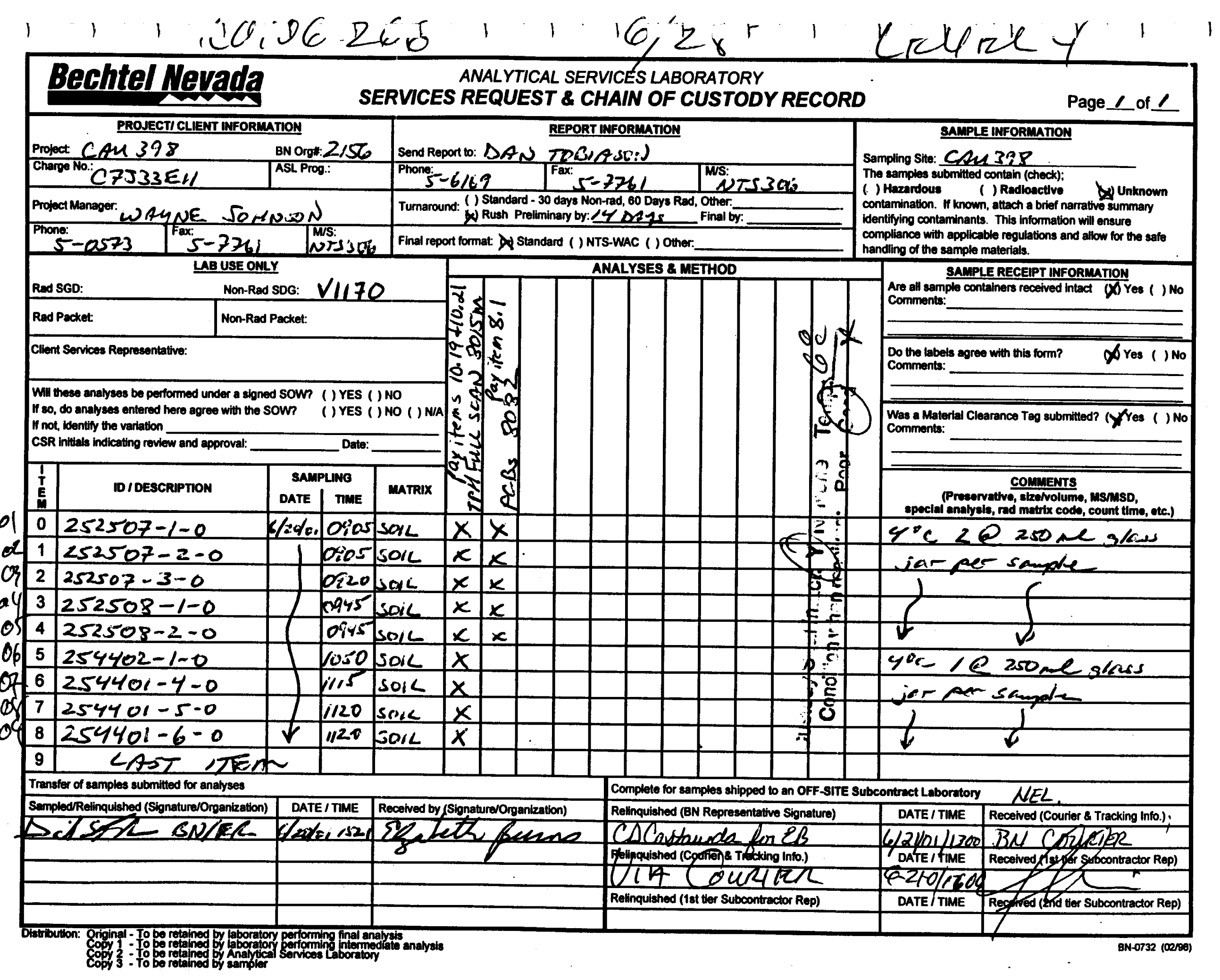




\section{APPENDIX A-2 \\ DATA QUALITY OBJECTIVES}




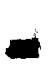

$=$

- 


\section{DATA QUALITY OBJECTIVES WORKSHEETS FOR CORRECTIVE ACTION UNIT 398: AREA 25 SPILL SITES (Presentation of Known Data Related to Corrective Action Unit 398)}

The information presented in this worksheet is based on historical data generated from preliminary assessment activities for Corrective Action Unit (CAU) 398 at the Nevada Test Site (NTS). Data quality objective (DQO) worksheets follow the U.S. Environmental Protection Agency (EPA) DQO guidance outline (EPA, 2000). The steps systematically build on the data acquired during preliminary assessment work and background research. Copies of the preliminary assessment work are retained in the project files.

Members of the Scoping Team:

1. U.S. Department of Energy, National Nuclear Security Administration, Nevada Operation Office (NNSANV) Janet Appenzeller-Wing

Sabine Curtis

2. Nevada Division of Environmental Protection (NDEP)

Ted Zaferatos

3. Bechtel Nevada (BN)

Thomas Fitzmaurice

Jeff Smith

Dan Tobiason

Kraig Knapp

Core Decision Team

Janet Appenzeller-Wing

Sabine Curtis

Thomas Fitzmaurice

Primary Decision Makers

Janet Appenzeller- Wing

Sabine Curtis

\subsection{PROBLEM STATEMENT}

\subsection{State the problem}

Thirteen sites have been identified for closure. In order to properly close these sites, currently available data must be evaluated to determine if the data adequately identify constituents of potential concern (COPC). These data will also be use to ascertain cleanup levels and verification sampling requirements. CAU 398 is comprised of the following 13 Corrective Action Sites (CASs): 
- CAS 25-44-01, Fuel Spill

- CAS 25-44-02, Spill

- CAS 25-44-03, Spill

- CAS 25-44-04, Acid Spill

- CAS 25-25-02, Oil Spills

- CAS 25-25-03, Oil Spills

- CAS 25-25-04, Oil Spills

- CAS 25-25-05, Oil Spills

- CAS 25-25-06, Oil Spills

- CAS 25-25-07, Hydraulic Oil Spill(s)

- CAS 25-25-08, Hydraulic Oil Spill(s)

- CAS 25-25-16, Diesel Fuel

- CAS 25-25-17, Subsurface Hydraulic Oil Spill

1.2 Summarize the problem - combine the relevant background information into a concise description of the problem to be resolved and known or suspected sources of disposed waste.

\subsubsection{CAS 25-44-01, Fuel Spill}

This site is located near the northwest corner of Road C and Road D in Area 25 of the NTS on a concrete pad at what was known as the Fuel Storage Facility. During site visits in 1996 and 1998, the spill was determined to be fuel spilled on soil that covers the concrete pad. The dimensions of the spill were measured to be 18 by 12 meters $(\mathrm{m})(60$ by 40 feet [ft]) with the depth ranging from 0 to 13 centimeters $(\mathrm{cm})(0$ to 5 inches [in]).

Two samples of the suspected spill material were collected on August 15, 1997, by International Technology Corporation (IT) and analyzed for volatile organic compounds (VOCs), semivolatile organic compounds (SVOCs), total petroleum hydrocarbons (TPH), polychlorinated biphenyls (PCBs), total Resource Conservation and Recovery Act (RCRA) metals, gross alpha and beta, and gamma spectroscopy. The results indicated that the only COPCs were the RCRA metals lead (186 milligrams per kilogram [mg/kg]) and chromium $(3,120 \mathrm{mg} / \mathrm{kg})$.

Sampling was conducted by BN on May 24, 2001. The spill material sampled was a dense, metallic material and was analyzed for Toxicity Characteristic Leaching Procedure (TCLP) metals and gamma spectroscopy. Samples were also collected of the soil surrounding the down gradient side of the pad and analyzed for full scan TPH. The results indicate that the dense metallic material did not contain TCLP metals or radiological constituents above regulatory levels. The soil surrounding the pad did not contain TPH above regulatory levels.

Historical information about this site is limited. It is unknown where the spill material came from or what it was used for. It is suspected to be railroad bedding material or shielding. There is no process knowledge of the area being used for fuel storage. The 
surrounding area (not part of the CAS) is littered with various construction debris and concrete. This indicates that the area may have been used as a dumping area for unused concrete and excess construction material.

Available information is adequate to show that this site contains no COPCs and can be properly closed with no further action.

\subsubsection{CAS 25-44-02, Spill}

This CAS is located on and near a concrete pad on the north side of Building 3117 at Test Cell A, Area 25, NTS. The CAS consists of a spill to the soil caused by leaking drums. A literature search and site visit by IT in 1993 determined that the barrels had been removed and shipped out of state for disposal as hazardous liquid waste. The shipping manifest indicated that the waste contained various oils, lead, and chromium. A previous contractor collected samples from one of the drums and of the soil in the spill area. The samples were analyzed for PCBs, TPH, and total chlorinated hydrocarbons. The only COPC detected was TPH in the soil.

BN conducted a site investigation on May 23, 2001, and collected soil samples from around the pad and the suspected spill area. It appeared that the area had been disturbed as if the impacted soil had been removed. The samples were analyzed for full-scan TPH, TCLP chromium and lead, and gamma spectroscopy. The sample results indicate that THP as diesel/oil above regulatory levels exists on the edge of the spill area pad.

The source of the drums is unknown. Building 3117 was used to support activities at Test Cell A, but it is unclear if activities at Building 3117 created the waste in the drums.

Sufficient information exists to properly clean-close this site by excavation and disposal.

\subsubsection{CAS 25-44-03, Spill}

This CAS is located on a concrete pad on the southeast side of Test Cell C. This was a temporary drum storage area where two drums labeled as containing PCBs leaked onto the pad and surrounding soil. On December 14, 1990, the drums were removed and shipped to the Area 6 PCB storage facility. The drums were later shipped off site for disposal after being characterized as non-PCB.

IT conducted a site assessment on December 2, 1999, and identified the staining on the pad and soil staining extending approximately $2 \mathrm{~m}(7 \mathrm{ft})$ east of the pad. Sampling was conducted by BN on May 24, 2001. The spilled material and surrounding soil were sampled and analyzed for full scan TPH, PCBs, TCLP metals, and gamma spectroscopy. The results indicate that TPH at levels of up to $5,320 \mathrm{mg} / \mathrm{kg}$ (diesel/oil range) are in the spill material. 
The source of the drums and contents is unknown but likely resulted from the draining of transformers or other electrical equipment. Available documentation indicates that the oil was non-PCB.

Adequate information exists to sufficiently clean-close this site by excavation and disposal.

\subsubsection{CAS 25-44-04, Acid Spill}

This CAS is located on a concrete pad on the east side of Building 3320 at the Engine Test Stand-1 facility. The site consists of spills from two tanks which were used for a water demineralization process. Tank T-2002 contained sodium hydroxide and Tank T-2003 contained sulfuric acid. Each tank had a 18,927-liter (L) (5,000-gallon [gal]) capacity.

A site visit on December 2,1999, determined the size of the spill to be approximately 10 by $5 \mathrm{~m}$ ( 33 by $15 \mathrm{ft})$. The spill extended another $3 \mathrm{~m}(10 \mathrm{ft})$ east off the pad into a small gully. The spill was identified by the red-brown and yellow staining. The spill from the sulfuric acid tank was believed to have occurred over a period of time due to corrosion of the tank bottom. The spill was reported to the Nevada Division of Emergency Management (NDEM) as NDEM 980819-3014. The spill was estimated to be approximately $380 \mathrm{~L}$ (100 gal). The sodium hydroxide spill (NDEM 980811-3001) occurred on August 10, 1998, during tank removal activities. Approximately 380 L (100 gal) were spilled and mixed with the residue from the sulfuric acid spill. A pH meter measured the $\mathrm{pH}$ of the standing liquid to be 8.5. Approximately 2.3 cubic meters $\left(\mathrm{m}^{3}\right)(3$ cubic yards $\left[\mathrm{yd}^{3}\right]$ ) of impacted soil were excavated and disposed in the NTS U10c construction landfill on August 12, 1998.

BN collected samples of the material on the pad and soil on May 24, 2001. The samples were analyzed for soil $\mathrm{pH}$ and gamma spectroscopy. The results indicated that the $\mathrm{pH}$ of the samples ranged from 7.48 to 9.90 , which is not RCRA-corrosive. No radiological constituents were present above regulatory levels.

Sufficient information exists to properly close this site with no further action.

\subsubsection{CAS 25-25-02, Oil Spills}

CAS 25-25-02 is located adjacent to a small concrete loading ramp on the south side of the Engine-Maintenance, Assembly, and Disassembly (E-MAD) facility (Building 3900). Available documentation indicates that the spill was associated with leaking drums that were removed in 1992. No documentation exists to indicate that the spill was removed. The source of the drums is not listed in the documentation. The loading ramp leads to the boiler room, so the drums could have been waste oil or fuel from operations conducted 
there. The spill was described as 3 by $3 \mathrm{~m}$ ( 10 by $10 \mathrm{ft}$ ) and approximately $10 \mathrm{~cm}$ ( $4 \mathrm{in})$ below the surface, and appeared to have been covered with soil.

IT collected a sample of the spill on August 27, 1997. The sample was analyzed for total VOCs, total SVOCs, TPH, PCBs, total RCRA metals, gross alpha/beta, and gamma spectrometry. The results indicated that the only COPCs were lead $(137 \mathrm{mg} / \mathrm{kg})$ and PCBs (34 mg/kg).

$\mathrm{BN}$ collected two samples of the spill material and soil on May 31, 2001. One sample was collected from the original IT sample location. The other sample was collected just off the southwest corner of the loading ramp from an obvious spill area of a dark grey to black tar- like material. Both samples were analyzed for TPH full scan, PCBs, TCLP SVOCs, TCLP metals, and gamma spectroscopy.

The results indicate that the soil contains TPH at levels up to $566 \mathrm{mg} / \mathrm{kg}$ (diesel/oil range) and PCBs up to $5.7 \mathrm{mg} / \mathrm{kg}$. The spill material contained TPH in the diesel/oil range at levels up to $3,440 \mathrm{mg} / \mathrm{kg}$ and PCBs up to $9.2 \mathrm{mg} / \mathrm{kg}$. No other chemical or radiological constituents were present above regulatory levels.

Adequate information exists to sufficiently clean-close this site by excavation and disposal.

\subsubsection{CAS 25-25-03, Oil Spills}

This CAS is located south of Building 4838 (Gas Station) in Area 25 of the NTS. The site was originally described as being a spill adjacent to a tipped-over drum. The drum was documented as being removed in July 1991 and in 1993 the spill was measured at 2 by 1 by $1 \mathrm{~m}$ ( 6 by 3 by $3 \mathrm{ft})$.

IT collected a sample from the site on August 15, 1997, and analyzed the sample for VOCs, SVOCs, TPH, PCBs, RCRA metals, gross alpha/beta, and gamma spectrometry. No COPCs were detected.

$\mathrm{BN}$ collected samples from two locations on May 24, 2001. One sample was collected from the original IT sample location approximately $3 \mathrm{~m}(10 \mathrm{ft})$ off the southwest corner of the paved parking area. The second sample was collected approximately $15 \mathrm{~m}(50 \mathrm{ft})$ southwest of the first location near where the IT CAS identification stake was. Samples

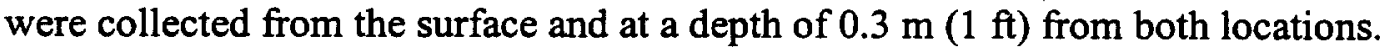
Samples were analyzed for TPH full scan and gamma spectroscopy. The results indicated that TPH levels were below detection limits at the surface and $0.3 \mathrm{~m}(1 \mathrm{ft})$ below the surface at the original IT sample location. At the sample near the IT CAS identification stake, TPH levels at the surface were $800 \mathrm{mg} / \mathrm{kg}$ diesel and $12,000 \mathrm{mg} / \mathrm{kg}$ oil. The TPH results at $0.3 \mathrm{~m}(1 \mathrm{ft})$ below ground surface were below regulatory limits. 
The source of the drum is not listed in the documentation although the drum was removed. The source of the drum was likely from vehicle maintenance operations at the gas station.

Adequate information exists to sufficiently clean-close this site by excavation and disposal.

\subsubsection{CAS 25-25-04, Oil Spills}

This site is located on the north side of the E-MAD facility near a flammable storage building. The site is described by the E-MAD facility engineer as an area where used oils and cooling fluids from metal machining operations were poured directly onto the ground. An estimated $133-151 \mathrm{~L}$ (35 - 40 gal) of waste liquids were discharged to the soil. The spill area is approximately 5 by $5 \mathrm{~m}$ ( 15 by $15 \mathrm{ft})$.

IT collected two samples on August 27, 1997, and analyzed the samples for VOCs, SVOCs, TPH, PCBs, total RCRA metals, gross alpha and beta, and gamma spectrometry. COPCs include TPH, PCBs $(2,300 \mathrm{mg} / \mathrm{kg})$, and the RCRA metals cadmium $(88.5 \mathrm{mg} / \mathrm{kg})$, chromium $(9,780 \mathrm{mg} / \mathrm{kg})$, and lead $(6,090 \mathrm{mg} / \mathrm{kg})$.

Samples of the area were collected by BN on May 26, 2001. Samples were collected from the original IT locations and from other locations within the CAS. Samples were collected from the surface and from a depth of $1-\mathrm{m}$ (3-ft). Surface samples were analyzed for TPH full scan, PCBs, TCLP VOCs, TCLP SVOCs, TCLP metals, and gamma spectroscopy. Subsurface samples were analyzed for only TPH full scan. The results indicated the presence of PCBs (up to $77 \mathrm{mg} / \mathrm{kg}$ ), TPH (up to $3,920 \mathrm{mg} / \mathrm{kg}$ in the diesel/oil range), and lead (up to $11 \mathrm{mg} / \mathrm{L}$ ). One sample was collected from a small area on the surface of what appeared to be machine shop trash. This appeared to be a very oily, greasy, absorbent material that contained metal turnings. The analytical results from this area were PCBs at $920 \mathrm{mg} / \mathrm{kg}$, TPH at $21,600 \mathrm{mg} / \mathrm{kg}$ total diesel and oil range, and cadmium at 1.1 milligram per liter $(\mathrm{mg} / \mathrm{L})$. This area was approximately 0.3 by $0.3 \mathrm{~m}(1$ by $1 \mathrm{ft}$ ) and appeared to be only surficial. All the analytical results for samples collected from the $1 \mathrm{~m}(3 \mathrm{ft})$ depth were below regulatory limits.

The source of the waste was from operations at the flammable storage building or the machine shop.

Adequate information exists to sufficiently clean-close this site by excavation and disposal. 


\subsubsection{CAS 25-25-05, Oil Spills}

This CAS is located on the northeast side of the E-MAD facility. The spills are oil and/or hydraulic fluid associated with heavy equipment that was stored there. There are four oil spills within the footprint of the heavy equipment. The spills are presumed to be from the same source (equipment oil tank) but leaked from different parts of the equipment.

IT collected two soil samples from the spills on August 27, 1997. The samples were analyzed for VOCs, SVOCs, TPH, PCBs, total RCRA metals, and gross alpha/beta. Possible COPCs include TPH and RCRA metals cadmium at $46.1 \mathrm{mg} / \mathrm{kg}$ and lead $208 \mathrm{mg} / \mathrm{kg}$.

BN collected samples from the spill on May 25, 2001. Samples were collected from the surface and from a depth of $0.6 \mathrm{~m}$ (2 ft). Surface samples were analyzed for TPH full scan, PCBs, TCLP VOCs, TCLP SVOCs, TCLP metals, pesticides, and gamma spectroscopy. Samples collected from the subsurface were analyzed for only TPH full scan. TPH results revealed concentrations as high as $46,500 \mathrm{mg} / \mathrm{kg}$ in the total diesel and oil range. Cadmium was detected in concentrations as high as $1.1 \mathrm{mg} / \mathrm{L}$. Results of samples collected from $60 \mathrm{~cm}(2 \mathrm{ft})$ depth were below regulatory levels.

Adequate information exists to sufficiently clean-close this site by excavation and disposal.

\subsubsection{CAS 25-25-06, Oil Spills}

CAS 25-25-06 is located at the Drill Hole Wash Monitoring Station which is past the Yucca Mountain facility in Area 25 of the NTS. The site was described as diesel fuel stains underneath two generators. The generators likely provided power for two trailers and monitoring equipment. A January 1996 site visit by IT determined that the generators were gone and no soil staining was apparent. The two trailers remained. A later IT site visit on March 5, 1998, revealed that the larger of the two trailers was gone. However, dark-brown staining was evident on the soil where the larger trailer had been. The staining was approximately 9 by $2 \mathrm{~m}$ ( 28 by $8 \mathrm{ft})$. No samples were collected.

A BN site visit on May 31, 2001, revealed no indication of the staining identified earlier. Based on descriptions and photographs, the location of the previously stained area was noted and samples were collected. Samples were analyzed for full scan TPH and gamma spectroscopy. The results indicated that TPH and radiological constituents are not present in the soil above regulatory limits. This CAS contains no COPCs and can be properly closed with no further action. 


\subsubsection{CAS 25-25-07, Hydraulic Oil Spill(s)}

This site is located adjacent to a boring machine in an escape drift of X-Tunnel. The boring machine was left in place when X-Tunnel was placed on inactive status in 1982. Over time, the hydraulic lines leaked fluid to the surrounding soil. The lines may also have leaked due to damage from removing salvageable parts from the boring machine. It is estimated that approximately 1,514 L ( $400 \mathrm{gal})$ of hydraulic fluid may have been released. The soil layer is estimated to be $0.3 \mathrm{~m}(1 \mathrm{ft})$ thick with bedrock beneath. Since the site is underground, there is no driving force for migration of the hydraulic fluid except for gravity.

A sample of the hydraulic reservoir was collected on January 31, 1997, and analyzed for PCBs. Results indicated that PCBs were below regulatory levels. The reservoir was later drained.

BN collected samples of the impacted soil on June 20, 2001. The samples were analyzed for full scan TPH, PCBs, and gamma spectroscopy. Due to the presence of fixed depleted uranium within the drift, a radiological control technician was present during sampling activities. No radiological levels above background were detected in the sampling areas. Sample results indicated TPH concentrations in the diesel/oil range of up to 105,000 $\mathrm{mg} / \mathrm{kg}$.

Based on the safety risks to personnel associated with cleaning up the hydrocarbonimpacted soil (confined mine shaft, limited lighting, hanging utility lines) as compared to the risk associated with leaving the soil in place, closure should consist of administrative controls using a use restriction with no further action.

\subsubsection{CAS 25-25-08, Hydraulic Oil Spill(s)}

This site is located adjacent to a boring machine at the end of the main drift of Y-Tunnel. The boring machine was left in place when Y-Tunnel was placed on inactive status in 1982. Over time, the hydraulic lines leaked fluid to the surrounding soil. The lines may also have leaked due to damage from removing salvageable parts from the boring machine. It is estimated that approximately $1,514 \mathrm{~L}(400 \mathrm{gal})$ of hydraulic fluid may have

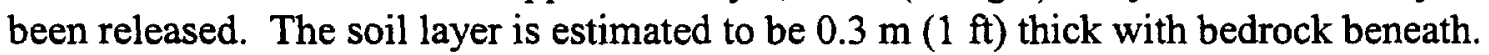
Since the site is underground, there is no driving force for migration of the hydraulic fluid except for gravity.

BN collected samples of the impacted soil on June 20, 2001. The samples were analyzed for full-scan TPH, PCBs, and gamma spectroscopy. A radiological control technician was present during sampling activities as a precautionary measure. No radiological levels above background were detected in the sampling areas. Sample results indicated TPH results in the diesel/oil range of up to $140,000 \mathrm{mg} / \mathrm{kg}$. 
Based on the safety risks to personnel associated with cleaning up the hydrocarbonimpacted soil (confined mine shaft, limited lighting, hanging utility lines) as compared to the risk associated with leaving the soil in place, closure should consist of administrative controls using a use restriction with no further action.

\subsubsection{CAS 25-25-16, Diesel Fuel}

This site is located east of the Engine Test Stand entrance in Area 25 of the NTS. The site consists of soil staining from a diesel release from an aboveground storage tank. Tank T-2001 was a 79,000-L (21,000-gal) capacity steel tank used to store diesel fuel for a boiler located in Building 3320.

The tank was removed during August 1998 closure activities for CAU 126. There was no evidence that the tank had leaked. However, the soil surrounding a valve connected to the drain pipe was moist with a strong diesel odor.

On August 10, 1998, an attempt was made to determine the extent of the spill. Approximately $10.7 \mathrm{~m}^{3}\left(14 \mathrm{yd}^{3}\right)$ were removed and disposed before it was determined that additional excavation was beyond the scope of that project.

BN collected samples from the surface and from a depth of up to $0.6 \mathrm{~m}(2 \mathrm{ft})$ in the area of the impacted soil on May 25, 2001. The samples were analyzed for full-scan TPH and gamma spectroscopy. The results indicated that diesel and oil range organics were present at a maximum concentration of $1,320 \mathrm{mg} / \mathrm{kg}$ at the surface.

Adequate information exists to sufficiently clean-close this site by excavation and disposal.

\subsubsection{CAS 25-25-17, Subsurface Hydraulic Oil Spill}

This CAS is located on the south side of the E-MAD facility. The spill was identified on December 1, 1998, during the housekeeping closure of CAU 297. Closure activities consisted of the removal of two $38 \mathrm{~L}$ (10 gal) metal containers used in a closed vacuum pump oil recovery system.

Completion of the closure activities identified a hydrocarbon release associated with historical operations of the oil recovery system. The spill is located in a significant power/utility corridor feeding into the building. The first of the utilities was located at a depth of approximately $1 \mathrm{~m}(3 \mathrm{ft})$. Impacted soil was removed to a maximum depth of approximately $46 \mathrm{~cm}$ (18 in). Work was discontinued due to the extent of the impacted area, confining work space limitations, and proximity to utilities. Clean soil was used to backfill over the excavated area. 
Samples of the soil were collected by BN for verification and waste disposition during CAU 297 closure activities. The samples were analyzed for TPH, TCLP VOCs, TCLP SVOCs, TCLP metals, PCBs, pesticides, and gamma spectroscopy. The results indicated COPCs as TPH and PCBs $(7.2 \mathrm{mg} / \mathrm{kg})$.

BN re-sampled the site on May 31, 2001. Soil samples were collected from a minimum depth of $0.3 \mathrm{~m}(1 \mathrm{ft})$ to get below the level of the backfill. The samples were analyzed for full-scan TPH, PCBs, and gamma spectroscopy. The results indicate the soil is impacted with petroleum hydrocarbons up to $647 \mathrm{mg} / \mathrm{kg}$.

Based on the risks (utility corridor) associated with cleaning up the hydrocarbon-impacted soil as compared to the risk associated with leaving the soil in place, closure should consist of administrative controls using a use restriction with no further action.

\subsection{DEVELOP AND REFINE THE CONCEPTUAL SITE MODEL (CSM)}

Available information, including site process knowledge and historical background information, is sufficient to support the CSMs for CAU 398. The CSMs describe the most probable scenarios for current conditions at each site and define the assumptions that are the basis for identifying appropriate data collection methods.

Ten of the eleven above-ground sites involve releases of petroleum hydrocarbons to surface or near-surface soil. Other COPCs are present at some of these sites and are associated with the petroleum hydrocarbons, which acts as a carrier for the other COPCs. The remaining above-ground site is an acid release to surface soil. In the $\mathrm{X}$ - and $\mathrm{Y}$-tunnel sites, petroleum has been released to the floor of the tunnel. The released substances will typically migrate downward due to gravity and will also flow downslope from the source if the soil conditions do not allow the fuel to seep in as quickly as the release is occurring. Previously disturbed ground, such as occurs along buried piping and utility corridors, will also serve as a preferential pathway. After the initial release has stopped, the fuel typically continues to migrate downward with gravity until equilibrium is reached. If additional pressure is added to the system after equilibrium is reached, such as what occurs with a new release or as a result of rainfall, downward migration will continue.

\subsection{Primary CSMs}

The primary CSMs are considered the most probable scenarios for current conditions at the CAU 398 sites. Available information from which the CSMs are based were derived from site process knowledge, historical background information, and site sampling and analysis. The proposed activities are based on the assumption that diesel- and oil- range petroleum hydrocarbons are the most prevalent COPCs at the sites. The petroleum hydrocarbons are also assumed to act as a carrier for the other COPCs which will not extend beyond the limits of the petroleum hydrocarbons. All of the sites are expected to 
fit the basic CSM with minor variations caused by site-specific preferential pathways, as identified below for each CAS:

- CAS 25-44-01, Fuel Spill: A CSM has not been developed for this site because sample analysis indicated that COPCs are not present above regulatory levels. No further action is recommended for this site.

- CAS 25-44-02, Spill: The primary CSM assumes that only petroleum hydrocarbons were released to the soil. Sample analysis supports thisCSM and indicates that petroleum hydrocarbons extend to a depth of $0.6 \mathrm{~m}(2 \mathrm{ft})$ below ground surface. There are no preferential pathways identified for this site.

- CAS 25-44-03, Spill: The primary CSM assumes that only petroleum hydrocarbons were released to the soil. Sample analysis supports this CSM. It is assumed that the hydrocarbons extend to a maximum depth of $0.3 \mathrm{~m}(1 \mathrm{ft})$ below the ground surface. There are no preferential pathways identified for this site.

- CAS 25-44-04, Acid Spill: A CSM has not been developed for this site because sample analysis indicated that COPCs are not present above regulatory levels. No further action is recommended for this site.

- CAS 25-25-02, Oil Spills: The primary CSM assumes that only petroleum hydrocarbons and associated PCBs were released to the soil. It is also assumed that the PCBs did not extend beyond the limits of the hydrocarbon release and that the PCBs present are below land ban concentration of $50 \mathrm{mg} / \mathrm{kg}$. It is assumed that the COPCs extend to a maximum depth of $0.3 \mathrm{~m}(1 \mathrm{ft})$ below the ground surface. The preferential pathway for this site may be down along the foundation of the E-MAD Building.

- CAS 25-25-03, Oil Spills: The primary CSM assumes that only petroleum hydrocarbons were released to the soil. Sample analysis supports this CSM and indicates that petroleum hydrocarbons extend to a depth of $0.3 \mathrm{~m}(1 \mathrm{ft})$ below ground surface. There are no preferential pathways identified for this site.

- CAS 25-25-04, Oil Spills: The primary CSM assumes that only petroleum hydrocarbons and associated COPCs (PCBs, lead, cadmium) were released to the soil and that the COPCs did not extend beyond the limits of the hydrocarbon release. Sample analysis supports this CSM and indicates that petroleum hydrocarbons extend

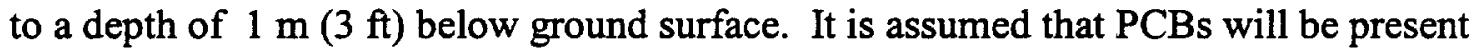
in concentrations above the land ban limit of $50 \mathrm{mg} / \mathrm{kg}$. Lead and cadmium are also assumed to be above the land ban concentrations of $7.5 \mathrm{mg} / \mathrm{kg}$ and $1.1 \mathrm{mg} / \mathrm{kg}$, respectively. The preferential pathway for this site may be the nearby (within $3 \mathrm{~m}$ $[10 \mathrm{ft}])$ storm drain.

- CAS 25-25-05, Oil Spills: The primary CSM assumes that only petroleum hydrocarbons and associated COPCs (cadmium) were released to the soil and that the COPCs did not extend beyond the limits of the hydrocarbon release. Sample analysis 
supports this model and indicates that petroleum hydrocarbons extend to a depth of $0.6 \mathrm{~m}(2 \mathrm{ft})$ below ground surface. It is assumed that cadmium is present above the land ban concentration of $1.1 \mathrm{mg} / \mathrm{kg}$. There are no preferential pathways identified for this site.

- CAS 25-25-06, Oil Spills: A CSM has not been developed for this site because sample analysis indicated that COPCs are not present above regulatory levels. No further action is recommended for this site.

- CAS 25-25-07, Hydraulic Oil Spill(s): The primary CSM assumes that only petroleum hydrocarbons were released to the soil in X-Tunnel. Sample analysis supports this CSM. It is assumed that the hydrocarbons extend to a maximum depth of $0.3 \mathrm{~m}(1 \mathrm{ft})$ below the ground surface. There are no preferential pathways identified for this site.

- CAS 25-25-08, Hydraulic Oil Spill(s): The primary CSM assumes that only petroleum hydrocarbons were released to the soil in Y-Tunnel. Sample analysis supports this CSM. It is assumed that the hydrocarbons extend to a maximum depth of $0.3 \mathrm{~m}(1 \mathrm{ft})$ below the ground surface. There are no preferential pathways identified for this site.

- CAS 25-25-16, Diesel Spill: The primary CSM assumes that only petroleum hydrocarbons were released to the soil. Sample analysis supports this CSM. It is

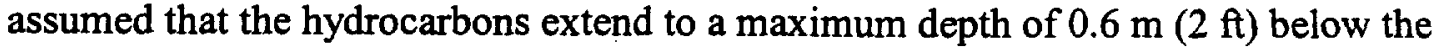
ground surface. There are no preferential pathways identified for this site.

- CAS 25-25-17, Subsurface Hydraulic Oil Spill: The primary CSM assumes that only petroleum hydrocarbons were released to the soil. Sample analysis supports this CSM.

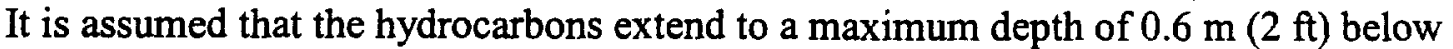
the ground surface. Preferential pathways for this site may be the utility corridor and the building foundation.

\section{$2.2 \quad$ Alternate CSMs}

The conditions under the alternate CSM are considered less likely than conditions outlined in the primary CSM.

- CAS 25-44-01, Fuel Spill: An alternate CSM is not necessary for this site because sample analysis indicated that COPCs are not present above regulatory levels.

- CAS 25-44-02, Spill: An alternate CSM has not been developed for this site because existing data show the primary CSM to be an adequate representation of current site conditions.

- CAS 25-44-03, Spill: The alternate CSM provides for a more extensive petroleum hydrocarbon release (depth) than assumed in the primary CSM. 
- CAS 25-44-04, Acid Spill: An alternate CSM is not necessary for this site because sample analysis indicated that COPCs are not present above regulatory levels.

- CAS 25-25-02, Oil Spills: The alternate CSM provides for a more extensive release of COPCs than assumed in the primary CSM. The preferential pathway remains the same as in the primary CSM.

- CAS 25-25-03, Oil Spills: The alternate CSM provides for a more extensive release of petroleum hydrocarbons than assumed in the primary CSM.

- CAS 25-25-04, Oil Spills: The alternate CSM provides for a more extensive release of COPCs than assumed in the primary CSM. The preferential pathway remains the same as in the primary CSM.

- CAS 25-25-05, Oil Spills: An alternate CSM has not been developed for this site because existing data show the primary CSM to be an adequate representation of current site conditions.

- CAS 25-25-06, Oil Spills: An alternate CSM is not necessary for this site because sample analysis indicated that COPCs are not present above regulatory levels.

- CAS 25-25-07, Hydraulic Oil Spill(s): The alternate CSM for the X-Tunnel spill provides for a more extensive release of petroleum hydrocarbons that has impacted the bedrock.

- CAS 25-25-08, Hydraulic Oil Spill(s): The alternate CSM for the Y-Tunnel spill provides for a more extensive release of petroleum hydrocarbons that has impacted the bedrock.

- CAS 25-25-16, Diesel Spill: The alternate CSM provides for a more extensive release of petroleum hydrocarbons than assumed in the primary CSM.

- CAS 25-25-17, Subsurface Hydraulic Oil Spill: The alternate CSM provides for a more extensive release of petroleum hydrocarbons than assumed in the primary CSM and for PCB concentrations above the cleanup level. The preferential pathways remain the same as in the primary CSM.

\subsection{IDENTIFY THE DECISION}

Development of a Streamlined Approach for Environmental Restoration (SAFER) plan can begin based on the currently available process knowledge, historical data, and sampling data. 
Decisions regarding the closure alternatives for the CASs can be made based on the available site data. The CASs have been grouped into three closure alternatives based on site conditions. The most probable closure decisions are identified below:

\subsection{No Further Action}

The CASs included in the no further action alternative are as follows:

- CAS 25-44-01, Fuel Spill

- CAS 25-44-04, Acid Spill

- CAS 25-25-06, Oil Spills

Are existing data sufficient to support the no further action alternative?

\subsection{Clean Closure}

The CASs and associated COPCs included in the clean closure alternative are as follows:

- CAS 25-44-02, Spill

- CAS 25-44-03, Spill

- CAS 25-25-02, Oil Spills

- CAS 25-25-03, Oil Spills

- CAS 25-25-04, Oil Spills

- CAS 25-25-05, Oil Spills

- CAS 25-25-16, Diesel Fuel
$\mathrm{COPC}=\mathrm{TPH}$ as diesel/oil

$\mathrm{COPC}=\mathrm{TPH}$ as diesel/oil

COPCs=TPH as diesel/oil; PCBs (below land ban)

$\mathrm{COPC}=\mathrm{TPH}$ as diesel/oil

$\mathrm{COPCs}=\mathrm{TPH}$ as diesel/oil; lead, cadmium, PCBs (above land ban)

COPCs=TPH as diesel/oil; cadmium (above land ban)

$\mathrm{COPC}=\mathrm{TPH}$ as diesel/oil

Are existing data sufficient to support the clean closure by excavation and disposal alternative?

\subsection{Administrative Closure}

The sites included in this group are as follows:

- CAS 25-25-07, Hydraulic Oil Spill(s)

- CAS 25-25-08, Hydraulic Oil Spill(s)

- CAS 25-25-17, Subsurface Hydraulic Oil Spill

Are existing data sufficient to support administrative closure of these sites with no further action other than implementation of a Use Restriction? 


\subsection{IDENTIFY THE INPUTS TO THE DECISION}

4.1 Identify the information inputs needed and resolve the decision.

\subsubsection{No Further Action}

- CAS 25-44-01, Fuel Spill

- CAS 25-44-04, Acid Spill

- CAS 25-25-06, Oil Spills

At these CASs, sample results revealed no evidence of COPCs above action levels.

- Therefore, these sites can be properly closed with no further action.

\subsubsection{Clean Closure}

- CAS 25-44-02, Spill

- CAS 25-44-03, Spill

- CAS 25-25-02, Oil Spills

- CAS 25-25-03, Oil Spills

- CAS 25-25-04, Oil Spills

- CAS 25-25-05, Oil Spills

- CAS 25-25-16, Diesel Fuel

Sufficient information exists for these CASs to be clean closed by excavation and disposal of impacted soil. All of these sites consist of TPH-impacted soil. Three sites contain COPCs in addition to TPH and are associated with the TPH. CAS 25-25-02 also contains PCBs below the land ban restrictions; CAS 25-25-05 also contains cadmium above land ban restrictions; CAS 25-25-04 contains lead, cadmium, and PCBs above land ban restrictions in addition to the TPH. CASs with COPCs above the land ban restrictions must be sent to an off-site treatment and disposal facility.

\subsubsection{Administrative Closure}

- CAS 25-25-07, Hydraulic Oil Spill(s)

- CAS 25-25-08, Hydraulic Oil Spill(s)

- CAS 25-25-17, Subsurface Hydraulic Oil Spill

$\mathrm{TPH}$ as diesel/oil is the only COPC at sites included in this group. Given specific site conditions (utilities, limited space, limited lighting), it is likely that a risk assessment of each of these sites, based on the "A through K" evaluation as presented in Nevada Administrative Code (NAC) 445A.227, would show that there is no significant risk to human health or the environment from the hydrocarbon-impacted soil. This "A through $\mathrm{K}$ " evaluation is recommended for inclusion in the SAFER Plan, so data supporting administrative closure of the site must be gathered. If risk-based closure is supported, the 
sites would then be recommended for administrative closure with no further action and a use restriction prepared for inclusion in the Closure Report (CR).

\subsection{List types of COPCs and affected media.}

The CASs and their associated COPCs are listed below:

- CAS 25-44-01, Fuel Spill - No COPCs above action levels

- CAS 25-44-04, Acid Spill - No COPCs above action levels

- CAS 25-25-06, Oil Spills - No COPCs above action levels

- CAS 25-44-02, Spill - TPH as diesel/oil

- CAS 25-44-03, Spill - TPH as diesel/oil

- CAS 25-25-02, Oil Spills - TPH as diesel/oil, PCBs

- CAS 25-25-03, Oil Spills - TPH as diesel/oil

- CAS 25-25-04, Oil Spills - TPH as diesel/oil, lead, cadmium, PCBs

- CAS 25-25-05, Oil Spills - TPH as diesel/oil, cadmium

- CAS 25-25-16, Diesel Fuel - TPH as diesel/oil

- CAS 25-25-07, Hydraulic Oil Spill(s) - TPH as diesel/oil

- CAS 25-25-08, Hydraulic Oil Spill(s) - TPH as diesel/oil

- CAS 25-25-17, Subsurface Hydraulic Oil Spill - TPH as oil

For all of the CASs, the affected media is soil.

\subsection{Identify potential sampling approaches and appropriate analytical methods.}

Existing documentation, process knowledge, and sample data are adequate to close the sites under the no further action and administrative closure alternatives without collecting additional data. For these sites, existing data will be referenced in the SAFER Plan and documented in the closure documentation to demonstrate adequate closure of the sites. The sites under the clean closure by excavation and disposal alternative can also be closed using existing data but will also require confirmation samples to verify that all COPCs have been removed to below action levels. The confirmation sampling approach will be discussed in the SAFER Work Plan. 


\subsection{DEFINE THE BOUNDARIES OF THE STUDY}

5.1 Define the geographic areas of the field investigation.

5.1.1 Define the geographic area within which all decisions must apply (in some cases this may be defined by the $\mathrm{CAU}$ ).

The geographic areas of the field investigation are those areas of each CAS which are impacted by COPCs as identified by the CSM. Descriptions of each area are found in Section 1.2 of this report.

\subsubsection{Specify the characteristics that define the population of interest.}

The population of interest consists of soil containing COPCs at concentrations above action levels.

\subsection{Define the time frame of the decision.}

\subsubsection{Determine the time frame to which the study data apply.}

- The study data should be relevant with the length of time allowed for by the SAFER process under the Federal Facility Agreement and Consent Order (FFACO) (FFACO, 1996).

- Migration (if occurring) is assumed to be imperceptibly slow. This is based on minimal surface water infiltration and the constraints of the CSM.

\subsubsection{Determine when to collect data.}

Field activities are scheduled to take place in Fiscal Year 2002 after approval of the final SAFER Work Plan. Field activities will be conducted at times that meet the security and safety constraints of the NTS.

\subsubsection{Define relevant time constraints.}

The FFACO deadline for delivery of the final SAFER Work Plan is December 31, 2001.

The FFACO deadline for delivery of the final CR is December 31, 2002.

\subsection{Identify any practical constraints on data collection.}

- Approval of the DQO process and the SAFER Plan by the NDEP.

- Site operations - NTS operational and security constraints.

- Equipment and personnel access. 
- Meteorological.

- Availability of heavy equipment.

- Health and safety of workers.

6.0 DEVELOP A DECISION RULE - DEFINE A LOGICAL BASIS FOR CHOOSING AMONG ALTERNATIVE ACTIONS

6.1 Specify the action level or preliminary action level for the decision.

Sufficient analytical data and process knowledge exists to support the CSM. The action level is $100 \mathrm{mg} / \mathrm{kg}$ for TPH based on NAC 445A.2272. Based on Preliminary

Remediation Goals for EPA Region 9 for Industrial Soils, the action levels are $1.0 \mathrm{mg} / \mathrm{kg}$ for PCBs, $750 \mathrm{mg} / \mathrm{kg}$ for lead, and $810 \mathrm{mg} / \mathrm{kg}$ for cadmium.

7.0 OPTIMIZE THE DESIGN - OUTLINE A SAMPLING DESIGN, SPECIFYING THE OPERATIONAL DETAILS OF THE SAMPLING PLAN WHICH FALLS WITHIN THE PROJECT'S CONSTRAINTS

7.1 Develop general sampling and analysis design alternatives.

Refer back to Section 4.3 for sampling and analysis alternatives.

7.2 Select the most resource-effective design that satisfies all of the DQOs.

- For those sites requiring clean closure by excavation and disposal, excavate impacted to lateral and vertical extent and collect confirmation samples to verify that all soil impacted with COPCs above action levels has been removed.

- Survey and implement Use Restrictions for those sites to be administratively closed.

7.3 Document the operational details and theoretical assumptions of the selected design in the sampling and analysis plan.

Detailed documentation of sampling and analysis will be discussed in the SAFER Work Plan. 
TABLE A1-1 - LABORATORY ANALYTICAL REQUIREMENTS FOR CAU 398 SOIL SAMPLES

\begin{tabular}{|c|c|c|c|c|c|c|}
\hline $\begin{array}{l}\text { Porrameter or } \\
\text { Analyte }\end{array}$ & Moringm & 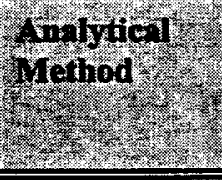 & $\begin{array}{l}\text { Minimum } \\
\text { Reporting } \\
\text { Ifint }\end{array}$ & 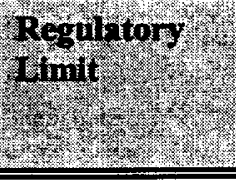 & $\begin{array}{l}\text { Relutwe } \\
\text { Percent } \\
\text { mimerence } \\
\text { (RPD) }\end{array}$ & $\begin{array}{l}\text { Percent } \\
\text { Recovery } \\
(\text { far }\end{array}$ \\
\hline $\begin{array}{l}\text { Polychlorinated } \\
\text { Biphenyls } \\
\text { (PCBs) }\end{array}$ & Soil & $8082^{c}$ & $1 \mathrm{mg} / \mathrm{kg}$ & $2 \mathrm{mg} / \mathrm{kg}$ & $\begin{array}{l}\text { Lab - } \\
\text { specific }\end{array}$ & $\begin{array}{l}\text { Lab - } \\
\text { specific }^{d}\end{array}$ \\
\hline $\begin{array}{l}\text { Total } \\
\text { Petroleum } \\
\text { Hydrocarbons } \\
\text { (TPH) }\end{array}$ & Soil Diesel & $\begin{array}{l}8015 \mathrm{~B} \\
\text { modified }^{\mathrm{c}}\end{array}$ & $25 \mathrm{mg} / \mathrm{kge}^{\mathrm{e}}$ & $100 \mathrm{mg} / \mathrm{kg}$ & $\begin{array}{l}\text { Lab - } \\
\text { specific }^{d}\end{array}$ & $\begin{array}{l}\text { Lab - } \\
\text { specific }^{d}\end{array}$ \\
\hline
\end{tabular}

${ }^{a} \mathrm{RPD}$ is used to calculate precision

b\% $\mathrm{R}$ is used to calculate accuracy

'U.S. Environmental Protection Agency (EPA) Test Methods for Evaluating Solid Waste, $3^{\text {rd }}$ Edition, Parts 1-4, SW-846 (EPA, 1996b)

${ }^{\mathrm{d}}$ In-House Generated RPD and \%R Performance Criteria

'Industrial Sites Quality Assurance Project Plan (DOE/NV, 1996) 


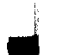




\section{APPENDIX A-3 PROJECT ORGANIZATION}


The U.S. Department of Energy, National Nuclear Security Administration Nevada Operations Office (NNSA/NV) Project Manager or Task Manager will serve as the primary point of contact for all activities conducted for this project. The NNSA/NV Project Manager is responsible for seeing that all activities conducted during the project fulfill the obligations of NNSA/NV, as described in the Federal Facility Agreement and Consent Order (FFACO) (FFACO, 1996) and the Nevada Division of Environmental Protection (NDEP) approved work plan. The NNSA/NV Project Manager will plan, authorize, and control project work so that activities are completed in accordance with the work plan on schedule and within budget. The NNSA/NV Project Manager will be the primary point of contact with the NDEP. The NNSA/NV points of contact for this project are as follows:

Project Manager: Janet Appenzeller-Wing

Telephone Number: (702) 295-0461

Task Manager: Sabine Curtis

Telephone Number: (702) 295-0542

The identification of the project Health and Safety Officer and the Quality Assurance Officer can be found in both the Field Management Plan and the Site-Specific Health and Safety Plan. However, personnel are subject to change and it is suggested that the Project Manager be contacted for further information. 
THIS PAGE INTENTIONALLY LEFT BLANK

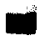




\section{APPENDIX A-4 NEDP DOCUMENT REVIEW SHEET}





\section{DOCUMENT REVIEW SHEET}

Document Title/Number: SAFER Plan for Corrective Action Unit 398: Area 25 Spill Sites, Nevada Test Site, Nevada

Document Date: October 2001

Revision Number: 0

Originator/Organization: Bechtel Nevada

Reviewer/Organization: Nevada Division of Environmental Protection

\begin{tabular}{|c|c|c|c|c|}
\hline $\begin{array}{l}\text { Comment } \\
\text { Number/ } \\
\text { Location } \\
\end{array}$ & Type & Comment & Comment Response & Accept \\
\hline $\begin{array}{l}\text { 1. Document } \\
\text { Cover }\end{array}$ & M & Change "Areas" to "Area" in title. & Change made. & YES \\
\hline 2. pg v - vii & $\mathbf{M}$ & $\begin{array}{l}\text { Check that document Section Headings have } \\
\text { correct page numbers in Table of Contents. }\end{array}$ & $\begin{array}{l}\text { Table of Contents checked and } \\
\text { corrections made. }\end{array}$ & YES \\
\hline 3. pg. vi & $\mathbf{M}$ & $\begin{array}{l}\text { Include Sections } 2.3 .6 \text { and } 3.2 .3 \text { in Table of } \\
\text { Contents. }\end{array}$ & Sections included in Table of Contents & YES \\
\hline 4. pg. viii & M & $\begin{array}{l}\text { Table } 7 \text { description should refer to CUA } 398 \\
\text { and not to CAU } 392\end{array}$ & $\begin{array}{l}\text { Correction made. Tables } 1 \text { - } 4 \text { have been } \\
\text { consolidated into a single Table } 1 \text {. Table } \\
7 \text { is now Table } 4\end{array}$ & YES \\
\hline 5. pg. $9-29$ & M & $\begin{array}{l}\text { Table of Contents headings should match the } \\
\text { headings on the figures. }\end{array}$ & $\begin{array}{l}\text { Changes made. All figure headings in } \\
\text { both the Table of Contents and on figure } \\
\text { match. }\end{array}$ & YES \\
\hline 6. pg. 27,29 & M & $\begin{array}{l}\text { Figures labeled } 13,14 \text { and } 15 \text { should be } \\
\text { labeled Figure } 11,12 \text { and } 13 .\end{array}$ & Changes made. & YES \\
\hline
\end{tabular}

a. Comment Types: $M=$ Mandatory $S=$ Suggested

Page 1 of 3 
DOCUMENT REVIEW SHEET

\begin{tabular}{|c|c|c|c|c|}
\hline $\begin{array}{l}\text { Comment } \\
\text { Number/ } \\
\text { Location } \\
\end{array}$ & Type $^{2}$ & Comment & Comment Response & Accept \\
\hline 7.pg. 38 & $\mathbf{M}$ & $\begin{array}{l}\text { In table } 5 \text { CAS indicated as } 25-27-07 \text { should } \\
\text { be referred to as } 25-25-07 \text {. }\end{array}$ & Change made. & YES \\
\hline $\begin{array}{l}\text { 8. pg. } 39, \\
\text { Section } 3.2 .2\end{array}$ & $\mathbf{M}$ & $\begin{array}{l}\text { Change the first sentence to read "...ten } \\
\text { CASs show the presence of COCs above..." }\end{array}$ & Change made. & YES \\
\hline $\begin{array}{l}9 . \text { pg. } 39 \text {, } \\
\text { bullet } 6\end{array}$ & $\mathbf{M}$ & $\begin{array}{l}\text { Excavated waste from } 25-25-05 \text { must be } \\
\text { disposed of as hazardous waste. }\end{array}$ & $\begin{array}{l}\text { Text added to clarify. Two soil samples } \\
\text { were collected and analyzed for RCRA } \\
\text { metals from this site. One was just } \\
\text { slightly above the RCRA level for } \\
\text { Cadmium }(1.0 \mathrm{mg} / \mathrm{L}) \text { at } 1.1 \mathrm{mg} / \mathrm{L} \text {. One } \\
\text { was below the RCRA level. Waste will } \\
\text { be managed as hazardous waste on site, } \\
\text { i.e., containerized and stored in a } 90 \text {-day } \\
\text { accumulation area. Characterization } \\
\text { sampling will be done to determine the } \\
\text { final disposal of waste, as hazardous or } \\
\text { non-hazardous waste. }\end{array}$ & YES \\
\hline 10. pg. 49 & $\mathbf{M}$ & $\begin{array}{l}\text { Table } 7 \text {, remove final note regarding the } \\
\text { pending modification for Area } 23 \text { landfill } \\
\text { permit regarding PCBs. }\end{array}$ & Note has been deleted. & YES \\
\hline
\end{tabular}

\footnotetext{
a. Comment Types: $M=$ Mandatory $S=$ Suggested $\quad$ Page 2 of 3
} 


\section{DOCUMENT REVIEW SHEET}

\begin{tabular}{||c|c|c|c|c|}
\hline $\begin{array}{c}\text { Comment } \\
\text { Number/ } \\
\text { Location }\end{array}$ & Type & Comment & Comment Response & Accept \\
\hline \hline $\begin{array}{l}\text { 11.pg. A1-18 } \\
\text { Section 5.2.3 }\end{array}$ & $\mathrm{M}$ & $\begin{array}{l}\text { The FFACO deadline for delivery of the } \\
\text { final SAFER Plan is December 31, 2001 and } \\
\text { not November 16,2001 }\end{array}$ & Change made. \\
\hline
\end{tabular}




\section{DISTRIBUTION LIST}




\section{DISTRIBUTION LIST}

*Provide copy of initial distribution of Revision 0; remainder of list gets Revision 0 if approved without changes. The entire list receives Revision 1, if issued.

\section{Nevada Division of Environmental Protection}

Paul Liebendorfer

2 (Controlled)*

Bureau of Federal Facilities

Division of Environmental Protection

333 W. Nye Lane, Room 138

Carson City, NV 89706-0866

Supervisor, Las Vegas Office

1 (Controlled)*

Bureau of Federal Facilities

Division of Environmental Protection

555 E. Washington, Suite 4300

Las Vegas, NV 89010-1043

\section{U.S. Department of Energy}

Janet Appenzeller-Wing

1 (Uncontrolled)*

Project Manager

Environmental Restoration Division

U.S. Department of Energy

National Nuclear Security Administration

Nevada Operations Office

P.O. Box 98518, M/S 505

Las Vegas, NV 89193-8518

Sabine Curtis

1 (Uncontrolled)*

Environmental Restoration Division

U.S. Department of Energy

National Nuclear Security Administration

Nevada Operations Office

P.O. Box 98518, M/S 505

Las Vegas, NV 89193-8518

Sabrina Lawrence

Environmental Restoration Division

1 (Controlled)*

U.S. Department of Energy

National Nuclear Security Administration

Nevada Operations Office

P.O. Box 98518 , M/S 505

Las Vegas, NV 89193-8518 


\section{DISTRIBUTION LIST (continued)}

\section{U.S. Department of Energy (continued)}

U.S. Department of Energy

1 (Controlled \&

National Nuclear Security Administration

1 Uncontrolled)

Nevada Operations Office

Public Reading Facility

P.O. Box 98521, M/S NLV040

Las Vegas, NV 89193-8521

U.S. Department of Energy

1 (Uncontrolled

National Nuclear Security Administration

Nevada Operations Office

Technical Information Resource Center

P.O. Box 98518, M/S 505

Las Vegas, NV 89193-8518

U.S. Department of Energy

Office of Scientific and Technical Information

1 (Uncontrolled \&

P.O. Box 62

Oak Ridge, TN 37831-0062

\section{Bechtel Nevada}

Correspondence Control

1 (Uncontrolled)*

Bechtel Nevada

P.O. Box 98521, M/S NLV008

Las Vegas, NV 89193-8521

Environmental Management Library

1 (Uncontrolled)*

Bechtel Nevada

P.O. Box 98521, M/S NLV080

Las Vegas, NV 89193-8521

Kevin Campbell

1 (Uncontrolled)*

Bechtel Nevada

P.O. Box 98521, M/S NTS306

Las Vegas, NV 89193-8521 


\section{DISTRIBUTION LIST (continued)}

\section{Bechtel Nevada (continued)}

Tom Fitzmaurice

1 (Uncontrolled)*

Bechtel Nevada

P.O. Box 98521, M/S NTS306

Las Vegas, NV 89193-8521

Ann Heidema

1 (Uncontrolled)

Bechtel Nevada

P.O. Box 98521, M/S NLV022

Las Vegas, NV 89193-8521

Wayne Johnson

1 (Uncontrolled)*

Bechtel Nevada

P.O. Box 98521, M/S NTS306

Las Vegas, NV 89193-8521

Steve Nacht

1 (Uncontrolled)*

Bechtel Nevada

P.O. Box $98521, \mathrm{M} / \mathrm{S}$ NTS306

Las Vegas, NV 89193-8521

\section{IT Corporation}

Lynn Kidman

IT Corporation

1 (Uncontrolled)*

P.O. Box 93838, M/S 439

Las Vegas, NV 89193-8521

Garry Romano

1 (Controlled)

IT FFACO Support Office

IT Corporation

P.O. Box 93838, M/S 439

Las Vegas, NV 89193-8521

\section{State of Nevada}

Manager, Northern Nevada 1 (Uncontrolled) FFACO Public Reading Room

Nevada State Library and Archives Federal Publications 100 North Stewart Street

Carson City, NV 89701-4285 
